\title{
ZUR SEQUENTIELLEN UMWANDLUNG \\ VON BICYCLOBUTYLIDENEN IN BICYCLOOCTENE: SYNTHESE UND UMLAGERUNG FUNKTIONALISIERTER SPIRO[3.4]OCTANE
}

\author{
DISSERTATION \\ Zur Erlangung des Doktorgrades \\ der Mathematisch-Naturwissenschaftlichen Fakultäten \\ der Georg-August-Universität zu Göttingen
}

\author{
vorgelegt von \\ Imelda Meyer \\ aus Emstek
}

Göttingen 2001 
D7

Referent: Prof. Dr. L. Fitjer

Korreferent: Prof. Dr. A. de Meijere

Tag der mündlichen Prüfung: 26.06.2001 
Die vorliegende Arbeit wurde unter Leitung von Herrn Prof. Dr. L. Fitjer in der Zeit von April 1999 bis April 2001 am Organisch-Chemischen Institut der Georg-August-Universität zu Göttingen durchgeführt.

An dieser Stelle möchte ich Herrn Prof. Dr. L. Fitjer für die Anregung zu diesem Thema, sein stetes Interesse am Fortgang der Arbeit, die ausgezeichneten Arbeitsbedingungen und zahlreichen fördernden Diskussionen danken. 
Meinen Eltern 


\section{INHALTSVERZEICHNIS}

EINLEITUNG 1

ALLGEMEINER TEIL

1 Zur sequentiellen Transformation von unsubstituiertem Bicyclobutyliden: 9

Synthese und Umlagerung mono-bis trimethylierter Spiro[3.4]octanole

1.1 Synthese von 5-Methyl-spiro[3.4] octan-5-ol (21), 6-Methyl-spiro- $\quad 10$ [3.4] octan-5-ol (22a,b), 5,6-Dimethyl-spiro[3.4] octan-5-ol (23a,b), 6,6-Dimethyl-spiro[3.4]octan-5-ol (24) und 5,6,6-Trimethyl-spiro[3.4] octan-5-ol (25)

1.2 Säurekatalysierte Umlagerung von 21, 22a, 22b, 23a, 23b, 24 und 14 25: 3a-Methyl-1,2,3,3a,4,5-hexahydro-pentalen (56), 6a-Methylhexahydro-pentalen-3a-ol (57), (3aR*,6aR*)-6-Methyl-1,2,3,3a,4,6ahexahydro-pentalen (58), (3aR*,6aR*)-Dimethyl-1,2,3, 3a,4,6a-hexahydro-pentalen $(\mathbf{5 9}),\left(3 \mathrm{aR}^{*}, 6 \mathrm{aR} *\right)-6,6 \mathrm{a}-\mathrm{Dimethyl}-1,2,3,3 \mathrm{a}, 4,6 \mathrm{a}-\mathrm{hexa}$ hydro-pentalen $(\mathbf{6 0})$ und (3aR,6aS*)-Trimethyl-1,2,3,3a,4,6a-hexahydro-pentalen (61)

2 Zur sequentiellen Transformation substituierter Bicyclobutylidene: Versuche zur Synthese von Partialstrukturen der Sesquiterpene ( \pm )-Ceratopicanol (32) und ( \pm )-Ptychanolid (33)

2.1 Synthese und sequentielle Transformation von 2,2-Dimethyl-bicyclo18 butyliden zu 1,1-Dimethyl-spiro[3.4]octan-5-ol (27) und 8,8-Dimethylspiro[3.4] octan-5-on (67)

2.2 Selektive Darstellung von 1,1-Dimethyl-spiro[3.4] octan-5-on (27) und 8,8-Dimethyl-spiro[3.4]octan-5-on (67) über $\beta$-Hydroxyselenide

2.2.1 Darstellung von 1,1-Dimethyl-spiro[3.4]octan-5-on (27) 
2.3 Säurekatalysierte Umlagerung von 27, 51 und 67: 3a,6a-Dimethylhexahydro-pentalen-1-on (30)

3 Zur sequentiellen Transformation in komplexe Systeme eingebundener

Bicyclobutylidene: Synthese und Umlagerung potentieller Vorläufer von

(士)-endo-Capnellen (39)

3.1 (1R*,2S*,5R*)-7-Cyclobutyliden-2-methyl-bicyclo[3.2.0]heptan

(40): Darstellung, Epoxidierung, Umlagerung und erschöpfende Methylierung

3.2 Synthese von $\left(1 \mathrm{R}^{*}, 4 \mathrm{~S}^{*}, 5 \mathrm{R}^{*}, 6 \mathrm{R}^{*}, \mathrm{2}^{\prime} \mathrm{R}^{*}\right)-(\mathbf{4 1}),\left(1 \mathrm{R}^{*}, 4 \mathrm{~S}^{*}, 5 \mathrm{R}^{*}, 6 \mathrm{R}^{*}\right.$, $\left.2^{\prime} \mathrm{S}^{*}\right)-(42),\left(1 \mathrm{R}^{*}, 4 \mathrm{~S}^{*}, 5 \mathrm{R}^{*}, 6 \mathrm{~S}^{*}, 2^{\prime} \mathrm{R}^{*}\right)-(44)$ und $\left(1 \mathrm{R}^{*}, 4 \mathrm{~S}^{*}, 5 \mathrm{R}^{*}, 6 \mathrm{~S}^{*}\right.$, $\left.2^{\prime} \mathrm{S}^{*}\right)-4,2^{\prime}, 3^{\prime}, 3^{\prime}$-Tetramethyl-spiro $\{$ bicyclo[3.2.0]heptan-6,1'-cyclopentan-2'-ol\} (45)

3.3 Synthese von $\left(1 R^{*}, 4 S^{*}, 5 R^{*}, 6 S^{*}\right)-(\mathbf{1 0 3})$ und $\left(1 R^{*}, 4 S^{*}, 5 R^{*}, 6 R^{*}\right)-$ 36 $2^{\prime}$-Methylen-4,3',3’-trimethyl- spiro\{ bicyclo[3.2.0] heptan-6,1'cyclopentan\} (104)

3.4 Säurekatalysierte Umlagerung der Alkohole 41, 42, 44 und 45 sowie 38 der Olefine 103 und 104 zu linearen Triquinanen

3.4.1 Produkte und zeitlicher Verlauf der Umlagerungen von 41, 42 und 104

3.4.2 Produkte und zeitlicher Verlauf der Umlagerungen von 44, 45 und 103

ZUSAMMENFASSUNG

Allgemeine Angaben

Arbeitsvorschriften 
$\begin{array}{ll}\text { IR-Spektren } & 96\end{array}$

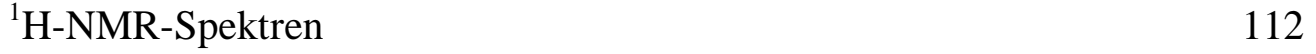

${ }^{13}$ C-NMR-Spektren $\quad 134$

ABBILDUNGSVERZEICHNIS 182

LITERATURVERZEICHNIS 190 


\section{EINLEITUNG}

Die säurekatalysierte Umlagerung von Bicyclobutyl-1-olen und Bicyclobutylidenen [2(4)-5-6-3] ist eine potentiell wertvolle Methode zum Aufbau von Bicyclo[3.3.0]octenen ${ }^{1}$ (Schema 1). Allerdings können substituierte Vertreter aufgrund der Bildungsweise der benötigten Edukte [1-2(4)] nur über substituierte Cyclobutanone und/oder substituierte Wittigbzw. Grignard-Reagenzien dargestellt werden.

\section{Schema 1}

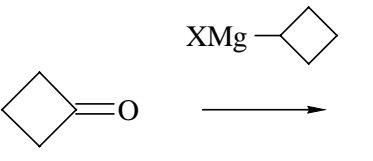

1

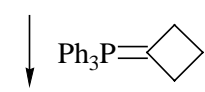

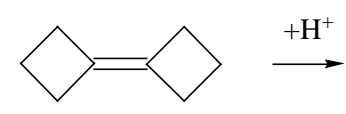

4
Bicyclo[3.3.0]octene

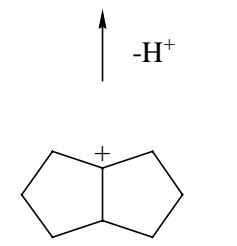

3

2

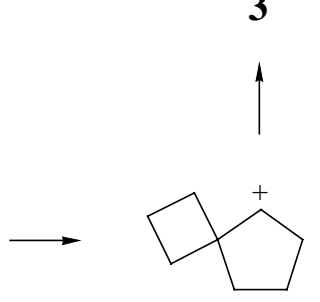

6

Substituierte Cyclobutanone sind in großer Zahl verfügbar ${ }^{2}$, auf substituierten Cyclobutanen basierende Wittig- und Grignard-Reagenzien dagegen sind rar. Bisher einzige Vertreter sind die in der Abteilung entwickelten Reagenzien $\mathbf{7}^{3}, \mathbf{8}^{4}$ und $\mathbf{9}^{5}$, sowie 10-12 ${ }^{6}$ (Schema 2). Von diesen wurden 7-9 zu Naturstoffsynthesen der Sesquiterpene $\left( \pm\right.$ )-Ceratopicanol ${ }^{7},( \pm)$ Hirsuten $^{7},( \pm)$-Cerapicol ${ }^{4},( \pm)$-Cuparen ${ }^{5}$ und $( \pm)$-Herberten ${ }^{5}$ genutzt. Dagegen schlossen mangelnde Selektivität $(\mathbf{1 0}, \mathbf{1 1})$ bzw. mangelnde Reaktivität (12) eine Verwendung von 10-12 für ähnliche Zwecke aus ${ }^{6}$. Es wäre daher von Vorteil, Substituenten auf andere Weise als über Wittig- oder Grignard-Reagenzien einführen zu können. 


\section{Schema 2}

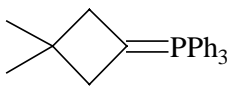

7

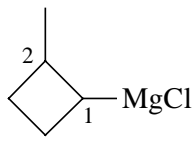

10a $1 R^{*}, 2 S^{*}$

b $1 R^{*}, 2 R^{*}$

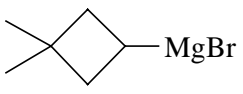

8

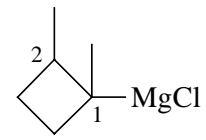

11a $1 R^{*}, 2 S^{*}$

b $1 R^{*}, 2 R^{*}$

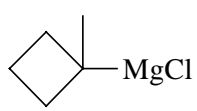

9

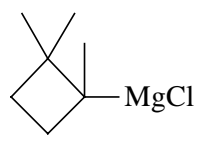

12

Eine Möglichkeit hierzu ist eine sequentielle Umwandlung von Bicyclobutylidenen in Bicyclooctene unter zwischenzeitlicher Einführung von Substituenten. Hierzu bietet sich an, das Bicyclobutyliden zunächst durch Epoxidierung und Umlagerung in ein Spiro[3.4]octan-5on zu überführen (4-13-14) und dieses dann über Alkylierungs- und/oder Additionsreaktionen so zu modifizieren, daß nach einer zweiten Umlagerung das gewünschte Substitutionsmuster resultiert (14-15-16-17) (Schema 3).

\section{Schema 3}

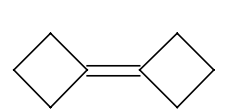

4

MCPB
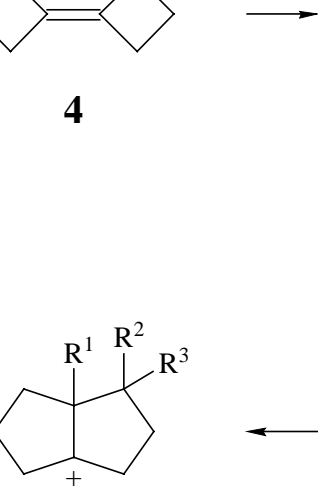

17

$\downarrow-\mathrm{H}^{+}$

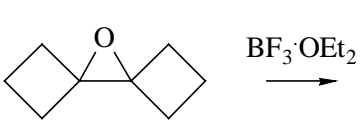

13

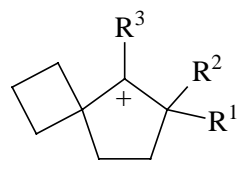

16

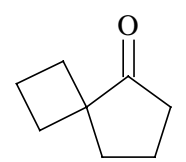

14 \begin{tabular}{l}
$+\mathrm{H}^{+}$ \\
$-\mathrm{H}_{2} \mathrm{O}$ \\
\hline
\end{tabular}

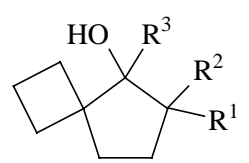

15

$\mathrm{R}^{1}, \mathrm{R}^{2}, \mathrm{R}^{3}=\mathrm{H}$, alkyl

Bicyclo[3.3.0]octene 
Die Epoxidierung und Umlagerung des Grundkörpers 4 zu Spiro[3.4]octan-5-on (14) ${ }^{8}$ (4-13-14) sowie die Umlagerung einiger von 14 abgeleiteter Derivate waren bekannt ${ }^{9-14}$. Eindrucksvollstes Beispiel ist die Umlagerung des Dispiro[3.0.4.2]undecan-6-ols 18 zu den Sesquiterpenen Modhephen (19) und Isocomen (20) ${ }^{11-14}$ (Schema 4). Gleichwohl stand eine sysstematische Untersuchung des Synthesepotentials sequentieller Transformationen von Bicyclobutylidenen in Bicyclo[3.3.0]octene noch aus. Im ersten Teil der vorliegenden Arbeit wurde versucht, diese Lücke zu schließen.

\section{Schema 4}

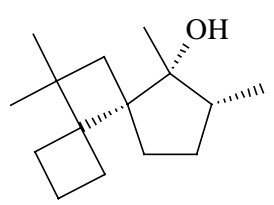

18
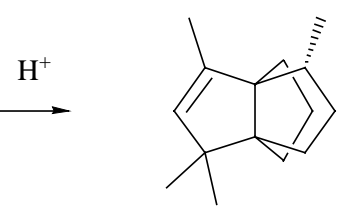

19

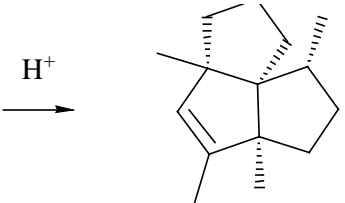

20

Hierzu war geplant, Spiro[3.4]octan-5-on (14) durch Standardreaktionen in die monobis trimethylierten Spiro[3.4]octan-5-ole 21-25 zu überführen und diese dann säurekatalysiert in Bicyclo[3.3.0]octene umzulagern (Schema 5). Hierbei rechneten wir in allen Fällen mit eindeutigen Reaktionsabläufen.

\section{Schema 5}

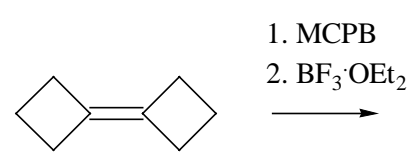

4

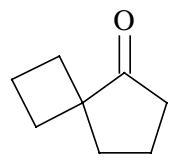

14
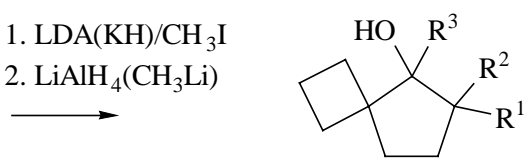

\begin{tabular}{l|lll} 
& $\mathrm{R}^{1}$ & $\mathrm{R}^{2}$ & $\mathrm{R}^{3}$ \\
\hline $\mathbf{2 1}$ & $\mathrm{H}$ & $\mathrm{H}$ & $\mathrm{CH}_{3}$ \\
$\mathbf{2 2}$ & $\mathrm{H}$ & $\mathrm{CH}_{3}$ & $\mathrm{H}$ \\
$\mathbf{2 3}$ & $\mathrm{H}$ & $\mathrm{CH}_{3}$ & $\mathrm{CH}_{3}$ \\
$\mathbf{2 4}$ & $\mathrm{CH}_{3}$ & $\mathrm{CH}_{3}$ & $\mathrm{H}$ \\
$\mathbf{2 5}$ & $\mathrm{CH}_{3}$ & $\mathrm{CH}_{3}$ & $\mathrm{CH}_{3}$
\end{tabular}


Weiterhin sollte versucht werden, aus 2,2-Dimethylcyclobutanon (29) ${ }^{6}$ über eine Reaktionsfolge aus Cyclobutylidenierung, Epoxidierung, Oxaspirohexan-CyclopentanonUmlagerung, Monomethylierung und Addition von Methyllithium das tetramethylierte Spiro[3.4] octan-5-ol 28 darzustellen (29-26-27-28) und dieses säurekatalysiert in das tetramethylierte Bicyclo[3.3.0]octen $\mathbf{3 1}$ als potentiellen Vorläufer des Sesquiterpens ( \pm )-Ptychanolid $(\mathbf{3 3})^{15}$ umzulagern. Für dieses Sesquiterpen gibt es bis heute keine Synthese ${ }^{16}$. Darüber sollte auch das Keton 27 säurekatalysiert umgelagert werden. Hier erwarteten wir mit $\mathbf{3 0}$ die Bildung einer Partialstruktur des Sesquiterpens $( \pm)$-Ceratopicanol ${ }^{17}$ (32) (Schema 6).

\section{Schema 6}

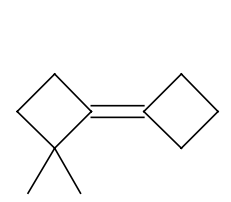

26

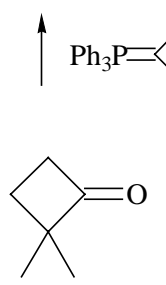

29

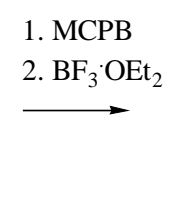

$$
\text { 西 }
$$$$
\text { (1) }
$$

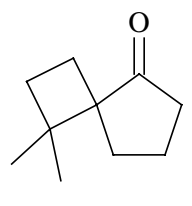

27

$\downarrow \mathrm{H}^{+}$

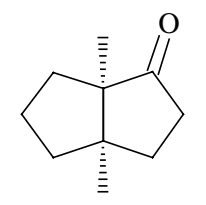

30
1. $\mathrm{LDA} / \mathrm{CH}_{3} \mathrm{I}$

2. $\mathrm{CH}_{3} \mathrm{Li}$

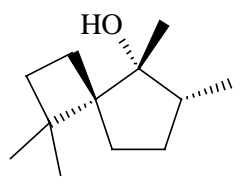

28

$\downarrow \mathrm{H}^{+}$

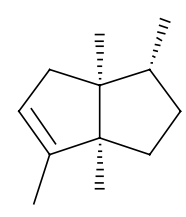

31

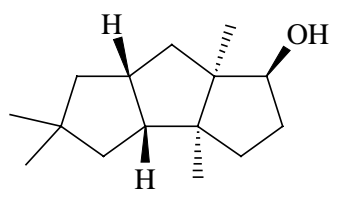

32 ( \pm -Ceratopicanol

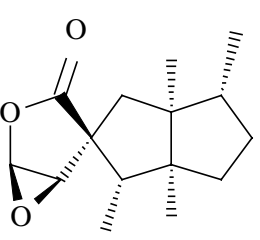

33 ( \pm )-Ptychanolid

Natürlich war es interessant, die Verwendbarkeit sequentieller Transformationen von Bicyclobutylidenen auch an komplexeren Systemen zu erproben. Hier war aus einer früheren Arbeit bekannt ${ }^{6}$, daß die aus dem Bicyclobutyliden $\mathbf{3 4}$ in vier Stufen zugänglichen Alkohole 35 und 36 bei Behandlung mit Säure unter thermodynamischer Kontrolle in identische Gemische 
des tricyclopentanoiden Sesquiterpens $( \pm)$-endo-Capnellen ${ }^{18}(39)$ mit seinen Doppelbindungsisomeren 37 und 38 übergehen (Schema 7).

\section{Schema 7}

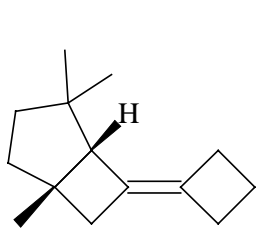

34
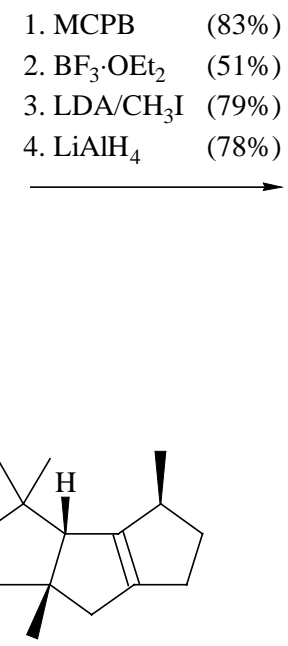

$37(33 \%)$

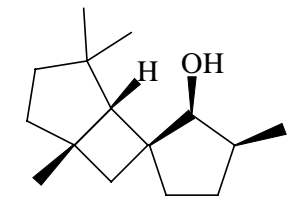

$35(49 \%)$
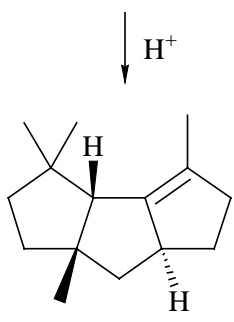

$38(25 \%)$

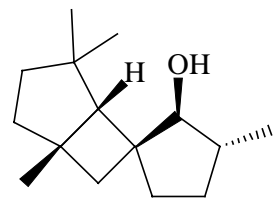

$36(29 \%)$
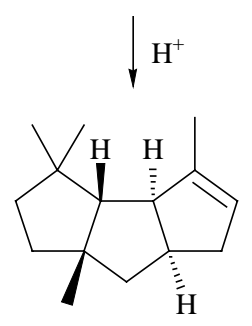

$39(42 \%)$

$( \pm)$-endo-Capnellen

Eine Umlagerung zu demselben Gemisch schien auch bei Alkoholen mit einer gegenüber 35 und 36 spezifisch veränderten Stellung der Methylgruppen möglich. So hatte eine Analyse potentieller Umlagerungswege zu ( \pm )-endo-Capnellen (39) mit unserem Retrosyntheseprogramm CARESY ${ }^{19}$ in Verbindung mit $\mathrm{HUNTER}^{20}$ und $\mathrm{MM}^{21}$ ergeben, daß das tricyclische Kation 47 über eine 1,3-Hydridverschiebung mit anschließender Deprotonierung unmittelbar zu 38 und 39, und damit mittelbar auch zu 37 führen konnte.

Denkbare Edukte für eine Erzeugung von 47 waren die Alkohole 41, 42, 44 und 45, und die Bedingung für einen Erfolg eine regio- und stereospezifische Ringerweiterung zu 46 mit anschließender Methylverschiebung. Aussichtsreichste Kandidaten unter Freie-IonenBedingungen waren damit $\mathbf{4 4}$ und $\mathbf{4 5}$, die allerdings wegen der Stereochemie der im Zuge ihrer Synthese notwendigen Epoxidierung und der sich anschließenden OxaspirohexanCyclopentanon-Umlagerung nur als Minoritätsprodukte zu erwarten waren. Trotz dieses Risikos sollte $\mathbf{4 3}^{22}$ cyclobutylideniert ${ }^{23}$ und das zu erwartende Bicyclobutyliden $\mathbf{4 0}$ einer sequenti- 
ellen Transformation zu den Alkoholen 41, 42, 44 und 45 unterworfen werden (Schema 8). Ferner sollten die den Alkoholen zugrunde liegenden Ketone methyleniert ${ }^{23}$ und die Methylenierungsprodukte ebenso wie die Alkohole säurekatalysiert umgelagert werden. Ziel war, in beiden Fällen zu klären, in welcher Weise die Stereochemie der Edukte die Struktur und Stereochemie der Produkte bestimmt. Im günstigsten Fall erwarteten wir eine Bildung von 37, 38 und 39, in jedem Fall aber eine Bildung von Triquinanen.

\section{Schema 8}

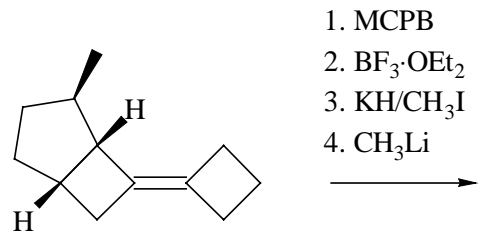

40

$\uparrow \mathrm{Ph}_{3} \mathrm{P}=\bigcirc$

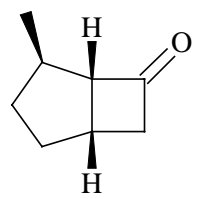

43

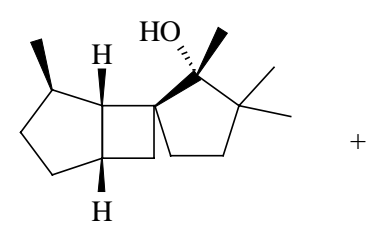

41

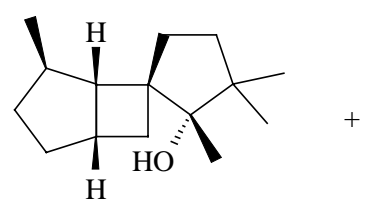

44

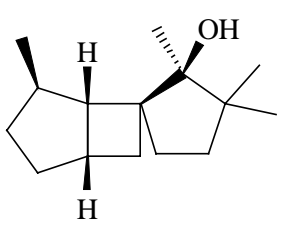

42

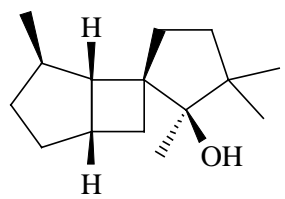

45 $\downarrow \mathrm{C}_{4}-\mathrm{C}_{5}$
46
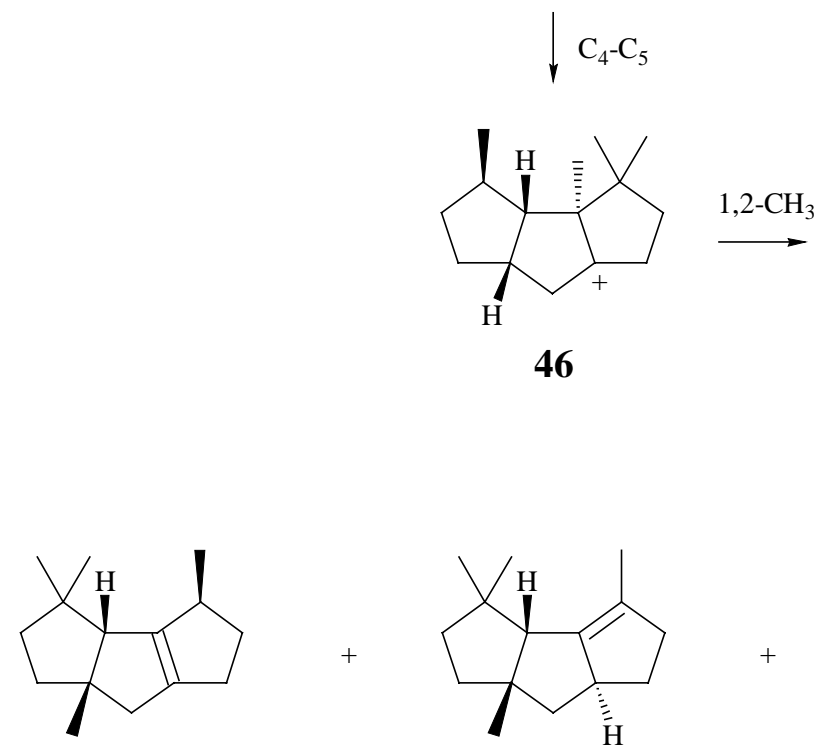

37

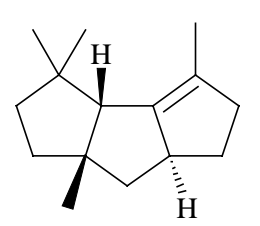

38

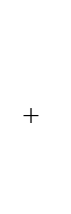

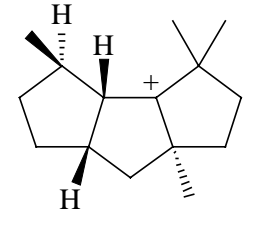

47

$\sqrt{-\mathrm{H}^{+}}$

77

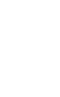


Wie später gezeigt werden wird (vgl. 1), verlief die Synthese und Umlagerung der mono- bis trimethylierten Spiro[3.4] octan-5-ole 21-25 problemlos. Dabei kam es in allen Fällen zu der erwartet einheitlichen Produktbildung. In Fällen, in denen bekannte Bicyclo[3.3.0]octene gebildet wurden, erwiesen sich die neuen Synthesen den bisher bekannten überlegen.

Schwierigkeiten ergaben sich bei der Synthese des Bicyclobutylidens 26 sowie, nach dessen Epoxidierung, bei der sich anschließenden Oxaspirohexan-Cyclopentanon-Umlagerung zu 27 als Vorläufer des tetramethylierten Spiro[3.4]octan-5-ols 28 (vgl. 2). Hier war eine einheitliche Produktbildung nur über eine durch m-Chlorperbenzoesäure bewirkte Umlagerung des $\beta$-Hydroxyselenids 49 zu erreichen (29-48-49-27) ${ }^{24}$ (Schema 9). Aber auch dann waren die Ausbeuten zu gering, um eine Fortführung der Synthese zu 28 zu erlauben.

\section{Schema 9}

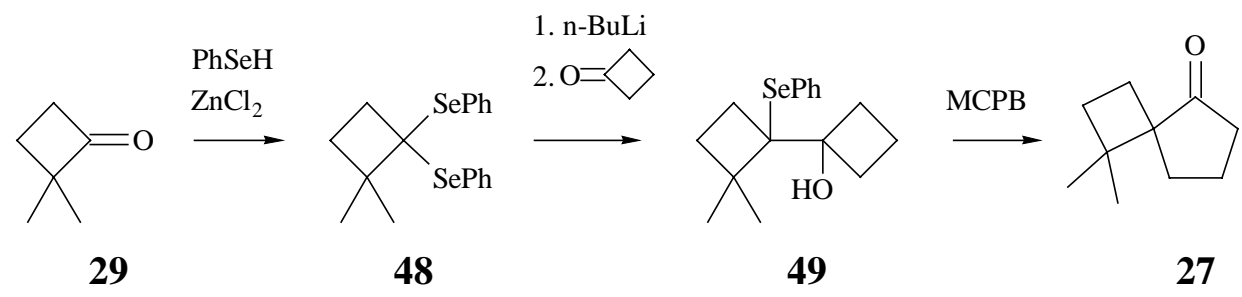

Die Versuche zur sequentiellen Transformation des Bicyclobutylidens 40 in ( \pm )-endoCapnellen (39) (vgl. 3) lieferten ein komplexes Bild. Bereits die OxaspirohexanCyclopentanon-Umlagerung nahm nicht den erwarteten Verlauf und lieferte drei statt zwei Ketone. Gleichwohl wurden die Synthesen bis zur Stufe der Alkohole 41, 42, 44 und 45 sowie der zugehörigen Olefine fortgeführt. Leider gelang es in keinem Fall, für eine kristallographische Untersuchung geeignete Kristalle zur Klärung der Struktur und Stereochemie zu erhalten. Gleiches gilt für die Umlagerungsprodukte. Die angegebenen Strukturen waren daher in vielen Fällen nur durch umfangreiche NMR-Untersuchungen zu ermitteln und müssen als vorläufig gelten. In keinem Fall bildete sich das erwünschte ( \pm )-endo-Capnellen (39). 
Insgesamt wird die vorliegende Arbeit zeigen, daß eine sequentielle Transformation von Bicyclobutylidenen über Spiro[3.4]octan-5-ole in übersichtlich gelagerten Fällen anderen Methoden zur Darstellung von Bicyclo[3.3.0]octenen überlegen ist. In komplexeren Fällen, insbesondere bei Einbindung des Bicyclobutylidens in höher kondensierte Systeme, ist das Risiko eines Auftretens von der Erwartung abweichender Umlagerungen dagegen erheblich. 


\section{ALLGEMEINER TEIL}

\section{Zur sequentiellen Transformation von unsubstituiertem Bicyclobutyliden: Syn-} these und Umlagerung mono- bis trimethylierter Spiro[3.4]octan-5-ole

Die säurekatalysierte Umlagerung von Bicyclobutyl-1-olen und Bicyclobutylidenen ist eine potentiell wertvolle Methode zum Aufbau von Bicyclo[3.3.0]octenen. Nachteilig ist, daß substituierte Vertreter aufgrund der Bildungsweise der benötigten Edukte nur über substituierte Cyclobutanone und/oder substituierte Wittig- bzw. Grignard-Reagenzien zugänglich sind. Insbesondere bei letzteren ist die Auswahl beschränkt ${ }^{4,5,6}$. Als Alternative bietet sich an, das Bicyclobutyliden 4 zunächst durch Epoxidierung und Umlagerung in ein Spiro[3.4]octan-5-on zu überführen (4-13-14) und dieses dann durch Alkylierungs- und/oder Additionsreaktionen so zu modifizieren, daß nach einer zweiten Umlagerung das gewünschte Substitutionsmuster resultiert (14-15-16-17) (Schema 10).

\section{Schema 10}

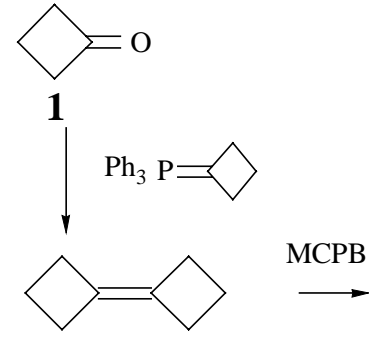

4

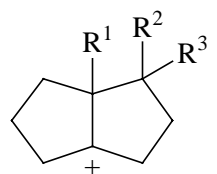

17

$-\mathrm{H}^{+}$

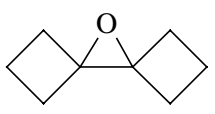

13

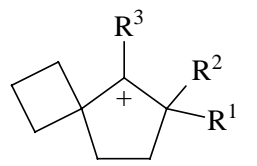

16

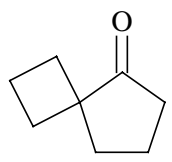

$14 \mathrm{~B} / \mathrm{RX}$

und/oder
$\mathrm{H}^{-} / \mathrm{R}^{-}$

$\stackrel{+\mathrm{H}^{+}}{-\mathrm{H}_{2} \mathrm{O}}$

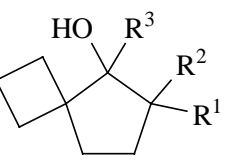

15

$\mathrm{R}^{1}, \mathrm{R}^{2}, \mathrm{R}^{3}=\mathrm{H}$, alkyl

Bicyclo[3.3.0]octene 
Im ersten Teil der vorliegenden Arbeit sollte das Synthesepotential sequentieller Transformationen von unsubstituiertem Bicyclobutyliden $^{25}$ (4) in substituierte Bicyclo[3.3.0]octene durch Synthese und Umlagerung einer Reihe bisher unbekannter mono- bis trimethylierter Spiro[3.4] octan-5-ole 15 geprüft werden. Das hierzu benötigte Spiro[3.4]octan5-on ${ }^{26}$ (14) wurde auf bekanntem Weg durch Epoxidierung von Bicyclobutyliden (4) und anschließender Oxaspirohexan-Cyclopentanon-Umlagerung dargestellt ${ }^{8}$.

\subsection{Synthese von 5-Methyl-spiro[3.4]octan-5-ol (21), 6-Methyl-spiro[3.4]octan- 5-ol (22a,b), 5,6-Dimethyl-spiro[3.4]octan-5-ol (23a,b), 6,6-Dimethyl-spiro[3.4]- octan-5-ol (24) und 5,6,6-Trimethyl-spiro[3.4]octan-5-ol (25)}

Zur Darstellung des monomethylierten Alkohols 5-Methylspiro[3.4]octan-5-ol (21) wurde Spiro[3.4] octan-5-on (14) mit einem Moläquivalent einer 0.5 M Lösung von Methyllithium in Ether 15 min bei $0{ }^{\circ} \mathrm{C}$ gehalten. Aufarbeitung und Chromatographie lieferten den gewünschten Alkohol in einer Ausbeute von 66\% (Schema 11). Das ${ }^{1} \mathrm{H}-\mathrm{NMR}-\mathrm{Spektrum}$ (Abb. 21) zeigt bei $\delta=1.21 \mathrm{ppm}$ das erwartete Singulett für die eingeführte Methylgruppe. Das ${ }^{13} \mathrm{C}$ NMR-Spektrum (Abb. 88) belegt mit Resonanzen bei $\delta=22.59\left(\mathrm{C}_{\text {prim }}\right)$ und $80.31\left(\mathrm{C}_{\text {quart }}\right)$ sowohl die Einführung einer Methylgruppe als auch das Vorliegen eines tertiären Alkohols.

Zur Darstellung der ebenfalls monomethylierten Alkohole (5R*,6R*)-6-Methyl-spiro[3.4] octan-5-ol (22a) und (5S*,6R*)-6-Methyl-spiro[3.4]octan-5-ol (22b) mußte 14 zuerst monomethyliert und dann reduziert werden (Schema 11). Dabei erwies sich als vorteilhaft, zur Generierung des Lithiumenolats genau ein Moläquivalent Lithiumdiisopropylamin (LDA) zu verwenden. Unter diesen Bedingungen war der Umsatz zwar unvollständig, dafür war der Anteil an dimethyliertem Produkt gering. Ein präparativer Ansatz mit je einem Moläquivalent LDA und 14 und 5 Moläquivalenten Methyliodid enthielt laut gaschromatographischer Analyse nach 2.5 h bei $-25{ }^{\circ} \mathrm{C}$ neben $8 \%$ Ausgangsprodukt 89\% 6-Methyl- (50) und 3\% 6,6-Dimethylspiro[3.4] octan-5-on (51). Aufarbeitung und Chromatographie lieferte das gewünschte $\mathbf{5 0}$ in einer Ausbeute von $80 \%$. Daß es sich bei dem isolierten Produkt tatsächlich um $\mathbf{5 0}$ handelte, machte bereits das ${ }^{1} \mathrm{H}-\mathrm{NMR}-$ Spektrum (Abb. 44) deutlich. Es zeigt für die eingeführte Methylgruppe ein Dublett bei $\delta=1.06 \mathrm{ppm}$. 
Schema 11

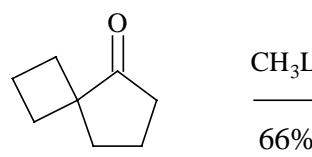

14
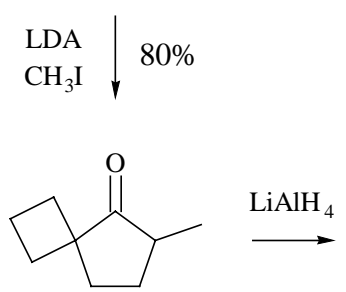

50

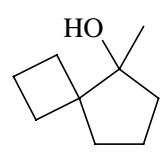

21

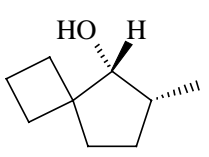

22a $\left(5 R^{*}, 6 R^{*}, 55 \%\right)$

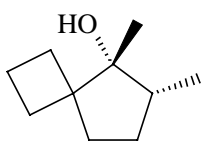

23a $\left(5 R^{*}, 6 R^{*}, 59 \%\right)$

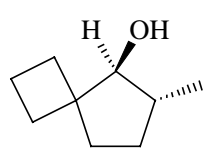

22b $\left(5 S^{*}, 6 R^{*}, 9 \%\right)$

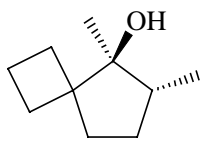

23b $\left(5 S^{*}, 6 R^{*}, 7 \%\right)$

Zur Reduktion wurde das monomethylierte Keton 50 mit zwei Moläquivalenten Lithiumaluminiumhydrid in Ether solange unter Rückfluß gekocht, bis eine begleitende gaschromatographische Analyse vollständigen Umsatz anzeigte $(1 \mathrm{~h})$. Aufarbeitung und Chromatographie lieferte zwei stereoisomere Alkohole in isolierten Ausbeuten von 55 bzw. 9\%. Beide Verbindungen zeigten in ihren IR-Spektren (Abb. 15 und 16) Banden für freie $\left[3620 \mathrm{~cm}^{-1}\right.$ (22a und 22b)] und assoziierte OH-Valenzschwingungen [3600-3140 (22a) und 3580-3080 $\mathrm{cm}^{-1}$ (22b)] und in ihren ${ }^{13} \mathrm{C}-\mathrm{NMR}-$ Spektren (Abb. 91 und 92) Resonanzen für sekundäre Alkohole $\left[\delta=82.19\left(\mathrm{C}_{\text {tert }}\right)(\mathbf{2 2 a})\right.$ und $\left.85.33\left(\mathrm{C}_{\text {tert }}\right)(\mathbf{2 2 b})\right]$. Zur stereochemischen Zuordnung wurde der $\gamma$ gauche-Effekt ${ }^{27}$, d.h. der Hochfeldshift der ${ }^{13}$ C-Resonanzen von 1,2-cis-orientierten Substituenten herangezogen. Danach stehen die Hydroxyl- und Methylgruppe im Majoritätsalkohol 22a $\left[\delta=14.39\left(\mathrm{C}_{\text {prim }}\right)\right]$ cis, im Minoritätsalkohol 22b $\left[\delta=19.08\left(\mathrm{C}_{\text {prim }}\right)\right]$ trans.

Durch Addition von Methyllithium an das monomethylierte Keton 50 sollten nun die beiden dimethylierten Alkohole (5R*,6R*)-5,6-Dimethyl-spiro[4.3]octan-5-ol (23a) und (5S*,6R*)-5,6-Dimethyl-spiro[4.3]octan-5-ol (23b) dargestellt werden (Schema 11). Dazu 
wurde 50 mit einem Moläquivalent einer $0.5 \mathrm{M}$ Lösung von Methyllithium in Ether versetzt und 15 min bei $0{ }^{\circ} \mathrm{C}$ gehalten. Aufarbeitung und Chromatographie lieferte auch hier zwei stereoisomere Alkohole in isolierten Ausbeuten von 59 bzw. 7\%. Beide Verbindungen zeigten in ihren IR-Spektren (Abb. 13 und 14) Banden für freie [3610 $\mathrm{cm}^{-1}$ (23a und 23b)] und assoziierte OH-Valenzschwingungen [3590-3120 (23a) und 3600-3100 $\left.\mathrm{cm}^{-1}(\mathbf{2 3 b})\right]$, in ihren ${ }^{13} \mathrm{C}$ NMR-Spektren (Abb. 89 und 90) Resonanzen für tertiäre Alkohole $\left[\delta=81.19\left(\mathrm{C}_{\text {quart }}\right)(\mathbf{2 3 a})\right.$ und $\left.80.37\left(\mathrm{C}_{\text {quart }}\right)(\mathbf{2 3 b})\right]$.

Zur Klärung der Stereochemie wurden zunächst die Methylgruppen über ${ }^{13} \mathrm{C}-{ }^{1} \mathrm{H}-$ Korrelationen (Abb. 119 und 120) als gekoppelt oder ungekoppelt identifiziert. Anschließend wurde wiederum nach $\gamma$-gauche-Effekten abgefragt, diesmal allerdings nicht mit der gekoppelten, sondern mit der ungekoppelten Methylgruppe als stereochemischer Sonde. Dies war deswegen erforderlich, weil die gekoppelte Methylgruppe in beiden Konfigurationen einen $\gamma$ gauche-Effekt erfährt. Das Ergebnis war eindeutig: im Majoritätsalkohol 23a stehen die ungekoppelte $(\delta=20.70)$ und die gekoppelte Methylgruppe $(\delta=13.26)$ trans, im Minoritätsalkohol 23b stehen die ungekoppelte $(\delta=16.60)$ und die gekoppelte Methylgruppe $(\delta=14.79)$ cis. Die Selektivitäten bei der Addition von Hydrid und Methyl sind daher diegleichen. In beiden Fällen erfolgt die Übertragung bevorzugt von der sterisch weniger gehinderten Seite.

Zur Darstellung des dimethylierten Alkohols 6,6-Dimethyl-spiro[3.4]octan-5-ol (24) mußte das Keton 14 zunächst erschöpfend methyliert und dann reduziert werden (Schema 12). Dazu wurde 14 mit je drei Moläquivalenten Kaliumhydrid und Methyliodid in wasserfreiem Ether solange bei $0{ }^{\circ} \mathrm{C}$ gehalten, bis eine begleitende gaschromatographische Kontrolle vollständigen Umsatz anzeigte (45 min). Aufarbeitung und Destillation lieferten das gewünschte 6,6-Dimethyl-spiro[3.4]octan-5-on (51) in einer Ausbeute von 68\%. Daß es sich bei dem isolierten Produkt tatsächlich um $\mathbf{5 1}$ handelte, machte bereits das ${ }^{1} \mathrm{H}-\mathrm{NMR}$-Spektrum (Abb. 33) deutlich. Es zeigte für die neu eingeführten Methylgruppen ein Singulett bei $\delta=0.96 \mathrm{ppm}$. 


\section{Schema 12}

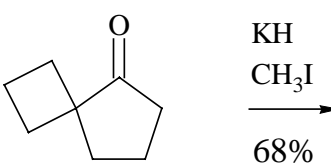

14

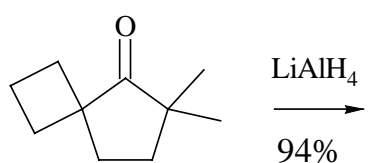

51

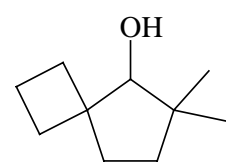

24

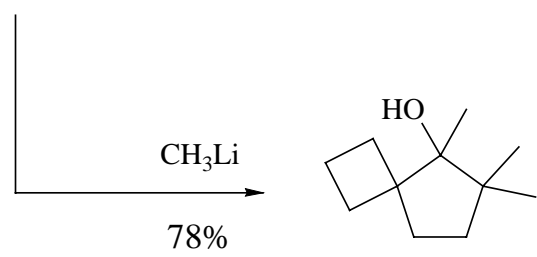

25

Zur Reduktion wurde $\mathbf{5 1}$ mit zwei Moläquivalenten Lithiumaluminiumhydrid in Ether solange unter Rückfluß erhitzt, bis eine gaschromatographische Kontrolle vollständigen Umsatz anzeigte $(1 \mathrm{~h})$. Aufarbeitung und Chromatographie lieferte das gewünschten Spiro[4.3]octan-5-ol 24 in einer Ausbeute von 94\%. Wie schon bei 22a,b zeigten sich auch bei 24 im IRSpektrum (Abb. 2) Banden für eine freie $\left(3620 \mathrm{~cm}^{-1}\right)$ sowie für assoziierte OH-Valenzschwingungen (3600-3120 $\mathrm{cm}^{-1}$ ) und im ${ }^{13} \mathrm{C}-\mathrm{NMR}$-Spektrum (Abb. 77) die Resonanz für einen sekundären Alkohol $\left[\delta=86.66\left(\mathrm{C}_{\text {tert }}\right)\right]$.

Die Umsetzung des Ketons 51 mit einem Moläquivalent einer 0.5 M Lösung von Methyllithium in Ether lieferte innerhalb $15 \mathrm{~min}$ bei $0{ }^{\circ} \mathrm{C}$ den trimethylierten Alkohol 5,6,6Trimethyl-spiro[3.4] octan-5-ol (25) (Schema 12). Nach Aufarbeitung und Chromatographie lag die isolierte Ausbeute bei 78\%. Auch hier finden sich im IR-Spektrum (Abb. 3) Banden für eine freie $\left(3620 \mathrm{~cm}^{-1}\right)$ sowie für assoziierte $\mathrm{OH}-$ Valenzschwingungen $\left(3600-3180 \mathrm{~cm}^{-1}\right)$. Das ${ }^{1}$ H-NMR-Spektrum (Abb. 35) zeigt u.a. drei Singuletts für die Methylgruppen $(\delta=0.78,0.92$, 1.06) und das ${ }^{13} \mathrm{C}$-NMR-Spektrum (Abb. 78) die Resonanz für einen tertiären Alkohol $[\delta=$ $\left.83.08\left(\mathrm{C}_{\text {quart }}\right)\right]$. 
1.2 Säurekatalysierte Umlagerung von 21, 22a, 22b, 23a, 23b, 24 und 25: 3a-Methyl1,2,3,3a,4,5-hexahydro-pentalen (56), 6a-Methyl-hexahydo-pentalen-3a-ol (57), (3aR*,6aR*)-6-Methyl-1,2,3,3a,4,6a-hexahydro-pentalen (58), (3aR*,6aR*)-3a,6Dimethyl-1,2,3,3a,4,6a-hexa-hydro-pentalen (59), (3aR*,6aR*)-6,6a-Dimethyl-1,

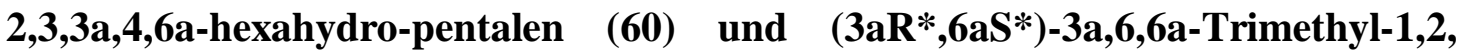

\section{3,3a4,6a-hexahydro-pentalen (61)}

Nachdem die mono- bis trimethylierten Spiro[3.4] octan-5-ole 21, 22a, 22b, 23a, 23b, 24 und 25 erfolgreich synthetisiert waren und in ausreichender Menge zur Verfügung standen, sollten sie säurekatalysiert umgelagert werden, um so das Potential einer sequentiellen Umwandlung von unsubstituiertem Bicyclobutyliden (4) in mono- bis trimethylierte Bicyclo[3.3.0]octene (Hexahydropentalene) auszuloten. Zur experimentellen Realisierung wurden die Spiro[3.4] octan-5-ole mit jeweils einem Moläquivalent einer 0.074-molaren Lösung von wasserfreier p-Toluolsulfonsäure in Benzol versetzt und $3 \mathrm{~h}$ bei $70{ }^{\circ} \mathrm{C}$ gehalten. Dabei bildete sich, außer bei 21, jeweils ein einziges Produkt. Die gebildeten Produkte wurden gaschromatographisch isoliert und spektroskopisch identifiziert.

\section{Schema 13}

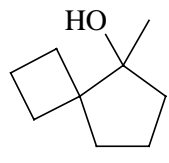

21
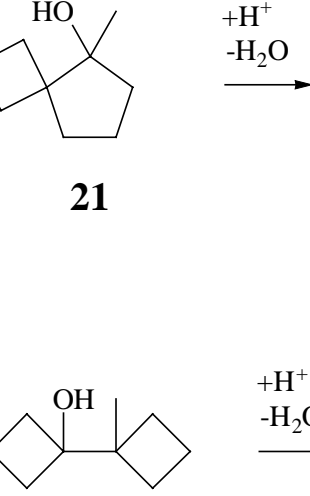

54

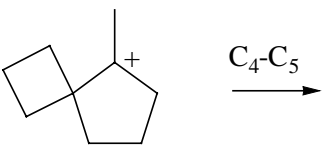

52

$\mathrm{C}_{4}-\mathrm{C}_{5}$

$\stackrel{+\mathrm{H}^{+}}{-\mathrm{H}_{2} \mathrm{O}}$
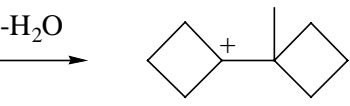

55

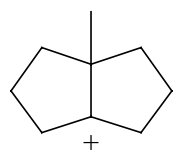

53
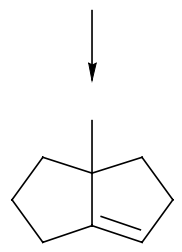

$56(94 \%)$

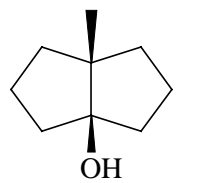

$57(6 \%)$ 
5-Methyl-spiro[3.4] octan-5-ol (21) lieferte als einziger Alkohol mit 3a-Methyl1,2,3,3a,4,5-hexahydro-pentalen ${ }^{5}$ (56) in 94\% Ausbeute ein Brückenkopfolefin und mit 6aMethyl-hexahyrdo-pentalen-3a-ol ${ }^{5}$ (57) in 6\% Ausbeute einen tertiären Alkohol (Schema 13). Dieses Ergebnis ist das gleiche wie bei der bereits früher untersuchten säurekatalysierten Umlagerung von 1'-Methyl-bicyclobutyl-1-ol ${ }^{5}(\mathbf{5 4})$ und war aufgrund ab 52 identischer Intermediate (21-52-53 bzw. 54-55-52-53) zu erwarten. Wahrscheinlich läßt sich, wie bei 54 beobachtet, die Bildung von 57 durch Umlagerung mit Thionylchlorid in Pyridin unterdrücken. Dieses wurde jedoch nicht erprobt.

Unabhängig von ihrer Stereochemie lieferten die Alkohole 22a und 22b sowie 23a und 23b jeweils dasselbe Produkt (Schema 14). Dabei handelte es sich nach Ausweis ihrer spektroskopischen Daten um die cis-konfigurierten Hexahydropentalene $\mathbf{5 8}^{28-32}$ bzw. 59 ${ }^{33-36}$, die ebenfalls bereits früher durch säurekatalysierte Umlagerung aus entsprechend substituierten Bicyclobutyl-1-olen erhalten worden waren ${ }^{5}$. Ob die Umlagerungen auf den angegebenen kürzesten Wegen erfolgen oder ob zwischenzeitlich Deprotonierungen und Reprotonierungen auftreten, muß offenbleiben. In jedem Fall scheint sicher, daß die Umlagerungen thermodynamisch kontrolliert ablaufen.

\section{Schema 14}

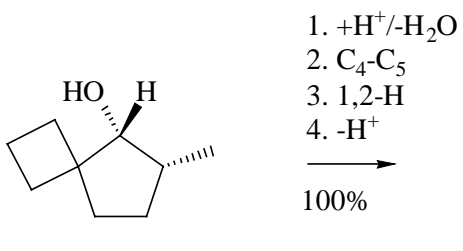

$22 a$

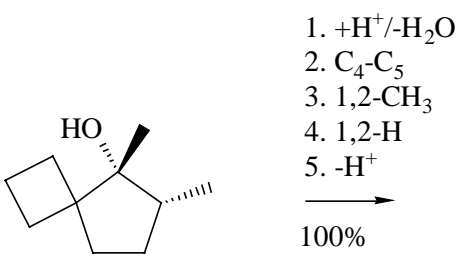

23a

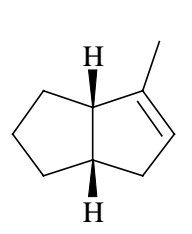

58

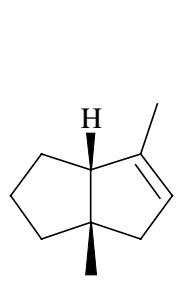

59

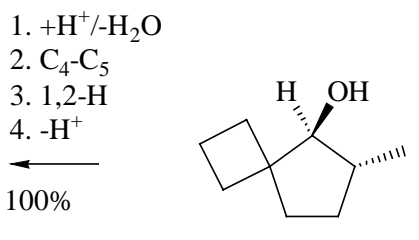

22b

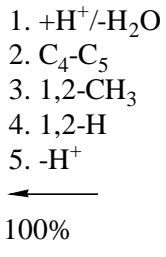

2. $\mathrm{C}_{4}-\mathrm{C}_{5}$

3. $1,2-\mathrm{CH}_{3}$

4. $1,2-\mathrm{H}$ $\leftarrow$

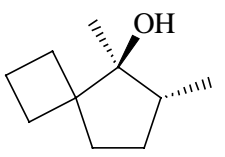

23b

Das dimethylierte Spiro[3.4]octan-5-ol 24 lieferte als einziger Alkohol ein neues Produkt. Hierbei handelte es sich nach Ausweis seiner spektroskopischen Daten um das Hexahy- 
dropentalen 60 (Schema 15). So zeigt sein ${ }^{1}$ H-NMR-Spektrum (Abb. 37) u.a. ein Singulett bei $\delta=1.05$ für eine Methylgruppe am Brückenkopf, ein bei $\delta=1.57$ zentriertes Multiplett für eine vinylisch gebundene Methylgruppe sowie ein breites Singulett bei $\delta=5.12$ für ein vinylisch gebundenes Proton. Auch das ${ }^{13} \mathrm{C}-\mathrm{NMR}-$ Spektrum (Abb. 80) ist mit der angegebenen Struktur vereinbar. Es zeigt die nach Anzahl und Lage zu erwartenden zehn Resonanzen für zwei primäre, vier sekundäre, zwei tertiäre und zwei quartäre Kohlenstoffatome, darunter die charakteristischen Resonanzen für ein trisubstituiertes Olefin $\left[\delta=122.30\left(\mathrm{C}_{\text {tert }}\right)\right.$ und 145.33 $\left.\left(\mathrm{C}_{\text {quart }}\right)\right]$.

\section{Schema 15}

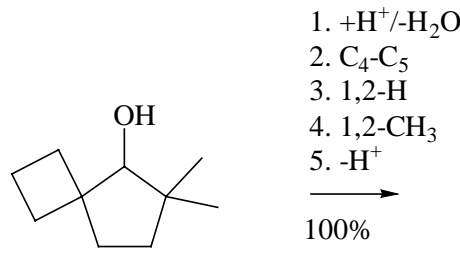

24

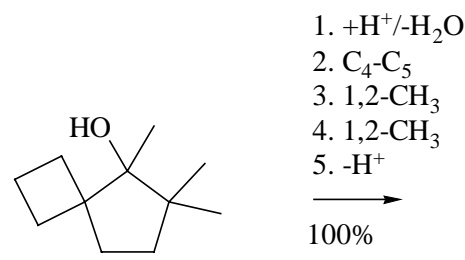

25

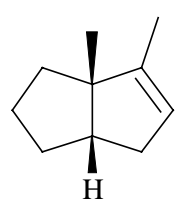

60

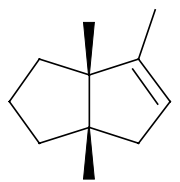

61

Das trimethylierte Spiro[3.4]octan-5-ol 25 lieferte in quantitativer Ausbeute das bekannte Hexahydropentalen 61. Seine spektroskopischen Daten stimmten mit Literaturdaten ${ }^{5}$ überein. Auch bei $\mathbf{2 4}$ und $\mathbf{2 5}$ ist nicht auszuschließen, daß die Umlagerungen nicht auf den angegebenen kürzesten Wegen, sondern unter zwischenzeitlicher Deprotonierung und Reprotonierung erfolgen. In jedem Fall sind sie auch hier thermodynamisch kontrolliert.

Insgesamt ist festzustellen, daß die sequentielle Transformation von unsubstituiertem Bicyclobutyliden (4) zu den mono- bis trimethylierten Bicyclo[3.3.0]octenen (Hexahydropentalenen) 56, 58, 59 und 61 der direkten Transformation entsprechender mono- bis trimethylierter Bicyclobutyl-1-ole überlegen ist. Der Grund dafür ist, daß die zur Umlagerung eingesetzten mono- bis trimethylierten Spiro[3.4] octan-5-ole 21, 22a,b, 23a,b und 25 leicht und mit 
Standardreagenzien darstellbar sind. Dieses gilt für die entsprechenden mono- bis trimethylierten Bicyclobutyl-1-ole nicht. Die Umlagerungen selbst verlaufen in beiden Fällen gleich effektiv.

\section{Zur sequentiellen Transformation substituierter Bicyclobutylidene: Versuche zur Synthese von Partialstrukturen der Sesquiterpene ( \pm )-Ceratopicanol (32) und ( \pm )-Ptychanolid (33).}

Wie unter 1 gezeigt, ist die sequentielle Transformation von unsubstituiertem Bicyclobutyliden (4) über Spiro[3.4]octan-5-on (14) eine präparativ einfache und ergiebige Methode zur Darstellung mono- bis trimethylierter Bicyclo[3.3.0]octene (Hexahydropentalene). Höher als trimethylierte Vertreter lassen sich mit $\mathbf{4}$ allerdings nicht darstellen. Hierzu benötigt man methylierte Bicyclobutylidene. Im zweiten Teil der vorliegenden Arbeit sollte daher versucht werden, das bisher unbekannte 2,2-Dimethyl-bicyclobutyliden (26) darzustellen und über eine Reaktionsfolge aus Epoxidierung, Oxaspirohexan-Cyclopentanon-Umlagerung, Monomethylierung und Addition von Methyllithium so zu modifizieren (26-27-28), daß aus einer abschlieBenden säurekatalysierten Umlagerung ein tetramethyliertes Bicyclo[3.3.0]octen resultieren mußte (Schema 16). Wunschprodukt war das all-cis-konfigurierte Bicyclo-[3.3.0]octen 31 als Partialstruktur und potentieller Vorläufer von $( \pm)$-Ptychanolid ${ }^{15}$. Für dieses Sesquiterpen gibt es bis heute keine Synthese ${ }^{16}$ (Schema 16).

\section{Schema 16}

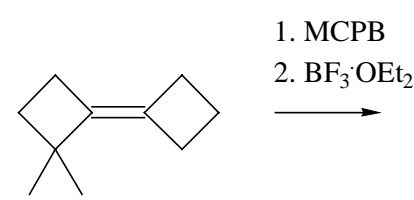

26

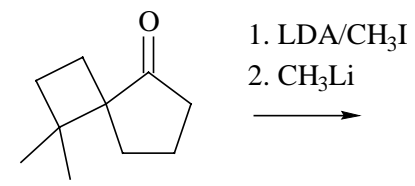

27

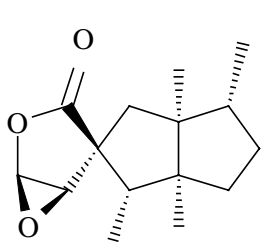

33 ( \pm )-Ptychanolid

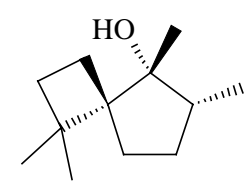

28
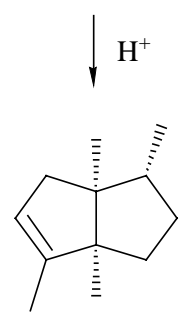

31 
Darüber hinaus sollte versucht werden, auch die Spiro[3.4]octan-5-one 27, 51 und 67 einer säurekatalysierten Umlagerung zu unterwerfen (Schema 17). Hier erwarteten wir eine Bildung des bicyclischen Ketons 30 als Partialstruktur des Sesquiterpens $\left( \pm\right.$ )-Ceratopicanol ${ }^{17}$ (32) und damit Hinweise auf dessen mögliche Synthese.

\section{Schema 17}

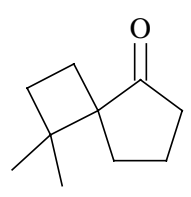

27
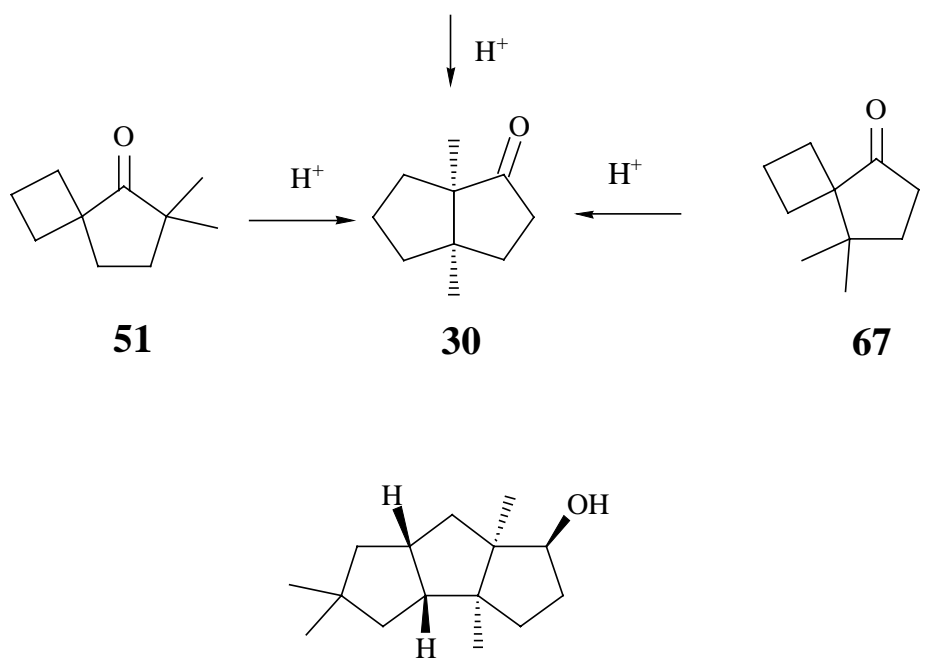

$32( \pm)$-Ceratopicanol

Wie nachstehend gezeigt werden wird, konnte die geplante Synthese nicht bis zur Stufe des Alkohols 28 gefördert werden. Dies lag an Schwierigkeiten bei der Darstellung von 27 (vgl. 2.1), die sich auch durch alternativ erprobte Wege über $\beta$-Hydroxyselenide (vgl. 2.2) nur teilweise beheben ließen. Die prospektierte Umlagerung von 27 gelang nicht, 30 ließ sich allerdings durch Umlagerung der Ketone 51 und $\mathbf{6 7}$ darstellen (vgl. 2.3). 


\subsection{Synthese und sequentielle Transformation von 2,2-Dimethyl-bicyclobutyliden} (26) zu 1,1-Dimethyl-spiro[4.3]octan-5-on (27) und 8,8-Dimethyl-spiro[4.3]octan5-on (67)

Die Synthese des zur sequentiellen Transformation zu 27 benötigten 2,2-Dimethylbicyclobutylidens (26) sollte durch Cyclobutylidenierung ${ }^{23}$ von 2,2-Dimethyl-cyclobutanon (29) erfolgen (Schema 18). Zur experimentellen Realisierung versetzte man eine aus 4Brombutyl-triphenylphosponiumbromid mit 2 Moläquivalenten Kalium-tert-butanolat generierte Suspension von Cyclobutyliden-triphenylphosphoran ${ }^{23}$ in Benzol bei $70^{\circ} \mathrm{C}$ mit $0.83 \mathrm{Mo}-$ läquivalenten $\mathbf{2 9}^{6}$ und verfolgte den Fortgang der Reaktion gaschromatographisch. Nach $3 \mathrm{~h}$ war 29 umgesetzt und ein $51: 17: 13$ Gemisch aus drei Produkten entstanden. Bei dem Hauptprodukt handelte es sich um das erwünschte 2,2-Dimethyl-bicyclobutyliden (26), bei den

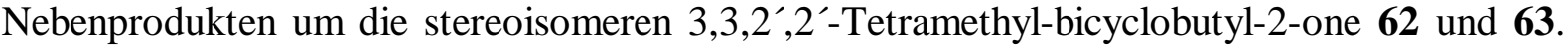
Der Versuch einer destillativen Trennung mißlang. Eine daraufhin durchgeführte chromatographische Trennung lieferte 26, 62 und $\mathbf{6 3}$ in Reinausbeuten von 20, 26 und 22\% bezogen auf eingesetztes Keton.

\section{Schema 18}

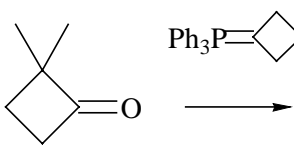

29

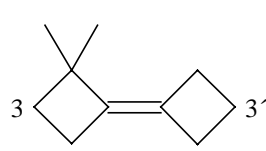

$26(20 \%)$

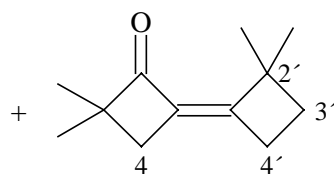

$62(26 \%)$

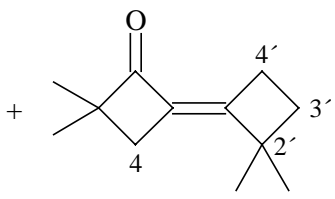

$63(22 \%)$

Von den genannten Produkten gab sich das Bicyclobutyliden 26 bereits im ${ }^{1} \mathrm{H}-\mathrm{NMR}$ Spektrum (Abb. 38) durch charakteristische Resonanzen für die Methylgruppen $(\delta=1.16$, s, $6 \mathrm{H})$ sowie die Protonen an C-3 $(\delta=1.66, \mathrm{t}, \mathrm{J}=8 \mathrm{~Hz}, 2 \mathrm{H})$ und C-3' $(\delta=1.94$, quint, $\mathrm{J}=9 \mathrm{~Hz}$, 2H) zu erkennen. Die allylischen Methylengruppen zeigten komplexe Multipletts $(\delta=2.36$, 2.54, 2.64, je 2H). Das ${ }^{13} \mathrm{C}-\mathrm{NMR}-$ Spektrum (Abb. 81) bestätigt die angegebene Struktur. Es zeigt die zu fordernden neun Resonanzen für zwei magnetisch äquivalente primäre, sowie fünf sekundäre und drei quartäre Kohlenstoffatome, darunter die charakteristischen Resonanzen für ein tetrasubstituiertes Olefin $\left[\delta=128.19\right.$ und $\left.137.37\left(\mathrm{C}_{\text {quart }}\right)\right]$. 
Die hier nicht erwarteten Kondensationsprodukte 62 und 63 zeigen in den IR-Spektren (Abb. 6 und 7) eine starke Bande für eine $\mathrm{C}=\mathrm{O}-$ Valenzschwingung [1745 (62), $1750 \mathrm{~cm}^{-1}$ (63)], und in den ${ }^{1} \mathrm{H}-\mathrm{NMR}-S p e k t r e n$ (Abb. 39 und 40) neben einem bereits bei 26 beobachteten Triplett für die Protonen an C-3' $[\delta=1.86, \mathrm{t}, \mathrm{J}=8 \mathrm{~Hz}, 2 \mathrm{H}(\mathbf{6 2}), 1.84, \mathrm{t}, \mathrm{J}=8 \mathrm{~Hz}, 2 \mathrm{H}(\mathbf{6 3})]$ zwei Singuletts für die Methylgruppen $[\delta=1.18,1.30$, je $6 \mathrm{H}(62), 1.16,1.20$, je $6 \mathrm{H}(63)]$, ein Triplett für die Protonen an C-4 $[\delta=2.20, \mathrm{t}, \mathrm{J}=4 \mathrm{~Hz}, 2 \mathrm{H}(\mathbf{6 2}), 2.30, \mathrm{t}, \mathrm{J}=4 \mathrm{~Hz}, 2 \mathrm{H}(\mathbf{6 3})]$ sowie ein Multiplett für die Protonen an $\mathrm{C}^{-} 4^{\prime}[\delta=2.56, \mathrm{~m}, 2 \mathrm{H}(\mathbf{6 2}), 2.86, \mathrm{~m}, 2 \mathrm{H}(\mathbf{6 3})]$. In Übereinstimmung mit den angegebenen Strukturen finden sich in den ${ }^{13} \mathrm{C}-\mathrm{NMR}-$ Spektren (Abb. 82 und 83) acht Resonanzen für zwei primäre, drei sekundäre und fünf quartäre Kohlenstoffatome, darunter Resonanzen für eine Carbonylgruppe $\left[\delta=203.39\left(\mathbf{C}_{\text {quart }}\right)(\mathbf{6 2}), 206.35\right.$ $\left.\left(\mathrm{C}_{\text {quart }}\right)(\mathbf{6 3})\right]$ sowie ein tetrasubstituiertes Olefin $\left[\delta=133.74,162.52\left(\mathrm{C}_{\text {quart }}\right)(\mathbf{6 2}), 132.70\right.$, $\left.160.32\left(\mathrm{C}_{\text {quart }}\right)(\mathbf{6 3})\right]$. Zur Ermittlung der Stereochemie haben wir die chemischen Verschiebungen der Protonen der Methylgruppen an C-2' $[\delta=1.30$ (62), $1.20(63)]$ und der Protonen an C-4' $[\delta=2.56(62), 2.86(63)]$ herangezogen und die jeweils bei tieferem Feld liegende Resonanz den zur Carbonylgruppe syn-orientierten Protonen zugeschrieben. Bezüglich des Bildungsmechanismus von 62 und 63 ist klar, daß Cyclobutyliden-triphenylphosphoran gegenüber 29 nicht nur als Nucleophil sondern auch als Base gewirkt haben mußte. Ersetzte man das zur Generierung des Phosphorans eingesetzte Kalium-tert-butylat durch Phenyllithium, so bildeten sich bei der anschließenden Umsetzung mit 29 anstelle der Kondensationsprodukte 62 und 63 die entsprechenden Additionsprodukte.

Zur Synthese des für Umlagerungsversuche und als Intermediat zur Darstellung von 28 benötigten 1,1-Dimethyl-spiro[3.4] octan-5-ons (27) sollte das Bicyclobutyliden 26 zunächst

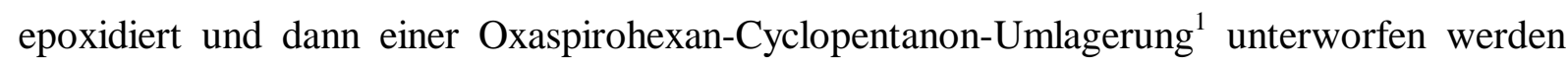
(Schema 19). Die Epoxidierung von 26 gelang problemlos. Mit 1.25 Moläquivalenten mChlorperbenzoesäure in Dichlormethan war die Umsetzung nach 15 min komplett. Aufarbeitung und Chromatographie lieferten das gewünschte Epoxid 64 in einer Ausbeute von 86\%. Sein ${ }^{1}$ H-NMR-Spektrum (Abb. 41) zeigt Resonanzen für insgesamt sechzehn Protonen, darunter zwei Singuletts $\left(\delta=1.06\right.$ und 1.08 , je $3 \mathrm{H}$ ) für die Methylgruppen. Sein ${ }^{13} \mathrm{C}-\mathrm{NMR}$ Spektrum (Abb. 84) belegt mit Resonanzen bei $\delta=67.77\left(\mathrm{C}_{\text {quart }}\right)$ und $71.61\left(\mathrm{C}_{\text {quart }}\right)$ das Vorliegen eines unsymmetrisch substituierten Oxirans. 
Zur Umlagerung wurde 64 in Dichlormethan gelöst und bei $0^{\circ} \mathrm{C}$ mit 0.06 Moläquivalenten einer 48\%-igen Lösung von Bortrifluoridetherat in Cyclohexan versetzt. Laut gaschromatographischer Analyse war 64 nach 15 min vollständig umgesetzt. Allerdings waren nicht ein, sondern zwei Produkte entstanden. Aufarbeitung und Chromatographie lieferten diese Produkte in isolierten Ausbeuten von 17 und 6\%. Laut spektroskopischer Daten war keines von ihnen mit dem unter 1.1 beschriebenen 6,6-Dimethyl-spiro[3.4] octan-5-on (51) identisch. Damit war ein Entscheid aufgrund von Symmetrieüberlegungen einfach. Bei dem Hauptprodukt mit zehn ${ }^{13} \mathrm{C}$-Resonanzen (Abb. 85) mußte es sich um 1,1-Dimethyl-spiro[3.4]octan-5-on (27), bei dem Nebenprodukt mit lediglich acht ${ }^{13} \mathrm{C}$-Resonanzen (Abb. 86) dagegen um 8,8Dimethyl-spiro[3.4] octan-5-on (67) handeln. In Übereinstimmung damit zeigt das ${ }^{13} \mathrm{C}-\mathrm{NMR}$ Spektrum von $\mathbf{6 7}$ ebenso wie das von $\mathbf{5 1}$ eine für periphere Kohlenstoffatome unsubstituierter spiroanellierter Cyclobutane charakteristische Resonanz ${ }^{45}$ bei hohem Feld $\left[\delta=14.36\left(\mathrm{C}_{\mathrm{sek}}\right)\right.$ (67), $\left.15.86\left(\mathrm{C}_{\mathrm{sek}}\right)(\mathbf{5 1})\right]$, die bei 27 fehlt. Die Ergebnisse zeigen, daß 64 von Bortrifluoridetherat unspezifisch geöffnet wird [64-66(65)], daß 65 allerdings unter selektiver 1,2Verschiebung der höher substituierten Bindung weiterreagiert. Gleichwohl war das Ergebnis der Oxaspirohexan-Cyclopentanon-Umlagerung aufgrund der geringen Ausbeuten an 27 und 67 wenig befriedigend. Wie später gezeigt werden wird (vgl. 2.3) waren 27 wie 67 als Objekte einer säurekatalysierten Keton-Keton-Umlagerung interessant. Wir haben daher nach anderen Wegen gesucht, diese Verbindungen darzustellen.

\section{Schema 19}

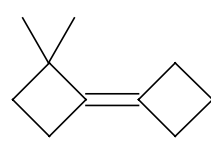

26

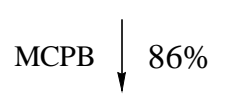

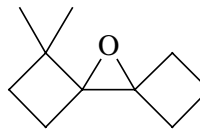

64
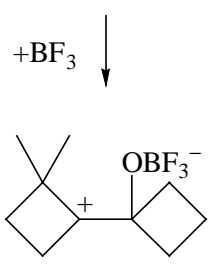

66

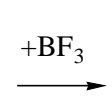

$\mathrm{C}_{4}-\mathrm{C}_{5}$ $-\mathrm{BF}_{3}$

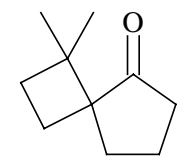

27 (17\%)
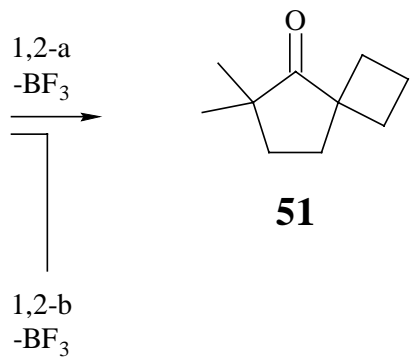

51

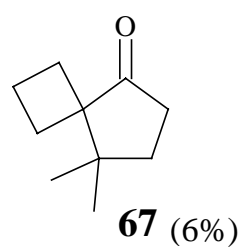




\subsection{Selektive Darstellung von 1,1-Dimethyl-spiro[3.4]octan-5-on (27) und 8,8-Dimethyl-spiro[3.4] octan-5-on (67) über $\beta$-Hydroxyselenide}

Ringerweiterungen über $\beta$-Hydroxyselenide sind bekannt ${ }^{37 / 24}$, solche unter Insertion eines Cyclobutans allerdings selten. Ein Beispiel ist die Addition von 1-Lithio-1-phenylselenocyclobutan $^{38 / 39}$ (71) an das Trispiroketon 68 unter nachfolgender Ringerweiterung des gebildeten $\beta$-Hydroxyselenids 72 durch Oxidation ${ }^{39 / 40}$ (68-72-73-69-70) (Schema 20). Dabei ist wesentlich, daß durch Anwendung eines Überschusses an Persäure die Oxidationsstufe des Selenons 69 erreicht wird, da nur aus dieser Stufe unter Abspaltung von Seleninsäure Ringerweiterung eintritt. Auf der Stufe des Selenoxids 73 kommt es unter Abspaltung von Selenensäure zur Bildung des Cyclobutens 74. Diese Reaktionsweise von Selenoxiden ist auch aus anderen Beispielen bekannt ${ }^{41}$.

\section{Schema 20}

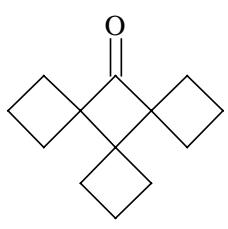

68
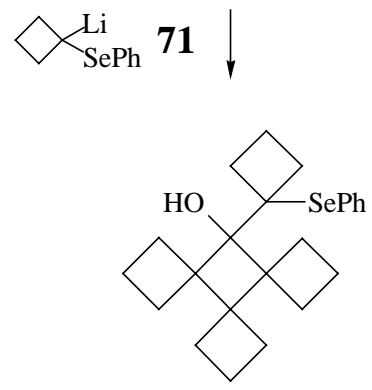

72

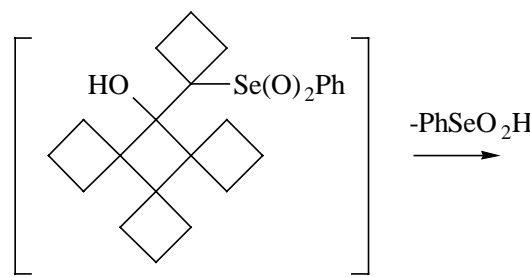

69

$\mathrm{MCPB} \uparrow$

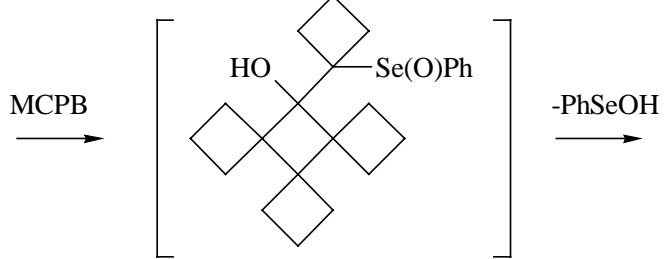

73

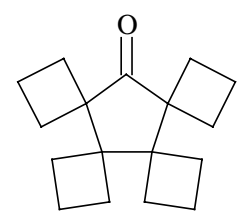

70

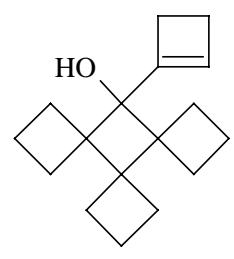

74

Vor diesem Hintergrund bot sich an, das aus dem zugehörigen Selenoketal $\mathbf{7 5}^{38}$ durch reduktive Metallierung generierbare 1-Lithio-1-phenylseleno-cyclobutan (71) $)^{39}$ an 2,2Dimethyl-cyclobutanon $^{6}$ (29) zu addieren und das zu erwartende $\beta$-Hydroxyselenid 76 durch Oxidation in 8,8-Dimethyl-spiro[3.4]octan-5-on (67) umzulagern. Ganz analog konnte aus dem bisher unbekannten Selenoketal 48 nach reduktiver Metallierung zu $\mathbf{7 7}^{39}$ und Addition an Cy- 
clobutanon (1) durch Umlagerung des $\mathrm{zu}$ erwartenden $\beta$-Hydroxyselenids 49 1,1Dispiro[3.4]octan-5-on (27) entstehen (Schema 21). Natürlich mußte dazu zunächst das Selenoketal 48 hergestellt werden. Dies sollte analog der Synthese von 75 durch Zinkchloridkatalysierte Umsetzung von 29 mit Phenylselenol geschehen ${ }^{38}$.

\section{Schema 21}

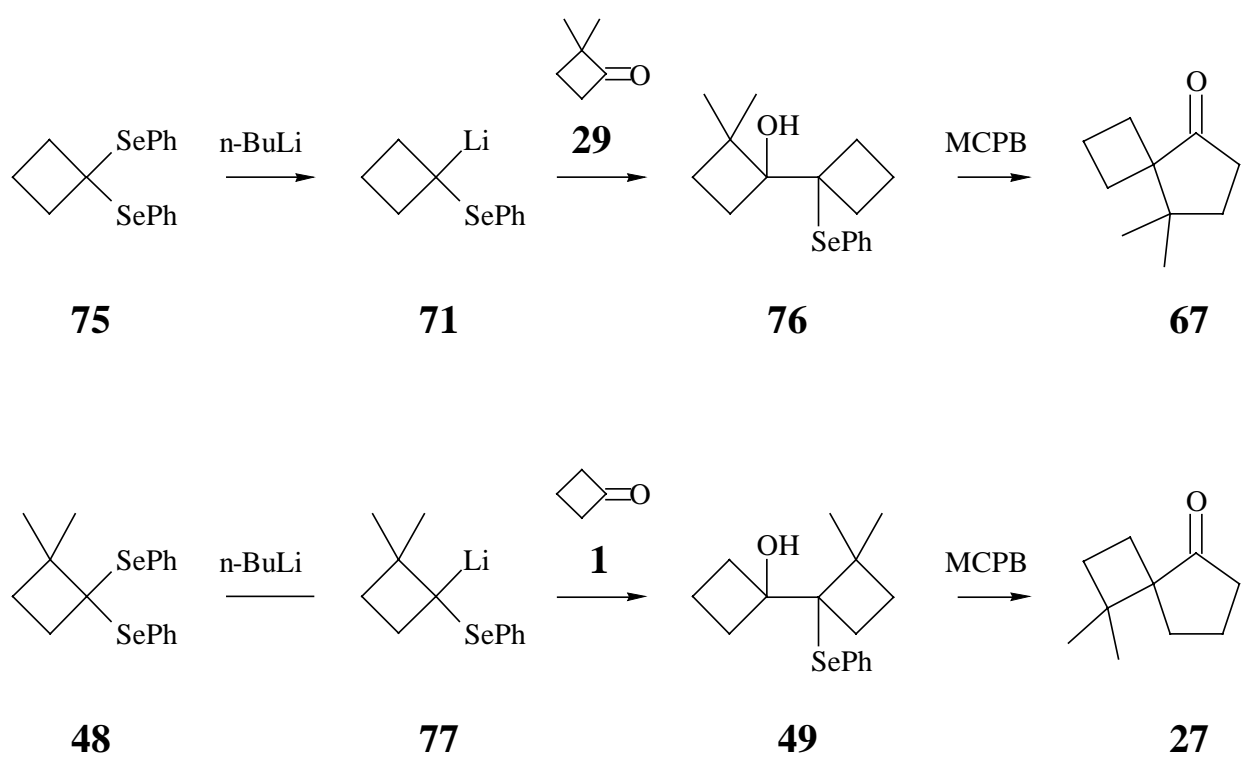

\subsubsection{Darstellung von 8,8-Dimethyl-spiro[3.4]octan-5-on (67)}

Zur Darstellung des für Umlagerungsversuche zu 8,8-Dimethyl-spiro[3.4]octan-5-on (67) benötigten $\beta$-Hydroxyselenids 76 (Schema 22) wurde das Selenoketal 75 bei $-78^{\circ} \mathrm{C}$ in Tetrahydrofuran metalliert und nach Zugabe von 0.67 Moläquivalenten 2,2-Dimethylcyclobutanon (29) $5.5 \mathrm{~h}$ bei $-40^{\circ} \mathrm{C}$ gerührt. Aufarbeitung und Chromatographie lieferte in $48 \%$ Ausbeute eine gelbliche Flüssigkeit, bei der es sich nach Ausweis ihrer spektroskopischen Daten nur um 76 handeln konnte. So zeigt das ${ }^{1}$ H-NMR-Spektrum (Abb. 65) neben zwei Singuletts für die Methylgruppen ( $\delta=1.10$ und 1.30 , je $3 \mathrm{H}$ ) zwei charakteristische Multipletts für die Protonen des Phenylrings $\left(\delta=6.96,3 \mathrm{H}\right.$ und 7.68, 2H). Das ${ }^{13} \mathrm{C}-\mathrm{NMR}-$ Spektrum (Abb. 108) zeigt im aliphatischen Bereich den vollen Satz von zehn Resonanzen, darunter bei höchstem Feld die Resonanz für das periphere Kohlenstoffatom des nicht methylierten Cyclobutanrings $\left[\delta=16.95\left(\mathrm{C}_{\text {sek }}\right)\right.$ ], sowie bei tiefstem Feld die Resonanzen für die Kohlenstoffatome, die die Phenylseleno- $\left[\delta=62.75\left(\mathrm{C}_{\text {quart }}\right)\right]$ bzw. Hydroxylgruppe tragen $\left[\delta=83.09\left(\mathrm{C}_{\text {quart }}\right)\right]$. 


\section{Schema 22}

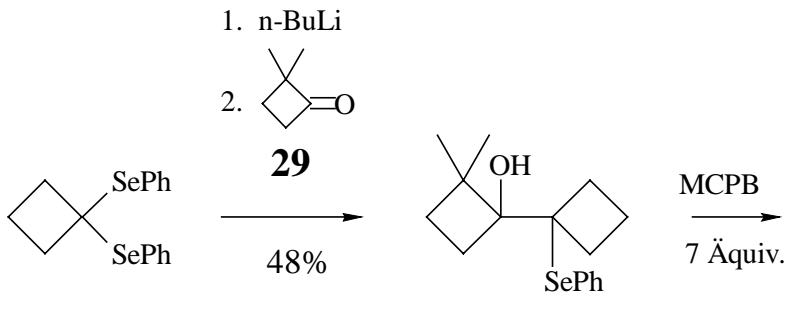

75
76
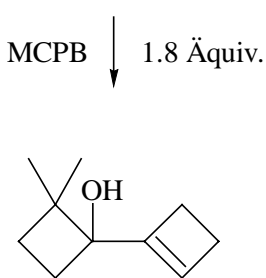

$78(58 \%)$

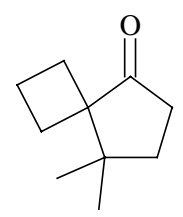

$67(50 \%)$

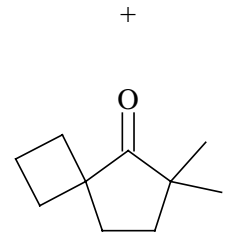

$51(10 \%)$

Zur Umlagerung wurde 76 in Dichlormethan gelöst und zu einer auf $0^{\circ} \mathrm{C}$ gekühlten Suspension von 7 Moläquivalenten m-Chlorperbenzoesäure in Dichlormethan getropft. Laut dünnschichtchromatographischer Kontrolle war 76 nach 40 min unter Bildung von zwei Produkten verbraucht. Aufarbeitung und Chromatographie lieferte diese Produkte in Ausbeuten von 50 bzw. 10\%. Nach Ausweis ihrer spektroskopischen Daten handelte es sich bei dem Majoritätsprodukt um 67 (vgl. 2.1), bei dem Minoritätsprodukt um 51 (vgl. 1.1). Demnach war die Selektivität der Umlagerung zugunsten einer 1,2-Verschiebung der höher substituierten Bindung zwar ausgeprägt, aber nicht vollständig. Gleichwohl ist das Verfahren dem Weg über das Epoxid 64 (vgl. 2.1) überlegen.

Wie zu erwarten, ließ sich der Einsatz von Persäure nicht wesentlich reduzieren ${ }^{41}$. Verwendete man unter sonst gleichen Bedingungen lediglich 1.8 Äquivalente, so entstand in $58 \%$ isolierter Ausbeute das Cyclobuten 78. Diese Verbindung zeigt im ${ }^{1} \mathrm{H}-\mathrm{NMR}-$ Spektrum (Abb. 66) u.a. zwei Singuletts für die Methylgruppen $(\delta=0.98$ und 1.14 , je $3 \mathrm{H})$, eine breite Resonanz für das Hydroxylproton $(\delta=1.74,1 \mathrm{H})$ sowie ein bei sehr tiefem Feld liegendes Singulett für das vinylische Proton des Cyclobutenringes $(\delta=5.90,1 \mathrm{H})$. Das ${ }^{13} \mathrm{C}-\mathrm{NMR}$-Spektrum (Abb. 109) belegt mit Resonanzen für das die Hydroxylgruppe tragende Kohlenstoffatom $[\delta=$ $\left.76.54\left(\mathrm{C}_{\text {quart }}\right)\right]$ sowie die Kohlenstoffatome der Doppelbindung $\left[\delta=127.50\left(\mathrm{C}_{\text {tert }}\right), 151.86\right.$ $\left.\left(\mathrm{C}_{\text {quart }}\right)\right]$ die Richtigkeit der angegebenen Struktur. 


\subsubsection{Darstellung von 1,1-Dimethyl-spiro[3.4] octan-5-on (27)}

Für die Versuche zur Synthese von 1,1-Dimethyl-spiro[3.4]octan-5-on (27) durch Umlagerung des Hydroxyselenids 49 mußte zunächst das Selenoketal 48 hergestellt werden. Wir erhielten diese Verbindung nach einer allgemeinen Vorschrift von Krief ${ }^{38}$ durch Zinkchlorid-katalysierte Ketalisierung von 2,2-Dimethyl-cyclobutanon (29) mit Phenylselenol in 78\% Ausbeute als orange Kristalle vom Schmp. $68^{\circ} \mathrm{C}$ (Schema 23). Daß es sich um das gewünschte Selenoketal handelte, zeigte schon das ${ }^{1} \mathrm{H}-\mathrm{NMR}$-Spektrum (Abb. 50) mit einem Singulett für die Methylgruppen $(\delta=1.24,6 \mathrm{H})$, einem $\mathrm{A}_{2} \mathrm{~B}_{2}$-Sytem für die Methylenprotonen $(\delta=1.93$ und $2.19, \mathrm{~J}_{\mathrm{AB}}=6 \mathrm{~Hz}$, je $2 \mathrm{H}$ ) und zwei charakterischen Multipletts für die Protonen der Phenylringe $(\delta=7.22-7.38,6 \mathrm{H}$ und $7.56-7.62,4 \mathrm{H})$.

\section{Schema 23}

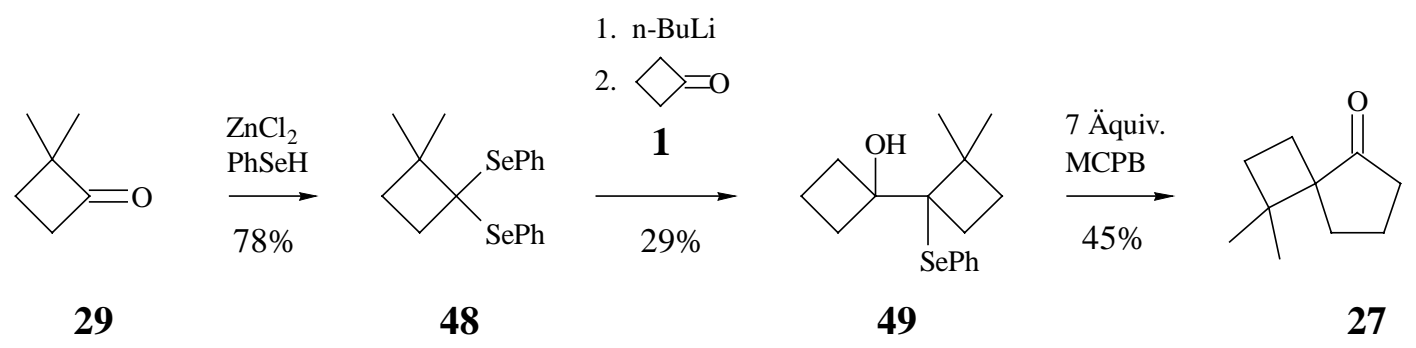

Zur Darstellung des Hydroxyselenids 49 wurde 48 in Tetrahydrofuran gelöst, bei -78C mit n-Butyllithium reduktiv metalliert ${ }^{39}$ und anschließend mit 0.67 Moläquivalenten Cyclobutanon (1) $5.5 \mathrm{~h}$ bei $-40^{\circ} \mathrm{C}$ gerührt. Aufarbeitung und Chromatographie lieferte in $29 \%$ isolierter Ausbeute eine hellgelbe Flüssigkeit, bei der es sich nach Ausweis ihrer spektroskopischen Daten um das gewünschte 49 handelte. So zeigt das ${ }^{1}$ H-NMR-Spektrum (Abb. 67) u.a. zwei Singuletts für die Methylgruppen $(\delta=1.18$ und 1.44, je $3 \mathrm{H}$ ), ein Singulett für das Hydroxylproton $(\delta=2.25,1 \mathrm{H})$ sowie zwei charakteristische Multipletts für die Protonen des Phenylringes $(\delta=$ 6.98, $3 \mathrm{H}$ und 7.60, 2H). Das ${ }^{13} \mathrm{C}-\mathrm{NMR}-$ Spektrum (Abb. 110) zeigt im aliphatischen Bereich den vollen Satz von zehn Resonanzen, darunter bei höchstem Feld die Resonanz für das periphere Kohlenstoffatom des nicht methylierten Cyclobutanrings $\left[\delta=15.15\left(\mathrm{C}_{\mathrm{sek}}\right)\right]$, sowie bei tiefstem Feld die Resonanzen für die Kohlenstoffatome, die die Phenylseleno- $[\delta=71.55$ $\left.\left(\mathrm{C}_{\text {quart }}\right)\right]$ bzw. Hydroxylgruppe tragen $\left[\delta=83.70\left(\mathrm{C}_{\text {quart }}\right)\right]$. 
Die Umlagerung von 49 wurde wie bei 76 (vgl. 2.2.1) mit sieben Moläquivalenten mChlorperbenzoesäure in Dichlormethan durchgeführt und lieferte nach 40 min bei $0^{\circ} \mathrm{C}$ in $45 \%$ isolierter Ausbeute mit 1,1-Dimethyl-spiro[3.4] octan-5-on (27) ein einziges Produkt. Seine ${ }^{1} \mathrm{H}$ NMR-Daten stimmten mit denen einer authentischen Probe überein.

Zusammenfassend ist festzustellen, daß die Synthese von 27 über das Hydroxyselenid 49 im Vergleich zur Synthese über das Epoxid 64 (vgl. 2.1) nur unwesentliche Vorteile bietet. Dies könnte sich ändern, wenn die Ausbeute im Additionsschritt verbessert werden kann. So aber waren die verfügbaren Mengen an 27 zu gering, um die geplante Synthese des Spiro[3.4]octan-5-ols 28 (vgl. 2) mit Aussicht auf Erfolg fortführen zu können. Gleichwohl reichten sie aus, um über eine Keton-Keton-Umlagerung einen Zugang zu einer Partialstruktur des Sesquiterpens ( \pm )-Ceratopicanol (32) zu suchen (vgl. 2.3).

\subsection{Säurekatalysierte Umlagerung von 27, 51 und 67: 3a,6a-Dimethyl-hexahydro- pentalen-1-on (30)}

Ketone können ebenso wie Olefine protoniert und in thermodynamisch günstigere Isomere umgelagert werden. Voraussetzung sind geeignete Umlagerungswege sowie die Möglichkeit zur Rückbildung der Carbonylgruppe. So ist z.B. bekannt, daß die Dispiroketone 79, $\mathbf{8 1}$ und $\mathbf{8 2}$ bei Einwirkung von Säure sämtlich in das [3.3.3]Propellan $\mathbf{8 0}$ als thermodynamisch günstigeres Keton übergehen ${ }^{9 / 10 / 42}$. Die Einzelschritte umfassen dabei eine Serie von Ringerweiterungen und Ringverengungen, sowie bei 81, und möglicherweise auch bei 79, auch eine 1,2-Hydroxyl-Transposition (Schema 24). Es schien daher lohnend zu untersuchen, ob die mit 79, 81 und 82 strukturell verwandten Monospiroketone 27, 51 und 67 analoge Umlagerungen eingehen und mit 3a,6a-Dimethyl-hexahydro-pentalen-1-on (30) eine Partialstruktur des Sesquiterpens ( \pm )-Ceratopicanol (32) liefern würden. 


\section{Schema 24}

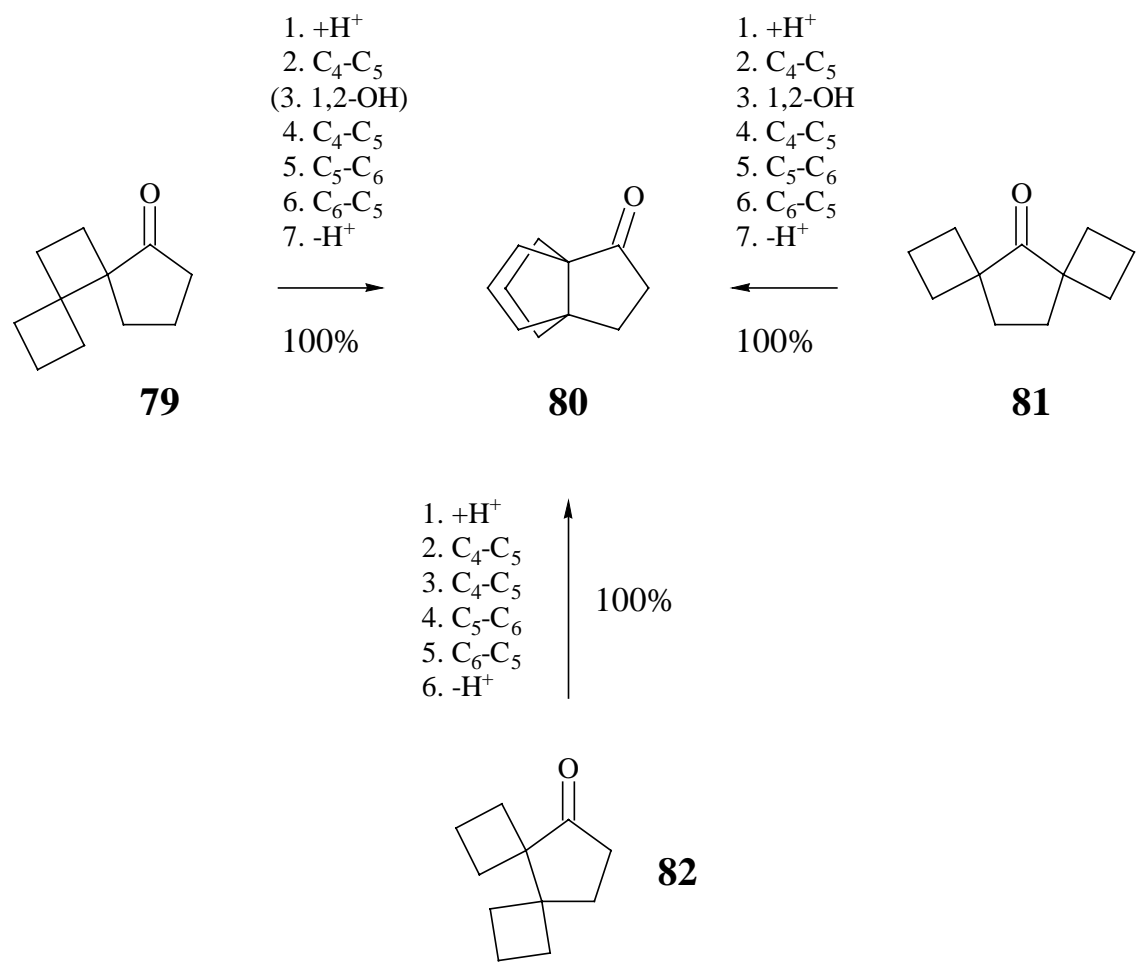

Vorversuche mit einer Lösung von wasserfreier p-Toluolsulfonsäure in Benzol (0.074molar, $70^{\circ} \mathrm{C}$ ), mit einer Suspension von mit 5\% konzentrierter Schwefelsäure dotiertem Kieselgel in Hexan $\left(70^{\circ} \mathrm{C}\right)$ sowie mit Suspensionen des perfluorierten Ionenaustauscher-Harzes Nafion $^{43}$ in Benzol $\left(70^{\circ} \mathrm{C}\right)$ zeigten, daß Umlagerungen nur durch Nafion, und auch dann nicht durch Nafion NR 50 (Perlkörner), sondern nur durch Nafion R SAC-13 mit einer gegenüber Nafion NR 50 40-mal größeren Oberfläche auszulösen waren. Dann allerdings lieferten die zwei Monospiroketone 51 und 67 mit 3a,6a-Dimethyl-hexahydro-pentalen-1-on (30) innerhalb 24-45 h die erwünschte Partialstruktur von ( \pm )-Ceratopicanol (32) (Schema 25). Am schnellsten war die Umlagerung von $67\left(100 \%\right.$ Umsatz nach 24 h bei $\left.70^{\circ} \mathrm{C}\right)$, am langsamsten die von 51 (91\% Umsatz nach 24 h bei $70^{\circ} \mathrm{C}$ ). 27 war nach $6 \mathrm{~h}$ unter der Bildung nicht gaschromatographisch-flüchtiger Produkte verbraucht. Dies deutet darauf hin, daß 1,2-HydroxylVerschiebungen eine relativ hohe Aktivierungsenergie besitzen. 


\section{Schema 25}
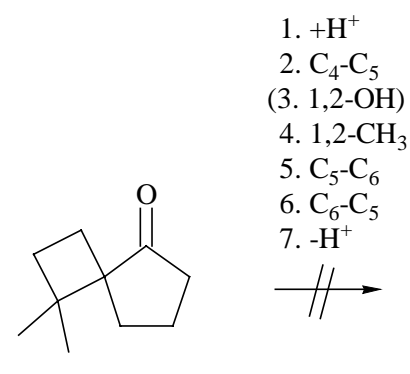

27

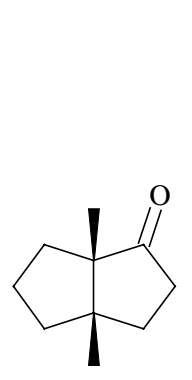

30
1. $+\mathrm{H}^{+}$

2. $\mathrm{C}_{4}-\mathrm{C}_{5}$

3. $1,2-\mathrm{OH}$

4. $1,2-\mathrm{CH}_{3}$

5. $\mathrm{C}_{5}-\mathrm{C}_{6}$

6. $\mathrm{C}_{6}-\mathrm{C}_{5}$

7. $-\mathrm{H}^{+}$

$100 \%$

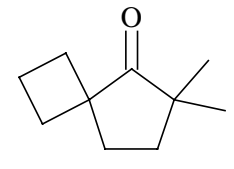

51

$$
\begin{array}{l|l}
\text { 1. }+\mathrm{H}^{+} & \\
\text {2. } \mathrm{C}_{4}-\mathrm{C}_{5} & \\
\text { 3. } 1,2-\mathrm{CH}_{3} & 100 \% \\
\text { 4. } \mathrm{C}_{5}-\mathrm{C}_{6} & \\
\text { 5. } \mathrm{C}_{6}-\mathrm{C}_{5} & \\
\text { 6. }-\mathrm{H}^{+} &
\end{array}
$$

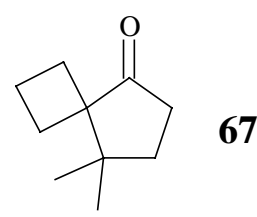

Bei dem neuen Keton handelte es sich um einen farblosen Feststoff (Schmp. 96 ${ }^{\circ}$ ) mit einer für Fünfringketone typischen $\mathrm{C}=\mathrm{O}-$ Valenzschwingung ${ }^{45}$ bei $1735 \mathrm{~cm}^{-1}$ (Abb. 4). Sein ${ }^{1} \mathrm{H}-$ NMR-Spektrum (Abb. 36) zeigt neben zwei Singuletts für ungekoppelte Methylgruppen $(\delta=$ 0.88 und 1.00, je $3 \mathrm{H})$, eine Serie von Multipletts für cycloaliphatische Protonen $(\delta=1.4-2.3$, $10 \mathrm{H}$ ). Sein ${ }^{13}$ C-NMR-Spektrum (Abb. 79) zeigt die nach Anzahl, Lage und Multiplizität zu erwartenden zehn Resonanzen für zwei primäre, fünf sekundäre und drei quartäre Kohlenstoffatome und schließt damit symmetrische Alternativstrukturen aus. Es bleibt zu prüfen, ob sich 51 und/oder 67 so verändern lassen, daß bei analogem Umlagerungsverlauf mit $86^{7 / 17}$ ein direkter Vorläufer von ( \pm )-Ceratopicanol (32) resultiert. Denkbare Kandidaten (ohne Angabe der Stereochemie) sind $\mathbf{8 3 ,} 84$ und 85 (Schema 26). 
Schema 26

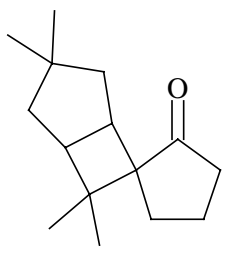

83

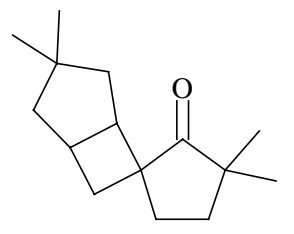

84

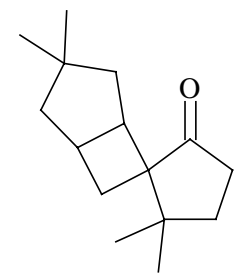

85

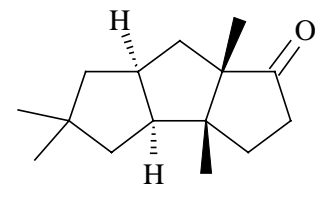

86

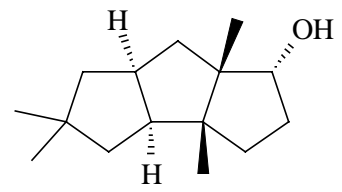

(士)-Ceratopicanol

3 Zur sequentiellen Transformation in komplexe Systeme eingebundener Bicyclobutylidene: Synthese und Umlagerung potentieller Vorläufer von (士)-endo-Capnellen (39)

Nach der erfolgreichen sequentiellen Transformation von Bicyclobutyliden (4) zu mono- bis trimethylierten Bicyclo[3.3.0]octenen (Hexahydropentalenen) (vgl. 1) und entsprechenden Versuchen mit 2,2-Dimethyl-bicyclobutyliden (26) (vgl. 2), sollten im dritten Teil dieser Arbeit Anwendungsmöglichkeiten für in komplexere Systemen eingebundene Bicyclobutylidene untersucht werden. Hier war bekannt, daß das Bicyclobutyliden 34 über eine Reaktionsfolge aus Epoxidierung, Oxaspirohexan-Cyclopentanon-Umlagerung ${ }^{1}$, Monomethylierung und Reduktion in die Alkohole $\mathbf{3 5}$ und $\mathbf{3 6}$ überführt werden kann, die bei Einwirkung von Säure identische Gemische des Sesquiterpens ( \pm )-endo-Capnellen (39) mit den Doppelbindungsisomeren 37 und 38 liefern $^{6}$ (Schema 27). 
Schema 27

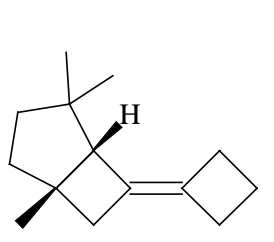

34

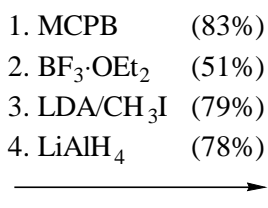

$(78 \%)$

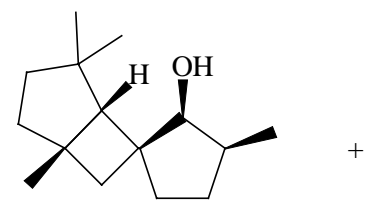

$\mathbf{3 5}(49 \%)$

$\downarrow \mathrm{H}^{+}$

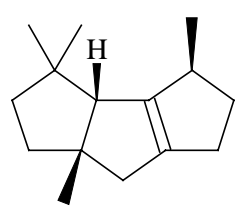

$37(33 \%)$

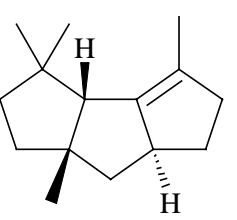

$38(25 \%)$

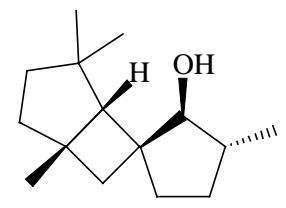

$36(29 \%)$ $\downarrow \mathrm{H}^{+}$

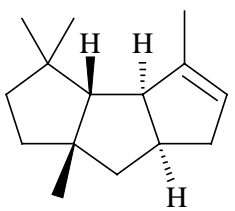

$39(42 \%)$

$( \pm)$-endo-Capnellen

Es schien allerdings auch möglich, andere Alkohole für diesen Zweck einzusetzen. So hatte eine Analyse denkbarer Umlagerungswege zu ( \pm )-endo-Capnellen (39) mit dem in unserer Abteilung entwickelten Retrosyntheseprogramm CARESY ${ }^{19}$ ergeben, daß das tricyclische Kation 47 über eine 1,3-Hydridverschiebung mit anschließender Deprotonierung unmittelbar zu 38 und 39, und damit mittelbar auch zu 37 führen konnte (Schema 27). Aussichtsreichste Edukte für eine Erzeugung von 47 waren die Alkohole 44 und 45 [44(45)-46-47], die ihrerseits nach Cyclobutylidenierung des bekannten bicyclischen Ketons $\mathbf{4 3}^{22}$ über eine Reaktionsfolge aus Epoxidierung, Oxaspirohexan-Cyclopentanon-Umlagerung ${ }^{1}$, erschöpfender Methylierung und Addition von Methyllithium zugänglich schienen [43-40-87-44(45)]. Als größtes Risiko sahen wir den Umstand an, daß Oxaspirohexan-Cyclopentanon-Umlagerungen in der Regel unter Inversion der Konfiguration am Endpunkt der Umlagerung ablaufen und deshalb das 44 und 45 zugrunde liegende Keton 87 gegenüber dem Diastereomer 88 eher als Minoritätsprodukt zu erwarten war. Trotz dieses Risikos sollte der prospektierte Syntheseweg beschritten und auf der Stufe der 41, 42, 44 und 45 zugrunde liegenden Ketone auch Methylenierungsprodukte hergestellt und umgelagert werden. Ziel war, zu klären, in welcher Weise die Stereochemie der Edukte die Struktur und Stereochemie der Produkte bestimmt. Im günstigsten Fall erwarteten wir eine Bildung von 37, 38 und 39, in jedem Fall aber eine Bildung von Triquinanen. 


\section{Schema 28}

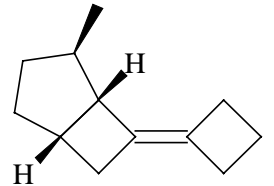

40
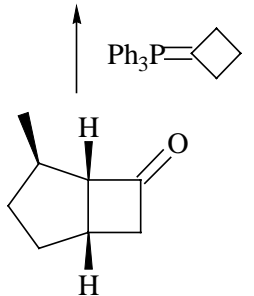

43
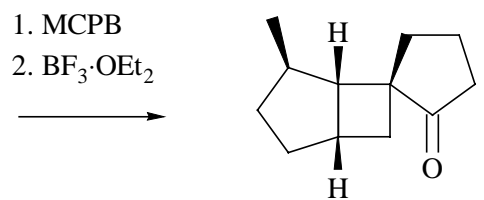

87

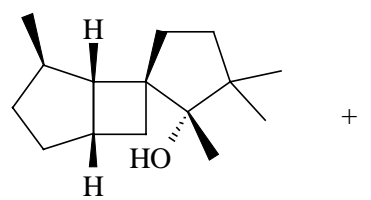

44

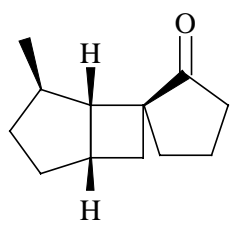

88

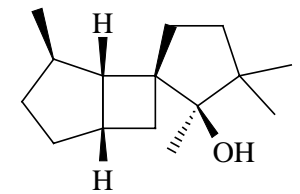

45

1. $+\mathrm{H}^{+} /-\mathrm{H}_{2} \mathrm{O}$
2. $\mathrm{C}_{4}-\mathrm{C}_{5}$

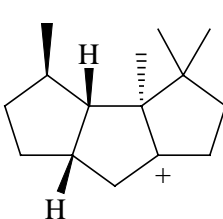

46

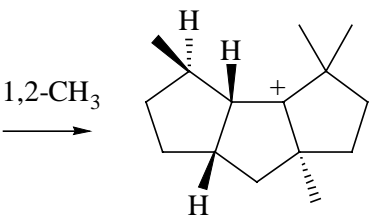

47

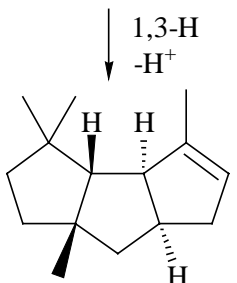

39

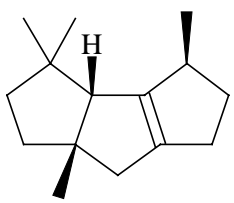

37

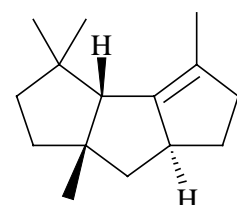

38

(士)-endo-Capnellen

\section{1 (1R*,2S*,5R*)-7-Cyclobutyliden-2-methyl-bicyclo[3.2.0]heptan (40): Darstellung, Epoxidierung, Umlagerung und erschöpfende Methylierung}

Das zur Darstellung von 7-Cyclobutyliden-2-methyl-bicyclo[3.2.0]heptan (40) benötigte bicyclische Keton 43 fällt bei seiner Darstellung als nicht trennbares 84:16-Gemisch mit dem Epimer 90 an $^{22}$ und mußte als solches eingesetzt werden. Bei der Cyclobutylidenierung mit Cyclobutyliden-triphenylphosphoran ${ }^{23}$ in Benzol bildeten sich daher mit 40 und 91 zwei Produkte, die jedoch durch Chromatographie an mit 10\% Sibernitrat dotiertem Kieselgel getrennt und in 67 bzw. 12\% Ausbeute als farblose Flüssigkeiten isoliert werden konnten (Schema 29). Beide Produkte zeigten in ihren ${ }^{1}$ H-NMR-Spektren (Abb. 51 und 52) neben dem er- 
warteten Dublett für die Methylgruppe $[\delta=0.96, \mathrm{~J}=6 \mathrm{~Hz}, 3 \mathrm{H}(\mathbf{4 0}) \mathrm{bzw} . \delta=0.76, \mathrm{~J}=6 \mathrm{~Hz}$, $3 \mathrm{H}$ (91)] eine Serie von Multipletts für weitere fünfzehn Protonen. In den ${ }^{13} \mathrm{C}-\mathrm{NMR}-\mathrm{Spektren}$ (Abb. 94 und 95) finden sich die zu fordernden zwölf Resonanzen zutreffender Multiplizität, darunter bei höchstem Feld die Resonanz des peripheren Kohlenstoffatoms des eingeführten Cyclobutylidenrestes $\left[\delta=17.26\left(\mathrm{C}_{\text {sek }}\right)(\mathbf{4 0})\right.$ bzw. $\left.18.81\left(\mathrm{C}_{\text {sek }}\right)(\mathbf{9 1})\right]$, und bei tiefstem Feld die Resonanzen für die Doppelbindung $\left[\delta=127.97,132.10\left(\mathrm{C}_{\text {quart }}\right)(\mathbf{4 0})\right.$ bzw. 130.60, 130.95 $\left.\left(\mathrm{C}_{\text {quart }}\right)(\mathbf{9 1})\right]$.

\section{Schema 29}

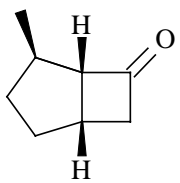

$43(84 \%)$

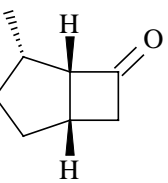

$90(16 \%)$
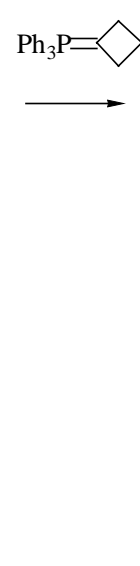

)

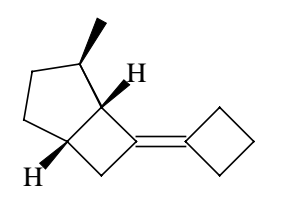

$40(67 \%)$

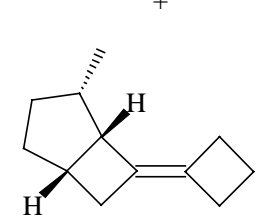

$91(12 \%)$
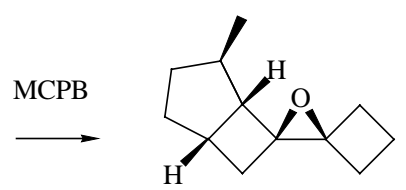

$89(75 \%)$

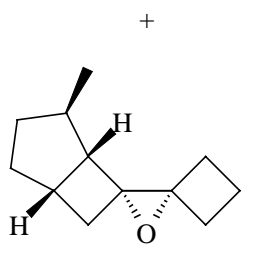

$92(12 \%)$

Als nächstes wurde 40 epoxidiert. Hierzu wurde es in Dichlormethan gelöst und mit 1.2 Moläquivalenten m-Chlorperbenzoesäure umgesetzt ${ }^{8}$. Nach 15 min war die Umsetzung beendet, und Aufarbeitung und Chromatographie lieferten zwei diastereomere Epoxide 89 und 92 in Ausbeuten von 75 bzw. 12\%. Beide Epoxide zeigten in ihren ${ }^{1} \mathrm{H}-\mathrm{NMR}-$ Spektren (Abb. 54 und 53) neben einem Dublett für die Methylgruppe $[\delta=1.08, \mathrm{~J}=6 \mathrm{~Hz}, 3 \mathrm{H}(\mathbf{8 9})$ bzw. 1.02, $\mathrm{J}=$ $6 \mathrm{~Hz}, 3 \mathrm{H}(\mathbf{9 2})$ Resonanzen für weitere fünfzehn Protonen, und in ihren ${ }^{13} \mathrm{C}-\mathrm{NMR}-\mathrm{Spektren}$ (Abb. 97 und 96) unter insgesamt zwölf Resonanzen zutreffender Multiplizität charakteristische Resonanzen für das periphere Kohlenstoffatom des spiroanellierten Cyclobutanringes ${ }^{45}[\delta$ $=12.93\left(\mathrm{C}_{\text {sek }}\right)(\mathbf{8 9})$ bzw. $\left.11.91\left(\mathrm{C}_{\text {sek }}\right)(\mathbf{9 2})\right]$ sowie für die Kohlen-stoffatome des Oxiranringes $\left[\delta=64.91,65.75\left(\mathrm{C}_{\text {quart }}\right)(\mathbf{9 2})\right.$ bzw. 67.43, $\left.69.66\left(\mathrm{C}_{\text {quart }}\right)(\mathbf{8 9})\right]$. Leider erwies sich eine stereochemische Zuordnung auf der Grundlage der spektroskopischen Daten als nicht möglich. Die angegebene Stereochemie beruht daher auf der Annahme, daß die Majoritätskomponente durch einen exo-Angriff der Persäure auf $\mathbf{4 0}$ gebildet wird. 
Zur Umlagerung wurde das 9:1-Gemisch der beiden Epoxide in Dichlormethan gelöst und bei $0^{\circ} \mathrm{C}$ mit 0.06 Moläquivalenten einer $48 \%$-igen Lösung von Bortrifluoridetherat in Cyclohexan versetzt. Nach 15 min war die Reaktion beendet, und Aufarbeitung und Chromatographie lieferten in 7\% Ausbeute ein reines Keton (88), und in $48 \%$ Ausbeute ein nicht trennbares 1 : 1.4-Gemisch zweier weiterer Ketone (87 und 93). Da die Ketone ohnehin erschöpfend methyliert werden sollten, wurden das reine Keton und das Ketongemisch in getrennten Ansätzen mit einem Überschuß an Kaliumhydrid und Methyliodid umgestzt. Aus dem reinen Keton resultierte in $82 \%$ Ausbeute ein reines, dimethyliertes Keton (95), und aus dem Ketongemisch ein Gemisch zweier dimethylierter Ketone, die sich allerdings jetzt als trennbar erwiesen und nach Chromatographie in Ausbeuten von 31 (94) bzw. 53\% (96) erhalten wurden (Schema 30).

Bis zu diesem Zeitpunkt war die Struktur und Stereochemie der dargestellten Ketone keineswegs klar. Immerhin lag jetzt jedes der drei dimethylierten Ketone rein vor, so daß neben ${ }^{1} \mathrm{H}$ - (Abb. 57, 58, 59) und ${ }^{13} \mathrm{C}-\mathrm{NMR}-$ Spektren (Abb. 100, 101 und 102) auch ${ }^{1} \mathrm{~J}_{\mathrm{CH}^{-}}$(HMQC) (Abb. 121, 123 und 125), ${ }^{2,3} \mathrm{~J}_{\mathrm{CH}^{-}}$(HMBC) (Abb. 127) und ${ }^{2,3} \mathrm{~J}_{\mathrm{HH}^{-}}$Korrela-tionsspektren (COSY) (Abb. 122, 124 und 126) aufgenommen und die Strukturen gelöst werden konnten.

\section{Schema 30}

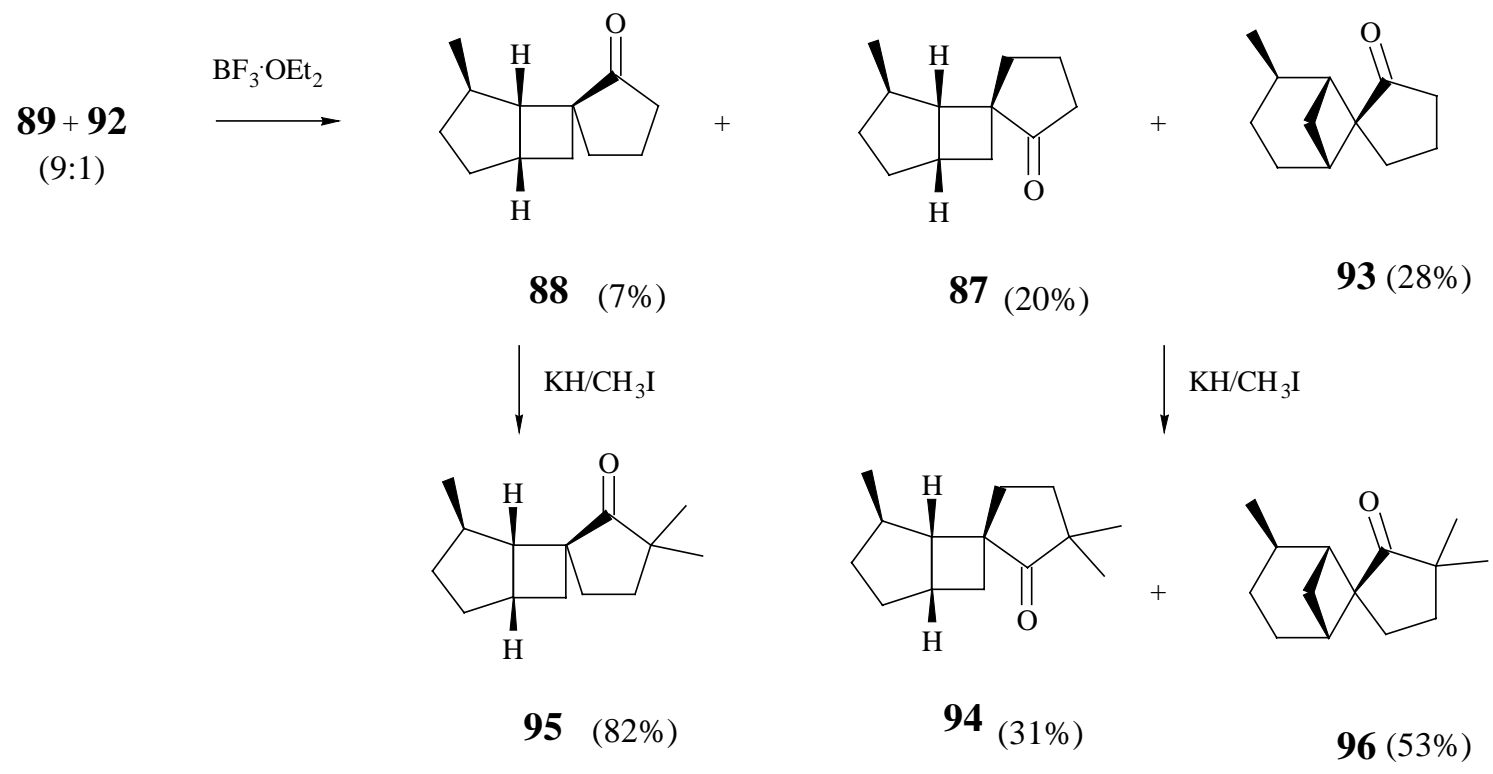

Zur Auswertung wurden die ${ }^{13} \mathrm{C}$-Resonanzen von hohem zu tiefem Feld durchnumeriert und jeder Resonanz die laut ${ }^{1} \mathrm{~J}_{\mathrm{CH}}$-Korrelation zugehörigen Protonen zugeordnet. Die resultierenden $\mathrm{CH}_{3^{-}}, \mathrm{CH}_{2^{-}}$und $\mathrm{CH}-$ Gruppen wurden anschließend über ${ }^{2,3} \mathrm{~J}_{\mathrm{HH}^{-}}$Korrelationen (bei 94 
auch über ein NOESY) zu größeren Fragmenten (fett gezeichnet) zusammengefügt und mit den verbliebenen Einzelfragmenten zu den angegebenen Gesamtstrukturen ergänzt. Es resultierten mit 94 und 95 die erwarteten Cyclopentanone mit einem spiroanellierten Bicyclo[3.2.0]heptan, und mit $\mathbf{9 6}$ ein unerwartetes Cyclopentanon mit einem spiroanellierten Bicyclo[3.1.1]heptan (Schema 31).

Bei 96 und damit bei 93 folgte die angegebene Stereochemie aus einem Vergleich seiner ${ }^{13} \mathrm{C}$-Daten mit Literaturdaten für die stereoisomeren Bicyclo[3.1.1]heptane 97 und $\mathbf{9 8}^{\mathbf{4 4}}$, bei $\mathbf{9 4}$ und 95 und damit bei $\mathbf{8 7}$ und $\mathbf{8 8}$ blieb die Frage der Stereochemie offen.

\section{Schema 31}

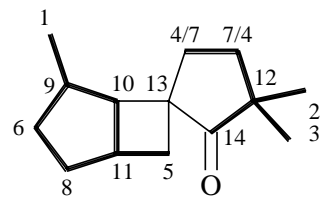

94

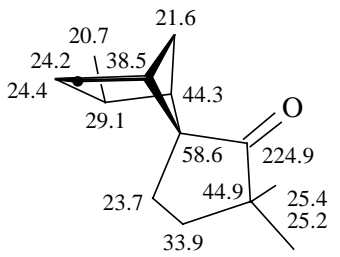

96

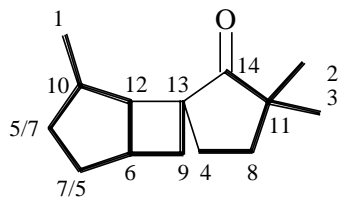

95

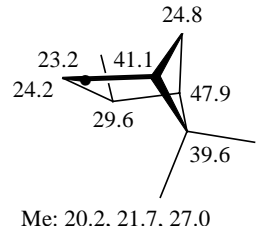

97

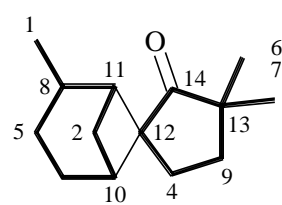

96

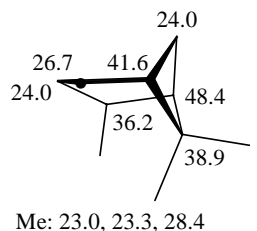

98

Bezüglich der Bildung von 93 nehmen wir an, daß die Epoxide 89 und 92 nach regiospezifischer Ringöffnung zu 99 konkurrierend zur $\mathrm{C}_{4}-\mathrm{C}_{5}$-Ringerweiterung unter Bildung von 87 und 88 eine Cyclobutyl-Homoallyl-Cyclopropylmethyl-Cyclobutyl-Umlagerungen eingehen, ehe eine abschließende $\mathrm{C}_{4}$ - $\mathrm{C}_{5}$-Ringerweiterung 93 liefert (99-100-101-102-93) (Schema 32). 


\section{Schema 32}

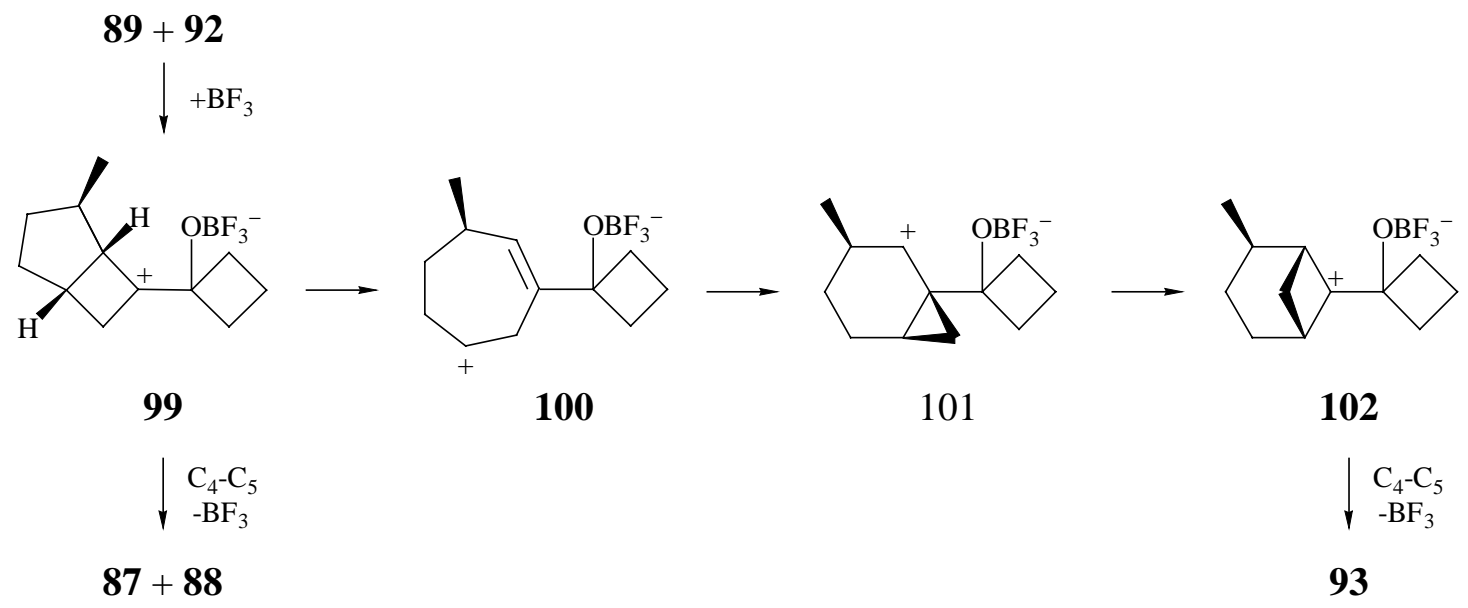

3.2 Synthese von $\left(1 R^{*}, 4 S^{*}, 5 R^{*}, 6 R^{*}, 2^{\prime} R^{*}\right)-\quad(41),\left(1 R^{*}, 4 S^{*}, 5 R^{*}, 6 R^{*}, 2^{\prime} S^{*}\right)-\quad(42)$, $\left(1 R^{*}, 4 S^{*}, 5 R^{*}, 6 S^{*}, 2^{\prime} R^{*}\right)-(44)$ und $\left(1 R^{*}, 4 S^{*}, 5 R^{*}, 6 S^{*}, 2^{\prime} S^{*}\right)-4,2^{\prime}, 3^{\prime}, 3^{\prime}$-Tetramethyl-spiro $\{$ bicyclo[3.2.0]heptan-6,1'-cyclopentan-2'-ol\} (45)

Für die geplanten säurekatalysierten Umlagerungen mit dem Ziel einer Synthese von ( \pm )-endo-Capnellen (39) benötigten wir die Alkohole 44 und 45. Da die sterochemische $\mathrm{Zu}$ ordnung der dimethylierten Ketone $\mathbf{9 4}$ und $\mathbf{9 5}$ nicht sicher war (vgl. 3.1), sollte bei beiden Verbindungen das noch fehlende Kohlenstoffatom durch Addition von Methyllithium eingeführt und versucht werden, fest anfallende Alkohole kristallographisch zu untersuchen. Die Additionen wurden mit einem Moläquivalent einer 0.5 M Lösung von Methyllithium in Ether durchgeführt und waren nach 15 min bei $0^{\circ} \mathrm{C}$ komplett (Schema 33).

\section{Schema 33}

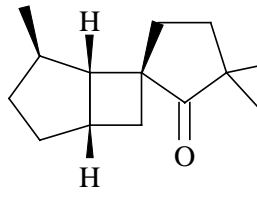

94

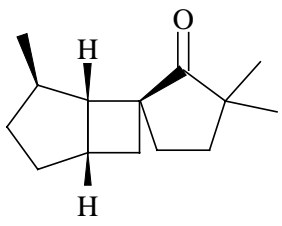

95
$\mathrm{CH}_{3} \mathrm{Li}$

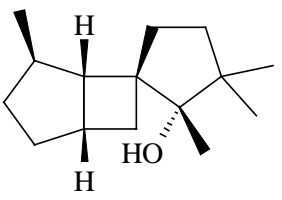

44 (59\%)

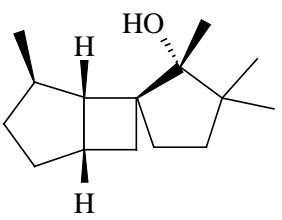

41

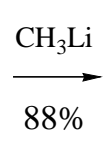

$88 \%$

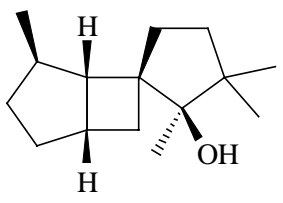

45 (9\%)

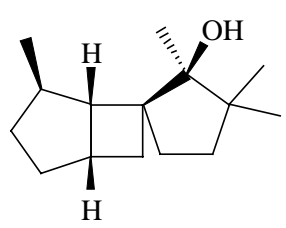

42 
95 lieferte in 88\% Ausbeute ein chromatographisch nicht trennbares 1:1-Gemisch der stereoisomeren Alkohole 41 und $\mathbf{4 2}$ in Form einer Flüssigkeit. Das IR-Spektrum (Abb. 29) zeigt Banden für eine freie $\left(3620 \mathrm{~cm}^{-1}\right)$ sowie für assoziierte $\mathrm{OH}-\mathrm{Valenzschwingungen} \mathrm{(3600-}$ $3420 \mathrm{~cm}^{-1}$ ), und das ${ }^{1} \mathrm{H}-\mathrm{NMR}-$ Spektrum (Abb. 64) Resonanzen für zweimal sechsundzwanzig Protonen, darunter sechs Singuletts $(\delta=0.76,0.80,1.02,1.03,1.08$ und 1.10 , je $3 \mathrm{H})$ und zwei Dubletts $(\delta=0.90, \mathrm{~J}=6 \mathrm{~Hz}, 3 \mathrm{H}$ und $0.91, \mathrm{~J}=6 \mathrm{~Hz}, 3 \mathrm{H})$ für die Methylgruppen. Das ${ }^{13} \mathrm{C}$ NMR-Spektrum (Abb. 107) belegt das Vorliegen tertiärer Alkohole $\left[\delta=83.74,83.83\left(\mathrm{C}_{\text {quart }}\right)\right]$.

94 lieferte in 59\% Ausbeute eine Flüssigkeit (44), und in 9\% Ausbeute einen Feststoff (Schmp. 59 $\left.{ }^{\circ} \mathrm{C}\right)(\mathbf{4 5})$. Auch hier zeigen die IR-Spektren (Abb. 28 und 27) Banden für eine freie [3610 (44) und $3600 \mathrm{~cm}^{-1}$ (45)] sowie für assoziierte OH-Valenzschwingungen [3580-3480 (44) und 3580-3400 cm-1 (45)] und die ${ }^{1} \mathrm{H}-\mathrm{NMR}-$ Spektren (Abb. 63 und 62 ) Resonanzen für jeweils sechsundzwanzig Protonen, darunter je drei Singuletts $[\delta=0.94,0.96,1.12$, je $3 \mathrm{H}$ (44) bzw. 0.83, 0.86, 0.97, je 3H (45)] und ein Dublett [ $\delta=1.16, \mathrm{~J}=6 \mathrm{~Hz}, 3 \mathrm{H}$ (44) bzw. 1.08, $\mathrm{J}=6 \mathrm{~Hz}, 3 \mathrm{H}(\mathbf{4 5})]$ für die Methylgruppen. Weiterhin belegen auch hier die ${ }^{13} \mathrm{C}-\mathrm{NMR}-$ Spektren (Abb. 106 und 105) das Vorliegen tertiärer Alkohole [ $\delta=83.70$ (44) bzw. 93.53 (45)].

Leider gelang es nicht von dem als Feststoff angefallenen Alkohol röntgenographisch verwertbare Kristalle zu züchten. Daraufhin unternommene Versuche zur Derivatisierung von 41-44 mit 3,5-Dinitrobenzoylchlorid schlugen ebenfalls fehl. Daher mußte die angegebene Stereochemie der vier Alkohole weiterhin als vorläufig gelten. Gleichwohl sollten alle Alkohole umgelagert werden, um aus der Struktur und Stereochemie der Produkte möglicherweise auf die Stereochemie der Edukte rückschließen zu können.

\subsection{Synthese von $\left(1 R^{*}, 4 S^{*}, 5 R^{*}, 6 S^{*}\right)-(103)$ und $\left(1 R^{*}, 4 S^{*}, 5 R^{*}, 6 R^{*}\right)-2^{\prime}$-Methylen-4,3', $3^{\prime}$-trimethyl-spiro\{ bicyclo[3.2.0]heptan-6,1'-cyclopentan\} (104)}

Als weitere Möglichkeit zur Einführung des noch fehlenden Kohlenstoffatoms wurden 94 und 95 methyleniert. Dies hatte gegenüber einer Addition von Methyllithium den Vorteil, daß keine neuen Stereozentren auftraten und gleichwohl umlagerungsfähige Verbindungen entstanden. Zur experimentellen Realisierung wurden 94 und 95 mit jeweils vier Moläquiva- 
lenten Methylen-triphenylphosphoran unter begleitender gaschromatographischer Kontrolle bei $110^{\circ} \mathrm{C}$ umgesetzt ${ }^{23}$. Nach $3 \mathrm{~h}$ waren die Umsetzungen komplett. Aufarbeitung und Chromatographie lieferte bei 94 in 84\% Ausbeute das Olefin 103 und bei 95 in 67\% Ausbeute das Olefin 104 (Schema 34).

\section{Schema 34}

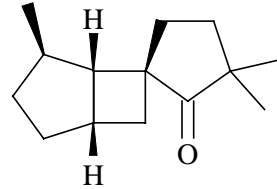

94

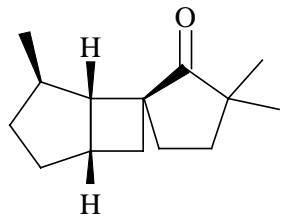

95
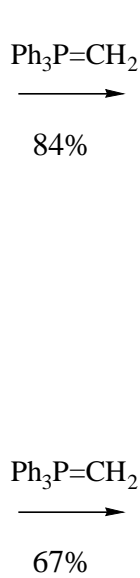

$67 \%$

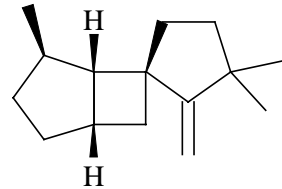

103

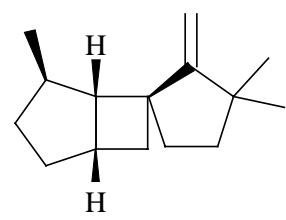

104

$\mathrm{Da} ß$ es sich bei den isolierten Produkten tatsächlich um die gewünschten Olefine handelte, zeigte sich in den ${ }^{1} \mathrm{H}-\mathrm{NMR}-$ Spektren (Abb. 60 und 61) durch das Auftreten von je zwei Singuletts für die vinylischen Protonen $[\delta=4.74$ und 5.02, je $1 \mathrm{H}(\mathbf{1 0 4}) \mathrm{bzw} .4 .74$ und 5.04, je $1 \mathrm{H} \mathrm{(103)]} \mathrm{und} \mathrm{in} \mathrm{den}{ }^{13} \mathrm{C}-\mathrm{NMR}-S p e k t r e n(\mathrm{Abb} .103$ und 104) durch charakteristische Resonanzen für die exocyclische Doppelbindung $\left[\delta=99.91\left(\mathrm{C}_{\mathrm{sek}}\right)\right.$ und $172.99\left(\mathrm{C}_{\text {quart }}\right)(\mathbf{1 0 4})$ bzw. $99.83\left(\mathrm{C}_{\text {sek }}\right)$ und $\left.173.47\left(\mathrm{C}_{\text {quart }}\right)(\mathbf{1 0 4})\right]$.

\subsection{Säurekatalysierte Umlagerung der Alkohole 41, 42, 44 und 45 sowie der Olefine 103 und $104 \mathrm{zu}$ linearen Triquinanen}

Nachdem die Alkohole 41, 42, 44 und 45 sowie die Olefine 103 und 104 in ausreichender Menge zur Verfügung standen, sollten sie säurekatalysiert umgelagert werden. Der Fortgang der Umlagerungen sollte zeitaufgelöst gaschromatographisch dokumentiert und entstehende Produkte bezüglich ihrer Struktur und Stereochemie untersucht werden. Da alle Edukte einleitend unter konkurrierender 1,2-Verschiebung der höher (a) und niedriger substituierten Bindung (b) reagieren konnten, war bei den Alkoholen 41 und 42 sowie dem Olefin 104 mit 
einem Auftreten der Kationen 105 und/oder 106 und bei den Alkoholen 44 und 45 sowie dem Olefin 103 mit einem Auftreten der Kationen 107 und/oder 46 zu rechnen (Schema 35). Von diesen Kationen war lediglich 46 ein potentieller Vorläufer des als Syntheseziel angestrebten ( \pm )-endo-Capnellens (39). Von den übrigen Kationen besitzt 107 die falsche Regiochemie, 105 die falsche Regio- und Stereochemie, und 106 die falsche Stereochemie. Gleichwohl schien auch hier eine Analyse der Umlagerungswege interessant.

\section{Schema 35}

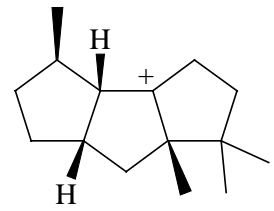

105

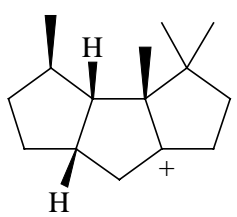

106

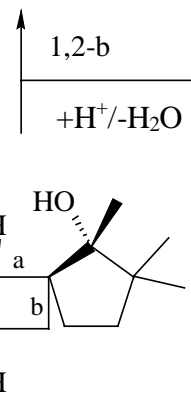

41

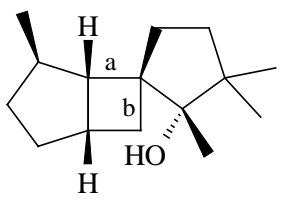

44

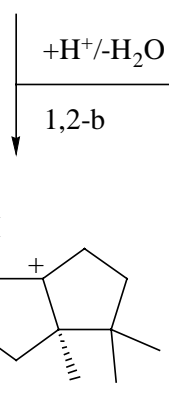

107

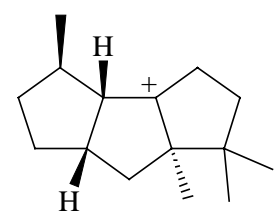

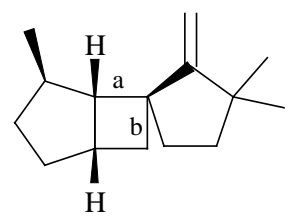

104

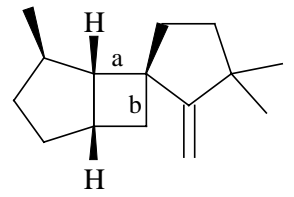

103

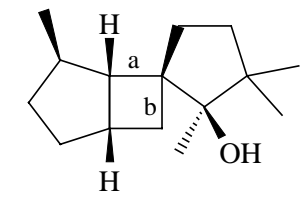

45

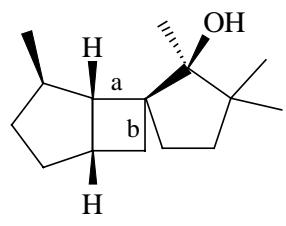

42

$+\mathrm{H}^{+} / \mathrm{H}_{2} \mathrm{O}$

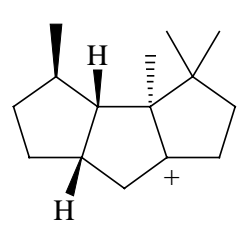

46
1. $1,2-\mathrm{CH}_{3}$

2. $1,3-\mathrm{H}$

3. $-\mathrm{H}^{+}$

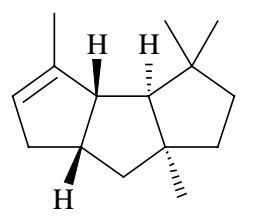

39

$( \pm)$-endo-Capnellen

Zur experimentellen Realisierung der einzelnen Umlagerungen wurden die Alkohole und Olefine mit jeweils einem Moläquivalent einer $0.074 \mathrm{M}$ Lösung von wasserfreier p- 
Toluolsulfonsäure in Benzol versetzt und $6 \mathrm{~h}$ bei $70^{\circ} \mathrm{C}$ (Alkohole) bzw. $45 \mathrm{~h}$ bei $25^{\circ} \mathrm{C}$ (Olefine) gerührt. Die Umlagerungen wurden gaschromatographisch überwacht und die gebildeten Produkte gaschromatographisch isoliert. Die Strukturaufklärung erfolgte durch Auswertung der ${ }^{1} \mathrm{H}$ - und ${ }^{13} \mathrm{C}-\mathrm{NMR}$-Spektren in Verbindung mit ${ }^{1} \mathrm{~J}_{\mathrm{CH}^{-}}(\mathrm{HMQC}),{ }^{2,3} \mathrm{~J}_{\mathrm{CH}^{-}}(\mathrm{HMBC})$ und ${ }^{2,3} \mathrm{~J}_{\mathrm{HH}^{-}}$ Korrelationsspektren (COSY) sowie NOESY-Spektren.

\subsubsection{Produkte und zeitlicher Verlauf der Umlagerung von 41, 42 und 104}

Als erstes wurde das 1:1-Gemisch der Alkohole 41 und 42, dann das Olefin 104 umgelagert. In beiden Fällen war eine Bildung von ( \pm )-endo-Capnellen (39) ausgeschlossen (vgl. Schema 34). Hielt man das Gemisch aus 41 und 42 mit einem Moläquivalent einer 0.074molaren Lösung von p-Toluolsulfonsäure bei $70^{\circ} \mathrm{C}$, so waren beide Alkohole nach 10 min verbraucht. Zu diesem Zeitpunkt enthielt das Reaktionsgemisch $31 \%$ eines nicht trennbaren Gemisches zweier tetrasubstituierter Olefine 108 und 109, 2, 28 und 20\% dreier trisubstituierter Olefine 110, 111 und 112, sowie 19\% nicht isolierte Produkte. Nach 6 h betrugen die Anteile $14 \% 108$ und 109, 18, 20 und 12\% 110, 111 und 112, und 36\% nicht isolierte Produkte. Bei allen isolierten Produkten handelte es sich um Monoolefine mit einer Summenformel $\mathrm{C}_{15} \mathrm{H}_{24}$ und damit um tricyclische Systeme. Erwartungsgemäß war keines der Produkte mit ( \pm )-endoCapnellen (39) oder einem seiner Doppelbindungsisomeren 37 oder 38 identisch.

Das Gemisch aus 108 und 109 bestand nach Ausweis seiner NMR-spektroskopischen Daten aus gleichen Anteilen zweier tetrasubstituierter Olefine. Das ${ }^{13} \mathrm{C}-\mathrm{NMR}-\mathrm{Spektrum}$ (Abb. 115) belegte mit Resonanzen zutreffender Lage und Multiplizität die vollständige Substitution $\left[\delta=144.19,144.32,149.86,149.98\left(\mathrm{C}_{\text {quart }}\right)\right]$, und das ${ }^{1} \mathrm{H}-\mathrm{NMR}-$ Spektrum (Abb. 72) machte mit Resonanzen für sechs ungekoppelte $(\delta=0.92,0.94,1.04,1.05,1.10,1.12,6$ x s, je $3 \mathrm{H})$ und zwei gekoppelte Methylgruppen $(\delta=0.99,1.01,2 \times \mathrm{d}, 2 \times \mathrm{J}=6 \mathrm{~Hz}, 3 \mathrm{H})$ aufgrund der chemischen Verschiebungen deutlich, daß keine der Methylgruppe vinylisch gebunden war.

Auffällig ist, daß nahezu alle Resonanzen im ${ }^{1} \mathrm{H}$ - und ${ }^{13} \mathrm{C}-\mathrm{NMR}-$ Spektrum als Paare auftreten. Es ist daher zu vermuten, daß es sich bei den Verbindungen nicht um Gerüstisomere, sondern um Stereoisomere handelt, die sich nur in der Stereochemie der gekoppelten Methylgruppe unterscheiden. Nimmt man noch hinzu, daß beide Verbindungen im Verlauf der Umla- 
gerung zugunsten von 110 abnehmen, dann folgen die für 108 und 109 angegebenen Strukturen zwanglos (Schema 36). Auf einen Versuch, die Strukturen über Korrelationsspektren zu bestätigen oder zu widerlegen, wurde wegen der zu erwartenden Komplexität der Spektren verzichtet.

\section{Schema 36}

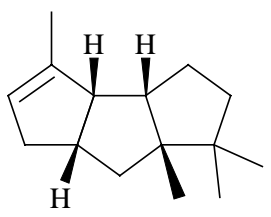

110

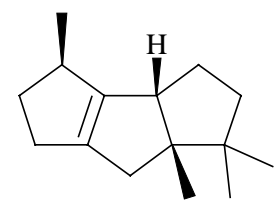

$108(109)$

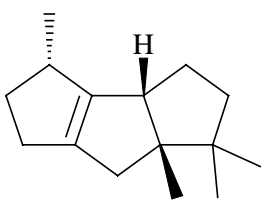

$109(108)$

Zur Klärung der im Vorgriff bereits mitgeteilten Struktur von 110 sowie der Strukturen von 111 und 112 wurden die ${ }^{1} \mathrm{H}-\mathrm{NMR}-$ Spektren (Abb. 73, 74 und 75) und ${ }^{13} \mathrm{C}-\mathrm{NMR}-$ Spektren (Abb. 116, 117 und 118) in Verbindung mit ${ }^{1} \mathrm{~J}_{\mathrm{CH}^{-}}$(HMQC) (Abb. 136, 138 und 141), ${ }^{2,3} \mathrm{~J}_{\mathrm{CH}^{-}}$ (HMBC) (Abb. 140 und 143) und ${ }^{2,3} \mathrm{~J}_{\mathrm{HH}}$-Korrelationsspektren (COSY) (Abb. 137, 139 und 142) herangezogen. Bei allen drei Verbindungen handelte es sich nach Ausweis ihrer ${ }^{13} \mathrm{C}$ NMR-Spektren um trisubstituierte Olefine $\left[\delta=123.63\left(\mathrm{C}_{\text {tert }}\right)\right.$ und $143.45\left(\mathrm{C}_{\text {quart }}\right)(\mathbf{1 1 0}), 120.80$ $\left(\mathrm{C}_{\text {tert }}\right)$ und $147.42\left(\mathrm{C}_{\text {quart }}\right)(\mathbf{1 1 1}), 122.50\left(\mathrm{C}_{\text {tert }}\right)$ und $\left.145.10\left(\mathrm{C}_{\text {quart }}\right)(\mathbf{1 1 2})\right]$, die laut ${ }^{1} \mathrm{H}-\mathrm{NMR}-$ Spektren jeweils eine Methylgruppe vinylisch gebunden enthielten $[\delta=1.68(\mathbf{1 1 0}), 1.60(\mathbf{1 1 1})$, 1.58 (112), br s, je 3H]. Damit schieden Brückenkopfolefine als denkbare Kandidaten aus. Von den restlichen drei Methylgruppen waren bei 110 alle ungekoppelt $(\delta=0.96,0.98,1.15$, s, je $3 \mathrm{H})$, und bei $111(\delta=0.76, \mathrm{~s}, 0.80, \mathrm{~d}, \mathrm{~J}=6 \mathrm{~Hz}, 0.86, \mathrm{~s}, \mathrm{je} 3 \mathrm{H})$ und $112(\delta=0.94, \mathrm{~d}, \mathrm{~J}=6 \mathrm{~Hz}$, 0.98, s, 1.00, s, je 3H) je eine gekoppelt. Eine Auswertung der Korrelationsspektren ergab die in Schema 37 fett gezeichneten Gerüste, die nur bei $\mathbf{1 1 0}$ durch verbliebene Einzelfragmente ergänzt werden mußten. Zur Festlegung der Konfiguration von 112 wurde ein NOESYSpektrum aufgenommen (Abb. 144). Es zeigte starke Crosspeaks zwischen H-3 und H-12 sowie H-4 und H-10 und belegte damit die angegebene cis-syn-cis-Konfiguration. Diese Konfiguration wurde für 110 und 111 übernommen. 


\section{Schema 37}
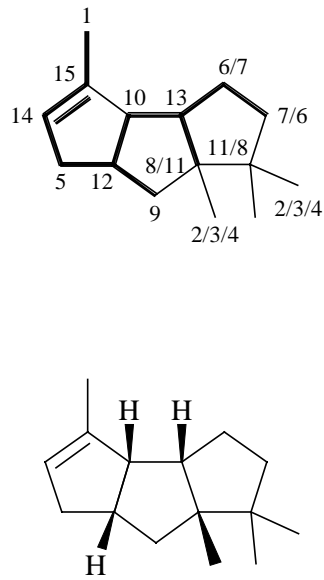

110
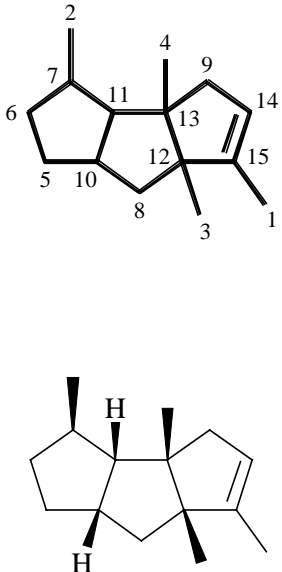

111
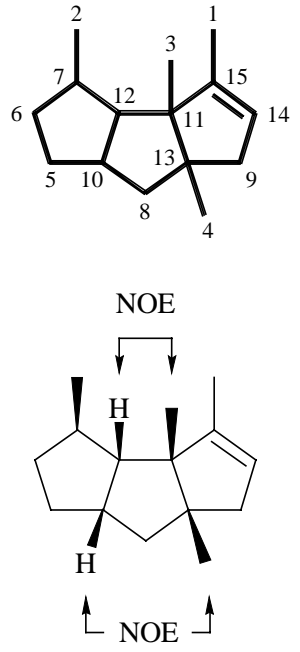

112

Nachdem die Struktur und Stereochemie der aus 41 und 42 entstandenen Triquinane geklärt war, wurde das Olefin 104 umgelagert. Hier erwarteten wir wegen der mit 41 und 42 vergleichbaren Stereochemie keine grundsätzlich neuen Produkte. Tatsächlich lieferte $\mathbf{1 0 4}$ mit einem Moläquivalent einer 0.074-molaren Lösung von p-Toluolsulfonsäure in Benzol innerhalb von 10 min bei $25^{\circ} \mathrm{C}$ mit 108 und $109(21 \%), 110(3 \%), 111(37 \%)$ und 112 (21\%) dieselben Produkte wie 41 und 42. Sämtliche Produkte wurden gaschromatographisch isoliert und durch Vergleich ihrer ${ }^{1} \mathrm{H}-\mathrm{NMR}$-Daten mit denen authentischer Proben identifiziert. Im weiteren Verlauf näherten sich die Konzentrationen den bei der Umlagerung von 41 und $\mathbf{4 2}$ beobachteten an, waren jedoch selbst nach $45 \mathrm{~h}$ bei $25^{\circ} \mathrm{C}$ noch leicht unterschiedlich. Einzelheiten zum zeitlichen Verlauf der Umlagerungen von $\mathbf{4 1}$ und $\mathbf{4 2}$ sowie von $\mathbf{1 0 4}$ finden sich in den Tabellen 1 und 2. 


\begin{tabular}{|c|c|c|c|c|c|}
\hline $\begin{array}{l}1 \text { Moläqui- } \\
\text { valent } 0.074 \\
\mathrm{M} \text { p-TsOH } \\
\text { in Benzol, } \\
70{ }^{\circ} \mathrm{C}\end{array}$ & \multirow[t]{2}{*}{$108 / 109$} & \multirow[t]{2}{*}{110} & \multirow[t]{2}{*}{111} & \multirow[t]{2}{*}{112} & \multirow[t]{2}{*}{$\begin{array}{l}\text { Nicht identi- } \\
\text { fizierte Pro- } \\
\text { dukte }\end{array}$} \\
\hline Zeit & & & & & \\
\hline $10 \mathrm{~min}$ & $31 \%$ & $2 \%$ & $28 \%$ & $20 \%$ & $19 \%$ \\
\hline $20 \mathrm{~min}$ & $30 \%$ & $2 \%$ & $15 \%$ & $20 \%$ & $33 \%$ \\
\hline $40 \mathrm{~min}$ & $30 \%$ & $5 \%$ & $19 \%$ & $19 \%$ & $27 \%$ \\
\hline $1 \mathrm{~h}$ & $24 \%$ & $11 \%$ & $22 \%$ & $17 \%$ & $26 \%$ \\
\hline $2 \mathrm{~h}$ & $22 \%$ & $14 \%$ & $22 \%$ & $17 \%$ & $25 \%$ \\
\hline $4 \mathrm{~h}$ & $24 \%$ & $17 \%$ & $21 \%$ & $15 \%$ & $23 \%$ \\
\hline $6 \mathrm{~h}$ & $14 \%$ & $18 \%$ & $20 \%$ & $12 \%$ & $36 \%$ \\
\hline
\end{tabular}

Tabelle 1: Produkte und zeitlicher Verlauf der Umlagerung von 41 und 42

\begin{tabular}{|c|c|c|c|c|c|}
\hline $\begin{array}{l}1 \text { Moläqui- } \\
\text { valent } 0.074 \\
\mathrm{M} \mathrm{p} \text {-TsOH } \\
\text { in Benzol, } \\
25{ }^{\circ} \mathrm{C}\end{array}$ & \multirow[t]{2}{*}{$109 / 108$} & \multirow[t]{2}{*}{110} & \multirow[t]{2}{*}{111} & \multirow[t]{2}{*}{112} & \multirow[t]{2}{*}{$\begin{array}{l}\text { Nicht identi- } \\
\text { fizierte Pro- } \\
\text { dukte }\end{array}$} \\
\hline Zeit & & & & & \\
\hline $10 \mathrm{~min}$ & $21 \%$ & $3 \%$ & $37 \%$ & $21 \%$ & $18 \%$ \\
\hline $20 \mathrm{~min}$ & $17 \%$ & $6 \%$ & $37 \%$ & $20 \%$ & $20 \%$ \\
\hline $40 \mathrm{~min}$ & $14 \%$ & $10 \%$ & $37 \%$ & $21 \%$ & $18 \%$ \\
\hline $1 \mathrm{~h}$ & $14 \%$ & $11 \%$ & $37 \%$ & $21 \%$ & $17 \%$ \\
\hline $4 \mathrm{~h}$ & $14 \%$ & $12 \%$ & $30 \%$ & $21 \%$ & $23 \%$ \\
\hline $21 \mathrm{~h}$ & $14 \%$ & $12 \%$ & $37 \%$ & $21 \%$ & $16 \%$ \\
\hline $45 \mathrm{~h}$ & $14 \%$ & $12 \%$ & $32 \%$ & $17 \%$ & $25 \%$ \\
\hline
\end{tabular}

Tabelle 2: Produkte und zeitlicher Verlauf der Umlagerung von 104 
Schema 38

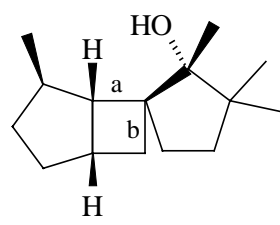

41

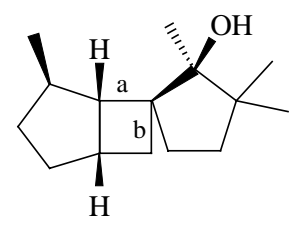

42

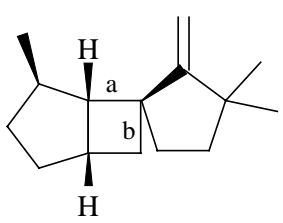

104

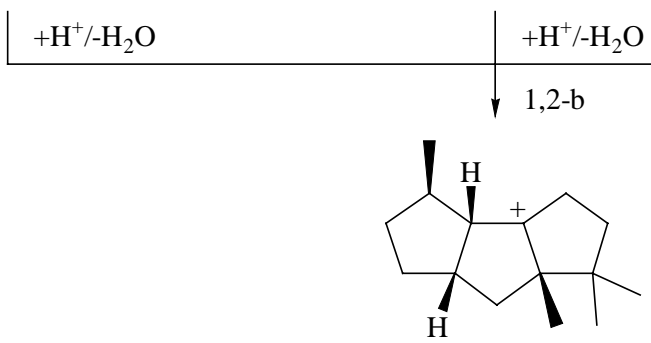

105

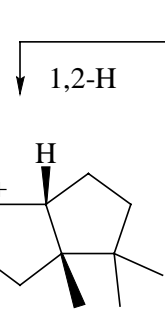

113

Deprotonierung Isomerisierung

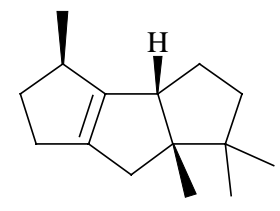

108 (109)

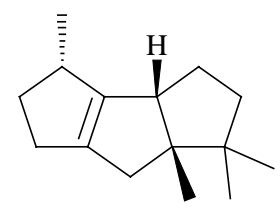

109 (108)

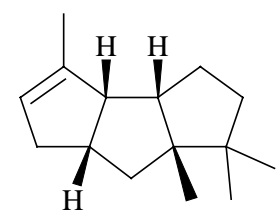

110

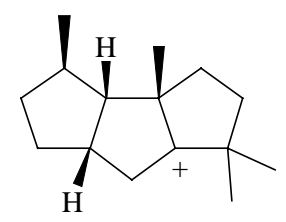

114

$\downarrow \begin{aligned} & 1,2-\mathrm{CH}_{3} \\ & -\mathrm{H}^{+}\end{aligned}$

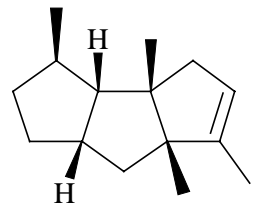

111 $+\mathrm{H}^{+}$ 1,2-a

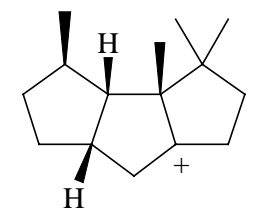

106

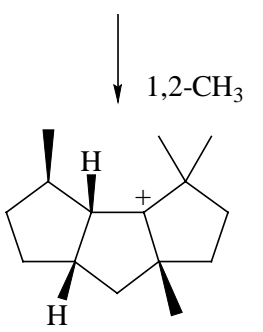

115

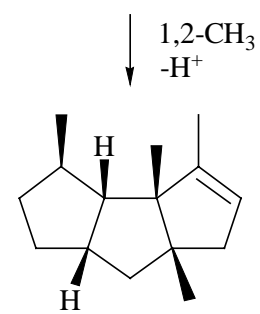

112 
Bezüglich des Mechanismus der Umlagerungen wurde bereits darauf hingewiesen, daß 41, 42 und 104 unter konkurrierender 1,2-Verschiebung der höher (a) und niedriger substituierten Bindung (b) 106 und/oder 105 als Primärkationen liefern können. 106 kann nur unter 1,2-Methylverschiebung unter Bildung von 115 weiterreagieren. Andernfalls müßten energiereiche sekundäre Kationen durchlaufen werden. Folgerichtig ist 112 eines der beobachteten Produkte. Im Gegensatz zu 105 stehen 104 mit einer 1,2-Hydridverschiebung zu 113 bzw. einer 1,2-Methylverschiebung zu 114 zwei Reaktionskanäle über tertiäre Kationen offen: ausgehend von 114 liefern eine weitere 1,2-Methylverschiebung und eine Deprotonierung 111, und ausgehend von 113 Deprotonierungs- und Isomerisierungsreaktionen 108, 109 und 110 (Schema 38). Wie die Produktverteilung zeigt (vgl. Tabelle 1 und 2), liegt die Selektivität zugunsten einer einleitenden 1,2-Verschiebung der niedriger substituierten Bindung (b) bei den Alkoholen wie bei dem Olefin bei ca. 3:1. Dies deutet daraufhin, daß die Umlagerungen unter Freie-Ionen-Bedingungen ablaufen und die Stereochemie der Alkohole für die Produktbildung keine Bedeutung hat.

\subsubsection{Produkte und zeitlicher Verlauf der Umlagerungen von 44, 45 und 103}

Als zweiter Satz von Verbindungen wurden die Alkohole 44 und $\mathbf{4 5}$ sowie das Olefin 103 umgelagert. Hier konnten im Erstschritt der Umlagerung unter konkurrierender 1,2Verschiebung der höher (a) und niedriger substituierten Bindung (b) die Primärkationen 46 und/oder 107 entstehen. Davon war 46 ein potentieller Vorläufer von ( \pm )-endo-Capnellen (39) (vgl. Schema 35). Da von den Alkoholen 44 in größerer Menge zur Verfügung stand, wurde er zuerst umgelagert.

Hielt man den Alkohol 44 mit einem Moläquivalent einer 0.074-molaren Lösung von pToluolsulfonsäure in Benzol bei $70^{\circ} \mathrm{C}$, so war er nach 10 min verbraucht. Zu diesem Zeitpunkt enthielt das Reaktionsgemisch 40\% eines nicht trennbaren 1: 1-Gemisches zweier unbekannter Olefine 116 und 117, 8, 10 und 36\% dreier trisubstituierter Olefine 118, 119 und 120 sowie 6\% nicht isolierte Produkte. Nach 20 min war 118 nicht mehr vorhanden, und die Zusammensetzung lag bei $33 \% 116$ und 117, $10 \% 119$ und 50\% 120. Nach 6 h enthielt das Gemisch 10\% 116 und 117, 46\% 119 und 37\% 120. Bei allen isolierten Produkten handelte es sich um Monoolefine mit einer Summenformel $\mathrm{C}_{15} \mathrm{H}_{24}$ und damit um tricyclische Systeme. Keines der Produkte war ( \pm )-endo-Capnellen (39) oder eines seiner Isomere 37 oder 38. 
Das nicht trennbare Gemisch aus 116 und 117 bestand nach Ausweis seines ${ }^{13} \mathrm{C}-\mathrm{NMR}$ Spektrums (Abb. 111) aus gleichen Anteilen zweier trisubstituierter Olefine $[\delta=123.80$, $\left.129.68\left(\mathrm{C}_{\text {tert }}\right), 142.86,152.02\left(\mathrm{C}_{\text {quart }}\right)\right]$. Allerdings war die Probe nicht rein und nicht alle Multiplizitäten ermittelbar. Da auch das ${ }^{1} \mathrm{H}-\mathrm{NMR}$-Spektrum (Abb. 68) keine sichere Interpretation zuließ, und von Korrelationsspektren wegen der zu erwartenden Komplexität keine Klärung zu erwarten war, wurde auf eine Klärung der Strukturen verzichtet.

Zur Ermittlung der Strukturen von 118, 119 und 120 wurden die ${ }^{1} \mathrm{H}-($ Abb. .71, 69, 70) und ${ }^{13} \mathrm{C}$-NMR-Spektren (Abb. 114, 112, 113) in Verbindung mit ${ }^{1} \mathrm{~J}_{\mathrm{CH}^{-}}$(HMQC) (Abb. 133, $129,131)$ und ${ }^{2,3} \mathrm{~J}_{\mathrm{HH}^{-}}$Korrelationsspektren (COSY) (Abb. 134, 130, 132) herangezogen. Bei 118 wurde zusätzlich ein ${ }^{2,3} \mathrm{~J}_{\mathrm{CH}}$-Korrelationsspektrum (HMBC) (Abb. 135) aufgenommen. Bei allen rein isolierten Verbindungen handelte es sich nach Ausweis ihrer ${ }^{13} \mathrm{C}-\mathrm{NMR}$-Spektren um trisubstituierte Olefine $\left[\delta=115.48\left(\mathrm{C}_{\text {tert }}\right)\right.$ und $158.73\left(\mathrm{C}_{\text {quart }}\right)(\mathbf{1 1 8}), 123.55\left(\mathrm{C}_{\text {tert }}\right)$ und 143.28 $\left(\mathrm{C}_{\text {quart }}\right)(\mathbf{1 1 9}), 124.62\left(\mathrm{C}_{\text {tert }}\right)$ und $\left.141.04\left(\mathrm{C}_{\text {quart }}\right)(\mathbf{1 2 0})\right]$. Ihre ${ }^{1} \mathrm{H}-\mathrm{NMR}-$ Spektren zeigen Resonanzen für jeweils vierundzwanzig Protonen. Charakteristisch für 118 sind Resonanzen für ein vinylisches Proton $(\delta=5.21,1 \mathrm{H})$ sowie eine gekoppelte $(\delta=1.14, \mathrm{~d}, \mathrm{~J}=6 \mathrm{~Hz}, 3 \mathrm{H})$ und drei ungekoppelte Methylgruppen $(\delta=0.96,0.98,1.02$, s, je $3 \mathrm{H})$ an gesättigten Kohlenstoffatomen. Charakteristisch für 119 und $\mathbf{1 2 0}$ sind Resonanzen für ein vinylisches Proton $[\delta=5.18$ (119), $5.24(\mathbf{1 2 0})$, je $1 \mathrm{H}]$, eine vinylisch gebundene Methylgruppe $[\delta=1.68(\mathbf{1 1 9}), 1.60(\mathbf{1 2 0})$, je $3 \mathrm{H}]$ sowie drei ungekoppelte Methylgruppen $[\delta=0.84,0.90,0.98(\mathbf{1 1 9}), 0.86,0.88,0.90$ (120), je 3H] an gesättigten Kohlenstoffatomen. Eine Auswertung der Korrelationsspektren ergab die in Schema 39 fett gezeichneten Gerüste, die bei 119 und 120 noch durch die fehlenden Einzelfragmente ergänzt werden mußten. Die für $\mathbf{1 2 0}$ angegebene cis-anti-cisKonfiguration folgt aus dem Umstand, daß diese Verbindung bei gleichem Gerüst nicht mit dem cis-syn-cis-konfigurierten 110 identisch ist und wurde für 118 und 119 übernommen. 


\section{Schema 39}
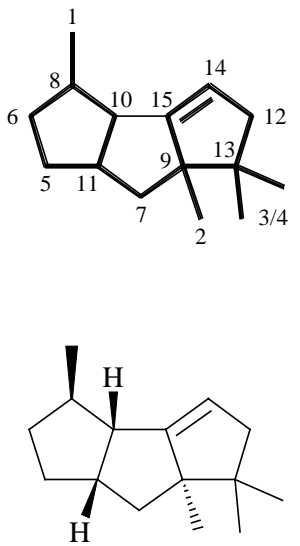

118
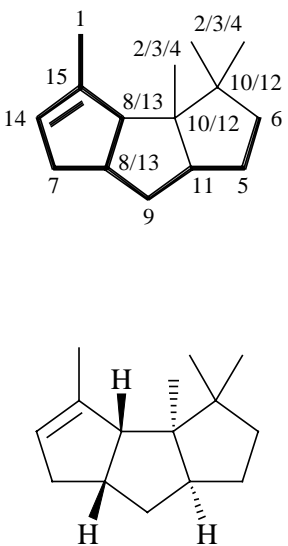

119
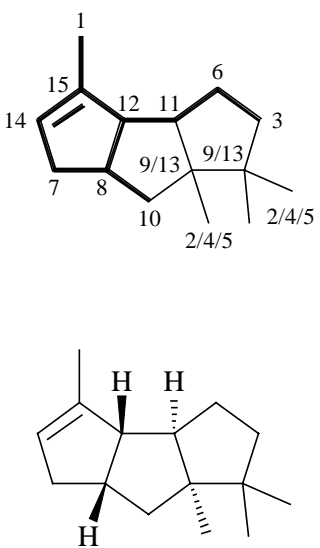

120

Nachdem die Struktur und Stereochemie der aus dem Alkohol $\mathbf{4 4}$ gebildeten Produkte geklärt war, wurde der Alkohol 45 und das Olefin 103 umgelagert. Da beide Verbindungen mit 46 und 107 dieselben Primärkationen wie 44 liefern mußten, erwarteten wir keine neuen Produkte.

Hielt man 45 mit einem Moläquivalent einer 0.074-molaren Lösung von pToluolsulfonsäure in Benzol 10 min bei $70^{\circ} \mathrm{C}$, so war 44 verbraucht und mit 116 und 117 (8\%), 118 (4\%), $119(11 \%)$ und 120 (75\%) dasselbe Gemisch an Olefinen entstanden wie schon bei 44. Gleiches galt für 103. Auch hier bildeten sich innerhalb 10 min bei $25^{\circ} \mathrm{C}$ unter vollständigem Verbrauch von 103 die Olefine 116 und 117 (19\%), 118 (9\%), 119 (14\%) und 120 (54\%). Im weiteren Verlauf wurde 118 vollständig verbraucht. Gleichzeitig nahm der Anteil an 120 deutlich ab, und der an 119 deutlich zu. Ausgehend von 45 (103) lag die Zusammenstzung nach $6 \mathrm{~h}$ bei $70^{\circ} \mathrm{C}\left(45 \mathrm{~h}\right.$ bei $\left.25^{\circ} \mathrm{C}\right)$ bei $6 \%$ (4\%) 116 und 117, 38\% (49\%) 119 und $44 \%$ (39\%) 120. Alle Produkte wurden gaschromatographisch isoliert und über einen Ver-

gleich ihrer ${ }^{1}$ H-NMR-Daten mit denen authentischer Proben identifiziert. Einzelheiten über den zeitlichen Verlauf der Umlagerungen von 44, 45 und 103 finden sich in den Tabellen 3, 4 und 5. 


\begin{tabular}{|c|c|c|c|c|c|}
\hline $\begin{array}{c}1 \text { Moläqui- } \\
\text { valent } 0.074 \\
\text { M p-TsOH } \\
\text { in Benzol, } \\
\quad 70{ }^{\circ} \mathrm{C}\end{array}$ & \multirow{2}{*}{$116 / 117$} & \multirow{2}{*}{119} & \multirow{2}{*}{120} & \multirow{2}{*}{118} & \multirow[t]{2}{*}{$\begin{array}{l}\text { Nicht identi-fi- } \\
\text { zierte Pro- } \\
\text { dukte }\end{array}$} \\
\hline Zeit & & & & & \\
\hline $10 \mathrm{~min}$ & $40 \%$ & $10 \%$ & $36 \%$ & $8 \%$ & $6 \%$ \\
\hline $20 \mathrm{~min}$ & $33 \%$ & $10 \%$ & $50 \%$ & - & $7 \%$ \\
\hline $40 \mathrm{~min}$ & $18 \%$ & $20 \%$ & $51 \%$ & - & $11 \%$ \\
\hline $60 \mathrm{~min}$ & $17 \%$ & $27 \%$ & $48 \%$ & - & $8 \%$ \\
\hline $2 \mathrm{~h}$ & $10 \%$ & $45 \%$ & $37 \%$ & - & $8 \%$ \\
\hline $4 \mathrm{~h}$ & $10 \%$ & $46 \%$ & $37 \%$ & - & $7 \%$ \\
\hline $6 \mathrm{~h}$ & $10 \%$ & $46 \%$ & $37 \%$ & - & $7 \%$ \\
\hline
\end{tabular}

Tabelle 3: Produkte und zeitlicher Verlauf der Umlagerung von 44

\begin{tabular}{|c|c|c|c|c|c|}
\hline $\begin{array}{l}1 \text { Moläqui- } \\
\text { valent } 0.074 \\
\mathrm{M} \text { p-TsOH } \\
\text { in Benzol, } \\
70{ }^{\circ} \mathrm{C}\end{array}$ & \multirow{2}{*}{ 116/117 } & \multirow{2}{*}{119} & \multirow{2}{*}{120} & \multirow{2}{*}{118} & \multirow[t]{2}{*}{$\begin{array}{l}\text { Nicht identi- } \\
\text { fizierte Pro- } \\
\text { dukte }\end{array}$} \\
\hline Zeit & & & & & \\
\hline $10 \mathrm{~min}$ & $8 \%$ & $11 \%$ & $75 \%$ & $4 \%$ & $2 \%$ \\
\hline $20 \mathrm{~min}$ & $6 \%$ & $11 \%$ & $84 \%$ & - & - \\
\hline $40 \mathrm{~min}$ & $6 \%$ & $26 \%$ & $54 \%$ & - & $14 \%$ \\
\hline $60 \mathrm{~min}$ & $6 \%$ & $33 \%$ & $48 \%$ & - & $13 \%$ \\
\hline $2 \mathrm{~h}$ & $6 \%$ & $34 \%$ & $48 \%$ & - & $12 \%$ \\
\hline $4 \mathrm{~h}$ & $6 \%$ & $35 \%$ & $47 \%$ & - & $12 \%$ \\
\hline $6 \mathrm{~h}$ & $6 \%$ & $38 \%$ & $44 \%$ & - & $12 \%$ \\
\hline
\end{tabular}

Tabelle 4: Produkte und zeitlicher Verlauf der Umlagerung von 45 


\begin{tabular}{|c|c|c|c|c|c|}
\hline $\begin{array}{c}1 \text { Moläqui- } \\
\text { valent } 0.074 \\
\mathrm{M} \mathrm{p}-\mathrm{TsOH} \\
\text { in Benzol, } \\
25^{\circ} \mathrm{C}\end{array}$ & $116 / 117$ & $\mathrm{H}_{\mathrm{H}}$ & 190 & 110 & $\begin{array}{l}\text { Nicht identi- } \\
\text { fizierte Pro- } \\
\text { dukte }\end{array}$ \\
\hline Zeit & & 119 & 120 & 110 & \\
\hline $10 \mathrm{~min}$ & $19 \%$ & $14 \%$ & $54 \%$ & $9 \%$ & $4 \%$ \\
\hline $20 \mathrm{~min}$ & $11 \%$ & $15 \%$ & $67 \%$ & $4 \%$ & $3 \%$ \\
\hline $60 \mathrm{~min}$ & $6 \%$ & $21 \%$ & $64 \%$ & - & $9 \%$ \\
\hline $2 \mathrm{~h}$ & $5 \%$ & $25 \%$ & $60 \%$ & - & $10 \%$ \\
\hline $4 \mathrm{~h}$ & $4 \%$ & $33 \%$ & $54 \%$ & - & $9 \%$ \\
\hline $21 \mathrm{~h}$ & $4 \%$ & $47 \%$ & $42 \%$ & - & $7 \%$ \\
\hline $45 \mathrm{~h}$ & $4 \%$ & $49 \%$ & $39 \%$ & - & $8 \%$ \\
\hline
\end{tabular}

Tabelle 5: Produkte und zeitlicher Verlauf der Umlagerung von 103

Bezüglich des Mechanismus wurde bereits darauf hingewiesen, daß 44, 45 und $103 \mathrm{im}$ Erstschritt der Umlagerung unter konkurrierender 1,2-Verschiebung der höher (a) und niedriger substituierten Cyclobutanbindung (b) 46 und/oder 107 als Primärkationen liefern können. Die Produktbildung zeigt, daß beide Wege beschritten werden. Ausgehend von 107 bildet sich durch Deprotonierung 118, sowie durch 1,3-Hydridverschiebung und Deprotonierung 120. 118 wird als energetisch ungünstiges Brückenkopfolefin reprotoniert und zugunsten von 120 verbraucht. Bei 46 ist die erwünschte 1,2-Methylverschiebung mit anschließender 1,3Hydridverschiebung unter Bildung von $( \pm$ )-endo-Capnellen (39) leider nicht zu beobachten. Den Grund dafür kennen wir nicht. Da 46 keine 1,2-Methylverschiebung eingeht und im Gegensatz zu 107 wegen ungünstiger Stereochemie auch keine 1,3-Hydridverschiebung eingehen kann, ist das zur Produktbildung notwendige Kation 122 nur über zwei konsekutive 1,2Verschiebungen und damit über ein energiereiches sekundäres Kation zugänglich. Deprotonierung und Isomerisierung liefern dann 119 (Schema 40). 
Schema 40

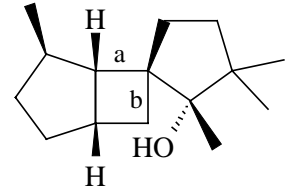

44

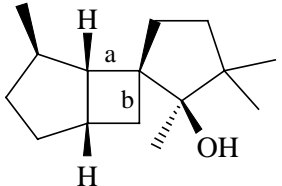

45

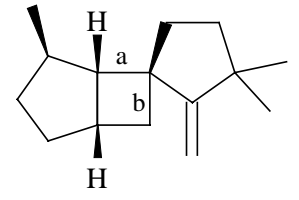

103

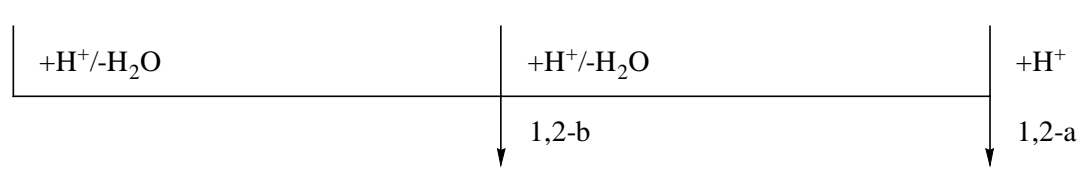

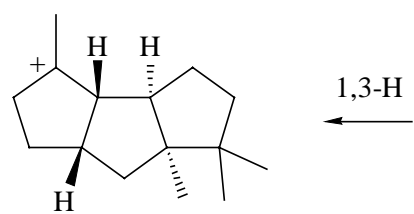

121

$$
+\mathrm{H}^{+} \uparrow-\mathrm{H}^{+}
$$

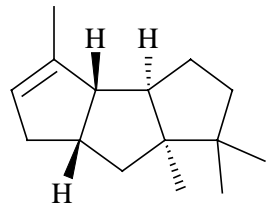

120

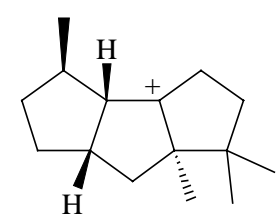

107

$+\mathrm{H}^{+} \mid \downarrow-\mathrm{H}^{+}$

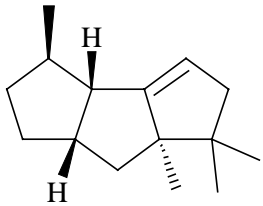

118

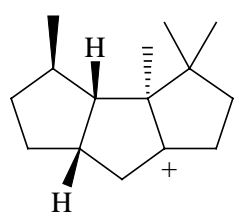

46

$1,2-\mathrm{H}$

$\sqrt{1,2-\mathrm{H}}$

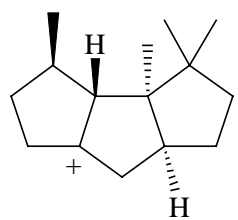

122 Deprotonierung
Isomerisierung

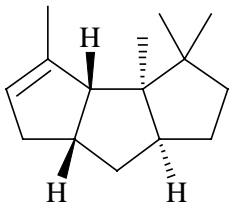

119

Eine Unsicherheit besteht: laut begleitender gaschromatographischer Analysen nimmt bei den Umlagerungen von 44, 45 und 103 der Gehalt an 120 mit zunehmender Zeit zugunsten von 119 ab (vgl. Tab. 3-5), obwohl für eine Umwandlung von 120 zu 119 kein plausibler Weg formuliert werden kann. Da die Analysen ohne internen Standard durchgeführt wurden könnte es sein, daß dieser Befund auf eine im Vergleich zu 119 schnellere Degradation von 120 zu gaschromatographisch nicht flüchtigen Produkten zurückzuführen ist. Sollten die Daten allerdings auch bei Verwendung eines internen Standards Bestand haben, so scheint eine Überprüfung der Struktur insbesondere von 119 geboten. 
In jedem Fall steht fest, daß ( \pm )-endo-Capnellen (39) auf den hier beschrittenen Wegen nicht zugänglich ist. Dieses macht deutlich, daß der Einsatz kationischer Umlagerungen in Totalsynthesen nach wie vor mit einem erheblichen Risiko behaftet ist. Dieses gilt insbesondere für mehrstufige Umlagerungen in komplexen Systemen. 


\section{ZUSAMMENFASSUNG}

Mit der vorliegenden Arbeit wurde versucht, das Potential einer sequentiellen Transformation von Bicyclobutylidenen in Bicyclo[3.3.0]octene auszuloten. Die zu prüfende Reaktionssequenz umfaßte eine Folge aus Epoxidierung, Oxaspirohexan-CyclopentanonUmlagerung, Methylierung und/oder Reduktion bzw. Addition von Methylithium mit abschlieBender säurekatalysierter Umlagerung. Unsubstituiertes Bicyclobutyliden (4) lieferte auf diese Weise über die Spiro[3.4] octan-5-one 14, 50 und 51 sowie die Spiro[3.4]octan-5-ole 21-25 die mono- bis trimethylierten Bicyclo[3.3.0] octene (Hexahydropentalene) 56, 58, 59, 60 und 61 (Schema 41).

Schema 41
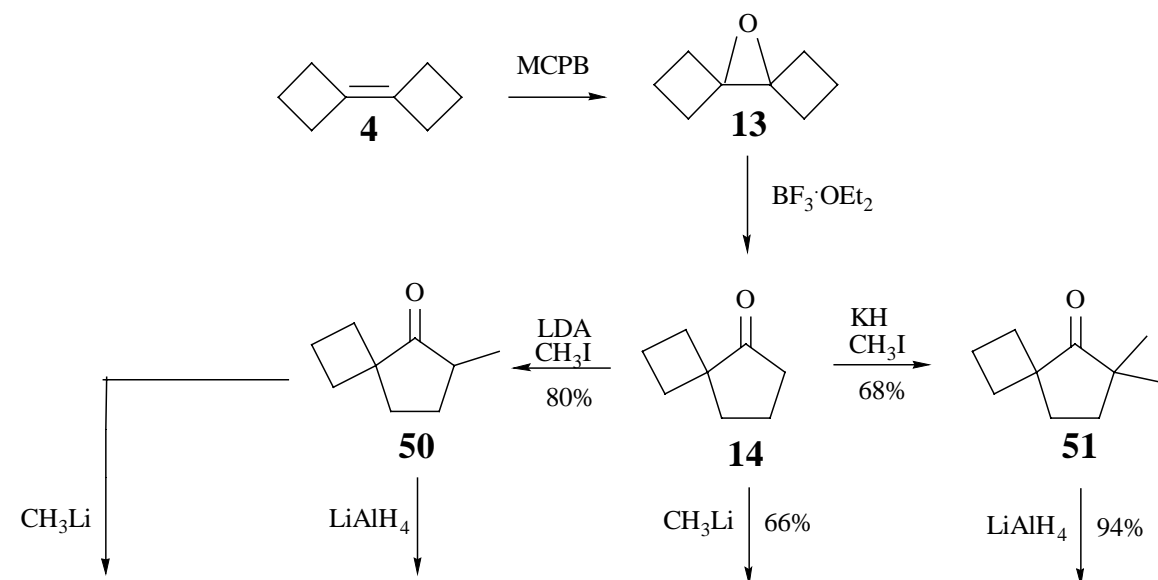

$$
50
$$

14

$$
\mathrm{LiAlH}_{4}
$$

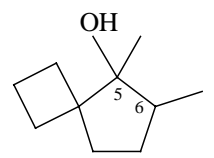

23a $\left(5 R^{*}, 6 R^{*}, 59 \%\right)$

23b $\left(5 S^{*}, 6 R^{*}, 7 \%\right)$

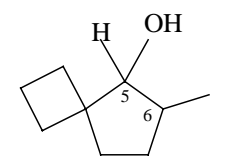

22a $\left(5 R^{*}, 6 R^{*}, 55 \%\right)$

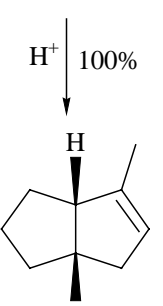

59 22b $\left(5 S^{*}, 6 R^{*}, 9 \%\right)$

$$
\mathrm{H}^{+} \mid 100 \%
$$

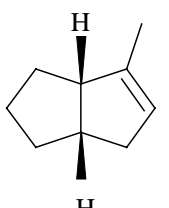

58

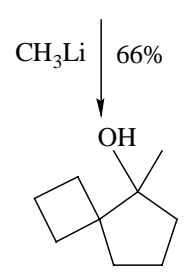

21
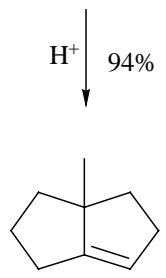

56

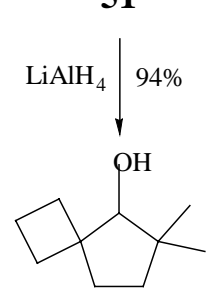

24
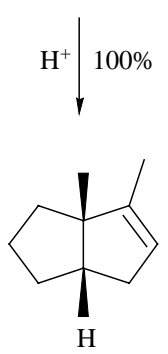

60

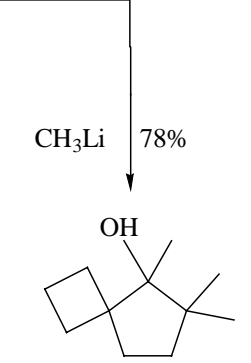

25

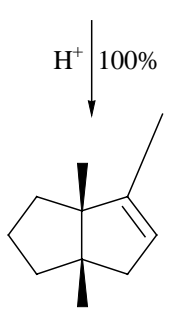

61 
Die mit dem Ziel einer Synthese von Partialstrukturen der Sesquiterpene $( \pm)$ Ptychanolid (33) und ( \pm )-Ceratopicanol (32) durchgeführten Arbeiten zur Synthese und sequentiellen Transformation von 2,2-Dimethyl-bicyclobutyliden (26) brachten nicht den gewünschten Erfolg. So traten bei der Cyclobutylidenierung von 29 neben 26 mit 62 und 63 auch Kondensationsprodukte auf, und das aus 26 durch Epoxidierung erhaltene Oxaspirohexan 64 öffnete nicht regiospezifisch und lieferte neben dem benötigten Spiroketon 27 auch das Isomer 67 (Schema 42). Daraufhin durchgeführte Versuche zur selektiven Darstellung von 27 und 67 über die $\beta$-Hydroxyselenide 49 (29-48-49-27) bzw. 76 (75-76-27) brachten zwar eine deutliche Verbesserung, aber bei 27 immer noch nicht genügend Material, um die angestrebte Synthese von 28 und dessen Umlagerung zu 31 als potentiellem Vorläufer von $\mathbf{3 3}$ führen zu können.

\section{Schema 42}

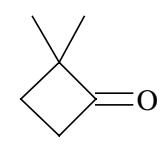

29
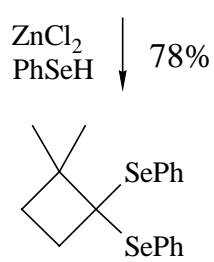

48

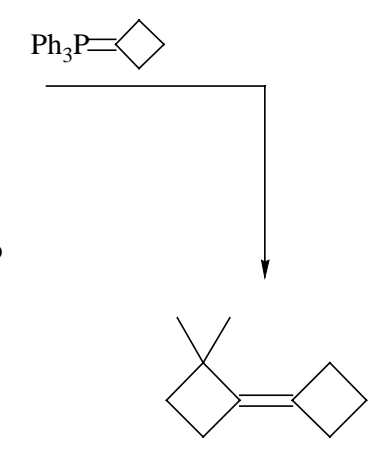

$26(20 \%)$

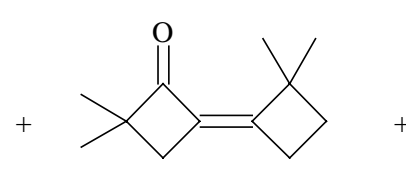

$62(26 \%)$

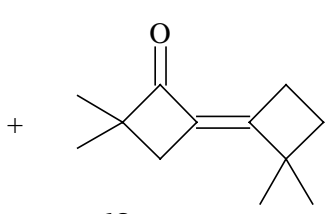

$63(22 \%)$

1. $\mathrm{n}-\mathrm{BuLi}=\mathrm{O} \mid 29 \% \quad$ MCРB $\mid 86 \%$

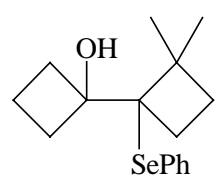

49
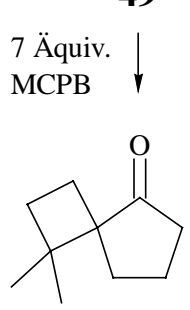

\begin{tabular}{l|l} 
& $\mathbf{2 7}$ \\
\hline $\mathbf{4 9}$ & $45 \%$ \\
$\mathbf{6 4}$ & $17 \%$ \\
$\mathbf{7 6}$ & -
\end{tabular}

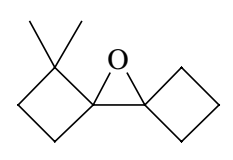

64

$\mathrm{BF}_{3} \mathrm{OEt}_{2}$

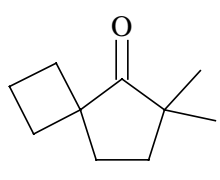

51

$10 \%$

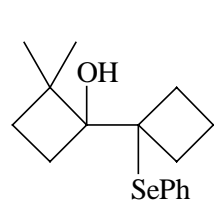

76

7 Äquiv. MCPB

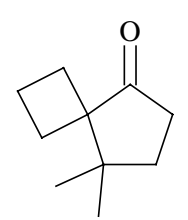

67

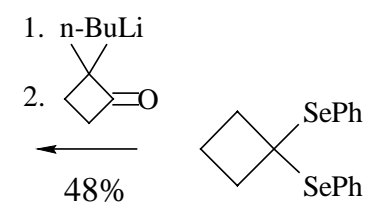

75 
Die Versuche zur säurekatalysierten Umlagerung der Ketone 51 und 67 dagegen verliefen erfolgreich. Unter der Einwirkung des stark sauren Ionenaustauschers Nafion R SAC-13 lieferten beide Verbindungen das bicyclische Keton $\mathbf{3 0}$ und damit die gewünschte Partialstruktur von ( \pm )-Ceratopicanol (32) (Schema 43). Das ohnehin nur in geringer Menge vorhandene 27 verharzte.

\section{Schema 43}

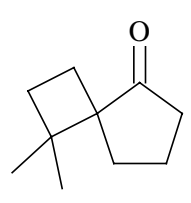

27

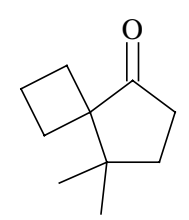

67

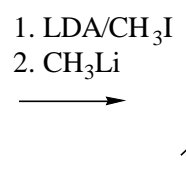

$\underset{100 \%}{\stackrel{\mathrm{H}^{+}}{\longrightarrow}}$

30

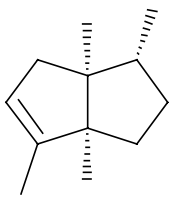

31

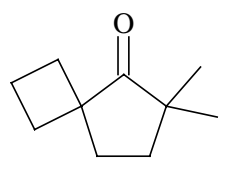

51

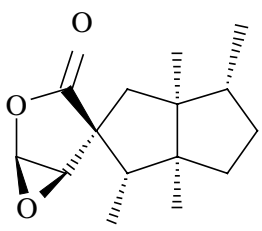

( \pm )-Ptychanolid (33)

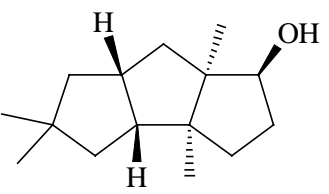

( \pm )-Ceratopicanol (32)

Nach Bicyclobutyliden (4) und 2,2-Dimethylbicyclobutyliden (26) wurde im dritten Teil der vorliegenden Arbeit mit $\mathbf{4 0}$ ein Bicyclobutyliden dargestellt und sequentiell verändert, das bei günstigem Verlauf der abschließenden Umlagerungen mit ( \pm )-endo-Capnellen (39) ein vollständiges Sesquiterpengerüst hätte liefern können. Allerdings zeigte sich hier, daß in komplexeren Fällen selbst das Ergebnis einer Oxaspirohexan-Cyclopentanon-Umlagerung nicht mehr vorhergesagt werden kann. So lieferte 40 nach Epoxidierung durch Bortrifluorid-katalysierte Umlagerung neben den stereoisomeren Cyclopentanonen $\mathbf{8 7}$ und $\mathbf{8 8}$ das hier unerwünschte Cyclopentanon 93 als Hauptprodukt. Die ausgehend von 87 und 88 durchgeführten Transformationen in Form einer erschöpfenden Methylierung gefolgt von einer Methylenierung bzw. einer Addition von Methyllithium verliefen problemlos und lieferten mit 44, 45 und 103 sowie 41, 42 und 104 Vorläufer der tricyclischen Kationen 46 und 107 bzw. 105 und 106. (Schema 44). 


\section{Schema 44}

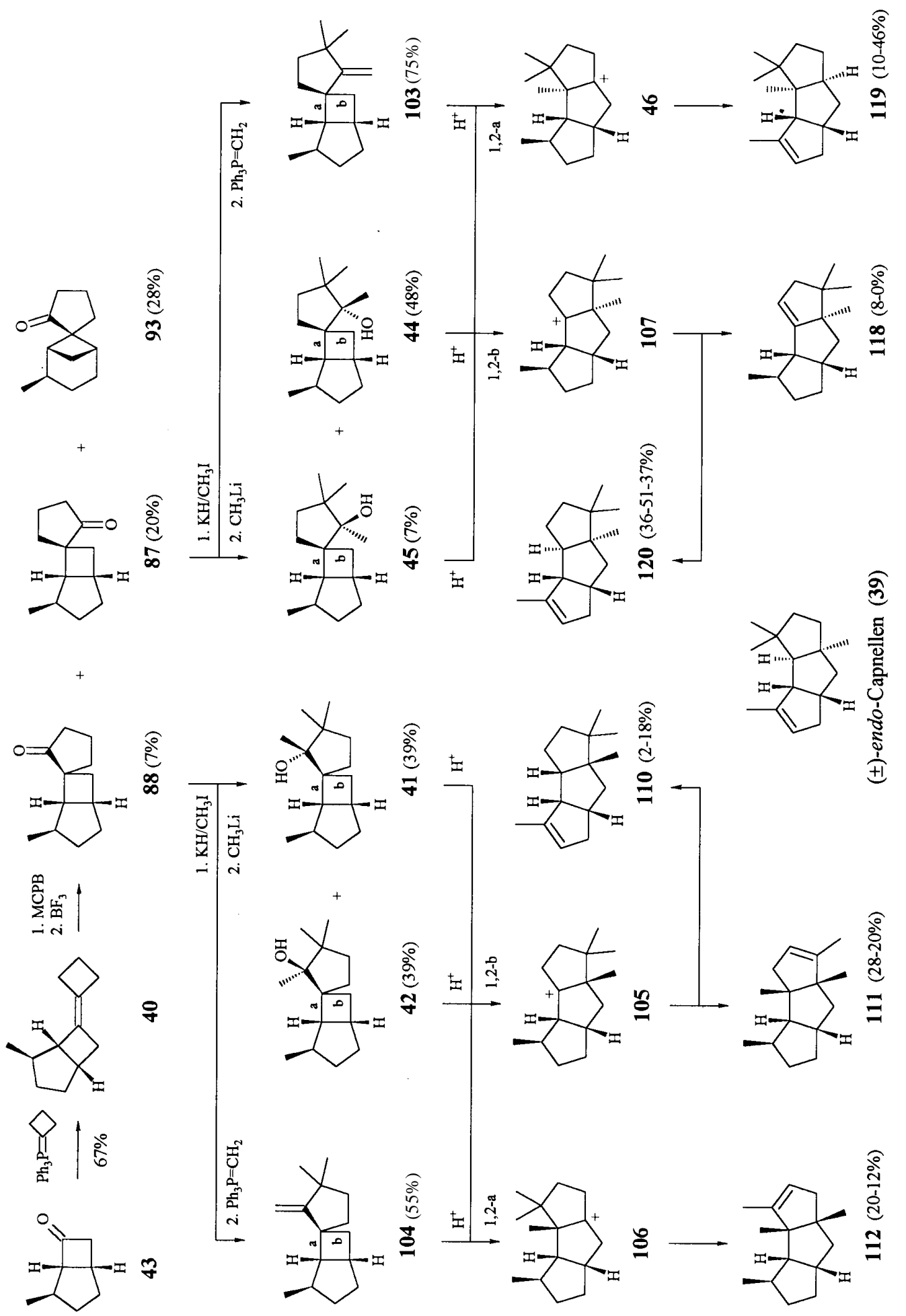


Von diesen Kationen konnte lediglich 46 über eine 1,2-Methylverschiebung, gefolgt von einer 1,3-Hydridverschiebung, in ( \pm )-endo-Capnellen (39) übergehen. Aber auch dieser Fall trat nicht ein. Zwar lieferte die säurekatalysierte Umlagerung von 44, 45 und 103 unter konkurrierender 1,2-Verschiebung der höher (a) und niedriger substituierten Cyclobutanbindung (b) neben 107 auch 46, doch blieb die erhoffte Bildung von ( \pm )-endo-Capnellen (39) aus. Die beobachteten Produkte waren 118, 119 und 120. Im Gegensatz zu 44, 45 und 103 war bei der säurekatalysierten Umlagerung von 41, 42 und 104 eine Bildung von ( \pm )-endo-Capnellen (39) von vornherein ausgeschlossen. Hier waren die beobachteten Produkte 110, 111 und 112. Sämtliche Strukturen wurden spektroskopisch gesichert und ihr Bildungsmechanismus diskutiert.

Insgesamt hat die vorliegende Arbeit gezeigt, daß die sequentielle Transformation von Bicyclobutylidenen über Spiro[3.4]octan-5-ole in übersichtlich gelagerten Fällen anderen Methoden zur Darstellung substituierter Bicyclo[3.3.0]octene überlegen ist. Bei Einbindung des Bicyclobutylidens in komplexere Systeme ist das Risiko eines Auftretens von der Erwartung abweichender Umlagerungen allerdings erheblich. 


\section{Experimenteller Teil}

\section{Allgemeine Angaben}

IR-Spektren wurden mit einem Spektrometer Modell 457 der Firma Perkin-Elmer aufgenommen.

${ }^{1}$ H- und ${ }^{13}$ C-NMR-Spektren wurden auf einem Gerät MERCURY 200, VXR 200, UNITY 300, INOVA 500 oder INOVA 600 der Firma Varian, bzw. einem Gerät AMX 300 der Firma Bruker aufgenommen. Chemische Verschiebungen sind als $\delta$-Werte in ppm angegeben und beziehen sich auf Tetramethylsilan $(\delta=0.00)$ als internen Standard. Bei Verwendung anderer Standards wurden folgende chemische Verschiebungen zugrunde gelegt: $\delta_{\mathrm{H}}$ $\left(\mathrm{CHCl}_{3}\right)=7.24, \delta_{\mathrm{C}}\left(\mathrm{CDCl}_{3}\right)=77.00, \delta_{\mathrm{H}}\left(\mathrm{C}_{6} \mathrm{D}_{5} \mathrm{H}\right)=7.15, \delta_{\mathrm{C}}\left(\mathrm{C}_{6} \mathrm{D}_{6}\right)=128$ ppm. Multiplizitäten wurden, soweit angegeben, mit den in der Literatur ${ }^{46}$ angegebenen Methoden bestimmt.

Massenspektren wurden auf einem Spektrometer Modell MAT 311 A der Firma Varian oder einem Modell MAT 95 der Firma Finnigan aufgenommen. Präzise Massen wurden mit dem Modell MAT 731 der Firma Varian oder dem Modell MAT 95 der Firma Finnigan bestimmt.

Präparative gaschromatographische Trennungen wurden mit einem Gerät GC 6000 Vega Series 2 der Firma Carlo Erba oder einem Gerät IGC 121 MLR der Firma Delsi mit Wärmeleitfähigkeitsdetektoren durchgeführt. Als Trägergas diente Wasserstoff (40-60 $\mathrm{ml} / \mathrm{min}$ ). Angaben über Art und Abmessungen der Säulen, Trägermaterialien, Art der Stationären Phasen, Belegung in Prozent und Säulentemperatur finden sich in den jeweiligen Versuchsbeschreibungen.

Integrationen wurden mit einem Gerät D 2000 der Firma Merck - Hitachi oder einem Gerät SP 4270 der Firma Carlo Erba durchgeführt. Responsefaktoren wurden nicht bestimmt. 
Dünnschichtchromatographische Untersuchungen erfolgten an DC-Plastikfolien Polygram SIL G/UV254 (Schichtdicke 0.25 mm) der Firma Macherey \& Nagel. Farblose Substanzen wurden durch Besprühen mit 4 prozentiger ethanolischer Molybdatophosphorsäure (Merck) und anschließendem Erwärmen sichtbar gemacht. Mit Silbernitrat dotierte DCPlastikfolien wurden durch Eintauchen (5 min) in eine 10 prozentiger Lösung von Silbernitrat in Methanol/Wasser $(2: 1)$ hergestellt und $1 \mathrm{~h}$ bei $110{ }^{\circ} \mathrm{C}$ getrocknet.

Für säulenchromatographische Trennungen stand ein Fraktionssammler Modell LINEAR II der Firma Serva Technik, sowie ein Modell FC 220 der Firma Gilson zur Verfügung. Mit Silbernitrat dotiertes Kieselgel wurde durch Aufschlämmen einer entsprechenden Menge Kieselgels (0.04 - $0.063 \mathrm{~mm})$ in einer Lösung von Silbernitrat in Acetonitril und Abziehen des Lösungsmittels im Rotationsverdampfer hergestellt. Das Material wurde bei $40{ }^{\circ} \mathrm{C} / 15$ Torr bis zur Gewichtskonstanz getrocknet. Angaben über das zur Trennung verwendete Material sowie über die Säulenabmessungen finden sich in den jeweiligen Versuchsbeschreibungen.

Schmelzpunkte wurden auf einem Mikroheiztisch der Firma Reichert bestimmt und sind nicht korrigiert.

Elementaranalysen wurden von Herrn Hambloch, mikroanalytische Abteilung des Institutes für Organische Chemie der Universität Göttingen, durchgeführt.

\section{Arbeitsvorschriften}

\section{Allgemeine Vorschrift zur Dimethylierung der Ketone 14, 88, 87 und 93}

$\mathrm{Zu}$ einer Suspension von Kaliumhydrid (0.12 g, $3.00 \mathrm{mmol} / 1.00 \mathrm{mmol}$ Keton) in wasserfreiem Ether (3.0 ml / 1.00 mmol Keton) gab man bei $0{ }^{\circ} \mathrm{C}$ das entsprechende Keton. Nach 30 min tropfte man Methyliodid (0.34 g, $3.00 \mathrm{mmol} / 1.00 \mathrm{mmol}$ Keton) zu. Nach $45 \mathrm{~min}$ zeigte gaschromatographische Analyse [Säule A: $3.3 \mathrm{~m} \mathrm{x} \mathrm{1/4"} \mathrm{Allglassystem,} \mathrm{15 \%} \mathrm{OV} 101$ auf Chromosorb W AW/DMCS 68/80 mesh; Säule B: 3 m x 1/4" Allglassystem, 15\% FFAP auf Chromosorb W AW/DMCS 60/80 mesh] für alle Proben vollständigen Umsatz an. Daraufhin hydrolysierte man vorsichtig mit gesättigter Natriumhydrogencarbonatlösung $(0.2 \mathrm{ml} / 1.00$ 
mmol Keton), trennte die Phasen und extrahierte die wässrige Phase zweimal mit Ether (1.0 ml / 1.00 mmol Keton). Nach dem Trocknen über Molekularsieb (3 Å) wurden die Lösungsmittel im Rotationsverdampfer entfernt (Badtemperatur $20{ }^{\circ} \mathrm{C} / 20$ Torr). In allen Fällen lieferte abschließende Chromatographie an Kieselgel $(0.05-0.20 \mathrm{~mm})$ in Pentan/Ether, bzw. fraktionierende Destillation das entsprechende reine Keton.

6,6-Dimethyl-spiro[3.4] ]octan-5-on (51): Eingesetzt: $3.60 \mathrm{~g}$ (29 mmol) 14; Ausbeute: $3.00 \mathrm{~g}$ (68\%) 51, farblose Flüssigkeit (Sdp.: $55^{\circ} \mathrm{C} / 5$ Torr), Retentionszeiten (min): 1.97 (14), 2.42 (51) ( Säule A, $150{ }^{\circ} \mathrm{C}$ ).

$\mathrm{C}_{10} \mathrm{H}_{16} \mathrm{O}$

Ber. C 79.89 H 10.59

Gef. C 79.16 H 10.59

IR (KBr): $2960 \mathrm{~cm}^{-1}(\mathrm{CH}), 1730(\mathrm{C}=\mathrm{O})$.

${ }^{1} \mathbf{H}-\mathbf{N M R}\left(300 \mathrm{MHz}, \mathrm{CDCl}_{3}, \mathrm{CHCl}_{3}\right.$ int.): $\delta=0.96 \mathrm{ppm}(\mathrm{s}, 6 \mathrm{H}), 1.60$ (t, J = $\left.6 \mathrm{~Hz}, 2 \mathrm{H}\right), 1.76$ $\left(\mathrm{m}_{\mathrm{c}}, 2 \mathrm{H}\right), 1.92\left(\mathrm{~m}_{\mathrm{c}}, 4 \mathrm{H}\right), 2.20\left(\mathrm{~m}_{\mathrm{c}}, 2 \mathrm{H}\right)$.

${ }^{13}$ C-NMR (50 MHz, $\mathrm{CDCl}_{3}, \mathrm{CDCl}_{3}$ int. $): \delta=15.86 \mathrm{ppm}\left(\mathrm{C}_{\mathrm{sek}}\right), 24.61\left(\mathrm{C}_{\text {prim }}\right), 30.58,33.42$, $34.73\left(\mathrm{C}_{\text {sek }}\right), 44.45,50.75,224.44\left(\mathrm{C}_{\text {quart }}\right)$.

MS $(70 \mathrm{eV}): \mathrm{m} / \mathrm{z}(\%)=152\left(\mathrm{M}^{+}, 68\right), 68(100)$.

$\left(1 R^{*}, 4 S^{*}, 5 R^{*}, 6 R^{*}\right)-4,3^{\prime}, 3^{\prime}-T^{\prime}$ rimethyl-spiro\{bicyclo[3.2.0]heptan-6,1'-cyclopentan-2'on\} (95): Eingesetzt: $100 \mathrm{mg} \mathrm{88}$; Ausbeute: $98 \mathrm{mg}$ (82\%) 95; farbloser Feststoff (Smp.: 28 ${ }^{\circ} \mathrm{C}$ ); Retentionszeiten (min): 4.49 (95) und 5.63 (88) (Säule B, $200{ }^{\circ} \mathrm{C}$ ); $\mathrm{R}_{\mathrm{f}}$-Wert in Pen$\tan /$ Ether 95: 5: 0.46 .

$\mathrm{C}_{14} \mathrm{H}_{22} \mathrm{O}$ 
Ber. 206.1671

Gef. 206.1670 HRMS

IR (KBr): $2950 \mathrm{~cm}^{-1}(\mathrm{CH}), 1725(\mathrm{C}=\mathrm{O})$.

${ }^{1} \mathbf{H}-\mathrm{NMR}\left(300 \mathrm{MHz}, \mathrm{CDCl}_{3}, \mathrm{CHCl}_{3}\right.$ int.): $\delta=0.84 \mathrm{ppm}(\mathrm{d}, \mathrm{J}=6 \mathrm{~Hz}, 3 \mathrm{H}), 0.98(\mathrm{~s}, 3 \mathrm{H}), 1.00$ $(\mathrm{s}, 3 \mathrm{H}), 1.24-2.00(\mathrm{~m}, 11 \mathrm{H}) ; 2.62\left(\mathrm{~m}_{\mathrm{c}}, 2 \mathrm{H}\right)$.

${ }^{13}$ C-NMR (50 MHz, $\mathrm{CDCl}_{3}, \mathrm{CDCl}_{3}$ int.): $\delta=13.09$ ppm, 24.98, $25.13\left(\mathrm{C}_{\text {prim }}\right)$, 27.32, 27.91, $27.99,33.20,34.65\left(\mathrm{C}_{\text {sek }}\right), 36.95,37.93,43.45\left(\mathrm{C}_{\text {tert }}\right), 44.67,49.64,226.08\left(\mathrm{C}_{\text {quart }}\right)$.

MS (70 eV): m/z (\%): $206\left(\mathrm{M}^{+}, 8\right), 81(100)$

$\left(1 R^{*}, 2 R^{*}, 5 R^{*}, 6 S^{*}\right)-2,3^{\prime}, 3^{\prime}$-Trimethyl-spiro\{bicyclo[3.1.1]heptan-6,1'-cyclopentan-2'on $\}$ (96) und $\left(1 R^{*}, 4 S^{*}, 5 R^{*}, 6 S^{*}\right)-4,3^{\prime}, 3^{\prime}$-Trimethyl-spiro\{bicyclo[3.2.0]heptan-6,1'cyclopentan-2'-on\} (94): Eingesetzt: 0.75 g 87 und 93 im Verhältnis 35 : 65; Ausbeute: 0.46 g (53\%) 96, 0.27 g (31\%) 94; Retentionszeiten (min): 4.34 (96/94), 6.25 (93/87) (Säule B $\left.200{ }^{\circ} \mathrm{C}\right) ; \mathrm{R}_{\mathrm{f}}$-Werte Pentan/Ether $98: 2: 0.23$ (96) und $0.19(\mathbf{9 4})$.

$\left(1 R^{*}, 2 R^{*}, 5 R^{*}, 6 S^{*}\right)-2,3^{\prime}, 3^{\prime}-T^{\prime}$ rimethyl-spiro\{bicyclo[3.1.1]heptan-6,1'-cyclopentan-2'on $)(96)$

$\mathrm{C}_{14} \mathrm{H}_{22} \mathrm{O}$

Ber. 206.1671

Gef. 206.1670 HRMS

IR (KBr): $2960 \mathrm{~cm}^{-1}(\mathrm{CH}), 1725(\mathrm{C}=\mathrm{O})$. 
${ }^{1} \mathrm{H}-\mathrm{NMR}\left(300 \mathrm{MHz}, \mathrm{CDCl}_{3}, \mathrm{CHCl}_{3}\right.$ int.): $\delta=0.80 \mathrm{ppm}(\mathrm{d}, \mathrm{J}=6 \mathrm{~Hz}, 3 \mathrm{H}), 0.94(\mathrm{~s}, 3 \mathrm{H}), 0.98$ $(\mathrm{s}, 3 \mathrm{H}), 1.18\left(\mathrm{~m}_{\mathrm{c}}, 1 \mathrm{H}\right), 1.30(\mathrm{~d}, \mathrm{~J}=9 \mathrm{~Hz}, 1 \mathrm{H}), 1.52\left(\mathrm{~m}_{\mathrm{c}}, 2 \mathrm{H}\right), 1.60$ - $1.78(\mathrm{~m}, 5 \mathrm{H}), 1.92\left(\mathrm{~m}_{\mathrm{c}}\right.$, $1 \mathrm{H}), 2.00\left(\mathrm{~m}_{\mathrm{c}}, 1 \mathrm{H}\right), 2.24\left(\mathrm{~m}_{\mathrm{c}}, 1 \mathrm{H}\right), 2.50\left(\mathrm{~m}_{\mathrm{c}}, 1 \mathrm{H}\right)$.

${ }^{13}$ C-NMR (50 MHz, $\mathrm{CDCl}_{3}, \mathrm{CDCl}_{3}$ int.): $\delta=20.66$ ppm $\left(\mathrm{C}_{\text {prim }}\right), 21.55,23.73,24.21,24.41$ $\left(\mathrm{C}_{\text {sek }}\right), 25.20,25.43\left(\mathrm{C}_{\text {prim }}\right), 29.13\left(\mathrm{C}_{\text {tert }}\right), 33.93\left(\mathrm{C}_{\text {sek }}\right), 38.53,44.32\left(\mathrm{C}_{\text {tert }}\right), 44.92,58.60$, $224.89\left(\mathrm{C}_{\text {quart }}\right)$.

MS (70 eV): m/z (\%): $206\left(\mathrm{M}^{+}, 93\right), 191(100)$.

$\left(1 R^{*}, 4 S^{*}, 5 R^{*}, 6 S^{*}\right)-4,3^{\prime}, 3^{\prime}$-Trimethyl-spiro\{bicyclo[3.2.0]heptan-6,1'-cyclopentan-2'on\} (94)

$\mathrm{C}_{14} \mathrm{H}_{22} \mathrm{O}$

Ber. 206.1671

Gef. 206.1670 HRMS

IR (KBr): $2950 \mathrm{~cm}^{-1}(\mathrm{CH}), 1730(\mathrm{C}=\mathrm{O})$.

${ }^{1} \mathbf{H}-\mathbf{N M R}\left(300 \mathrm{MHz}, \mathrm{CDCl}_{3}, \mathrm{CHCl}_{3}\right.$ int.): $\delta=0.92 \mathrm{ppm}(\mathrm{s}, 3 \mathrm{H}), 1.08$ (s, 3H), 1.10 (d, J = 6 $\mathrm{Hz}, 3 \mathrm{H}), 1.26(\mathrm{dd}, \mathrm{J}=12,6 \mathrm{~Hz}, 1 \mathrm{H}), 1.38-1.70(\mathrm{~m}, 5 \mathrm{H}), 1.72-2.16(\mathrm{~m}, 5 \mathrm{H}), 2.62\left(\mathrm{~m}_{\mathrm{c}}, 1 \mathrm{H}\right)$, $2.76\left(\mathrm{~m}_{\mathrm{c}}, 1 \mathrm{H}\right)$.

${ }^{13}$ C-NMR (50 MHz, $\mathrm{CDCl}_{3}, \mathrm{CDCl}_{3}$ int.): $\delta=15.50 \mathrm{ppm}, 24.42,26.17\left(\mathrm{C}_{\text {prim }}\right), 31.36,32.83$ $\left(\mathrm{C}_{\text {sek }}\right), 33.27\left(\mathrm{C}_{\text {tert }}\right), 33.67,34.68,37.32\left(\mathrm{C}_{\text {sek }}\right), 39.56\left(\mathrm{C}_{\text {tert }}\right), 43.61\left(\mathrm{C}_{\text {quart }}\right), 45.12\left(\mathrm{C}_{\text {tert }}\right), 51.65$, $225.24\left(\mathrm{C}_{\text {quart }}\right)$.

MS (70 eV): m/z (\%): $206\left(\mathrm{M}^{+}, 14\right), 125(100)$ 


\section{Allgemeine Vorschrift zur Reduktion der Ketone 50 und 51}

$\mathrm{Zu}$ einer Suspension aus Lithiumalanat $(0.61 \mathrm{~g}, 2 \mathrm{mmol} / 1 \mathrm{mmol}$ Keton $)$ in trockenem Ether (16 ml) gab man unter Rühren und Stickstoff eine Lösung des entsprechenden Ketons in wasserfreiem Ether ( $1 \mathrm{ml} / 1 \mathrm{mmol}$ Keton) so $\mathrm{zu}$, daß die Mischung gelinde siedete. Nach beendeter Zugabe erhitzte man unter Rückfluß. Nach 1 h zeigte gaschromatographische Kontrolle [3.3 m x 1/4" Allglassystem, 15\% OV 101 auf Chromosorb W AW/DMCS 68/80 mesh] quantitativen Umsatz an. Man kühlte auf $0{ }^{\circ} \mathrm{C}$ ab und hydrolysierte das Reaktionsgemisch vorsichtig mit Wasser (72 $\mu \mathrm{l} / 1 \mathrm{mmol}$ Keton), 15 \%iger Natriumhydroxidlösung (72 $\mu \mathrm{l} / 1$ mmol Keton) und Wasser (215 $\mu \mathrm{l} / 1 \mathrm{mmol}$ Keton). Der ausgefallene Feststoff wurde über Celite abfiltriert, der Rückstand mit Ether (2 $\mathrm{ml} / 1$ mmol Keton) gewaschen und das Lösungsmittel im Rotationsverdampfer (Badtemperatur $20{ }^{\circ} \mathrm{C} / 15$ Torr) entfernt. Abschließende Chromatographie an Kieselgel (0.05 - $0.20 \mathrm{~mm})$ lieferte die jeweiligen Alkohole als farblose Flüssigkeiten.

6,6-Dimethyl-spiro[3.4] octan-5-ol (24): Eingesetzt: 1.22 g 51; Ausbeute: 1.17 g (94\%) 24; Retentionszeiten (min): 2.38 (51), $3.52(\mathbf{2 4})\left(150{ }^{\circ} \mathrm{C}\right) ; \mathrm{R}_{\mathrm{f}}$-Wert in Pentan/Ether $8: 2: 0.3$.

\section{$\mathrm{C}_{10} \mathrm{H}_{18} \mathrm{O}$}

Ber. C 77.87 H 11.76

Gef. C 77.72 H 11.77

IR (KBr): $3620 \mathrm{~cm}^{-1}(\mathrm{OH}), 3600-3120\left(\mathrm{OH}_{\mathrm{ass}}\right), 2950(\mathrm{CH})$.

${ }^{1}$ H-NMR (300 MHz, $\mathrm{CDCl}_{3}, \mathrm{CHCl}_{3}$ int.): $\delta=0.82 \mathrm{ppm}(\mathrm{s}, 3 \mathrm{H}), 0.96$ (s, 3H), $1.30-1.61$ (m, $4 \mathrm{H}), 1.74\left(\mathrm{~m}_{\mathrm{c}}, 3 \mathrm{H}\right), 1.88\left(\mathrm{~m}_{\mathrm{c}}, 2 \mathrm{H}\right), 2.06\left(\mathrm{~m}_{\mathrm{c}}, 1 \mathrm{H}\right), 2.26\left(\mathrm{~m}_{\mathrm{c}}, 1 \mathrm{H}\right), 3.26(\mathrm{~s}, 1 \mathrm{H})$.

${ }^{13}$ C-NMR (50 MHz, $\mathrm{CDCl}_{3}, \mathrm{CDCl}_{3}$ int. $): \delta=16.72 \mathrm{ppm}\left(\mathrm{C}_{\mathrm{sek}}\right), 22.62,28.46\left(\mathrm{C}_{\text {prim }}\right), 29.03$, $33.13,35.74,36.79\left(\mathrm{C}_{\text {sek }}\right), 41.23,49.34\left(\mathrm{C}_{\text {quart }}\right), 86.66\left(\mathrm{C}_{\text {tert }}\right)$.

MS $(70 \mathrm{eV}): \mathrm{m} / \mathrm{z}(\%)=154\left(\mathrm{M}^{+}, 6\right), 139(100)$ 
$\left(5 R^{*}, 6 R^{*}\right)-6-M e t h y l-s p i r o[3.4]$ octan-5-ol $\quad(22 a)$ und $\quad\left(5 S^{*}, 6 R^{*}\right)-6$-Methyl-spiro[3.4]octan-5-ol (22b):Eingesetzt: 1.10 g 50; Ausbeute: 0.32 g (29\%) 22a, 0.62 g (55\%) 22b und 0.10 g (9\%) 48 : 52 Gemisch aus 22a und 22b; Retentionszeiten (min): 6.90 (50), 7.78 (22a/22b) $\left(130{ }^{\circ} \mathrm{C}\right) ; \mathrm{R}_{\mathrm{f}}-$ Werte in Pentan/Ether $8: 2: 0.22$ (22a), 0.18 (22b).

$\left(5 R^{*}, 6 R^{*}\right)-6-M e t h y l-s p i r o[3.4]$ octan-5-ol (22a)

\section{$\mathrm{C}_{9} \mathrm{H}_{16} \mathrm{O}$}

Ber. C 77.09 H 11.50

Gef. C 76.99 H 11.40

IR (KBr): $3620 \mathrm{~cm}^{-1}(\mathrm{OH}), 3600-3140\left(\mathrm{OH}_{\mathrm{ass}}\right), 2950(\mathrm{CH})$.

${ }^{1}$ H-NMR (300 MHz, $\mathrm{CDCl}_{3}, \mathrm{CHCl}_{3}$ int.): $\delta=0.98 \mathrm{ppm}(\mathrm{d}, \mathrm{J}=6 \mathrm{~Hz}, 3 \mathrm{H}), 1.18-1.34$ (m, $2 \mathrm{H}), 1.74\left(\mathrm{~m}_{\mathrm{c}}, 8 \mathrm{H}\right), 1.98\left(\mathrm{~m}_{\mathrm{c}}, 1 \mathrm{H}\right), 2.14\left(\mathrm{~m}_{\mathrm{c}}, 1 \mathrm{H}\right), 3.65(\mathrm{~d}, \mathrm{~J}=4 \mathrm{~Hz}, 1 \mathrm{H})$.

${ }^{13}$ C-NMR (50 MHz, $\mathrm{CDCl}_{3}, \mathrm{CDCl}_{3}$ int. $): \delta=14.39$ ppm $\left(\mathrm{C}_{\text {prim }}\right), 15.80,26.97,29.20,33.99$, $36.00\left(\mathrm{C}_{\text {sek }}\right), 36.19\left(\mathrm{C}_{\text {tert }}\right), 50.74\left(\mathrm{C}_{\text {quart }}\right), 82.19\left(\mathrm{C}_{\text {tert }}\right)$.

MS $(70 \mathrm{eV}): \mathrm{m} / \mathrm{z}(\%)=140\left(\mathrm{M}^{+}, 3\right), 97(100)$.

$\left(5 S^{*}, 6 R^{*}\right)-6-M e t h y l-s p i r o[3.4]$ octan-5-ol (22b)

\section{$\mathrm{C}_{9} \mathrm{H}_{16} \mathrm{O}$}

Ber. C 77.09 H 11.50

Gef. C 76.87 H 11.47

IR (KBr): $3620 \mathrm{~cm}^{-1}(\mathrm{OH}), 3580-3080\left(\mathrm{OH}_{\mathrm{ass}}\right), 2940(\mathrm{CH})$. 
${ }^{1}$ H-NMR (300 MHz, $\mathrm{CDCl}_{3}, \mathrm{CHCl}_{3}$ int.): $\delta=1.00 \mathrm{ppm}(\mathrm{d}, \mathrm{J}=6 \mathrm{~Hz}, 3 \mathrm{H}), 1.02-1.10$ (m, $1 \mathrm{H}), 1.52-2.01(\mathrm{~m}, 10 \mathrm{H}), 2.12\left(\mathrm{~m}_{\mathrm{c}}, 1 \mathrm{H}\right), 3.22(\mathrm{~d}, \mathrm{~J}=6 \mathrm{~Hz}, 1 \mathrm{H})$.

${ }^{13}$ C-NMR (50 MHz, $\mathrm{CDCl}_{3}, \mathrm{CDCl}_{3}$ int.): $\delta=16.44 \mathrm{ppm}\left(\mathrm{C}_{\mathrm{sek}}\right), 19.08\left(\mathrm{C}_{\text {prim }}\right), 27.12,28.62$, 30.61, $35.41\left(\mathrm{C}_{\text {sek }}\right), 40.12\left(\mathrm{C}_{\text {tert }}\right), 49.31\left(\mathrm{C}_{\text {quart }}\right), 85.33\left(\mathrm{C}_{\text {tert }}\right)$.

MS (70 eV): m/z (\%) = $140\left(\mathrm{M}^{+}, 3\right), 97(100)$.

Allgemeine Vorschrift zur Addition von Methyllithium an die Ketone 14, 50, 51, 94 und 95

$\mathrm{Zu}$ einer 0.5 M Lösung von Methyllithium in Ether (1.00 mmol, $2 \mathrm{ml} / 1.00 \mathrm{mmol}$ Keton) tropfte man bei $0{ }^{\circ} \mathrm{C}$ unter Stickstoff und Rühren eine Lösung des entsprechenden Ketons in wasserfreiem Ether (1 ml / 1.00 mmol Keton). Gaschromatographische Analyse [Säule A: 3.3 m x 1/4" Allglassystem, 15\% OV 101 auf Chromosorb W AW/DMCS 68/80 mesh; Säule B: $3 \mathrm{~m} x$ 1/4" Allglassystem, 15\% FFAP auf Chromosorb W AW/DMCS 60/80 mesh] zeigte nach 15 min für alle Proben quantitativen Umsatz. Man hydrolysierte das Reaktionsgemisch mit gesättigter Ammoniumchloridlösung ( $1 \mathrm{ml} / 1.00$ mmol Keton), trennte die Phasen, extrahierte die wässrige Phase mit Ether ( $2 \times 2 \mathrm{ml} / 1.00 \mathrm{mmol}$ Keton), trocknete die vereinigten organischen Phasen über Magnesiumsulfat und entfernte das Lösungsmittel im Rotationsverdampfer (Badtemperatur $20{ }^{\circ} \mathrm{C} / 15$ Torr). In allen Fällen lieferte abschließende Chromatographie der Rohprodukte an Kieselgel (0.05 - $0.20 \mathrm{~mm})$ die reinen Alkohole.

5,6,6-Trimethyl-spiro[3.4]octan-5-ol (25): Eingesetzt: 0.46 g, 3 mmol 51; Ausbeute: $0.40 \mathrm{~g}$ (78\%) 25; Retentionszeiten ( $\min ): 2.38$ (51), 4.16 (25) (Säule A, $150{ }^{\circ} \mathrm{C}$ ); $\mathrm{R}_{\mathrm{f}}$-Wert in Pen$\tan /$ Ether $8: 2: 0.35$.

\section{$\mathrm{C}_{11} \mathrm{H}_{20} \mathrm{O}$}

Ber. C 78.75 H 11.98

Gef. C 78.76 H 11.89

IR (KBr): $3620 \mathrm{~cm}^{-1}(\mathrm{OH}), 3600-3180\left(\mathrm{OH}_{\text {ass }}\right), 2960(\mathrm{CH})$. 
${ }^{1}$ H-NMR (300 MHz, $\mathrm{CDCl}_{3}, \mathrm{CHCl}_{3}$ int.): $\delta=0.78 \mathrm{ppm}(\mathrm{s}, 3 \mathrm{H}), 0.92$ (s, 3H), 1.06 (s, 3H), $1.14(\mathrm{~s}, 1 \mathrm{H}), 1.41\left(\mathrm{~m}_{\mathrm{c}}, 1 \mathrm{H}\right), 1.62\left(\mathrm{~m}_{\mathrm{c}}, 4 \mathrm{H}\right), 1.94\left(\mathrm{~m}_{\mathrm{c}}, 3 \mathrm{H}\right), 2.14\left(\mathrm{~m}_{\mathrm{c}}, 2 \mathrm{H}\right)$.

${ }^{13}$ C-NMR (50 MHz, $\mathrm{CDCl}_{3}, \mathrm{CDCl}_{3}$ int. $): \delta=15.75 \mathrm{ppm}\left(\mathrm{C}_{\mathrm{sek}}\right), 18.23,23.46,26.09\left(\mathrm{C}_{\text {prim }}\right)$, $29.89,33.90,36.70,37.10\left(\mathrm{C}_{\mathrm{sek}}\right), 44.84,52.92,83.08\left(\mathrm{C}_{\text {quart }}\right)$.

MS $(70 \mathrm{eV}): \mathrm{m} / \mathrm{z}(\%)=168\left(\mathrm{M}^{+}, 7\right), 97(100)$.

5-Methyl-spiro[3.4]octan-5-ol (21): Eingesetzt: $1.00 \mathrm{~g}, 8.05$ mmol 14; Ausbeute: $0.74 \mathrm{~g}$ (66\%); Retentionszeiten (min): 1.95 (14), 2.33 (21) (Säule A, $150{ }^{\circ} \mathrm{C}$ ); $\mathrm{R}_{\mathrm{f}}$-Wert in Pen$\tan /$ Ether $8: 2: 0.21$.

\section{$\mathrm{C}_{9} \mathrm{H}_{16} \mathrm{O}$}

Ber. C 77.09 H 11.50

Gef. C 76.95 H 11.37

IR (KBr): $3610 \mathrm{~cm}^{-1}(\mathrm{OH}), 3590-3120\left(\mathrm{OH}_{\mathrm{ass}}\right), 2960(\mathrm{CH})$.

${ }^{1}$ H-NMR (300 MHz, $\mathrm{CDCl}_{3}, \mathrm{CHCl}_{3}$ int.): $\delta=1.21 \mathrm{ppm}(\mathrm{s}, 3 \mathrm{H}), 1.36$ (br s, $\left.1 \mathrm{H}\right), 1.44-2.30$ $(\mathrm{m}, 12 \mathrm{H})$.

${ }^{13}$ C-NMR (50 MHz, $\mathrm{CDCl}_{3}, \mathrm{CDCl}_{3}$ int.): $\delta=14.98$ ppm, $16.72\left(\mathrm{C}_{\text {sek }}\right), 22.59\left(\mathrm{C}_{\text {prim }}\right), 26.18$, $29.10,37.00,37.66\left(\mathrm{C}_{\text {sek }}\right), 52.03,80.31\left(\mathrm{C}_{\text {quart }}\right)$.

MS $(70 \mathrm{eV}): \mathrm{m} / \mathrm{z}(\%)=140\left(\mathrm{M}^{+}, 6\right), 97(100)$

$\left(5 R^{*}, 6 R^{*}\right)-5,6-D i m e t h y l-s p i r o[3.4]$ octan-5-ol $\quad(23 a)$ und $\left(5 S^{*}, 6 R^{*}\right)-5,6-$ Dimethylspiro[3.4] octan-5-ol (23b): Eingesetzt: 490 mg, 3.50 mmol 50; Ausbeute: $317 \mathrm{mg}$ (59\%) 23a, $37 \mathrm{mg}$ (7\%) 23b und $137 \mathrm{mg}$ (25\%) $4: 1$ Gemisch aus 23a und 23b; Retentionszeiten 
(min): 4.59 (51), 8.25 (23a), 9.81 (23b) (Säule B, $140{ }^{\circ} \mathrm{C}$ ); $\mathrm{R}_{\mathrm{f}}$-Werte in Pentan/Ether 8 : 2: 0.79 (50), 0.33 (23a), 0.28 (23b).

$(5 \mathrm{R} *, 6 \mathrm{R} *)-5,6-D i m e t h y l-s p i r o[3.4]$ octan-5-ol (23a)

$\mathrm{C}_{10} \mathrm{H}_{18} \mathrm{O}$

Ber. C 77.87 H 11.76

Gef. C 76.97 H 11.76

IR (KBr): $3610 \mathrm{~cm}^{-1}(\mathrm{OH}), 3590-3120\left(\mathrm{OH}_{\text {ass }}\right), 2950(\mathrm{CH})$.

${ }^{1} \mathrm{H}-\mathrm{NMR}\left(300 \mathrm{MHz}, \mathrm{CDCl}_{3}, \mathrm{CHCl}_{3}\right.$ int.): $\delta=0.91 \mathrm{ppm}(\mathrm{d}, \mathrm{J}=6 \mathrm{~Hz}, 3 \mathrm{H}), 1.18(\mathrm{~s}, 3 \mathrm{H}), 1.20$ - $1.30(\mathrm{~m}, 1 \mathrm{H}), 1.49-1.96(\mathrm{~m}, 10 \mathrm{H}), 2.1\left(\mathrm{~m}_{\mathrm{c}}, 1 \mathrm{H}\right)$.

${ }^{13}$ C-NMR (50 MHz, $\mathrm{CDCl}_{3}, \mathrm{CDCl}_{3}$ int. $): \delta=13.26 \mathrm{ppm}\left(\mathrm{C}_{\text {prim }}\right), 15.18\left(\mathrm{C}_{\text {sek }}\right), 20.70\left(\mathrm{C}_{\text {prim }}\right)$, $26.34,28.63,31.77,36.57\left(\mathrm{C}_{\text {sek }}\right), 40.86\left(\mathrm{C}_{\text {tert }}\right), 52.79,81.19\left(\mathrm{C}_{\text {quart }}\right)$.

$\operatorname{MS}(70 \mathrm{eV}): \mathrm{m} / \mathrm{z}(\%)=154\left(\mathrm{M}^{+}, 18\right), 111(100)$

$(5 \mathrm{~S} *, 6 \mathrm{R} *)-5,6-$ Dimethyl-spiro[3.4] $]$ octan-5-ol (23b)

$\mathrm{C}_{10} \mathrm{H}_{18} \mathrm{O}$

Ber. C 77.87 H 11.76

Gef. C 77.72 H 11.77

IR (KBr): $3610 \mathrm{~cm}^{-1}(\mathrm{OH}), 3600-3100\left(\mathrm{OH}_{\mathrm{ass}}\right), 2950(\mathrm{CH})$.

${ }^{1}$ H-NMR $\left(300 \mathrm{MHz}, \mathrm{CDCl}_{3}, \mathrm{CHCl}_{3}\right.$ int.): $\delta=0.90 \mathrm{ppm}(\mathrm{d}, \mathrm{J}=4 \mathrm{~Hz}, 3 \mathrm{H}), 0.92$ (s, 3H), 1.06 $\left(\mathrm{m}_{\mathrm{c}}, 1 \mathrm{H}\right), 1.28($ br s, $1 \mathrm{H}) 1.43-2.26(\mathrm{~m}, 10 \mathrm{H})$. 
${ }^{13}$ C-NMR (50 MHz, $\mathrm{CDCl}_{3}, \mathrm{CDCl}_{3}$ int. $): \delta=14.79 \mathrm{ppm}\left(\mathrm{C}_{\text {prim }}\right), 15.19\left(\mathrm{C}_{\text {sek }}\right), 16.60\left(\mathrm{C}_{\text {prim }}\right)$, $27.45,27.55,28.47,34.68\left(\mathrm{C}_{\text {sek }}\right), 41.25\left(\mathrm{C}_{\text {tert }}\right), 52.23,80.37\left(\mathrm{C}_{\text {quart }}\right)$.

MS $(70 \mathrm{eV}): \mathrm{m} / \mathrm{z}(\%)=154\left(\mathrm{M}^{+}, 13\right), 111(100)$

$\left(1 R^{*}, 4 S^{*}, 5 R^{*}, 6 S^{*}, 2^{\prime} S^{*}\right)-4,2^{\prime}, 3^{\prime}, 3^{\prime}$-Tetramethyl-spiro\{bicyclo[3.2.0]heptan-6,1'-

cyclopentan-2'-ol $\} \quad(45) \quad$ und $\quad\left(1 R^{*}, 4 S^{*}, 5 R^{*}, 6 S^{*}, 2^{\prime} R^{*}\right)-4,2^{\prime}, 3^{\prime}, 3^{\prime}$-Tetramethylspiro\{bicyclo[3.2.0]heptan-6,1'-cyclopentan-2'-ol\} (44): Eingesetzt: 0,10 g, 0.48 mmol 94; Ausbeute: $10 \mathrm{mg}$ (9\%) 45, farbloser Feststoff, (Smp.: $46{ }^{\circ} \mathrm{C}$ ), $63 \mathrm{mg}$ (59\%) 44 und $26 \mathrm{mg}$ (24\%) 47 : 53 Gemisch aus 44 und 45, farblose Flüssigkeiten; Retentionszeiten (min): 4.96 (94), 9.95 (45), 10.55 (44) (Säule B, $\left.200{ }^{\circ} \mathrm{C}\right)$; $\mathrm{R}_{\mathrm{f}}$-Werte in Pentan/Ether 9 : 1: 0.30 (45), 0.19 (44).

$\left(1 R^{*}, 4 S^{*}, 5 R^{*}, 6 S^{*}, 2^{\prime} S^{*}\right)-4,2^{\prime}, 3^{\prime}, 3^{\prime}-T e t r a m e t h y l-s p i r o\left\{b i c y c l o[3.2 .0] h e p t a n-6,1^{\prime}-\right.$ cyclopentan-2'-ol\} (45)

$\mathrm{C}_{15} \mathrm{H}_{26} \mathrm{O}$

Ber. 222.1984

Gef. 222.1983 HRMS

IR (KBr): $3600 \mathrm{~cm}^{-1}(\mathrm{OH}), 3580-3400\left(\mathrm{OH}_{\mathrm{ass}}\right), 2940(\mathrm{CH})$.

${ }^{1} \mathbf{H}-\mathbf{N M R}\left(300 \mathrm{MHz}, \mathrm{CDCl}_{3}, \mathrm{CHCl}_{3}\right.$ int.): $\delta=0.83 \mathrm{ppm}(\mathrm{s}, 3 \mathrm{H}), 0.86$ (s, 3H), 0.97 (s, 3H), $1.08(\mathrm{dd}, \mathrm{J}=10,7 \mathrm{~Hz}, 1 \mathrm{H}), 1.18(\mathrm{~d}, \mathrm{~J}=6 \mathrm{~Hz}, 3 \mathrm{H}), 1.25-1.34(\mathrm{~m}, 2 \mathrm{H}), 1.45-1.80(\mathrm{~m}, 7 \mathrm{H})$, $2.00\left(\mathrm{~m}_{\mathrm{c}}, 1 \mathrm{H}\right), 2.20-2.34(\mathrm{~m}, 2 \mathrm{H}), 2.56\left(\mathrm{~m}_{\mathrm{c}}, 1 \mathrm{H}\right)$.

${ }^{13}$ C-NMR (75 MHz, $\mathrm{CDCl}_{3}, \mathrm{CDCl}_{3}$ int.): $\delta=13.93$ ppm, 14.37, 25.35, $26.17\left(\mathrm{C}_{\text {prim }}\right), 31.89$, 33.52, 33.78, $37.82\left(\mathrm{C}_{\text {sek }}\right), 37.92,39.02\left(\mathrm{C}_{\text {tert }}\right), 41.24\left(\mathrm{C}_{\text {quart }}\right), 42.71\left(\mathrm{C}_{\text {sek }}\right), 58.99\left(\mathrm{C}_{\text {tert }}\right)$, 59.57, $93.53\left(\mathrm{C}_{\text {quart }}\right)$. 
MS (70 eV): m/z (\%): $222\left(\mathrm{M}^{+}, 2\right), 111(100)$.

$\left(1 R^{*}, 4 S^{*}, 5 R^{*}, 6 S^{*}, 2^{\prime} R^{*}\right)-4,2^{\prime}, 3^{\prime}, 3^{\prime}-T^{\prime}$ etramethyl-spiro\{bicyclo[3.2.0]heptan-6,1'-

cyclopentan-2’ol\} (44)

$\mathrm{C}_{15} \mathrm{H}_{26} \mathrm{O}$

Ber. 222.1984

Gef. 222.1983 HRMS

IR (KBr): $3610 \mathrm{~cm}^{-1}(\mathrm{OH}), 3580-3480\left(\mathrm{OH}_{\mathrm{ass}}\right), 2935(\mathrm{CH})$.

${ }^{1}$ H-NMR (300 MHz, $\mathrm{CDCl}_{3}, \mathrm{CHCl}_{3}$ int.): $\delta=0.94$ ppm (s, 3H), 0.96 (s, 3H), 1.12 (s, 3H), $1.16(\mathrm{~d}, \mathrm{~J}=6 \mathrm{~Hz}, 3 \mathrm{H}), 1.18-1.32(\mathrm{~m}, 1 \mathrm{H}), 1.40-1.62(\mathrm{~m}, 6 \mathrm{H}), 1.68-1.78(\mathrm{~m}, 2 \mathrm{H}), 1.86$ $\left(\mathrm{m}_{\mathrm{c}}, 1 \mathrm{H}\right), 2.04-2.16(\mathrm{~m}, 1 \mathrm{H}), 2.42\left(\mathrm{~m}_{\mathrm{c}}, 2 \mathrm{H}\right), 2.58\left(\mathrm{~m}_{\mathrm{c}}, 1 \mathrm{H}\right)$.

${ }^{13}$ C-NMR (75 MHz, $\mathrm{CDCl}_{3}, \mathrm{CDCl}_{3}$ int.): $\delta=14.99$ ppm, 19.08, 26.13, $27.26\left(\mathrm{C}_{\text {prim }}\right), 31.38$, $32.78\left(\mathrm{C}_{\text {sek }}\right), 33.32\left(\mathrm{C}_{\text {tert }}\right), 33.70,35.10,37.39\left(\mathrm{C}_{\text {sek }}\right), 40.33\left(\mathrm{C}_{\text {tert }}\right), 43.10\left(\mathrm{C}_{\text {quart }}\right), 46.50\left(\mathrm{C}_{\text {tert }}\right.$, $53.25,83.70\left(\mathrm{C}_{\text {quart }}\right)$.

MS (70 eV): m/z (\%): $222\left(\mathrm{M}^{+}, 44\right), 81(100)$.

$\left(1 R^{*}, 4 S^{*}, 5 R^{*}, 6 R^{*}, 2^{\prime} R^{*}\right)-4,2^{\prime}, 3^{\prime}, 3^{\prime}-$ Tetramethyl-spiro\{bicyclo[3.2.0]heptan-6,1'-

cyclopentan-2'-ol $\} \quad(41) \quad$ und $\quad\left(1 R^{*}, 4 S^{*}, 5 R^{*}, 6 R^{*}, 2^{\prime} S^{*}\right)-4,2^{\prime}, 3^{\prime}, 3^{\prime}$-Tetramethylspiro\{bicyclo[3.2.0]heptan-6,1'-cyclopentan-2'-ol\} (42): Eingesetzt: $100 \mathrm{mg}, 0.48 \mathrm{mmol}$ 95; Ausbeute: $94 \mathrm{mg}$ (88\%) 50 : 50 Gemisch aus 41 und $42\left({ }^{1} \mathrm{H}-\mathrm{NMR}-\right.$-spektroskopische Analyse); Retentionszeiten (min): 4.64 (95), 8.74 (41 und 42) (Säule B, $200{ }^{\circ} \mathrm{C}$ ); $\mathrm{R}_{\mathrm{f}}$-Wert in Pentan/Ether $9: 1: 0.26$. 
$\mathrm{C}_{15} \mathrm{H}_{26} \mathrm{O}$

IR (KBr): $3620 \mathrm{~cm}^{-1}(\mathrm{OH}), 3600-3420\left(\mathrm{OH}_{\mathrm{ass}}\right), 2960(\mathrm{CH})$.

${ }^{1}$ H-NMR (300 MHz, $\mathrm{C}_{6} \mathrm{D}_{6}, \mathrm{C}_{6} \mathrm{D}_{5} \mathrm{H}$ int.): $\delta=0.76 \mathrm{ppm}(\mathrm{s}, 3 \mathrm{H}), 0.80$ (s, 3H), 0.90 (d, J = 6 Hz, 3H), 0.91 (d, J = 6 Hz, 3H), 1.02 (s, 3H), 1.03 (s, 3H), 1.08 (s, 3H), 1.10 (s, 3H), 1.32 $\left(\mathrm{m}_{\mathrm{c}}, 11 \mathrm{H}\right), 1.52-1.76(\mathrm{~m}, 9 \mathrm{H}), 1.78-2.02(\mathrm{~m}, 4 \mathrm{H}), 2.24\left(\mathrm{~m}_{\mathrm{c}}, 2 \mathrm{H}\right), 2.48\left(\mathrm{~m}_{\mathrm{c}}, 1 \mathrm{H}\right), 2.58\left(\mathrm{~m}_{\mathrm{c}}\right.$ $1 \mathrm{H})$.

${ }^{13}$ C-NMR (75 MHz, $\mathrm{C}_{6} \mathrm{D}_{6}, \mathrm{C}_{6} \mathrm{D}_{6}$ int.): $\delta=13.67 \mathrm{ppm}, 13.95,17.47,17.60,23.95,24.01$ $\left(\mathrm{C}_{\text {prim }}\right), 26.81\left(\mathrm{C}_{\text {sek }}\right), 27.19$ (Koinzidenz zweier Signale) $\left(\mathrm{C}_{\text {prim }}\right), 28.11,28.72,29.50,31.64$, 33.42, 33.68, $33.80\left(\mathrm{C}_{\text {sek }}\right), 37.43,37.55\left(\mathrm{C}_{\text {tert }}\right), 37.65,38.03\left(\mathrm{C}_{\text {sek }}\right), 38.46,38.69,42.09$, $44.71\left(\mathrm{C}_{\text {tert }}\right), 54.43,45.94,50.66,51.26,83.74,83.83\left(\mathrm{C}_{\text {quart }}\right)$.

MS (70 eV): m/z (\%): $222\left(\mathrm{M}^{+}, 73\right), 189$ (100).

\section{Allgemeine Vorschrift zur Umlagerung der Spiro[3.4]octan-5-ole 21, 22a,b, 23a,b und} 24

$\mathrm{Zu}$ einer $0.074 \mathrm{M}$ Lösung von wasserfreier p-Toluolsulfonsäure $(1.0 \mathrm{mmol}, 13.5 \mathrm{ml} / 1.0$ mmol Spiro[3.5] octan-5-ol) in Benzol tropfte man unter Stickstoff und Rühren bei $60{ }^{\circ} \mathrm{C}$ eine 0.5 M Lösung des entsprechenden Spiro[3.4]octan-5-ols in wasserfreiem Benzol (1.0 mmol, $1.5 \mathrm{ml} / 1.0 \mathrm{mmol}$ Spiro[3.5]octan-5-ol) und hielt $3 \mathrm{~h}$ am Rückfluß. Die Reaktionsmischung wurde mit gesättigter Natriumhydrogencarbonatlösung (3.5 ml / 1.0 mmol Spiro[3.5]octan-5ol) hydrolysiert und die Phasen getrennt. Die wässrige Phase wurde anschließend mit Pentan extrahiert ( $2 \times 3.0 \mathrm{ml} / 1.0 \mathrm{mmol}$ Spiro[3.5]octan-5-ol), die organische Phase mit gesättigter Natriumhydrogencarbonatlösung gewaschen $(2 \times 1.5 \mathrm{ml} / 1.0 \mathrm{mmol}$ Spiro[3.5]octan-5-ol) und über Magnesiumsulfat getrocknet. Die Lösungsmittel wurden über eine $20 \mathrm{~cm}$ VigreuxKolonne entfernt. Analytische Proben der farblosen Flüssigkeiten wurden durch präparative Gaschromatographie [3.3 m x 1/4" Allglassystem, 15\% OV 101 auf Chromosorb W AW/DMCS 68/80 mesh] erhalten. 
3a-Methyl-1,2,3,3a,4,5-hexahydro-pentalen (56) und 6a-Methyl-hexahydro-pentalen3a-ol (57): Eingesetzt: 0.42 g, 3 mmol 21; Retentionszeiten (min): 2.42 (56), 4.72 (57) (120 $\left.{ }^{\circ} \mathrm{C}\right)$. Die ${ }^{1} \mathrm{H}-\mathrm{NMR}$ und ${ }^{13} \mathrm{C}-\mathrm{NMR}$-Daten von $\mathbf{5 6}$ und 57 entsprachen den Literaturdaten ${ }^{5}$.

(3aR*,6aR*)-6-Methyl-1,2,3,3a,4,6a-hexahydro-pentalen (58): Eingesetzt: 0.14 g, 1.0 mmol 22a,b im Verhältnis 44 : 56; Retentionszeit (min): 2.85 (58) $\left(120{ }^{\circ} \mathrm{C}\right)$. Die ${ }^{1} \mathrm{H}-\mathrm{NMR}$ und ${ }^{13} \mathrm{C}$-NMR-Daten von $\mathbf{5 8}$ entsprachen den Literaturdaten ${ }^{28-32}$.

(3aR*,6aR*)-3a,6-Dimethyl-1,2,3,3a,4,6a-hexahydro-pentalen (59): Eingesetzt: $0.23 \mathrm{~g}$, 1.5 mmol 23a,b im Verhältnis 25 :75,;Retentionszeit (min): $2.81(\mathbf{5 9})\left(120{ }^{\circ} \mathrm{C}\right)$. Die ${ }^{1} \mathrm{H}-\mathrm{NMR}$ und ${ }^{13} \mathrm{C}-\mathrm{NMR}-$ Daten von $\mathbf{5 9}$ entsprachen den Literaturdaten ${ }^{33-36}$.

(3aR*,6aR*)-6,6a-Dimethyl-1,2,3,3a,4,6a-hexahydro-pentalen (60): Eingesetzt: $0.23 \mathrm{~g}$, 1.5 mmol 24; Retentionszeit (min), : $1.60(60)\left(150{ }^{\circ} \mathrm{C}\right)$.

$\mathrm{C}_{10} \mathrm{H}_{16}$

Ber. C 88.16 H 11.84

Gef. C 88.18 H 11.65

IR (KBr): $2940 \mathrm{~cm}^{-1}(\mathrm{CH}), 1620(\mathrm{C}=\mathrm{C})$.

${ }^{1}$ H-NMR (300 MHz, $\mathrm{CDCl}_{3}, \mathrm{CHCl}_{3}$ int.): $\delta=1.05 \mathrm{ppm}(\mathrm{s}, 3 \mathrm{H}), 1.18-1.55(\mathrm{~m}, 5 \mathrm{H}), 1.57$ $\left(\mathrm{m}_{\mathrm{c}}, 3 \mathrm{H}\right), 1.74-1.88(\mathrm{~m}, 2 \mathrm{H}), 2.12\left(\mathrm{~m}_{\mathrm{c}}, 1 \mathrm{H}\right), 2.52\left(\mathrm{~m}_{\mathrm{c}}, 1 \mathrm{H}\right), 2.12($ br s, $1 \mathrm{H})$.

${ }^{13}$ C-NMR (50 MHz, $\mathrm{CDCl}_{3}, \mathrm{CDCl}_{3}$ int.): $\delta=12.69$ ppm, $25.84\left(\mathrm{C}_{\text {prim }}\right), 26.00,36.05,37.98$, $38.51\left(\mathrm{C}_{\text {sek }}\right), 49.11\left(\mathrm{C}_{\text {tert }}\right), 58.18\left(\mathrm{C}_{\text {quart }}\right), 122.30\left(\mathrm{C}_{\text {tert }}\right), 145.33\left(\mathrm{C}_{\text {quart }}\right)$.

MS $(70 \mathrm{eV}): \mathrm{m} / \mathrm{z}(\%)=136\left(\mathrm{M}^{+}, 26\right), 107(100)$ 


\section{6-Methyl-spiro[3.4]octan-5-on (50)}

$\mathrm{Zu}$ einer Lösung von Diisopropylamin $(8.1 \mathrm{~g}, 80 \mathrm{mmol})$ in wasserfreiem Tetrahydrofuran $(160 \mathrm{ml})$ tropfte man unter Stickstoff und Rühren bei $5-10{ }^{\circ} \mathrm{C}$ eine $1.6 \mathrm{M}$ Lösung von $\mathrm{n}$ Butyllithium in n-Hexan (50 ml, $80 \mathrm{mmol})$. Anschließend fügte man 14 (9.9 g, $80 \mathrm{mmol}) \mathrm{zu}$, ließ auf Raumtemperatur erwärmen, hielt $0.5 \mathrm{~h}$ bei dieser Temperatur, kühlte auf $-78{ }^{\circ} \mathrm{C}$ ab und tropfte Methyliodid (56.8 g, $400 \mathrm{mmol}$ ) zu. Danach wurde das Reaktionsgemisch auf $25{ }^{\circ} \mathrm{C}$ erwärmt und $2.5 \mathrm{~h}$ bei dieser Temperatur gehalten, bis gaschromatographische Analyse [3.3 m x 1/4" Allglassystem, 15\% OV 101 auf Chromosorb W AW/DMCS 68/80 mesh, 130 ${ }^{\circ} \mathrm{C}$; Retentionszeiten (min): 9.19 (14) (8\%), 11.94 (50) (89\%), 12.93 (51) (3\%)] keinen weiteren Umsatz mehr zeigte. Man ließ auf Raumtemperatur erwärmen, hydrolysierte mit gesättigter Ammoniumchloridlösung (20 ml), dekantierte ab, extrahierte den Rückstand mit Pentan (3 x $75 \mathrm{ml}$ ), trocknete die vereinigten organischen Phasen über Magnesiumsulfat und engte über eine $30 \mathrm{~cm}$ Vigreux-Kolonne ein. Der Rückstand wurde mit Pentan (20 ml) versetzt und mit 1 N Salzsäure $(10 \mathrm{ml})$ extrahiert. Danach wurde die organische Phase mit Wasser $(2 \times 10$ ml) gewaschen und über Magnesiumsulfat getrocknet. Anschließende fraktionierte Destillation ergab $8.8 \mathrm{~g}(80 \%) \mathbf{5 0}$ (Sdp.: $85-87^{\circ} \mathrm{C} / 30$ Torr).

\section{6-Methyl-spiro[3.4]octan-5-on (50)}

\section{$\mathrm{C}_{9} \mathrm{H}_{14} \mathrm{O}$}

Ber. C $78.21 \mathrm{H} 10.21$

Gef. C 78.44 H 10.48

IR (KBr): $2940 \mathrm{~cm}^{-1}(\mathrm{CH}), 1730(\mathrm{C}=\mathrm{O})$.

${ }^{1}$ H-NMR (300 MHz, $\mathrm{CDCl}_{3}, \mathrm{CHCl}_{3}$ int.): $\delta=1.06 \mathrm{ppm}(\mathrm{d}, \mathrm{J}=6 \mathrm{~Hz}, 3 \mathrm{H}), 1.32\left(\mathrm{~m}_{\mathrm{c}}, 1 \mathrm{H}\right)$, $1.76\left(\mathrm{~m}_{\mathrm{c}}, 3 \mathrm{H}\right), 1.92\left(\mathrm{~m}_{\mathrm{c}}, 2 \mathrm{H}\right), 2.08\left(\mathrm{~m}_{\mathrm{c}}, 4 \mathrm{H}\right), 2.30\left(\mathrm{~m}_{\mathrm{c}}, 1 \mathrm{H}\right)$.

${ }^{13}$ C-NMR (50 MHZ, $\mathrm{CDCL}_{3}, \mathrm{CDCL}_{3}$ int.): $\delta=15.27 \mathrm{ppm}\left(\mathrm{C}_{\text {prim }}\right), 15.78,27.97,28.66$, $32.11,35.04\left(\mathrm{C}_{\text {sek }}\right), 42.93\left(\mathrm{C}_{\text {tert }}\right), 50.70,222.98\left(\mathrm{C}_{\text {quart }}\right)$. 
MS $(70 \mathrm{eV}): \mathrm{m} / \mathrm{z}(\%)=138\left(\mathrm{M}^{+}, 61\right), 67(100)$.

\section{Allgemeine Vorschrift zur Cyclobutylidenierung der Ketone 29, 40 und 91}

$\mathrm{Zu}$ einer Suspension von 4-Brombutyltriphenylphosphoniumbromid (0.52 g, $1.1 \mathrm{mmol} / 1.0$ mmol Keton) in trockenem Benzol (2 $\mathrm{ml} / 1.0 \mathrm{mmol}$ Keton) gab man in drei Portionen Kalium-tert-butylat $\left(0.25 \mathrm{~g}, 2.2 \mathrm{mmol} / 1.0 \mathrm{mmol}\right.$ Keton) und erhitzte auf $50{ }^{\circ} \mathrm{C}$. Nach drei Stunden kühlte man auf $40{ }^{\circ} \mathrm{C}$ ab, tropfte das entsprechende Keton zu und erhitzte auf $70{ }^{\circ} \mathrm{C}$, bis gaschromatographische Analyse [3 m x 1/4" Allglassystem, 15\% OV 101 auf Chromosorb W AW/DMCS 60/80 mesh], quantitativen Umsatz anzeigte. Es wurde mit Wasser $(0.1 \mathrm{ml} / 1.0$ mmol Keton) hydrolysiert und abdekantiert. Der Rückstand wurde mit Pentan (3 x $1 \mathrm{ml} / 1.0$ mmol Keton) extrahiert. Die Lösungsmittel wurden über eine $20 \mathrm{~cm}$ Vigreux-Kolonne bis zu einer Badtemperatur von $120{ }^{\circ} \mathrm{C}$ abdestilliert. Der Rückstand wurde nach Abkühlen in Pentan (5 $\mathrm{ml} / 1.0 \mathrm{mmol}$ Keton) aufgenommen, das ausgefallene Triphenylphosphinoxid abfiltriert und die Lösungsmittel abdestilliert. Der Rückstand wurde nochmals in Pentan (2.5 $\mathrm{ml} / 1.0$ mmol Keton) aufgenommen und das ausgefallene Triphenylphosphinoxid abfiltriert. Nach Abdestillieren der Lösungsmittel wurde der Rückstand chromatographiert und lieferte die reinen Olefine als farblose Flüssigkeiten.

2,2-Dimethylbicyclobutyliden (26), 3,3,2',2'-Tetramethyl-bicyclobutyliden-2-on (62) und 3,3,2',2'-Tetramethyl-bicyclobutyliden-2-on (63): Eingesetzt: 1.96 g, 20 mmol 29; Ausbeute: 0.54 g (20\%) 26, farblose Flüssigkeit, 0.48 g (13\%) 62 und 0.38 g (11\%) 63 als gelbe Flüssigkeiten; Reaktionsdauer: 3 h; Retentionszeiten (min): 2.87 (29), 7.33 (26), 9.19 (62), $11.76(63)$ (Temperaturprogramm: $80{ }^{\circ} \mathrm{C}, 10{ }^{\circ} \mathrm{C} / \mathrm{min}$, auf $220{ }^{\circ} \mathrm{C}$ ); Chromatographie an Kieselgel (0.05 - $0.20 \mathrm{~mm}$ ) in Pentan; $\mathrm{R}_{\mathrm{f}}$-Werte: 0.74 (26), 0.02, (62), 0.01 (63).

\section{2,2-Dimethylbicyclobutyliden (26)}

$\mathrm{C}_{10} \mathrm{H}_{16}$

Ber. C 88.16 H 11.84

Gef. C 87.96 H 11.62 
IR (KBr): $2960 \mathrm{~cm}^{-1}(\mathrm{CH}), 1660(\mathrm{C}=\mathrm{C})$.

${ }^{1} \mathbf{H}-\mathbf{N M R}\left(300 \mathrm{MHz}, \mathrm{CDCl}_{3}, \mathrm{CHCl}_{3}\right.$ int.): $\delta=1.16 \mathrm{ppm}(\mathrm{s}, 6 \mathrm{H}), 1.66$ (t, J = $\left.8 \mathrm{~Hz}, 2 \mathrm{H}\right), 1.94$ $(\mathrm{q}, \mathrm{J}=9 \mathrm{~Hz}, 2 \mathrm{H}), 2.36\left(\mathrm{~m}_{\mathrm{c}}, 2 \mathrm{H}\right), 2.54\left(\mathrm{~m}_{\mathrm{c}}, 2 \mathrm{H}\right), 2.64\left(\mathrm{~m}_{\mathrm{c}}, 2 \mathrm{H}\right)$.

${ }^{13}$ C-NMR (50 MHz, $\mathrm{CDCl}_{3}, \mathrm{CDCl}_{3}$ int. $): \delta=18.11 \mathrm{ppm}, 22.80\left(\mathrm{C}_{\text {sek }}\right), 26.79\left(\mathrm{C}_{\text {prim }}\right), 28.91$, $29.37,32.20\left(\mathrm{C}_{\text {sek }}\right), 43.44,128.19,137.37\left(\mathrm{C}_{\text {quart }}\right)$.

MS $(70 \mathrm{eV}): \mathrm{m} / \mathrm{z}(\%)=136\left(\mathrm{M}^{+}, 18\right), 93(100)$.

\section{$3,3,2^{\prime}, 2^{\prime}$-Tetramethyl-bicyclobutyliden-2-on (62)}

\section{$\mathrm{C}_{12} \mathrm{H}_{18} \mathrm{O}$}

Ber. 178.1358

Gef. 178. 1358 HRMS

IR (KBr): $2960 \mathrm{~cm}^{-1}(\mathrm{CH}), 1745(\mathrm{C}=\mathrm{O})$.

${ }^{1}$ H-NMR (200 MHz, $\mathrm{CDCl}_{3}, \mathrm{CHCl}_{3}$ int.) $\delta=1.18 \mathrm{ppm}(\mathrm{s}, 6 \mathrm{H}), 1.30$ (s, 6H), 1.86 (t, J = 8 $\mathrm{Hz}, 2 \mathrm{H}), 2.20(\mathrm{t}, \mathrm{J}=4 \mathrm{~Hz}, 2 \mathrm{H}), 2.56\left(\mathrm{~m}_{\mathrm{c}}, 2 \mathrm{H}\right)$.

${ }^{13}$ C-NMR (50 MHz, $\mathrm{CDCl}_{3}, \mathrm{CDCl}_{3}$ int. $): \delta=22.53 \mathrm{ppm}\left(\mathrm{C}_{\text {prim }}\right), 24.02\left(\mathrm{C}_{\text {sek }}\right), 26.36\left(\mathrm{C}_{\text {prim }}\right)$, 31.36, $35.36\left(\mathrm{C}_{\text {sek }}\right), 46.46,54.69,133.74,162.52,203.39\left(\mathrm{C}_{\text {quart }}\right)$.

MS $(70 \mathrm{eV}): \mathrm{m} / \mathrm{z}(\%)=178\left(\mathrm{M}^{+}, 70\right), 66(100)$

\section{$3,3,2^{\prime}, 2^{\prime}$-Tetramethyl-bicyclobutyliden-2-on (63)}

$$
\mathrm{C}_{12} \mathrm{H}_{18} \mathrm{O}
$$


Ber. 178.1358

Gef. 178. 1358 HRMS

IR (KBr): $2960 \mathrm{~cm}^{-1}(\mathrm{CH}), 1750(\mathrm{C}=\mathrm{O})$.

${ }^{1}$ H-NMR (300 MHz, $\mathrm{CDCl}_{3}, \mathrm{CHCl}_{3}$ int.): $\delta=1.16 \mathrm{ppm},(\mathrm{s}, 6 \mathrm{H}), 1.20(\mathrm{~s}, 6 \mathrm{H}), 1.84$ (t, J = 8 $\mathrm{Hz}, 2 \mathrm{H}), 2.30,(\mathrm{t}, \mathrm{J}=4 \mathrm{~Hz}, 2 \mathrm{H}), 2.86\left(\mathrm{~m}_{\mathrm{c}}, 2 \mathrm{H}\right)$.

${ }^{13}$ C-NMR (50 MHz, $\mathrm{CDCl}_{3}, \mathrm{CDCl}_{3}$ int.): $\delta=22.72 \mathrm{ppm}, 26.03\left(\mathrm{C}_{\text {prim }}\right), 27.79,32.56,35.92$ $\left(\mathrm{C}_{\text {sek }}\right), 45.59,57.30,134.70,160.32,206.35\left(\mathrm{C}_{\text {quart }}\right)$.

MS (70 eV): m/z (\%) = $178\left(\mathrm{M}^{+}, 100\right)$.

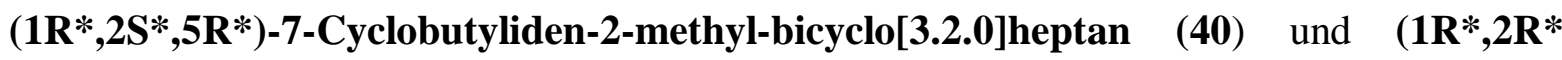
5R*)-7-Cyclobutyliden-2-methyl-bicyclo[3.2.0]heptan (91): Eingesetzt: 2.46 g, 20 mmol $\left(1 R^{*}, 4 S^{*}, 5 S^{*}\right)-4-M e t h y l b i c y c l o[3.2 .0]$ heptan-6-on $(43)$ und $\left(1 R^{*}, 4 R^{*}, 5 S^{*}\right)$-4-Methylbicyclo[3.2.0]hep-tan-6-on (90) im Verhältnis 84 : 16; Ausbeute: 2.15 g (67\%) 40 und 0.37 g (12\%) 91; Reaktionszeit: 15 min; Retentionszeiten (min): 4.90 (43/90), 7.75 (91), 8.33 (40) (Temperaturprogramm: $120{ }^{\circ} \mathrm{C}, 10{ }^{\circ} \mathrm{C} / \mathrm{min}$, auf $220{ }^{\circ} \mathrm{C}$ ); Chromatographie an mit $10 \%$ Silbernitrat dotiertem Kieselgel (0.040 - $0.063 \mathrm{~mm}$ ) in Pentan; $\mathrm{R}_{\mathrm{f}}$-Werte: 0.52 (40) und 0.19 (91).

$\left(1 R^{*}, 2 S *, 5 R *\right)-7-C y c l o b u t y l i d e n-2-m e t h y l-b i c y c l o[3.2 .0]$ heptan (40)

$\mathrm{C}_{12} \mathrm{H}_{18}$

Ber. 162.1409

Gef. 162.1408 HRMS

IR (KBr): $2955 \mathrm{~cm}^{-1}(\mathrm{CH}), 1730(\mathrm{C}=\mathrm{C})$. 
${ }^{1}$ H-NMR (300 MHz, $\mathrm{CDCl}_{3}, \mathrm{CHCl}_{3}$ int.): $\delta=0.96 \mathrm{ppm}(\mathrm{d}, \mathrm{J}=6 \mathrm{~Hz}, 3 \mathrm{H}), 1.36-1.72$ (m, 4H), 1.80 - 1.94 (m, 4H), 2.38 - 2.72 (m, 6H), 3.08 (br. s, 1H).

${ }^{13}$ C-NMR (50 MHz, $\mathrm{CDCl}_{3}, \mathrm{CDCl}_{3}$ int.): $\delta=16.15$ ppm $\left(\mathrm{C}_{\text {prim }}\right), 17.26,29.44,30.37,32.71$, $32.74,33.21\left(\mathrm{C}_{\text {sek }}\right), 35.25,39.87,50.85\left(\mathrm{C}_{\text {tert }}\right), 127.97,132.10\left(\mathrm{C}_{\text {quart }}\right)$.

MS (70 eV): m/z (\%): $162\left(\mathrm{M}^{+}, 32\right), 91(100)$.

$(1 \mathrm{R} *, 2 \mathrm{R} * 5 \mathrm{R} *)-7-$ Cyclobutyliden-2-methyl-bicyclo[3.2.0]heptan (91)

$\mathrm{C}_{12} \mathrm{H}_{18}$

Ber. 162.1409

Gef. 162.1408 HRMS

IR (KBr): $2960 \mathrm{~cm}^{-1}(\mathrm{CH}), 1640(\mathrm{C}=\mathrm{C})$.

${ }^{1}$ H-NMR $\left(300 \mathrm{MHz}, \mathrm{CDCl}_{3}, \mathrm{CHCl}_{3}\right.$ int.): $\delta=0.76 \mathrm{ppm}(\mathrm{d}, \mathrm{J}=6 \mathrm{~Hz}, 3 \mathrm{H}), 1.34-1.48$ (m, 2H), $1.70\left(\mathrm{~m}_{\mathrm{c}}, 1 \mathrm{H}\right), 1.82$ - $2.10(\mathrm{~m}, 5 \mathrm{H}), 2.42$ - $2.58(\mathrm{~m}, 4 \mathrm{H}), 2.60$ - $2.74(\mathrm{~m}, 2 \mathrm{H}) .2 .80$ (br. s, 1H).

${ }^{13}$ C-NMR $\left(50 \mathrm{MHz}, \mathrm{CDCl}_{3}, \mathrm{CDCl}_{3}\right.$ int. $): \delta=17.45 \mathrm{ppm}\left(\mathrm{C}_{\text {sek }}\right), 18.81\left(\mathrm{C}_{\text {prim }}\right), 29.13,29.23$, $30.36,31.94,32.18\left(\mathrm{C}_{\text {sek }}\right), 34.13,37.92,53.50\left(\mathrm{C}_{\text {tert }}\right), 130.60,130.95\left(\mathrm{C}_{\text {quart }}\right)$.

MS (70 eV): m/z (\%): $162\left(\mathrm{M}^{+}, 21\right), 91$ (100).

\section{Allgemeine Vorschrift zur Epoxidierung der Olefine 26 und 40}

Eine Lösung des entsprechenden Olefins in Dichlormethan (2.5 $\mathrm{ml} / 1.0 \mathrm{mmol}$ Olefin) wurde bei Raumtemperatur portionsweise mit m-Chlorperbenzoesäure $(0.22$ g, 70 - 75 \% ig, ca. 1.25 mmol / 1.0 mmol Olefin) versetzt. Nach 15 min zeigte gaschromatographische Kontrolle [3 m x 1/4" Allglassystem, 15\% OV 101 auf Chromosorb W AW/DMCS 60/80 mesh] vollständi- 
gen Umsatz an. Es wurde $1 \mathrm{~N}$ Natronlauge (1.25 $\mathrm{ml} / 1.0 \mathrm{mmol}$ Olefin) zugegeben und die Phasen getrennt. Die wässrige Phase wurde anschließend mit Dichlormethan ( 3 x $2.5 \mathrm{ml} / 1.0$ mmol Olefin) extrahiert und die vereinigten organischen Phasen über Molekularsieb (3 $\AA$ ) getrocknet. Nach Abdestillieren der Lösungsmittel über eine $20 \mathrm{~cm}$ Vigreux-Kolonne (Bad 60 $\left.{ }^{\circ} \mathrm{C}\right)$ lieferte abschließende Chromatographie an Kieselgel $(0.05-0.20 \mathrm{~mm})$ die reinen Epoxide als farblose Flüssigkeiten.

1,1-Dimethyl-9-oxa-dispiro[3.0.3.1]nonan (64): Eingesetzt: 0.54 g, 4.0 mmol 26; Ausbeute: $0.53 \mathrm{~g}(86 \%)$; Retentionszeiten (min): $3.64(\mathbf{2 6}), 4.76(\mathbf{6 4})\left(140{ }^{\circ} \mathrm{C}\right)$; $\mathrm{R}_{\mathrm{f}}$-Wert in Pentan/Ether $95: 5: 0.35$.

$\mathrm{C}_{10} \mathrm{H}_{16} \mathrm{O}$

Ber. C 78.89 H 10.59

Gef. C 78.99 H 10.31

IR (KBr): $2960 \mathrm{~cm}^{-1}(\mathrm{CH}), 825(\mathrm{C}-\mathrm{O}-\mathrm{C})$.

${ }^{1}$ H-NMR (300 MHz, $\mathrm{CDCl}_{3}, \mathrm{CHCl}_{3}$ int.): $\delta=1.06 \mathrm{ppm}$ (s, 3H), 1.08 (s, 3H), 1.52 - 1.96 (m, $4 \mathrm{H}), 2.02-2.26(\mathrm{~m}, 4 \mathrm{H}), 2.40\left(\mathrm{~m}_{\mathrm{c}}, 2 \mathrm{H}\right)$.

${ }^{13}$ C-NMR (50 MHz, $\mathrm{CDCl}_{3}, \mathrm{CDCl}_{3}$ int.): $\delta=12.84 \mathrm{ppm}\left(\mathrm{C}_{\mathrm{sek}}\right), 23.81,24.44\left(\mathrm{C}_{\text {prim }}\right), 24.51$, $28.83,29.82,30.14\left(\mathrm{C}_{\text {sek }}\right), 42.34,67.77,71.61\left(\mathrm{C}_{\text {quart }}\right)$.

MS $(70 \mathrm{eV}): \mathrm{m} / \mathrm{z}(\%)=152\left(\mathrm{M}^{+}, 2\right), 137(100)$.

\section{2,2-Spiro-cyclobutyl-3,3-spiro-[(1R*,4S*,5R*,6S*)-2-methyl-bicyclo[3.2.0]heptan-6,6-} yl]-oxiran (92) und 2,2-Spiro-cyclobutyl-3,3-spiro-[(1R*,4S*,5R*,6R*)-2-methylbicyclo[3.2.0]heptan-6,6-yl]-oxiran (89): Eingesetzt: 3.00 g, 18.6 mmol 40; Ausbeute: 0.40 g (12\%) 92 und 2.48 g (75\%) 89; Retentionszeiten: 8.33 (40), 8.65 (92) und 9.33 (89) (Temperaturprogramm: $120{ }^{\circ} \mathrm{C}, 10{ }^{\circ} \mathrm{C} / \mathrm{min}$, auf $220{ }^{\circ} \mathrm{C}$ ); $\mathrm{R}_{\mathrm{f}}$-Werte in Pentan/Ether 95 : 5 : 0.64 (92) und 0.29 (89). 
2,2-Spiro-cyclobutyl-3,3-spiro-[(1R*,4S*,5R*,6S*)-2-methyl-bicyclo[3.2.0]heptan-6,6yl]-oxiran (92)

$\mathrm{C}_{12} \mathrm{H}_{18} \mathrm{O}$

Ber. 178.1358

Gef. 178.1357 HRMS

IR (KBr): $2940 \mathrm{~cm}^{-1}(\mathrm{CH}), 1280$ (C-O-C), 820 (C-O-C).

${ }^{1}$ H-NMR (300 MHz, $\mathrm{CDCl}_{3}, \mathrm{CHCl}_{3}$ int.): $\delta=1.02 \mathrm{ppm}(\mathrm{d}, \mathrm{J}=6 \mathrm{~Hz}, 3 \mathrm{H}), 1.58-2.00$ (m, $8 \mathrm{H}), 2.02-2.18(\mathrm{~m}, 2 \mathrm{H}), 2.20-2.40(\mathrm{~m}, 3 \mathrm{H}), 2.44\left(\mathrm{~m}_{\mathrm{c}}, 1 \mathrm{H}\right), 2.70\left(\mathrm{~m}_{\mathrm{c}}, 1 \mathrm{H}\right)$.

${ }^{13}$ C-NMR (50 MHz, $\mathrm{CDCl}_{3}, \mathrm{CDCl}_{3}$ int.): $\delta=11.91$ ppm $\left(\mathrm{C}_{\text {sek }}\right), 14.78\left(\mathrm{C}_{\text {prim }}\right), 28.35,28.51$ $\left(\mathrm{C}_{\text {sek }}\right), 31.22\left(\mathrm{C}_{\text {tert }}\right), 32.87,33.09,33.40\left(\mathrm{C}_{\text {sek }}\right), 39.18,47.58\left(\mathrm{C}_{\text {tert }}\right), 64.91,65.75\left(\mathrm{C}_{\text {quart }}\right)$.

MS $(70 \mathrm{eV}): \mathrm{m} / \mathrm{z}(\%): 178\left(\mathrm{M}^{+}, 6\right), 83(100)$.

2,2-Spiro-cyclobutyl-3,3-spiro-[(1R*,4S*,5R*,6R*)-2-methyl-bicylco[3.2.0]heptan-6,6yl]-oxiran (89)

$\mathrm{C}_{12} \mathrm{H}_{18} \mathrm{O}$

Ber. 178.1358

Gef. 178.1357 HRMS

IR (KBr): $2940 \mathrm{~cm}^{-1}$ (CH), 1240 (C-O-C), 810 (C-O-C).

${ }^{1}$ H-NMR (300 MHz, $\mathrm{CDCl}_{3}, \mathrm{CHCl}_{3}$ int.): $\delta=1.08 \mathrm{ppm}(\mathrm{d}, \mathrm{J}=6 \mathrm{~Hz}, 3 \mathrm{H}), 1.46-2.20$ (m, $11 \mathrm{H}), 2.32-2.48(\mathrm{~m}, 2 \mathrm{H}), 2.72-2.92(\mathrm{~m}, 2 \mathrm{H})$. 
${ }^{13}$ C-NMR (50 MHz, $\mathrm{CDCl}_{3}, \mathrm{CDCl}_{3}$ int. $): \delta=12.93$ ppm $\left(\mathrm{C}_{\text {sek }}\right), 17.46\left(\mathrm{C}_{\text {prim }}\right), 30.29,31.73$, $31.96\left(\mathrm{C}_{\text {sek }}\right), 33.59\left(\mathrm{C}_{\text {tert }}\right), 33.76,34.63\left(\mathrm{C}_{\text {sek }}\right), 37.53,53.23\left(\mathrm{C}_{\text {tert }}\right), 67.43,69.66\left(\mathrm{C}_{\text {quart }}\right)$.

MS (70 eV): m/z (\%): $178\left(\mathrm{M}^{+}, 5\right), 93(100)$.

\section{Allgemeine Vorschrift zur Umlagerung der Epoxide 64, 88 und 92}

Eine Lösung des entsprechenden Epoxids in wasserfreiem Dichlormethan (1.5 ml / $1.00 \mathrm{mmol}$ Epoxid) wurde mit Bortrifluoridetheratlösung (7 $\mu$ l, ca. 48\%ig, ca. $0.06 \mathrm{mmol} / 1.00 \mathrm{mmol}$ Epoxid) versetzt. Nach 15 min zeigte gaschromatographische Kontrolle $33 \mathrm{~m}$ x 1/4" Allglassystem, 15\% FFAP auf Chromosorb W AW/DMCS 60/80 mesh] vollständigen Umsatz an. Daraufhin wurde $1 \mathrm{~N}$ Natronlauge $(2.5 \mathrm{ml} / 1.00$ mmol Epoxid) zugegeben und die Phasen getrennt. Die wässrige Phase wurde mit Dichlormethan (2 x $2.5 \mathrm{ml} / 1.00$ mmol Epoxid) extrahiert und die vereinigten organische Phasen über Molekularsieb ( $3 \AA$ ) getrocknet. Nach Abdestillieren der Lösungsmittel über eine $20 \mathrm{~cm}$ Vigreux-Kolonne (Bad $60{ }^{\circ} \mathrm{C}$ ) lieferte abschließende Chromatographie an Kieselgel $(0.02-0.50 \mathrm{~mm})$ die gewünschten Ketone als farblose Flüssigkeiten.

1,1-Dimethyl-spiro[3.4]octan-5-on (27) und 8,8-Dimethyl-spiro[3.4]octan-5-on (67): Eingesetzt: 0.53 g; $0.65 \mathrm{mmol}$ 64; Ausbeute: $90 \mathrm{mg}$ (17\%) 27, $33 \mathrm{mg}(6 \%)$ 67, $42 \mathrm{mg}(8 \%)$ 63 : 36 Gemisch aus 27 und 67; Retentionszeiten (min): 2.93 (64), 6.42 (27) und 7.81 (67) $\left(140{ }^{\circ} \mathrm{C}\right) ; \mathrm{R}_{\mathrm{f}}$-Werte in Pentan/Ether 95 : 5: 0.11 (27) und $0.10(\mathbf{6 7})$.

\section{1,1-Dimethyl-spiro[3.4]octan-5-on (27)}

$\mathrm{C}_{10} \mathrm{H}_{16} \mathrm{O}$

Ber. 152.1201

Gef. 152.1201 HRMS

IR (KBr): $2930 \mathrm{~cm}^{-1}(\mathrm{CH}), 1725(\mathrm{C}=\mathrm{O})$. 
${ }^{1}$ H-NMR $\left(300 \mathrm{MHz}, \mathrm{CDCl}_{3}, \mathrm{CHCl}_{3}\right.$ int.): $\delta=0.92 \mathrm{ppm}(\mathrm{s}, 3 \mathrm{H}), 1.14$ (s, 3H), 1.44 - 2.22 (m, $10 \mathrm{H})$.

${ }^{13}$ C-NMR (50 MHz, $\mathrm{CDCl}_{3}, \mathrm{CDCl}_{3}$ int.): $\delta=19.37 \mathrm{ppm}, 20.37\left(\mathrm{C}_{\mathrm{sek}}\right), 25.37,25.47\left(\mathrm{C}_{\text {prim }}\right)$, $30.57,32.56,37.98\left(\mathrm{C}_{\text {sek }}\right), 40.96,56.75,222.06\left(\mathrm{C}_{\text {quart }}\right)$.

MS $(70 \mathrm{eV}): \mathrm{m} / \mathrm{z}(\%)=152\left(\mathrm{M}^{+}, 34\right), 97(100)$.

\section{8,8-Dimethyl-spiro[3.4]octan-5-on (67)}

$\mathrm{C}_{10} \mathrm{H}_{16} \mathrm{O}$

Ber. 152.1201

Gef. 152.1201 HRMS

IR (KBr): $2960 \mathrm{~cm}^{-1}(\mathrm{CH}), 1735(\mathrm{C}=\mathrm{O})$.

${ }^{1}$ H-NMR (300 MHz, $\mathrm{CDCl}_{3}, \mathrm{CHCl}_{3}$ int.): $\delta=0.90 \mathrm{ppm}(\mathrm{s}, 6 \mathrm{H}), 1.58$ (t, J = $\left.6 \mathrm{~Hz}, 2 \mathrm{H}\right), 1.68$ $\left(\mathrm{m}_{\mathrm{c}}, 1 \mathrm{H}\right), 1.92\left(\mathrm{~m}_{\mathrm{c}}, 5 \mathrm{H}\right), 2.22(\mathrm{t}, \mathrm{J}=6 \mathrm{~Hz}, 2 \mathrm{H})$.

${ }^{13}$ C-NMR (75 MHz, $\mathrm{CDCl}_{3}, \mathrm{CDCl}_{3}$ int.): $\left.\delta=14.36 \mathrm{ppm}\left(\mathrm{C}_{\mathrm{sek}}\right), 22.94 \mathrm{C}_{\text {prim }}\right), 23.89,32.31$, $33.33\left(\mathrm{C}_{\text {sek }}\right), 39.39,58.84,221.12\left(\mathrm{C}_{\text {quart }}\right)$.

MS $(70 \mathrm{eV}): \mathrm{m} / \mathrm{z}(\%)=152\left(\mathrm{M}^{+}, 38\right), 137(100)$

$\left(1 R^{*}, 4 S^{*}, 5 R^{*}, 6 S^{*}\right)-4-M e t h y l-s p i r o\{b i c y c l o[3.2 .0]$ heptan-6,1'-cyclopentan-2'́-on\} $\quad(87)$, $\left(1 R^{*}, 2 R^{*}, 5 R^{*}, 6 S^{*}\right)-2-M e t h y l-s p i r o\left\{b i c y c l o[3.1 .1] h e p t a n-6,1^{\prime}\right.$-cyclopentan-2'-on\} (93) und $\quad\left(1 R^{*}, 4 S^{*}, 5 R^{*}, 6 R^{*}\right)-4-M e t h y l-s p i r o\left\{b i c y c l o[3.2 .0] h e p t a n-6,1^{\prime}\right.$-cyclopentan-2'-on\} (88): Eingesetzt: $2.43 \mathrm{~g}, 13.60 \mathrm{mmol} 92$ und 89 im Verhältnis 10 : 90; Ausbeute: $0.87 \mathrm{~g}$ (40\%) 87 und 93 im Verhältnis 41 : 59 ( ${ }^{1} \mathrm{H}$-NMR-spektroskopische Analyse), $0.14 \mathrm{~g}$ (7\%) 88, $1.04 \mathrm{~g}$ (48\%) 38:62 Gemisch aus 87,93 und 88; Retentionszeiten (min): 8.65 (92), 9.33 
(89), 10.58 (87, 93 und 88) (Temperaturprogramm: $120{ }^{\circ} \mathrm{C}, 10{ }^{\circ} \mathrm{C} / \mathrm{min}$, auf $220{ }^{\circ} \mathrm{C}$ ); $\mathrm{R}_{\mathrm{f}}$-Werte in Pentan/Ether $98: 2$ : 0.12 (87 und 93) und 0.11 (88), Identifizierung erfolgte gaschromatographisch: [3 m x 1/4" Allglassystem, 15\% FFAP auf Chromosorb W AW/DMCS 60/80 mesh; $200{ }^{\circ} \mathrm{C}$; Retentionszeiten ( $\mathrm{min}$ ): 5.63 (88) und 6.25 (87 und 93).

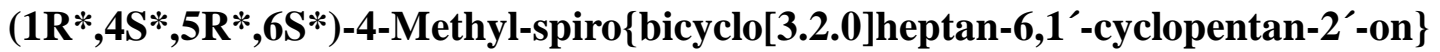

und $\left(1 R^{*}, 2 R^{*}, 5 R^{*}, 6 S^{*}\right)-2-M e t h y l-s p i r o\left\{b i c y c l o[3.1 .1] h e p t a n-6,1^{\prime}\right.$-cyclopentan-2'-on\} (93)

$\mathrm{C}_{12} \mathrm{H}_{18} \mathrm{O}$

Ber. 178.1358

Gef. 178.1357 HRMS

IR (KBr): $2940 \mathrm{~cm}^{-1}(\mathrm{CH}), 1720(\mathrm{C}=\mathrm{O})$.

${ }^{1}$ H-NMR (300 MHz, $\mathrm{CDCl}_{3}, \mathrm{CHCl}_{3}$ int.): $\delta=0.86 \mathrm{ppm}(\mathrm{d}, 6 \mathrm{~Hz}, 3 \mathrm{H}), 1.10(\mathrm{~d}, 6 \mathrm{~Hz}, 3 \mathrm{H})$, 1.18 - $2.34(\mathrm{~m}, 27 \mathrm{H}), 2.48\left(\mathrm{~m}_{\mathrm{c}}, 1 \mathrm{H}\right), 2.64\left(\mathrm{~m}_{\mathrm{c}}, 1 \mathrm{H}\right), 2.78\left(\mathrm{~m}_{\mathrm{c}}, 1 \mathrm{H}\right)$.

${ }^{13}$ C-NMR (50 MHz, $\mathrm{CDCl}_{3}, \mathrm{CDCl}_{3}$ int.): $\delta=15.49 \mathrm{ppm}\left(\mathrm{C}_{\text {prim }}\right), 18.04,18.87\left(\mathrm{C}_{\text {sek }}\right), 20.87$ $\left(\mathrm{C}_{\text {prim }}\right), 21.40,23.82,24.35,28.17\left(\mathrm{C}_{\text {sek }}\right), 29.32\left(\mathrm{C}_{\text {tert }}\right), 32.79\left(\mathrm{C}_{\text {sek }}\right), 33.05\left(\mathrm{C}_{\text {tert }}\right), 33.55$, 34.84, 35.50, 36.20, $37.28\left(\mathrm{C}_{\text {sek }}\right), 37.78,39.44,43.60,44.40\left(\mathrm{C}_{\text {tert }}\right), 51.52,58.70,221.44$, $221.83\left(\mathrm{C}_{\text {quart }}\right)$.

MS (70 eV): m/z (\%): $178\left(\mathrm{M}^{+}, 6\right), 81(100)$

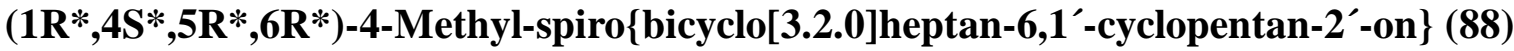

$\mathrm{C}_{12} \mathrm{H}_{18} \mathrm{O}$

Ber. 178.1358 
Gef. 178.1357 HRMS

IR (KBr): $2940 \mathrm{~cm}^{-1}(\mathrm{CH}), 1725(\mathrm{C}=\mathrm{O})$.

${ }^{1}$ H-NMR (300 MHz, $\mathrm{CDCl}_{3}, \mathrm{CHCl}_{3}$ int.): $\delta=0.84 \mathrm{ppm}(\mathrm{d}, \mathrm{J}=6 \mathrm{~Hz}, 3 \mathrm{H}), 1.26-2.00$ (m, $11 \mathrm{H}), 2.16\left(\mathrm{~m}_{\mathrm{c}}, 2 \mathrm{H}\right), 2.54-2.68(\mathrm{~m}, 2 \mathrm{H})$.

${ }^{13}$ C-NMR (50 MHz, $\mathrm{CDCl}_{3}, \mathrm{CDCl}_{3}$ int. $): \delta=13.09$ ppm $\left(\mathrm{C}_{\text {prim }}\right), 18.65,26.73,27.29,31.48$, $33.08,36.47\left(\mathrm{C}_{\text {sek }}\right), 36.90,37.60,42.71\left(\mathrm{C}_{\text {tert }}\right), 49.62,222.76\left(\mathrm{C}_{\text {quart }}\right)$.

MS (70 eV): m/z (\%): $178\left(\mathrm{M}^{+}, 6\right), 81(100)$.

\section{1,1-Bis(phenylseleno)-2,2-Dimethylcyclobutan (48)}

Eine Suspension von Zinkchlorid (1.39 g, $10.20 \mathrm{mmol})$ in wasserfreiem Tetrachlorkohlenstoff (5 ml) wurde auf $0{ }^{\circ} \mathrm{C}$ gekühlt und langsam mit einer Lösung von 2,2-Dimethylcyclobutanon (29) (2.00 g, $20.38 \mathrm{mmol})$ und Phenylselenol (6.33 g, $40.61 \mathrm{mmol})$ in trockenem Tetrachlorkohlenstoff ( $6 \mathrm{ml}$ ) versetzt. Das Reaktionsgemisch wurde fünf Stunden bei Raumtemperatur gerührt. Danach fügte man Ether $(40 \mathrm{ml})$ zu und extrahierte die organische Phase zuerst mit 5\% iger Salzsäure $(2 \times 12 \mathrm{ml})$, danach mit gesättigter Natriumhydrogencarbonatlösung $(2 \times 12$ ml) und dann mit Wasser ( 2 x $12 \mathrm{ml})$ und trocknete über Magnesiumsulfat. Nach Entfernen der Lösungsmittel im Rotationsverdampfer (Badtemperatur $50{ }^{\circ} \mathrm{C} / 20$ Torr) lieferte Säulenfiltration an Kieselgel $(0.02-0.50 \mathrm{~mm})$ in Pentan/Ether $98: 2,6.85 \mathrm{~g}$ eines orangenen Öls, das später auskristallisierte. Das so erhaltene Rohprodukt wurde in trockenem Ether (12 ml) gelöst und langsam zu einer Suspension aus Lithiumalanat $(0.38 \mathrm{~g}, 10.01 \mathrm{mmol})$ in trockenem Ether $(35 \mathrm{ml})$ gegeben. Nach beendeter Zugabe erhitzte man 30 min unter Rückfluß und hydrolysierte mit 50\%iger Kaliumhydroxidlösung (11 ml). Anschließend trennte man die Phasen, filtrierte die organische Phase über Cellite, trocknete über Magnesiumsulfat und entfernte die Lösungsmittel im Rotationsverdampfer (Badtemperatur $20{ }^{\circ} \mathrm{C} / 20$ Torr). Man erhielt so $6.23 \mathrm{~g}(78 \%) 48$ als orange-gelbe Kristalle (Smp.: $\left.68^{\circ} \mathrm{C}\right)$. 


\title{
1,1-Bis(phenylseleno)-2,2Dimethylcyclobutan (48)
}

\author{
$\mathrm{C}_{18} \mathrm{H}_{20} \mathrm{Se}_{2}$
}

Ber. 395.9890

Gef. 395.9895 HRMS

IR (KBr): $3060 \mathrm{~cm}^{-1}(\mathrm{CH}), 1575(\mathrm{C}-\mathrm{C})$.

${ }^{1}$ H-NMR (300 MHz, $\mathrm{CDCl}_{3}, \mathrm{CHCl}_{3}$ int.): $\delta=1.24 \mathrm{ppm}(\mathrm{s}, 6 \mathrm{H}), 1.92(\mathrm{t}, \mathrm{J}=6 \mathrm{~Hz}, 2 \mathrm{H}), 2.20$ $(\mathrm{t}, \mathrm{J}=6 \mathrm{~Hz}, 2 \mathrm{H}), 7.22-7.38(\mathrm{~m}, 6 \mathrm{H}), 7.56-7.62(\mathrm{~m}, 4 \mathrm{H})$.

${ }^{13}$ C-NMR (50 MHz, $\mathrm{CDCl}_{3}, \mathrm{CDCl}_{3}$ int. $): \delta=27.48 \mathrm{ppm}\left(\mathrm{C}_{\text {prim }}\right), 31.99,32.03\left(\mathrm{C}_{\text {sek }}\right), 49.02$, $63.90\left(\mathrm{C}_{\text {quart }}\right), 128.14,128.17,128.29,128.51\left(\mathrm{C}_{\text {tert }}\right), 130.83\left(\mathrm{C}_{\text {quart }}\right), 136.64,136.67\left(\mathrm{C}_{\text {tert }}\right)$.

MS (DCI): $\mathrm{m} / \mathrm{z}(\%)=412\left(\left[\mathrm{M}^{+}+18\right]^{+}, 6\right), 429\left(\left[\mathrm{M}^{+}+18+17\right]^{+}, 13\right), 806\left(\left[2 \mathrm{M}^{+}+18\right]^{+}, 19\right)$, $239(100)$.

\section{Allgemeine Vorschrift zur Methylenierung der Ketone 94 und 95}

$\mathrm{Zu}$ einer Suspension von Triphenylmethylenphosphoniumbromid (1.48 g, $4 \mathrm{mmol} / 1 \mathrm{mmol}$ Keton) in wasserfreiem Benzol (15 ml / 1 mmol Keton) gab man unter Stickstoff und Rühren Kalium-tert-butylat $(0.45 \mathrm{~g}, 4 \mathrm{mmol})$ und erhitzte eine Stunde auf $60{ }^{\circ} \mathrm{C}$. Danach wurde das entsprechende Keton zugetropft. Nach 15 min wurde der Hauptteil des Lösungsmittels bis zu einer Badtemperatur von $110{ }^{\circ} \mathrm{C}$ abdestilliert. Nach drei Stunden bei dieser Temperatur zeigte gaschromatographische Kontrolle [3 m x 1/4" Allglassystem, 15\% OV 101 auf Chromosorb W AW/DMCS 60/80 mesh] quantitativen Umsatz an. Man hydrolysierte mit Wasser (0.4 ml / 1 mmol Keton), dekantierte ab, extrahierte den Rückstand mit Pentan (3 x 15 ml / 1 mmol Keton), trocknete die vereinigten organischen Phasen über Molekularsieb (3 $\AA$ ) und entfernte die Lösungsmittel im Rotationsverdampfer (Badtemperatur $20{ }^{\circ} \mathrm{C} / 20$ Torr). Abschließende Chromatographie an Kieselgel $(0.05-0.20 \mathrm{~mm})$ ergab die reinen Olefine als farblose Flüssigkeiten. 
$\left(1 R^{*}, 4 S^{*}, 5 R^{*}, 6 R^{*}\right)-2^{\prime}$-Methylen-4,3' $3^{\prime}$-trimethyl-spiro\{ bicyclo[3.2.0]heptan-6,1'-

cyclopentan \} (104): Eingesetzt: 0.06 g, 0.29 mmol 95; Ausbeute: 0.08 g (67\%) 104; Retentionszeiten (min): 9.72 (104), 10.81 (95) (Temperaturprogramm: $120{ }^{\circ} \mathrm{C}, 10{ }^{\circ} \mathrm{C} / \mathrm{min}$, auf 220 $\left.{ }^{\circ} \mathrm{C}\right)$; $\mathrm{R}_{\mathrm{f}}$-Wert in Pentan: 0.71 .

$\mathrm{C}_{15} \mathrm{H}_{24}$

Ber. 204.1878

Gef. 204.1878 HRMS

${ }^{1}$ H-NMR (300 MHz, $\mathrm{CDCl}_{3}, \mathrm{CHCl}_{3}$ int.): $\delta=0.88 \mathrm{ppm}(\mathrm{d}, 6 \mathrm{~Hz}, 3 \mathrm{H}), 1.00$ (s, 3H), 1.04 (s, $3 \mathrm{H}), 1.28-1.88(\mathrm{~m}, 11 \mathrm{H}), 2.48\left(\mathrm{~m}_{\mathrm{c}}, 1 \mathrm{H}\right), 2.58\left(\mathrm{~m}_{\mathrm{c}}, 1 \mathrm{H}\right), 4.74(\mathrm{~s}, 1 \mathrm{H}), 5.02(\mathrm{~s}, 1 \mathrm{H})$.

${ }^{13}$ C-NMR (75 MHz, $\mathrm{CDCl}_{3}, \mathrm{CDCl}_{3}$ int. $): \delta=13.50 \mathrm{ppm}\left(\mathrm{C}_{\text {prim }}\right), 27.66\left(\mathrm{C}_{\text {sek }}\right), 30.22,30.47$ $\left(\mathrm{C}_{\text {prim }}\right), 31.79,32.94,33.26\left(\mathrm{C}_{\text {sek }}\right), 37.09,37.93\left(\mathrm{C}_{\text {tert }}\right), 38.27\left(\mathrm{C}_{\text {sek }}\right), 42.29,48.04\left(\mathrm{C}_{\text {quart }}\right)$, $48.45\left(\mathrm{C}_{\text {tert }}\right), 99.91\left(\mathrm{C}_{\text {sek }}\right), 172.99\left(\mathrm{C}_{\text {quart }}\right)$.

MS (70 eV): m/z (\%): $204\left(\mathrm{M}^{+}, 6\right), 107$ (100).

$\left(1 R^{*}, 4 S^{*}, 5 R^{*}, 6 S^{*}\right)-2^{\prime}$-Methylen-4,3',3'-trimethyl-spiro\{ bicyclo[3.2.0]heptan-6,1'-

cyclopentan \} (103): Eingesetzt: 0.06 g, 0.29 mmol 94; Ausbeute: 0.10 g (84\%); Retentionszeiten (min): 9.94 (103), 10.82 (94) (Temperaturprogramm: $120{ }^{\circ} \mathrm{C}, 10{ }^{\circ} \mathrm{C} / \mathrm{min}$, auf $220{ }^{\circ} \mathrm{C}$ ); $\mathrm{R}_{\mathrm{f}}$-Wert in Pentan: 0.69 .

$\mathbf{C}_{15} \mathbf{H}_{24}$

Ber. 204.1878

Gef. 204.1878 HRMS

${ }^{1} \mathbf{H}-\mathbf{N M R}\left(300 \mathrm{MHz}, \mathrm{CDCl}_{3}, \mathrm{CHCl}_{3}\right.$ int.): $\delta=0.98 \mathrm{ppm}$ (s, 3H), 1.08 (s, 3H), 1.14 (d, J = 6 $\mathrm{Hz}, 3 \mathrm{H}), 1.28$ - $2.04(\mathrm{~m}, 11 \mathrm{H}), 2.52-2.70(\mathrm{~m}, 2 \mathrm{H}), 4.74$ (s, 1H), 5.04 (s, 1H). 
${ }^{13}$ C-NMR (75 MHz, $\mathrm{CDCl}_{3}, \mathrm{CDCl}_{3}$ int.): $\delta=15.60$ ppm, 30.36, $31.08\left(\mathrm{C}_{\text {prim }}\right), 32.82\left(\mathrm{C}_{\text {sek }}\right)$, $33.13\left(\mathrm{C}_{\text {tert }}\right), 33.56,34.89,38.39\left(\mathrm{C}_{\text {sek }}\right), 40.38\left(\mathrm{C}_{\text {tert }}\right), 41.53\left(\mathrm{C}_{\text {quart }}\right), 42.08\left(\mathrm{C}_{\text {sek }}\right), 49.88\left(\mathrm{C}_{\text {tert }}\right)$, $50.24\left(\mathrm{C}_{\text {quart }}\right), 99.83\left(\mathrm{C}_{\text {sek }}\right), 173.47\left(\mathrm{C}_{\text {quart }}\right)$.

MS (70 eV): m/z (\%): $204\left(\mathrm{M}^{+}, 5\right), 189(100)$.

\section{1-[(1'-Phenylseleno)cyclobutyl]-2,2-dimethylcyclobutan-1-ol (76)}

$\mathrm{Zu}$ einer auf $-78{ }^{\circ} \mathrm{C}$ vorgekühlten Lösung von 1,1-Bis(phenylseleno)cyclobutan (75) (5.88 g, $15.24 \mathrm{mmol}$ ) in $15 \mathrm{ml}$ wasserfreiem Tetrahydrofuran tropfte man unter Rühren und Argon eine 1.6 M Lösung von n-Butyllithium in n-Hexan (9.5 ml, $15.24 \mathrm{mmol})$. Nach einer Stunde ließ man auf $-40{ }^{\circ} \mathrm{C}$ erwärmen und gab eine Lösung von 29 (0.98 g, $\left.10 \mathrm{mmol}\right)$ in wasserfreiem Tetrahydrofuran $(10 \mathrm{ml})$ zu. Nach $5.5 \mathrm{~h}$ ließ man auf Raumtemperatur erwärmen, hydrolysierte mit gesättigter Natriumhydrogencarbonatlösung $(10 \mathrm{ml})$, wusch die organische Phase mit gesättigter Natriumhydrogencarbonatlösung ( 2 x $10 \mathrm{ml})$, extrahierte die vereinigten wässrigen Phasen mit Ether $(2 \times 10 \mathrm{ml})$, trocknete die vereinigten organischen Phasen über Magnesiumsulfat und entfernte die Lösungsmittel im Rotationsverdampfer (Badtemperatur 50 ${ }^{\circ} \mathrm{C} / 20$ Torr). Chromatographie des Rohproduktes (7.49 g) an Kieselgel (0.05 - $0.20 \mathrm{~mm}$ ) in Pentan/Ether 95 : 5 [Säule 57 x 3 cm, DC-Kontrolle, $\mathrm{R}_{\mathrm{f}}$-Wert: 0.22 (76)] lieferte $1.47 \mathrm{~g}$ (48\%) 76 als gelbe Flüssigkeit.

\section{1-[(1'-Phenylseleno)cyclobutyl]-2,2-dimethylcyclobutan-1-ol (76)}

\section{$\mathrm{C}_{16} \mathrm{H}_{22} \mathrm{OSe}$}

Ber. 310.0830

Ber. 310.0535 HRMS

IR (KBr): $3580 \mathrm{~cm}^{-1}(\mathrm{OH}), 3560-3300\left(\mathrm{OH}_{\mathrm{ass}}\right), 2940(\mathrm{CH})$.

${ }^{1}$ H-NMR $\left(300 \mathrm{MHz}, \mathrm{C}_{6} \mathrm{D}_{6}, \mathrm{C}_{6} \mathrm{D}_{5} \mathrm{H}\right.$ int.): $\delta=1.10 \mathrm{ppm}(\mathrm{s}, 3 \mathrm{H}), 1.30(\mathrm{~s}, 3 \mathrm{H}), 1.32\left(\mathrm{~m}_{\mathrm{c}}, 1 \mathrm{H}\right)$, $1.40\left(\mathrm{~m}_{\mathrm{c}}, 1 \mathrm{H}\right), 1.88\left(\mathrm{~m}_{\mathrm{c}}, 4 \mathrm{H}\right), 2.10-2.34(\mathrm{~m}, 4 \mathrm{H}), 2.60\left(\mathrm{~m}_{\mathrm{c}}, 1 \mathrm{H}\right), 6.96\left(\mathrm{~m}_{\mathrm{c}}, 3 \mathrm{H}\right), 7.68\left(\mathrm{~m}_{\mathrm{c}}\right.$, $2 \mathrm{H})$. 
${ }^{13}$ C-NMR (50 MHz, $\mathrm{C}_{6} \mathrm{D}_{6}, \mathrm{C}_{6} \mathrm{D}_{6}$ int.): $\delta=16.95 \mathrm{ppm}\left(\mathrm{C}_{\text {sek }}\right), 25.17,25.35\left(\mathrm{C}_{\text {prim }}\right), 26.65$, $29.93,30.75,35.90\left(\mathrm{C}_{\text {sek }}\right), 44.19,62.75,83.09\left(\mathrm{C}_{\text {quart }}\right), 128.75\left(\mathrm{C}_{\text {tert }}\right), 128.98\left(\mathrm{C}_{\text {quart }}\right), 129.06$, $138.22\left(\mathrm{C}_{\text {tert }}\right)$.

MS $(\mathrm{DCI}): \mathrm{m} / \mathrm{z}(\%)=328\left(\left[\mathrm{M}^{+}+18\right]^{+}, 90\right), 345\left(\left[\mathrm{M}^{+}+18+17\right]^{+}, 49\right), 638\left(\left[2 \mathrm{M}^{+}+18\right]^{+}, 5\right)$, $293(100)$.

\section{1-[(1'-Phenylseleno)-2',2’-dimethylcyclobutyl]cyclobutan-1-ol (49)}

$\mathrm{Zu}$ einer auf $-78{ }^{\circ} \mathrm{C}$ vorgekühlten Lösung von 1,1-Bis(phenylseleno)-2,2-Dimethylcyclobutan (48) $(6.01 \mathrm{~g}, 15.24 \mathrm{mmol})$ in $15 \mathrm{ml}$ wasserfreiem Tetrahydrofuran tropfte man unter Rühren und Argon eine 1.6 M Lösung von n-Butyllithium in n-Hexan (9.5 ml, $15.24 \mathrm{mmol})$. Nach einer Stunde ließ man auf $-40{ }^{\circ} \mathrm{C}$ erwärmen und gab eine Lösung von Cyclobutanon (1) (0.70 g, $10 \mathrm{mmol})$ in wasserfreiem Tetrahydrofuran $(10 \mathrm{ml}) \mathrm{zu}$. Nach $5.5 \mathrm{~h}$ ließ man auf Raumtemperatur erwärmen, hydrolysierte mit gesättigter Natriumhydrogencarbonatlösung (10 ml), wusch die organische Phase mit gesättigter Natriumhydrogencarbonatlösung (2 x 10 ml), extrahierte die vereinigten wässrigen Phasen mit Ether $(2 \times 10 \mathrm{ml})$, trocknete die vereinigten organischen Phasen über Magnesiumsulfat und entfernte die Lösungsmittel im Rotationsverdampfer (Badtemperatur $50{ }^{\circ} \mathrm{C} / 20$ Torr). Chromatographie des hellgelben, öligen Rohproduktes (7.49 g) an Kieselgel $(0.05-0.20 \mathrm{~mm})$ in Pentan/Ether $95: 5$ [Säule 57 x $3 \mathrm{~cm}$, DC-Kontrolle, $\mathrm{R}_{\mathrm{f}^{-}}$ Wert: 0.17 (49)] lieferte $0.87 \mathrm{~g}(29 \%) 49$ als gelbe Flüssigkeit.

\section{1-[(1'-Phenylseleno)-2',2'-dimethylcyclobutyl]cyclobutan-1-ol (49)}

$$
\mathrm{C}_{16} \mathrm{H}_{22} \mathrm{OSe}
$$

Ber. 310.0830

Gef. 310.0835 HRMS

IR (KBr): $3605 \mathrm{~cm}^{-1}(\mathrm{OH}), 3600-3300\left(\mathrm{OH}_{\mathrm{ass}}\right), 2940(\mathrm{CH})$.

${ }^{1}$ H-NMR $\left(300 \mathrm{MHz}, \mathrm{C}_{6} \mathrm{D}_{6}, \mathrm{C}_{6} \mathrm{D}_{5} \mathrm{H}\right.$ int.): $\delta=1.18 \mathrm{ppm}(\mathrm{s}, 3 \mathrm{H}), 1.44(\mathrm{~s}, 3 \mathrm{H}), 1.48\left(\mathrm{~m}_{\mathrm{c}}, 1 \mathrm{H}\right)$, 1.72 - $2.14(\mathrm{~m}, 8 \mathrm{H}), 2.34(\mathrm{~s}, 1 \mathrm{H}), 2.42\left(\mathrm{~m}_{\mathrm{c}}, 1 \mathrm{H}\right), 6.98\left(\mathrm{~m}_{\mathrm{c}}, 3 \mathrm{H}\right), 7.60\left(\mathrm{~m}_{\mathrm{c}}, 2 \mathrm{H}\right)$. 
${ }^{13}$ C-NMR (75 MHz, $\mathrm{C}_{6} \mathrm{D}_{6}, \mathrm{C}_{6} \mathrm{D}_{6}$ int. $): \delta=15.15 \mathrm{ppm}\left(\mathrm{C}_{\text {sek }}\right), 25.98\left(\mathrm{C}_{\text {prim }}\right), 26.36\left(\mathrm{C}_{\text {sek }}\right), 30.88$ $\left(\mathrm{C}_{\text {prim }}\right), 32.06,33.81,36.41\left(\mathrm{C}_{\text {sek }}\right), 45.70,71.55,83.70\left(\mathrm{C}_{\text {quart }}\right), 128.79\left(\mathrm{C}_{\text {tert }}\right), 129.28\left(\mathrm{C}_{\text {quart }}\right)$, $138.81\left(\mathrm{C}_{\text {tert }}\right)$.

MS (DCI): m/z (\%) = $310\left(\mathrm{M}^{+}, 9\right), 328\left(\left[\mathrm{M}^{+}+18\right]^{+}, 34\right), 638\left(\left[2 \mathrm{M}^{+}+18\right]^{+}, 2\right), 293(100)$.

\section{2,2-Dimethyl-bicyclobutyl-1'́-en-1-ol (78)}

$\mathrm{Zu}$ einer auf $0{ }^{\circ} \mathrm{C}$ vorgekühlten Suspension von m-Chlorperbenzoesäure (1.20 g, 70 - 75\%ig, ca. $7 \mathrm{mmol})$ in Methylenchlorid (15 ml) gab man unter Rühren 76 (1.24 g, $4 \mathrm{mmol})$ und rührte $1 \mathrm{~h}$, bis dünnschichtchromatographische Kontrolle [Laufmittel Pentan/Ether $9: 1 ; \mathrm{R}_{\mathrm{f}^{-}}$ Werte: 0.37 (76), 0.16 (78)] vollständigen Umsatz anzeigte. Daraufhin ließ man auf Raumtemperatur erwärmen, wusch die organische Phase mit $1 \mathrm{M}$ Natriumhydroxidlösung $(2 \times 14$ $\mathrm{ml})$, extrahierte die wässrige Phase mit Dichlormethan $(2 \times 25 \mathrm{ml})$ und trocknete die vereinigten organischen Phasen über Magnesiumsulfat. Nach dem Entfernen des Lösungsmittels im Rotationsverdampfer (Badtemperatur $20{ }^{\circ} \mathrm{C} / 20$ Torr) lieferte abschließende Chromatographie an Kieselgel (0.05 - $0.20 \mathrm{~mm})$ in Pentan/Ether 9 : 1 [Säule 57 x 3 cm; R $\mathrm{R}_{\mathrm{f}}$-Werte: 0.37 (76), $0.16(78)] 0.35 \mathrm{~g}(58 \%) 78$ als eine farblose Flüssigkeit.

\section{2,2-Dimethyl-bicyclobutyl-1'́-en-1-ol (78)}

\section{$\mathrm{C}_{10} \mathrm{H}_{16} \mathrm{O}$}

Ber. 152.1201

Gef. 152.1201 HRMS

IR (KBr): $3600 \mathrm{~cm}^{-1}(\mathrm{OH}), 3580-3100\left(\mathrm{OH}_{\mathrm{ass}}\right), 2950(\mathrm{CH})$.

${ }^{1}$ H-NMR $\left(300 \mathrm{MHz}, \mathrm{CDCl}_{3}, \mathrm{CDCl}_{3}\right.$ int.): $\delta=0.98 \mathrm{ppm}(\mathrm{s}, 3 \mathrm{H}), 1.14(\mathrm{~s}, 3 \mathrm{H}), 1.40\left(\mathrm{~m}_{\mathrm{c}}, 1 \mathrm{H}\right)$, $154(\mathrm{ddd}, \mathrm{J}=12,9,3 \mathrm{~Hz}, 1 \mathrm{H}), 1.74(\mathrm{br} \mathrm{s}, 1 \mathrm{H}), 1.92\left(\mathrm{~m}_{\mathrm{c}}, 1 \mathrm{H}\right), 2.22\left(\mathrm{~m}_{\mathrm{c}}, 1 \mathrm{H}\right), 2.36\left(\mathrm{~m}_{\mathrm{c}}, 2 \mathrm{H}\right)$, $2.56\left(\mathrm{~m}_{\mathrm{c}}, 2 \mathrm{H}\right), 5.90(\mathrm{~s}, 1 \mathrm{H})$. 
${ }^{13}$ C-NMR (50 MHz, $\mathrm{CDCl}_{3}, \mathrm{CDCl}_{3}$ int. $): \delta=23.11 \mathrm{ppm}, 25.02\left(\mathrm{C}_{\text {prim }}\right), 25.56,28.08,29.59$, $30.03\left(\mathrm{C}_{\text {sek }}\right), 43.90,76,54\left(\mathrm{C}_{\text {quart }}\right), 127.50\left(\mathrm{C}_{\text {tert }}\right), 151.86\left(\mathrm{C}_{\text {quart }}\right)$.

MS (70 eV): m/z (\%): $152\left(\mathrm{M}^{+}, 5\right), 95(100)$.

\section{Allgemeine Vorschrift zur Umlagerung der $\beta$-Hydroxyselenide 49 und 76}

$\mathrm{Zu}$ einer auf $0{ }^{\circ} \mathrm{C}$ vorgekühlten Suspension von m-Chlorperbenzoesäure $(1.21 \mathrm{~g}, 70$ - 75\% $\mathrm{ig}$, ca. $7.0 \mathrm{mmol} / 1.0 \mathrm{mmol} \beta$-Hydroxyselenid) in Methylenchlorid (4 ml / $1.0 \mathrm{mmol} \beta$ Hydroxyselenid) gab man unter Rühren eine Lösung des entsprechenden $\beta$-Hydroxyselenids in Dichlormethan $(0.25 \mathrm{ml} / 1.0 \mathrm{mmol} \beta$-Hydroxyselenid) und rührte bis dünnschichtchromatographische vollständigen Umsatz anzeigte. Daraufhin ließ man auf Raumtemperatur erwärmen, wusch die organische Phase mit $1 \mathrm{M}$ Natriumhydroxidlösung $(2 \times 4 \mathrm{ml} / 1.0 \mathrm{mmol} \beta$ Hydroxyselenid), extrahierte die wässrige Phase mit Dichlormethan (2 x $6 \mathrm{ml} / 1.0 \mathrm{mmol} \beta$ Hydroxyselenid) und trocknete die vereinigten organischen Phasen über Magnesiumsulfat. Nach dem Entfernen des Lösungsmittel im Rotationsverdampfer (Badtemperatur $20{ }^{\circ} \mathrm{C} / 20$ Torr) lieferte abschließende Chromatographie an Kieselgel (0.05 - $0.20 \mathrm{~mm})$ die reinen Ketone als farblose Flüssigkeiten

6,6-Dimethy-spiro[3.4]octan-5-on (57) und 8.8-Dimethyl-spiro[3.4]octan-5-on (67): Eingesetzt: 1.24 g, 4.0 mmol 76; Ausbeute: 0.06 g (10\%) 51 und 0.30 g (50\%) 67; Reaktionsdauer: $40 \mathrm{~min}$; $\mathrm{R}_{\mathrm{f}}$-Werte in Pentan/Ether 9 : 1: 0.37 (76), 0.55 (51) und 0.33 (67). Die ${ }^{1} \mathrm{H}-$ NMR und ${ }^{13} \mathrm{C}$-NMR-Daten stimmten mit denen authentischer Proben überein.

1,1-Dimethyl-spiro[3.4]octan-5-on (27): Eingesetzt: 0.50 g, $1.6 \mathrm{mmol} 49$; Ausbeute: $0.11 \mathrm{~g}$ (45\%) 27; Reaktionsdauer: 40 min; $\mathrm{R}_{\mathrm{f}}$-Werte in Pentan/Ether 95 : 5: 0.17 (49) und 0.28 (27). Die ${ }^{1} \mathrm{H}-\mathrm{NMR}$ und ${ }^{13} \mathrm{C}$-NMR-Daten stimmten mit denen einer authentischen Probe überein.

\section{Allgemeine Vorschrift zur Umlagerung der Ketone 51 und 67}

Zu einer Suspension aus Nafion R Sac-13 ${ }^{43}$ (200 mg / 1.0 mmol Keton) in wasserfreiem Benzol (3 ml / 1.0 mmol Keton) gab man unter Rühren und Argon das entsprechende Keton und erhitzte auf $70{ }^{\circ} \mathrm{C}$ bis gaschromatographische Analyse [3 m x 1/4" Allglassystem, 15\% FFAP auf Chromosorb W AW/DMCS 60/80 mesh] vollständigen Umsatz anzeigte. Man ließ auf 
Raumtemperatur abkühlen, verdünnte mit Ether (10 ml / 1.0 mmol Keton) und engte die Lösung an einer $20 \mathrm{~cm}$ Vigreux-Kolonne (Badtemperatur $90{ }^{\circ} \mathrm{C}$ ) ein. Analytische Proben des farblosen Feststoffes (Smp.: $96^{\circ} \mathrm{C}$ ) wurden durch präparative Gaschromatographie erhalten.

Eingesetzt: 75 mg, 0.49 mmol 51; Reaktionsdauer: 45 h; Retentionszeiten (min): 4.00 (51) und $8.97(30)\left(140{ }^{\circ} \mathrm{C}\right)$.

Eingesetzt: 75 mg, 0.49 mmol 67; Reaktionsdauer: 24 h; Retentionszeiten (min): 7.69 (67) und $8.97(30)\left(140{ }^{\circ} \mathrm{C}\right)$.

\section{3a,6a-Dimethylhexahydro-pentalen-1-on (30)}

\section{$\mathrm{C}_{10} \mathrm{H}_{16} \mathrm{O}$}

Ber. 152.1201

Gef. 152.1201 HRMS

IR (KBr): $2960 \mathrm{~cm}^{-1}(\mathrm{CH}), 1735(\mathrm{C}=\mathrm{O})$.

${ }^{1}$ H-NMR (300 MHz, $\mathrm{CDCl}_{3}, \mathrm{CDCl}_{3}$ int.): $\delta=0.88 \mathrm{ppm}(\mathrm{s}, 3 \mathrm{H}), 1.00$ (s, 3H), $1.48\left(\mathrm{~m}_{\mathrm{c}}, 2 \mathrm{H}\right)$, $1.54-1.70(\mathrm{~m}, 4 \mathrm{H}), 1.80\left(\mathrm{~m}_{\mathrm{c}}, 1 \mathrm{H}\right), 1.98\left(\mathrm{~m}_{\mathrm{c}}, 1 \mathrm{H}\right), 2.30\left(\mathrm{~m}_{\mathrm{c}}, 2 \mathrm{H}\right)$.

${ }^{13}$ C-NMR (150 MHz, $\mathrm{CDCl}_{3}, \mathrm{CDCl}_{3}$ int. $): \delta=17.33$ ppm, $22.52\left(\mathrm{C}_{\text {prim }}\right), 22.73,31.92,35.61$, $37.05,39.82\left(\mathrm{C}_{\mathrm{sek}}\right), 49.63,58.69,224.96\left(\mathrm{C}_{\text {quart }}\right)$.

MS (70 eV): m/z (\%): $152\left(\mathrm{M}^{+}, 38\right), 95$ (100). 
116, 117, (3R*,3aS*,6aR*,7aR*)-3,6,6,6a-Tetramethyl-2,3,3a,5,6,6a,7,7a-octahydro-1Hcyclopenta[a]pentalen $(118),(3 \mathrm{aR} *, 3 \mathrm{bR} *, 6 \mathrm{aR} *, 7 \mathrm{aR} *)-3,3,3 \mathrm{a}, 4-$ Tetramethyl-2,3,3a,b,6, 6a,7,7a-octahydro-1H-cyclopenta[a]pentalen $(119), \quad\left(3 \mathrm{aR}^{*}, 3 \mathrm{bR}^{*}, 6 \mathrm{aS} *, 7 \mathrm{aS} *\right)-1,1,4,7 \mathrm{a}-$ Tetramethyl-2,3,3a,3b,6,6a,7,7a-octahydro-1H-cyclopenta[a]pentalen $\quad(120), \quad\left(3 R^{*}\right.$, 3bR*,6aR*)-3,6,6,6a-Tetramethyl-2,3,3a,5,6,6a,7,7a-octahydro-1H-cyclopenta[a]pentalen $(108 / 109)$, (3R*,3bS*,6aS*)-3,6,6,6a-Tetramethyl-2,3,3a,5,6,6a,7,7a-octahydro-1Hcyclopenta[a]pentalen $(108 / 109),\left(3 \mathrm{aR} *, 3 \mathrm{bS} *, 6 \mathrm{aR}^{*}, 7 \mathrm{aS} *\right)-1,1,4,7 \mathrm{a}-$ Tetramethyl-2,3,3a, 3b,6,6a,7,7a-octahydro-1H-cyclopenta[a]pentalen $\quad(110), \quad\left(3 R^{*}, 3 a S^{*}, 3 b S^{*}, 6 a S^{*}, 7 a S^{*}\right)-$ 3,3b,6,6a-Tetramethyl-2,3,3a,3b,4,-6a,7,7a-octahydro-1H-cyclopenta-[a]pentalen (111), $\left(3 \mathrm{R}^{*}, 3 \mathrm{aS} *, 3 \mathrm{bS} *, 6 \mathrm{aS} *, 7 \mathrm{aS} *\right)-3,3 \mathrm{~b}, 4,6 \mathrm{a}-$ Tetramethyl-2,3,3a,3b,6,6a,7,7a-octahydro-1Hcyclopenta[a]pentalen (112)

Man gab bei $70{ }^{\circ} \mathrm{C}$ Alkohol 44, 45, bzw. ein 50 : 50 Gemisch der beiden Stereoisomeren Alkohole 41,42 (10 mg, $0.045 \mathrm{mmol}$ ) zu einer $0.074 \mathrm{M}$ Lösung von wasserfreier pToluolsulfonsäure $(0.61 \mathrm{ml}, 0.045 \mathrm{mmol})$ in Benzol und untersuchte den Verlauf der Umlagerungen sowie deren Zusammensetzung durch Entnahme von Probehydrolysaten gaschromatographisch [3 m x 1/4" Allglassystem, 15\% FFAP auf Chromosorb W AW/DMCS 60/80 mesh, Temperaturprogramm: $8 \mathrm{~min}$ bei $150{ }^{\circ} \mathrm{C}, 20{ }^{\circ} \mathrm{C} / \mathrm{min}$ auf $220{ }^{\circ} \mathrm{C}$; Retentionszeiten (min): 3.21 (108/109), 5.30 (110), 5.90 (116/117), 6.68 (111), 7.29 (112), 7.99 (118), 8.73 (119), 9.33 (120), 14.72 (41/42) 15.39 (45) und 16.11 (44)]. Aufgrund der so erhaltenen Ergebnisse [s. S. 42 Tab. 1, S. 48 Tab. 3 und 4] wurden entsprechende präparative Ansätze zur Identifikation der Produkte durchgeführt:

116, 117, (3aR*,3bR*,6aR*,7aR*)-3,3,3a,4-Tetramethyl-2,3,3a,3b,6,6a,7,7a-octahydro$1 H$-cyclopenta[a]pentalen (119) und $\left(3 \mathrm{aR}^{*}, 3 \mathrm{bR} *, 6 \mathrm{aS} *, 7 \mathrm{aS} *\right)-1,1,4,7 \mathrm{a}-$ Tetramethyl-2,3, 3a,3b,6,6a,7,7a-octahydro-1H-cyclopenta[a]pentalen (120)

Man gab bei $70{ }^{\circ} \mathrm{C}$ Alkohol 44 (40 mg, $0.18 \mathrm{mmol}$ ) zu einer 0.074 M Lösung von wasserfreier p-Toluolsulfonsäure $(2.44 \mathrm{ml}, 0.18 \mathrm{mmol})$ in Benzol und untersuchte den Verlauf der Umlagerung [s. S. 48, Tab. 3] sowie die Zusammensetzung durch Entnahme von Probehydrolysaten gaschromatographisch [3 m x 1/4" Allglassystem, 15\% FFAP auf Chromosorb W AW/DMCS 60/80 mesh, Temperaturprogramm: $8 \mathrm{~min}$ bei $150{ }^{\circ} \mathrm{C}, 20{ }^{\circ} \mathrm{C} / \mathrm{min}$ auf $220{ }^{\circ} \mathrm{C}$; Retentionszeiten (min): 5.90 (116 und 117) (9\%), 8.73 (119) (30\%), 9.33 (120) (30\%)]. Nach 60 min verdünnte man mit Pentan $(4 \mathrm{ml})$, wusch mit gesättigter Natriumhydrogencarbonatlösung ( $3 \times 4 \mathrm{ml})$, extrahierte die wässrige Phase mit Pentan, trocknete die vereinigten 
organischen Phasen über Molekularsieb (3 Å) und engte die Lösung an einer 20 cm VigreuxKolonne ein (Badtemperatur $90{ }^{\circ} \mathrm{C}$ ). Präparative Gaschromatographie lieferte116, 117, 119 und 120als farblose Flüssigkeiten.

\section{$116 / 117$}

$\mathrm{C}_{15} \mathrm{H}_{24}$

${ }^{1}$ H-NMR (300 MHz, $\mathrm{C}_{6} \mathrm{D}_{6}, \mathrm{C}_{6} \mathrm{D}_{5} \mathrm{H}$ int.): $\delta=0.88 \mathrm{ppm}(\mathrm{s}, 3 \mathrm{H}), 0.94$ (s, 3H), 1.00 (s, 3H), $1.01(\mathrm{~s}, 3 \mathrm{H}), 1.04(\mathrm{~s}, 3 \mathrm{H}), 1.08(\mathrm{~d}, \mathrm{~J}=6 \mathrm{~Hz}, 3 \mathrm{H}), 1.32-1.46(\mathrm{~m}, 2 \mathrm{H}), 1.48\left(\mathrm{~m}_{\mathrm{c}}, 3 \mathrm{H}\right), 1.63$ $\left(\mathrm{m}_{\mathrm{c}}, 3 \mathrm{H}\right), 1.65-2.50(\mathrm{~m}, 19 \mathrm{H}), 2.64\left(\mathrm{~m}_{\mathrm{c}}, 1 \mathrm{H}\right), 2.86\left(\mathrm{~m}_{\mathrm{c}}, 1 \mathrm{H}\right), 5.32\left(\mathrm{~m}_{\mathrm{c}}, 1 \mathrm{H}\right)$.

${ }^{13}$ C-NMR (150 MHz, $\mathrm{C}_{6} \mathrm{D}_{6}, \mathrm{C}_{6} \mathrm{D}_{6}$ int.): $\delta=16.80 \mathrm{ppm}, 16.93,21.02,23.24,23.27,24.85$, 26.62, $26.65\left(\mathrm{C}_{\text {prim }}\right), 29.09,29.18,30.94,33.33,35.94,\left(\mathrm{C}_{\text {sek }}\right), 37.24\left(\mathrm{C}_{\text {tert }}\right), 37.90\left(\mathrm{C}_{\text {sek }}\right)$, $37.93\left(\mathrm{C}_{\text {tert }}\right), 39.24,39.30\left(\mathrm{C}_{\text {quart }}\right), 40.65 .41 .47\left(\mathrm{C}_{\text {sek }}\right), 42.63,43.22\left(\mathrm{C}_{\text {quart }}\right), 45.10\left(\mathrm{C}_{\text {tert }}\right)$, $47.01\left(\mathrm{C}_{\text {quart }}\right), 53.29\left(\mathrm{C}_{\text {tert }}\right), 63.05\left(\mathrm{C}_{\text {quart }}\right), 123.80,129.68\left(\mathrm{C}_{\text {tert }}\right), 142.86,152.02\left(\mathrm{C}_{\text {quart }}\right)$.

MS (70 eV): m/z (\%): $204\left(\mathrm{M}^{+}, 100\right)$.

$(3 \mathrm{aR} *, 3 \mathrm{bR} *, 6 \mathrm{aR} *, 7 \mathrm{aR} *)-3,3,3 \mathrm{a}, 4-$ Tetramethyl-2,3,3a,3b,6,6a,7,7a-octahydro-1Hcyclopenta[a]pentalen (119)

$\mathrm{C}_{15} \mathbf{H}_{24}$

${ }^{1}$ H-NMR (300 MHz, $\mathrm{C}_{6} \mathrm{D}_{6}, \mathrm{C}_{6} \mathrm{D}_{5} \mathrm{H}$ int.): $\delta=0.84 \mathrm{ppm}(\mathrm{s}, 3 \mathrm{H}), 0.90$ (s, 3H), 0.98 (s, 3H), $1.02\left(\mathrm{~m}_{\mathrm{c}}, 1 \mathrm{H}\right), 1.14\left(\mathrm{~m}_{\mathrm{c}}, 1 \mathrm{H}\right), 1.30\left(\mathrm{~m}_{\mathrm{c}}, 1 \mathrm{H}\right), 1.50-1.62(\mathrm{~m}, 1 \mathrm{H}), 1.68$ (br s, 3H), $1.90-2.20$ (m, 4H), $2.56-2.68(\mathrm{~m}, 1 \mathrm{H}), 2.76\left(\mathrm{~m}_{\mathrm{c}}, 2 \mathrm{H}\right), 5.18(\mathrm{br} \mathrm{s}, 1 \mathrm{H})$.

${ }^{13}$ C-NMR (75, $\mathrm{C}_{6} \mathrm{D}_{6}, \mathrm{C}_{6} \mathrm{D}_{6}$ int.): $\delta=15.49 \mathrm{ppm}\left(\mathrm{C}_{\text {prim }}\right), 22.51,23.36,26.17\left(\mathrm{C}_{\text {prim }}\right), 31.08$, 39.26, $41.78\left(\mathrm{C}_{\text {sek }}\right), 42.36\left(\mathrm{C}_{\text {tert }}\right), 45.23\left(\mathrm{C}_{\text {sek }}\right)$ [Koinzidenz mit $\left.\left(\mathrm{C}_{\text {quart }}\right)\right], 51.95\left(\mathrm{C}_{\text {tert }}\right), 57.22$ $\left(\mathrm{C}_{\text {quart }}\right), 64.63,123.55\left(\mathrm{C}_{\text {tert }}\right), 143.28\left(\mathrm{C}_{\text {quart }}\right)$. 
MS (70 eV): m/z (\%): $204\left(\mathrm{M}^{+}, 100\right)$.

$(3 \mathrm{aR} *, 3 \mathrm{bR} *, 6 \mathrm{aS} *, 7 \mathrm{aS} *)-1,1,4,7 \mathrm{a}-\mathrm{Tetramethyl-2,3,3a,3b,6,6a,7,7a-octahydro-1H-}$ cyclopenta[a]pentalen $(120)$

$\mathrm{C}_{15} \mathbf{H}_{24}$

${ }^{1}$ H-NMR (300 MHz, $\mathrm{C}_{6} \mathrm{D}_{6}, \mathrm{C}_{6} \mathrm{D}_{5} \mathrm{H}$ int.): $\delta=0.86 \mathrm{ppm}(\mathrm{s}, 3 \mathrm{H}), 0.88$ (s, 3H), 0.90 (s, 3H), $1.10-1.22(\mathrm{~m}, 1 \mathrm{H}), 1.30-1.56(\mathrm{~m}, 4 \mathrm{H}), 1.60$ (br s, 3H), $1.64-1.80$ (m, 1H), $1.88-2.12$ $(\mathrm{m}, 2 \mathrm{H}), 2.52\left(\mathrm{~m}_{\mathrm{c}}, 1 \mathrm{H}\right), 2.80\left(\mathrm{~m}_{\mathrm{c}}, 1 \mathrm{H}\right), 3.10\left(\mathrm{~m}_{\mathrm{c}}, 1 \mathrm{H}\right), 5.24$ (br s, $\left.1 \mathrm{H}\right)$.

${ }^{13}$ C-NMR (75 MHz, $\mathrm{C}_{6} \mathrm{D}_{6}, \mathrm{C}_{6} \mathrm{D}_{6}$ int. $): \delta=16.47 \mathrm{ppm}, 18.81\left(\mathrm{C}_{\text {prim }}\right), 22.51\left(\mathrm{C}_{\text {sek }}\right), 23.58$, $26.82\left(\mathrm{C}_{\text {prim }}\right), 38.59,40.40\left(\mathrm{C}_{\text {sek }}\right), 41.87\left(\mathrm{C}_{\text {tert }}\right), 43.43\left(\mathrm{C}_{\text {quart }}\right), 43.78\left(\mathrm{C}_{\text {sek }}\right), 50.82,58.35\left(\mathrm{C}_{\text {tert }}\right)$, $59.88\left(\mathrm{C}_{\text {quart }}\right), 124.62\left(\mathrm{C}_{\text {tert }}\right), 141.04\left(\mathrm{C}_{\text {quart }}\right)$.

MS (70 eV): m/z (\%): $204\left(\mathrm{M}^{+}, 53\right), 94(100)$.

$\left(3 \mathrm{R}^{*}, 3 \mathrm{aS} *, 6 \mathrm{aR} *, 7 \mathrm{aR} *\right)-3,6,6,6 \mathrm{a}-$ Tetramethyl-2,3,3a,5,6,6a,7,7a-octahydro-1 $\mathrm{H}$-cyclopenta[a]pentalen (118)

Man gab bei $70{ }^{\circ} \mathrm{C}$ Alkohol 44 (40 mg, $0.18 \mathrm{mmol}$ ) zu einer 0.074 M Lösung von wasserfreier p-Toluolsulfonsäure $(2.44 \mathrm{ml}, 0.18 \mathrm{mmol})$ in Benzol und untersuchte den Verlauf der Umlagerung [s. S. 48, Tab. 3] sowie die Zusammensetzung durch Entnahme von Probehydrolysaten gaschromatographisch [3 m x 1/4" Allglassystem, 15\% FFAP auf Chromosorb W AW/DMCS 60/80 mesh, Temperaturprogramm: $8 \mathrm{~min}$ bei $150{ }^{\circ} \mathrm{C}, 20{ }^{\circ} \mathrm{C} / \mathrm{min}$ auf $220{ }^{\circ} \mathrm{C}$; Retentionszeiten (min): 5.90 (116/117) (43\%), 7.99 (118) (12\%), 9.33 (120) (34\%)]. Nach 5 min verdünnte man mit Pentan (4 ml), wusch mit gesättigter Natriumhydrogencarbonatlösung ( $3 \times 4 \mathrm{ml}$ ), extrahierte die wässrige Phase mit Pentan, trocknete die vereinigten organischen Phasen über Molekularsieb ( $3 \AA$ ) und engte die Lösung an einer 20 cm Vigreux-Kolonne ein (Badtemperatur $90{ }^{\circ} \mathrm{C}$ ). Präparative Gaschromatographie lieferte116, 117, 118 und 120 als farblose Flüssigkeiten. 


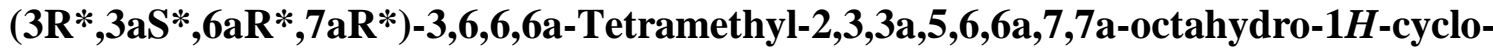
penta[a]pentalen (118)

$\mathbf{C}_{15} \mathbf{H}_{24}$

${ }^{1}$ H-NMR (500 MHz, $\mathrm{C}_{6} \mathrm{D}_{6}, \mathrm{C}_{6} \mathrm{D}_{5} \mathrm{H}$ int.): $\delta=0.96 \mathrm{ppm}(\mathrm{s}, 3 \mathrm{H}) ; 0.98$ (s, 3H), 1.02 (s, 3H), $1.14(\mathrm{~d}, \mathrm{~J}=6 \mathrm{~Hz}, 3 \mathrm{H}), 1.16-1.32(\mathrm{~m}, 3 \mathrm{H}), 1.40\left(\mathrm{~m}_{\mathrm{c}}, 1 \mathrm{H}\right), 1.50\left(\mathrm{~m}_{\mathrm{c}}, 2 \mathrm{H}\right), 1.86\left(\mathrm{~m}_{\mathrm{c}}, 1 \mathrm{H}\right)$, $2.12\left(\mathrm{~m}_{\mathrm{c}}, 1 \mathrm{H}\right), 2.66\left(\mathrm{~m}_{\mathrm{c}}, 1 \mathrm{H}\right), 2.74\left(\mathrm{~m}_{\mathrm{c}}, 1 \mathrm{H}\right), 2.94\left(\mathrm{~m}_{\mathrm{c}}, 1 \mathrm{H}\right), 5.21\left(\mathrm{~m}_{\mathrm{c}}, 1 \mathrm{H}\right)$.

${ }^{13}$ C-NMR (150 MHz, $\mathrm{C}_{6} \mathrm{D}_{6}, \mathrm{C}_{6} \mathrm{D}_{6}$ int. $): \delta=16.08$ ppm, 21.28, 23.99, $26.93\left(\mathrm{C}_{\text {prim }}\right), 32.76$, 34.43, $37.84\left(\mathrm{C}_{\text {sek }}\right), 38.09\left(\mathrm{C}_{\text {tert }}\right), 42.87\left(\mathrm{C}_{\text {quart }}\right), 43.29,48.82\left(\mathrm{C}_{\text {tert }}\right), 51.86\left(\mathrm{C}_{\text {sek }}\right), 62.11$ $\left(\mathrm{C}_{\text {quart }}\right), 115.48\left(\mathrm{C}_{\text {tert }}\right), 158.73\left(\mathrm{C}_{\text {quart }}\right)$.

MS (70 eV): m/z (\%): $204\left(\mathrm{M}^{+}, 27\right), 198$ (100).

116, 117, $\left(3 \mathrm{R}^{*}, 3 \mathrm{aS} *, 6 \mathrm{aR} *, 7 \mathrm{aR} *\right)-3,6,6,6 \mathrm{a}-\mathrm{Tetramethyl-2,3,3a,5,6,6a,7,7a-octahydro-1H}$ cyclopenta[a]pentalen (118), (3aR*,3bR*,6aR*,7aR*)-3,3,3a,4-Tetramethyl-2,3,3a,3b, 6,6a,7,7a-octahydro-1H-cyclopenta[a]pentalen (119) und $\left(3 \mathrm{aR}^{*}, 3 \mathrm{bR} *, 6 \mathrm{aS} *, 7 \mathrm{aS} *\right)$ 1,1,4,7a-Tetramethyl-2,3,3a,3b,6,6a,7,7a-octahydro-1 $H$-cyclopenta[a]pentalen (120)

Man gab bei $70{ }^{\circ} \mathrm{C}$ Alkohol 45 (20 mg, $0.09 \mathrm{mmol}$ ) zu einer $0.074 \mathrm{M}$ Lösung von wasserfreier p-Toluolsulfonsäure $(1.22 \mathrm{ml}, 0.09 \mathrm{mmol})$ in Benzol und untersuchte den Verlauf der Umlagerung [s. S. 48, Tab. 4] sowie die Zusammensetzung durch Entnahme von Probehydrolysaten gaschromatographisch [3 m x 1/4" Allglassystem, 15\% FFAP auf Chromosorb W AW/DMCS $60 / 80$ mesh, Temperaturprogramm: $8 \mathrm{~min}$ bei $150{ }^{\circ} \mathrm{C}, 20{ }^{\circ} \mathrm{C} / \mathrm{min}$ auf $220{ }^{\circ} \mathrm{C}$; Retentionszeiten (min): 5.90 (116/117) (11\%), 8.73 (119) (16\%), 9.33 (120) (60\%)] Nach 60 min verdünnte man mit Pentan $(2 \mathrm{ml})$, wusch mit gesättigter Natriumhydrogencarbonatlösung ( 3 x $2 \mathrm{ml}$ ), extrahierte die wässrige Phase mit Pentan, trocknete die vereinigten organischen Phasen über Molekularsieb ( $3 \AA$ ) und engte die Lösung an einer $20 \mathrm{~cm}$ Vigreux-Kolonne ein (Badtemperatur $90^{\circ} \mathrm{C}$ ). Präparative Gaschromatographie lieferte 116, 117, 119 und 120 als farblose Flüssigkeiten. Die ${ }^{1} \mathrm{H}-\mathrm{NMR}-$ Daten entsprachen denen authentischer Proben. 
$(3 \mathrm{R} *, 3 \mathrm{bR} *, 6 \mathrm{aR} *)-3,6,6,6 \mathrm{a},-$ Tetramethyl-2,3,3a,5,6,6a,7,7a-octahydro-1H-cyclopenta[a]

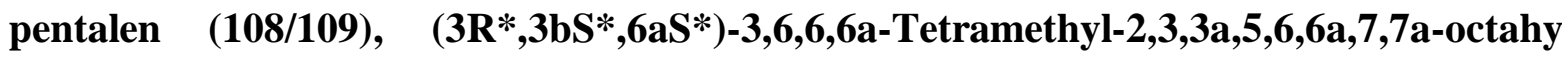
dro-1H-cyclopenta[a]pentalen $(108 / 109)\left(3 \mathrm{aR}^{*}, 3 \mathrm{bS} *, 6 \mathrm{aR} *, 7 \mathrm{aS} *\right)-1,1,4,7 \mathrm{a}-$ Tetra-methyl2,3,3a,3b,6,6a,7,7a-octahydro- $1 H$-cyclopenta[a]pentalen $\quad(110), \quad\left(3 R^{*}, 3 a S^{*}, 3 b b^{*}\right.$, 6aS*,7aS*)-3,3b,6,6a-Tetramethyl-2,3,3a,3b,4,6a,7,7a-octahydro-1H-cyclopenta[a]pentalen (111), (3R*,3aS*,3bS*,6aS*,7aS*)-3,3b,4,6a-Tetramethyl-2,3,3a,3b,6,6a,7,7a-octahydro-1H-cyclopenta[a]pentalen (112)

Man gab bei $70{ }^{\circ} \mathrm{C}$ ein 50 : 50 Gemisch der beiden stereoisomeren Alkohole 41 und 42 (40 $\mathrm{mg}, 0.18 \mathrm{mmol}) \mathrm{zu}$ einer $0.074 \mathrm{M}$ Lösung von wasserfreier p-Toluolsulfonsäure $(2.44 \mathrm{ml}$, $0.18 \mathrm{mmol}$ ) in Benzol und untersuchte den Verlauf der Umlagerung [s. S. 42, Tab. 1] sowie die Zusammensetzung durch Entnahme von Probehydrolysaten gaschromatographisch [3 $\mathrm{m} \mathrm{x}$ 1/4" Allglassystem, 15\% FFAP auf Chromosorb W AW/DMCS 60/80 mesh, Temperaturprogramm: $8 \mathrm{~min}$ bei $150{ }^{\circ} \mathrm{C}, 20{ }^{\circ} \mathrm{C} / \mathrm{min}$ auf $220{ }^{\circ} \mathrm{C}$; Retentionszeiten (min): $3.21(\mathbf{1 0 8} / \mathbf{1 0 9})$ (23\%), 5.30 (110) (19\%), 6.68 (111) (18\%), 7.29 (112) (9\%)]. Nach 4 h verdünnte man mit Pentan (4 ml), wusch mit gesättigter Natriumhydrogencarbonatlösung (3 x $4 \mathrm{ml})$, extrahierte die wässrige Phase mit Pentan, trocknete die vereinigten organischen Phasen über Molekularsieb ( $3 \AA$ ) und engte die Lösung an einer $20 \mathrm{~cm}$ Vigreux-Kolonne ein. Präparative Gaschromatographie lieferte 108, 109, 110, 111 und 112 als farblose Flüssigkeiten.

$\left(3 R^{*}, 3 b R^{*}, 6 a R *\right)-3,6,6,6 a-T e t r a m e t h y l-2,3,3 a, 5,6,6 a, 7,7 a-o c t a h y d r o-1 H$-cyclopenta[a]pentalen $(108 / 109)$ und $\left(3 R^{*}, 3 b S^{*}, 6 a S^{*}\right)-3,6,6,6 a-T e t r a m e t h y l-2,3,3 a, 5,6,6 a, 7,7 a-$ octahydro- $1 H$-cyclopenta[a]pentalen $(108 / 109)$

$\mathrm{C}_{15} \mathrm{H}_{24}$

${ }^{1}$ H-NMR (300 MHz, $\mathrm{C}_{6} \mathrm{D}_{6}, \mathrm{C}_{6} \mathrm{D}_{5} \mathrm{H}$ int.): $\delta=0.92 \mathrm{ppm}(\mathrm{s}, 3 \mathrm{H}), 0.94$ (s, 3H), 0.99 (d; J = 6 $\mathrm{Hz}, 3 \mathrm{H}), 1.01$ (d, J = 6 Hz, 3H), 1.04 (s, 3H), 1.05 (s, 3H), 1.10 (s, 3H), 1.12 (s, 3H), 1.36 $1.76(\mathrm{~m}, 11 \mathrm{H}), 1.88\left(\mathrm{~m}_{\mathrm{c}}, 1 \mathrm{H}\right), 2.02\left(\mathrm{~m}_{\mathrm{c}}, 3 \mathrm{H}\right), 2.08-2.32(\mathrm{~m}, 5 \mathrm{H}), 2.38\left(\mathrm{~m}_{\mathrm{c}}, 2 \mathrm{H}\right), 2.56\left(\mathrm{~m}_{\mathrm{c}}\right.$, $2 \mathrm{H})$.

${ }^{13}$ C-NMR (150 MHz, $\mathrm{C}_{6} \mathrm{D}_{6}, \mathrm{C}_{6} \mathrm{D}_{6}$ int.): $\delta=19.90 \mathrm{ppm}, 20.42,25.36,25.66\left(\mathrm{C}_{\text {prim }}\right)$, 29.63, $29.65\left(\mathrm{C}_{\text {sek }}\right), 30.10,30.13,30.95,31.02\left(\mathrm{C}_{\text {prim }}\right), 36.43,37.03\left(\mathrm{C}_{\text {tert }}\right), 37.49,37.69,40.02$, 
$40.13,42.12,42.13\left(\mathrm{C}_{\text {sek }}\right), 42.48,42.70\left(\mathrm{C}_{\text {quart }}\right), 44.65,44.91,\left(\mathrm{C}_{\text {sek }}\right), 57.18,57.22\left(\mathrm{C}_{\text {quart }}\right)$, $66.74,67.06\left(\mathrm{C}_{\text {tert }}\right), 144.19,144.32,149.86,149.98\left(\mathrm{C}_{\text {quart }}\right)$.

MS (70 eV): m/z (\%): $204\left(\mathrm{M}^{+}, 42\right), 135$ (100).

$\left(3 \mathrm{aR}^{*}, 3 \mathrm{bS} *, 6 \mathrm{aR} *, 7 \mathrm{aS} *\right)-1,1,4,7 \mathrm{a}-\mathrm{Tetramethyl}-2,3,3 \mathrm{a}, 3 \mathrm{~b}, 6,6 \mathrm{a}, 7,7 \mathrm{a}-\mathrm{octahydro}-1 \mathrm{H}$-cyclopenta[a]pentalen (110)

$\mathrm{C}_{15} \mathrm{H}_{24}$

${ }^{1} \mathbf{H}-\mathbf{N M R}\left(300 \mathrm{MHz}, \mathrm{C}_{6} \mathrm{D}_{6}, \mathrm{C}_{6} \mathrm{D}_{5} \mathrm{H}\right.$ int.): $\delta=0.96 \mathrm{ppm}(\mathrm{s}, 3 \mathrm{H}), 0.98$ (s, 3H), 1.15 (s, 3H), $1.34-1.64(\mathrm{~m}, 6 \mathrm{H}), 1.68$ (br s, 3H), $1.74\left(\mathrm{~m}_{\mathrm{c}}, 1 \mathrm{H}\right), 2.08\left(\mathrm{~m}_{\mathrm{c}}, 1 \mathrm{H}\right), 2.48\left(\mathrm{~m}_{\mathrm{c}}, 1 \mathrm{H}\right), 2.57\left(\mathrm{~m}_{\mathrm{c}}\right.$, $1 \mathrm{H}), 2.86\left(\mathrm{~m}_{\mathrm{c}}, 1 \mathrm{H}\right), 5.22$ (br s, 1H).

${ }^{13}$ C-NMR (150 MHz, $\mathrm{C}_{6} \mathrm{D}_{6}, \mathrm{C}_{6} \mathrm{D}_{6}$ int.): $\delta=15.15$ ppm, 25.45, 30.57, $31.58\left(\mathrm{C}_{\text {prim }}\right), 40.73$, $40.78,41.14\left(\mathrm{C}_{\text {sek }}\right), 41.81\left(\mathrm{C}_{\text {quart }}\right), 45.37\left(\mathrm{C}_{\text {sek }}\right), 45.85\left(\mathrm{C}_{\text {tert }}\right), 52.40\left(\mathrm{C}_{\text {quart }}\right), 56.32,71.67$, $123.63\left(\mathrm{C}_{\text {tert }}\right), 143.45\left(\mathrm{C}_{\text {quart }}\right)$.

MS (70 eV): m/z (\%): $204\left(\mathrm{M}^{+}, 10\right), 133$ (100).

$\left(3 \mathrm{R}^{*}, 3 \mathrm{aS} *, 3 \mathrm{bS} *, 6 \mathrm{aS} *, 7 \mathrm{aS} *\right)-3,3 \mathrm{~b}, 6,6 \mathrm{a}-\mathrm{Tetramethyl-2,3,3a,3b,4,6a,7,7a-octahydro-1H}$ cyclopenta[a]pentalen (111)

$\mathrm{C}_{15} \mathrm{H}_{24}$

${ }^{1}$ H-NMR (300 MHz, $\mathrm{C}_{6} \mathrm{D}_{6}, \mathrm{C}_{6} \mathrm{D}_{5} \mathrm{H}$ int.): $\delta=0.76 \mathrm{ppm}(\mathrm{s}, 3 \mathrm{H}), 0.80(\mathrm{~d}, \mathrm{~J}=6 \mathrm{~Hz}, 3 \mathrm{H}), 0.86$ $(\mathrm{s}, 3 \mathrm{H}), 1.02-1.40(\mathrm{~m}, 3 \mathrm{H}), 1.54\left(\mathrm{~m}_{\mathrm{c}}, 3 \mathrm{H}\right), 1.60\left(\right.$ br s, 3H), $1.62-1.68(\mathrm{~m}, 1 \mathrm{H}), 1.72\left(\mathrm{~m}_{\mathrm{c}}\right.$, $1 \mathrm{H}), 2.02\left(\mathrm{~m}_{\mathrm{c}}, 1 \mathrm{H}\right), 2.18-2.32(\mathrm{~m}, 2 \mathrm{H}), 4.96\left(\mathrm{~m}_{\mathrm{c}}, 1 \mathrm{H}\right)$. 
${ }^{13}$ C-NMR (150 MHz, $\mathrm{C}_{6} \mathrm{D}_{6}, \mathrm{C}_{6} \mathrm{D}_{6}$ int.): $\delta=13.93$ ppm, 15.98, 19.78, $26.17\left(\mathrm{C}_{\text {prim }}\right), 28.25$, $33.30\left(\mathrm{C}_{\text {sek }}\right), 37.71\left(\mathrm{C}_{\text {tert }}\right), 39.05,41.26\left(\mathrm{C}_{\text {sek }}\right), 48.48,55.37\left(\mathrm{C}_{\text {tert }}\right), 56.15,59.93\left(\mathrm{C}_{\text {quart }}\right), 120.80$ $\left(\mathrm{C}_{\text {tert }}\right), 147.42\left(\mathrm{C}_{\text {quart }}\right)$.

MS (70 eV): m/z (\%): $204\left(\mathrm{M}^{+}, 47\right), 108(100)$.

$\left(3 \mathrm{R}^{*}, 3 \mathrm{aS} *, 3 \mathrm{bS} *, 6 \mathrm{aS} *, 7 \mathrm{aS} *\right)-3,3 \mathrm{~b}, 4,6 \mathrm{a}-\mathrm{Tetramethyl-2,3,3a,3b,6,6a,7,7a-octahydro-1H}$ cyclopenta[a]pentalen (112)

$\mathrm{C}_{15} \mathrm{H}_{24}$

${ }^{1}$ H-NMR (300 MHz, $\mathrm{C}_{6} \mathrm{D}_{6}, \mathrm{C}_{6} \mathrm{D}_{5} \mathrm{H}$ int.): $\delta=0.94 \mathrm{ppm}(\mathrm{d}, \mathrm{J}=6 \mathrm{~Hz}, 3 \mathrm{H}), 0.98(\mathrm{~s}, 3 \mathrm{H}), 1.00$ (s, 3H), $1.24-1.34(\mathrm{~m}, 1 \mathrm{H}), 1.36(\mathrm{~d}, \mathrm{~J}=9 \mathrm{~Hz}, 2 \mathrm{H}), 1.50-1.54(\mathrm{~m}, 2 \mathrm{H}), 1.58\left(\mathrm{~m}_{\mathrm{c}}, 3 \mathrm{H}\right), 1.68$ $\left(\mathrm{m}_{\mathrm{c}}, 1 \mathrm{H}\right), 1.88-2.04(\mathrm{~m}, 2 \mathrm{H}), 2.06-2.22(\mathrm{~m}, 1 \mathrm{H}), 2.28\left(\mathrm{~m}_{\mathrm{c}}, 2 \mathrm{H}\right), 5.18$ (br s, 1H).

${ }^{13}$ C-NMR (150 MHz, $\mathrm{C}_{6} \mathrm{D}_{6}, \mathrm{C}_{6} \mathrm{D}_{6}$ int.): $\delta=15.10 \mathrm{ppm}, 17.02,23.16,25.21\left(\mathrm{C}_{\text {prim }}\right), 27.07$, $32.13\left(\mathrm{C}_{\text {sek }}\right), 36.04\left(\mathrm{C}_{\text {tert }}\right), 42.57,47.88\left(\mathrm{C}_{\text {sek }}\right), 48.90\left(\mathrm{C}_{\text {tert }}\right), 52.07\left(\mathrm{C}_{\text {quart }}\right), 56.52\left(\mathrm{C}_{\text {tert }}\right), 62.63$ $\left(\mathrm{C}_{\text {quart }}\right), 122.50\left(\mathrm{C}_{\text {tert }}\right), 145.10\left(\mathrm{C}_{\text {quart }}\right)$.

MS (70 eV): m/z (\%): $204\left(\mathrm{M}^{+}, 26\right), 108(100)$. 
116, 117, (3R*,3aS*,6aR*,7aR*)-3,6,6,6a-Tetramethyl-2,3,3a,5,6,6a,7,7a-octahydro-1Hcyclopenta[a]pentalen $(118),(3 \mathrm{aR} *, 3 \mathrm{bR} *, 6 \mathrm{aR} *, 7 \mathrm{aR} *)-3,3,3 \mathrm{a}, 4-$ Tetramethyl-2,3,3a,3b,6, 6a,7,7a-octahydro- $1 H$-cyclopenta[a]pentalen $\quad(119), \quad\left(3 \mathrm{aR}^{*}, 3 \mathrm{bR} *, 6 \mathrm{aS} *, 7 \mathrm{aS} *\right)-1,1,4,7 \mathrm{a}-$ Tetramethyl-2,3,3a,3b,6,6a,7,7a-octahydro-1H-cyclopenta[a]pentalen $\quad(120), \quad\left(3 R^{*}\right.$, 3bR*,6aR*)-3,6,6,6a,-Tetramethyl-2,3,3a,5,6,6a,7,7a-octahydro-1H-cyclopenta[a]pentalen $(108 / 109), \quad\left(3 \mathrm{R} *, 3 \mathrm{bS}^{*}, 6 \mathrm{aS} *\right)-3,6,6,6 \mathrm{a}-\mathrm{Tetramethyl-2,3,3a,5,6,6a,7,7a-octahydro-}$ $1 H$-cyclopenta[a]pentalen $\quad(108 / 109), \quad\left(3 \mathrm{aR}^{*}, 3 \mathrm{bS}^{*}, 6 \mathrm{aR}^{*}, 7 \mathrm{aS} *\right)-1,1,4,7 \mathrm{a}-$ Tetramethyl2,3,3a,3b,6,6a,7,7a-octahydro-1H-cyclopenta[a]pentalen $(110), \quad\left(3 R^{*}, 3 a S^{*}, 3 b S^{*}, 6 a S^{*}\right.$, 7aS*)-3,3b,6,6a-Tetramethyl-2,3,3a,3b,4,6a,7,7a-octahydro- $1 H$-cyclopenta[a]pentalen (111), (3R*,3aS*,3bS*,6aS*,7aS*)-3,3b,4,6a-Tetramethyl-2,3,3a,3b,6,6a,7,7a-octahydro-1H-cyclopenta[a]pentalen (112)

Man gab bei $25^{\circ} \mathrm{C}$ Olefin 103 bzw. 104 (10 mg, $\left.0.049 \mathrm{mmol}\right)$ zu einer 0.074 M Lösung von wasserfreier p-Toluolsulfonsäure $(0.66 \mathrm{ml}, 0.049 \mathrm{mmol})$ in Benzol und untersuchte den Verlauf der Umlagerungen sowie die Zusammensetzung durch Entnahme von Probehydrolysaten gaschromatographisch [3 m x 1/4" Allglassystem, 15\% FFAP auf Chromosorb W AW/DMCS 60/80 mesh, Temperaturprogramm: $8 \mathrm{~min}$ bei $150{ }^{\circ} \mathrm{C}, 20{ }^{\circ} \mathrm{C} / \mathrm{min}$ auf $220{ }^{\circ} \mathrm{C}$; Retentionszeiten (min): 3.21 (108/109), 5.30 (110), 5.90 (116/117), 6.48 (104), 6.57 (103), 6.68 (111), 7.29 (112), 8.73 (119) und 9.33 (120)]. Die so erhaltenen Olefine [s. S. 42 Tab. 2 und S. 49 Tab.5] entsprachen laut ${ }^{1}$ H-NMR-spektroskopischer Analyse denen der Umlagerungen der entsprechenden Alkohole 41, 42, 44 und 45. 


\section{Spektrenanhang}

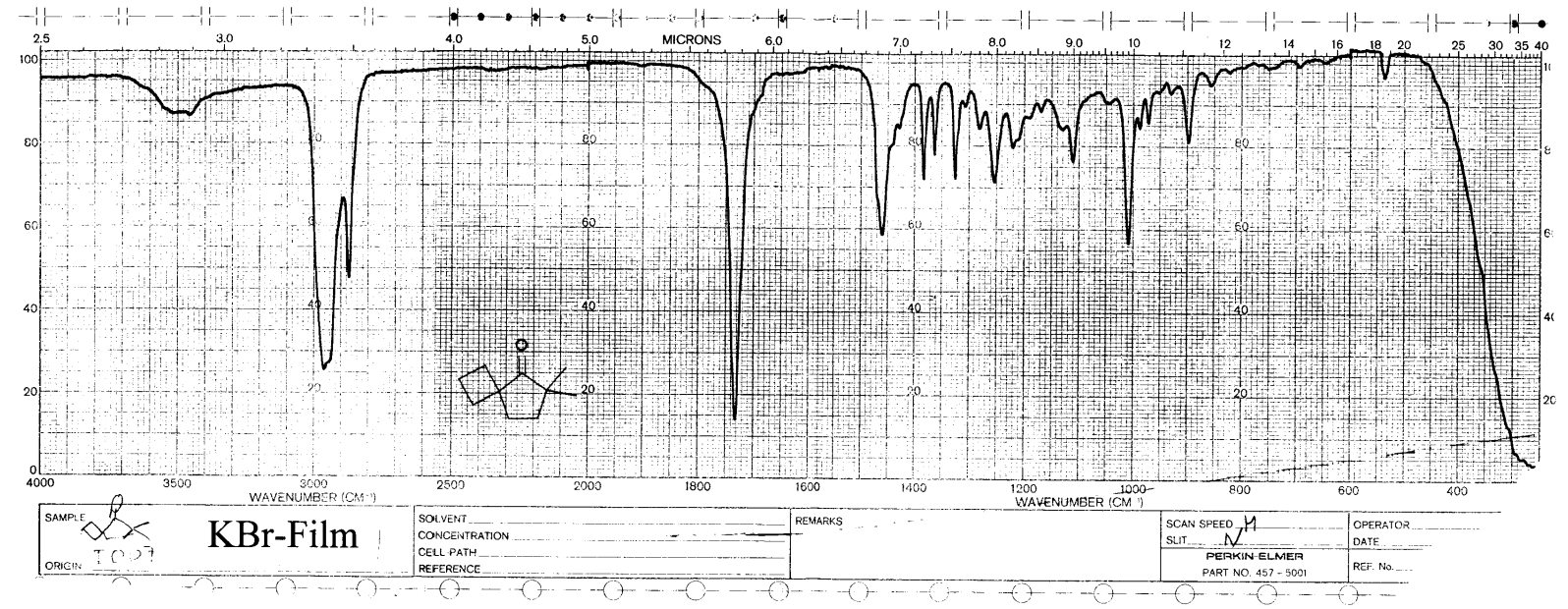

Abb. 1: IR-Spektrum von 6,6-Dimethyl-spiro[3.4]octan-5-on (51)

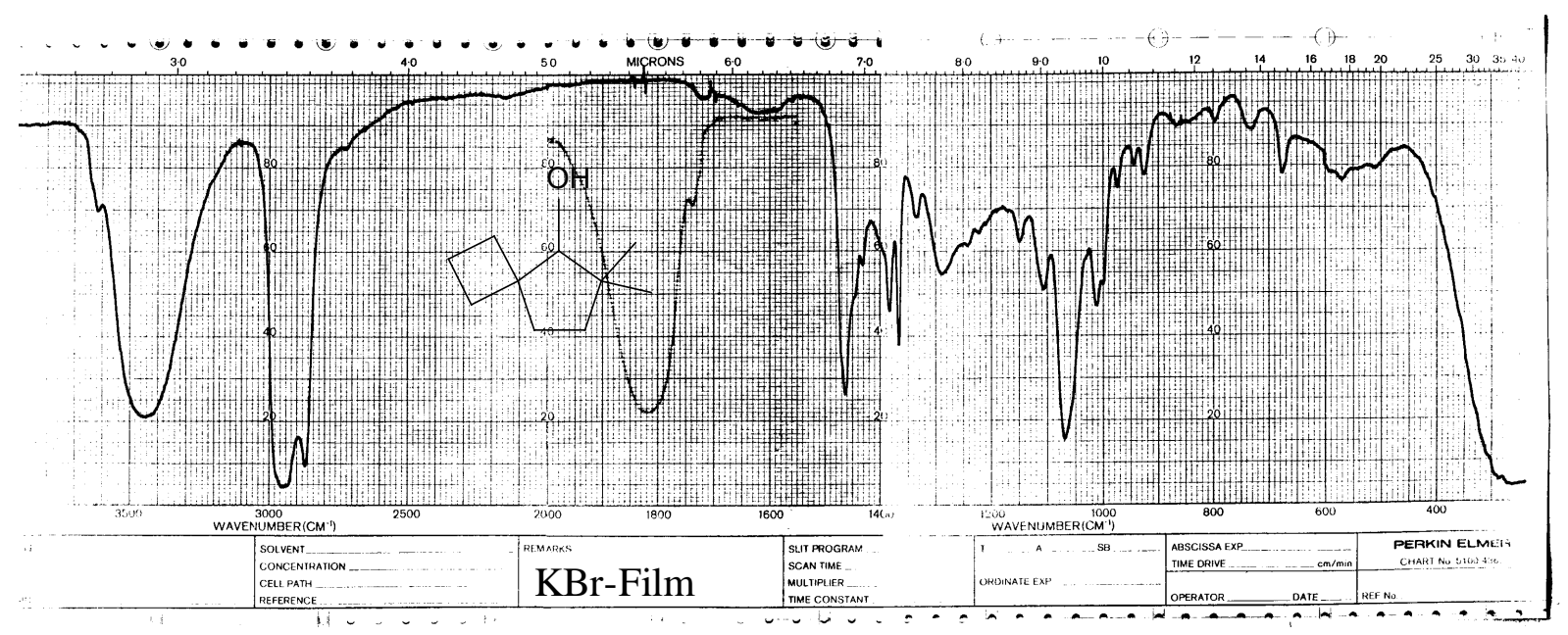

Abb.2: IR-Spektrum von 6,6-Dimethyl-spiro[3.4]octan-5-ol (24) 


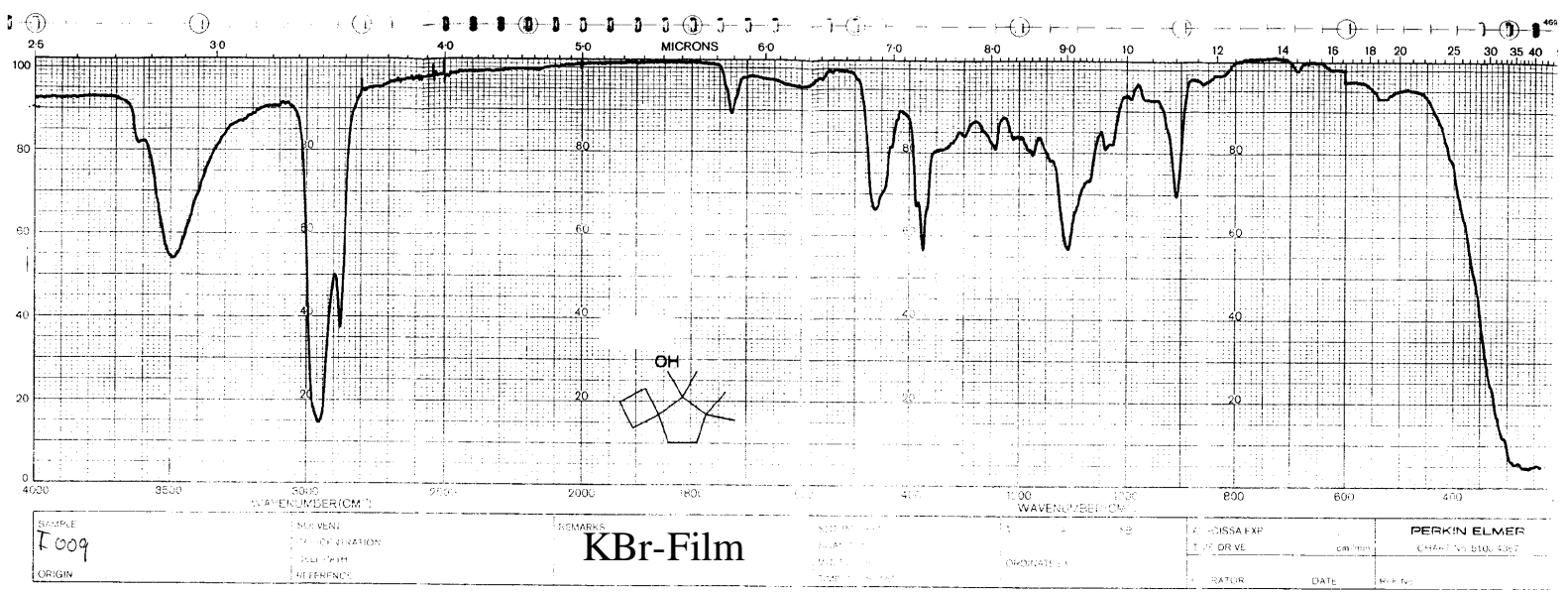

Abb. 3: IR-Spektrum von 5,6,6-Trimethyl-spiro[3.4]octan-5-ol (25)

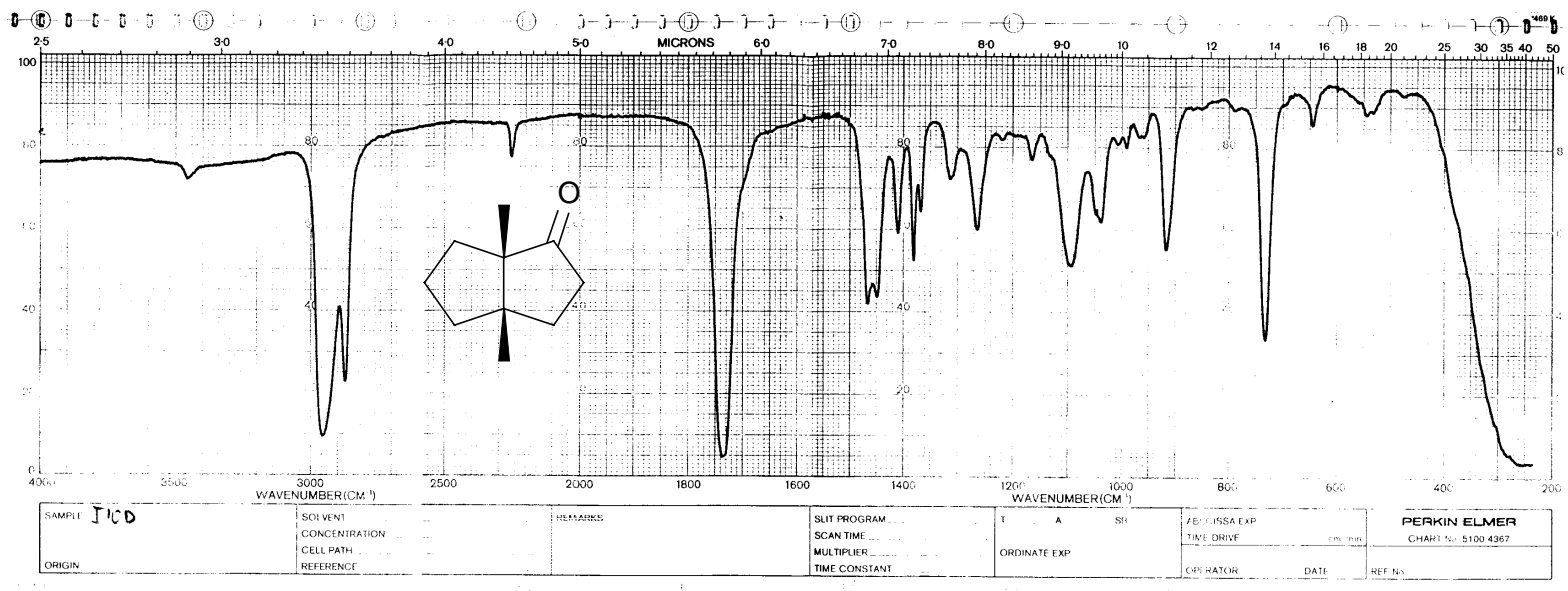

Abb. 4: IR-Spektrum von 3a,6a-Dimethylhexahydro-pentalen-1-on (30) 


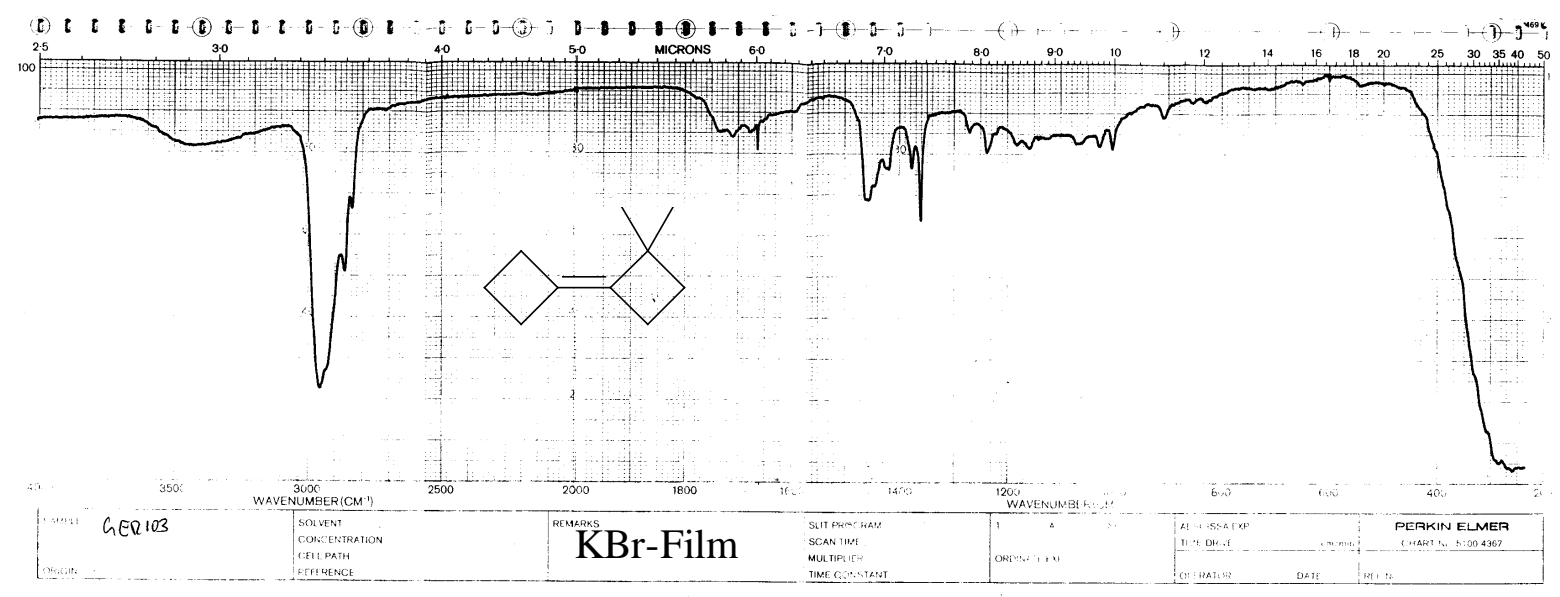

Abb. 5: IR-Spektrum von 2,2-Dimethylbicyclobutyliden (26)

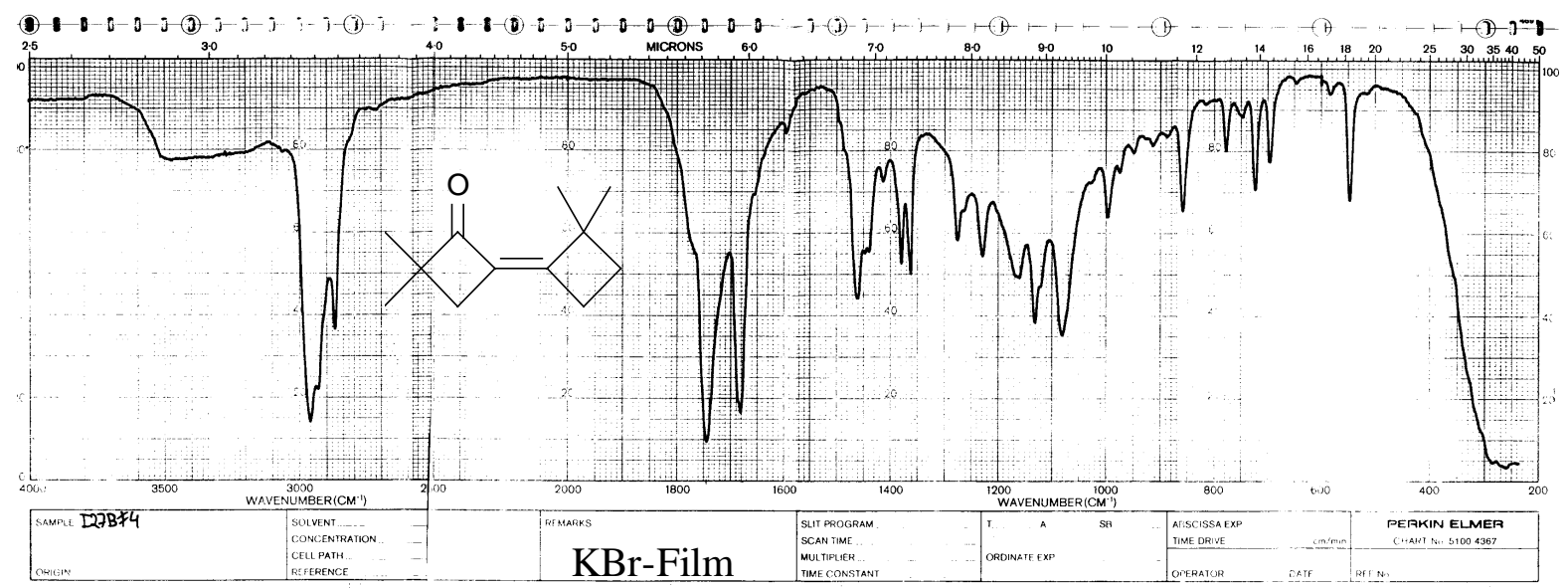

Abb. 6: IR-Spektrum von 3,3,2',2'-Tetramethyl-bicyclobutyliden-2-on (62) 


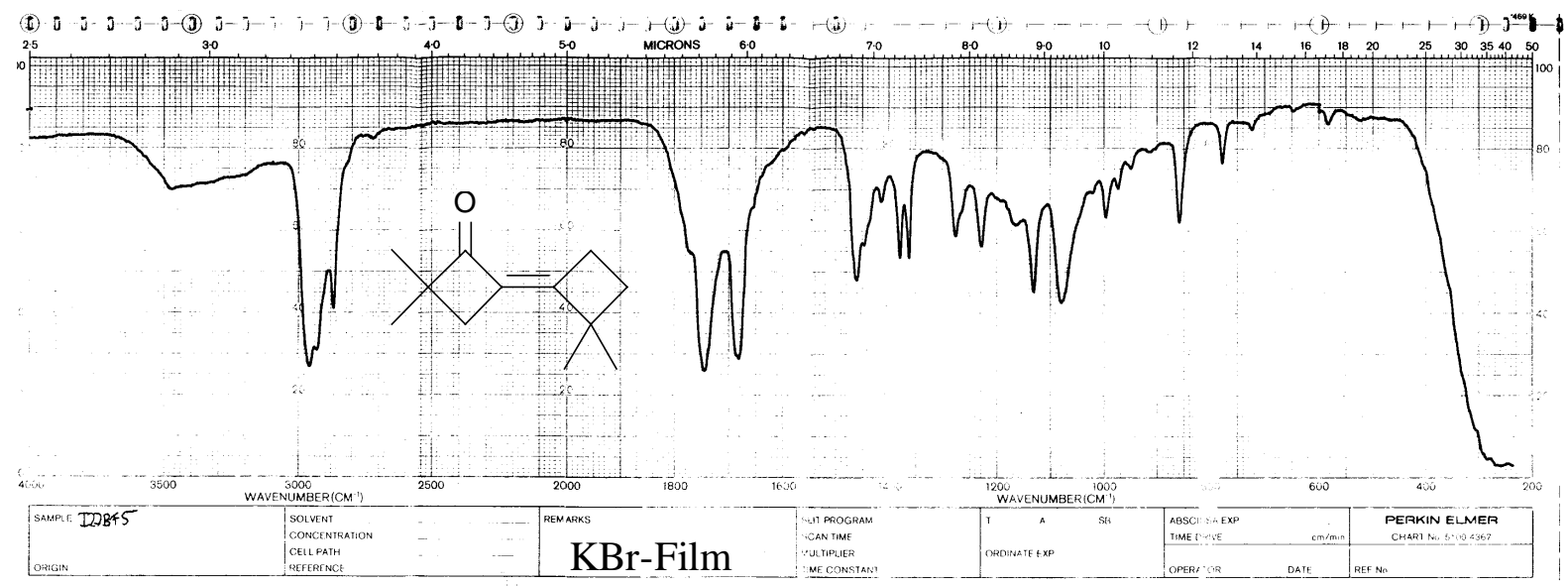

Abb. 7: IR-Spektrum von 3,3,2',2'-Tetramethyl-bicyclobutyliden-2-on (63)

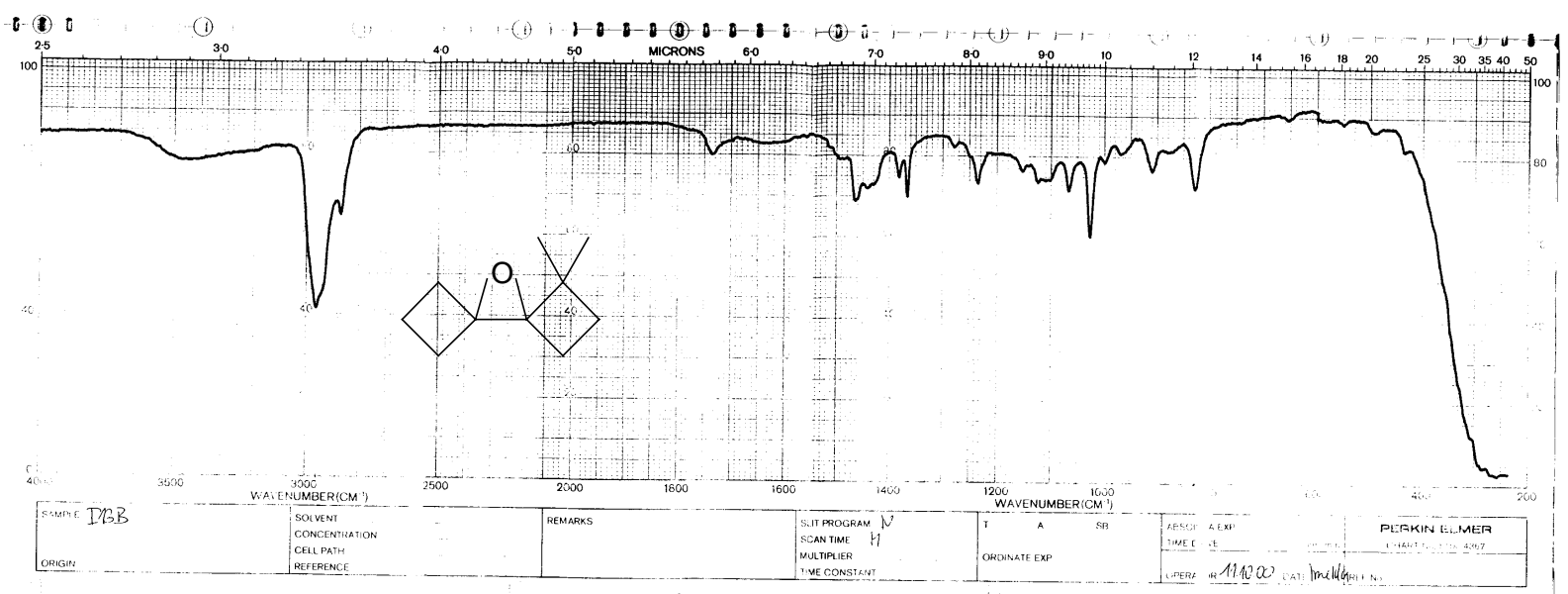

Abb. 8: IR-Spektrum von 1,1-Dimethyl-9-oxa-dispiro[3.0.3.1]nonan (64) 


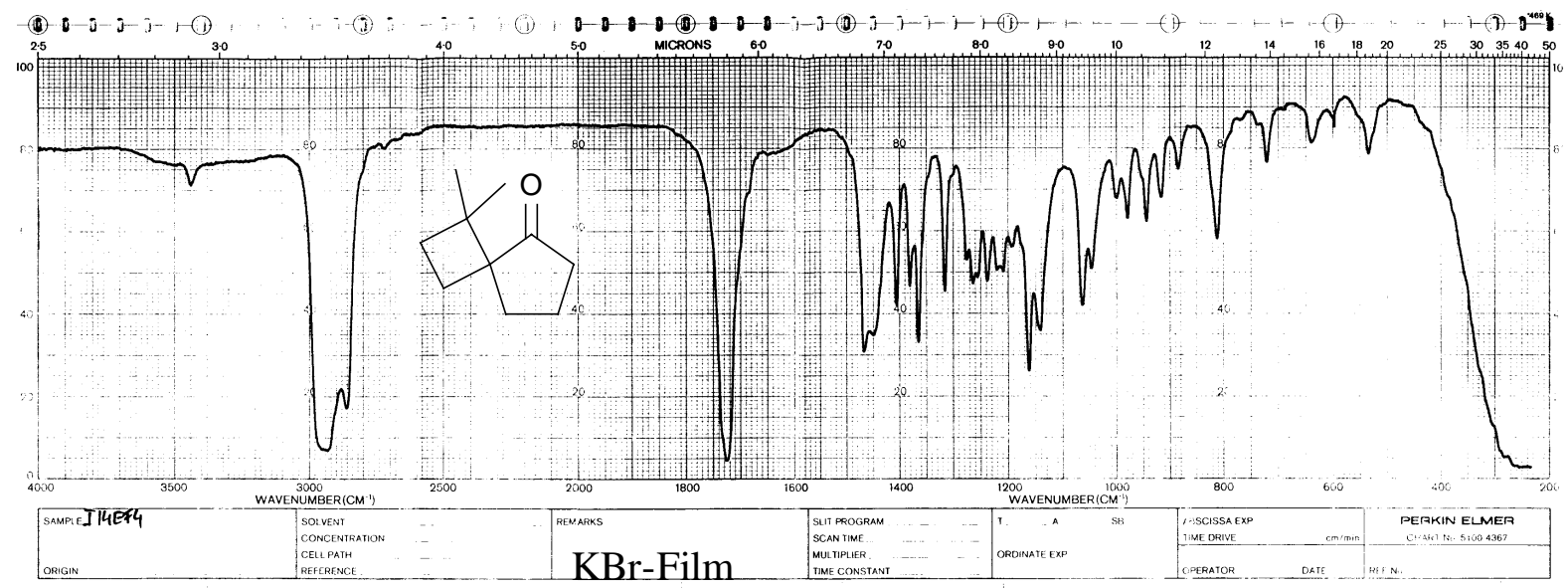

Abb. 9: IR-Spektrum von 1,1-Dimethyl-spiro[3.4]octan-5-on (27)

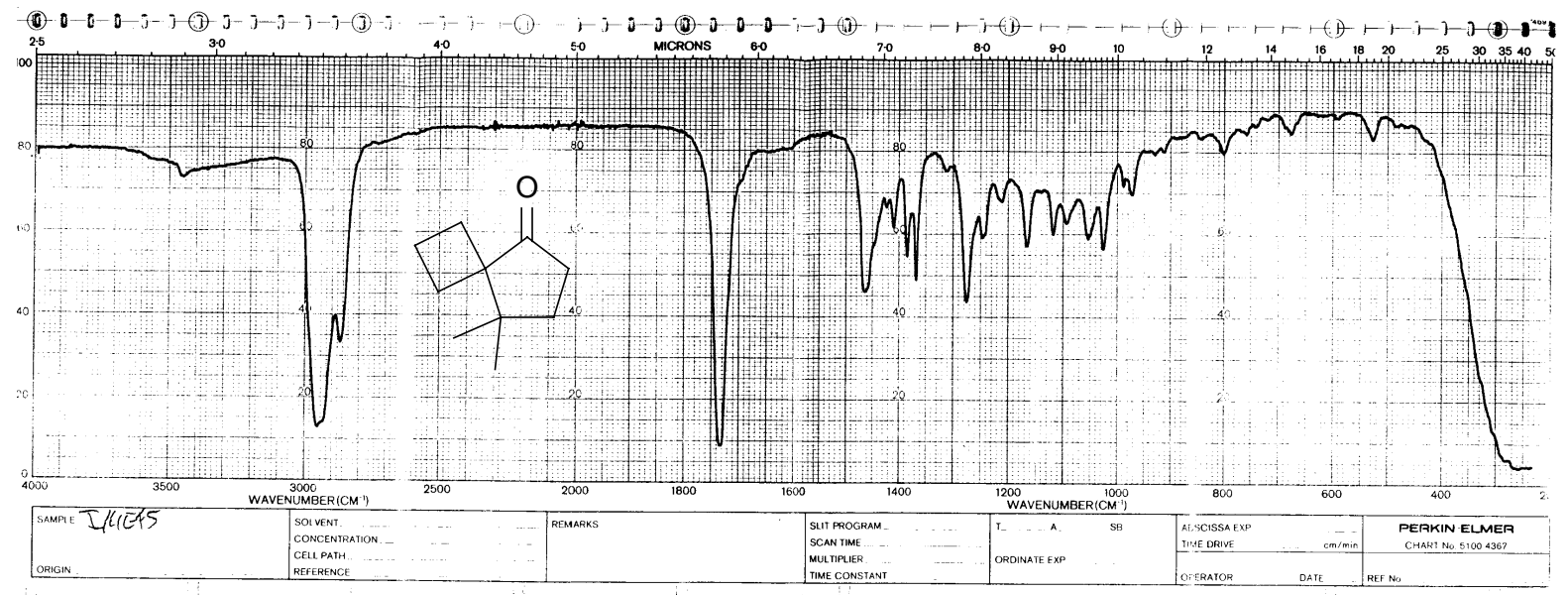

Abb. 10: IR-Spektrum von 8,8-Dimethyl-spiro[3.4] octan-5-on (67) 


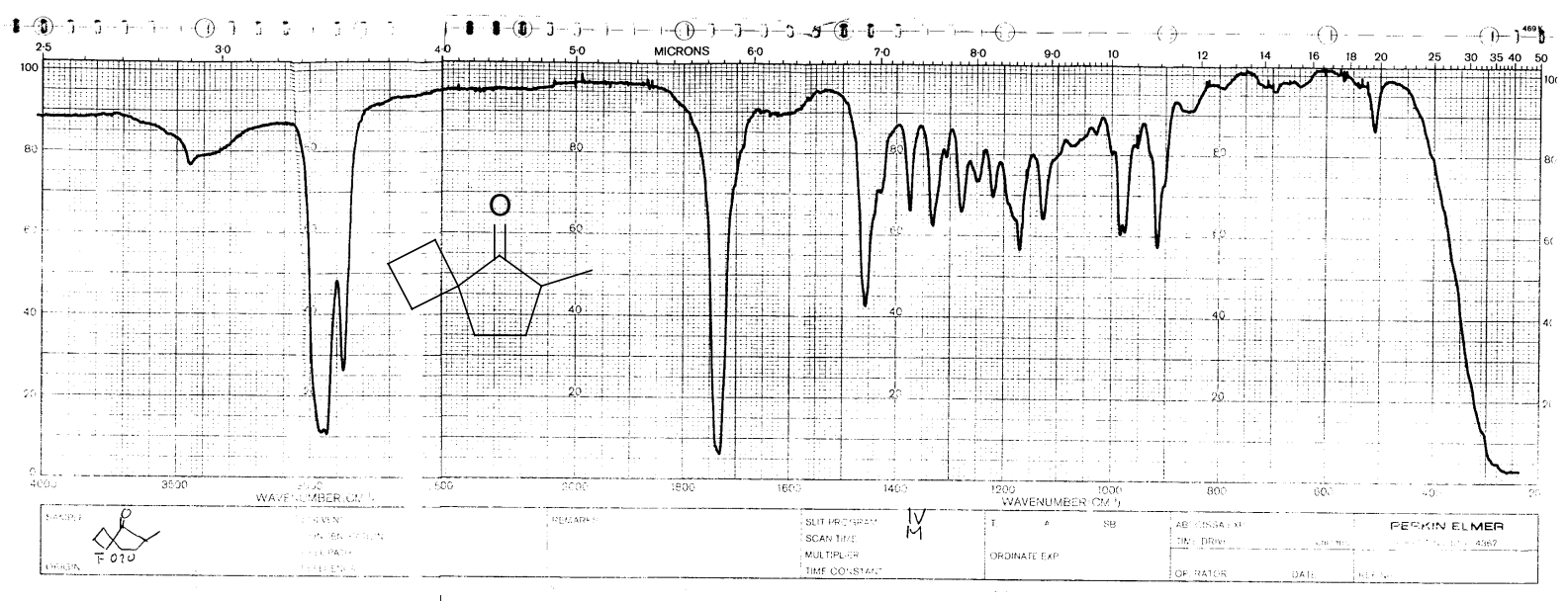

Abb. 11: IR-Spektrum von 6-Methyl-spiro[3.4]octan-5-on (50)

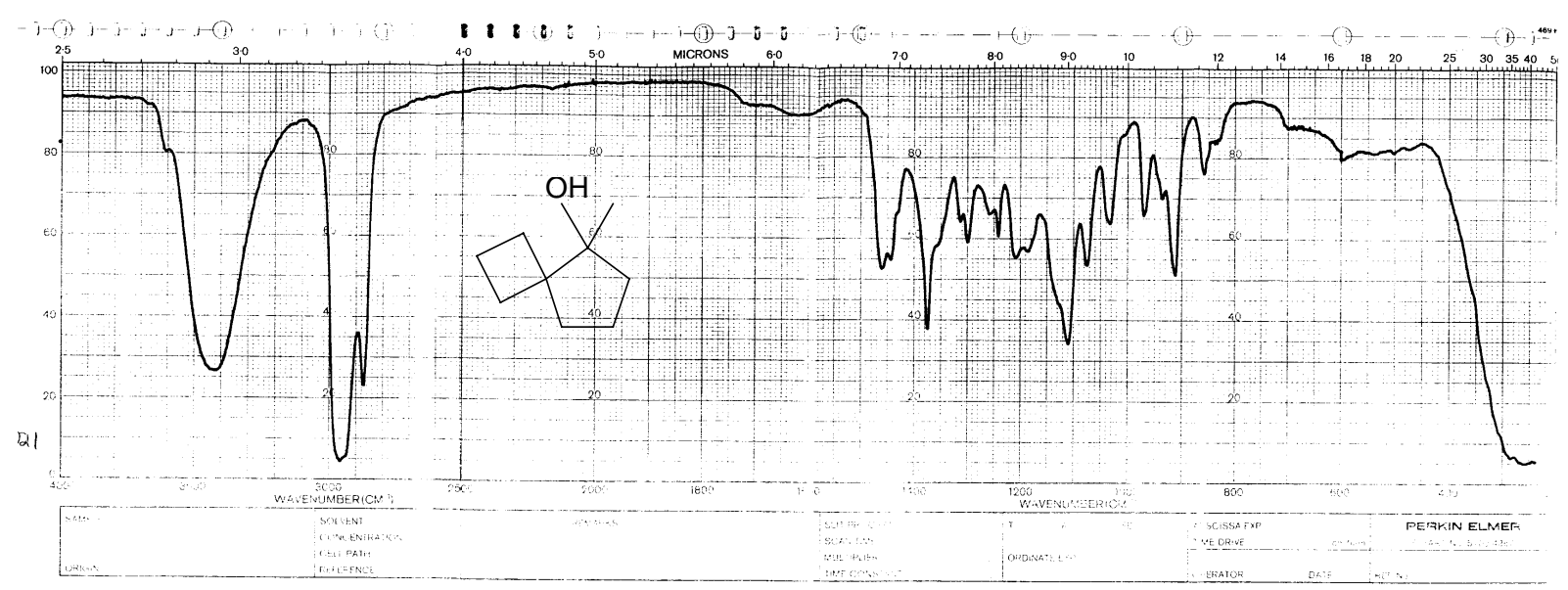

Abb. 12: IR-Spektrum von 5-Methyl-spiro[3.4]octan-5-ol (21) 


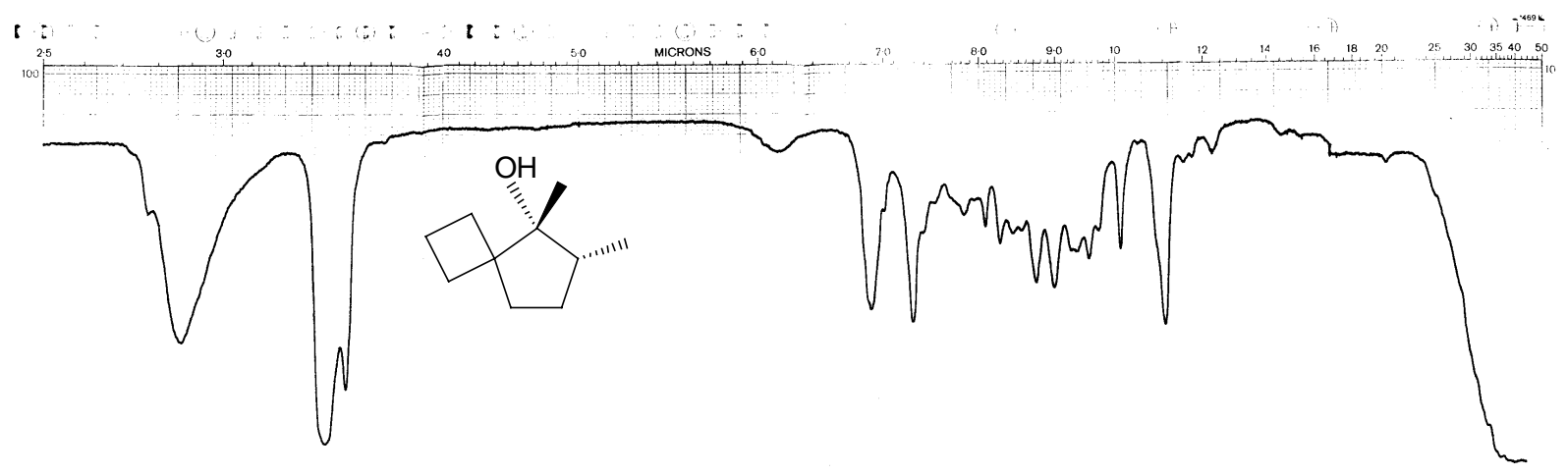

7231

KBr-Film

Abb. 13: IR-Spektrum von (5R*,6R*)-5,6-Dimethyl-spiro[3.4]octan-5-ol (23a)

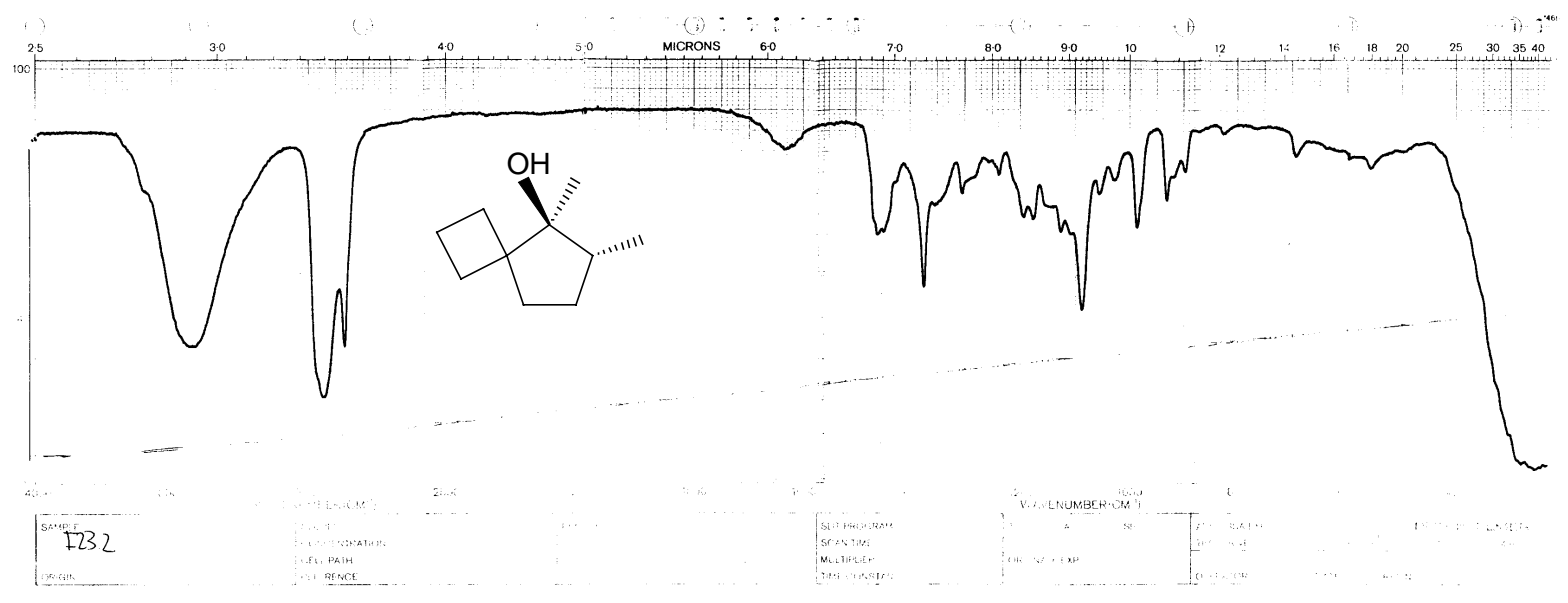

Abb. 14: IR-Spektrum von (5S*,6R*)-5,6-Dimethyl-spiro[3.4]octan-5-ol (23b) 


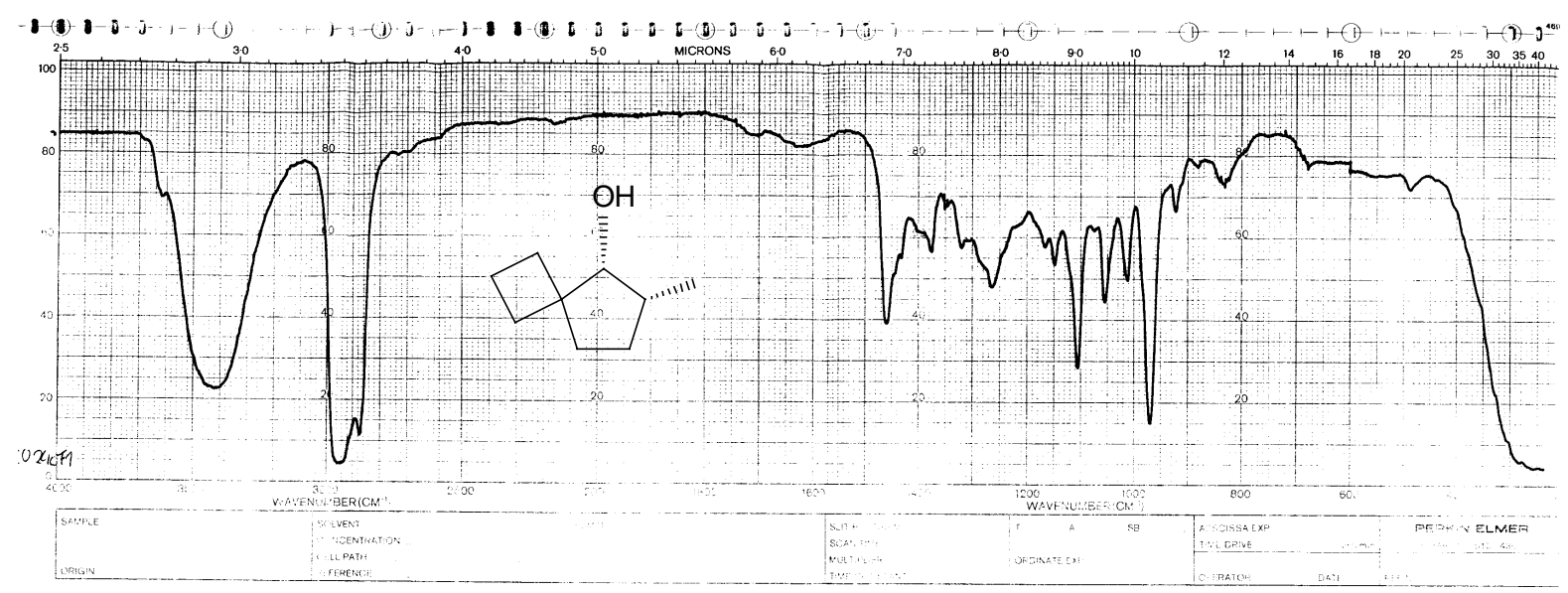

Abb. 15: IR-Spektrum von (5R*,6R*)-6-Methyl-spiro[3.4] octan-5-ol (22a)

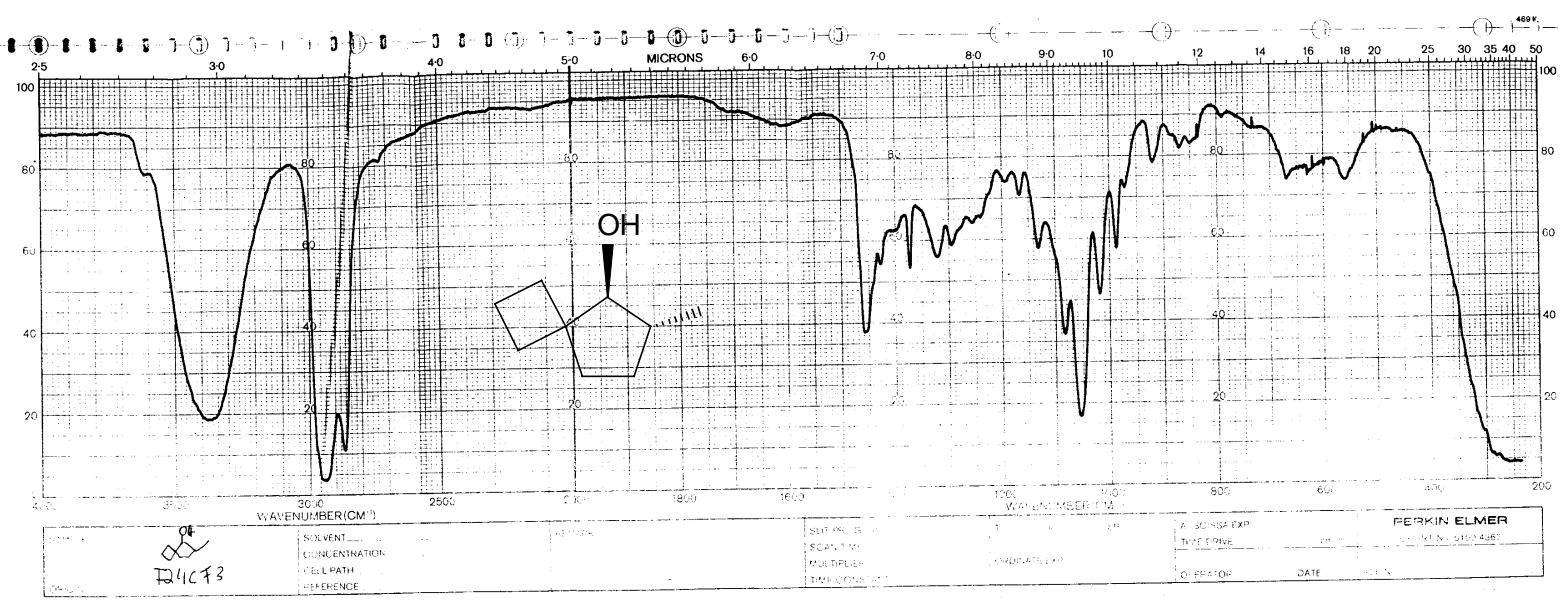

Abb. 16: IR-Spektrum von (5S*,6R*)-6-Methyl-spiro[3.4]octan-5-ol (22b) 


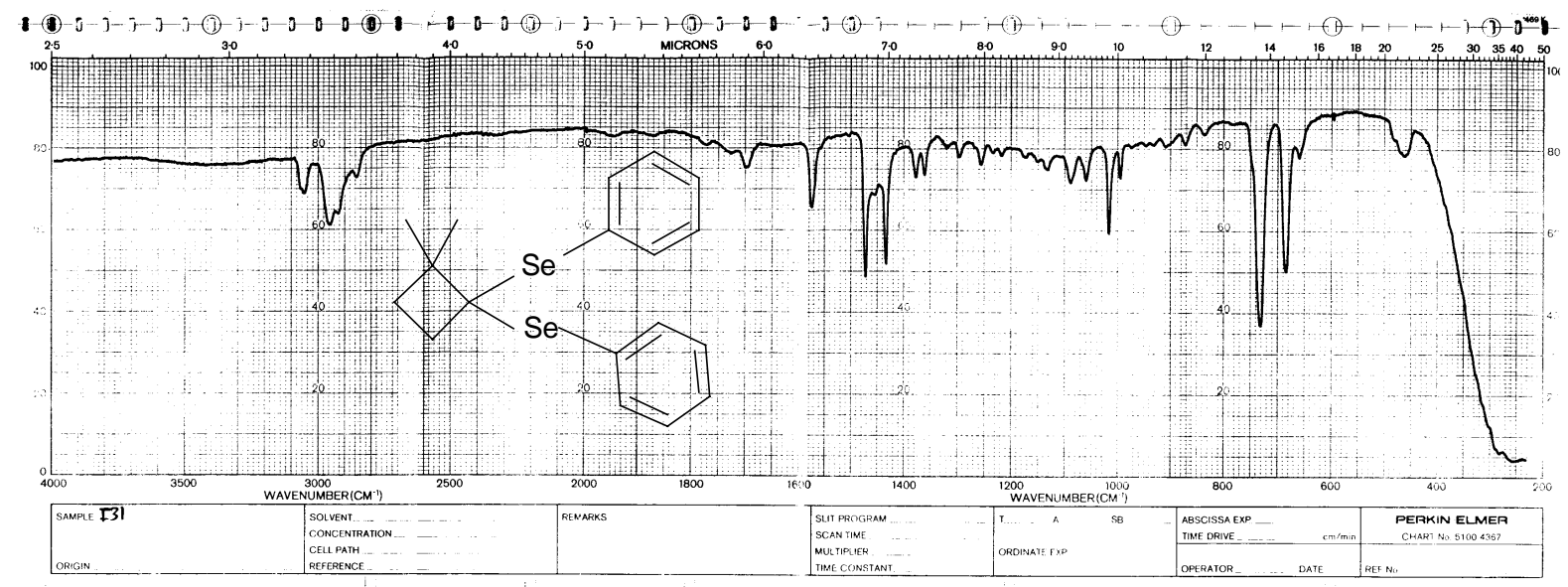

Abb. 17: IR-Spektrum von 1,1-Bis(phenylseleno)-2,2-Dimethylcyclobutan (48)

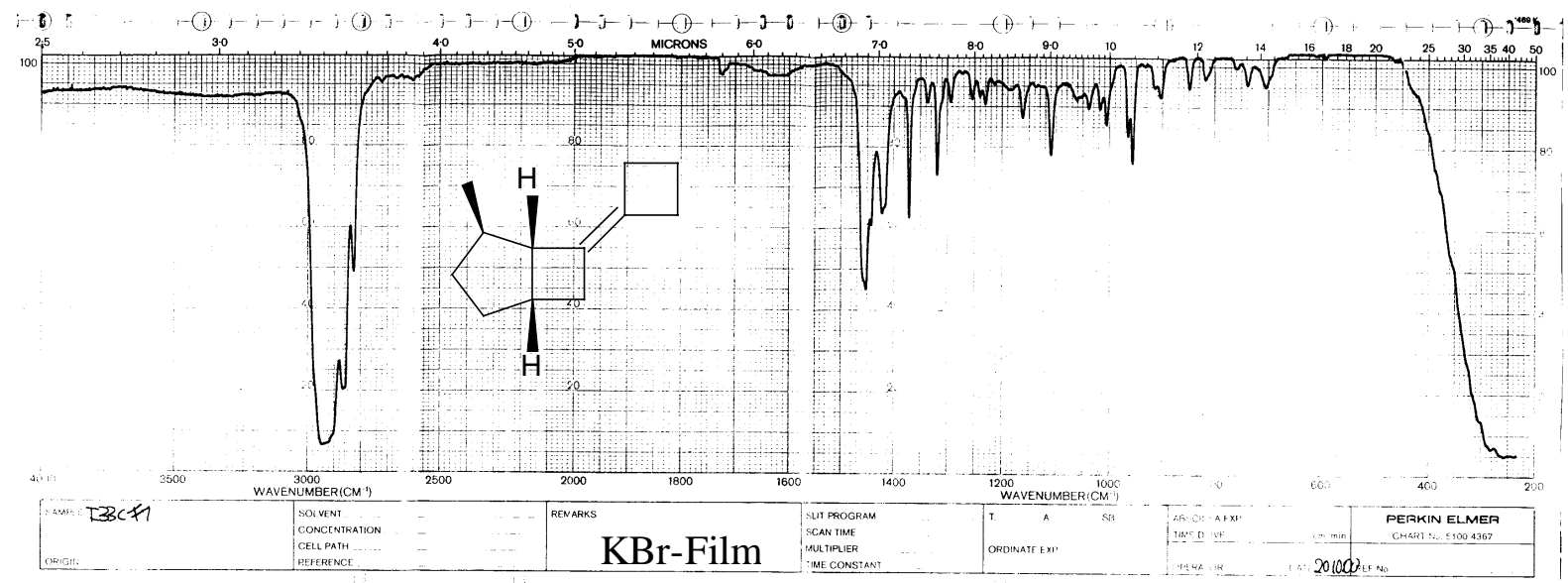

Abb. 18: IR-Spektrum von (1R*,2S*,5R*)-7-Cyclobutyliden-2-methyl-bicyclo[3.2.0]heptan (40) 


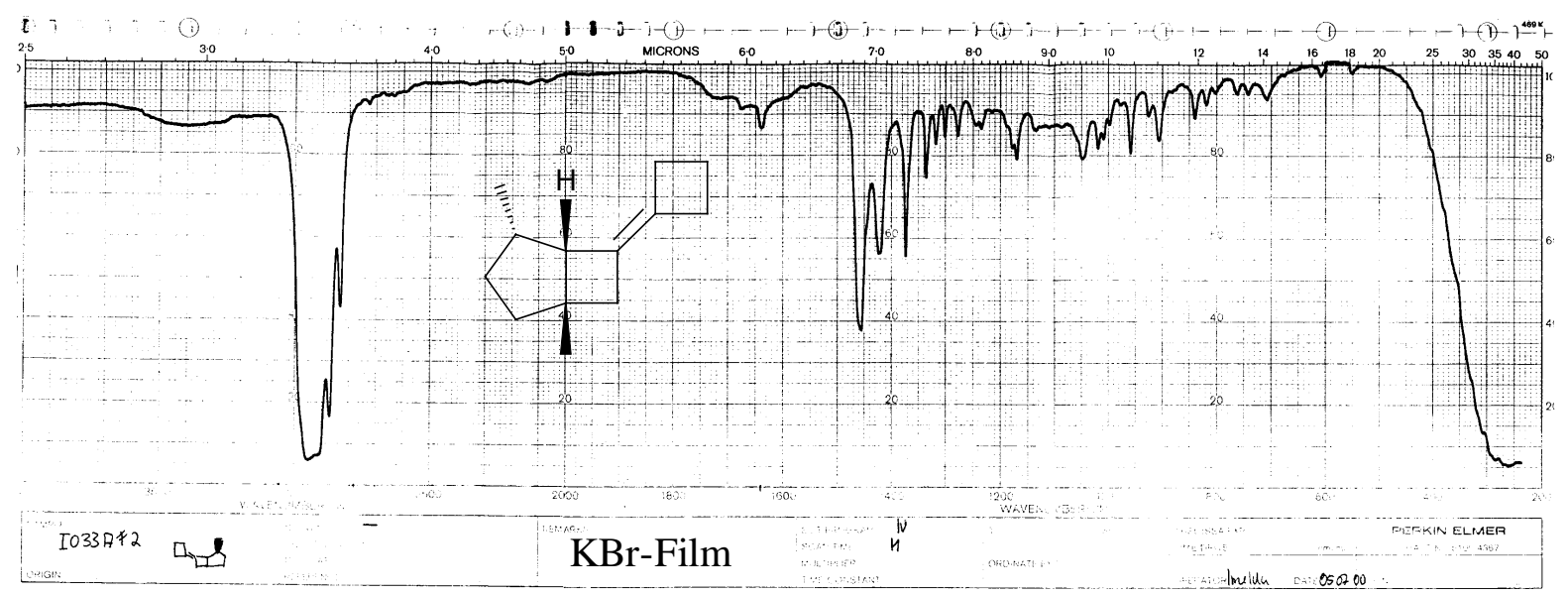

Abb. 19: IR-Spektrum von (1R*,2R*5R*)-7-Cyclobutyliden-2-methyl-bicyclo[3.2.0]heptan (91)

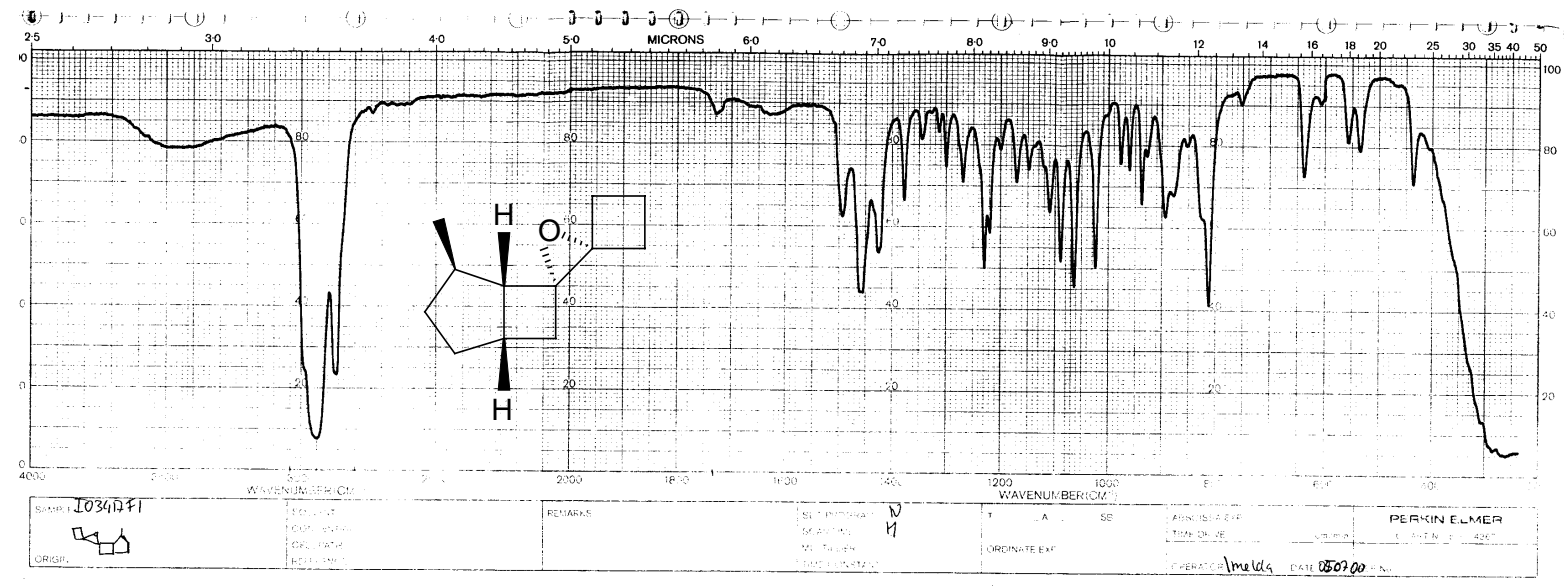

Abb. 20: IR-Spektrum von 2,2-Spiro-cyclobutyl-3,3-spiro-[(1R*,4S*,5R*,6S*)-2methyl-bicyclo[3.2.0]heptan-6,6-yl]-oxiran (92) 


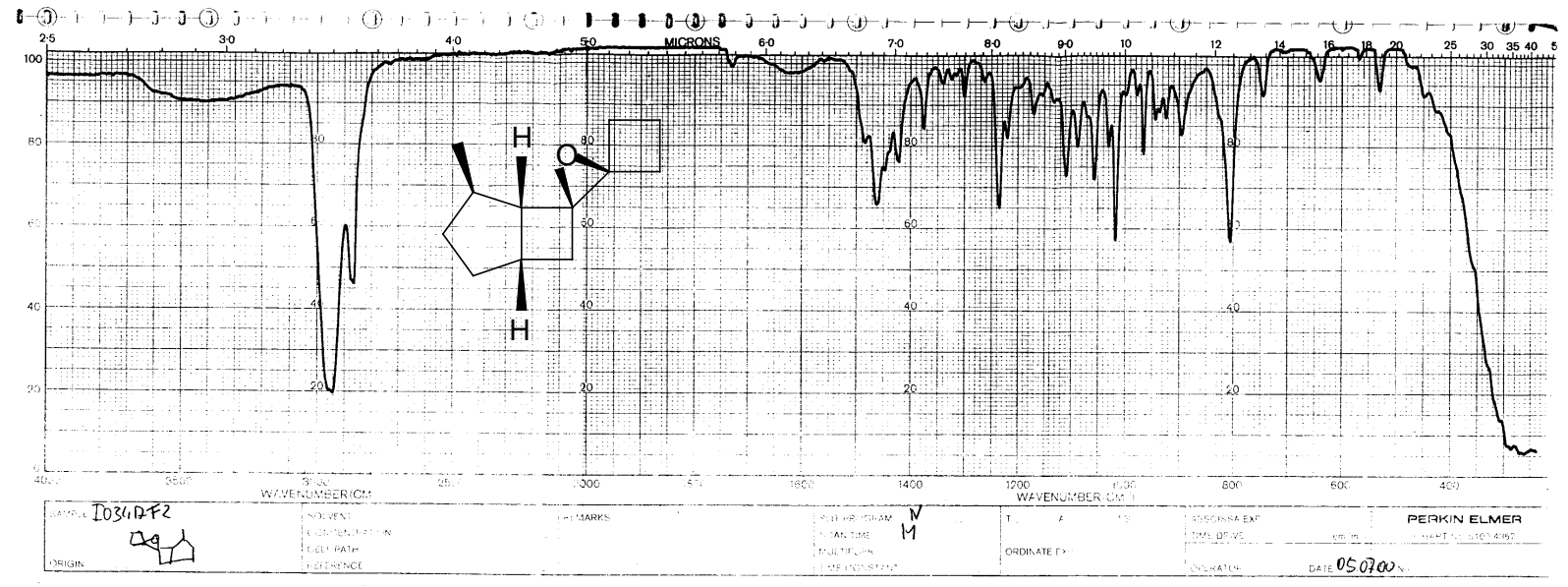

Abb. 21: IR-Spektrum von 2,2-Spiro-cyclobutyl-3,3-spiro-[(1R*,4S*,5R*,6R*)-2methyl-bicyclo[3.2.0]heptan-6,6-yl]-oxiran (89)

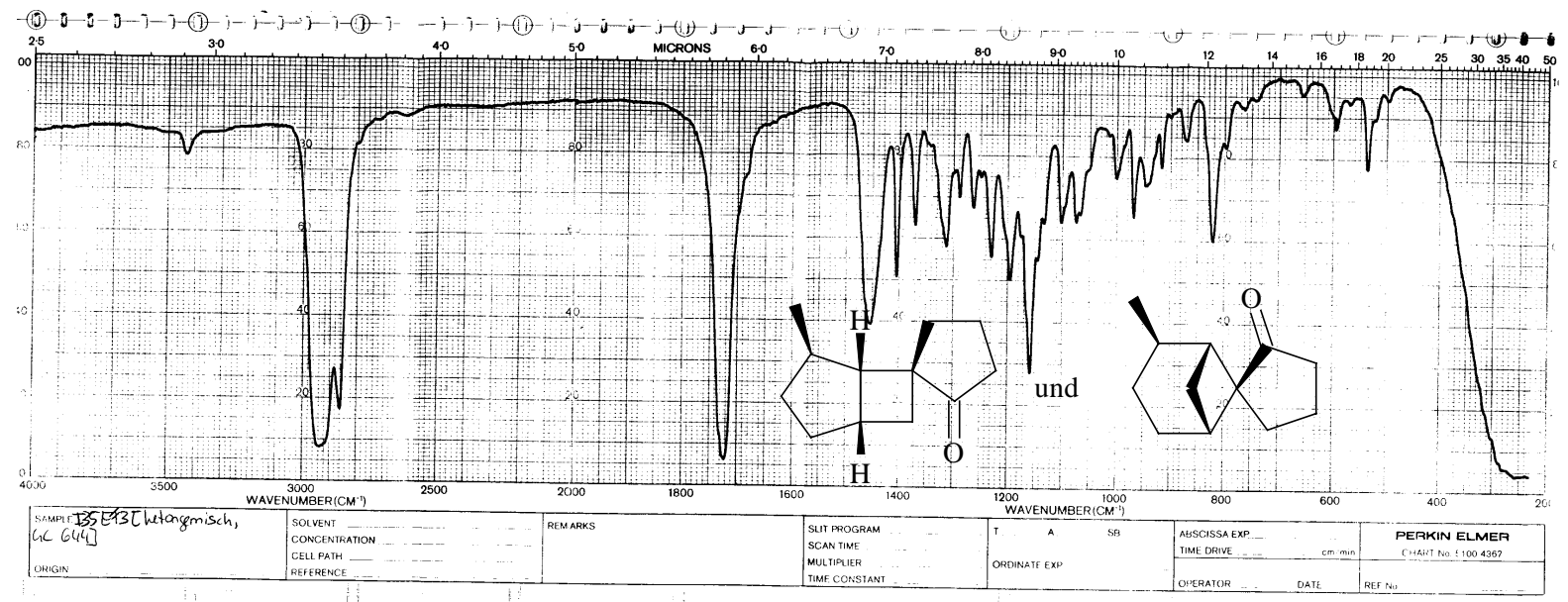

Abb. 22: IR-Spektrum von (1R*,4S*,5R*,6S*)-4-Methyl-spiro \{ bicyclo[3.2.0]heptan-6,1'cyclopentan-2'-on $\}(87)$ und $\left(1 \mathrm{R}^{*}, 2 \mathrm{R}^{*}, 5 \mathrm{R}^{*}, 6 \mathrm{~S}^{*}\right)$-2-Methyl-spiro $\{$ bicyclo[3.1.1]heptan-6, $1^{\prime}$-cyclopentan-2'-on $\}$ (93) 


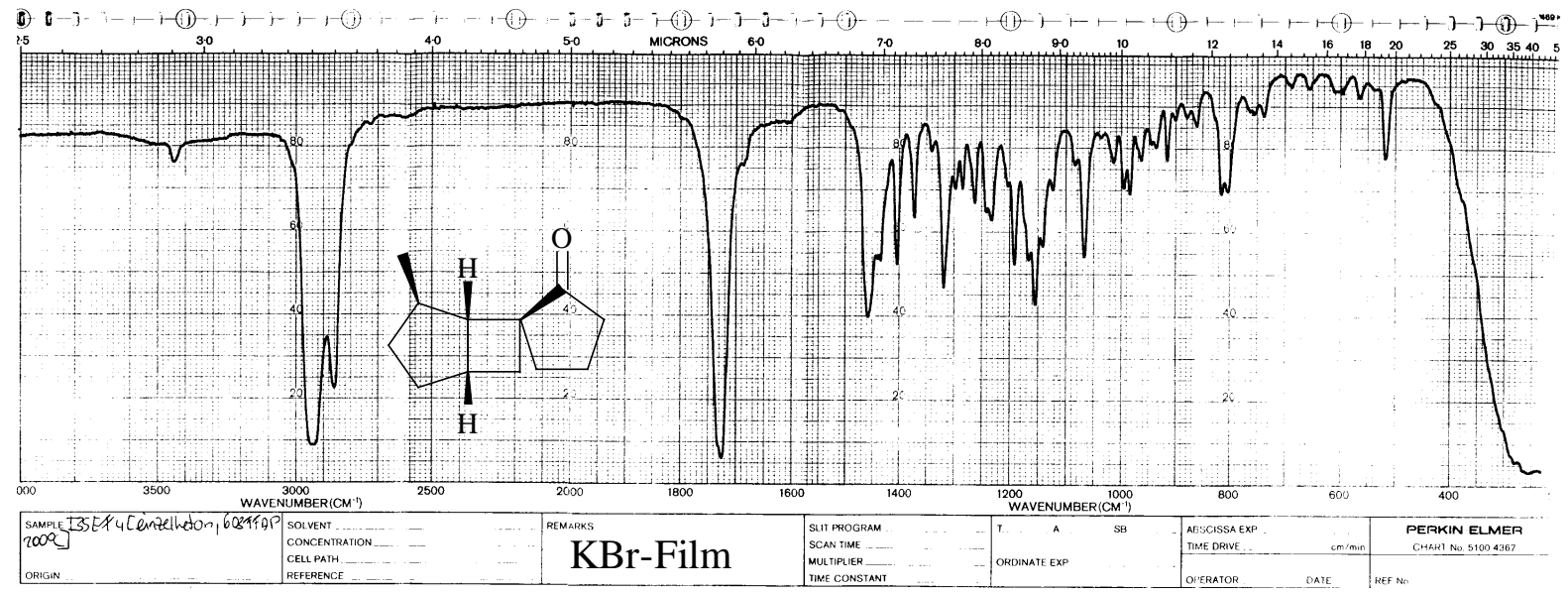

Abb. 23: IR-Spektrum von (1R*,4S*,5R*,6R*)-4-Methyl-spiro\{ bicyclo[3.2.0]heptan-6,1'cyclopentan-2'-on\} (88)

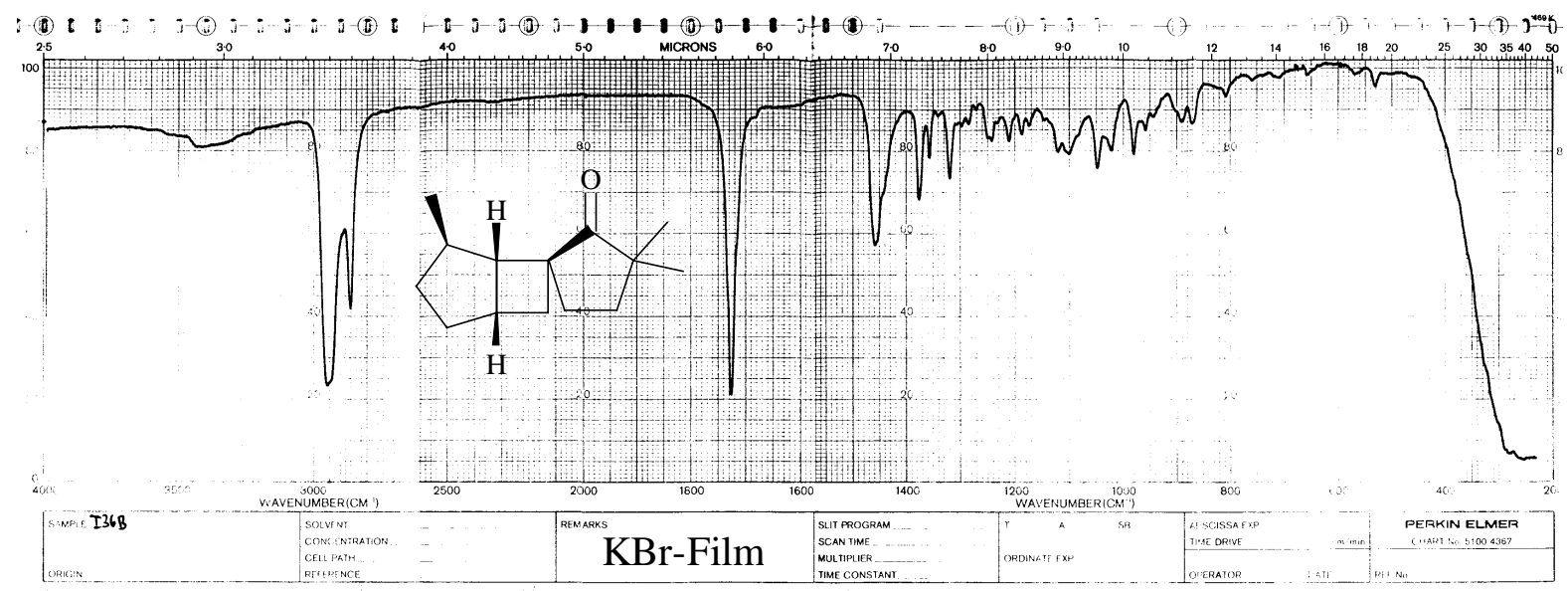

Abb. 24: IR-Spektrum von (1R*,4S*,5R*,6R*)-4,3',3'-Trimethyl-spiro \{bicyclo[3.2.0]heptan-6, 1'-cyclopentan-2'-on\} (95) 


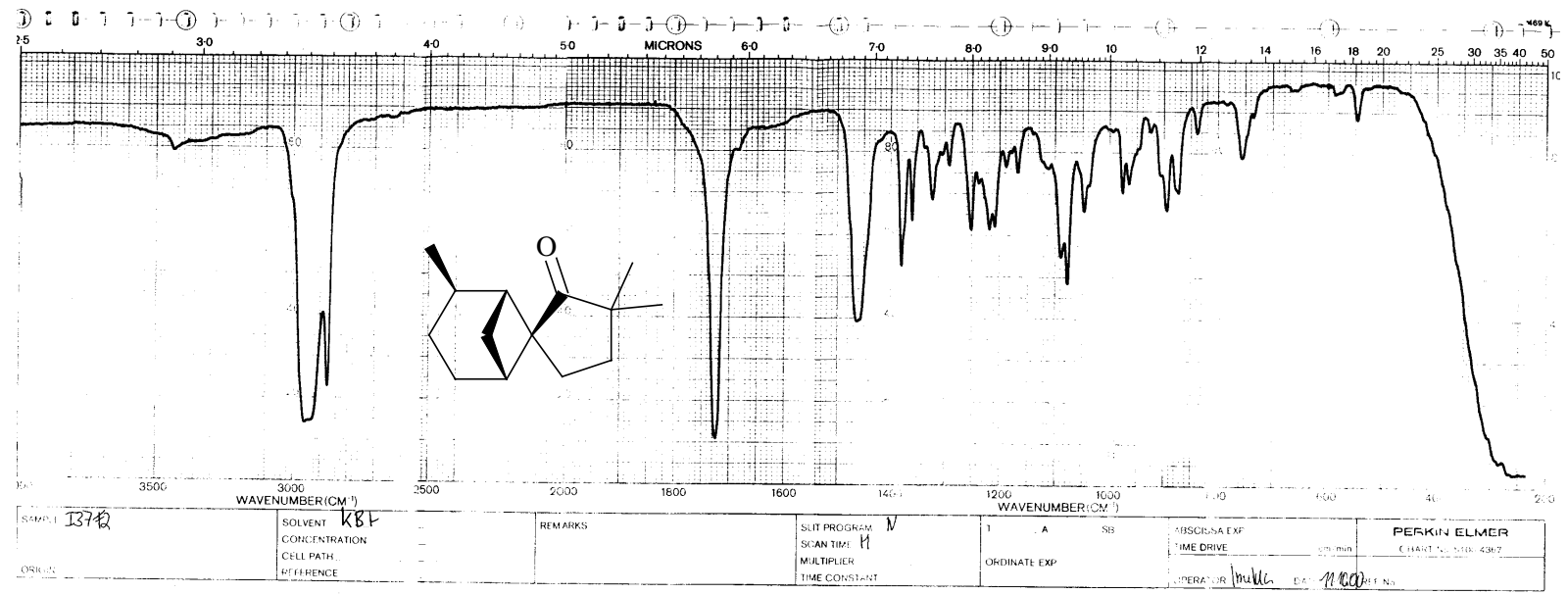

Abb. 25: IR-Spektrum von (1R*,2R*,5R*,6S*)-2,3',3'-Trimethyl-spiro \{bicyclo[3.1.1] heptan-6,1'-cyclopentan-2'-on $\}$ (96)

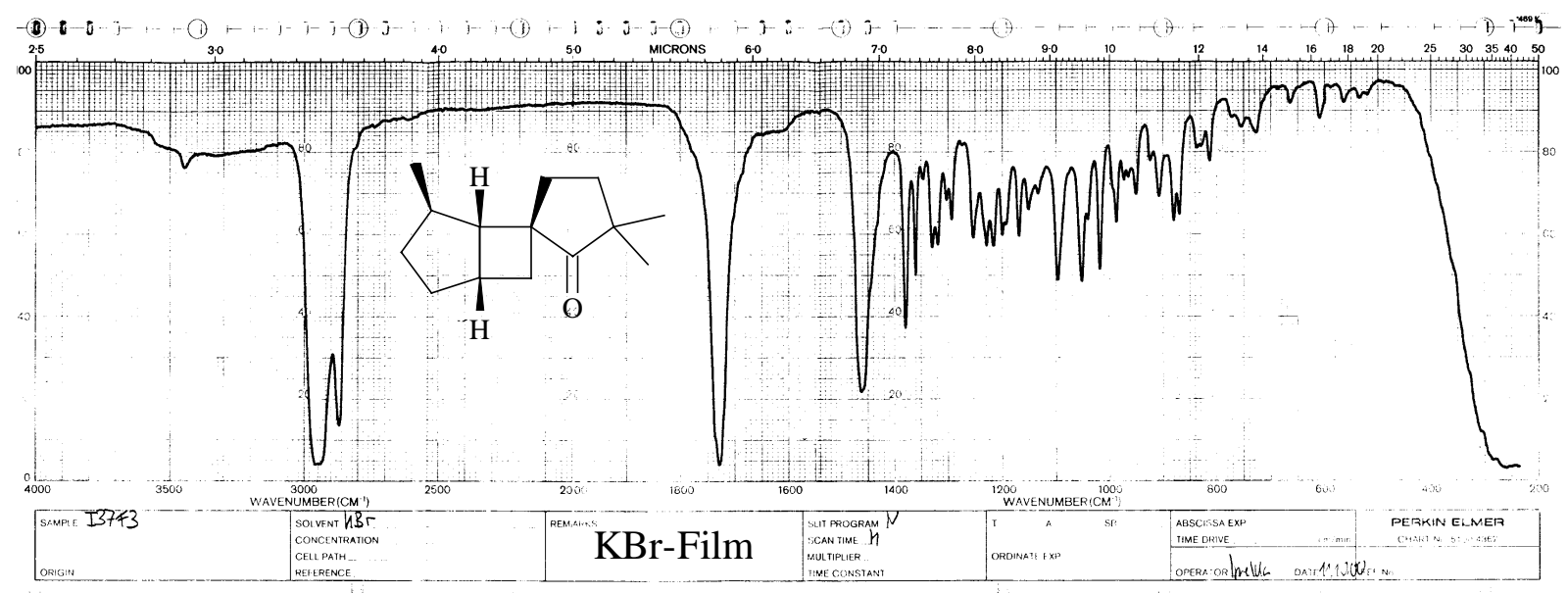

Abb. 26: (1R*,4S*,5R*,6S*)-4,3',3'-Trimethyl-spiro\{bicyclo[3.2.0]heptan-6,1'cyclopentan-2'-on\} (94) 


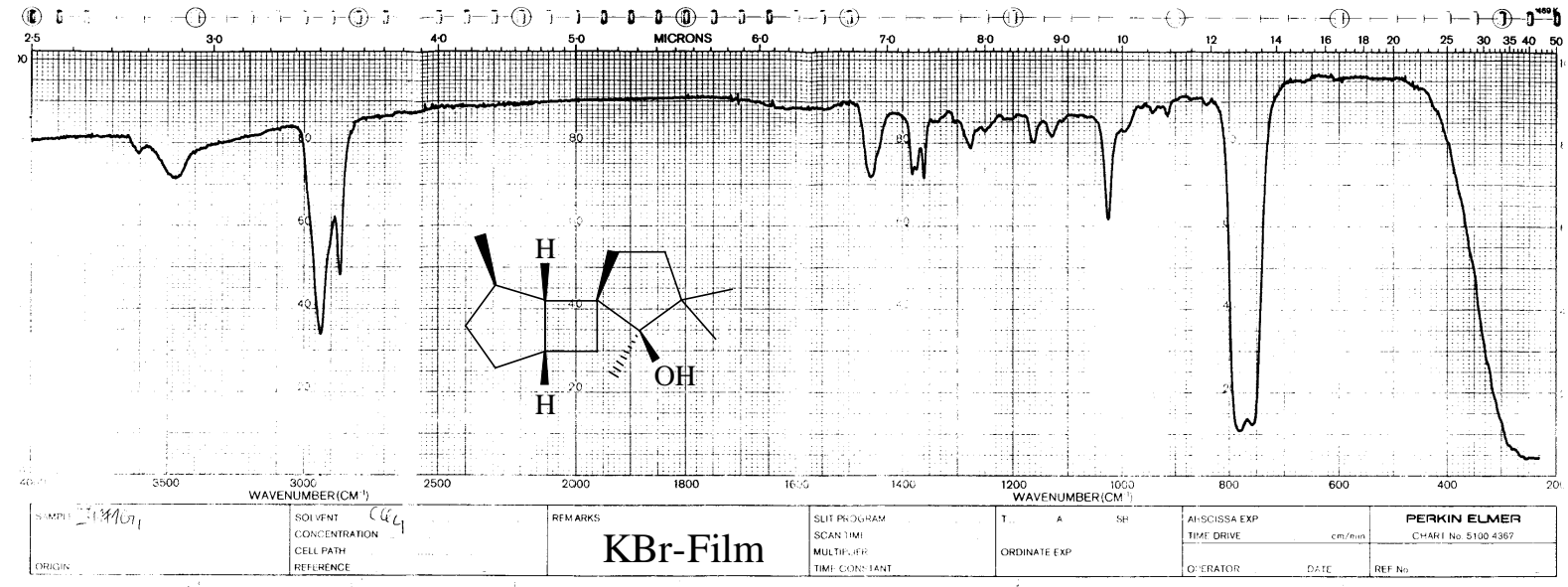

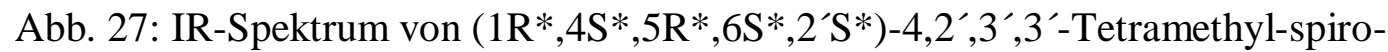
$\left\{\right.$ bicyclo[3.2.0]heptan-6, $1^{\prime}$-cyclopentan-2'-ol $\}$ (45)

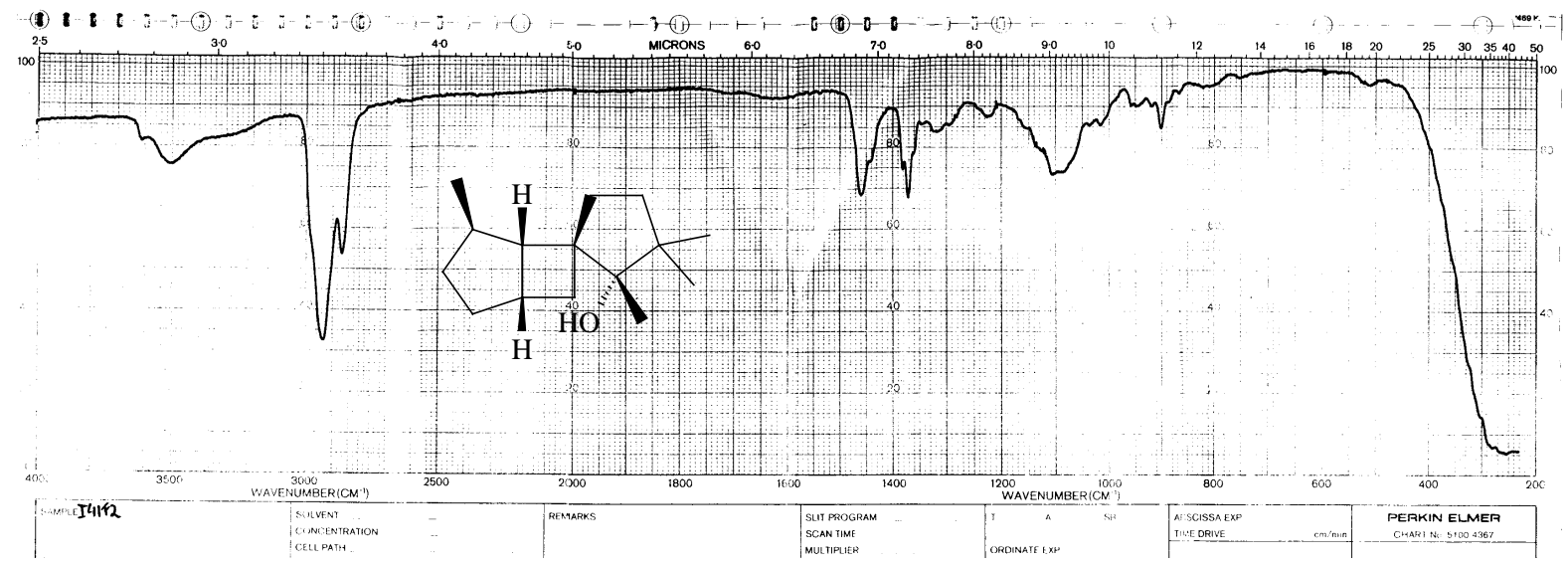

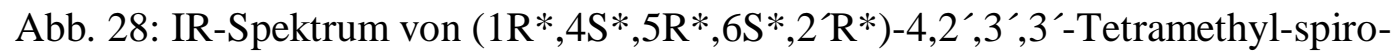

\{bicyclo[3.2.0]heptan-6,1'-cyclopentan-2'-ol\} (44) 


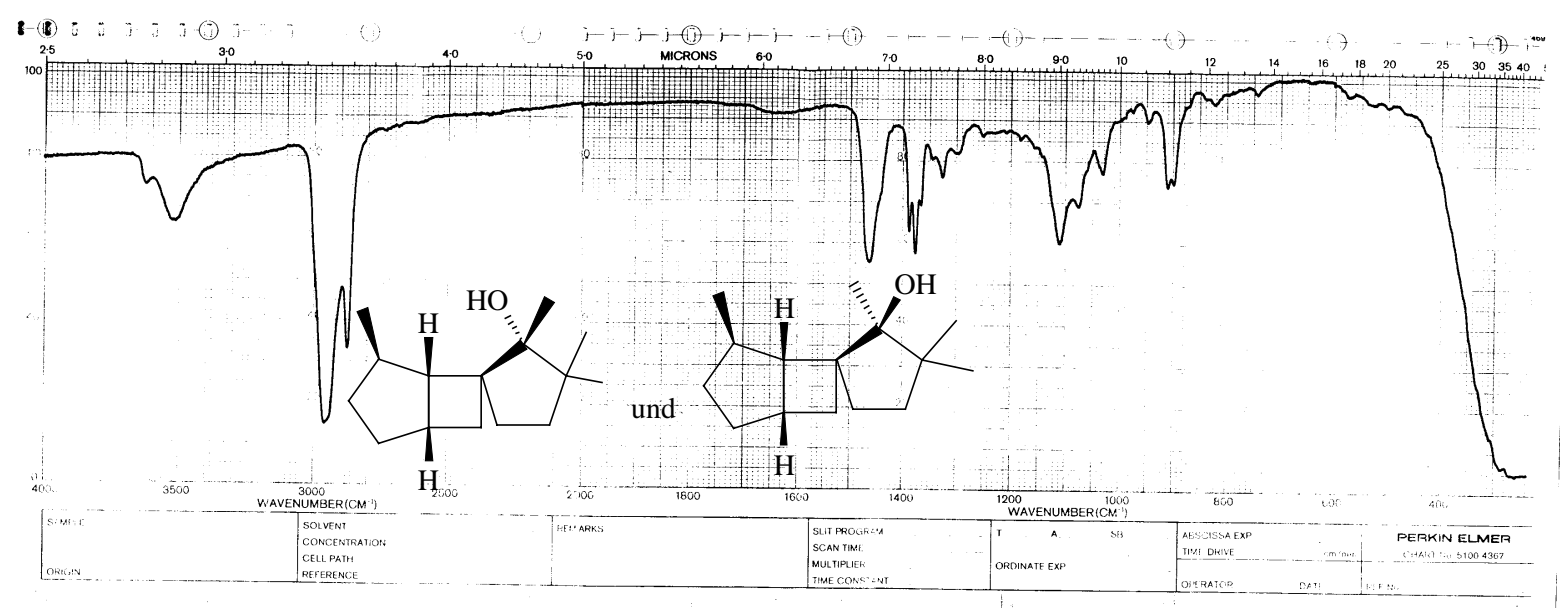

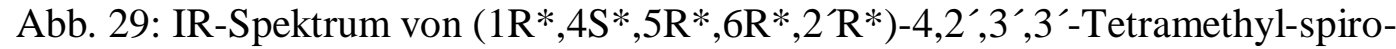

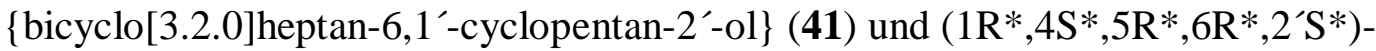

4,2',3',3'-Tetramethyl-spiro \{bicyclo[3.2.0]heptan-6,1'-cyclopentan-2'-on\} (42)

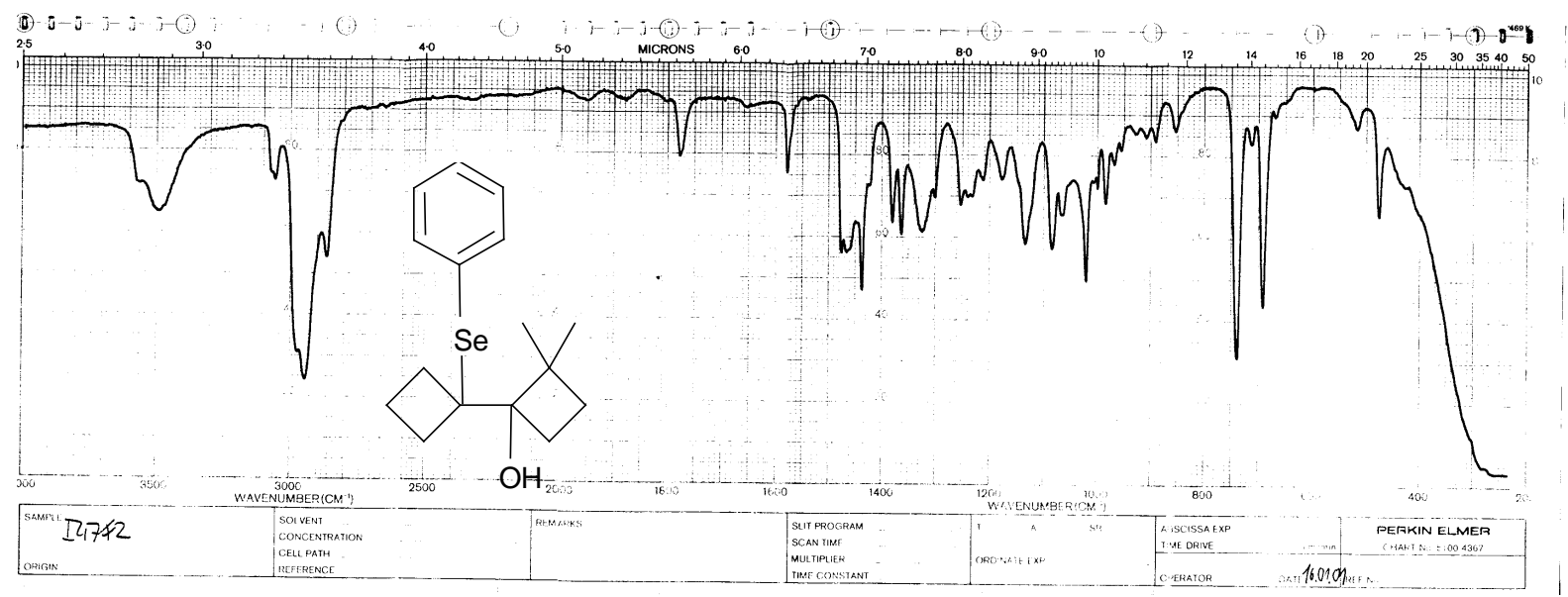

Abb. 30: IR-Spektrum von 1-[(1-Phenylseleno)cyclobutyl]-2,2-dimethylcyclobutan-1-ol’ (76) 


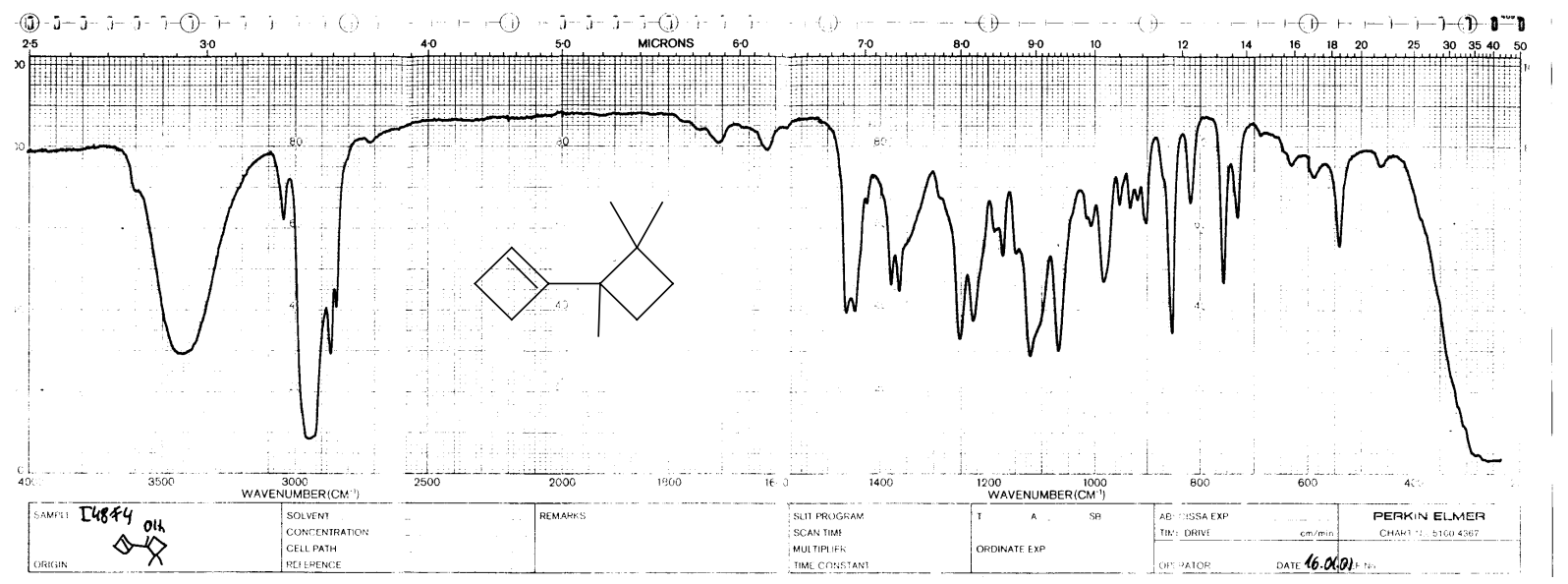

Abb. 31: IR-Spektrum von 2,2-Dimethyl-bicyclobutyl-1'-en-1-ol (78)

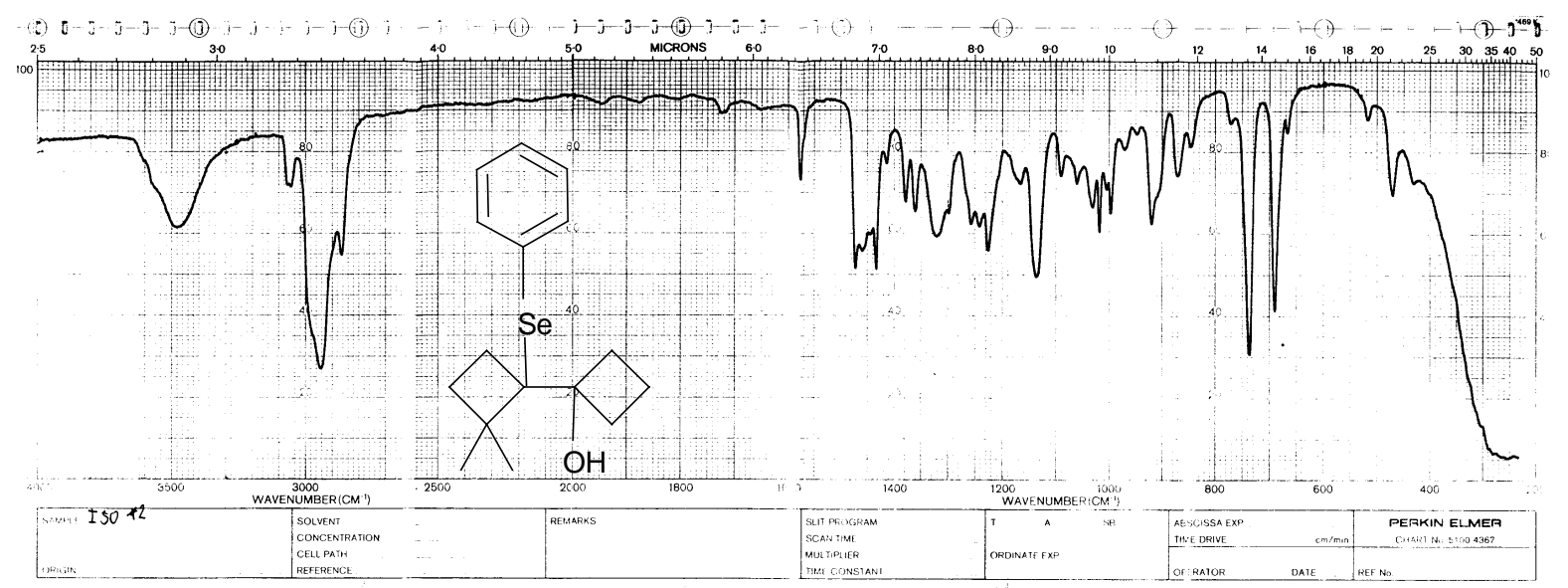

Abb. 32: IR-Spektrum von 1-[(1'-Phenylseleno)-2',2'-dimethylcyclobutyl]cyclobutan-1-ol (49) 


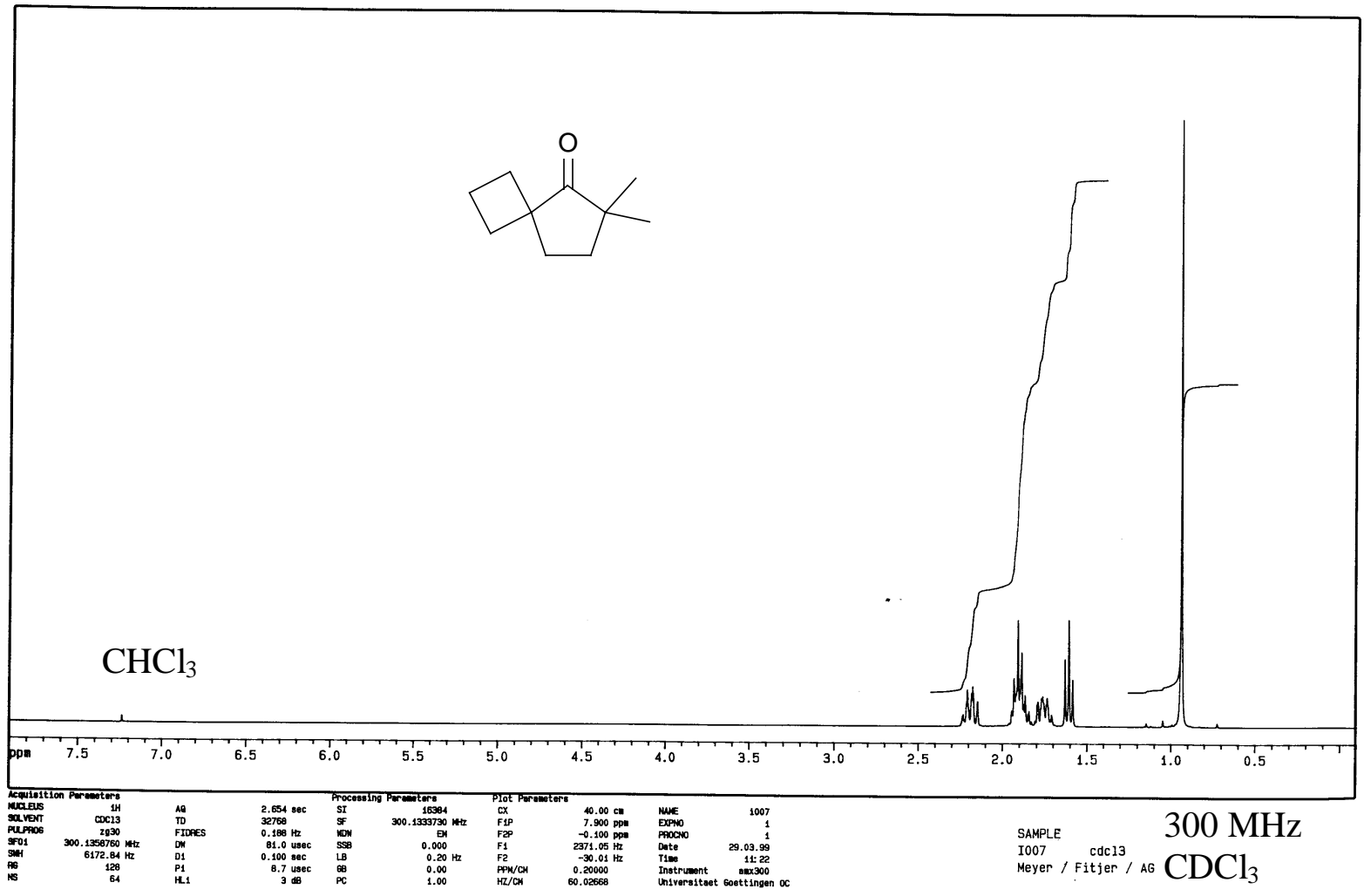

Abb. 33: ${ }^{1} \mathrm{H}-\mathrm{NMR}-$ Spektrum von 6,6-Dimethyl-spiro[3.4] octan-5-on (51)

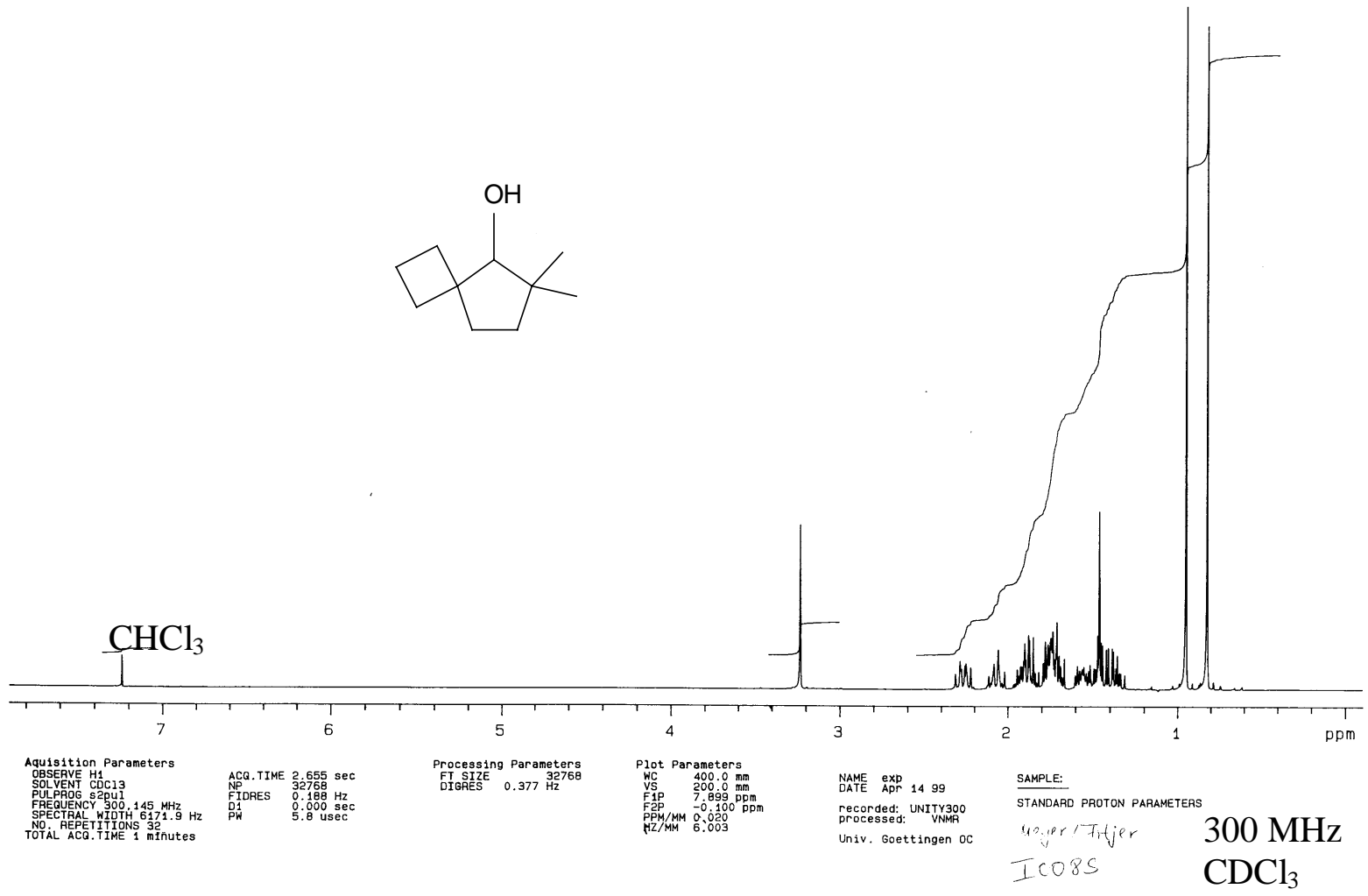

Abb. 34: ${ }^{1}$ H-NMR-Spektrum von 6,6-Dimethyl-spiro[3.4]octan-5-ol (24) 


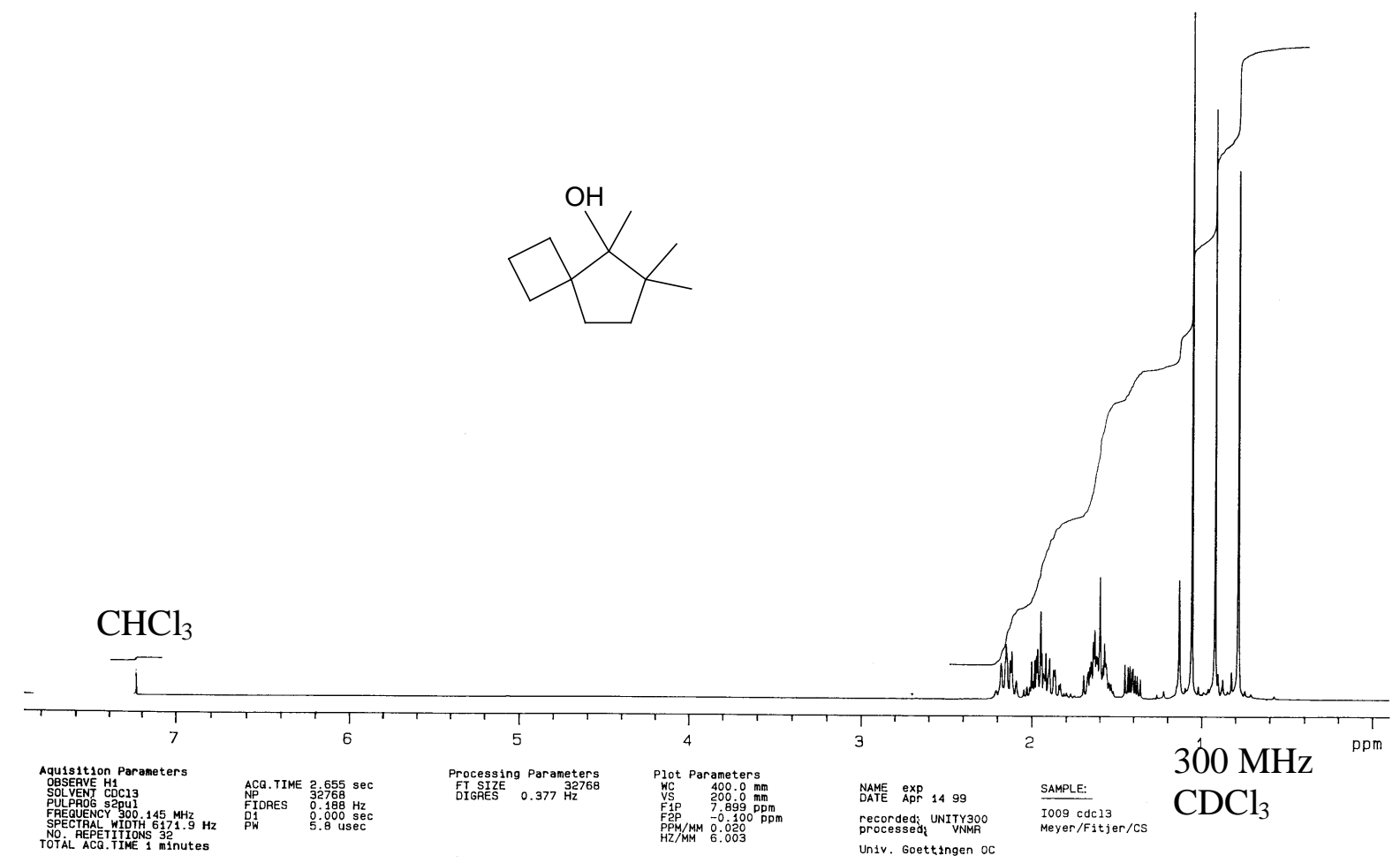

Abb. 35: ${ }^{1} \mathrm{H}-\mathrm{NMR}-$ Spektrum von 5,6,6-Trimethyl-spiro[3.4] octan-5-ol (25)

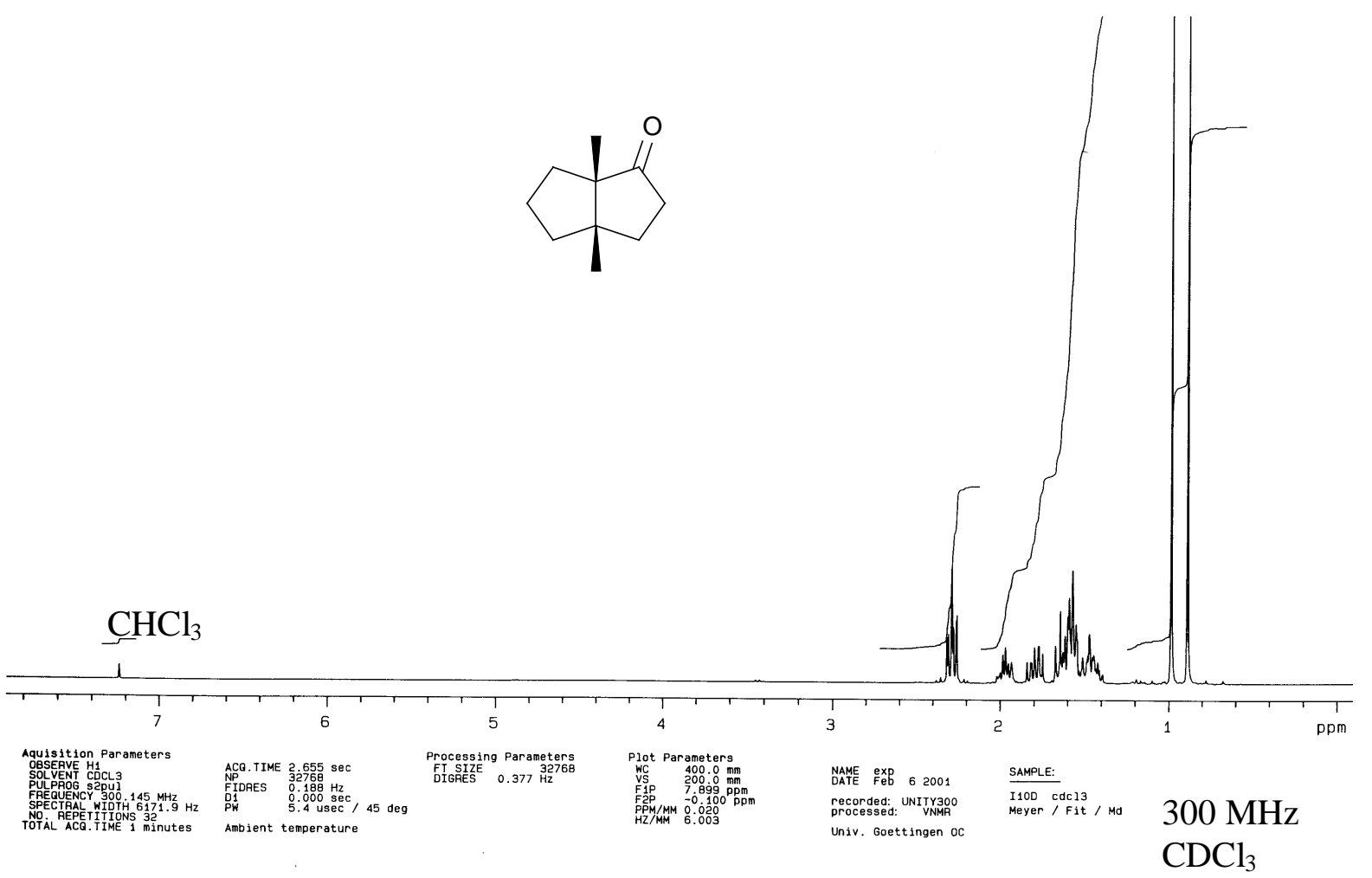

Abb. 36: ${ }^{1}$ H-NMR-Spektrum von 3a,6a-Dimethylhexahydro-pentalen-1-on (30) 


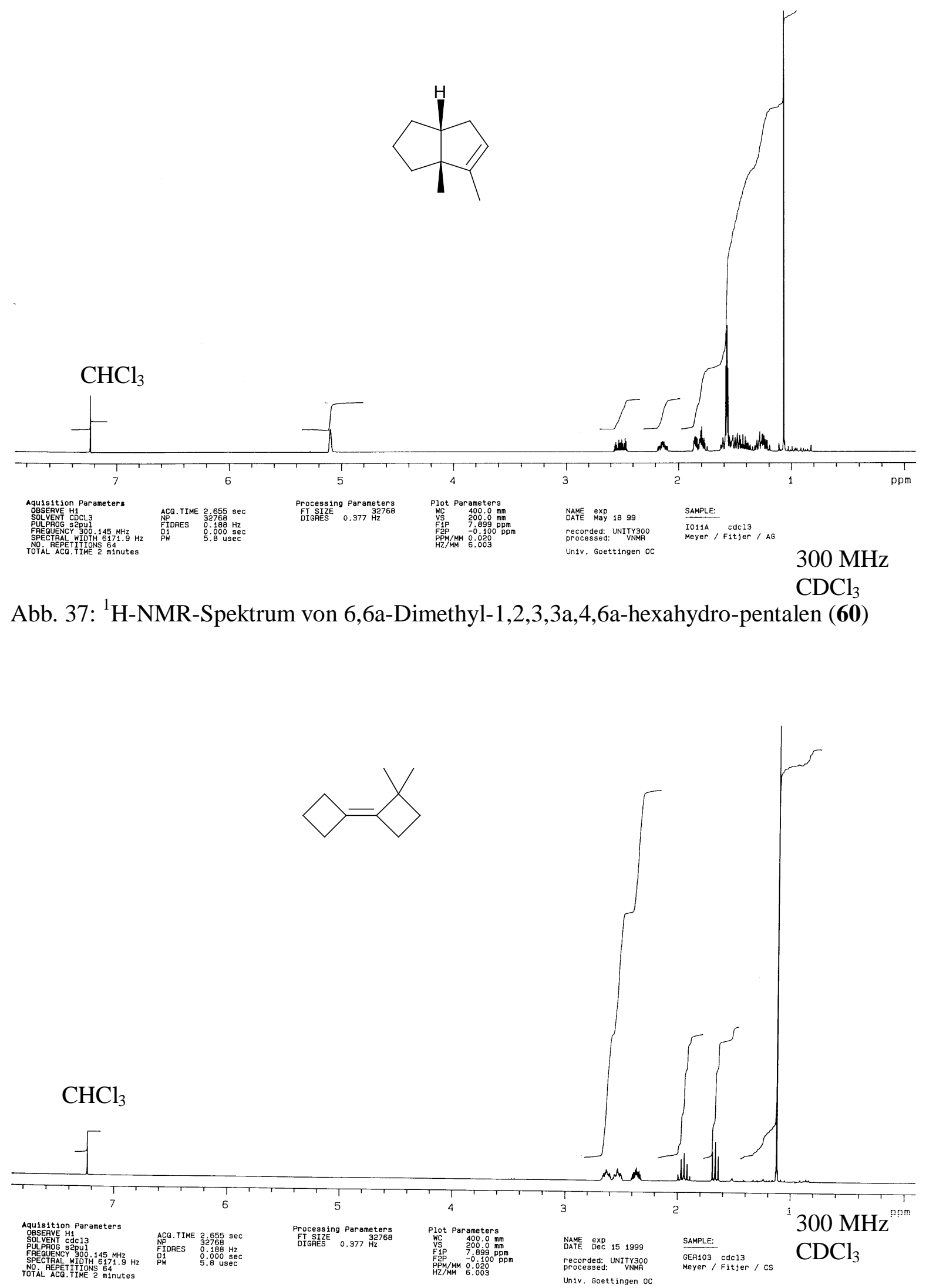

Abb. 38: ${ }^{1} \mathrm{H}-\mathrm{NMR}-$ Spektrum von 2,2-Dimethylbicyclobutyliden (26) 


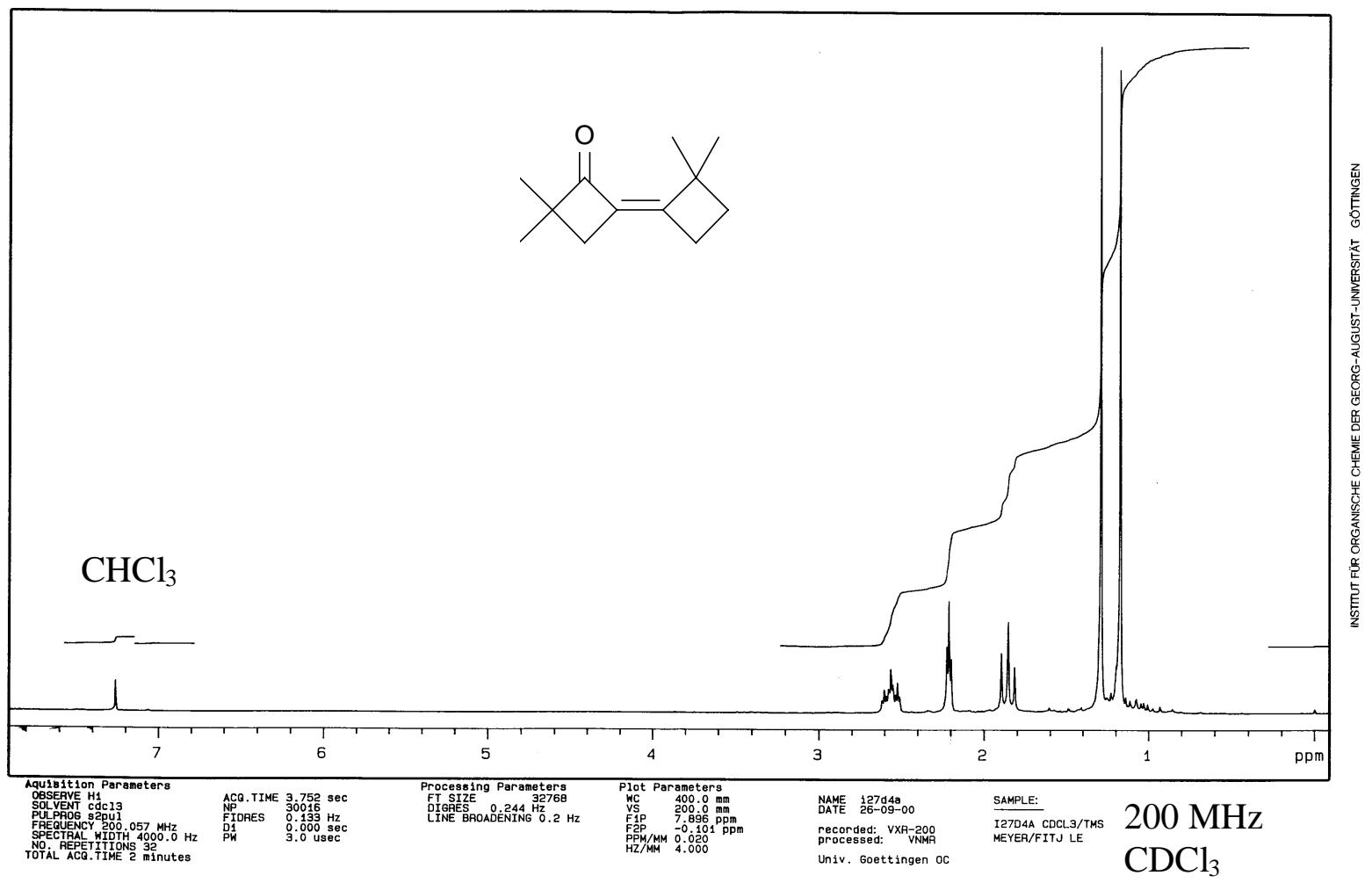

Abb. 39: ${ }^{1}$ H-NMR-Spektrum von 3,3,2',2'-Tetramethyl-bicyclobutyliden-2-on (62)
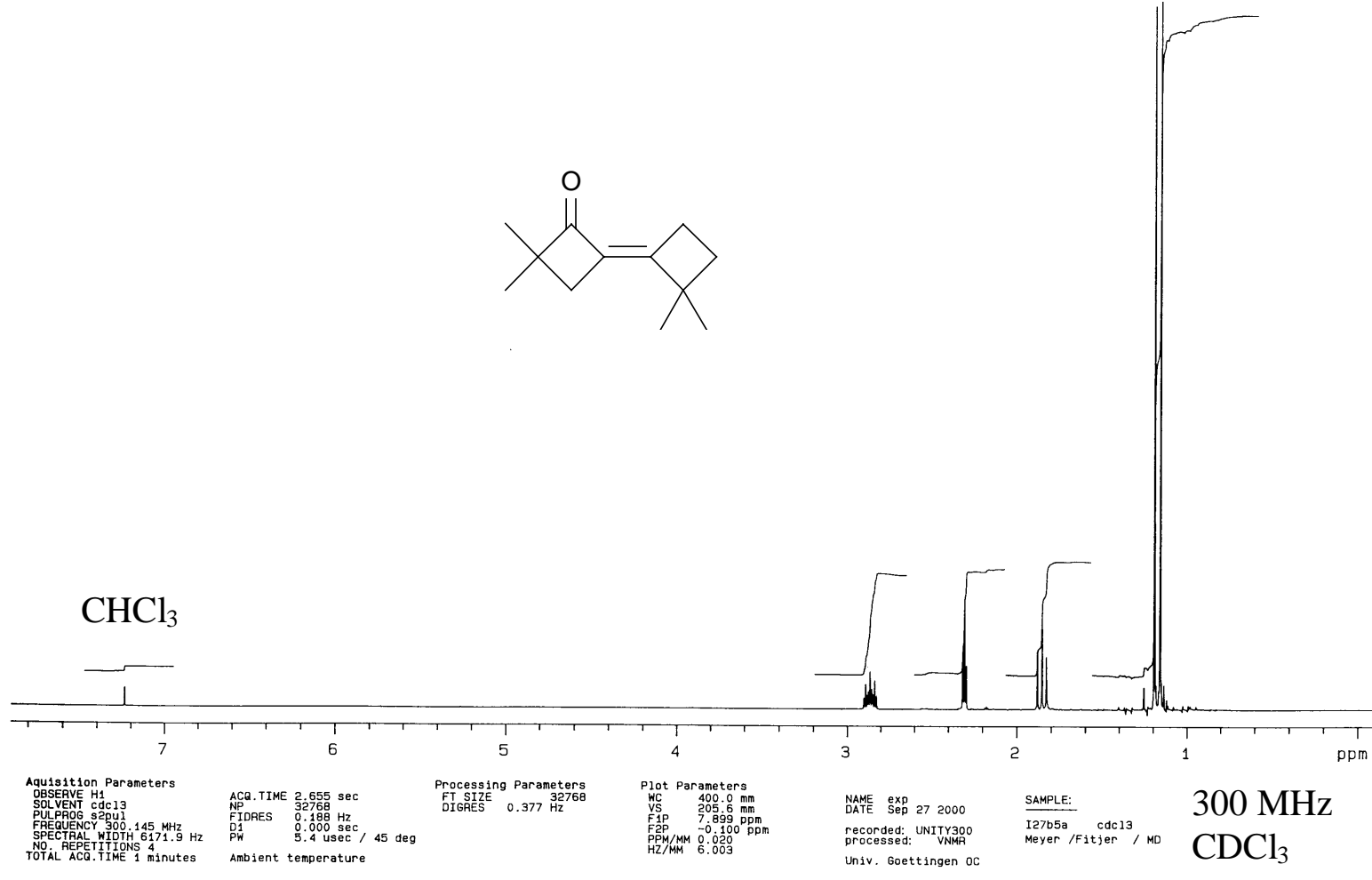

Abb. 40: ${ }^{1}$ H-NMR-Spektrum von 3,3,2' $2^{\prime}$-Tetramethyl-bicyclobutyliden-2-on (63) 


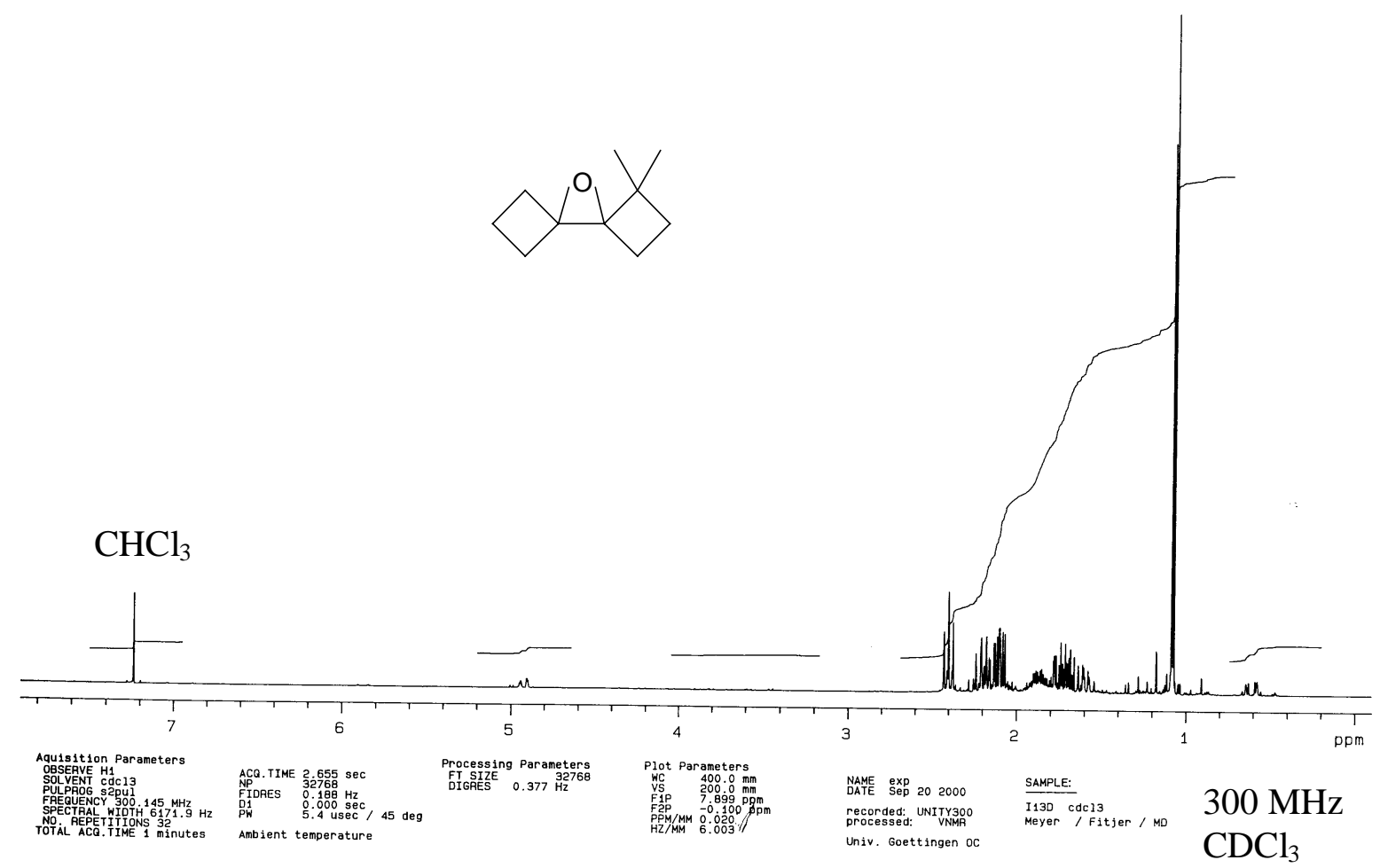

Abb. 41: ${ }^{1}$ H-NMR-Spektrum von 1,1-Dimethyl-9-oxa-dispiro[3.0.3.1]nonan (64)

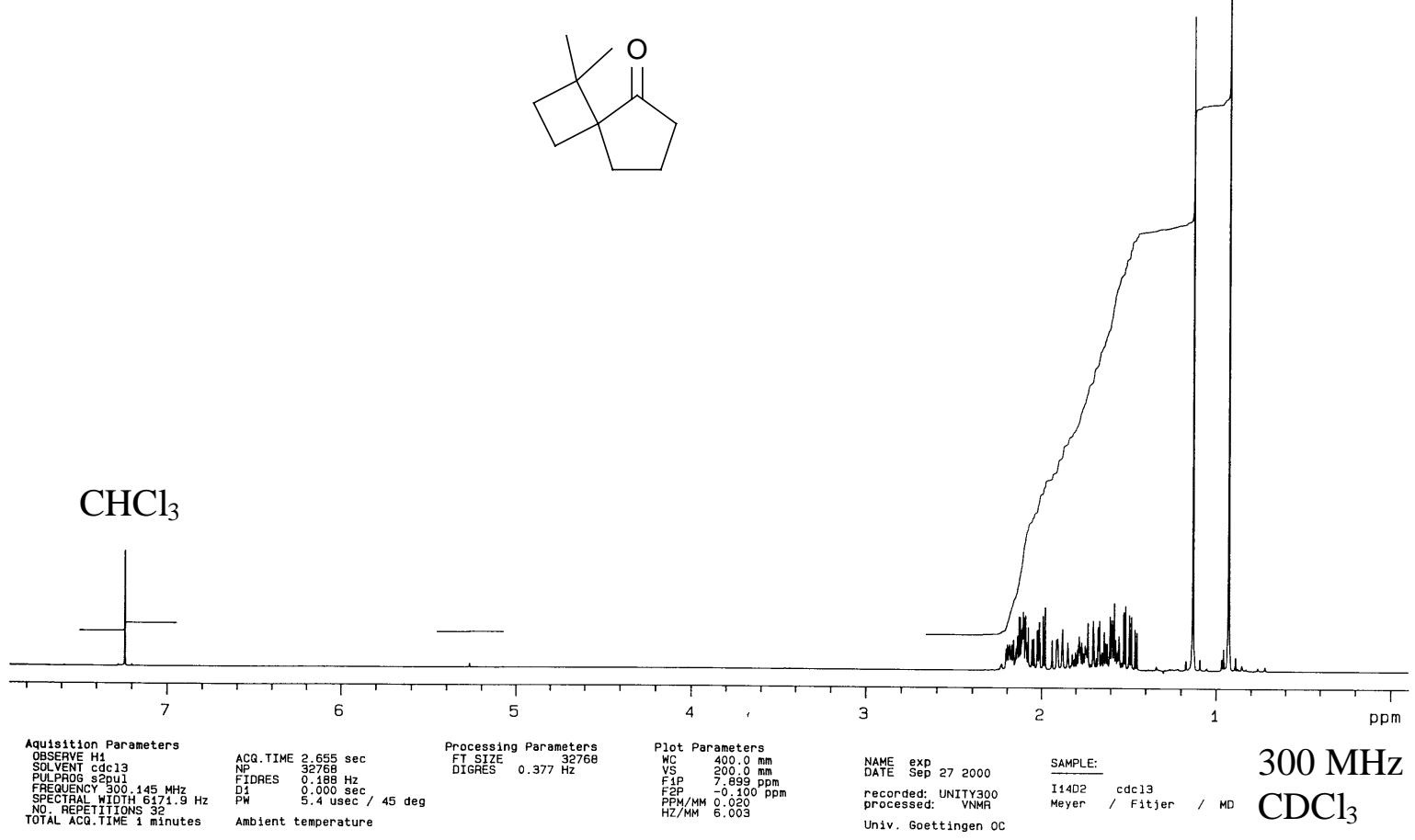

Abb. 42: ${ }^{1} \mathrm{H}-\mathrm{NMR}-$ Spektrum von 1,1-Dimethyl-spiro[3.4] octan-5-on (27) 


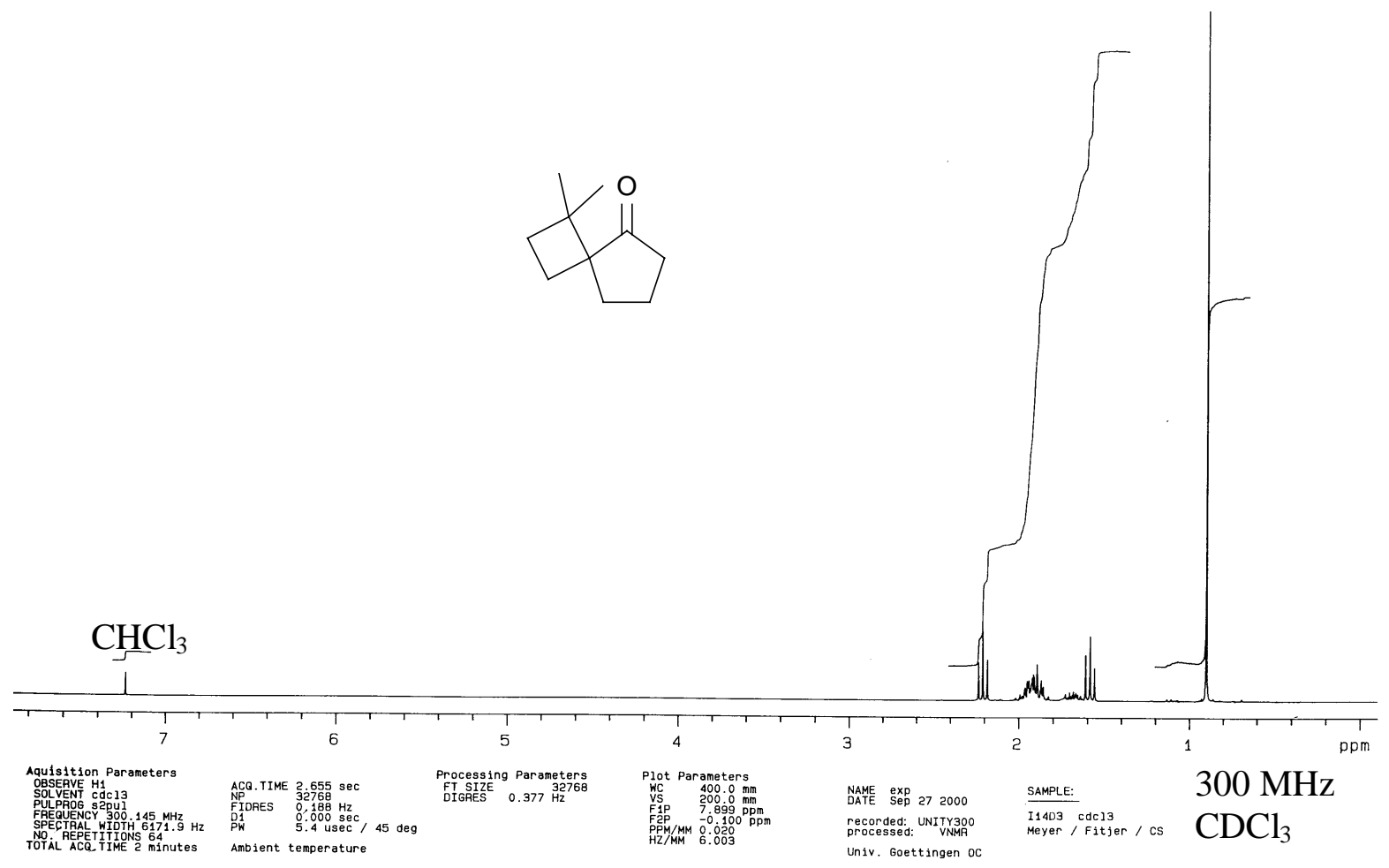

Abb. 43: ${ }^{1}$ H-NMR-Spektrum von 8,8-Dimethyl-spiro[3.4] octan-5-on (67)

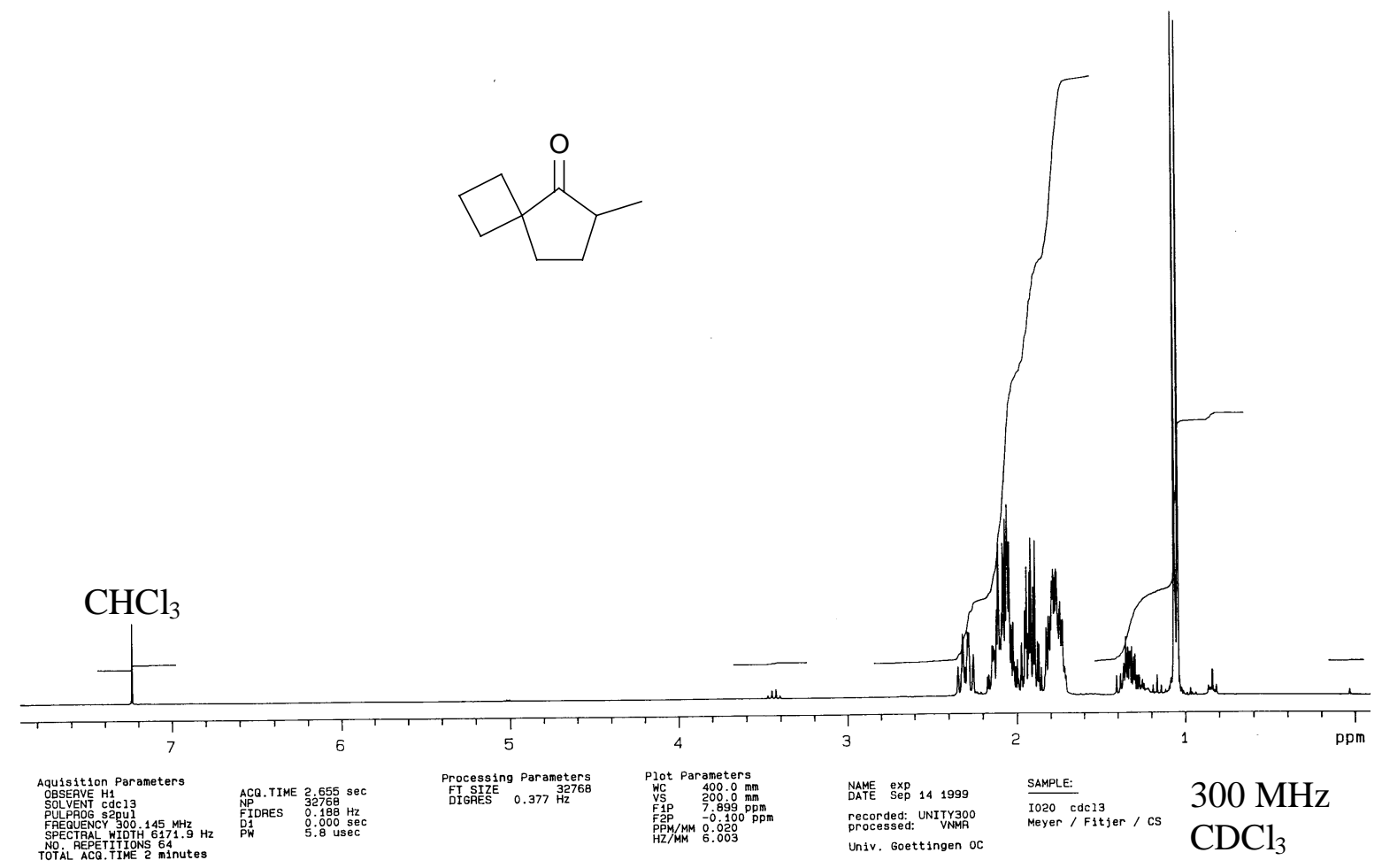

Abb. 44: ${ }^{1} \mathrm{H}-\mathrm{NMR}-$ Spektrum von 6-Methyl-spiro[3.4] octan-5-on (50) 


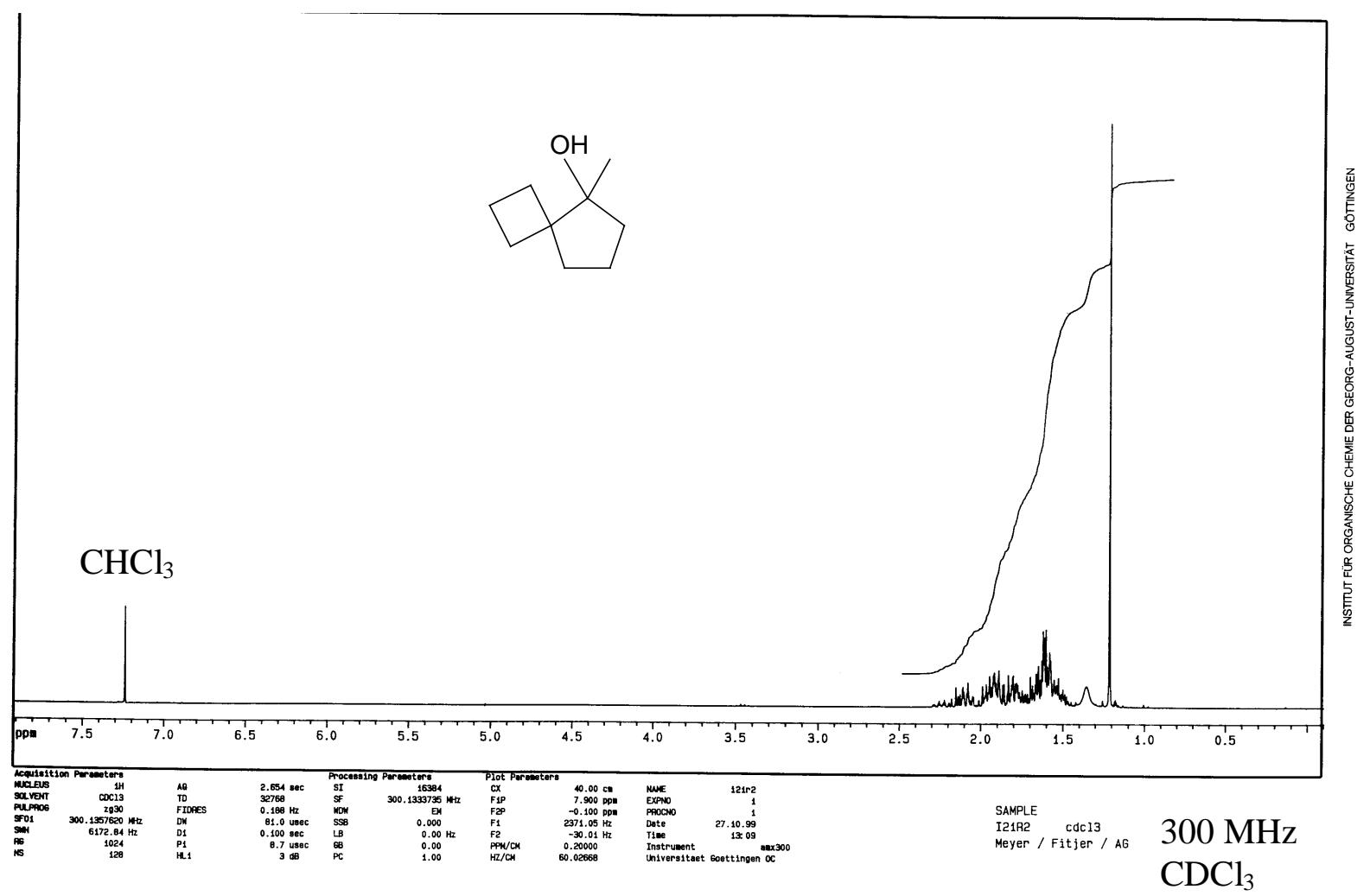

Abb. 45: ${ }^{1} \mathrm{H}-\mathrm{NMR}-$ Spektrum von 5-Methyl-spiro[3.4] octan-5-ol (21)

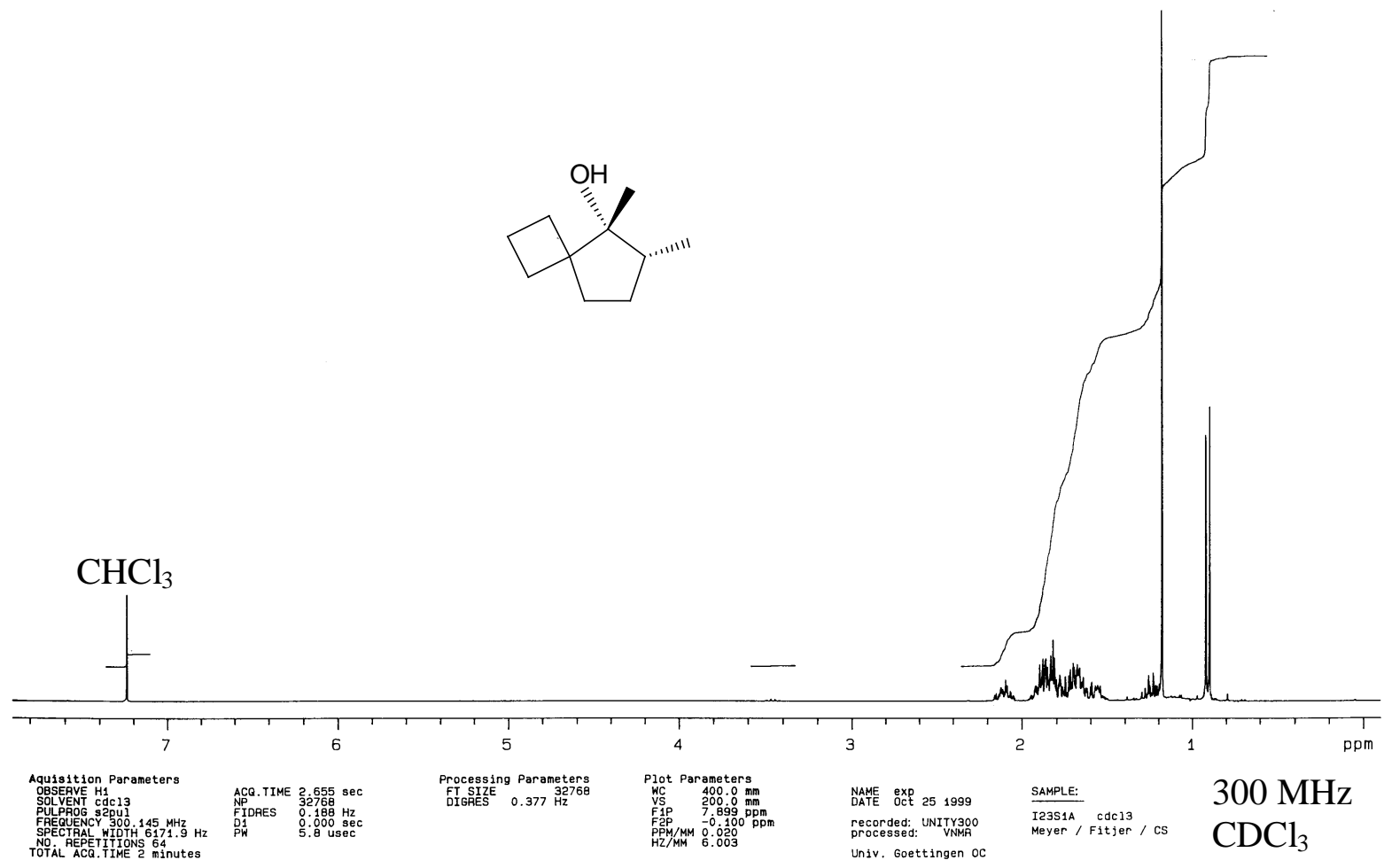

Abb. 46: ${ }^{1} \mathrm{H}-\mathrm{NMR}-$ Spektrum von $\left(5 \mathrm{R}^{*}, 6 \mathrm{R}^{*}\right)-5,6-$ Dimethyl-spiro[3.4] octan-5-ol (23a) 


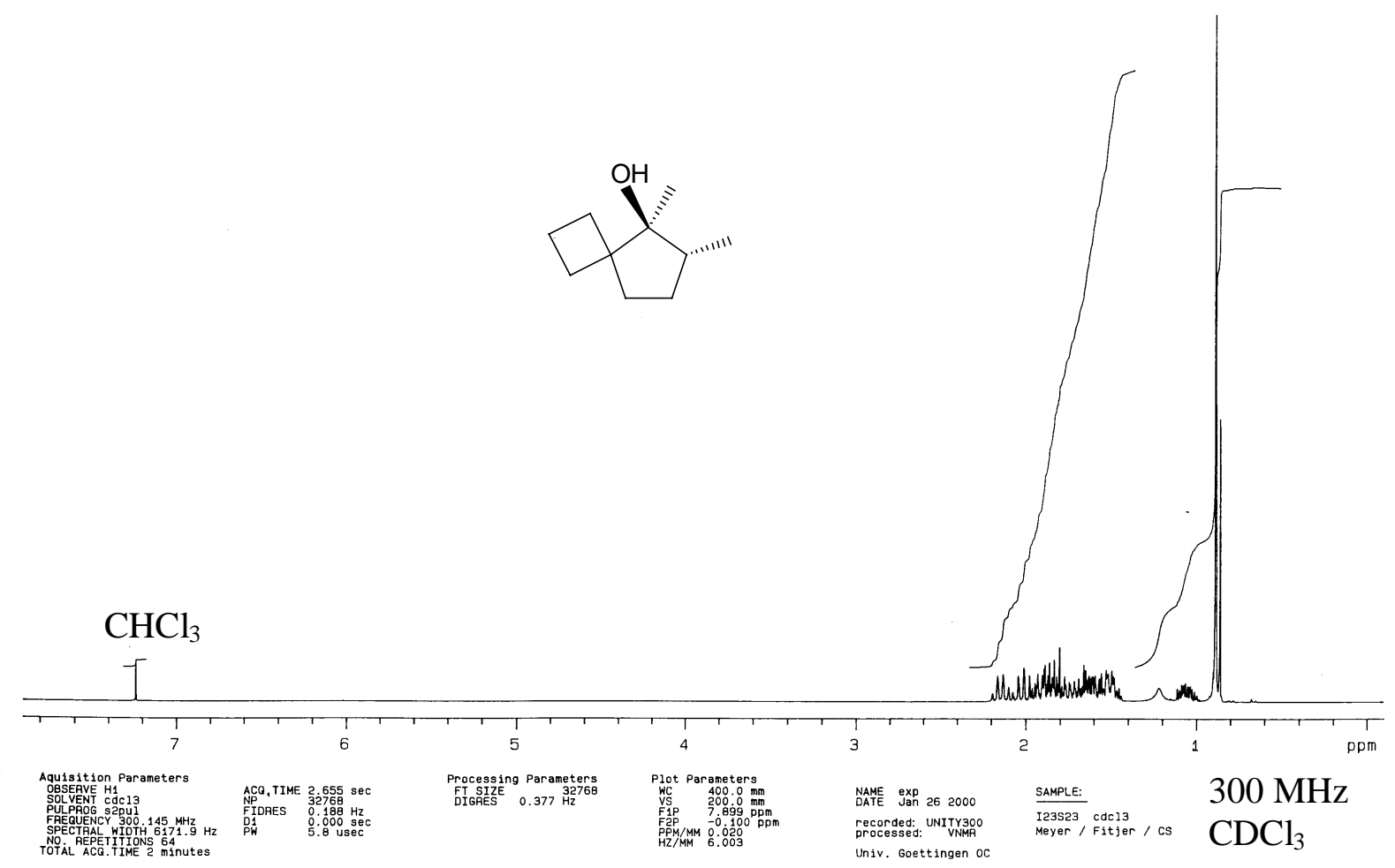

Abb. 47: ${ }^{1} \mathrm{H}-\mathrm{NMR}-$ Spektrum von $\left(5 \mathrm{~S}^{*}, 6 \mathrm{R}^{*}\right)$-5,6-Dimethyl-spiro[3.4]octan-5-ol (23b)

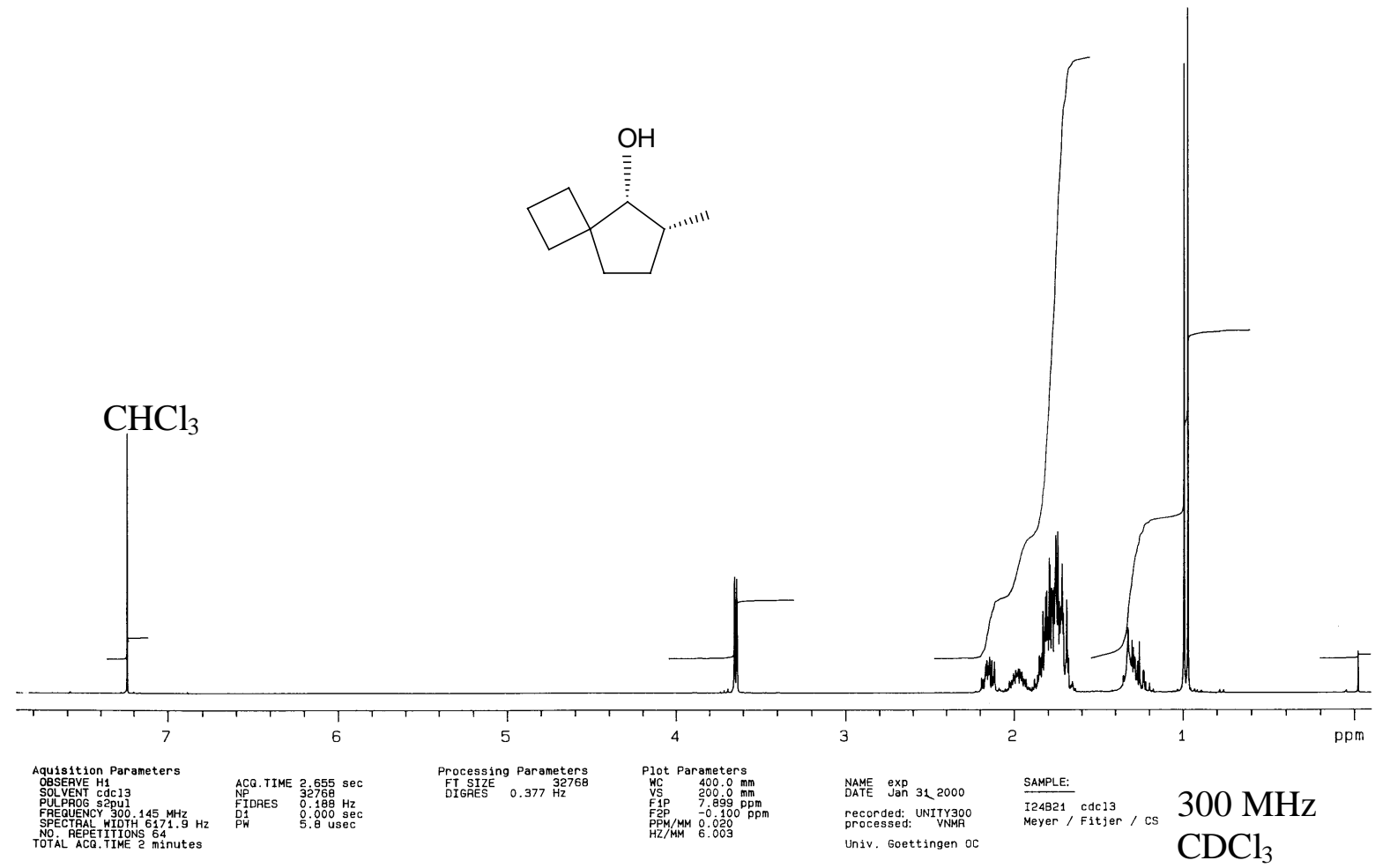

Abb. 48: ${ }^{1}$ H-NMR-Spektrum von (5R*,6R*)-6-Methyl-spiro[3.4] octan-5-ol (22a) 


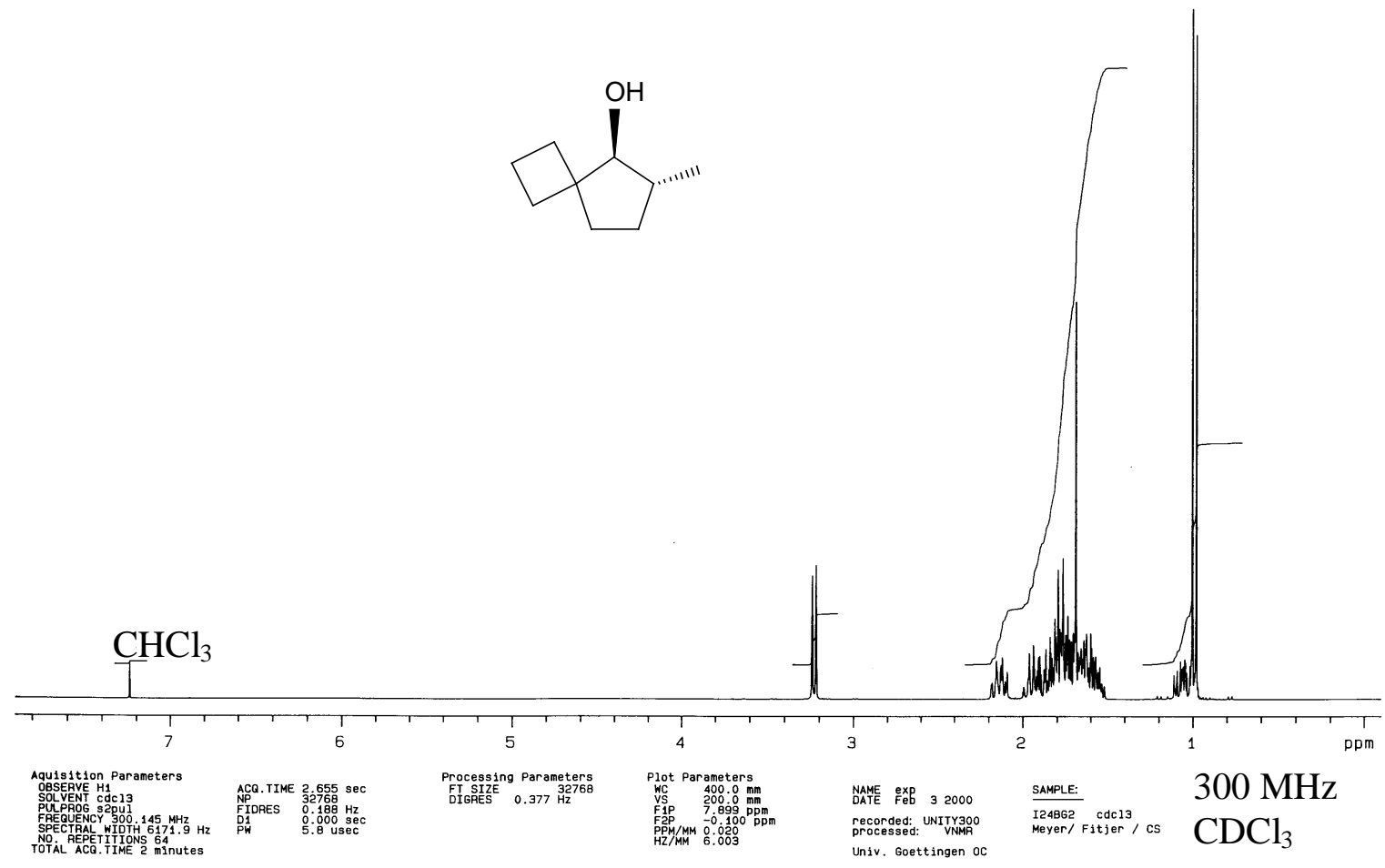

Abb. 49: ${ }^{1}$ H-NMR-Spektrum von (5S*,6R*)-6-Methyl-spiro[3.4] octan-5-ol (22b)

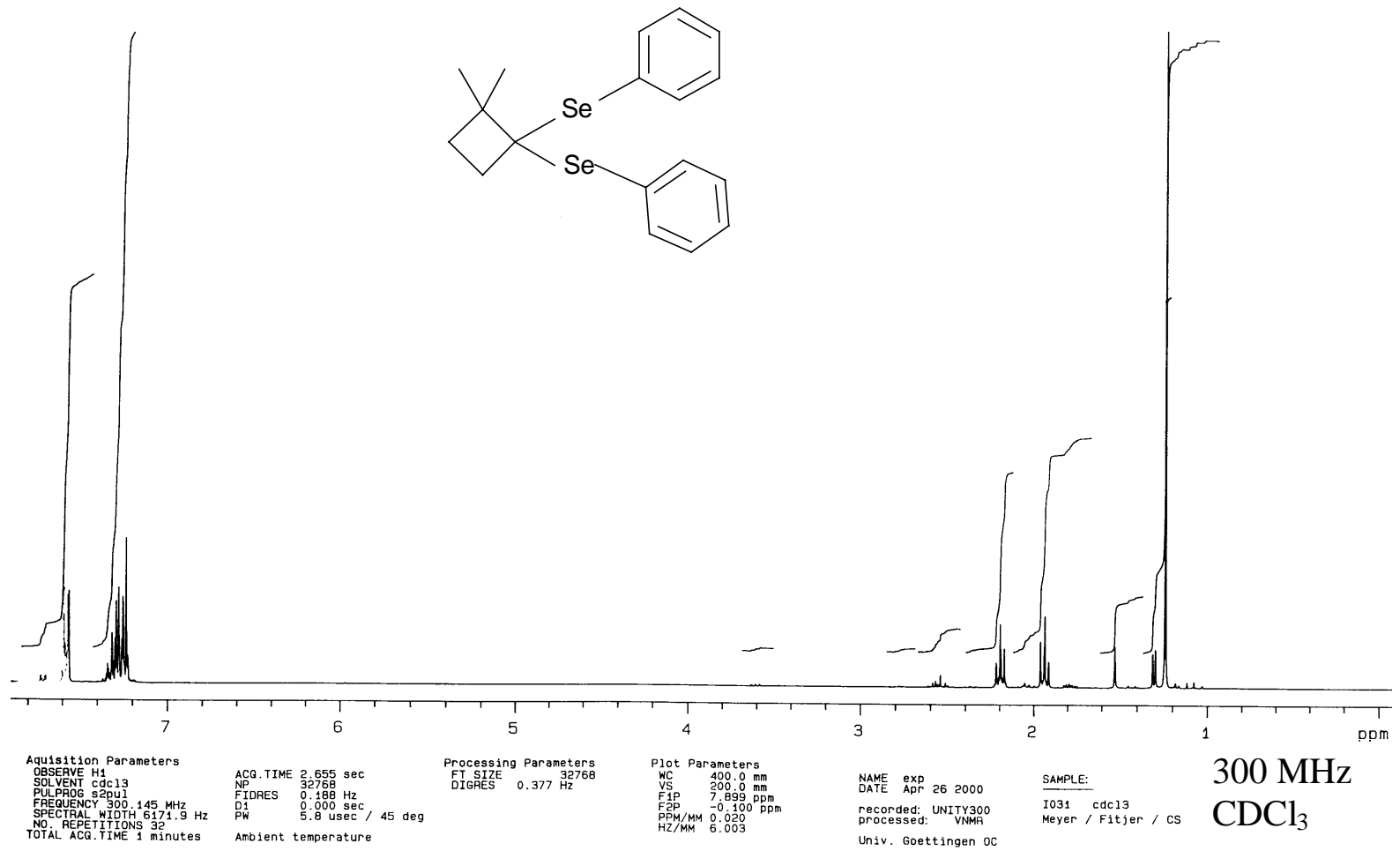

Abb. 50: ${ }^{1} \mathrm{H}-\mathrm{NMR}-$ Spektrum von 1,1-Bis(phenylseleno)-2,2-Dimethylcyclobutan (48) 


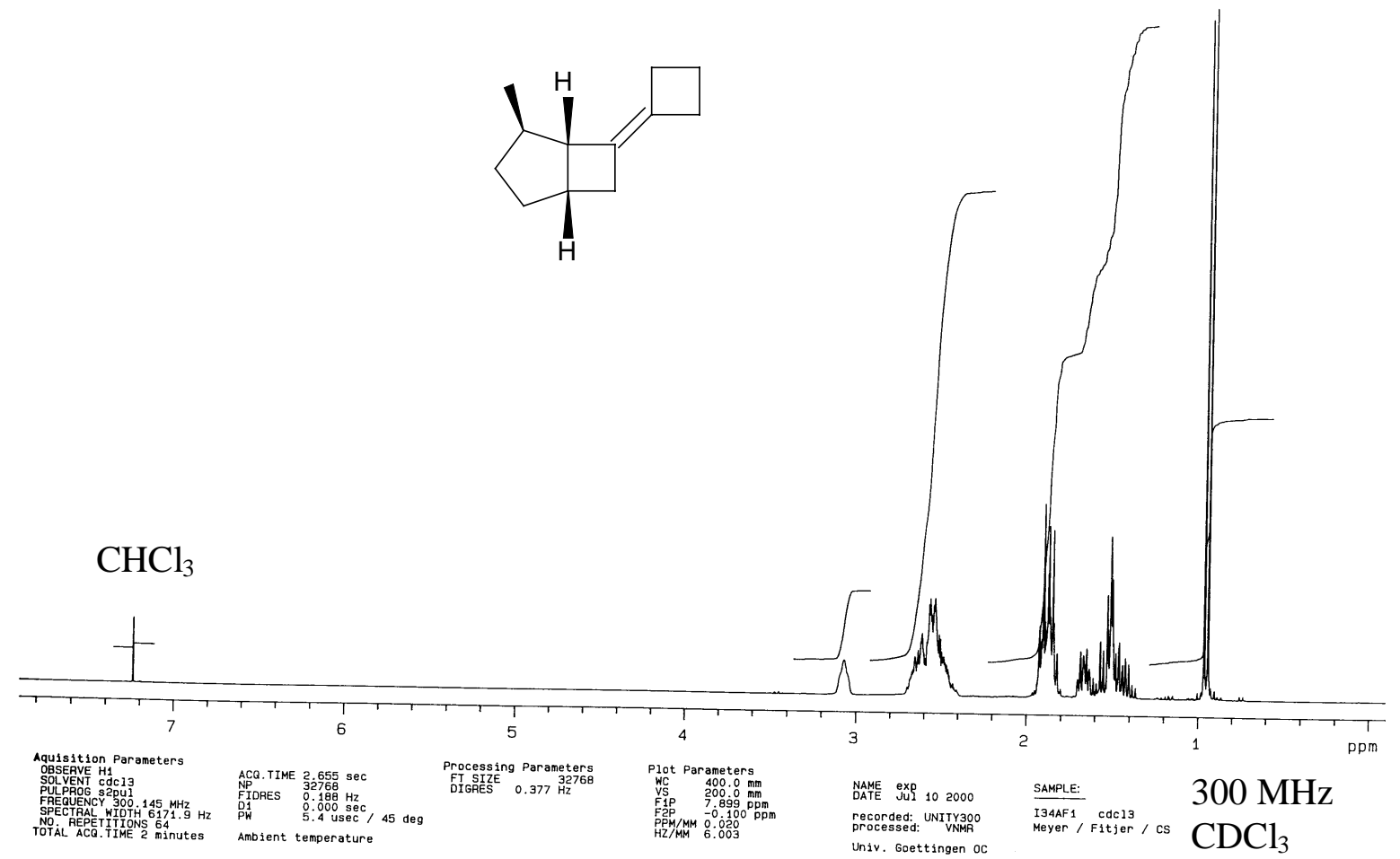

Abb. 51: ${ }^{1} \mathrm{H}-\mathrm{NMR}-$ Spektrum von $\left(1 \mathrm{R}^{*}, 2 \mathrm{~S}^{*}, 5 \mathrm{R}^{*}\right)$-7-Cyclobutyliden-2-methyl-bicyclo[3.2.0]heptan (40)

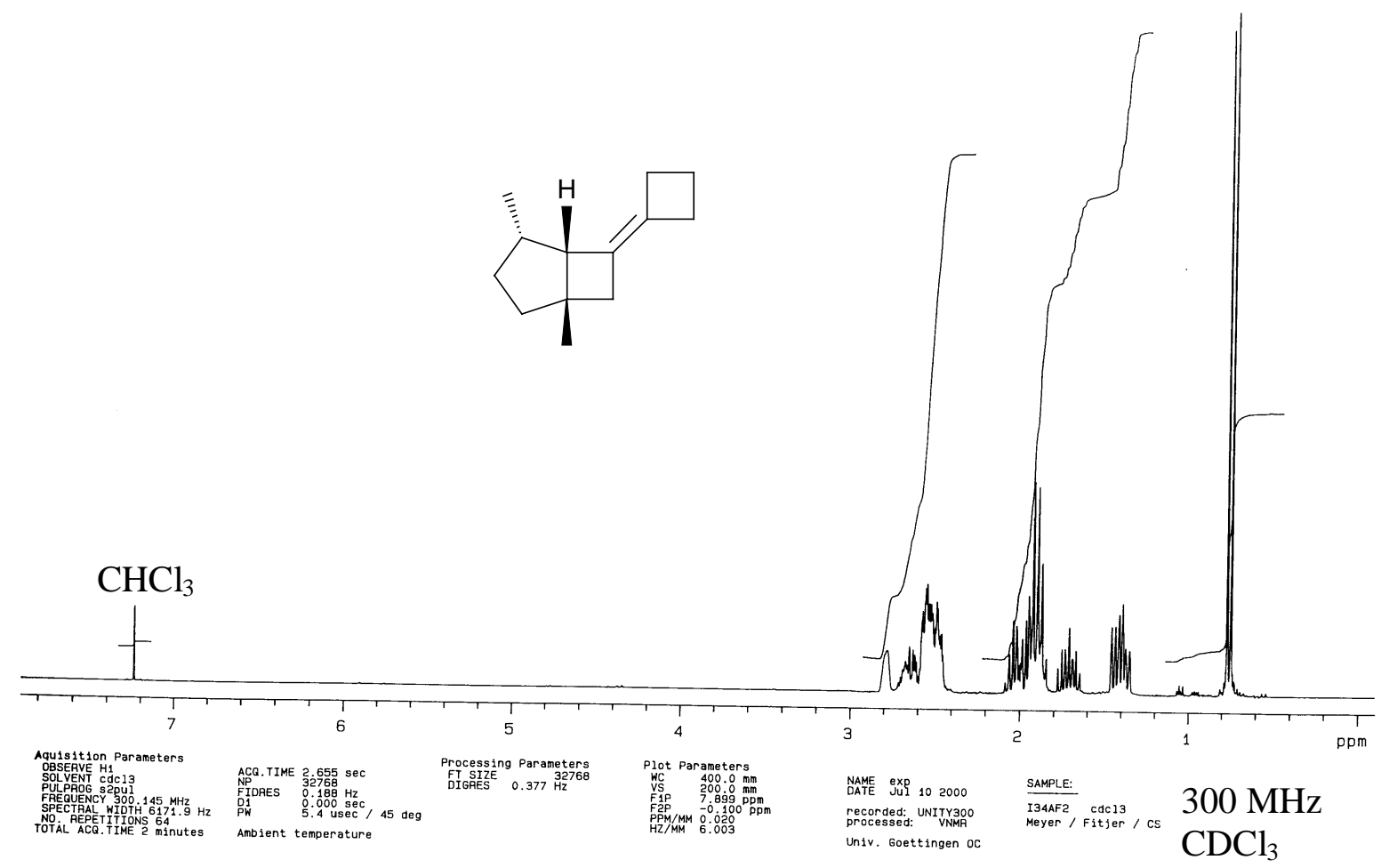

Abb. 52: ${ }^{1} \mathrm{H}-\mathrm{NMR}-$ Spektrum von $\left(1 \mathrm{R}^{*}, 2 \mathrm{R} * 5 \mathrm{R} *\right)$-7-Cyclobutyliden-2-methyl-bicyclo[3.2.0]heptan (91) 


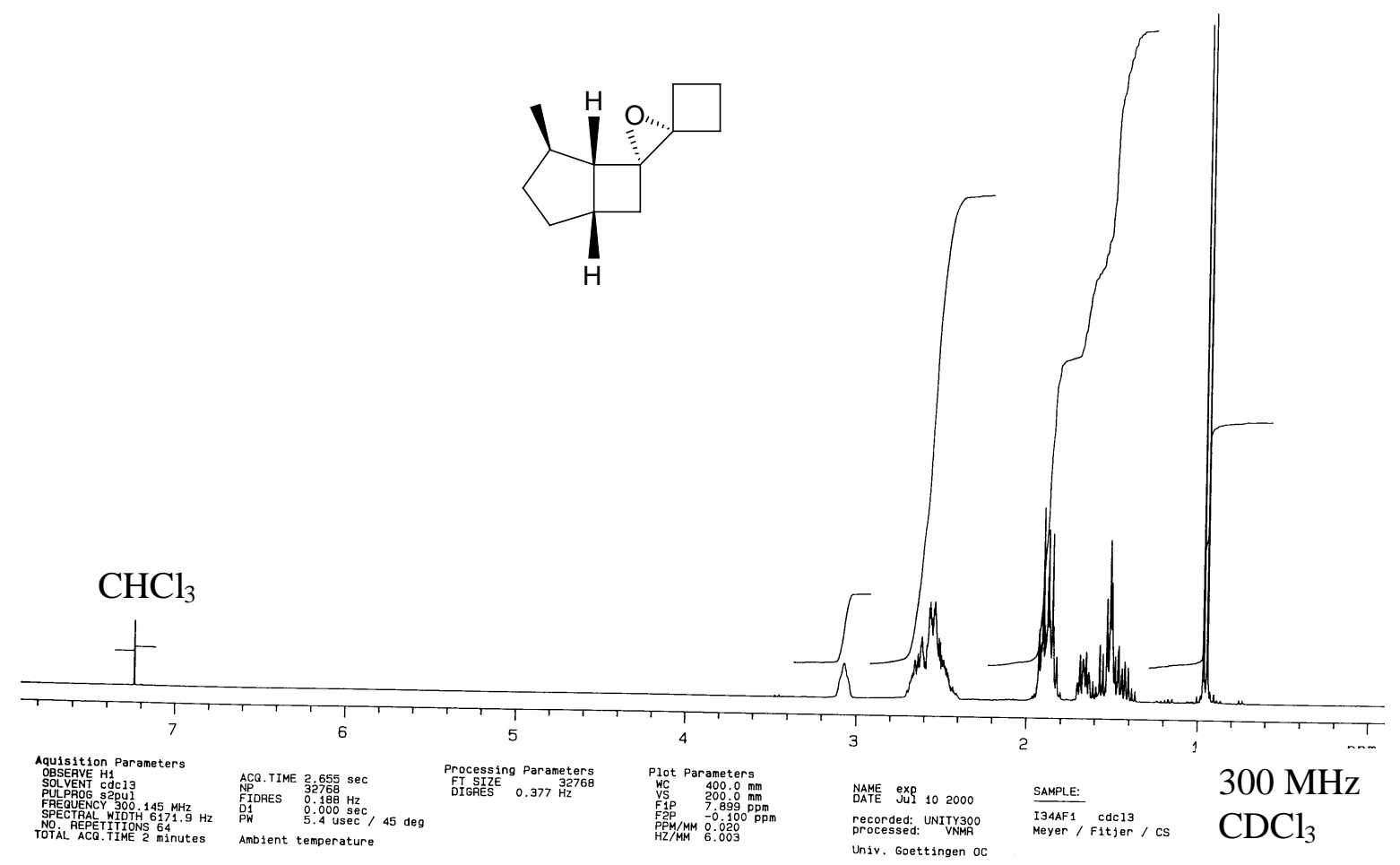

Abb. 53: ${ }^{1} \mathrm{H}-\mathrm{NMR}-$ Spektrum von 2,2-Spiro-cyclobutyl-3,3-spiro-[(1R*,4S*,5R*,6S*)-2methyl-bicyclo[3.2.0]heptan-6,6-yl]-oxiran (92)

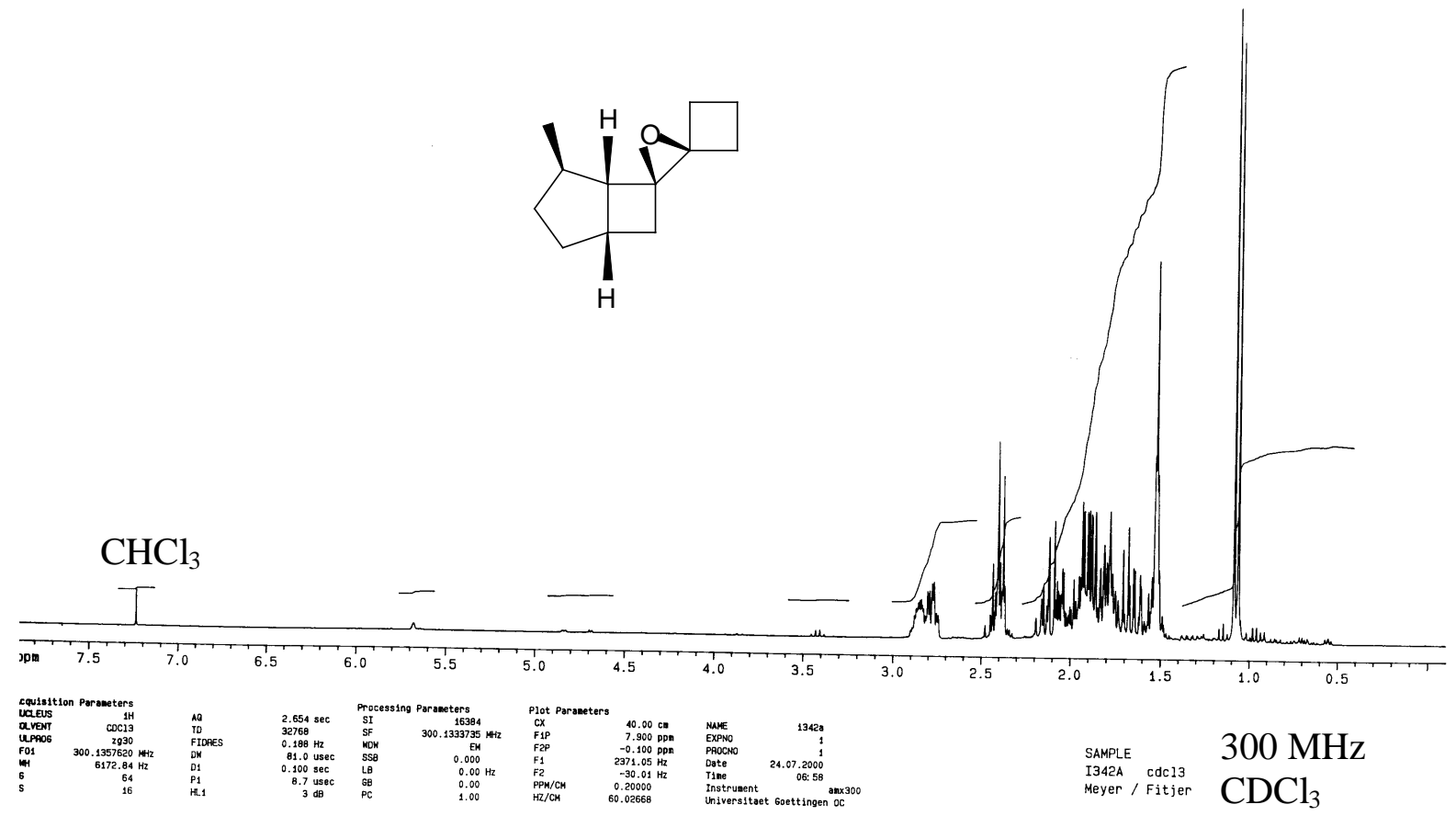

Abb. 54: ${ }^{1} \mathrm{H}-\mathrm{NMR}-$ Spektrum von 2,2-Spiro-cyclobutyl-3,3-spiro-[(1R*,4S*,5R*,6R*)-2methyl-bicyclo[3.2.0]heptan-6,6-yl]-oxiran (89) 

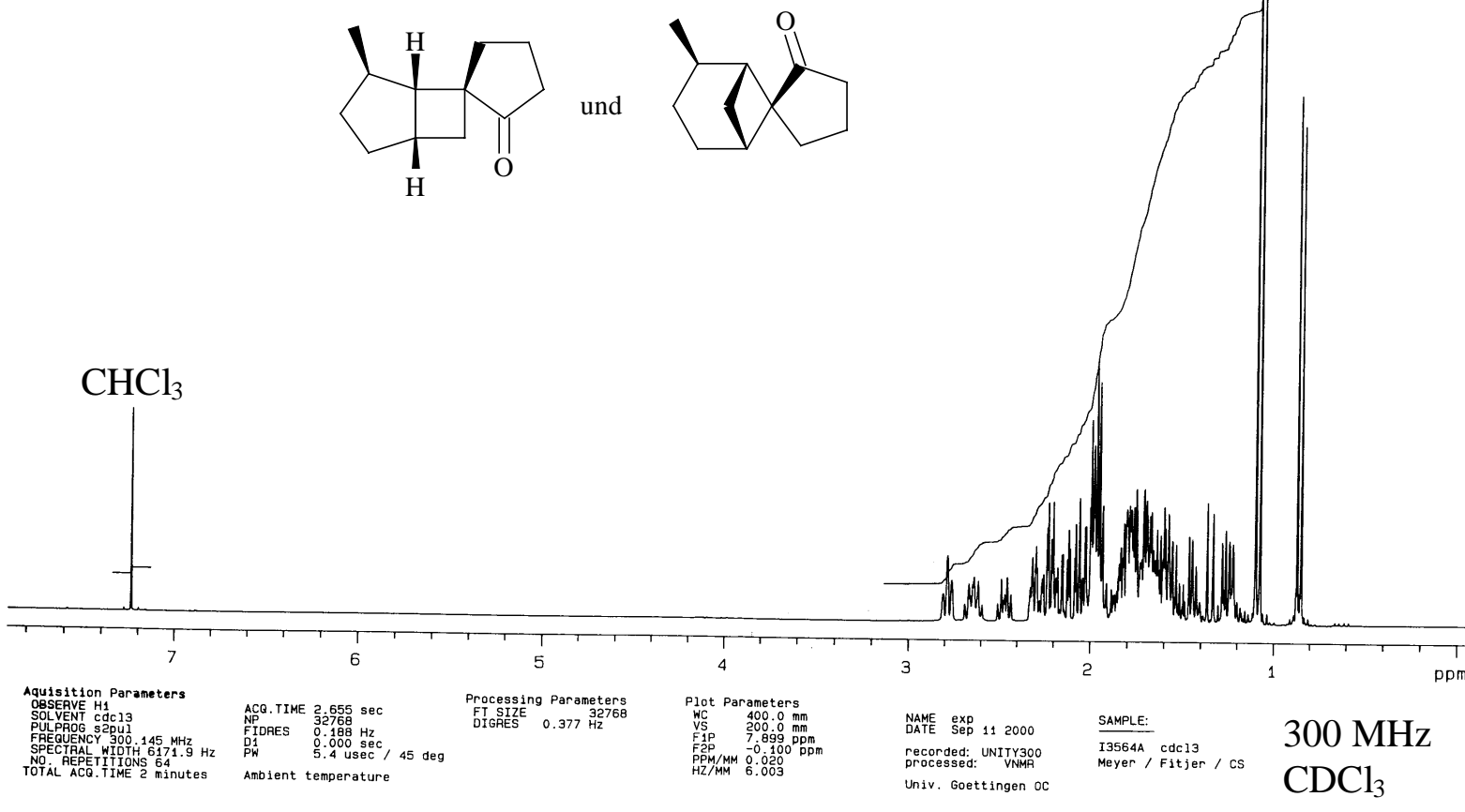

Abb. 55: ${ }^{1} \mathrm{H}-\mathrm{NMR}-\mathrm{Spektrum}$ von $\left(1 \mathrm{R}^{*}, 4 \mathrm{~S}^{*}, 5 \mathrm{R}^{*}, 6 \mathrm{~S}^{*}\right)-4-$ Methyl-spiro $\{$ bicyclo[3.2.0]heptan6,1'-cyclopentan-2'-on\} (87) und (1R*,2R*,5R*,6S*)-2-Methyl-spiro \{bicyclo[3.1.1]heptan-6,1'-cyclopentan-2'-on\} (93)

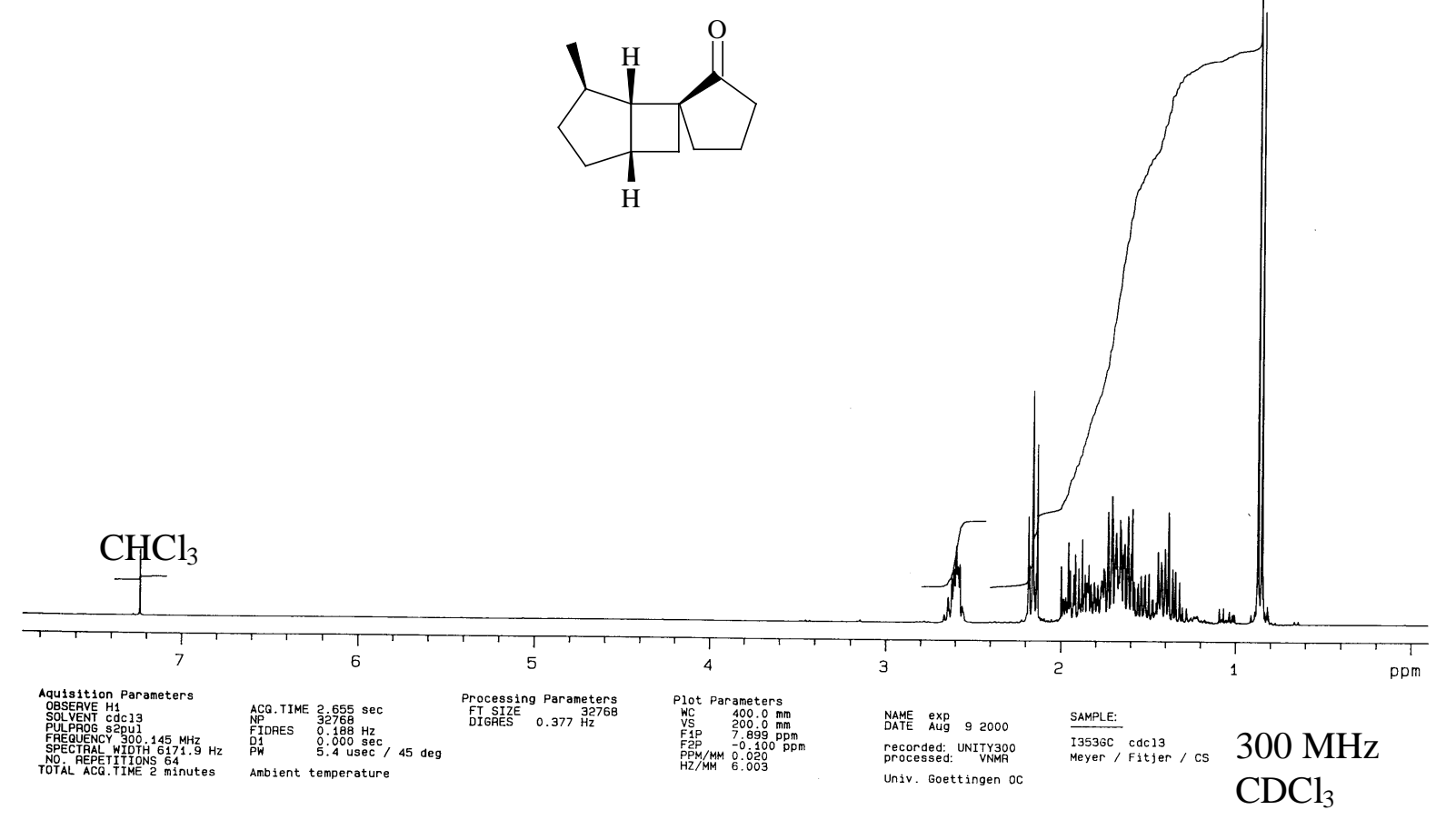

Abb. 56: ${ }^{1} \mathrm{H}-\mathrm{NMR}-\mathrm{Spektrum}$ von $\left(1 \mathrm{R}^{*}, 4 \mathrm{~S}^{*}, 5 \mathrm{R}^{*}, 6 \mathrm{R}^{*}\right)$-4-Methyl-spiro $\{$ bicyclo[3.2.0]heptan6,1'-cyclopentan-2'-on $\}(\mathbf{8 8})$ 

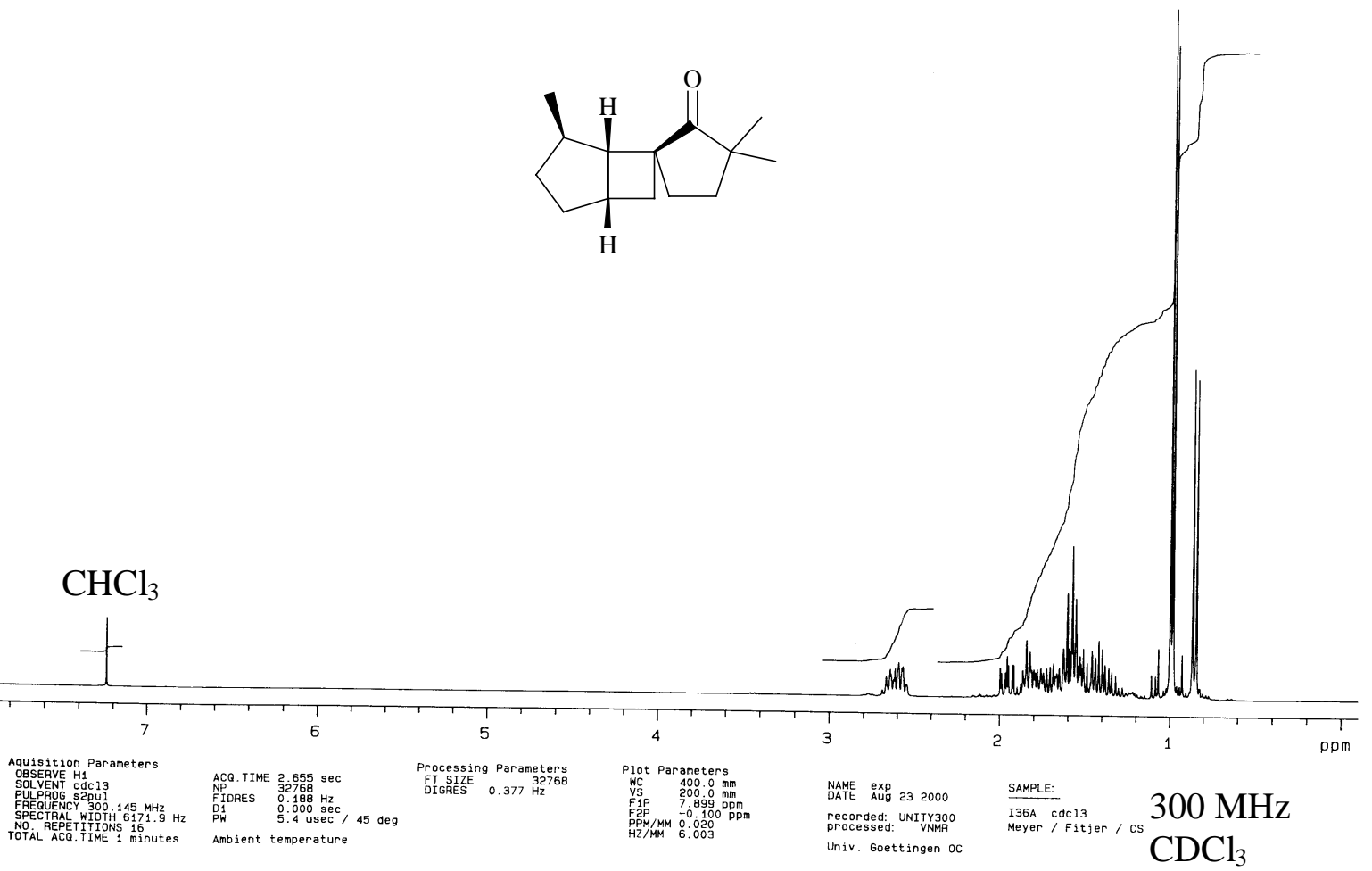

Abb. 57: ${ }^{1} \mathrm{H}-\mathrm{NMR}-\mathrm{Spektrum}$ von $\left(1 \mathrm{R}^{*}, 4 \mathrm{~S}^{*}, 5 \mathrm{R}^{*}, 6 \mathrm{R}^{*}\right)-4,3^{\prime}, 3^{\prime}$-Trimethyl-spiro $\{$ bicyclo[3.2.0]heptan-6, $1^{\prime}$-cyclopentan-2'-on\} (95)

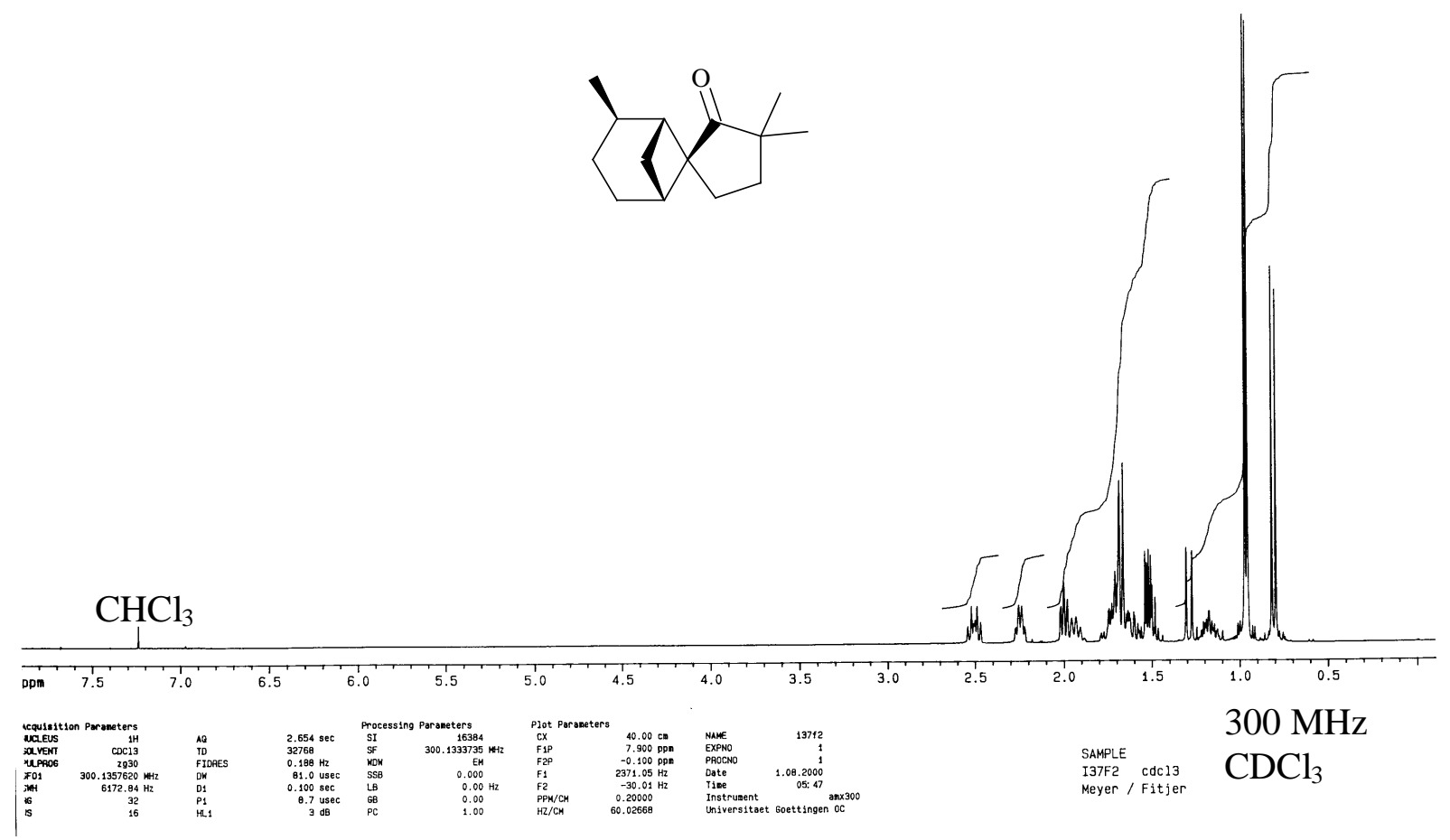

Abb. 58: ${ }^{1} \mathrm{H}-\mathrm{NMR}-\mathrm{Spektrum}$ von $\left(1 \mathrm{R}^{*}, 2 \mathrm{R}^{*}, 5 \mathrm{R}^{*}, 6 \mathrm{~S}^{*}\right)-2,3^{\prime}, 3^{\prime}$-Trimethyl-spiro \{ bicyclo[3.1.1]heptan-6,1'-cyclopentan-2'-on\} (96) 

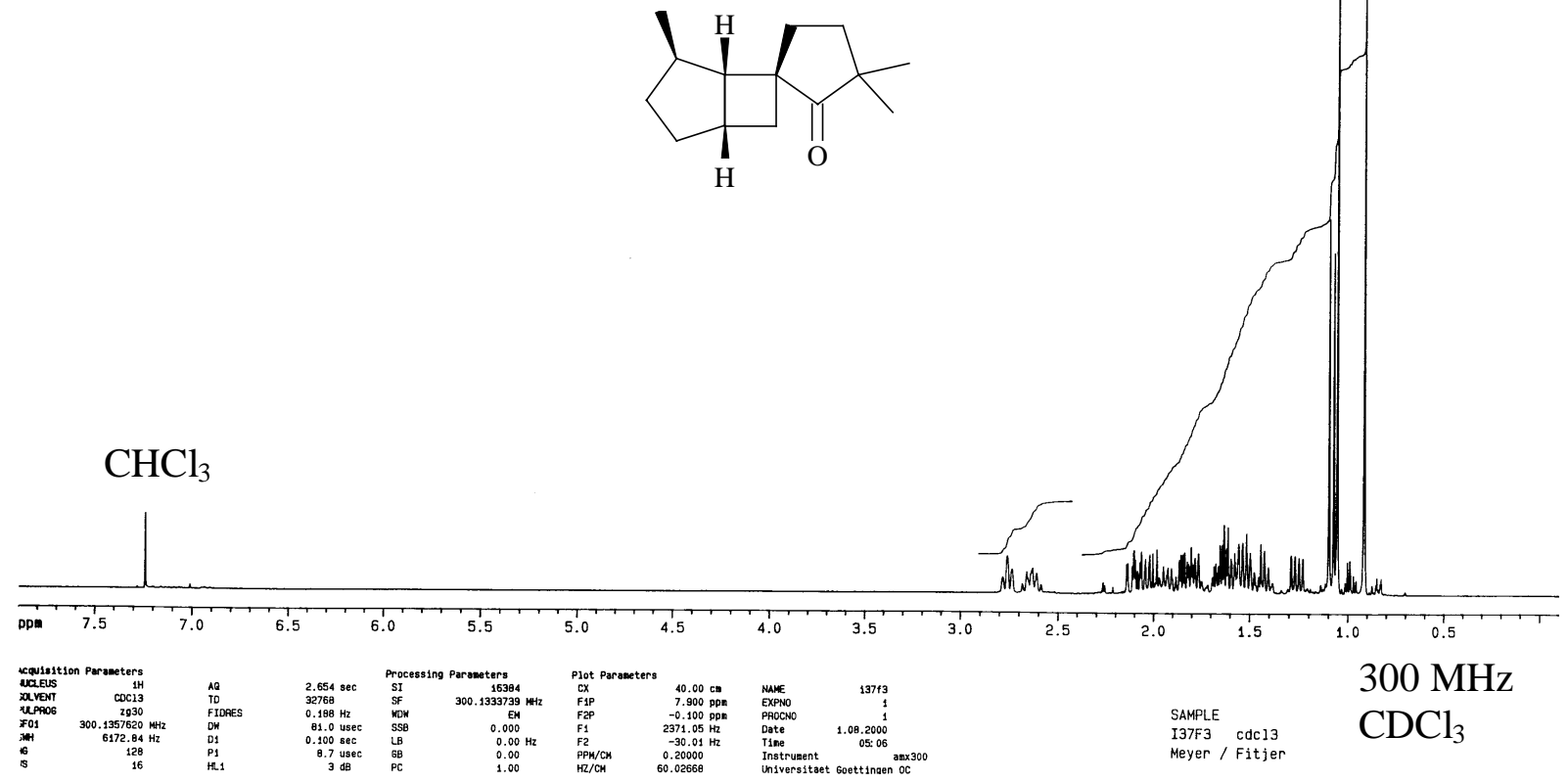

Abb. 59: ${ }^{1} \mathrm{H}-\mathrm{NMR}-$ Spektrum von $\left(1 \mathrm{R}^{*}, 4 \mathrm{~S}^{*}, 5 \mathrm{R}^{*}, 6 \mathrm{~S}^{*}\right)-4,3^{\prime}, 3^{\prime}$-Trimethyl-spiro \{ bicyclo[3.2.0]heptan-6,1'-cyclopentan-2'-on\} (94)
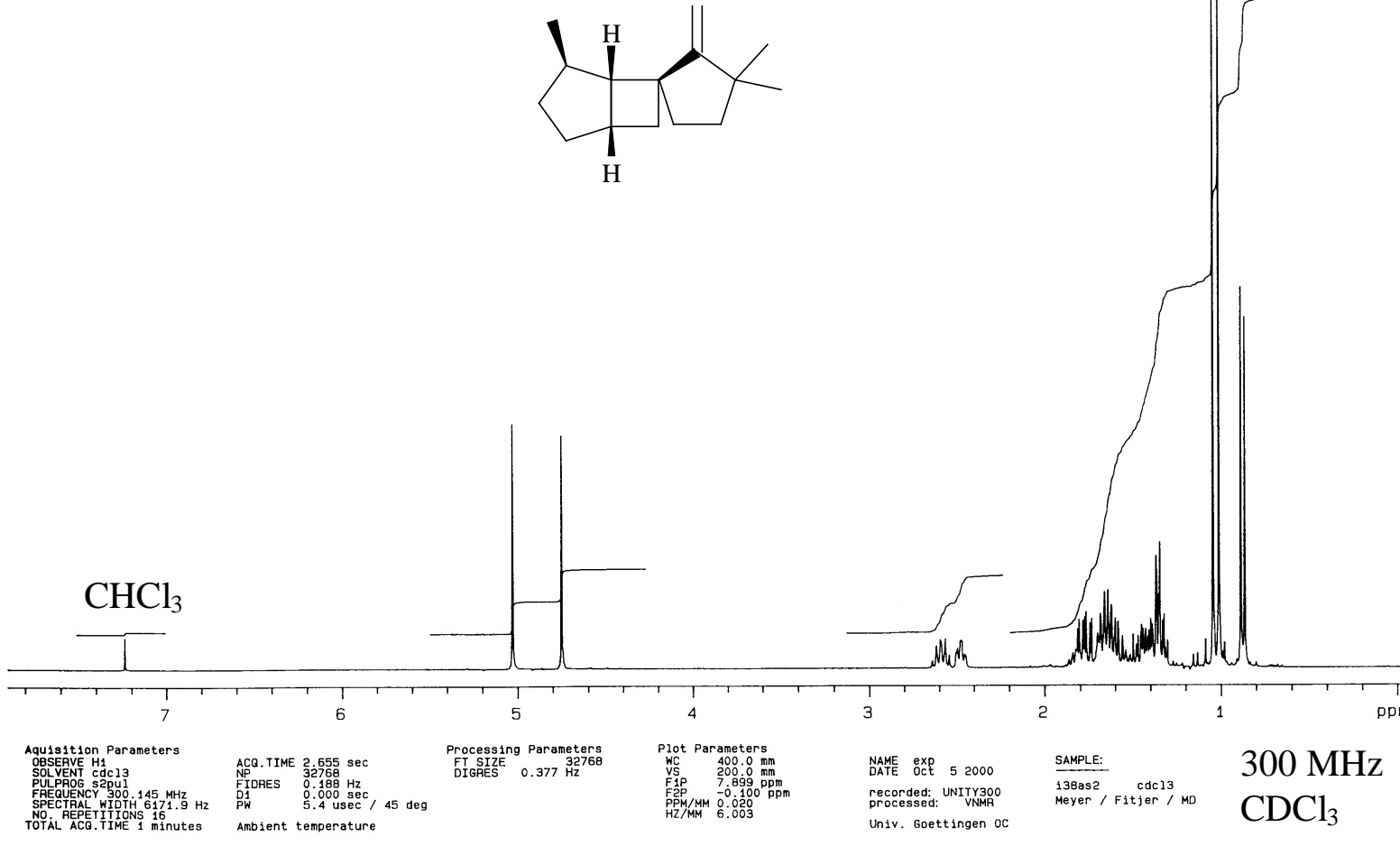

Abb. 60: ${ }^{1} \mathrm{H}-\mathrm{NMR}-$ Spektrum von $\left(1 \mathrm{R}^{*}, 4 \mathrm{~S}^{*}, 5 \mathrm{R}^{*}, 6 \mathrm{R}^{*}\right)-2^{\prime}$-Methylen-4,3',3'-Trimethylspiro\{ bicyclo[3.2.0]heptan-6,1'-cyclopentan\} (104) 

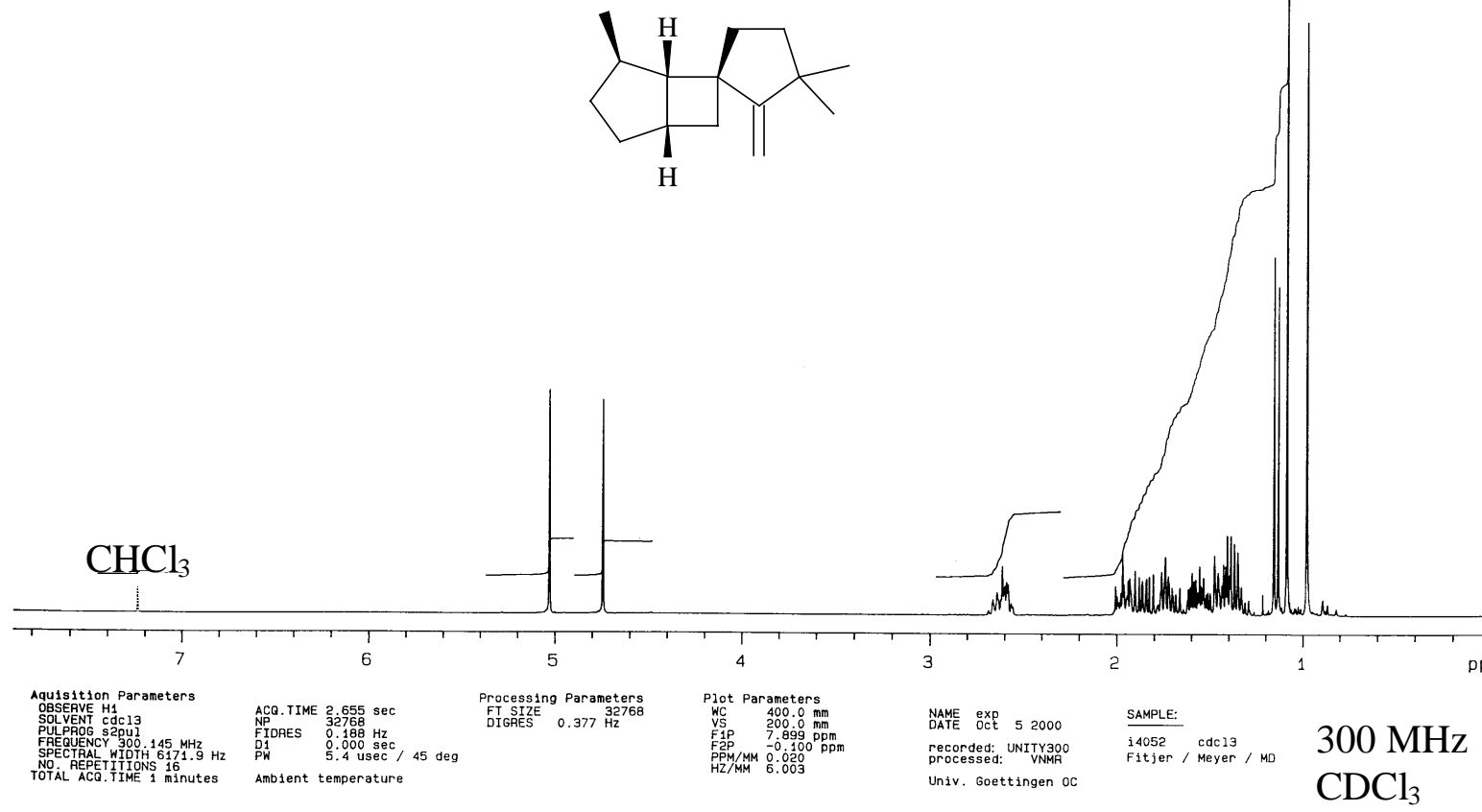

Abb. 61: ${ }^{1} \mathrm{H}-\mathrm{NMR}-$ Spektrum von $\left(1 \mathrm{R}^{*}, 4 \mathrm{~S}^{*}, 5 \mathrm{R}^{*}, 6 \mathrm{~S}^{*}\right)-2^{\prime}$-Methylen-4,3',3'-Trimethylspiro\{bicyclo[3.2.0]heptan-6,1'-cyclopentan\} (103)
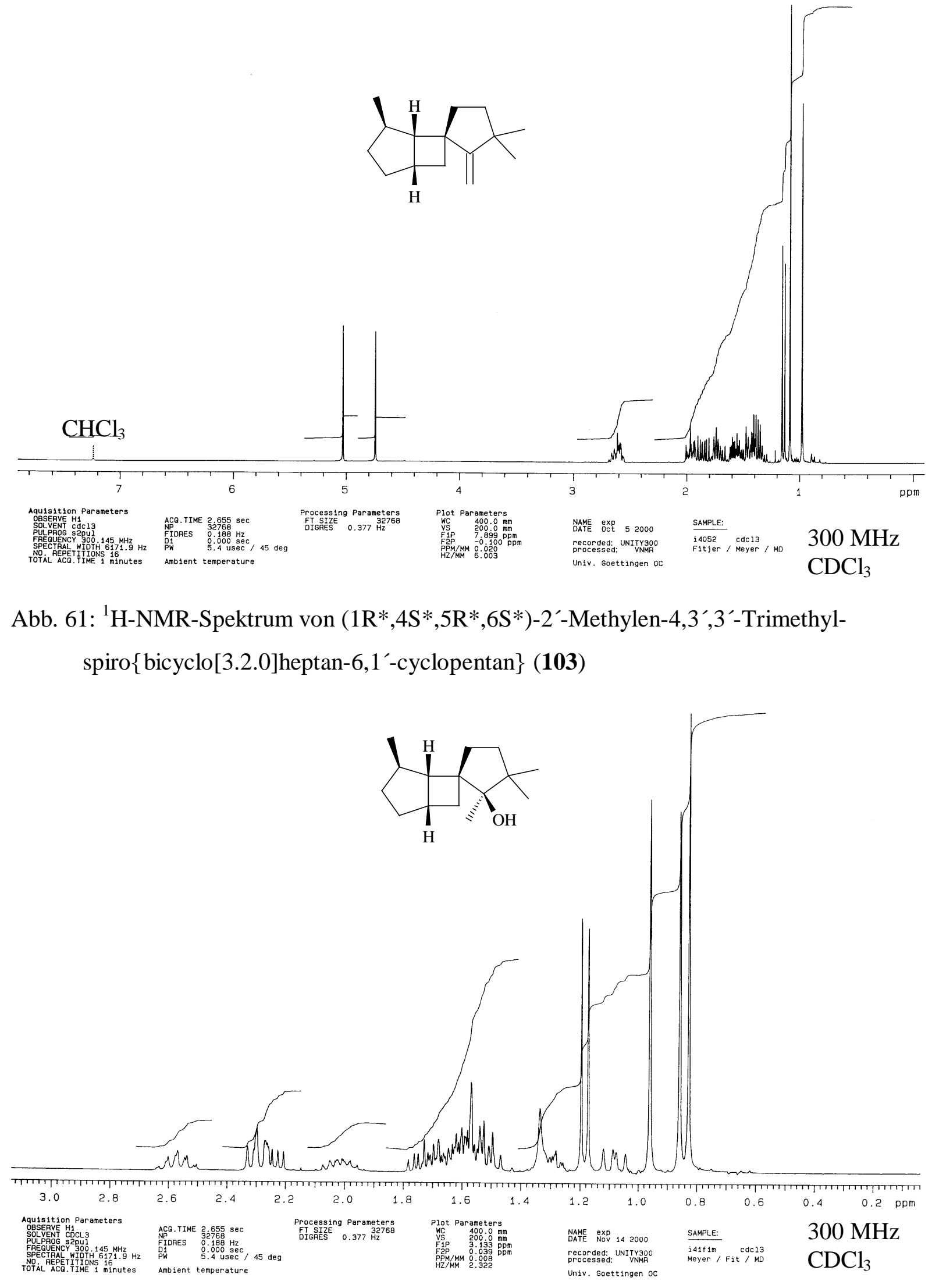

Abb. 61: 'H-NMR-Spektrum von $\left(1 \mathrm{R}^{*}, 4 \mathrm{~S}^{*}, 5 \mathrm{R}^{*}, 6 \mathrm{~S}^{*}\right)-2^{\prime}-$ Methylen-4,3',3'-Trimethyl-

Abb. 62: ${ }^{1} \mathrm{H}-\mathrm{NMR}-$ Spektrum von $\left(1 \mathrm{R}^{*}, 4 \mathrm{~S}^{*}, 5 \mathrm{R}^{*}, 6 \mathrm{~S}^{*}, 2^{\prime} \mathrm{S}^{*}\right)-4,2^{\prime}, 3^{\prime}, 3^{\prime}-$ Tetramethyl-spiro\{bicyclo[3.2.0]heptan-6,1'-cyclopentan-2'-ol (45) 

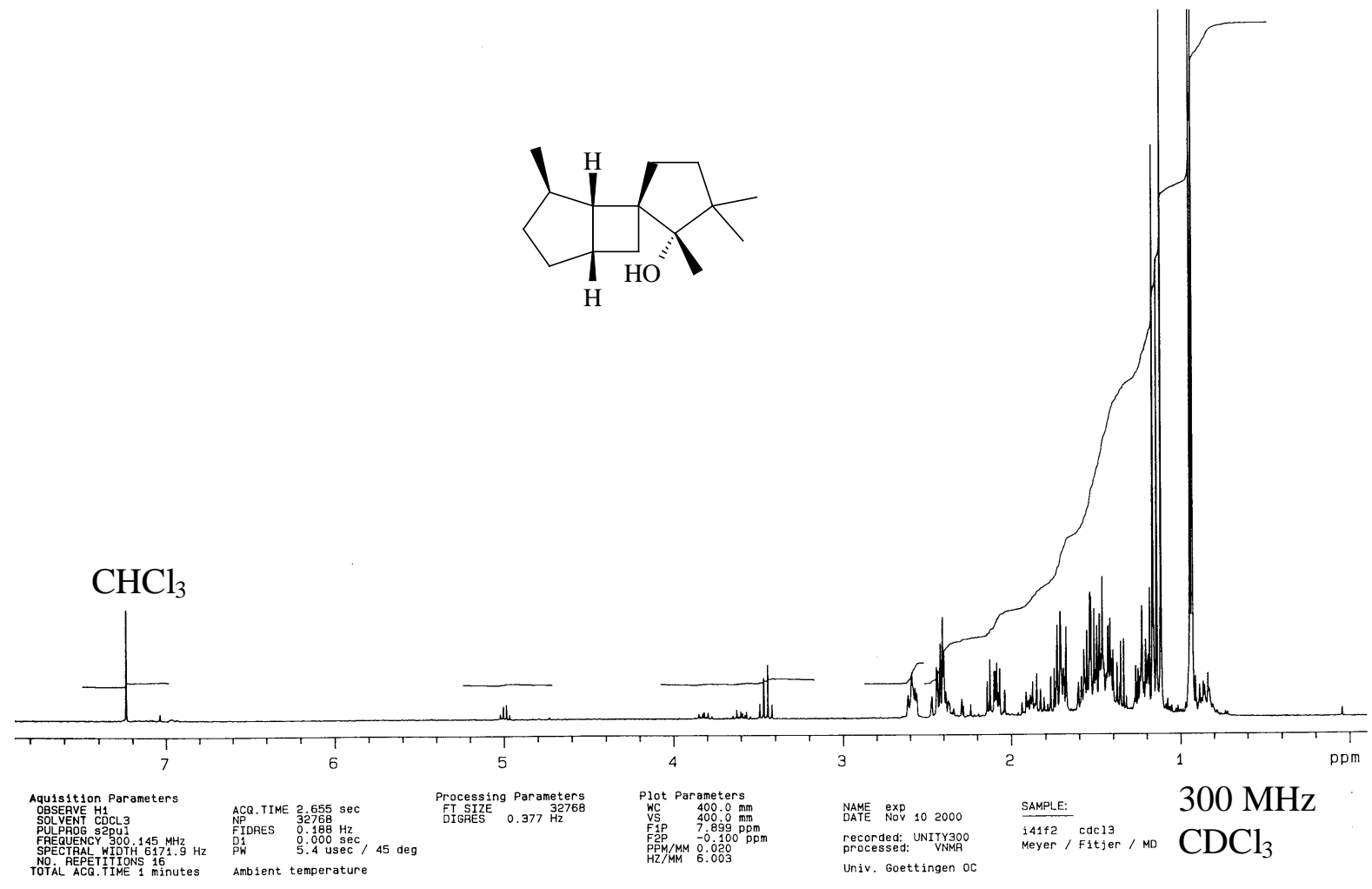

Abb. 63: ${ }^{1} \mathrm{H}-\mathrm{NMR}-\mathrm{Spektrum}$ von $\left(1 \mathrm{R}^{*}, 4 \mathrm{~S}^{*}, 5 \mathrm{R}^{*}, 6 \mathrm{~S}^{*}, 2^{\prime} \mathrm{R}^{*}\right)-2^{\prime}, 3^{\prime}, 3^{\prime}, 4-\mathrm{T}$ etramethyl-spiro$\left\{\right.$ bicyclo[3.2.0]heptan-6, $1^{\prime}$-cyclopentan-2'-ol\} (44)
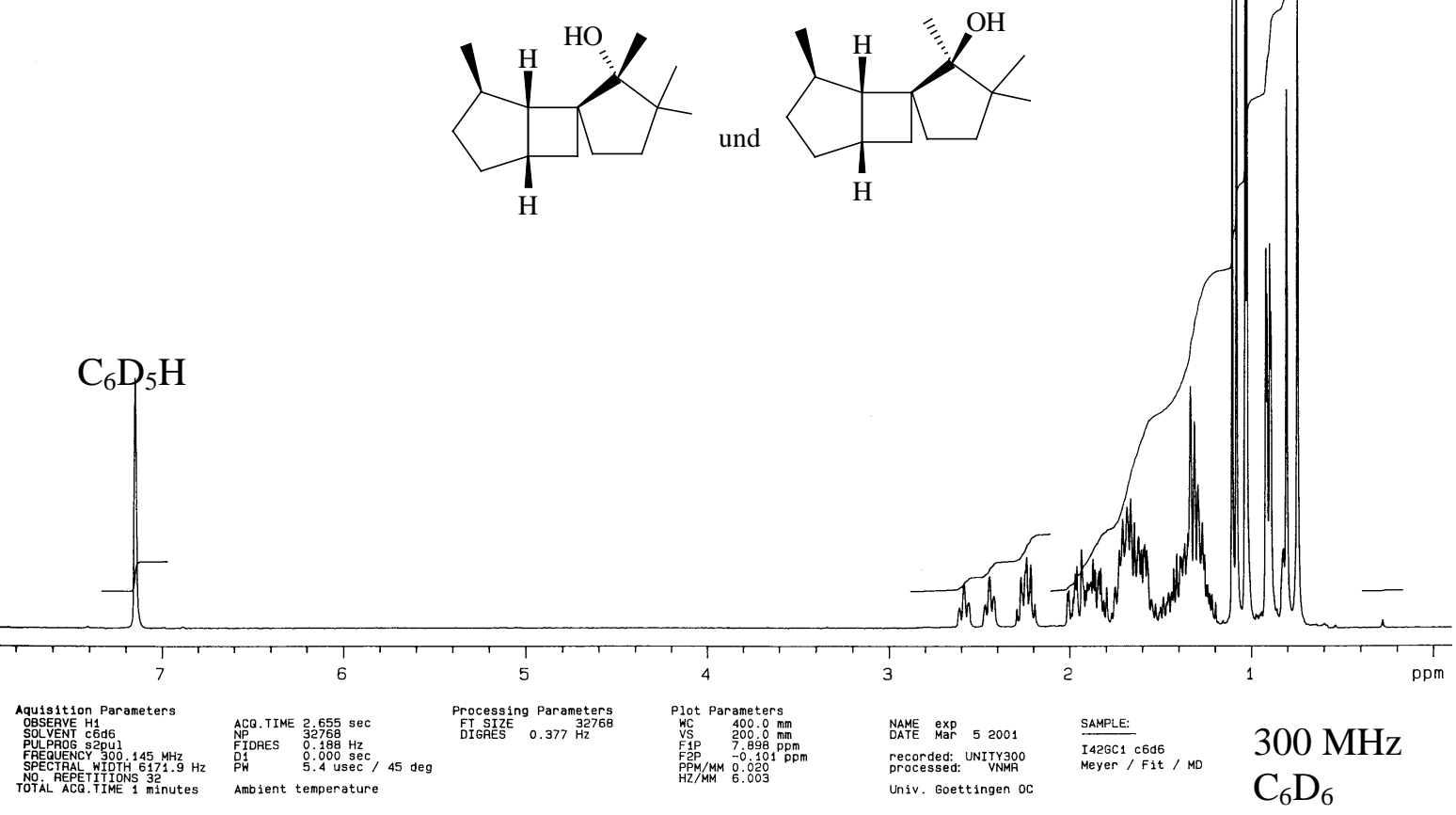

Abb. 64: ${ }^{1} \mathrm{H}-\mathrm{NMR}-\mathrm{Spektrum}$ von $\left(1 \mathrm{R}^{*}, 4 \mathrm{~S}^{*}, 5 \mathrm{R}^{*}, 6 \mathrm{R}^{*}, 2^{\prime} \mathrm{R}^{*}\right)-4,2^{\prime}, 3^{\prime}, 3^{\prime}-\mathrm{Tetramethyl}$-spiro-

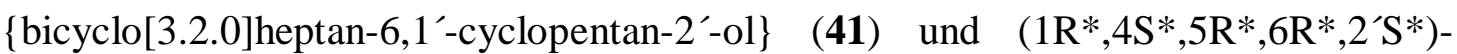
4,2', 3',3'-Tetramethyl-spiro $\{$ bicyclo[3.2.0]heptan-6,1'-cyclopentan-2'-on $\}$ (42) 


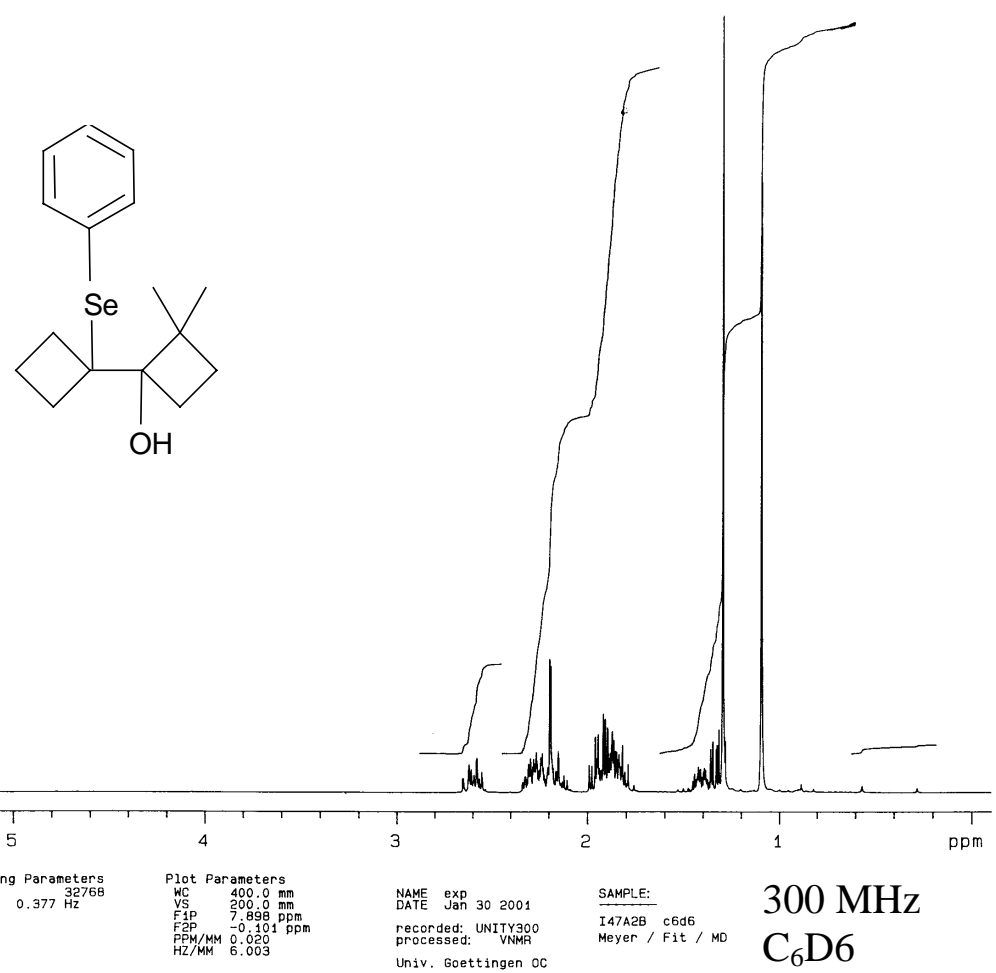

Abb. 65: ${ }^{1}$ H-NMR-Spektrum von 1-[(1'-Phenylseleno $)$ cyclobutyl]-2,2-dimethylcyclobutan-1ol (76)

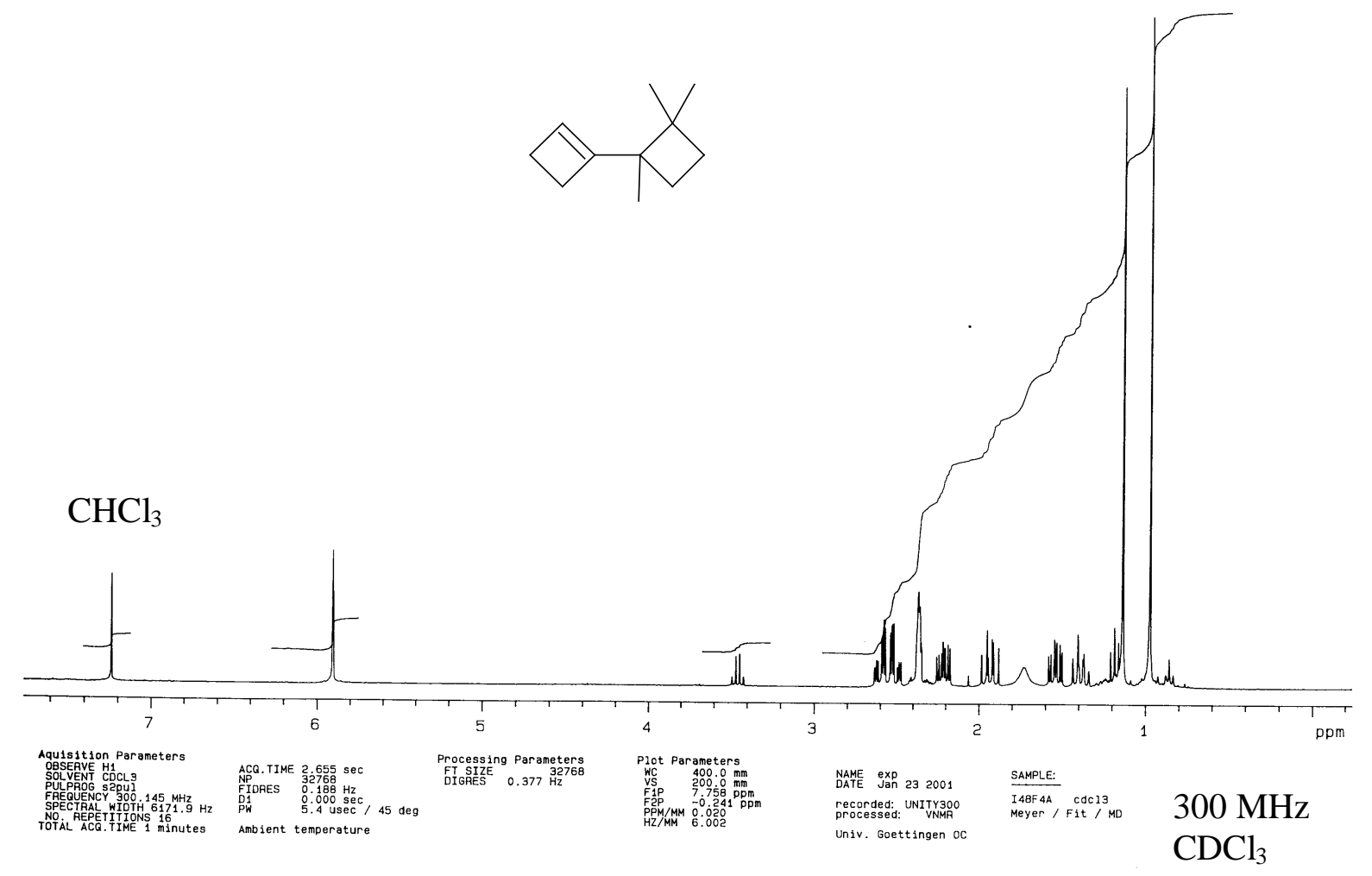

Abb. 66: ${ }^{1}$ H-NMR-Spektrum von 2,2-Dimethyl-bicyclobutyl-1'-en-1-ol (78) 


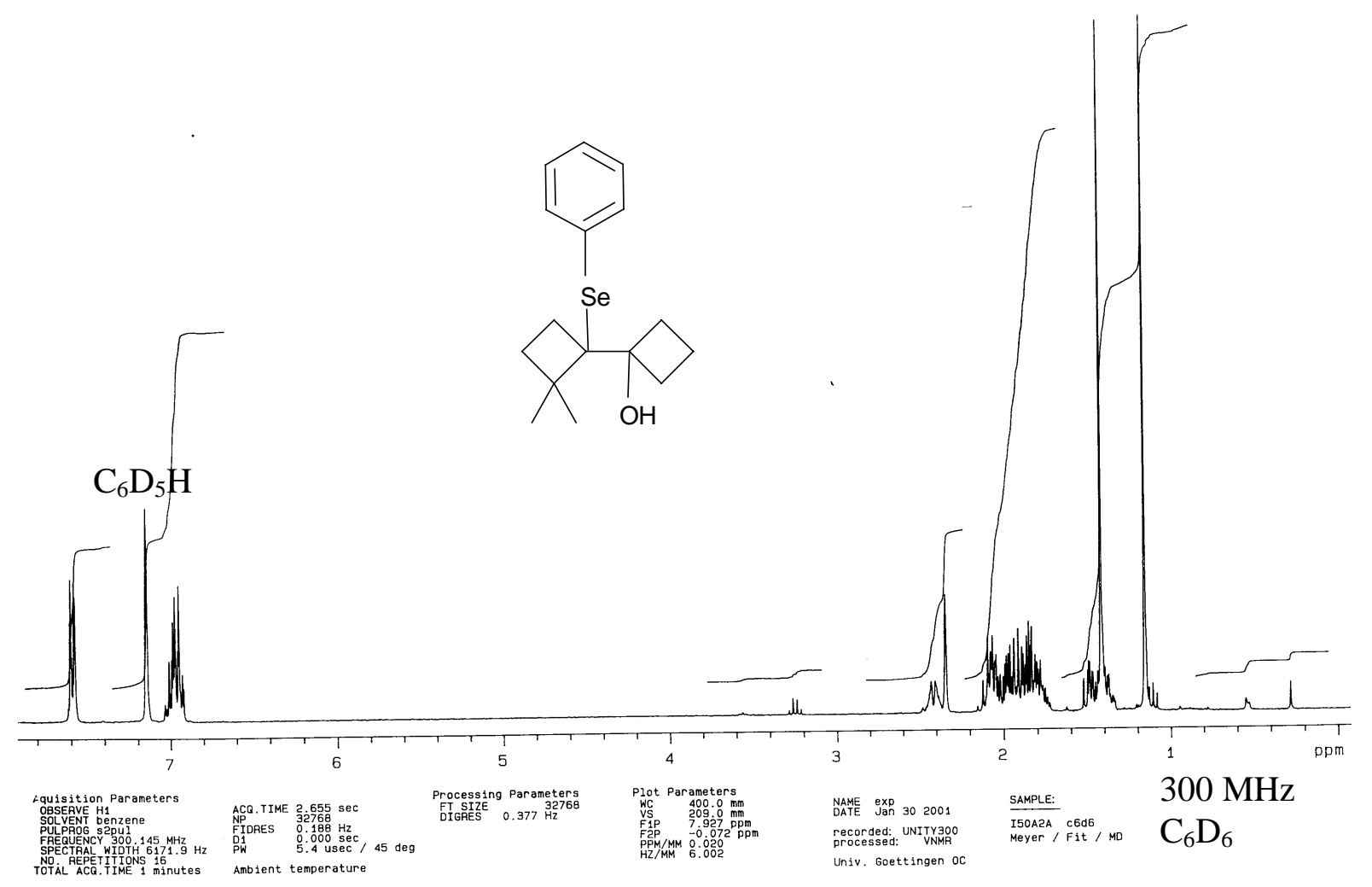

Abb. 67: ${ }^{1} \mathrm{H}-\mathrm{NMR}-$ Spektrum von 1-[(1'-Phenylseleno)-2',2'-dimethylcyclobutyl]cyclobutan1-ol (49)

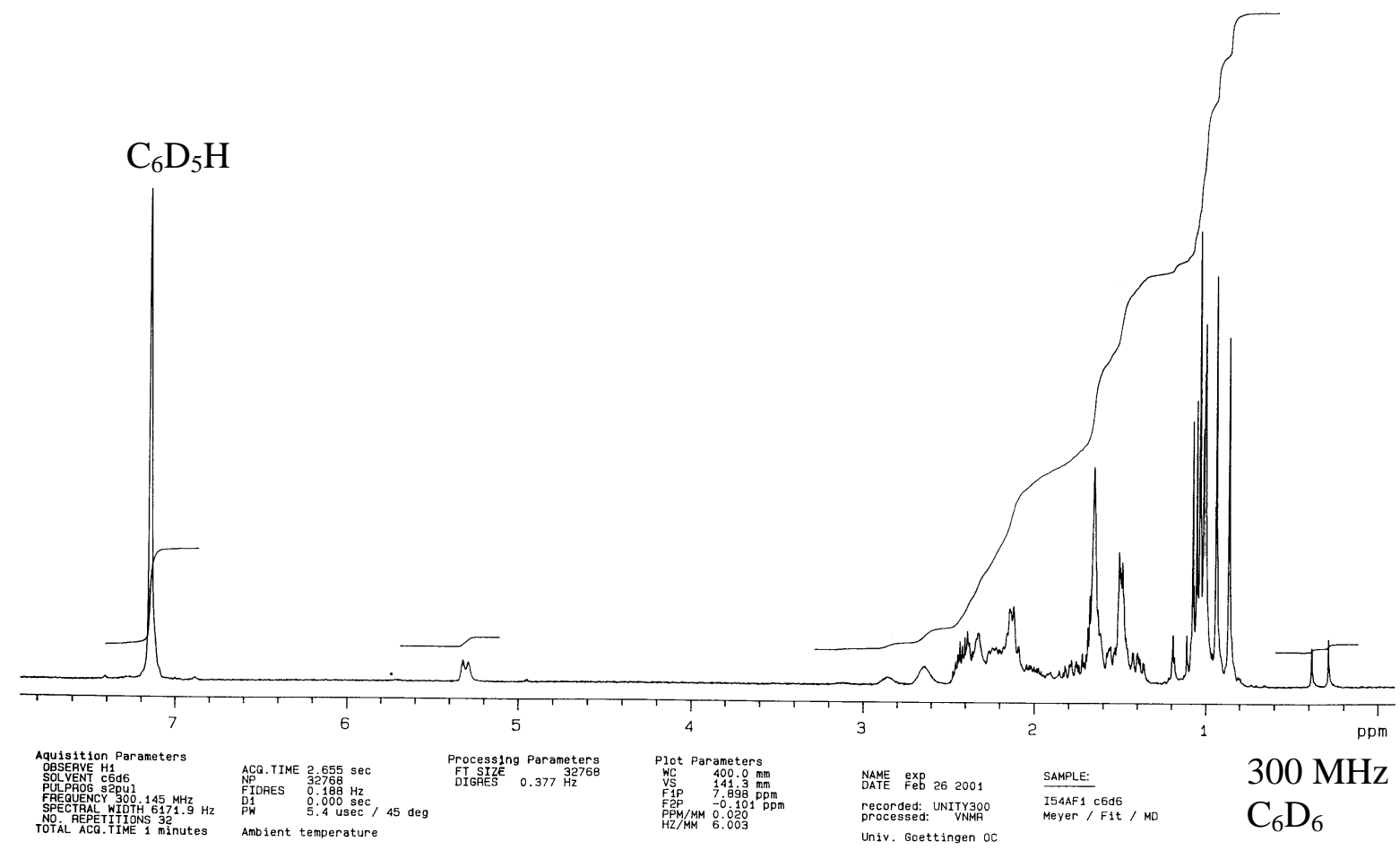

Abb. 68: ${ }^{1} \mathrm{H}-\mathrm{NMR}-$ Spektrum von 116/117 

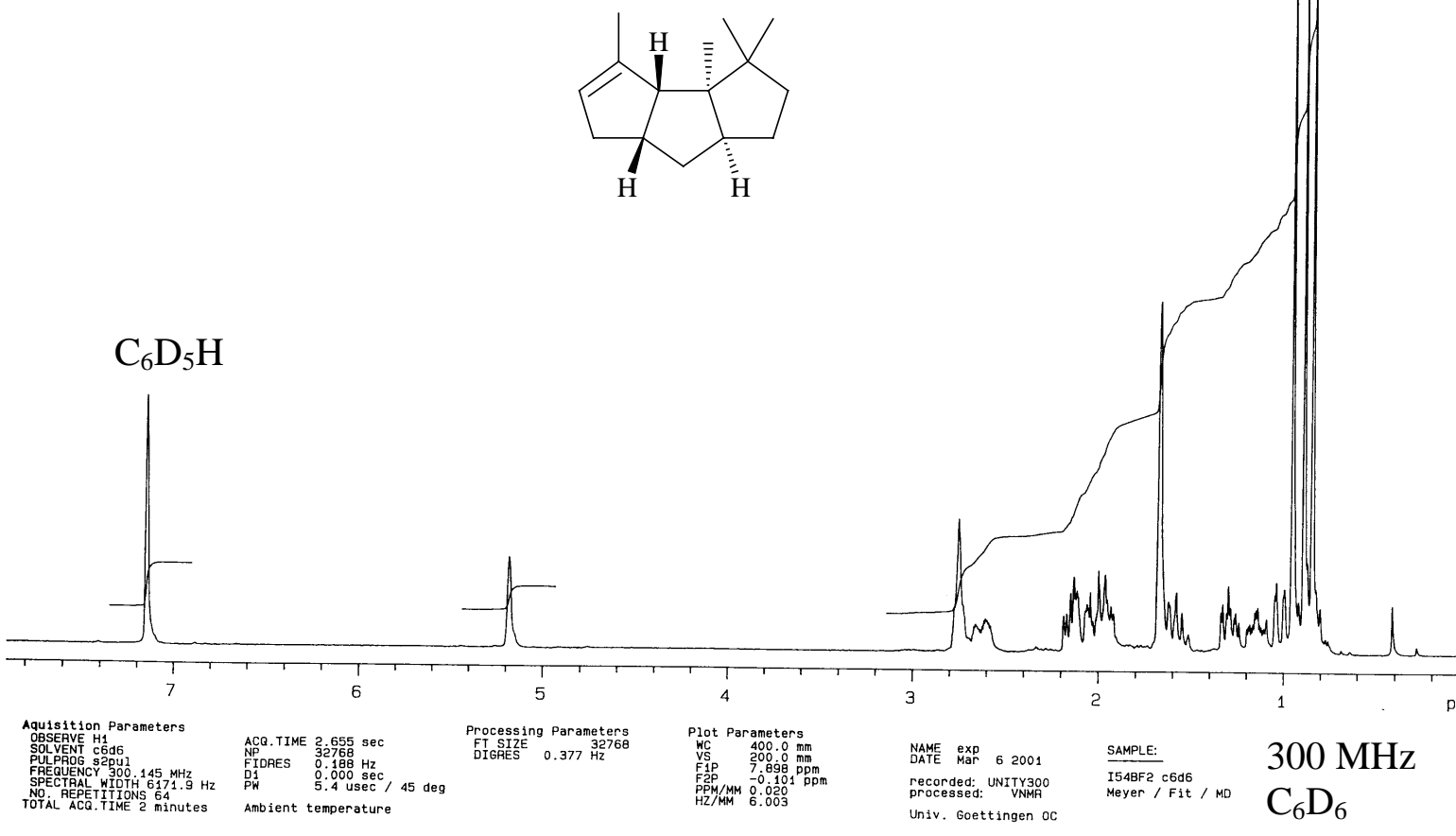

Abb. 69: ${ }^{1} \mathrm{H}-\mathrm{NMR}-$ Spektrum von $\left(3 \mathrm{aR}^{*}, 3 \mathrm{bR}^{*}, 6 \mathrm{aR}^{*}, 7 \mathrm{aR} *\right)-3,3,3 \mathrm{a}, 4-$ Tetramethyl-2,3,3a,3b,6, 6a, 7,7a-octahydro-1H-cyclopenta[a]pentalen (119)
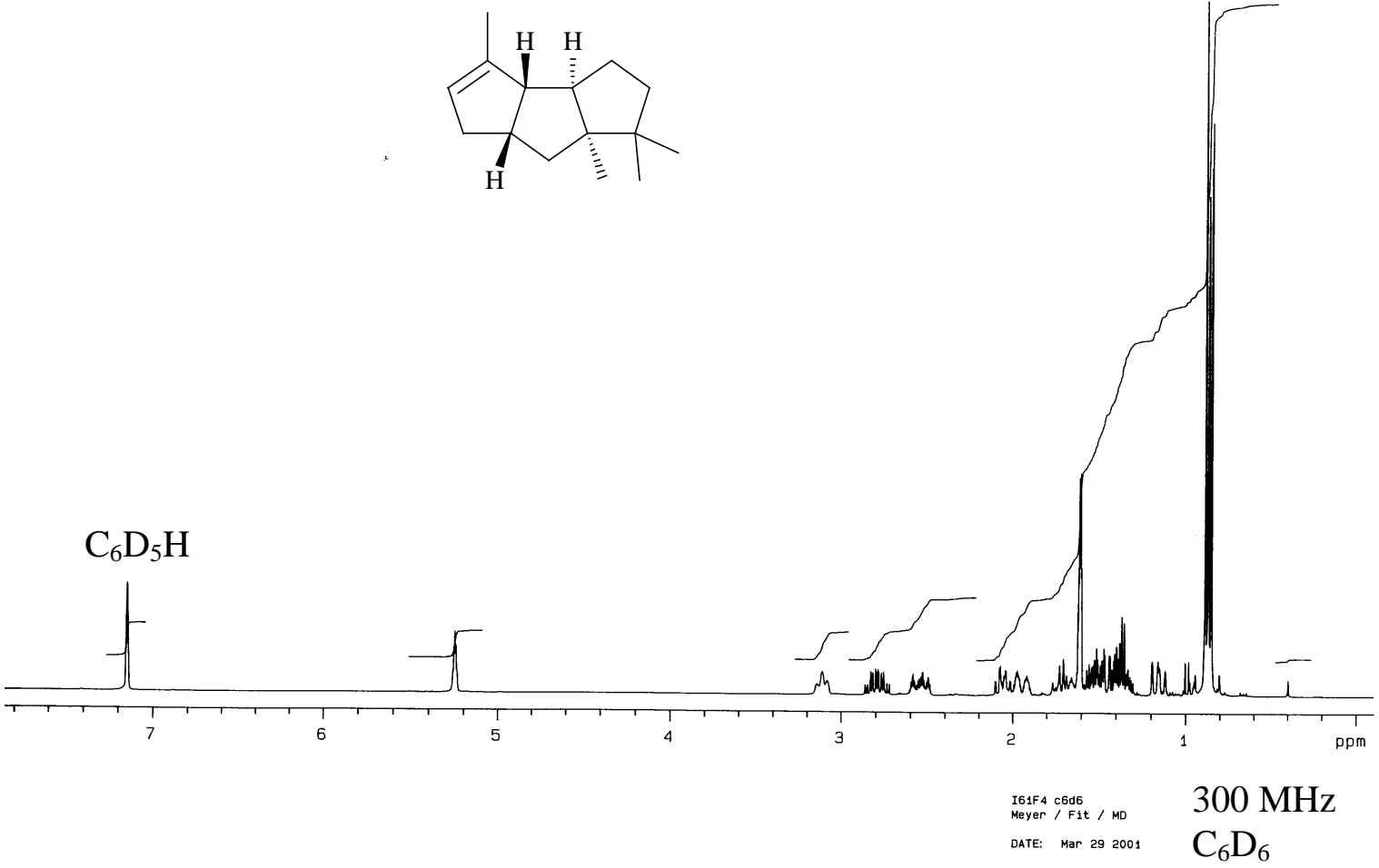

Abb. 70: ${ }^{1} \mathrm{H}-\mathrm{NMR}-$ Spektrum von (3aR*,3bR*,6aS*,7aS*)-1,1,4,7a-Tetramethyl-2,3,3a,3b,6, 6a, 7,7a-octahydro-1H-cyclopenta[a]pentalen (120) 


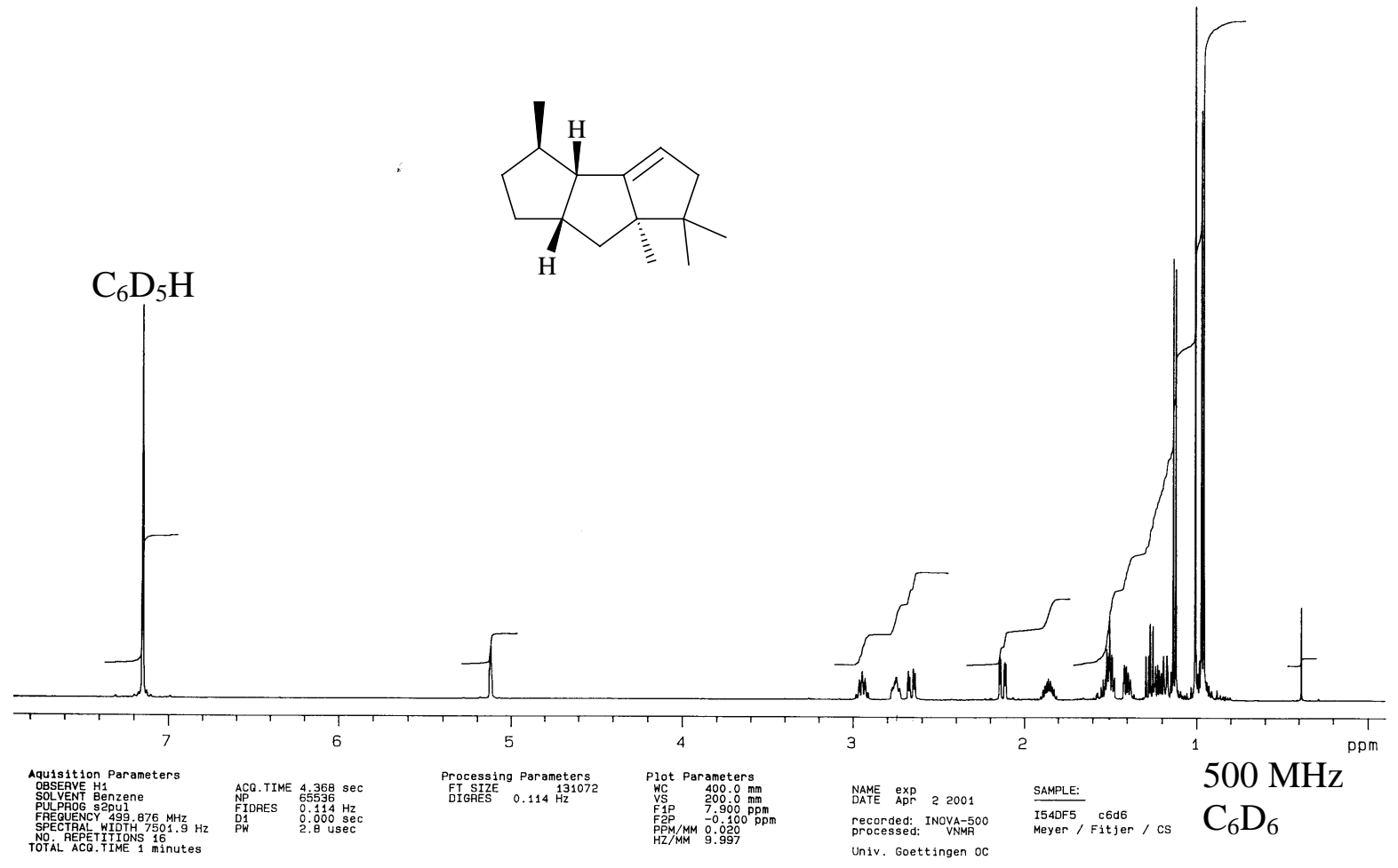

Abb. 71: ${ }^{1} \mathrm{H}-\mathrm{NMR}-$ Spektrum von (3R*,3aS*,6aR*,7aR*)-3,6,6,6a-Tetramethyl-2,3,3a,5,6, 6a,7,7a-octahydro-1H-cyclopenta[a]pentalen (118)
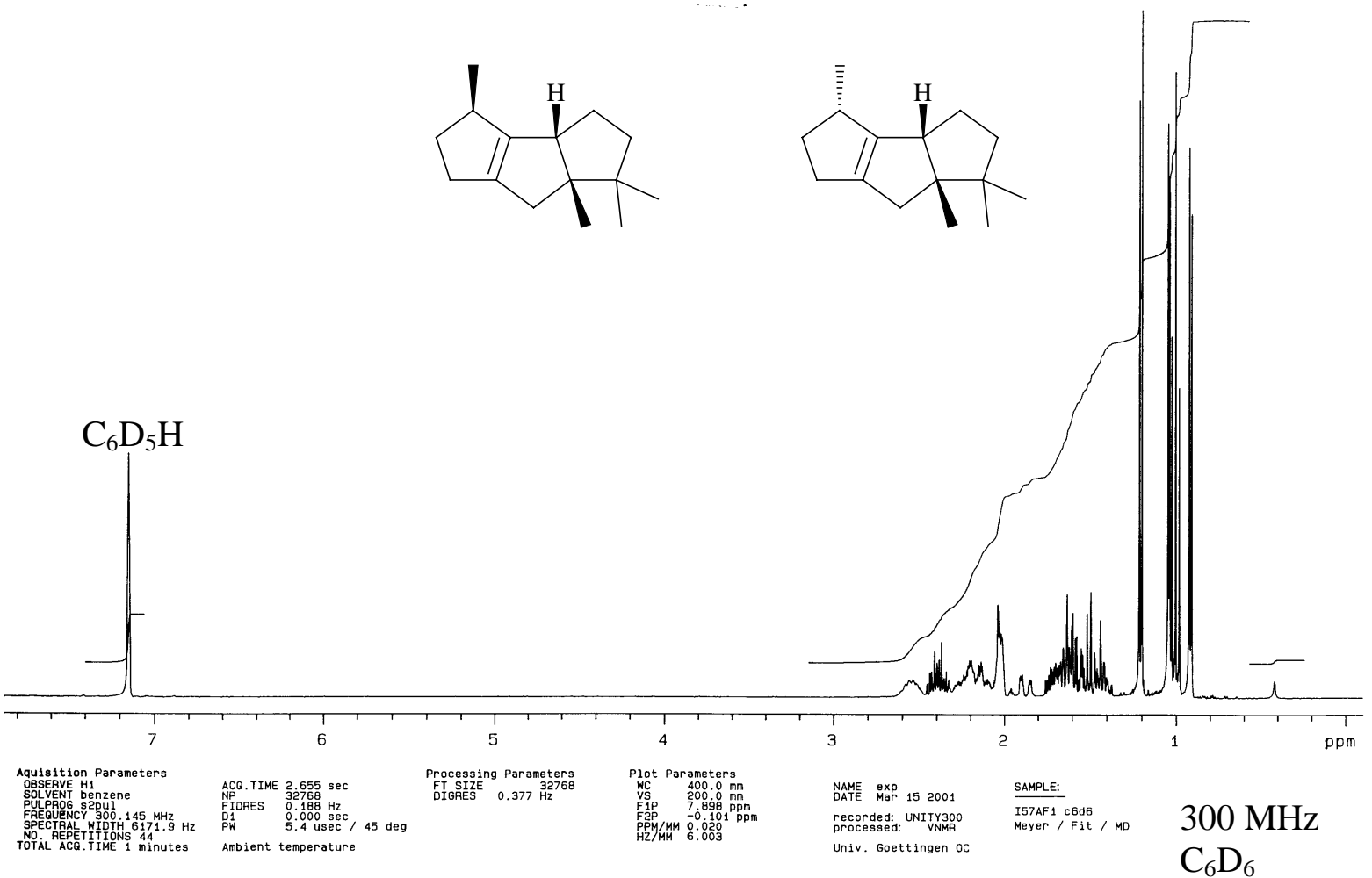

Abb. 72: ${ }^{1} \mathrm{H}-\mathrm{NMR}-$ Spektrum von $\left(3 \mathrm{R}^{*}, 3 \mathrm{bS} *, 6 \mathrm{aS} *\right)$ - und $\left(3 \mathrm{R}^{*}, 3 \mathrm{bR}^{*}, 6 \mathrm{aR} *\right)-3,6,6,6 \mathrm{a}-\mathrm{Tetra}-$ methyl-2,3,3a,5,6,6a, 7,7a-octahydro-1H-cyclopenta[a]pentalen (108/109) 


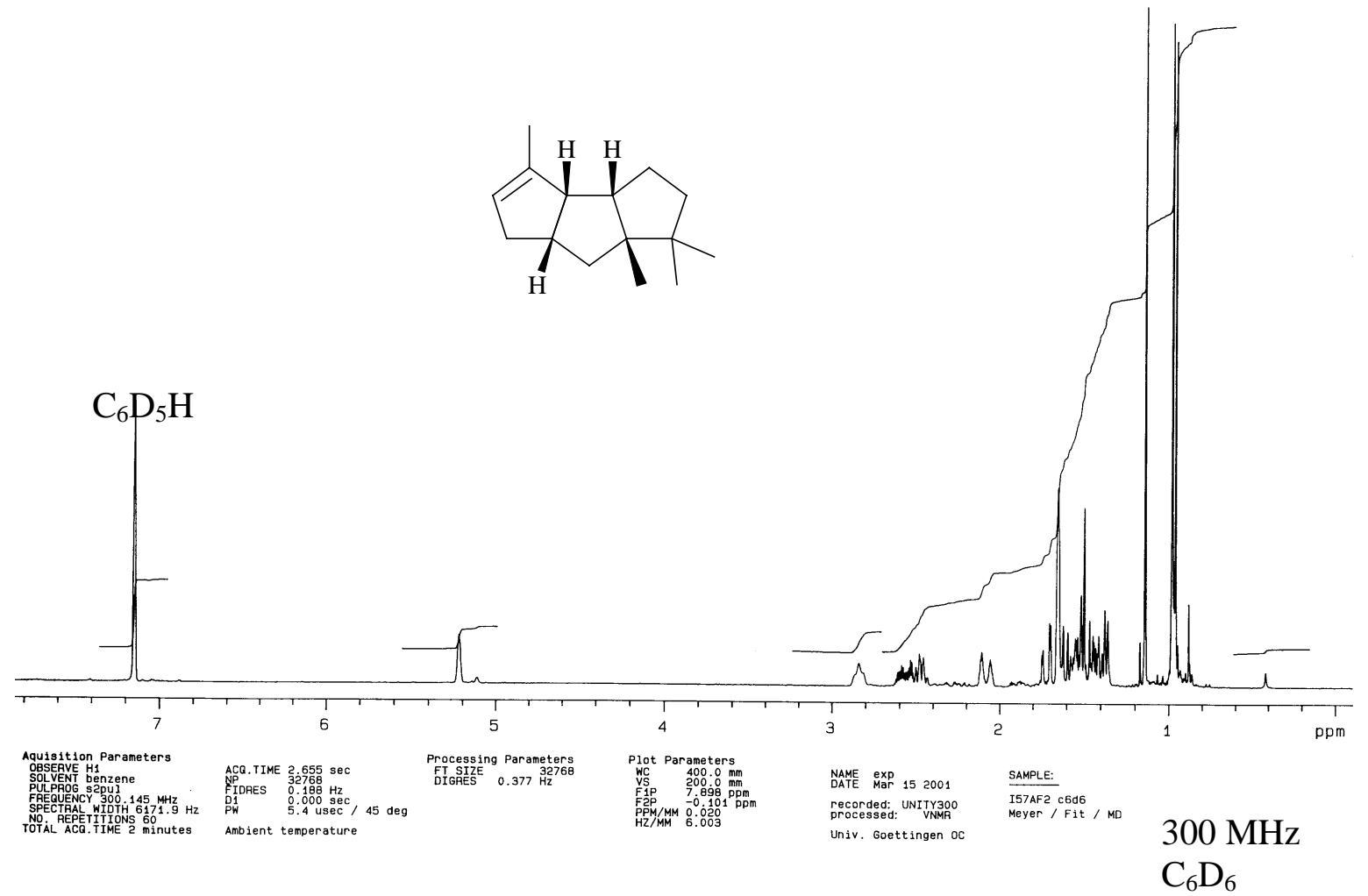

Abb. 73: ${ }^{1} \mathrm{H}-\mathrm{NMR}-$ Spektrum von $\left(3 \mathrm{aR}^{*}, 3 \mathrm{bS} *, 6 \mathrm{aR} *, 7 \mathrm{aS} *\right)-1,1,4,7 \mathrm{a}-$ Tetramethyl-2,3,3a,3b,6, 6a, 7,7a-octahydro- $1 H$-cyclopenta[a]pentalen (110)

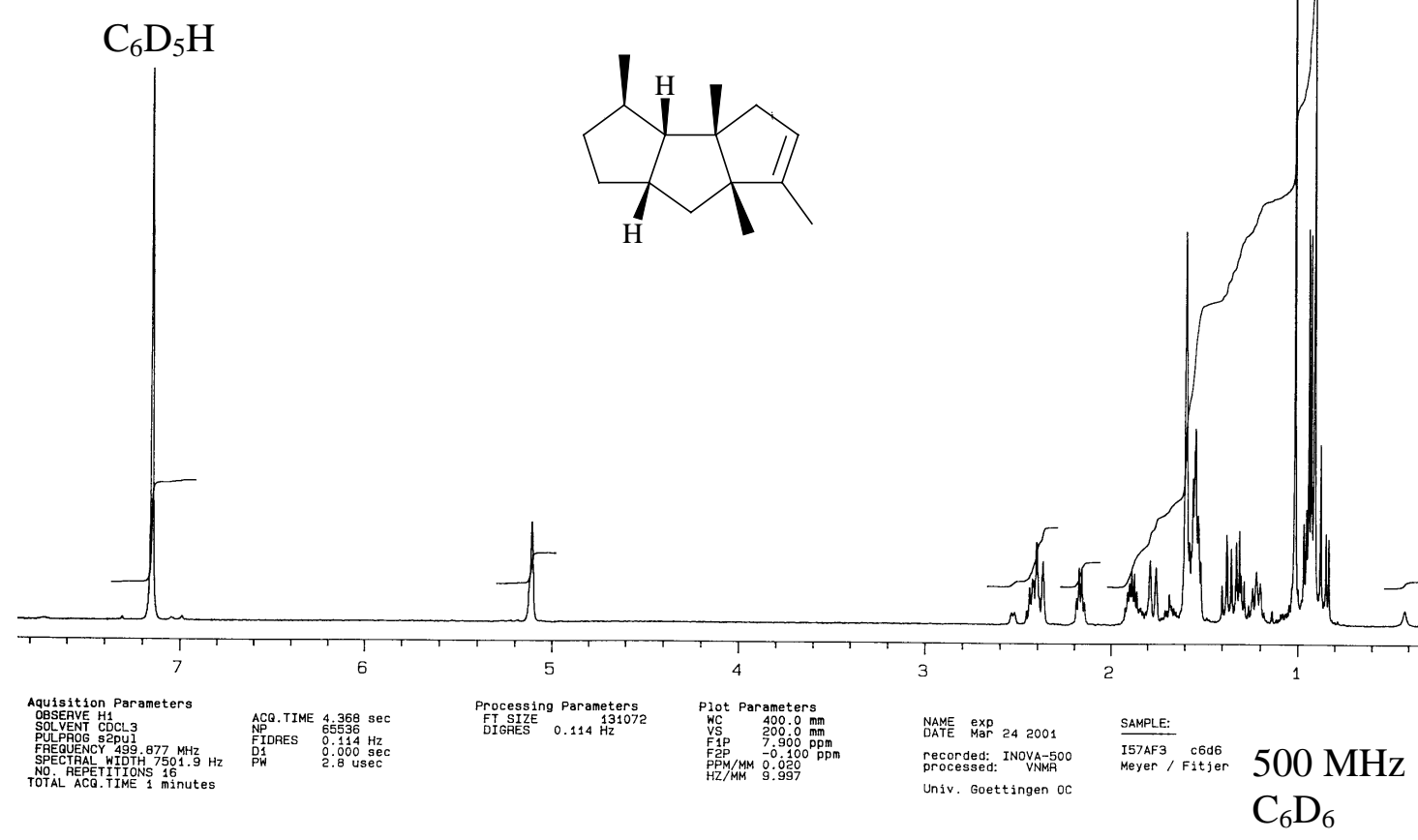

Abb. 74: ${ }^{1} \mathrm{H}-\mathrm{NMR}-$ Spektrum von $\left(3 \mathrm{R}^{*}, 3 \mathrm{aS} *, 3 \mathrm{bS} *, 6 \mathrm{aS} *, 7 \mathrm{aS} *\right)-3,3 \mathrm{~b}, 6,6 \mathrm{a}-\mathrm{Tetramethyl-}$ 2,3,3a,3b,4,6a,7,7a-octahydro-1H-cyclopenta[a]pentalen (111) 

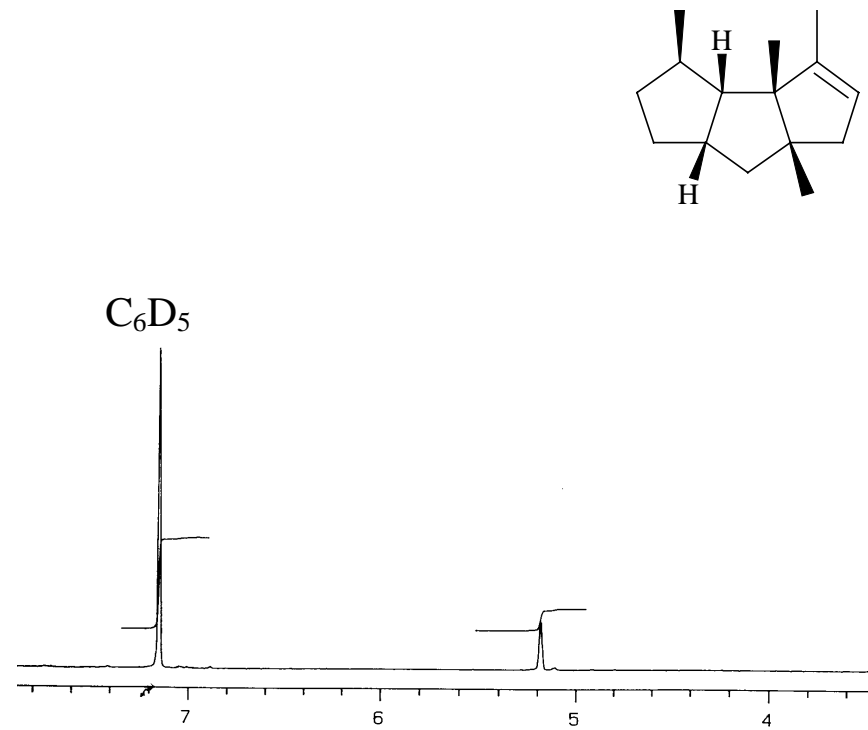

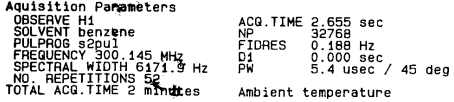

Processing Parameters
FI SIIE Par 32768
DIGAES $0.377 \mathrm{~Hz}$

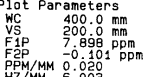

NAME EXP
DATE Mar 152001
recorded: UNIIY3

recorded: UNITY300
processed: VNMA

SAMPLE:

$500 \mathrm{MHz}$

Abb. 75: ${ }^{1} \mathrm{H}-\mathrm{NMR}-$ Spektrum von $\left(3 \mathrm{R}^{*}, 3 \mathrm{aS} *, 3 \mathrm{bS} *, 6 \mathrm{aS} *, 7 \mathrm{aS} *\right)-3,3 \mathrm{~b}, 4,6 \mathrm{a}-$ Tetramethyl-

2,3,3a,3b,6,6a,7, 7a-octahydro-1H-cyclopenta[a]pentalen (112) 


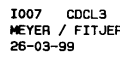

INSTRUMENT: VXR-200
OQSERVE C13

Frequency 50.309 MHZ

Spectral width 12004.8 Hz
Acquisition time 1.357 sec
Aelaxat ion delay 0.000 sec

Pulse 30.3 degrees

5120 repetition

Frequency 200.057 Mnt

H1gh power 56

Decoupler continuously on

WATAZ-16 modulated

Line brosdening $1.0 \mathrm{~Hz}$

Fr size 32768 $1.0 \mathrm{~Hz}$
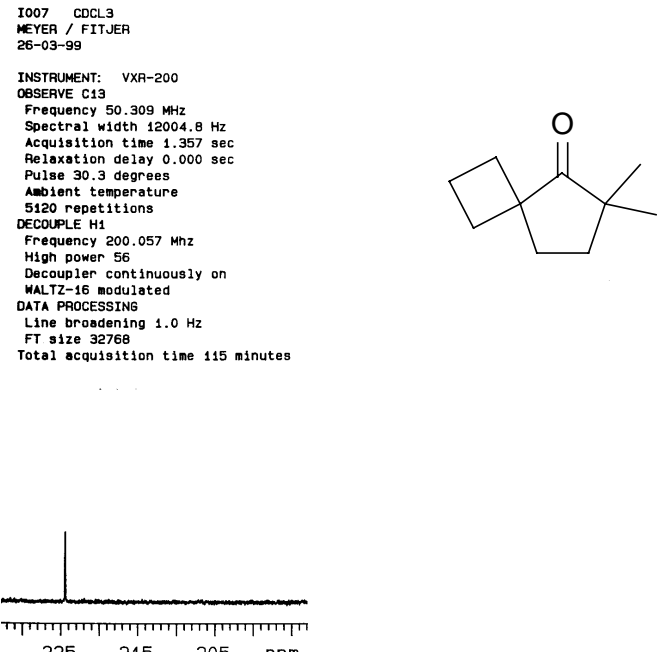

$225 \quad 215 \quad 205 \quad \mathrm{ppm}$

(1)

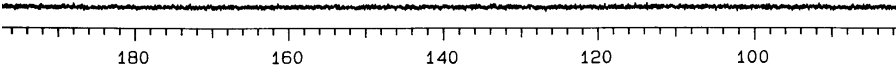

Bo

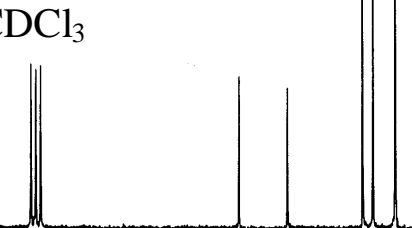

160

140

60

40

Abb. 76: ${ }^{13}$ C-NMR-Spektrum von 6,6-Dimethyl-spiro[3.4] octan-5-on (51)

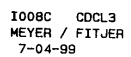

INSTRUMENT: VXA-200
OBSEAVE C13

Frequency $50.309 \mathrm{MHz}$

Spectral width $11061.9 \mathrm{~Hz}$
Acquisition time $1.357 \mathrm{sec}$
Reesaxation delay $0.000 \mathrm{sec}$

Relaxation delay 0.000

Ambient temperature

ECOUPLE H1

Frequency $200.057 \mathrm{Mhz}$

Decoup per continuousiy on

MALTZ-16 modulated

Line broadening $1.0 \mathrm{~Hz}$

FT size 32768
Total acquisition time 69 minutes

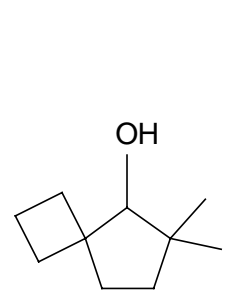

$\mathrm{CDCl}_{3}$

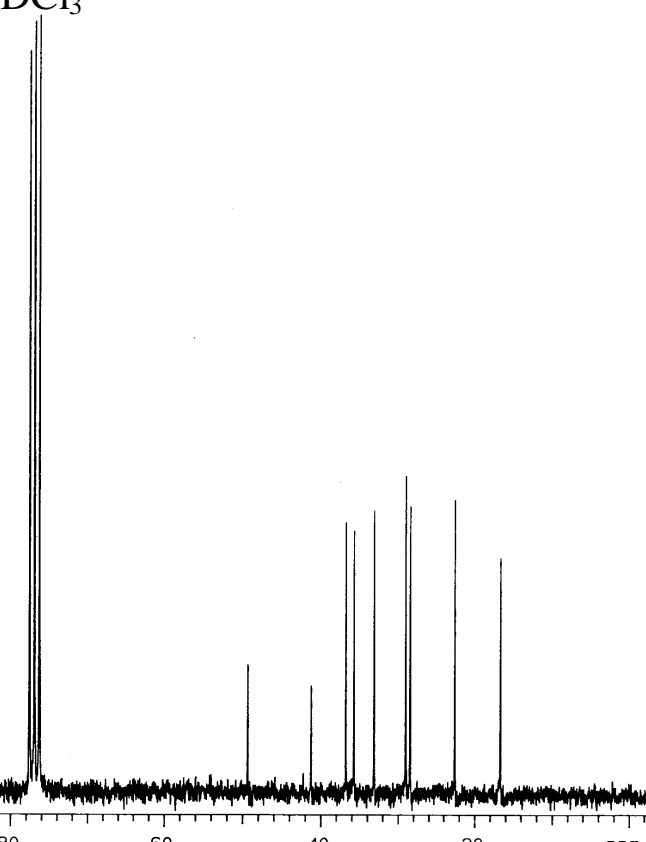

180

160

140

120

100

40

$50 \mathrm{MHz}$

Abb. 77: ${ }^{13}$ C-NMR-Spektrum von 6,6-Dimethyl-spiro[3.4] octan-5-ol (24)

$\mathrm{CDCl}_{3}$ 


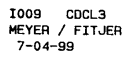

INSTRUMENT: VXR-200

INSTRUMENT: VXA-200
OBSERVE C13

Frequency $50.309 \mathrm{MHz}$
Spectral width $11061.9 \mathrm{~Hz}$
Acquisition t t 1 me $1.357 \mathrm{sz}$

Acquisition time $1.357 \mathrm{sec}$
Relaxation delay $0.000 \mathrm{sec}$

Pulse 30.3 degrees
Ambient temperature

Ambient temperature
3072 repetitions

Frequency $200.057 \mathrm{Mh}$

Hign power 56 .

Decoupler cont inuously
HALTZ-16 modulated

DATA PROCESSING

Fi size 32769 1.0

Total acquisition time 69 minute

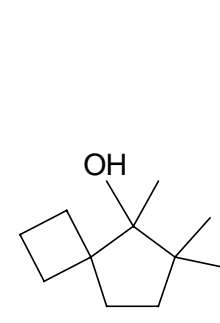

$\mathrm{CDCl}_{3}$

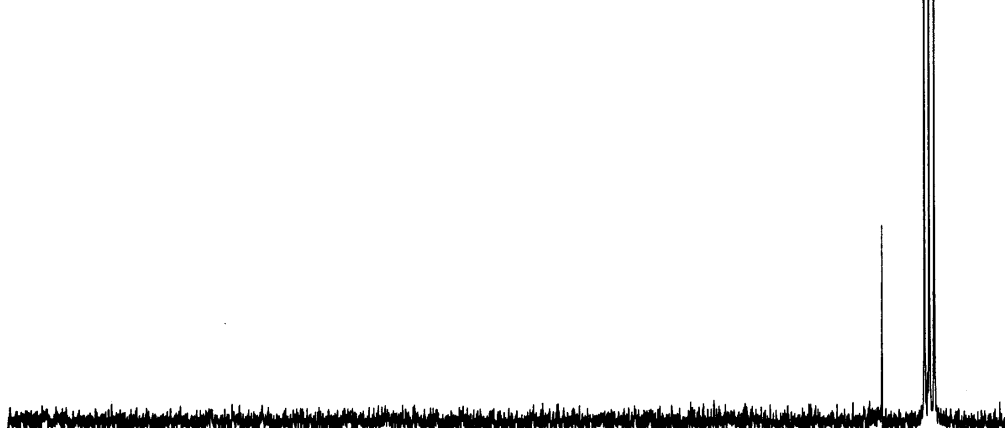

Abb. 78: ${ }^{13}$ C-NMR-Spektrum von 5,6,6-Trimethyl-spiro[3.4] octan-5-ol (25)

\section{$50 \mathrm{MHz}$}

$\mathrm{CDCl}_{3}$

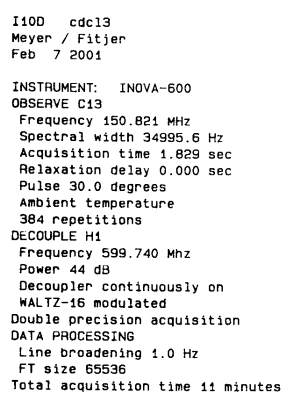

Total acquisition time 11 minutes
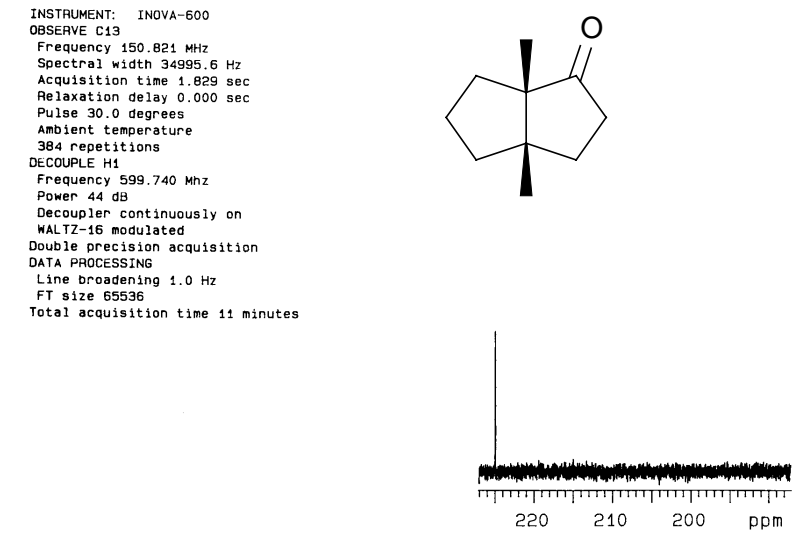

160

140

120

100

80

$\mathrm{CDCl}_{3}$

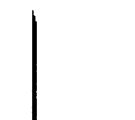

$150 \mathrm{MHz}$

$\mathrm{CDCl}_{3}$

Abb. 79: ${ }^{13}$ C-NMR-Spektrum von 3a,6a-Dimethylhexahydro-pentalen-1-on (30) 


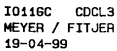

INSTRUMENT: VXA-200 OBSERVE C13 Frequency $50.309 \mathrm{MHz}$
Spectral Kidth $11061.9 \mathrm{~Hz}$
Acquisstion time 1.357 Acquisition time $1.357 \mathrm{sec}$
Relaxation delay $0.000 \mathrm{sec}$ Pulse 30.3 degrees Ambient temperature 5120 repetitions Frequency $200.057 \mathrm{Mnz}$ High pouer 56. WALTZ-16 modulated DATA PROCESSING thane broadening $1.0 \mathrm{~Hz}$

$\mathrm{CDCl}_{3}$

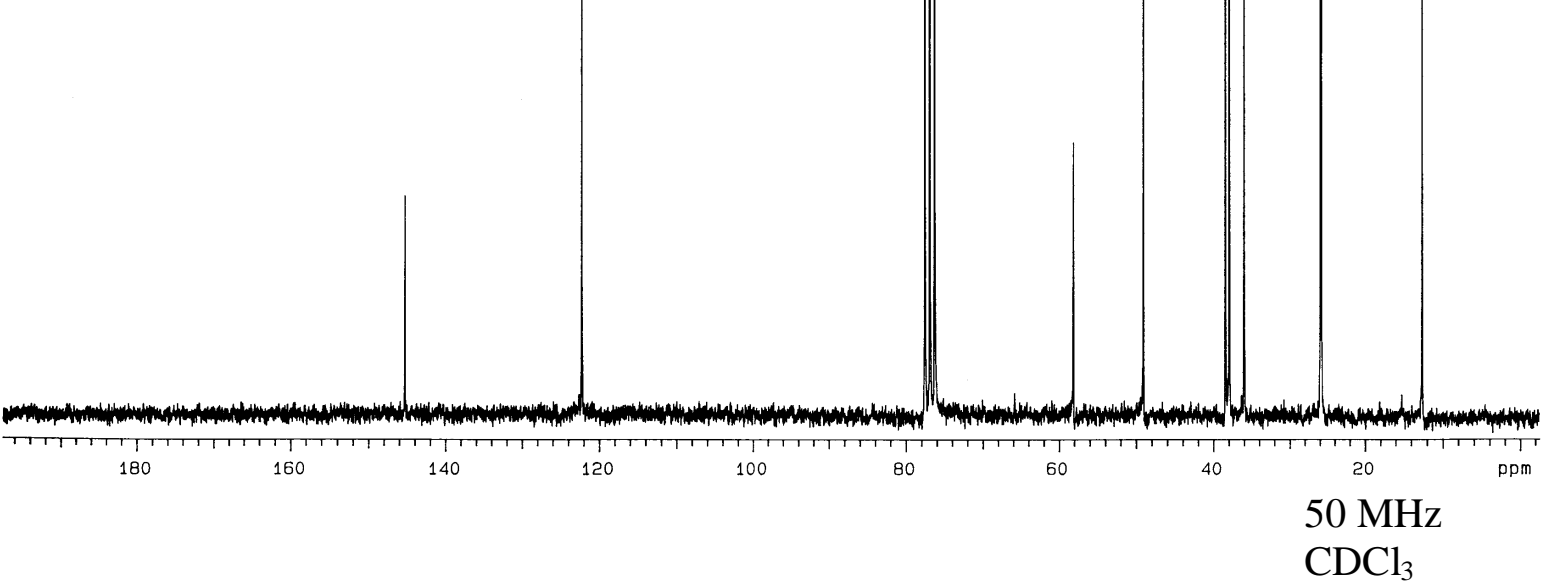

Abb. 80: ${ }^{13}$ C-NMR-Spektrum von 6,6a-Dimethyl-1,2,3,3a,4,6a-hexahydro-pentalen (60)
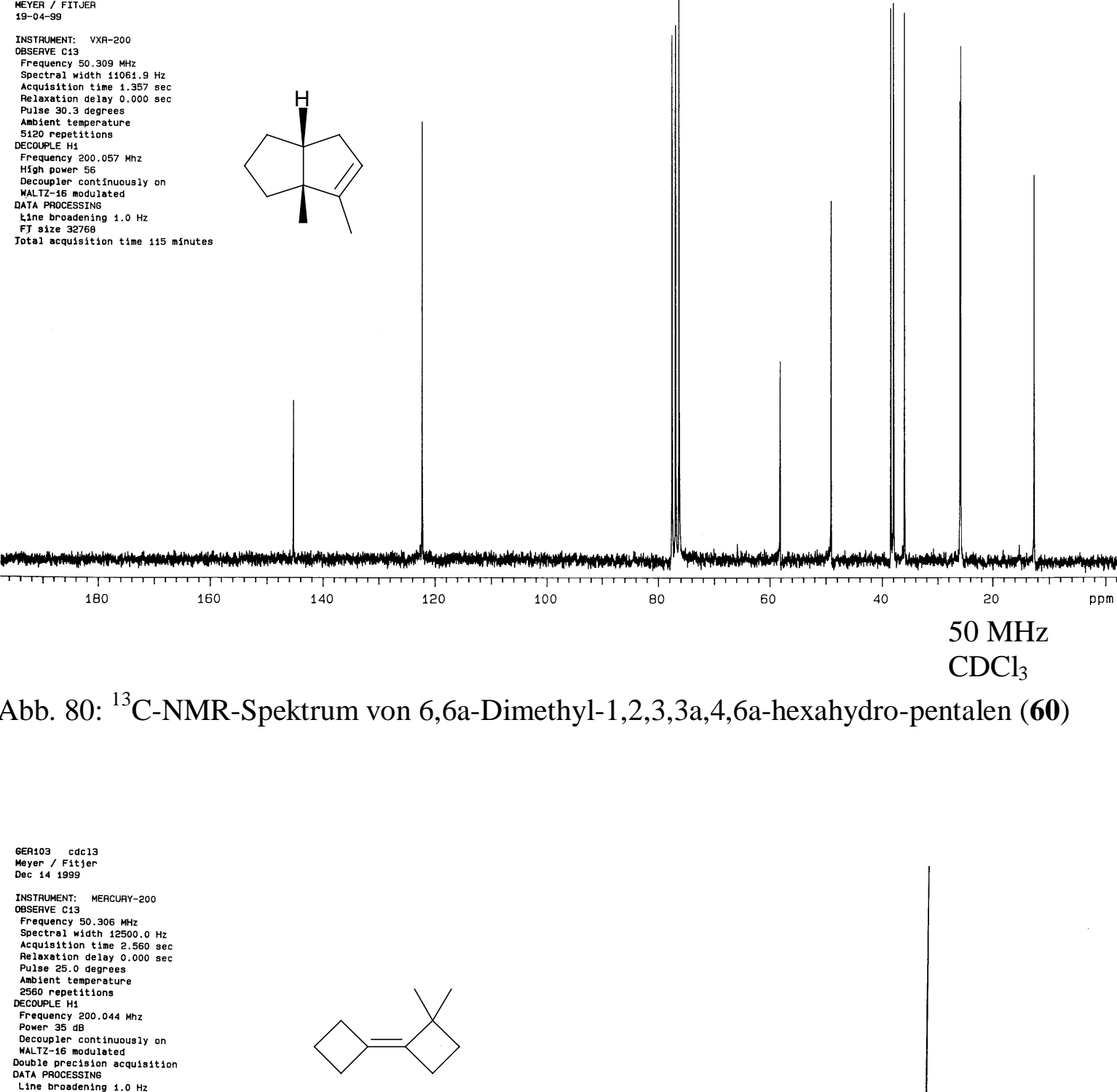

$50 \mathrm{MHz}$

Total acquisition time 109 minutes

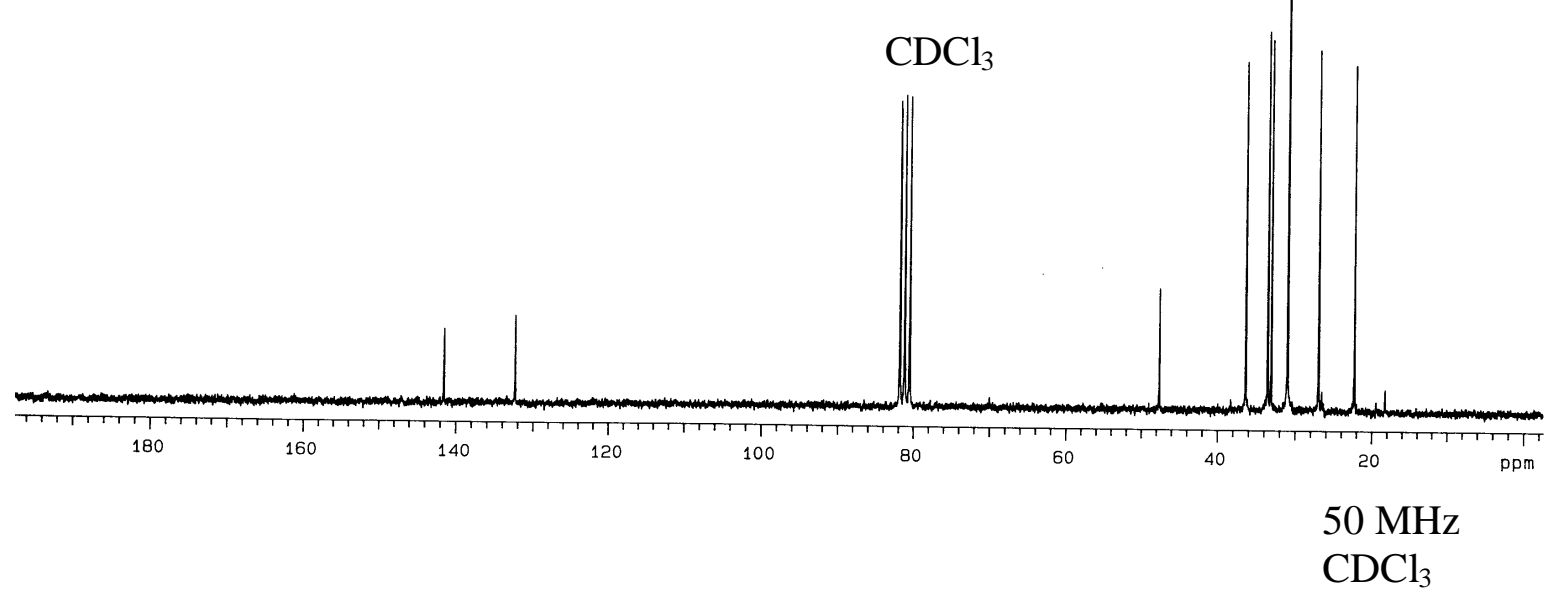

Abb. 81: ${ }^{13}$ C-NMR-Spektrum von 2,2-Dimethylbicyclobutyliden (26) 


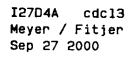

INSTRUMENT: MEACURY-200
OBSEAVE C13 $^{-13}$

Frequency 50.306 MHZ

Acquisition time $2.560 \mathrm{sec}$

Relaxation delay $0.000 \mathrm{sec}$

Ambient temperature

2355 repetitions

Frequency 200.044 Mhz

Frequency $200.044 \mathrm{Mhz}$
Power $35 \mathrm{~dB}$

Decoupler continuously on

Double precision acquisition

DATA PROCESSING

Line broadening $1.0 \mathrm{~Hz}$

Total acquisition time 100 minutes

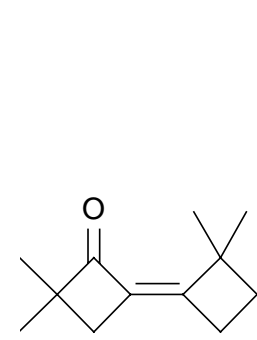

$\mathrm{CDCl}_{3}$

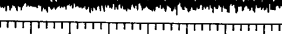

$220 \quad 210 \quad 200 \quad 0010$

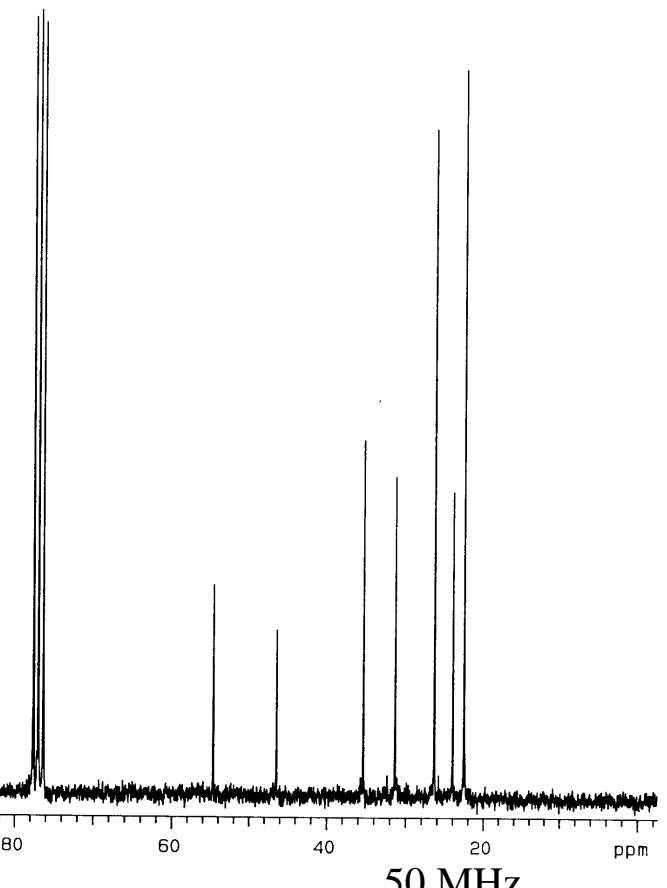

180

160

$140 \quad 120$

100

$50 \mathrm{MHz}$

$\mathrm{CDCl}_{3}$

Abb. 82: ${ }^{13}$ C-NMR-Spektrum von 3,3,2',2'-Tetramethyl-bicyclobutyliden-2-on (62)
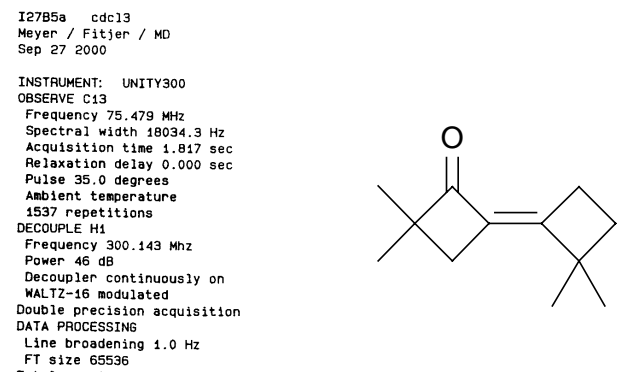

Line broadening $1.0 \mathrm{~Hz}$

total acquisition time 46 minutes
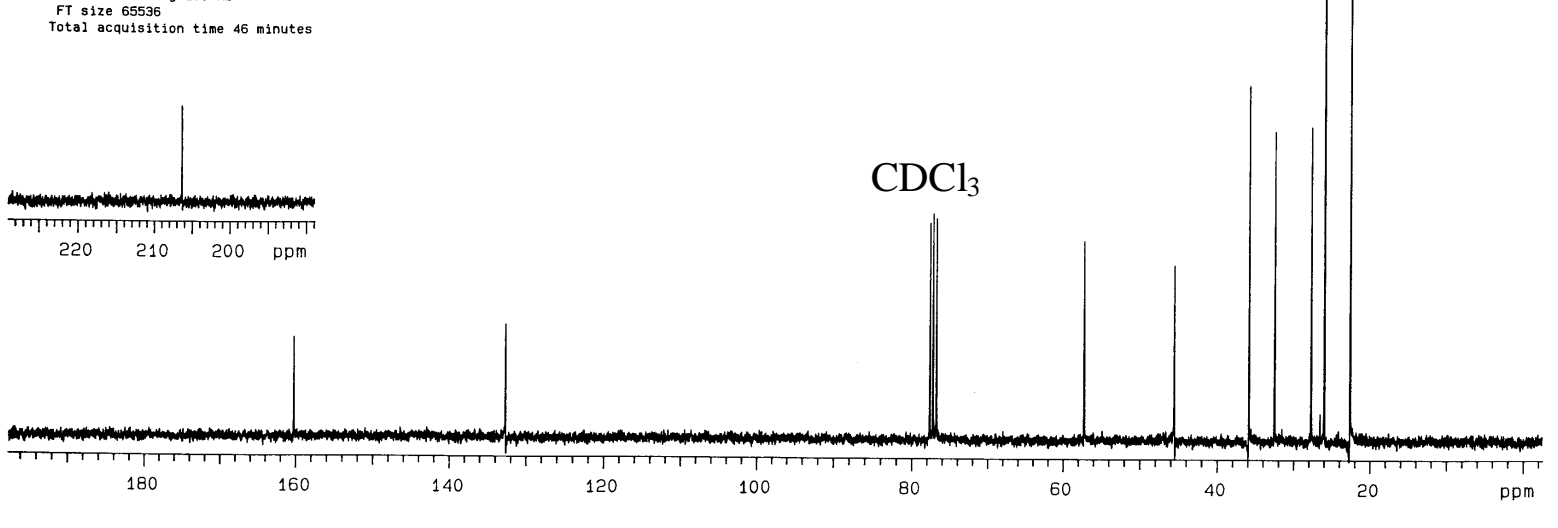

$75 \mathrm{MHz}$

$\mathrm{CDCl}_{3}$

Abb. 83: ${ }^{13}$ C-NMR-Spektrum von 3,3,2', $2^{\prime}$-Tetramethyl-bicyclobutyliden-2-on (63) 


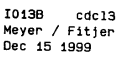

INSTRUMENT: MERCUAY-200
OBSERVE C13 MERT

Frequency $50.306 \mathrm{MHz}$

Spectral width $12500.0 \mathrm{~Hz}$
Acquisition time $2.560 \mathrm{sec}$ Relaxat ín delay $0.000 \mathrm{sec}$ Ambient temperature 2384 repetitions DECOUPLE H1
Frequency $200.044 \mathrm{Mhz}$
Power $35 \mathrm{~dB}$ Decoupler continuously on waLTZ-16 modulated
oubie precision acquisition

ATA PROCESSING

ITe broadening $1.0 \mathrm{~Hz}$

rotal acquisition time 101 minutes
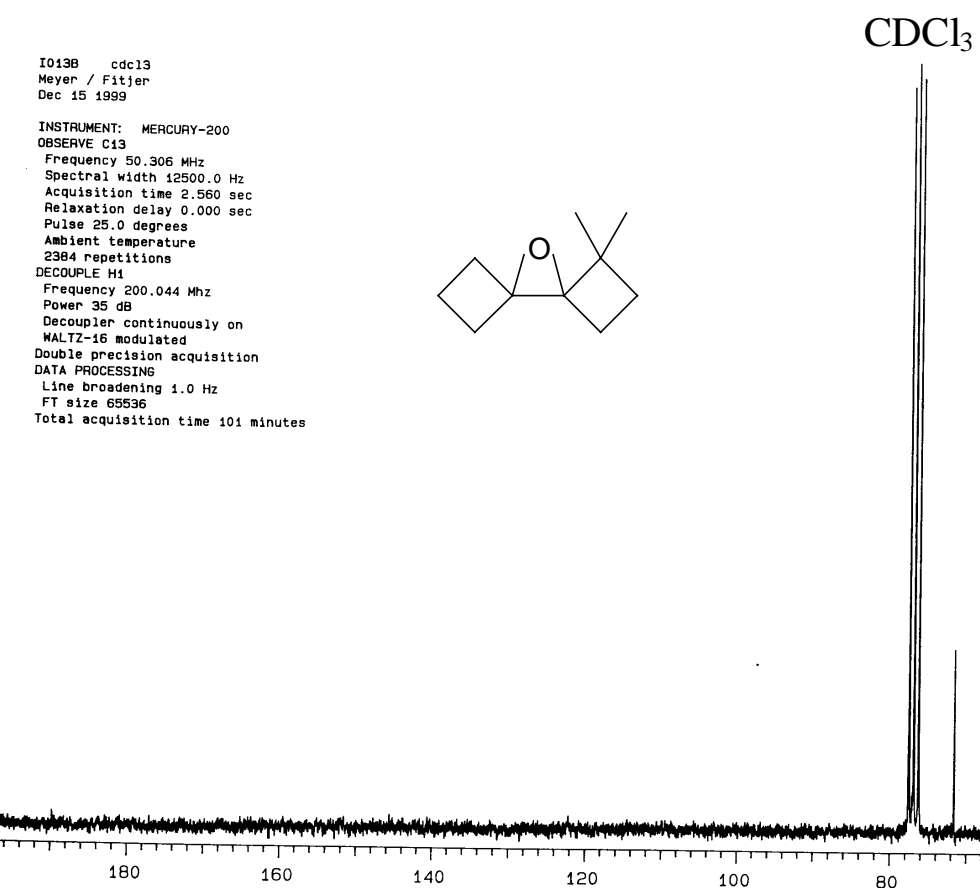

Abb. 84: ${ }^{13} \mathrm{C}-\mathrm{NMR}-\mathrm{Spektrum}$ von 1,1-Dimethyl-9-oxa-dispiro[3.0.3.1]nonan (64)
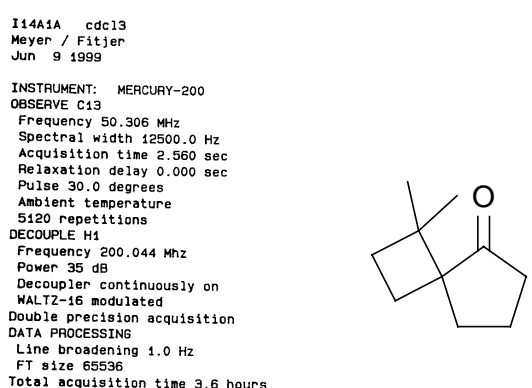

$\mathrm{CDCl}_{3}$

Total acquisition time 3.6 hours
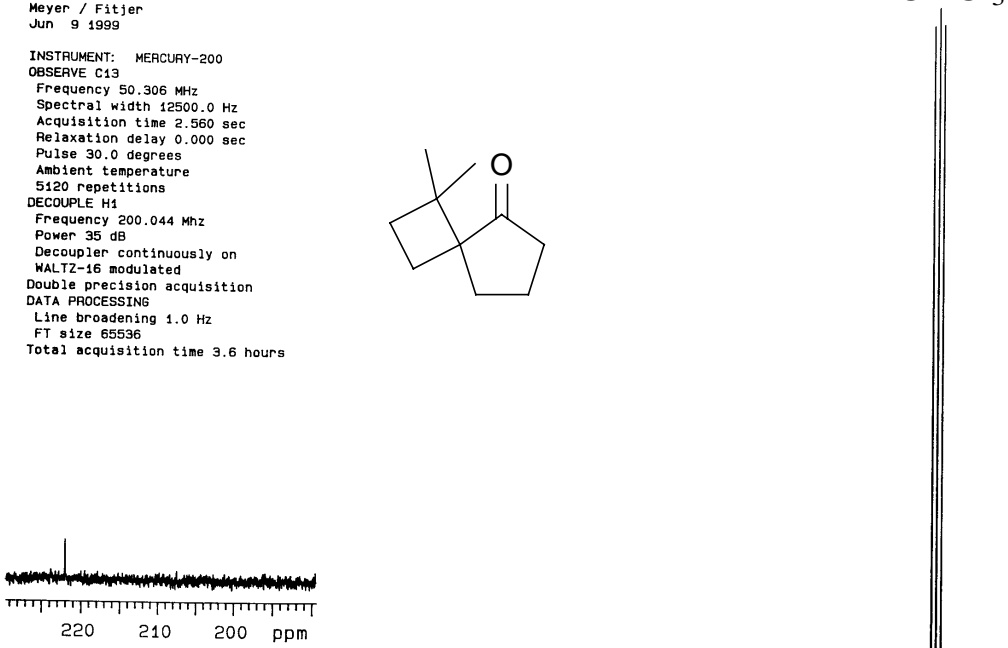

$220 \quad 210 \quad 200 \quad$ ppm

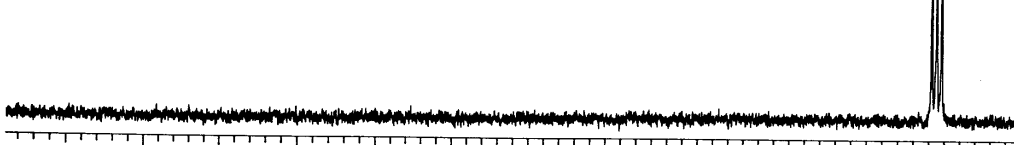

Abb. 85: ${ }^{13} \mathrm{C}-\mathrm{NMR}-$ Spektrum von 1,1-Dimethyl-spiro[3.4] octan-5-on (27) 


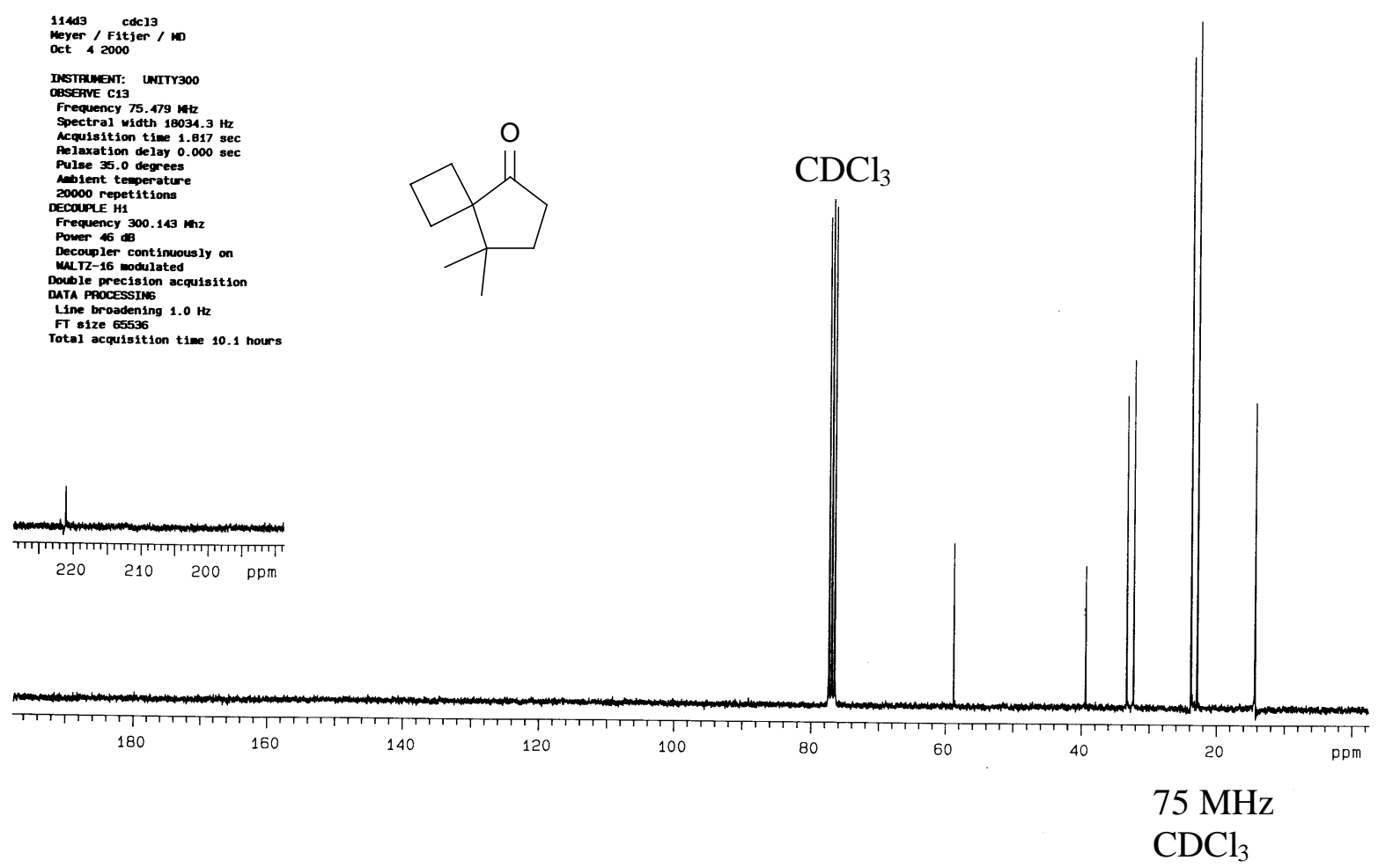

Abb. 86: ${ }^{13}$ C-NMR-Spektrum von 8,8-Dimethyl-spiro[3.4] octan-5-on (67)
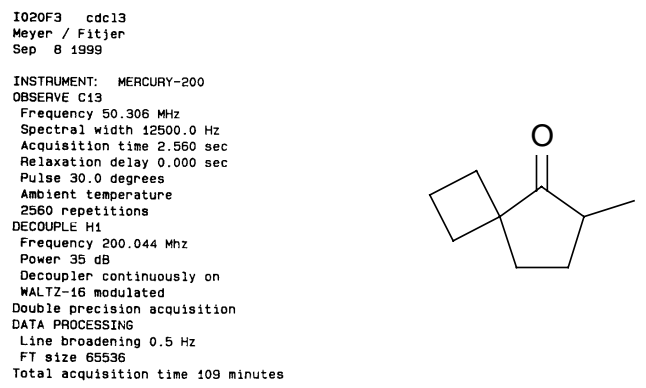

$\mathrm{CDCl}_{3}$
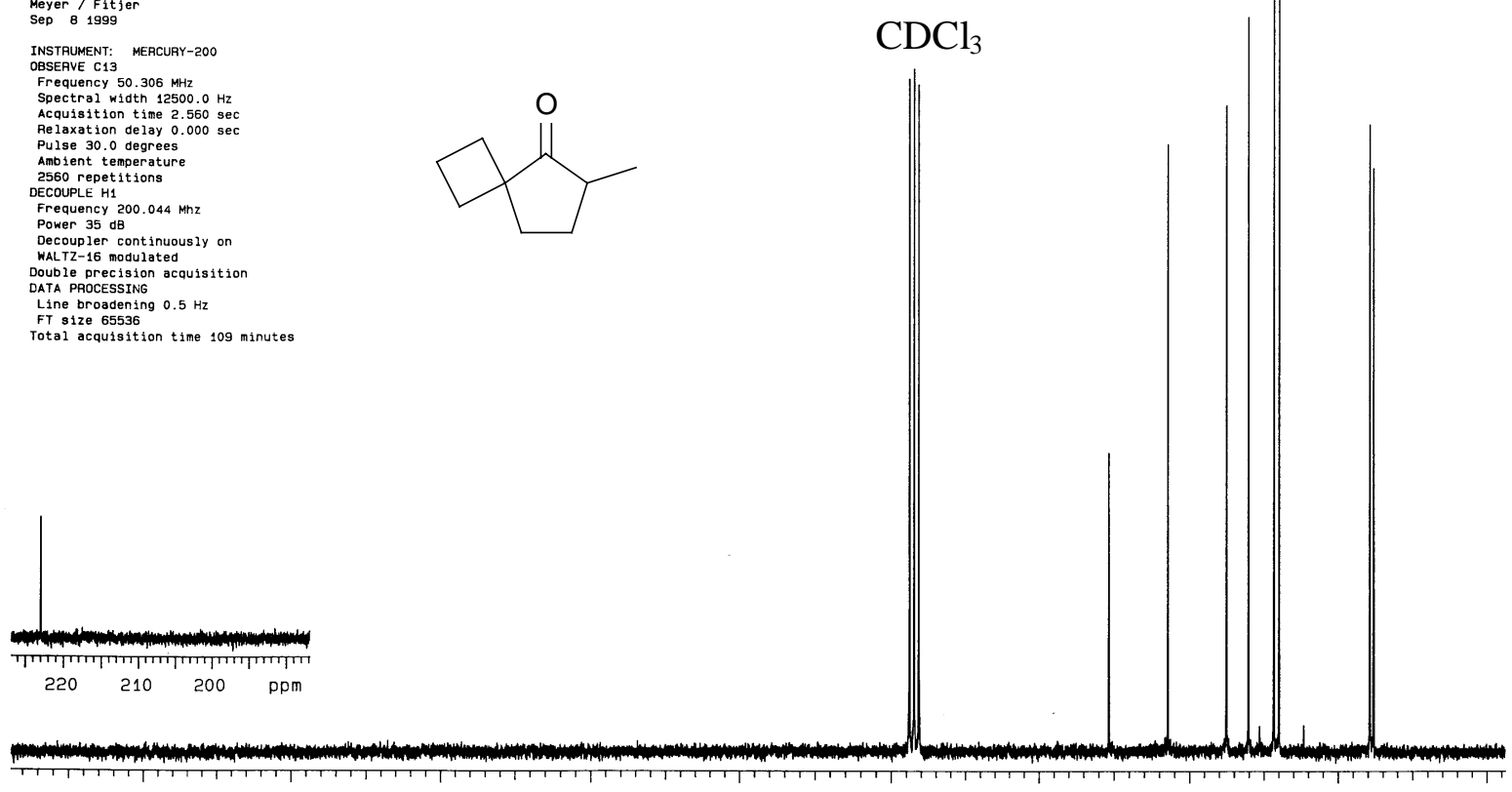

Abb. 87: ${ }^{13}$ C-NMR-Spektrum von 6-Methyl-spiro[3.4] octan-5-on (50) 


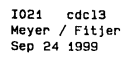

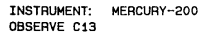

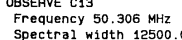

Acquisition time $2.560 \mathrm{sec}$
Relaxation delay $0.000 \mathrm{sec}$

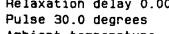

Ambient temperatur

DECOUPLE H1

Frequency 200.04
Power 35 dB

Decoupler cont tnuously on

MALLZ-16 modulated
Double precision acquisitio

DATA PROCESSING
Line broadening $1.0 \mathrm{~Hz}$

FT 9 size 65536
Total acquisition time 54 minutes
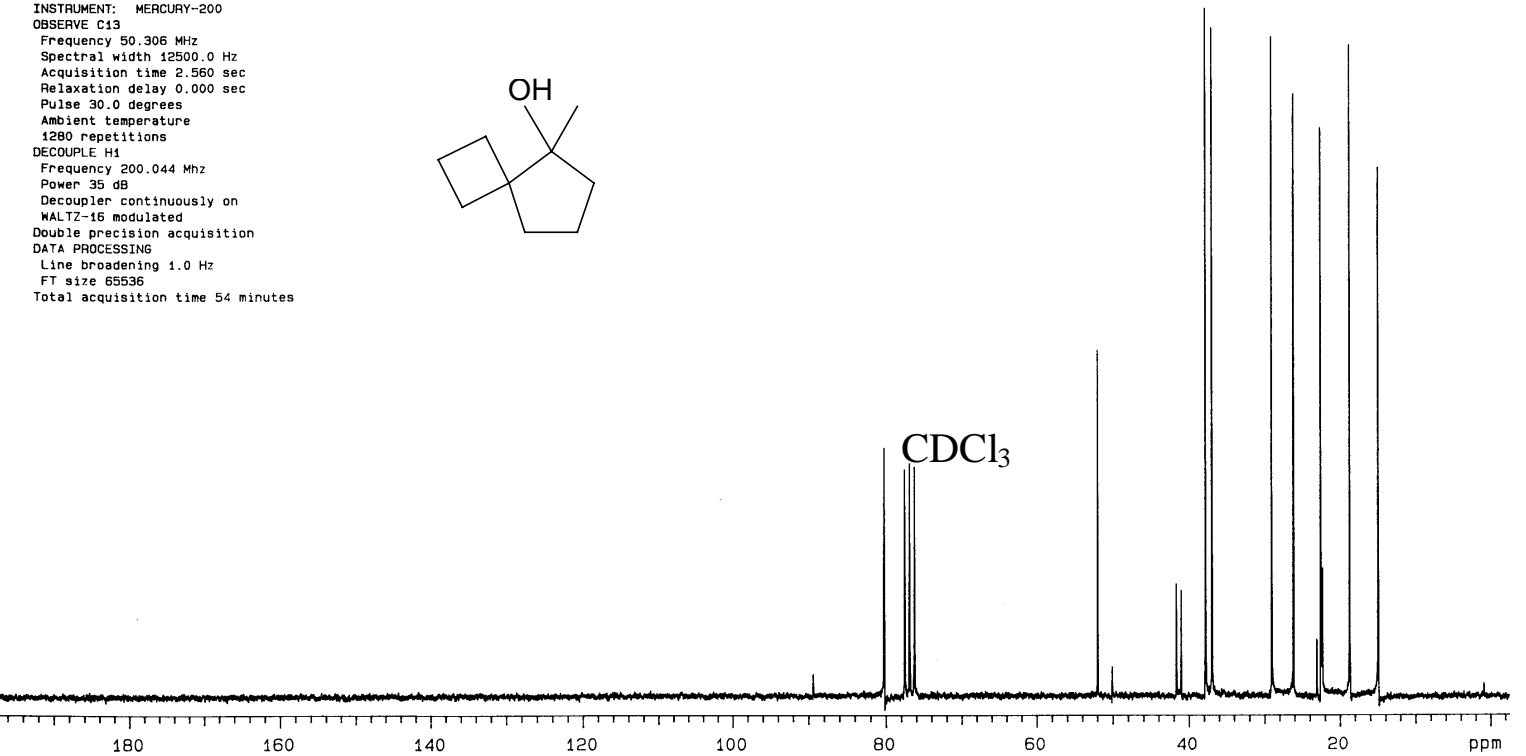

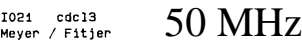

date: Sep 24 1999 $\mathrm{CDCl}_{3}$

Abb. 88: ${ }^{13}$ C-NMR-Spektrum von 5-Methyl-spiro[3.4] octan-5-ol (21)

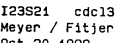

INSTRUMENT: MERCUAY-200

Frequency $50.306 \mathrm{MHz}$
Spectral width 12500.0

Spectral width $12500.0 \mathrm{~Hz}$
Acquisition time $2.560 \mathrm{sec}$

Pulse 30.0 degrees

Ambient temperatur

DECOUPLE H1

Decoupler cont inuousiy on

HALTZ-16 modulated
Double precision acquisition

DATA PROCESSING
Line broadening $1.0 \mathrm{H}$

FT size 65536

Total acquisition time 60 minutes

$\mathrm{CDCl}_{3}$
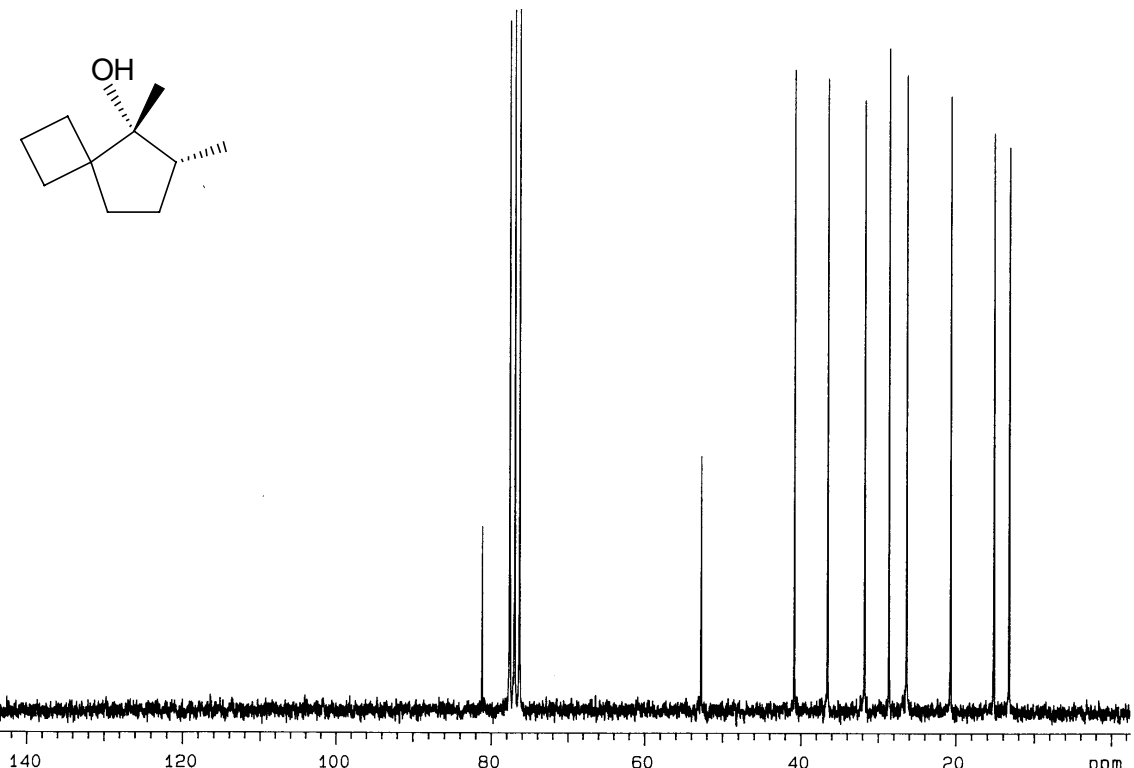

180

160

140

120

100

I23s21, cdc13

$50 \mathrm{MHz}$

date: oct $201999 \mathrm{CDCl}_{3}$

Abb. 89: ${ }^{13}$ C-NMR-Spektrum von (5R*,6R*)-5,6-Dimethyl-spiro[3.4] octan-5-ol (23a) 
I23623 Cdic13
Mever $/$ Fitjer
Jan 242000

INSTRUMENT: MEACUAY-200

Frequency $50.306 \mathrm{NHZ}$

Spectral width $12500.0 \mathrm{~Hz}$ Relaxation delay $0.000 \mathrm{sec}$ Pulse 30.0 degrees Ambient temperature
2560 repet it tions OECOUPLE HI Frequency $200.044 \mathrm{Mnz}$ Decoupler continuously on HALTZ-16 modulated DATA PROCESSING Line broadening $1.0 \mathrm{~Hz}$

年
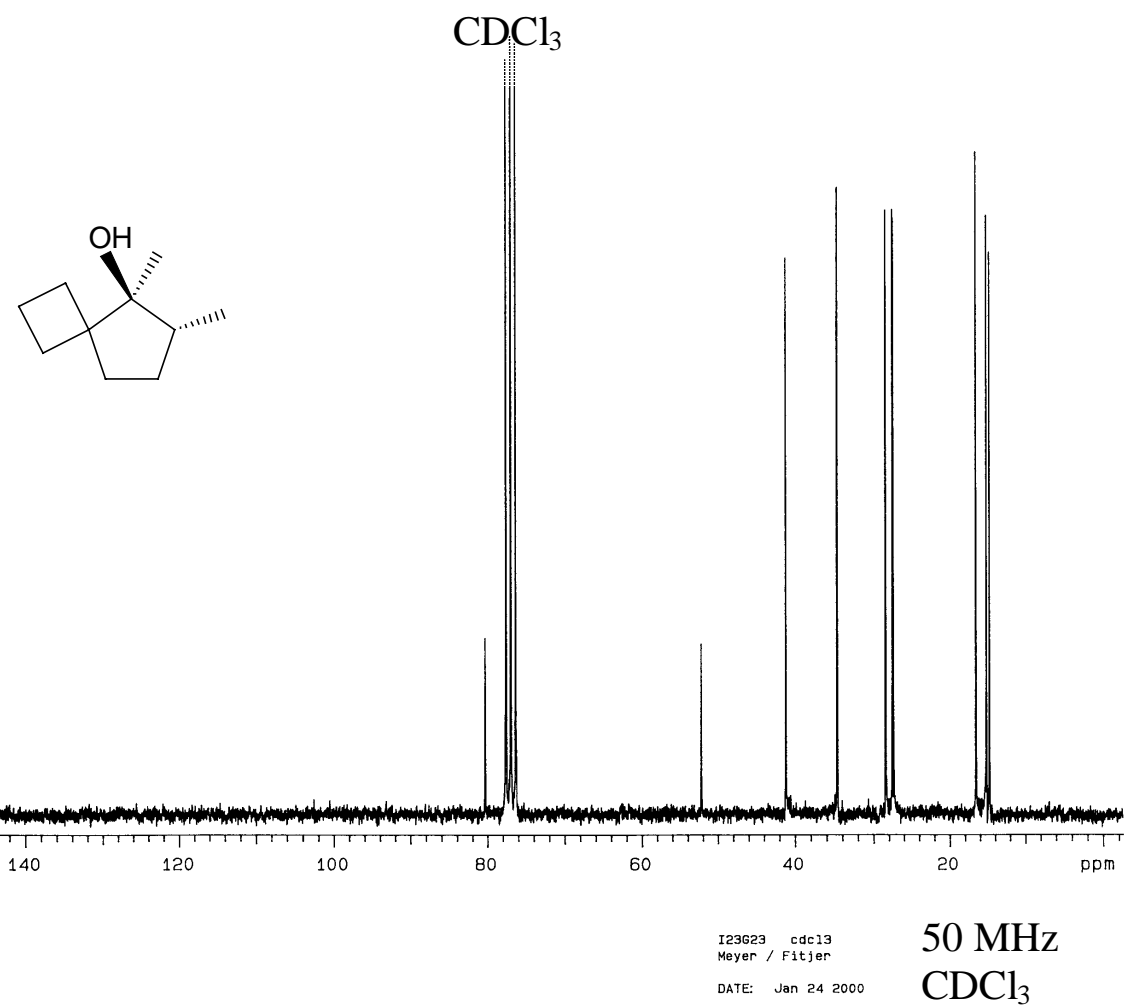

Abb. 90: ${ }^{13}$ C-NMR-Spektrum von $\left(5 S^{*}, 6 R^{*}\right)-5,6-$ Dimethyl-spiro[3.4] octan-5-ol (23b)
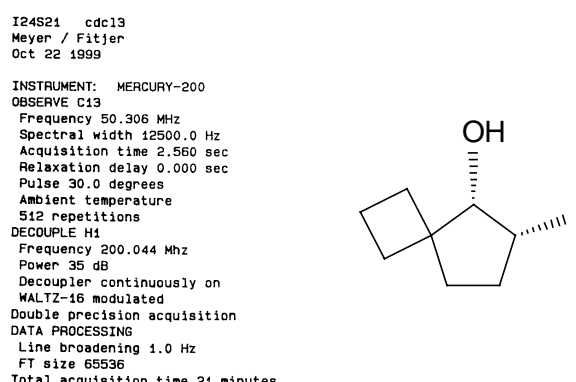

on time 21 minutes

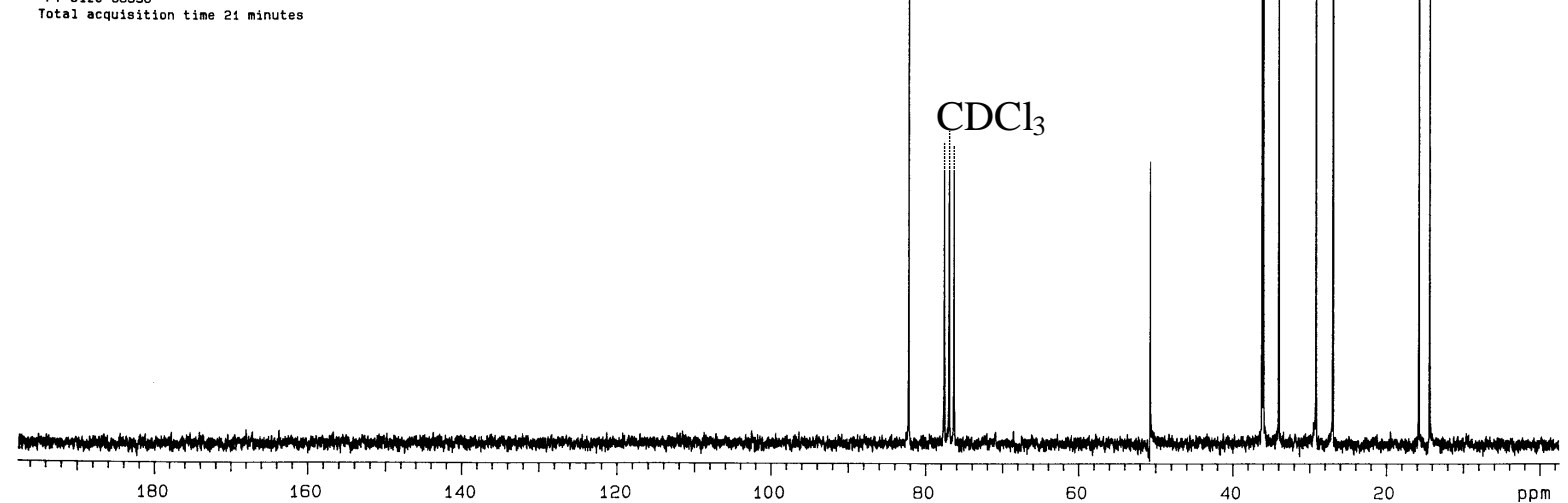

Abb. 91: ${ }^{13} \mathrm{C}-\mathrm{NMR}$-Spektrum von $\left(5 \mathrm{R}^{*}, 6 \mathrm{R}^{*}\right)$-6-Methyl-spiro[3.4] octan-5-ol (22a) 


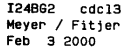

INSTRUMENT: MERCURY-200
OASEREE C13

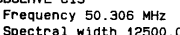

Accuisistion time 2.560 sec

Aeraxatton delay 0.000

Antise 30.0 degrees temperature

2455 repetition

DECOUPLE H1
Frequency 200.044 Mhz

Power 35 dB
Decoupler cont inuously on

KALTZ-16 modulated
Double precision acquisition

DATA PROCESSING

Line broadening $1.0 \mathrm{H}$

Total acquisition time 104 minutes
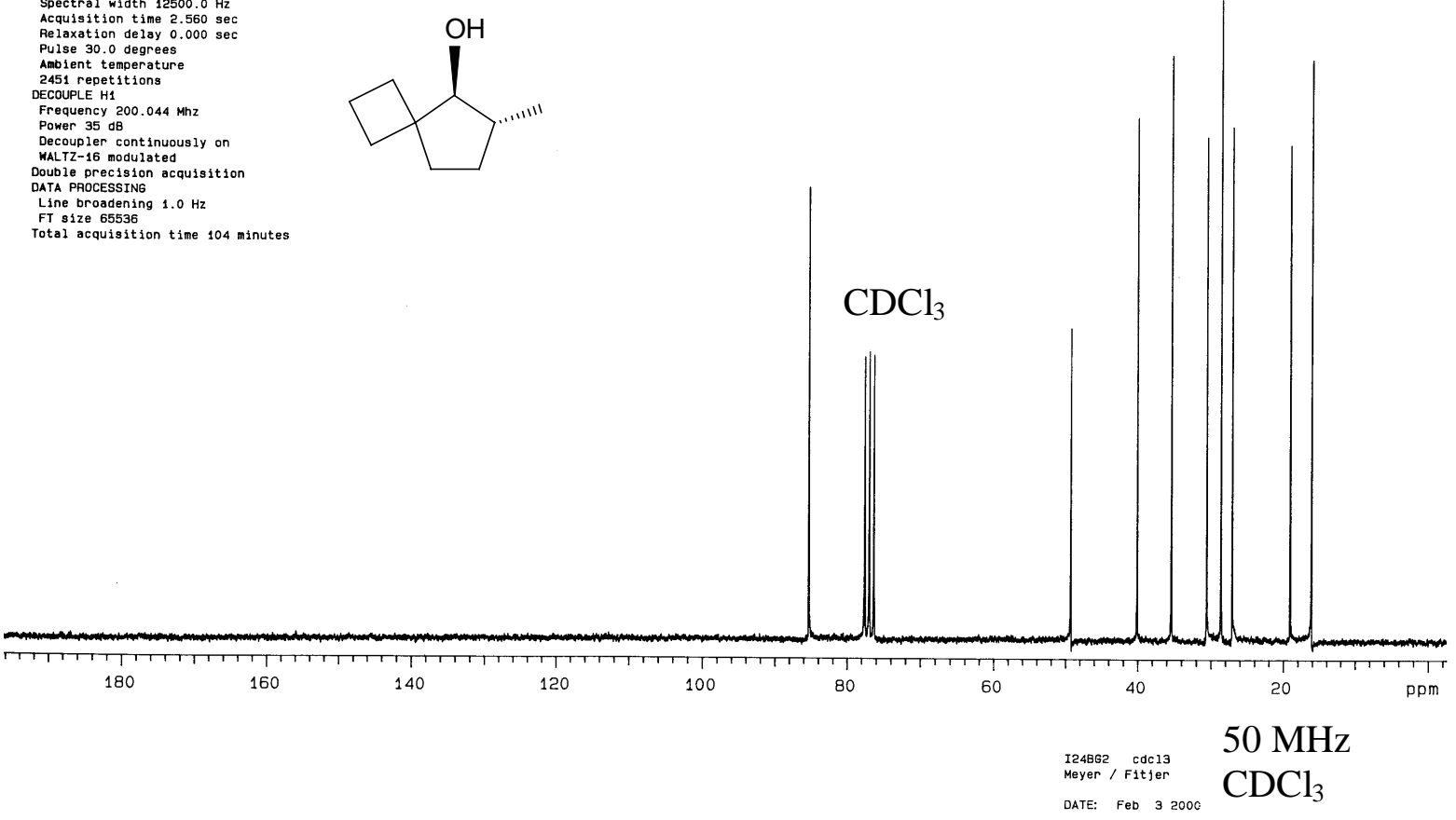

Abb. 92: ${ }^{13}$ C-NMR-Spektrum von (5S*,6R*)-6-Methyl-spiro[3.4] octan-5-ol (22b)
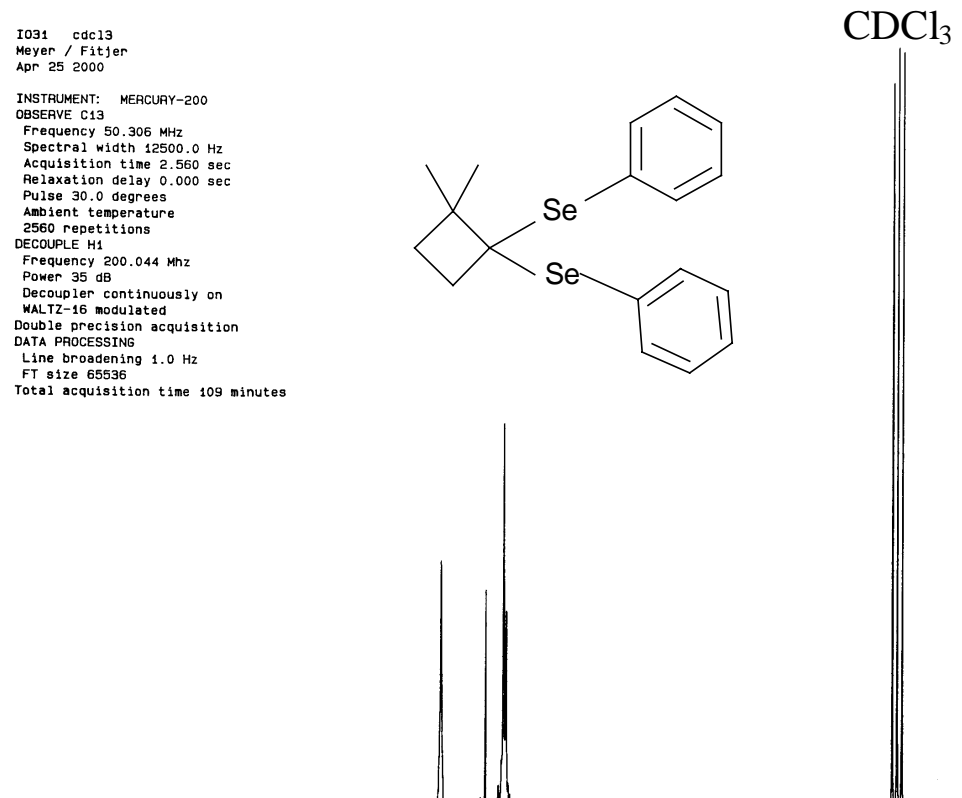

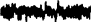

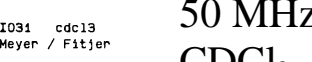

$$
\begin{aligned}
& \text { DATE: App } 25 \text { s } 2000 \quad \mathrm{CDCl}_{3}
\end{aligned}
$$

Abb. 93: ${ }^{13} \mathrm{C}-\mathrm{NMR}-$ Spektrum von 1,1-Bis(phenylseleno)-2,2-Dimethylcyclobutan (48) 


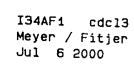

INSTRUMENT: MERCURY-200

Frequency $50.306 \mathrm{MHz}$
Spectral Hidth $12500.0 \mathrm{~Hz}$
Acquistion time $2.560 \mathrm{sec}$
Relaxation delag Relaxation delay 0.000
Pulse 30.0 degrees Ambient temperature
5120 repetitions DECOUPLE H1 Frequency $200.044 \mathrm{Mhz}$ Decoupler continuous $1 y$ on
WALTZ-16 modulated WALTZ-16 modulated
Double precision acquisition

Double precision acquis
DATA PRocESING

Line broadening $1.0 \mathrm{~Hz}$

Total acquisition time 3.6 hours
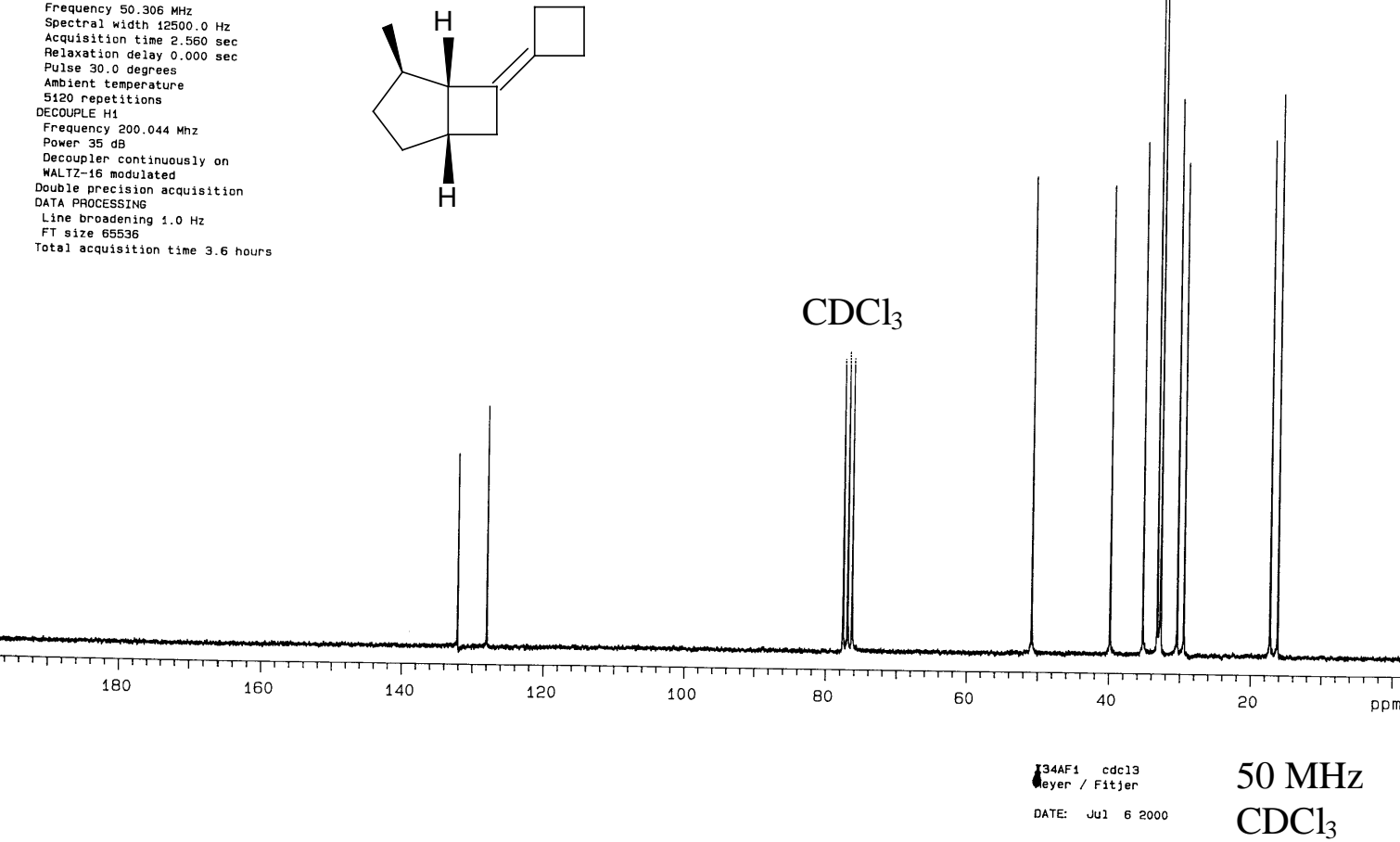

Abb. 94: ${ }^{13} \mathrm{C}-\mathrm{NMR}-$ Spektrum von $\left(1 \mathrm{R}^{*}, 2 \mathrm{~S} *, 5 \mathrm{R}^{*}\right)$-7-Cyclobutyliden-2-methyl-bicyclo[3.2.0]heptan (40)
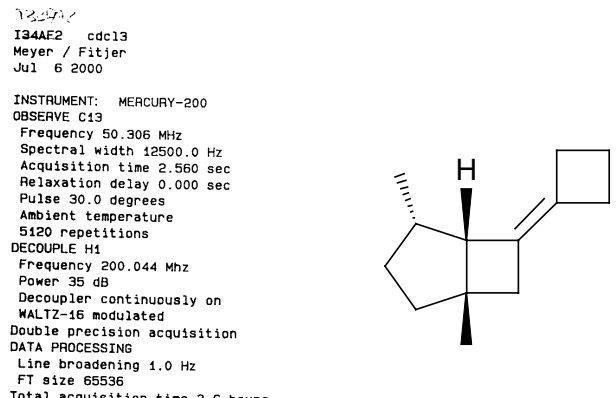

Total acquisition time 3.6 hours

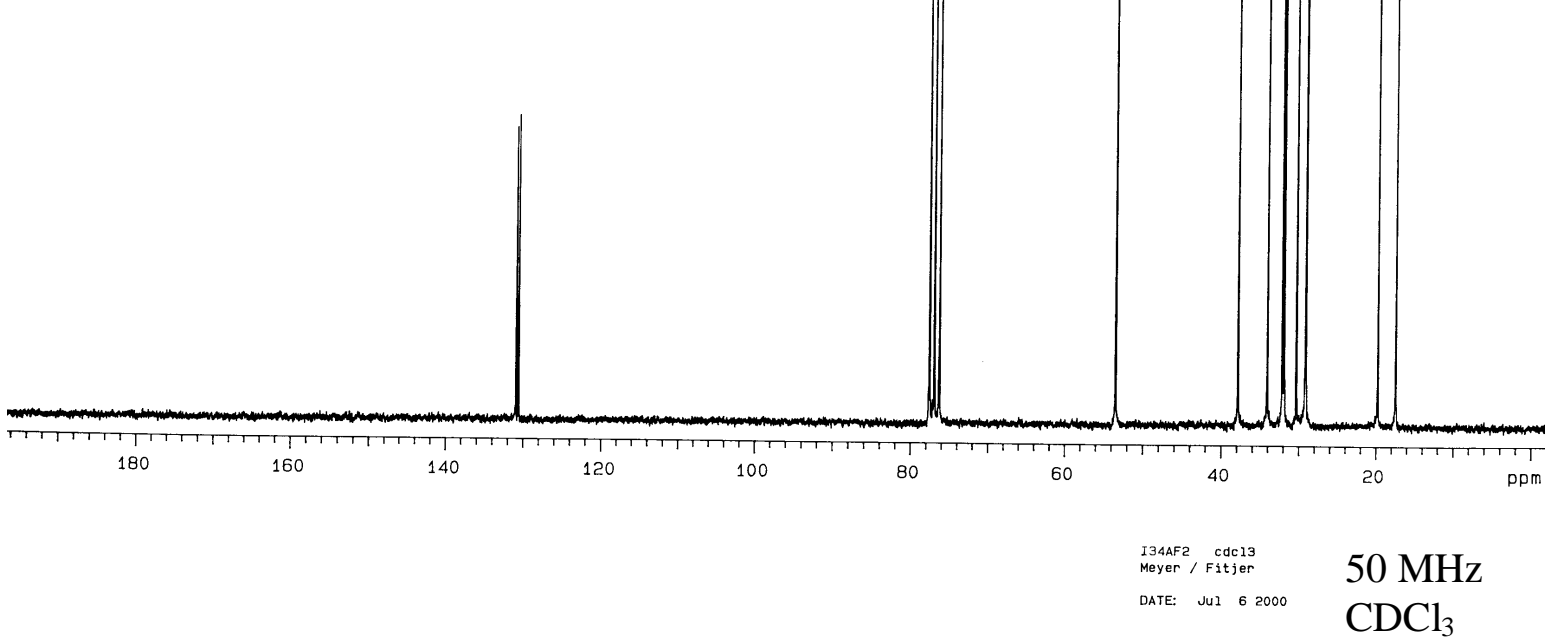

Abb. 95: ${ }^{13} \mathrm{C}-\mathrm{NMR}-$ Spektrum von $(1 \mathrm{R} *, 2 \mathrm{R} * 5 \mathrm{R} *)$-7-Cyclobutyliden-2-methyl-bicyclo[3.2.0]heptan (91) 
I341 cod13
Meyer $\quad$ Fitjer
Ju1 15 2000

INSTRUMENT: MERCUAY-200

Frequency $50.306 \mathrm{MHz}$

Spectral width $12500.0 \mathrm{~Hz}$
Acquisition time $2.560 \mathrm{sec}$
Relaxation delay $0.000 \mathrm{sec}$

Alse

2560 repetitions

Frequency $200.044 \mathrm{Mhz}$

Decoupler continuously on

WALTZ-16 modulated

Line broadening $1.0 \mathrm{~Hz}$

Total acquisition time 109 minutes
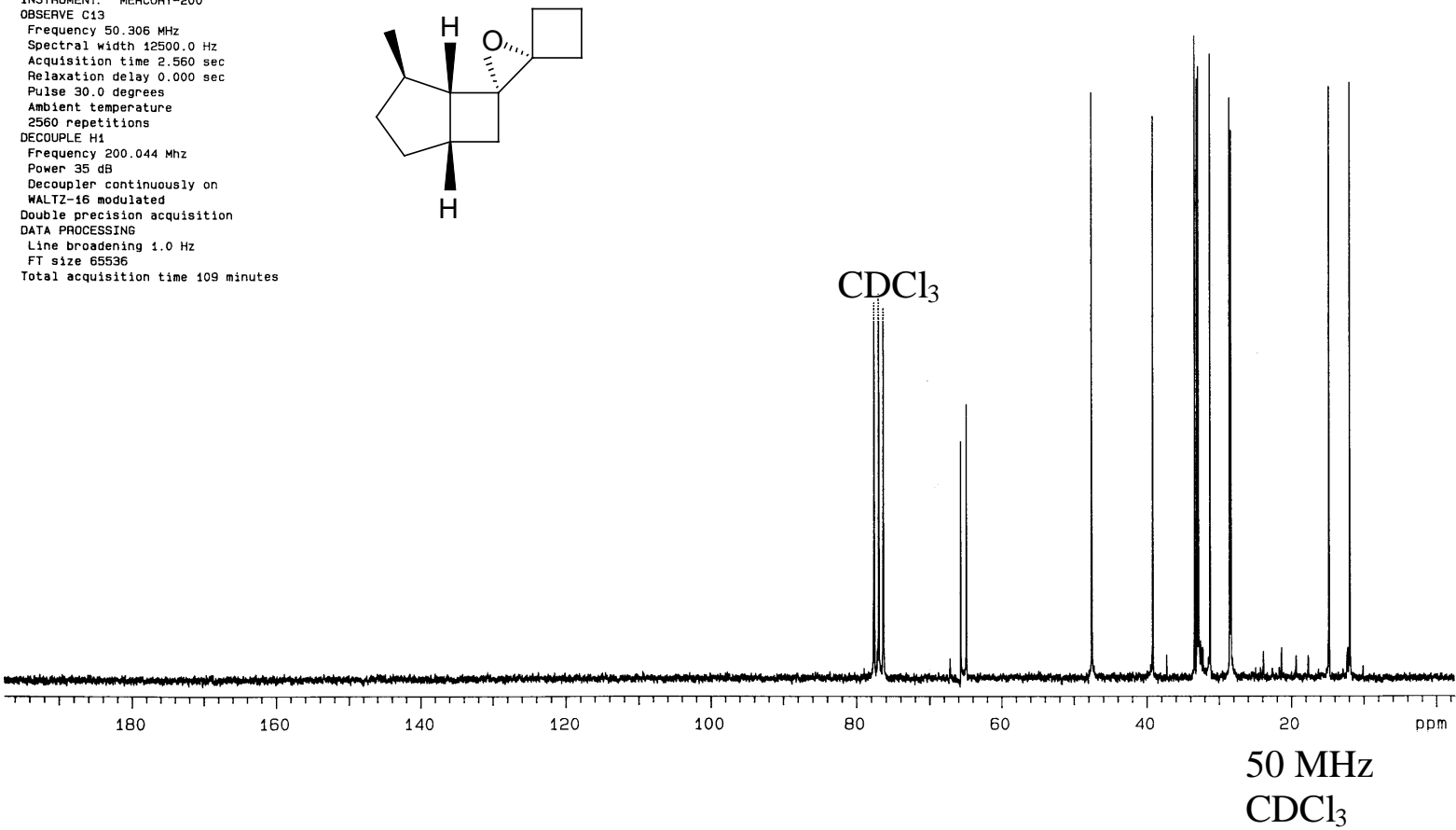

Abb. 96: ${ }^{13} \mathrm{C}$-NMR-Spektrum von 2,2-Spiro-cyclobutyl-3,3-spiro-[(1R*,4S*,5R*,6S*)-2methyl-bicyclo[3.2.0]heptan-6,6-yl]-oxiran (92)

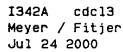

INSTRUMENT: MEACUAY-200

Frequency $50.306 \mathrm{MHz}$
Spectral width $12500.0 \mathrm{~Hz}$
Acquisition time $2.560 \mathrm{sec}$

Aetation delay 0.000 sec

Pulse 30.0 degrees
Ambient temperature

3840 repetit 10

Frequency $200.044 \mathrm{Mhz}$

Power 35 de

Decoupler continuously on

Double precision acquisition

DATA PROCESSING

FT s1ze 6553

Total acquisition time 2.7 hours
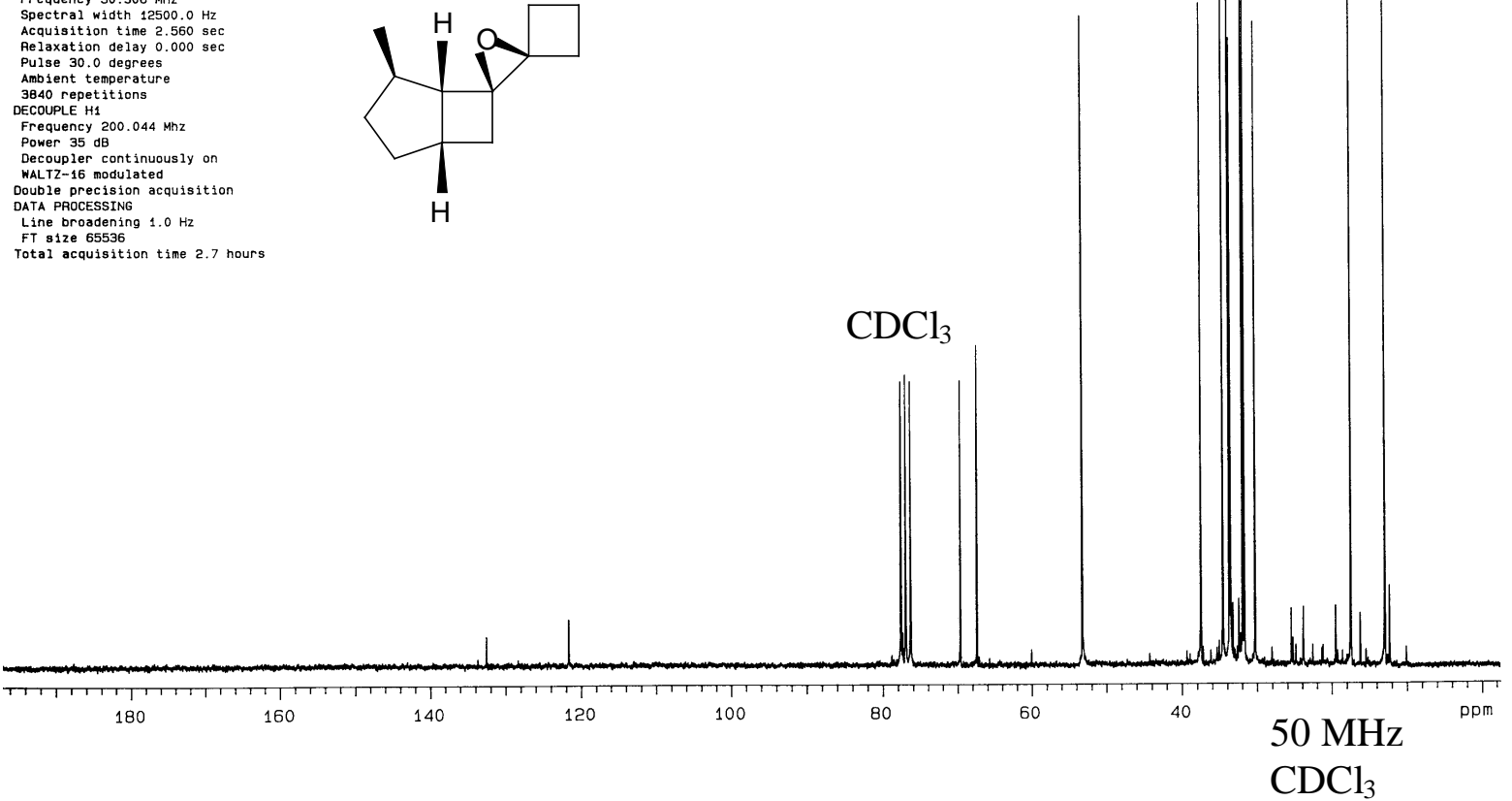

Abb. 97: ${ }^{13}$ C-NMR-Spektrum von 2,2-Spiro-cyclobutyl-3,3-spiro-[(1R*,4S*,5R*,6R*)-2methyl-bicyclo[3.2.0]heptan-6,6-yl]-oxiran (89) 


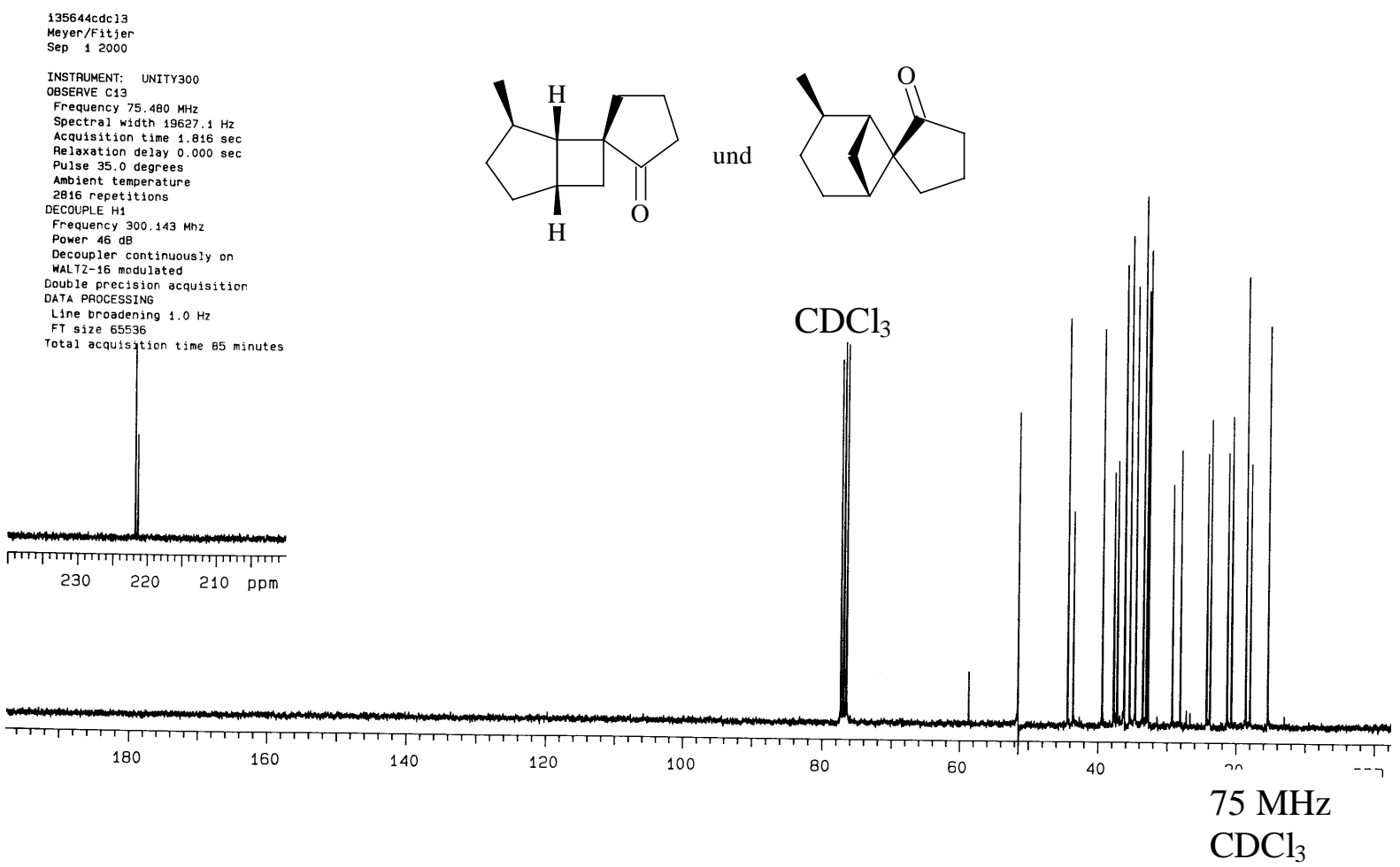

Abb. 98: ${ }^{13} \mathrm{C}-\mathrm{NMR}-\mathrm{Spektrum}$ von $\left(1 \mathrm{R}^{*}, 4 \mathrm{~S}^{*}, 5 \mathrm{R}^{*}, 6 \mathrm{~S}^{*}\right)$-4-Methyl-spiro\{bicyclo[3.2.0]heptan6,1'-cyclopentan-2'-on\} (87) und $\left(1 \mathrm{R}^{*}, 2 \mathrm{R}^{*}, 5 \mathrm{R}^{*}, 6 \mathrm{~S}^{*}\right)$-2-Methyl-spiro\{bicyclo[3.1.1]heptan-6,1'-cyclopentan-2'-on\} (93)

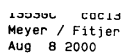

INSTRUMENT: MERCUAY-200

Frequency $50.306 \mathrm{MHz}$

Spectral width $12500.0 \mathrm{~Hz}$
Accuisitition time 2.560 sec
Relaxation

Relaxation

Ambient temperature

5120 repetitions
DECOUPLE H1

Frequency 200.044 Mhz

Decoupler continuousiy on

Double precision acquisition

Line broadening $0.5 \mathrm{~Hz}$

Total acquisition time 3.6 hours
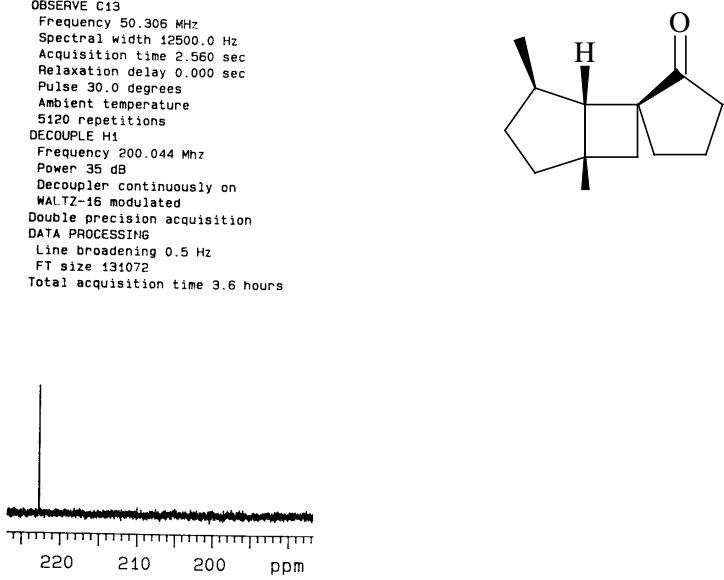


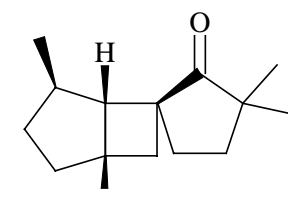

$\mathrm{CDCl}_{3}$

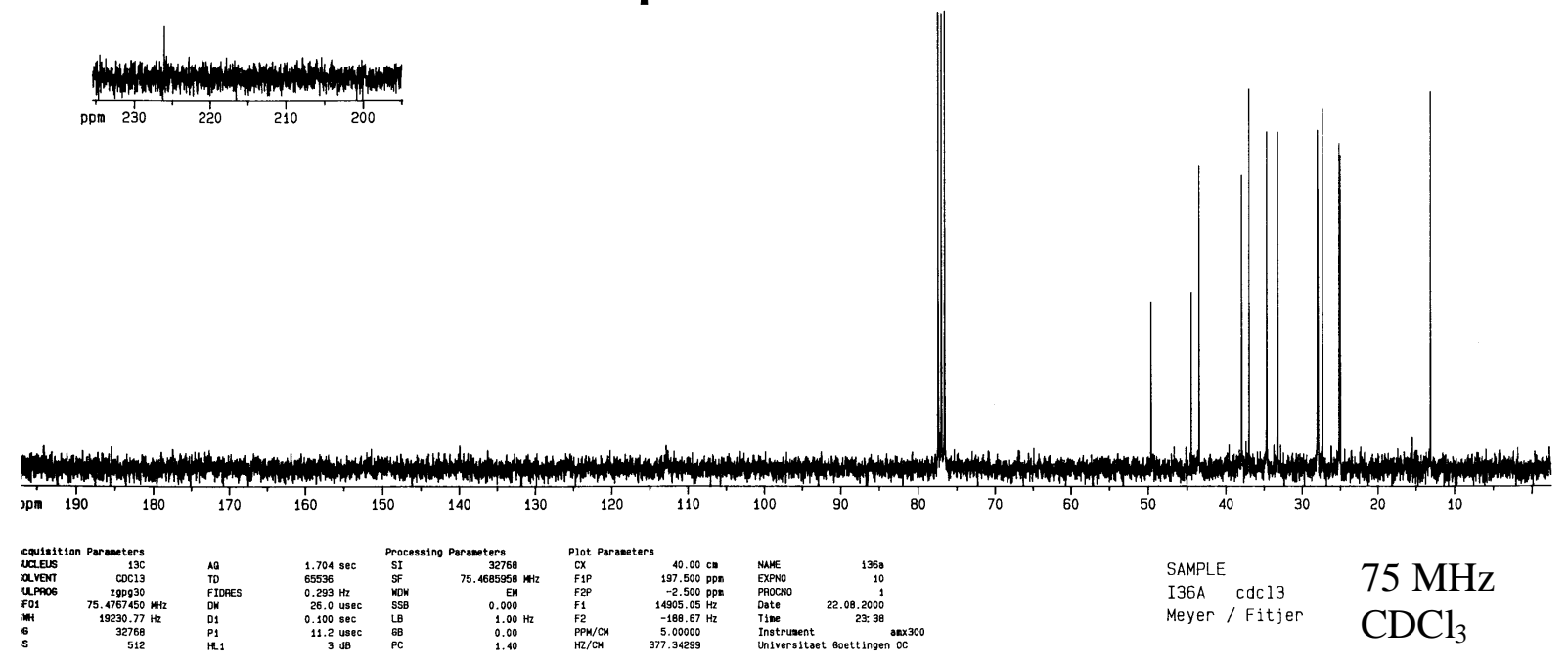

Abb. $100:{ }^{13} \mathrm{C}-\mathrm{NMR}-$ Spektrum von $\left(1 \mathrm{R}^{*}, 4 \mathrm{~S}^{*}, 5 \mathrm{R}^{*}, 6 \mathrm{R}^{*}\right)-4,3^{\prime}, 3^{\prime}$-Trimethyl-spiro \{ bicyclo[3.2.0]heptan-6, $1^{\prime}$-cyclopentan-2'-on\} (95)
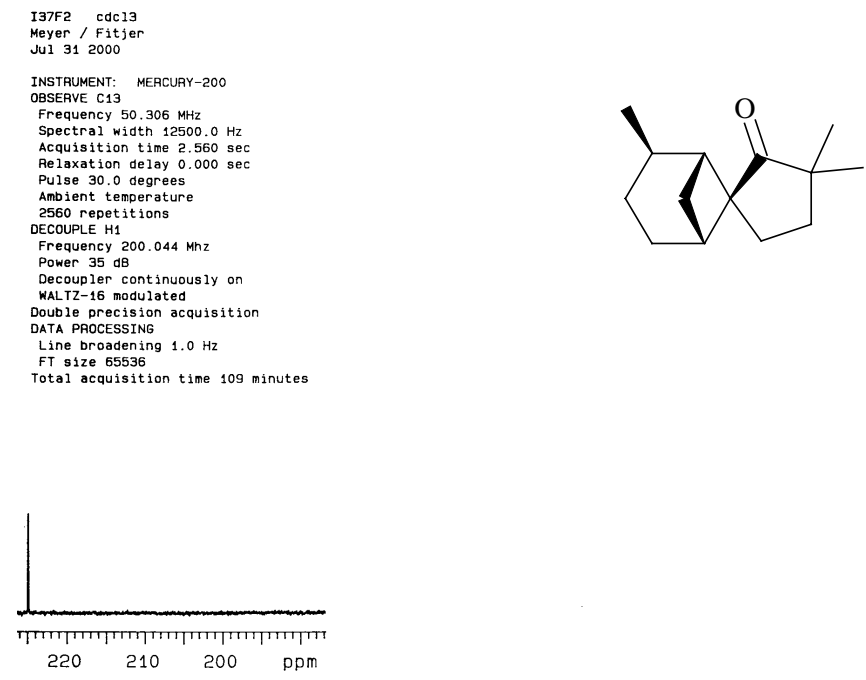

Decoupler continuousiy on

Total acquisition time 109 minutes

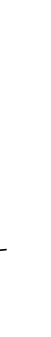



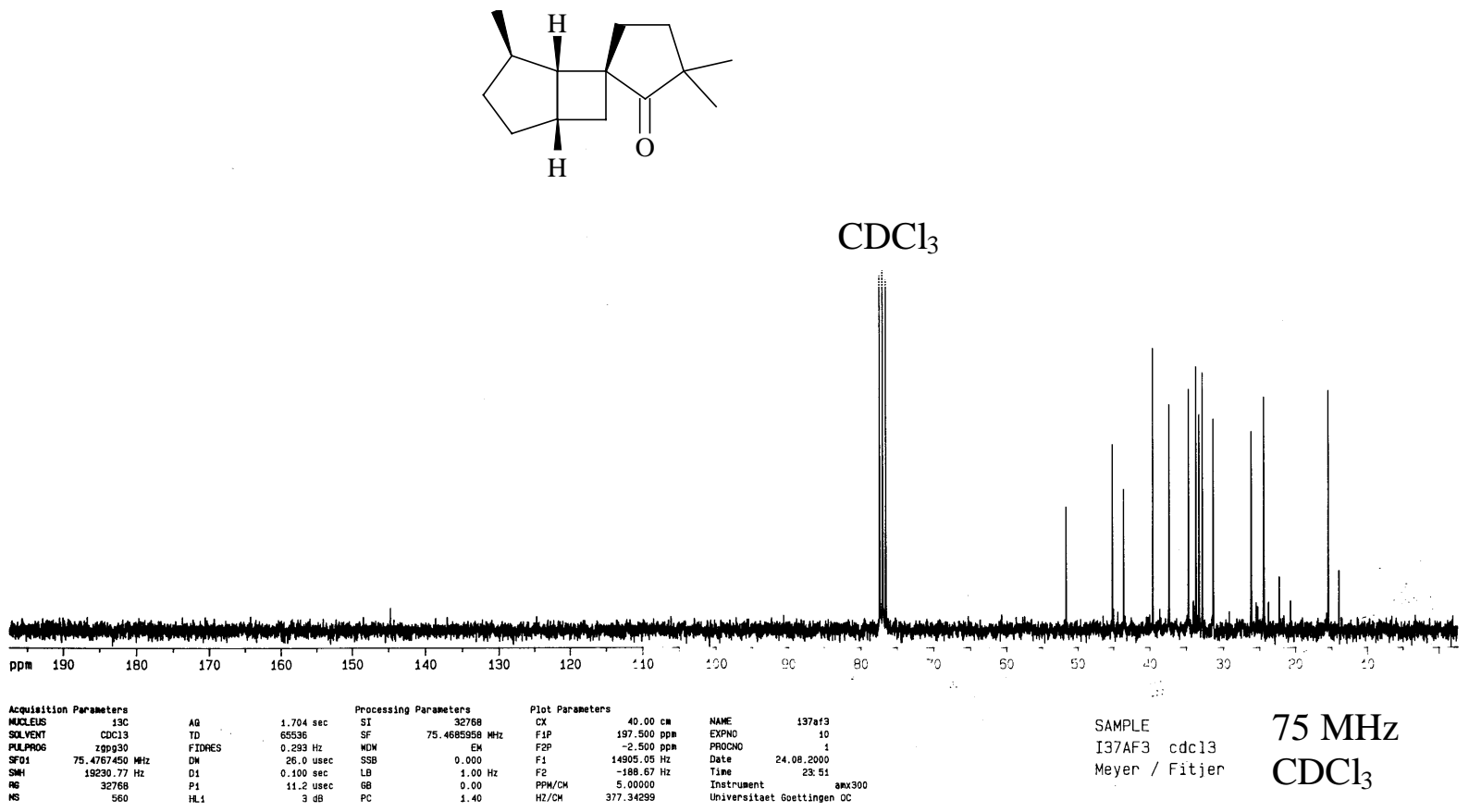

Abb. 102: ${ }^{13} \mathrm{C}-\mathrm{NMR}-$ Spektrum von $\left(1 \mathrm{R}^{*}, 4 \mathrm{~S}^{*}, 5 \mathrm{R}^{*}, 6 \mathrm{~S}^{*}\right)-4,3^{\prime}, 3^{\prime}$-Trimethyl-spiro \{ bicyclo[3.2.0]heptan-6,1'-cyclopentan-2'-on $\}$ (94)
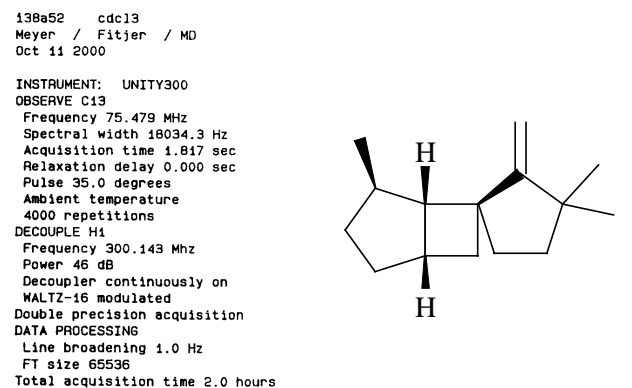

Total acquisition time 2.0 hours

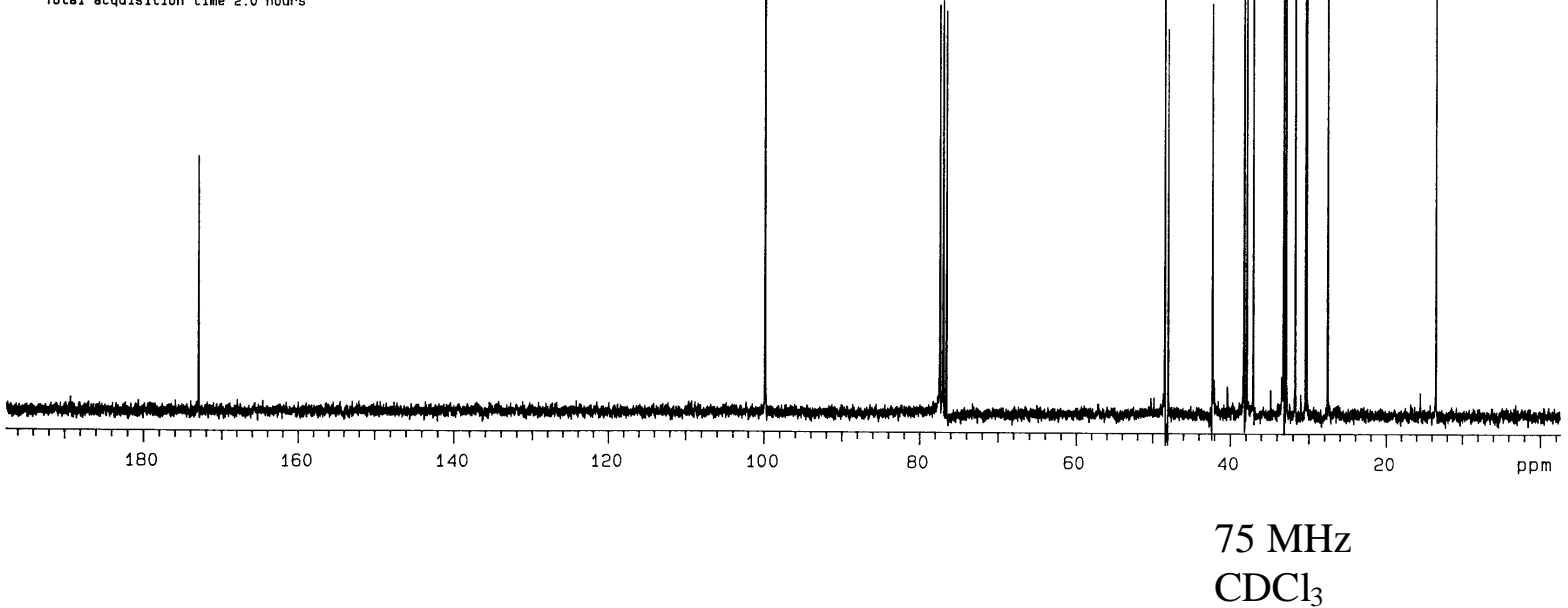

Abb. 103: ${ }^{13} \mathrm{C}-\mathrm{NMR}-$ Spektrum von $\left(1 \mathrm{R}^{*}, 4 \mathrm{~S}^{*}, 5 \mathrm{R}^{*}, 6 \mathrm{R}^{*}\right)-2^{\prime}-$ Methylen-4,3',3'-Trimethylspiro\{bicyclo[3.2.0]heptan-6,1'-cyclopentan\} (104) 


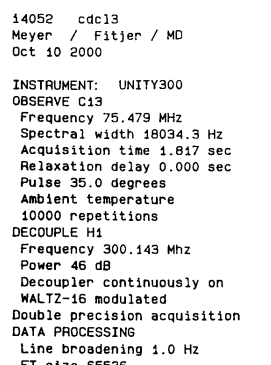

FT size 65536
Total acquisition time 5.0 nours
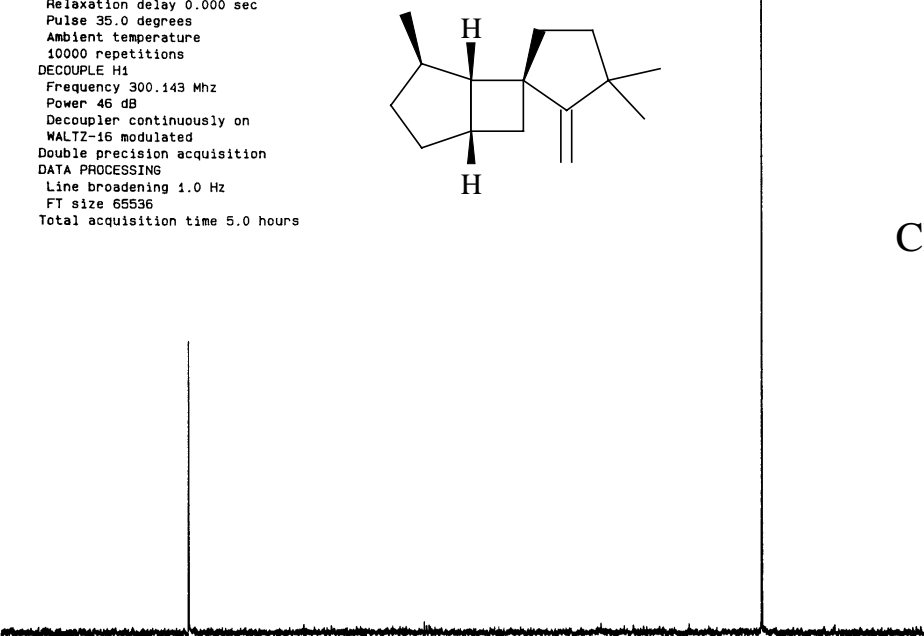

80

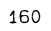

140

120

100

80

60
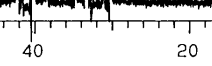

$75 \mathrm{MHz}$

$\mathrm{CDCl}_{3}$

Abb. 104: ${ }^{13} \mathrm{C}-\mathrm{NMR}-\mathrm{Spektrum}$ von $\left(1 \mathrm{R}^{*}, 4 \mathrm{~S}^{*}, 5 \mathrm{R}^{*}, 6 \mathrm{~S}^{*}\right) \mathrm{2}^{\prime}$-Methylen-4,3',3'-Trimethylspiro $\{$ bicyclo[3.2.0]heptan-6,1'-cyclopentan\} (103)
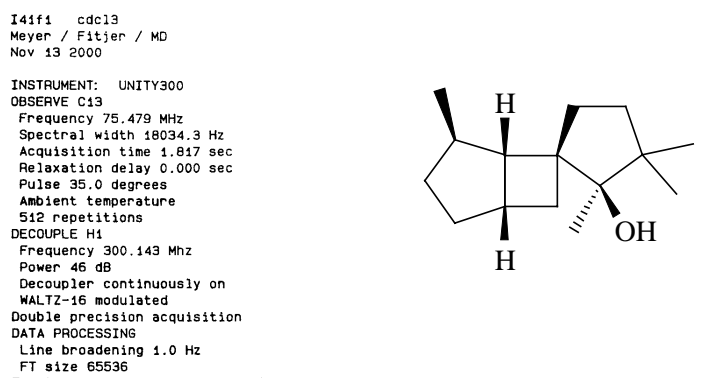

Total acquisition time 15 minutes

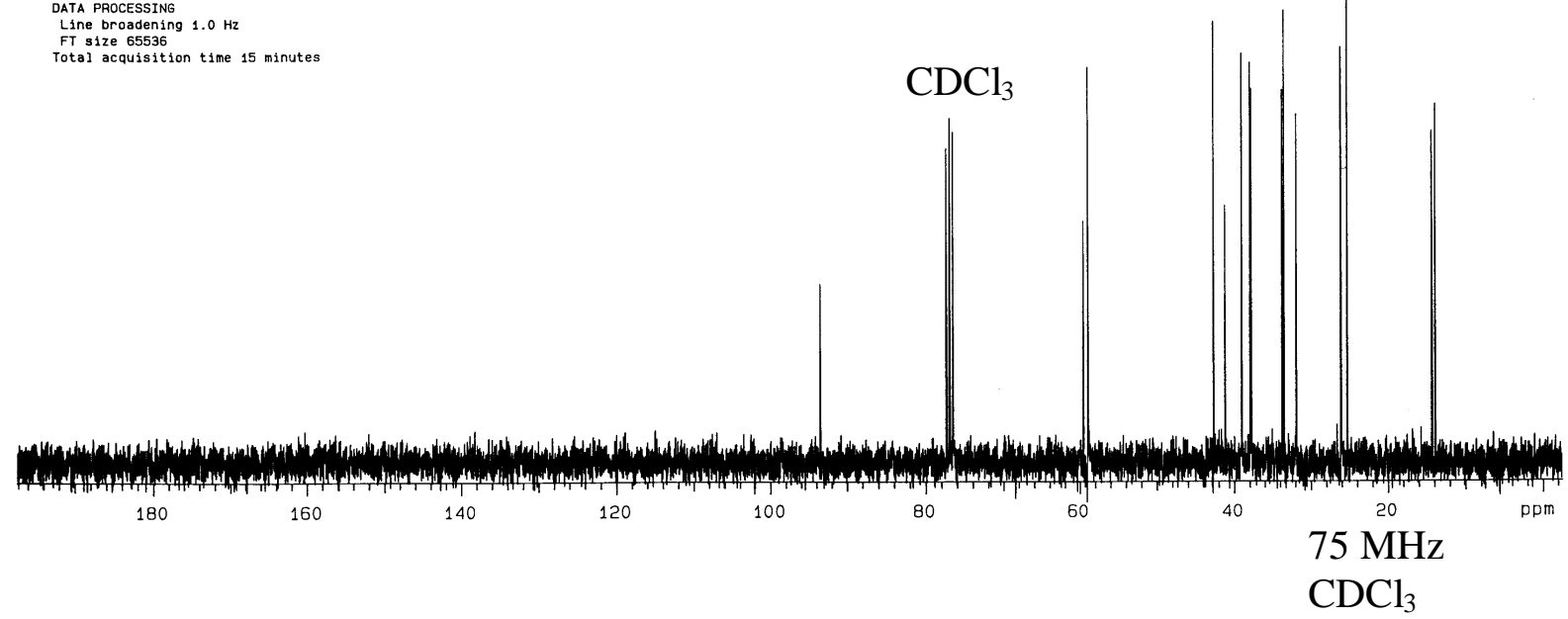

Abb. 105: ${ }^{13} \mathrm{C}-\mathrm{NMR}-\mathrm{Spektrum}$ von $\left(1 \mathrm{R}^{*}, 4 \mathrm{~S}^{*}, 5 \mathrm{R}^{*}, 6 \mathrm{~S}^{*}, 2^{\prime} \mathrm{S}\right)-4,2^{\prime} \mathbf{3}^{\prime}, 3^{\prime}-$-Tetramethyl-spiro $\{$ bi- 
cyclo[3.2.0]heptan-6, $1^{\prime}$-cyclopentan-2'-ol\}

$\mathrm{CDCl}_{3}$
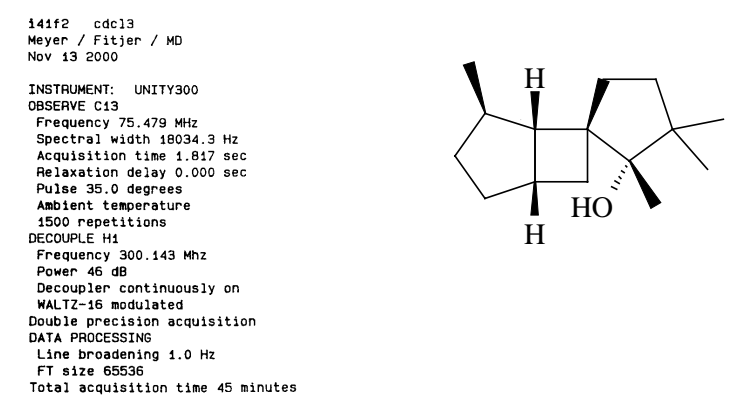

Total acquisition time 45 minutes

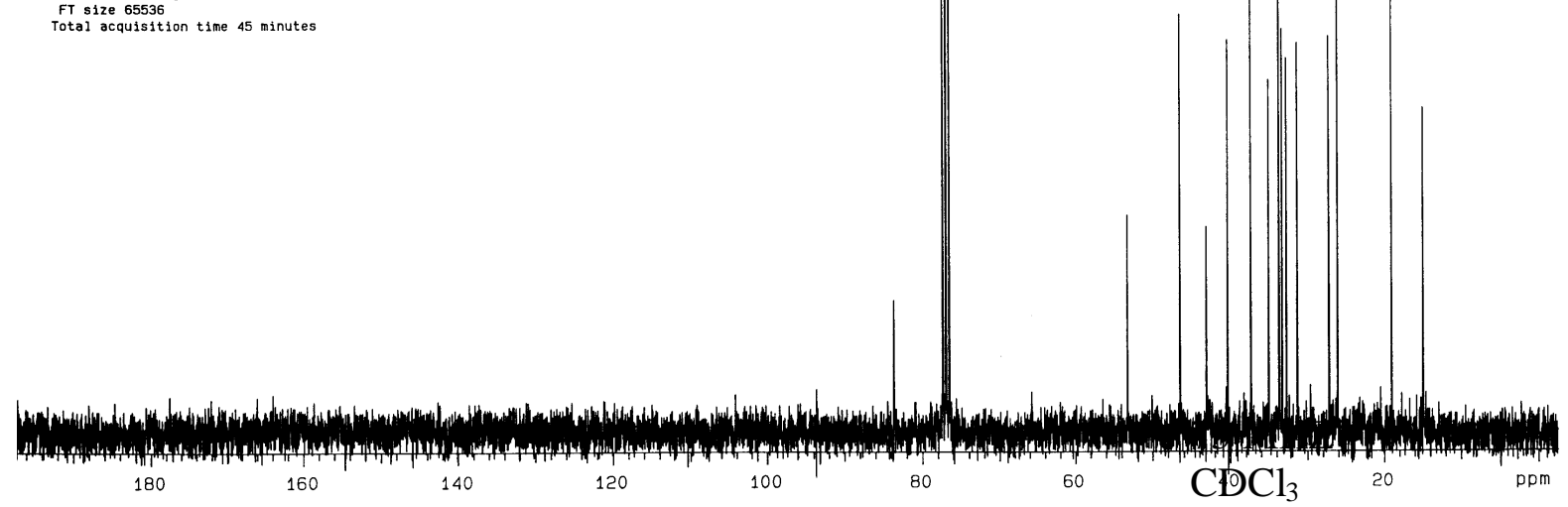

Abb. 106: ${ }^{13} \mathrm{C}-\mathrm{NMR}-\mathrm{Spe}$ trum von $\left(1 \mathrm{R}^{*}, 4 \mathrm{~S}^{*}, 5 \mathrm{R}^{*}, 6 \mathrm{~S}^{*}, 2^{\prime} \mathrm{R}^{*}\right)-4,2^{\prime}, 3^{\prime}, 3^{\prime}-$ Tetramethylspiro $\{$ bicyclo[3.2.0]heptan-6,1'-cyclopentan-2'-ol $\}$ (44)

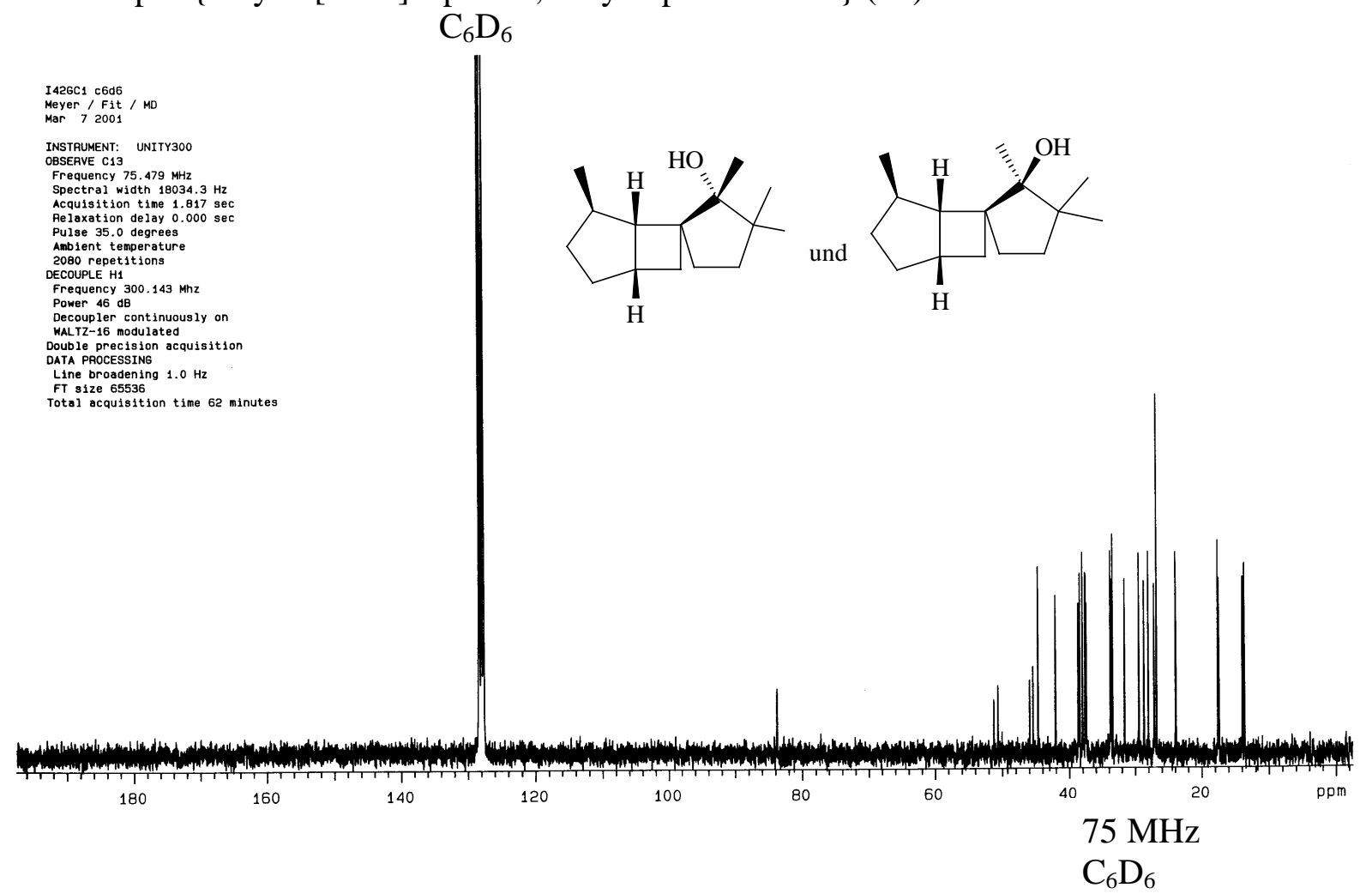

Abb. 107: ${ }^{13} \mathrm{C}-\mathrm{NMR}-$ Spektrum von $\left(1 \mathrm{R}^{*}, 4 \mathrm{~S}^{*}, 5 \mathrm{R}^{*}, 6 \mathrm{R}^{*}, 2^{\prime} \mathrm{R}^{*}\right)-4,2^{\prime}, 3^{\prime}, 3^{\prime}$-Tetramethyl-spiro

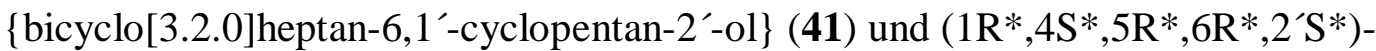


4,2',3',3'-Tetramethyl-spiro $\left\{\right.$ bicyclo[3.2.0]heptan-6, $1^{\prime}$-cyclopentan-2'-on $\}$ (42)

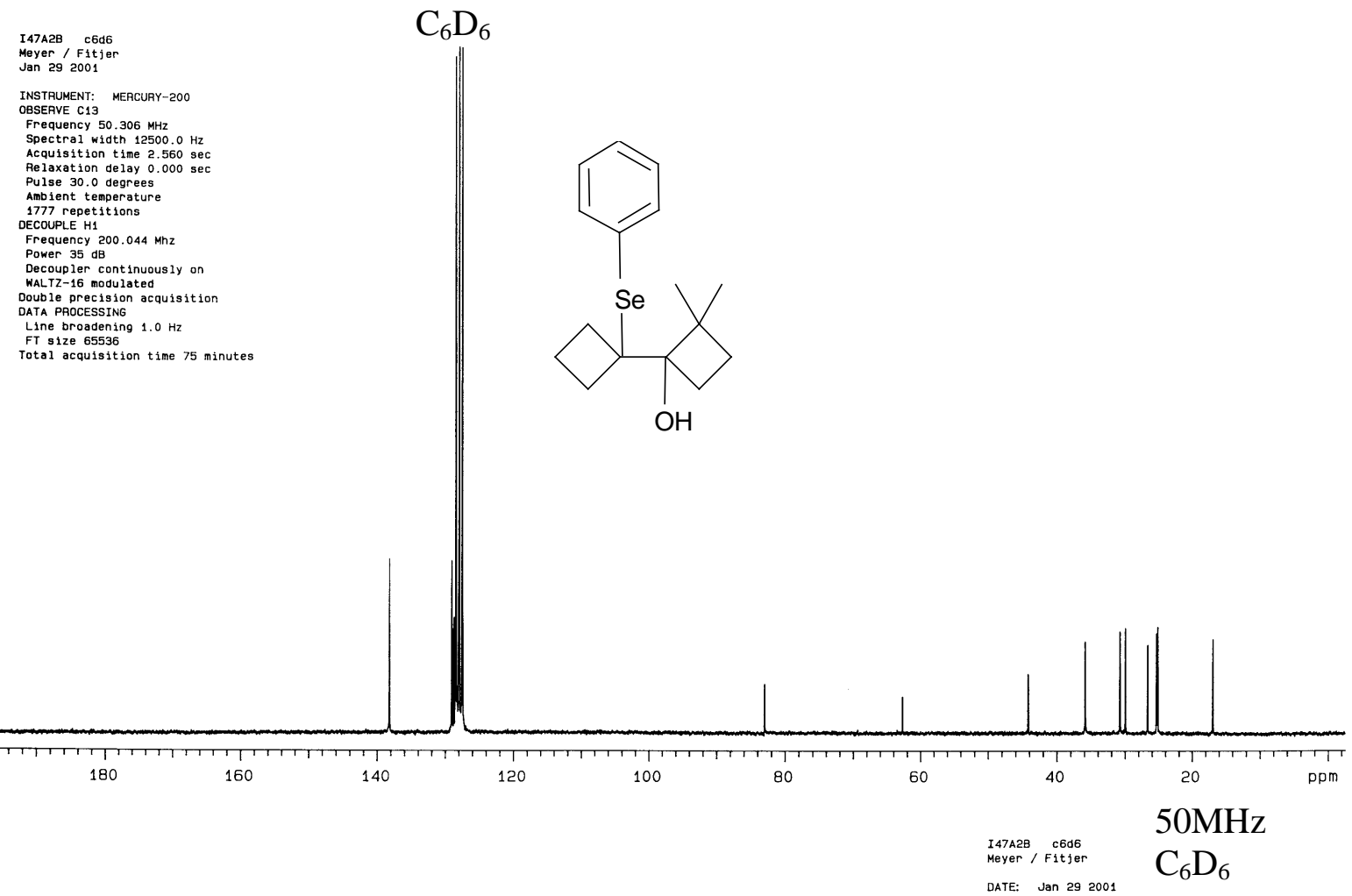

Abb. 108: ${ }^{13}$ C-NMR-Spektrum von 1-[(1'-Phenylseleno)cyclobutyl]-2,2-dimethylcyclobutan-1-ol (76)
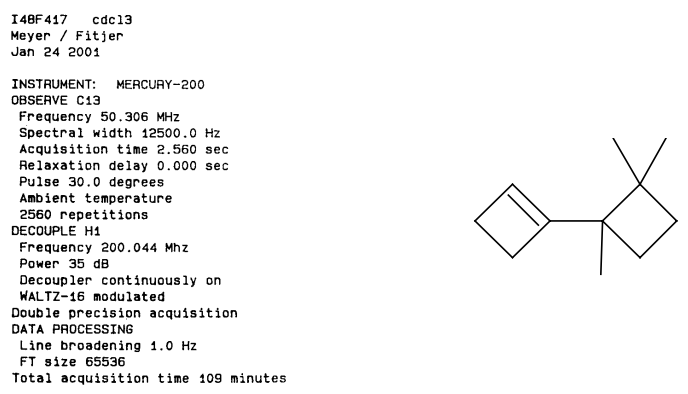

FT size 65536
Total acquisition time 109 minutes

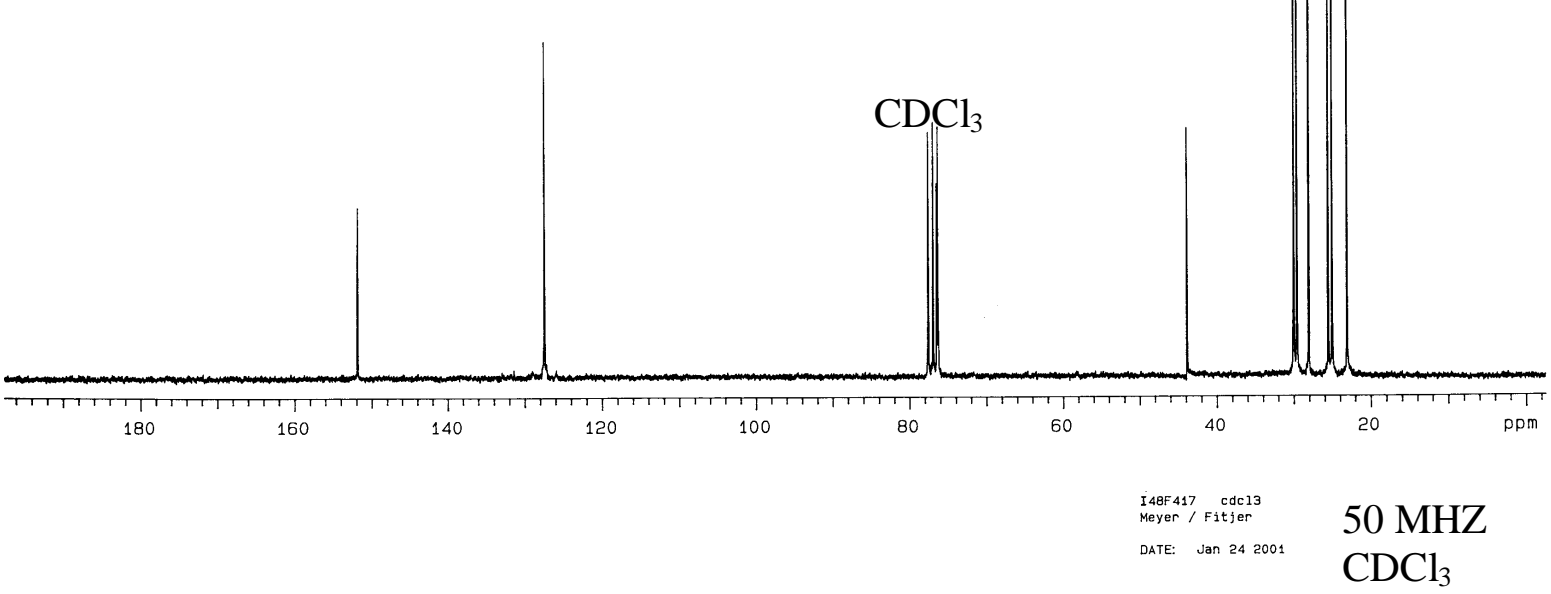

Abb. 109: ${ }^{13}$ C-NMR-Spektrum von 2,2-Dimethyl-bicyclobutyl-1'-en-1-ol (78) 


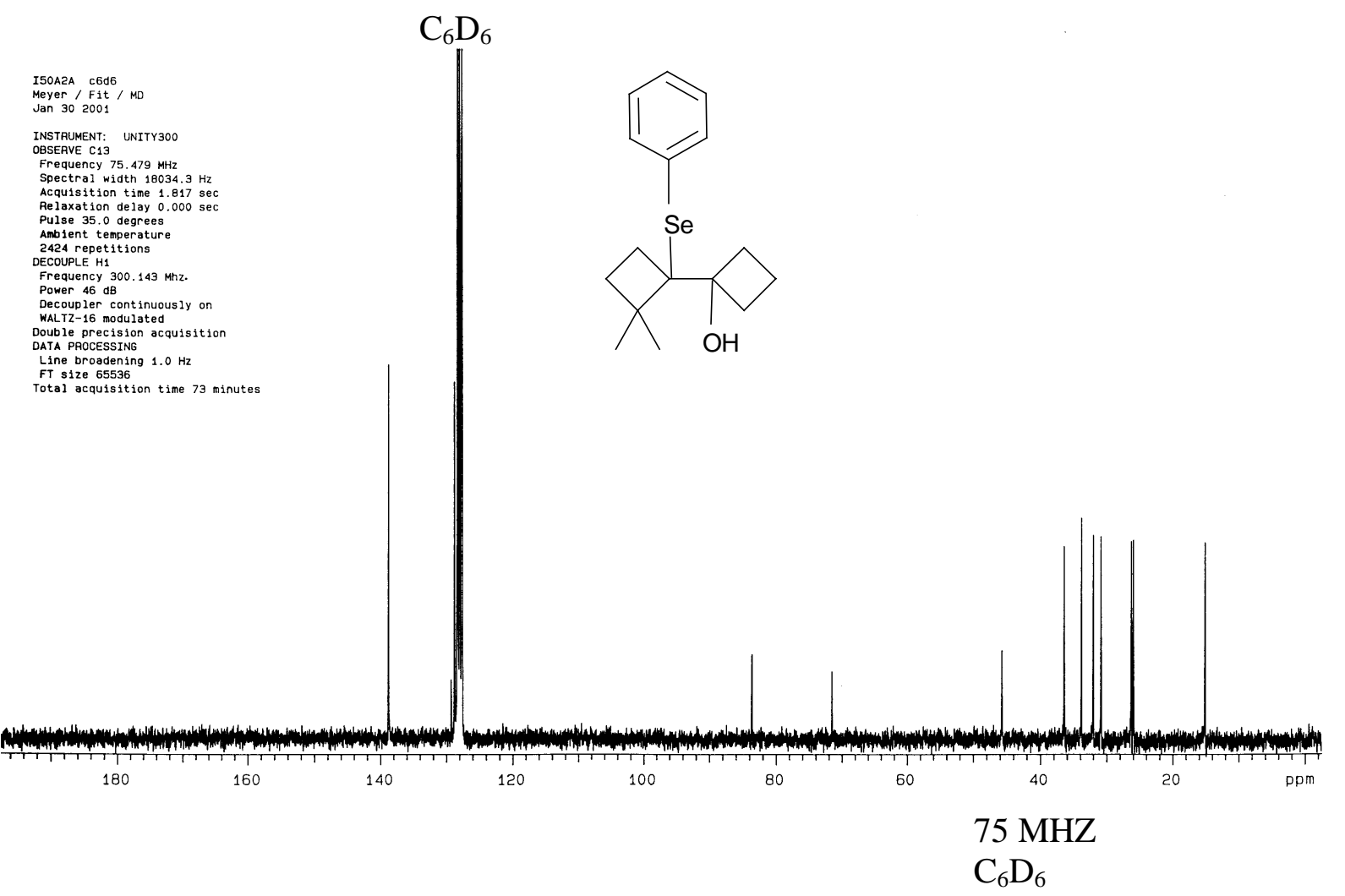

Abb. 110: ${ }^{13} \mathrm{C}-\mathrm{NMR}-\mathrm{Spektrum}$ von 1-[(1'-Phenylseleno)-2',2'-dimethylcyclobutyl $]$ cyclobutan-1-ol (49)

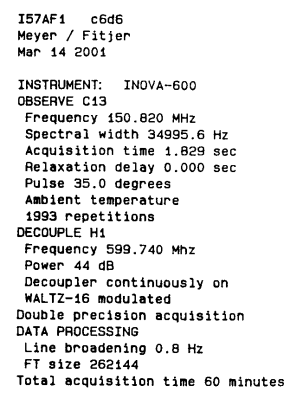

$$
\mathrm{C}_{6} \mathrm{D}_{6}
$$

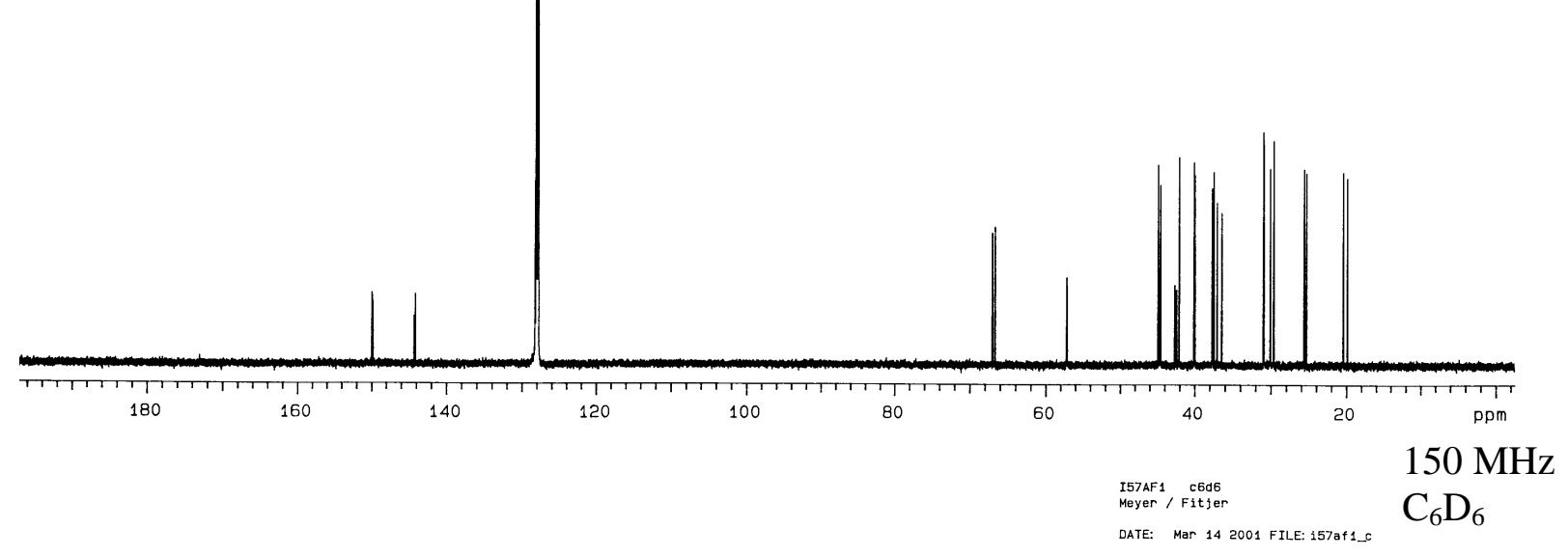

Abb. 110: ${ }^{13}$ C-NMR-Spektrum von 116/117 

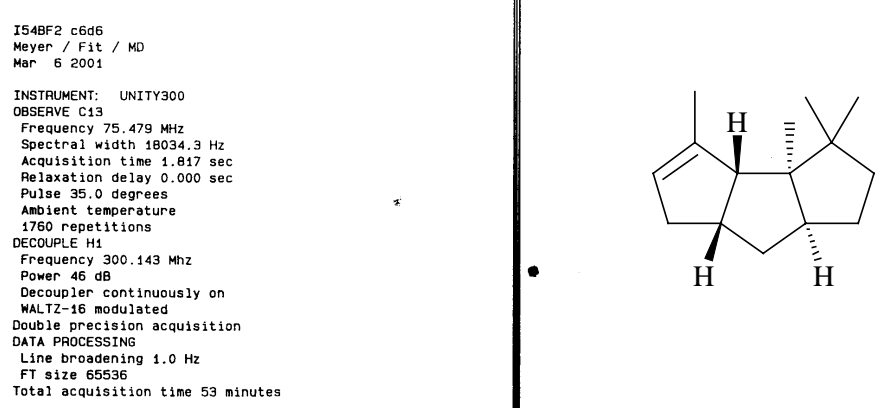

Total acquisition time 53 minutes

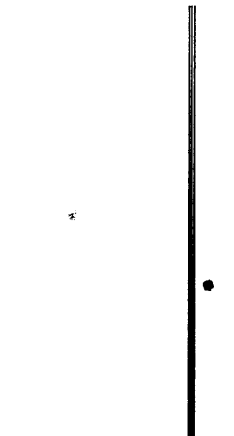

$$
\text { H }
$$

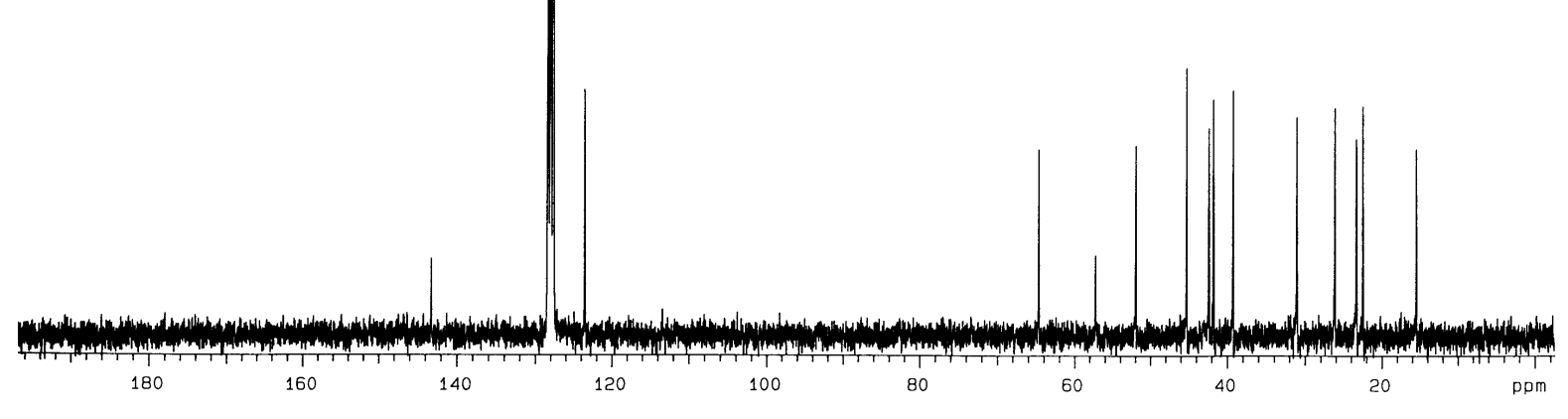

$75 \mathrm{MHz}$

$\mathrm{C}_{6} \mathrm{D}_{6}$

Abb.112: ${ }^{13}$ C-NMR-Spektrum von (3aR*,3bR*,6aR*,7aR*)-3,3,3a,4-Tetramethyl-2,3,3a,3b,

6,6a,7,7a-octahydro- $1 H$-cyclopenta[a]pentalen (119)

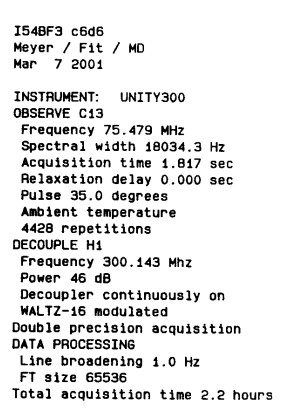

$\mathrm{C}_{6} \mathrm{D}_{6}$

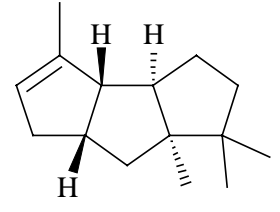

Line broadening 1.0

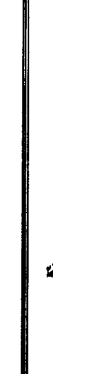




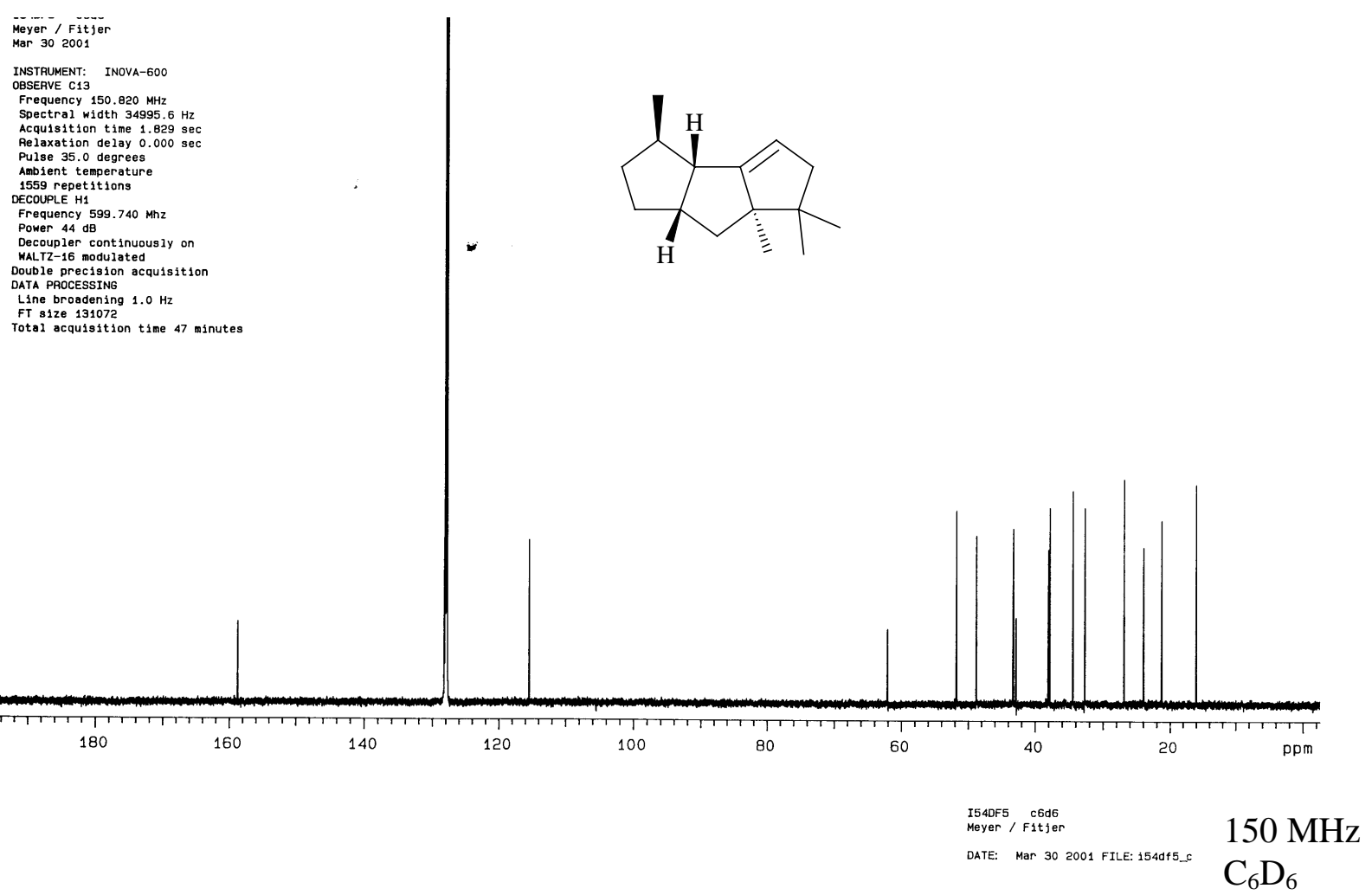

Abb.114: ${ }^{13} \mathrm{C}-\mathrm{NMR}-$ Spektrum von $\left(3 \mathrm{R}^{*}, 3 \mathrm{aS} *, 6 \mathrm{aR} *, 7 \mathrm{aR} *\right)-3,6,6,6 \mathrm{a}-$ Tetramethyl-2,3,3a,5,6, 6a,7,7a-octahydro-1H-cyclo-penta[a]pentalen (118)

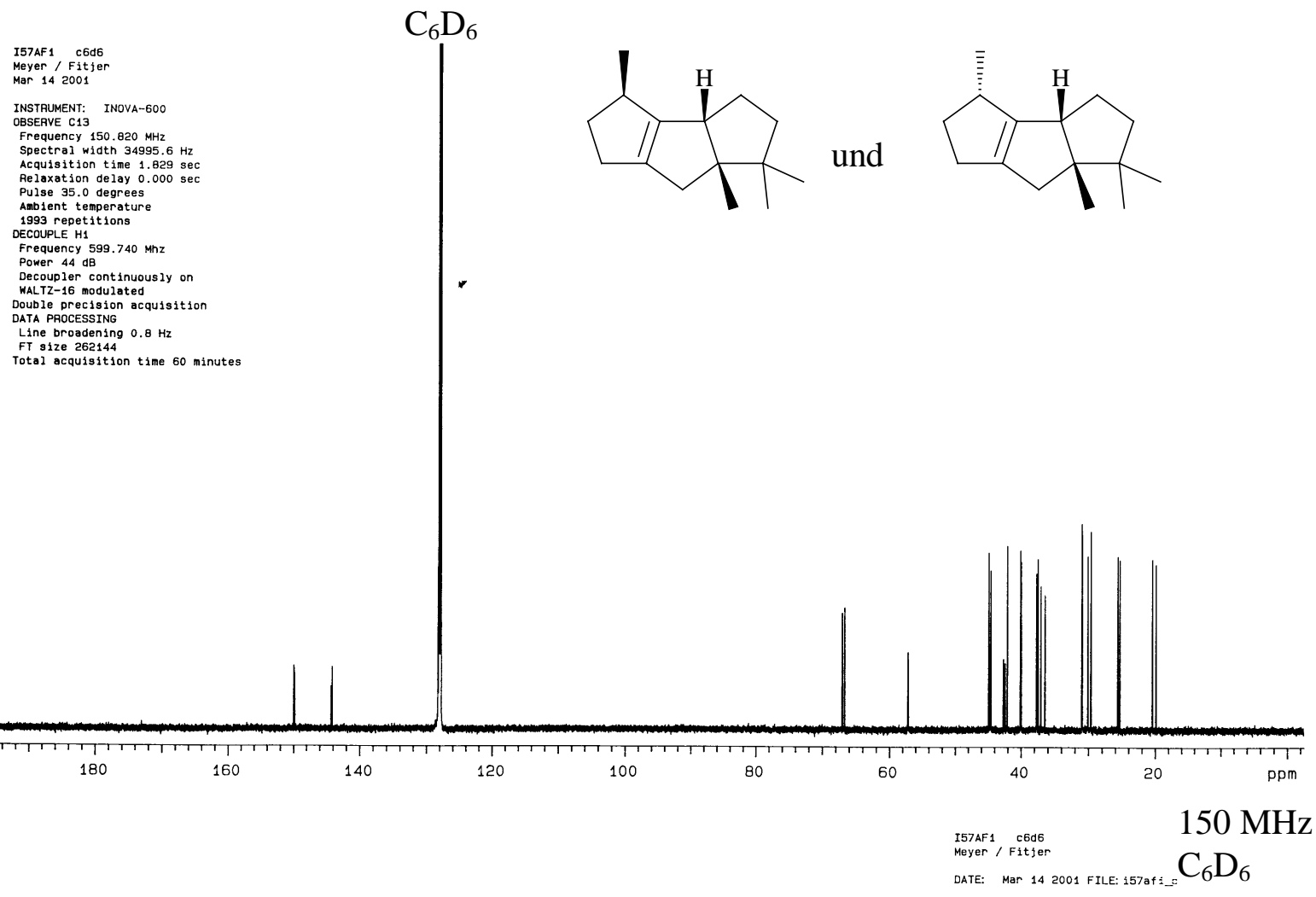

Abb.115: ${ }^{13} \mathrm{C}-\mathrm{NMR}-$ Spektrum von $\left(3 \mathrm{R}^{*}, 3 \mathrm{bS} *, 6 \mathrm{a} \mathrm{S}^{*}\right)$ - und (3R*,3bR*,6aR*)-3,6,6,6a-

Tetramethyl-2,3,3a,5,6,6a,7,7a-octahydro-1H-cyclopenta[a]pentalen (108/109) 


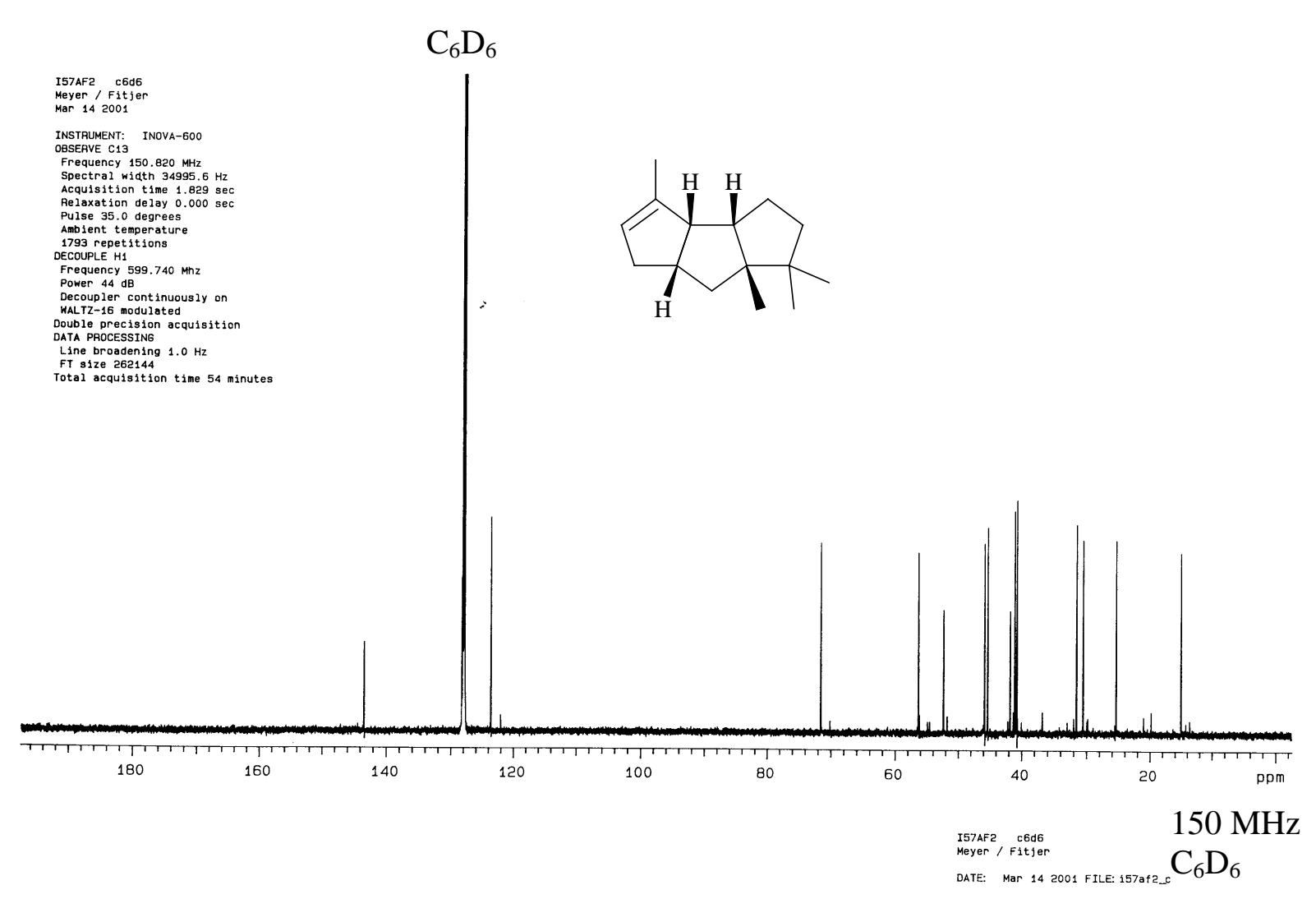

Abb.116: ${ }^{13} \mathrm{C}-\mathrm{NMR}-$ Spektrum von $\left(3 \mathrm{aR}^{*}, 3 \mathrm{bS} *, 6 \mathrm{aR} *, 7 \mathrm{aS} *\right)-1,1,4,7 \mathrm{a}-$ Tetramethyl-2,3,3a,3b,6, 6a,7,7a-octahydro-1H-cyclopenta[a]pentalen (110)
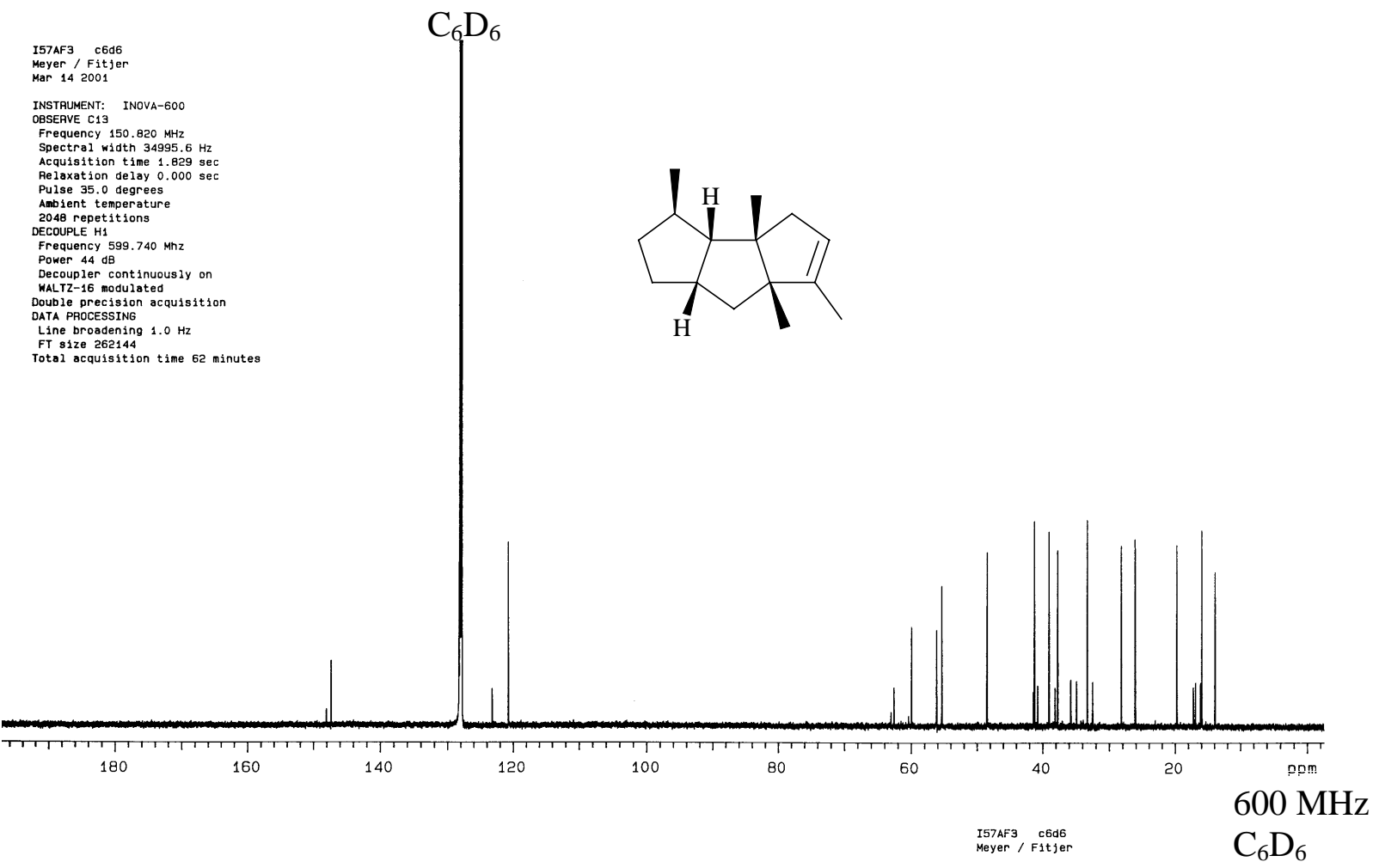

Abb. 117: ${ }^{13} \mathrm{C}-\mathrm{NMR}-$ Spektrum von $\left(3 \mathrm{R}^{*}, 3 \mathrm{aS} *, 3 \mathrm{bS} *, 6 \mathrm{aS} *, 7 \mathrm{aS} *\right)-3,3 \mathrm{~b}, 6,6 \mathrm{a}-$ Tetramethyl2,3,3a,3b,4,6a,7,7a-octahydro-1H-cyclopenta[a]pentalen (111) 


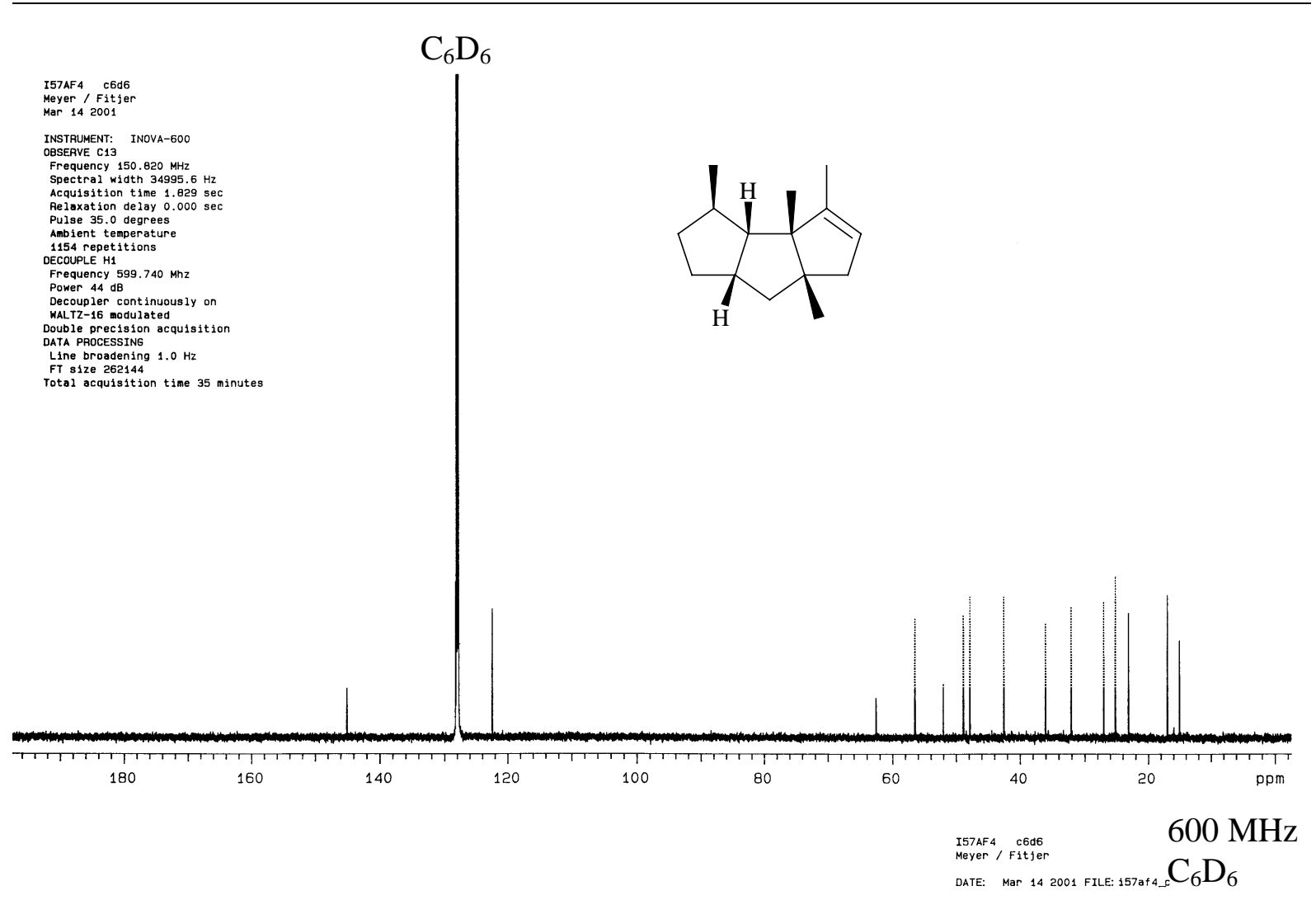

Abb. 120: ${ }^{13}$ C-NMR-Spektrum von (3R*,3aS*,3bS*,6aS*,7aS*)-3,3b,4,6a-Tetramethyl2,3,3a,3b,6,6a,7,7a-octahydro-1H-cyclopenta[a]pentalen (112) 

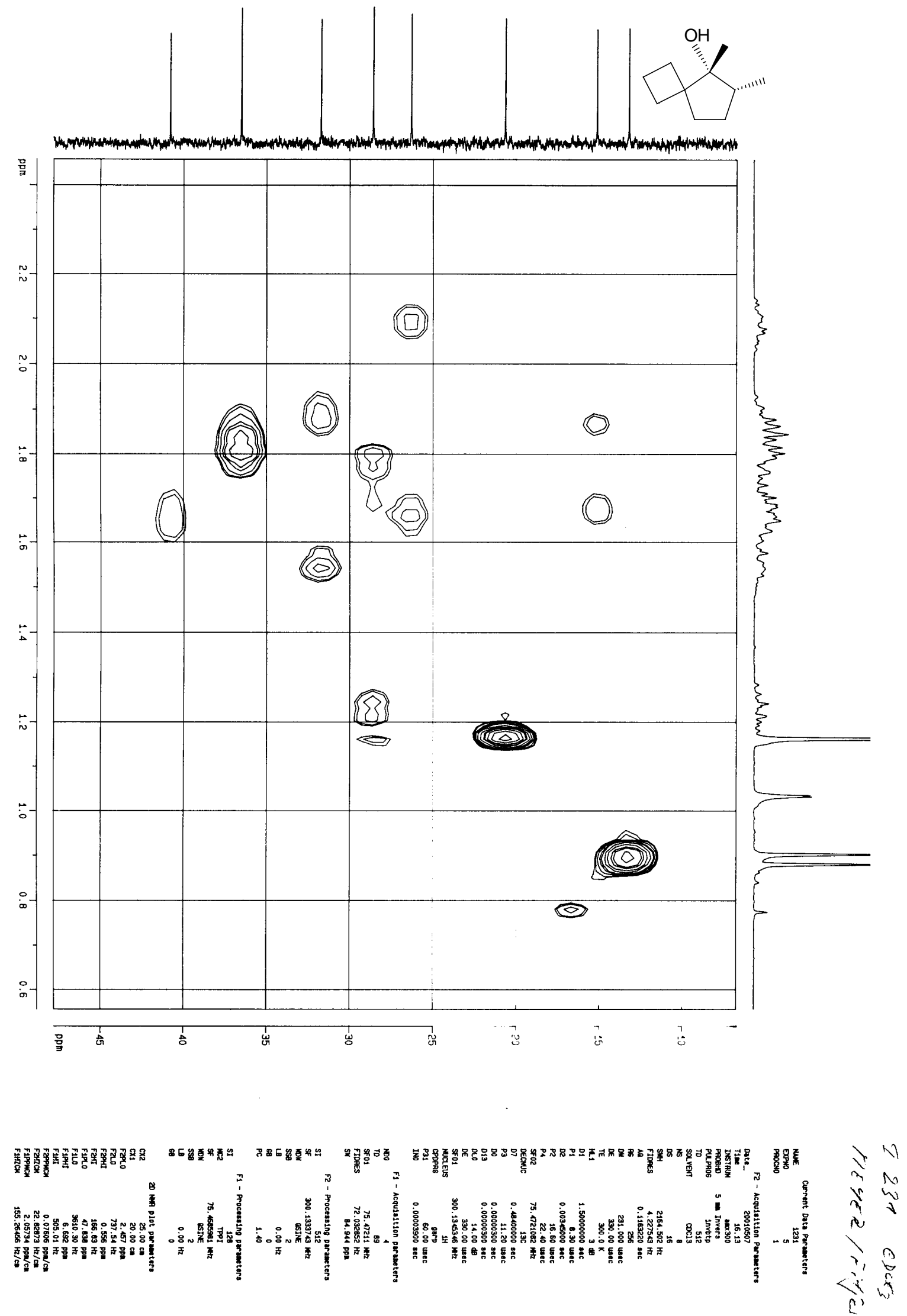

1)

Abb. 119: HMQC-Spektrum von (5R*,6R*)-5,6-Dimethyl-spiro[3.4] octan-5-ol (23a) 


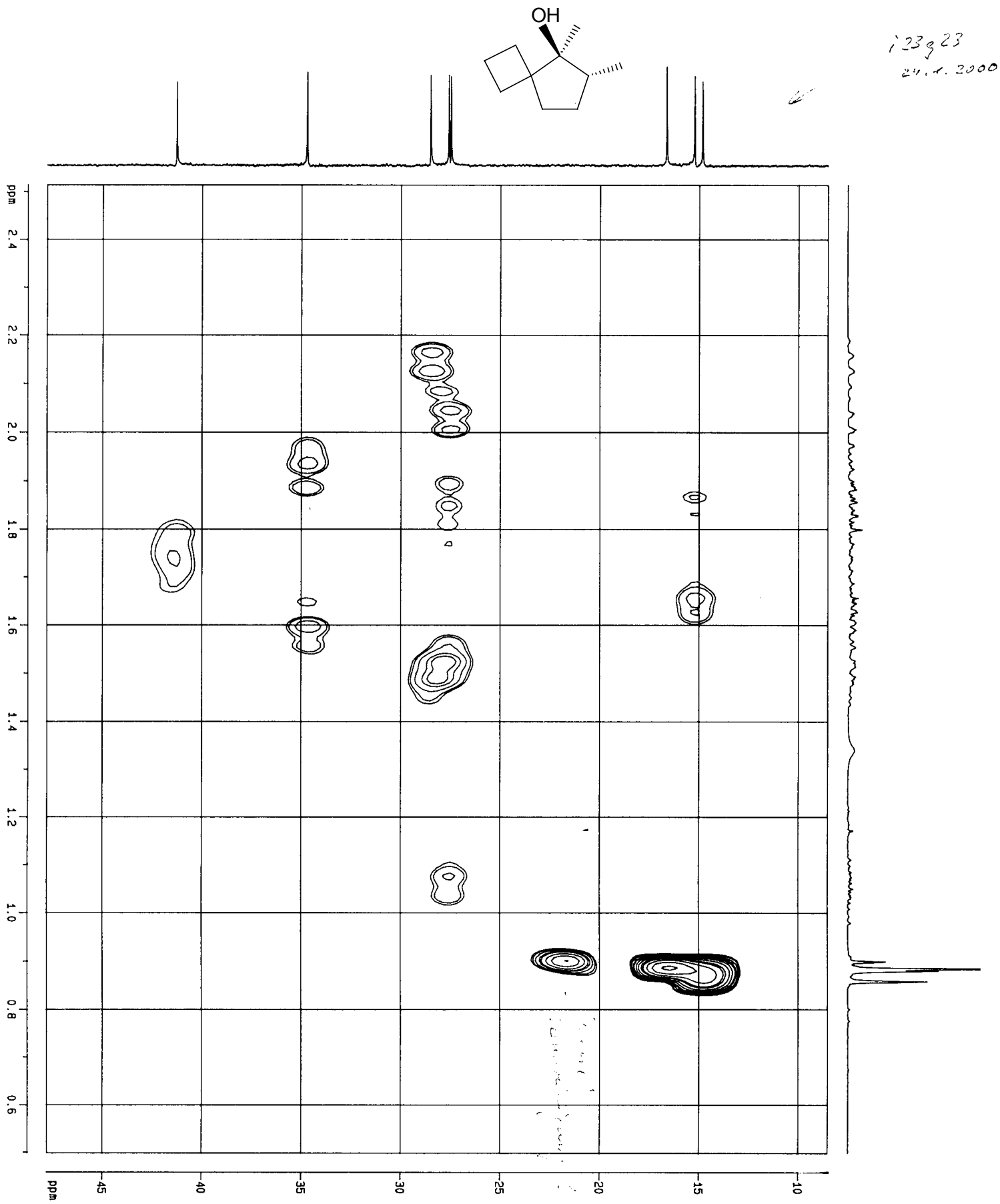

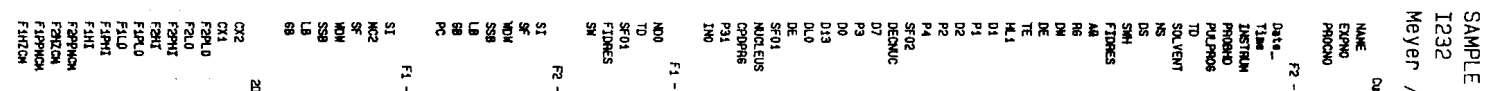

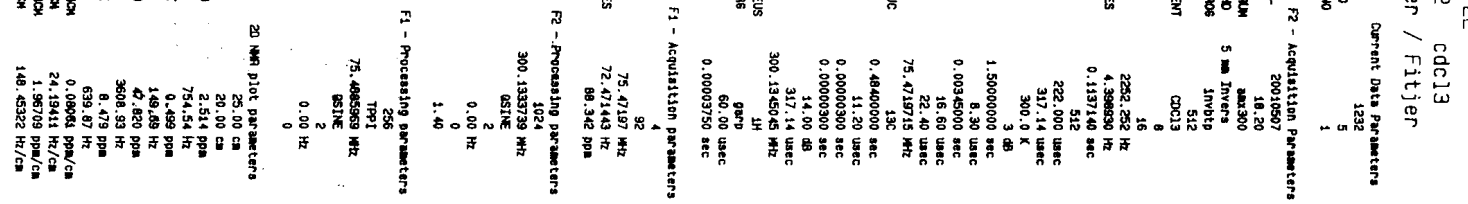

Abb. 120: HMQC-Spektrum von (5S*,6R*)-5,6-Dimethyl-spiro[3.4]octan-5-ol (23b) 

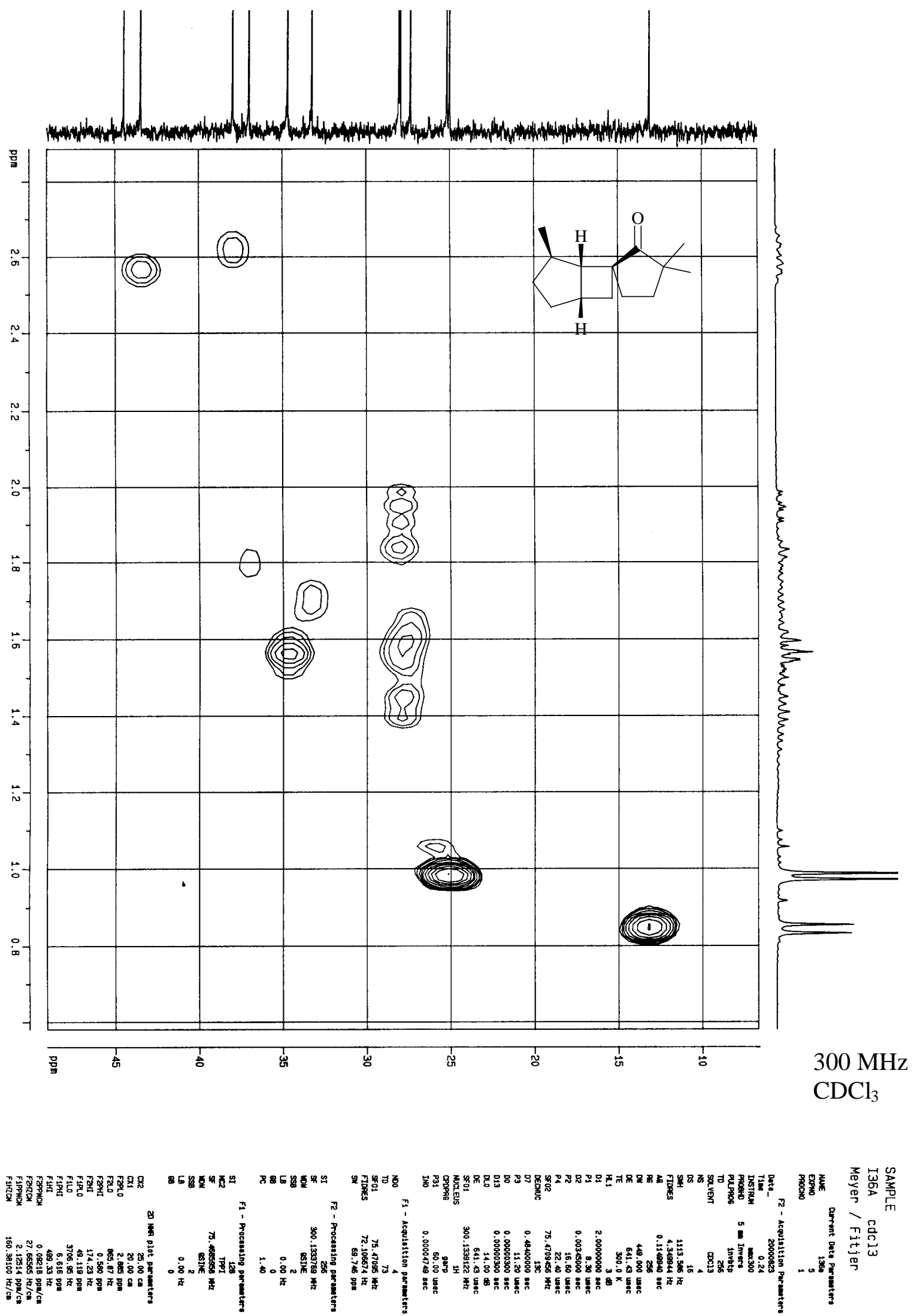

Abb. 121: HMQC-Spektrum von (1R*,4S*,5R*,6R*)-4,3',3'-Trimethyl-spiro \{ bicyclo[3.2.0]heptan-6, $1^{\prime}$-cyclopentan-2'-on $\}$ (95) 

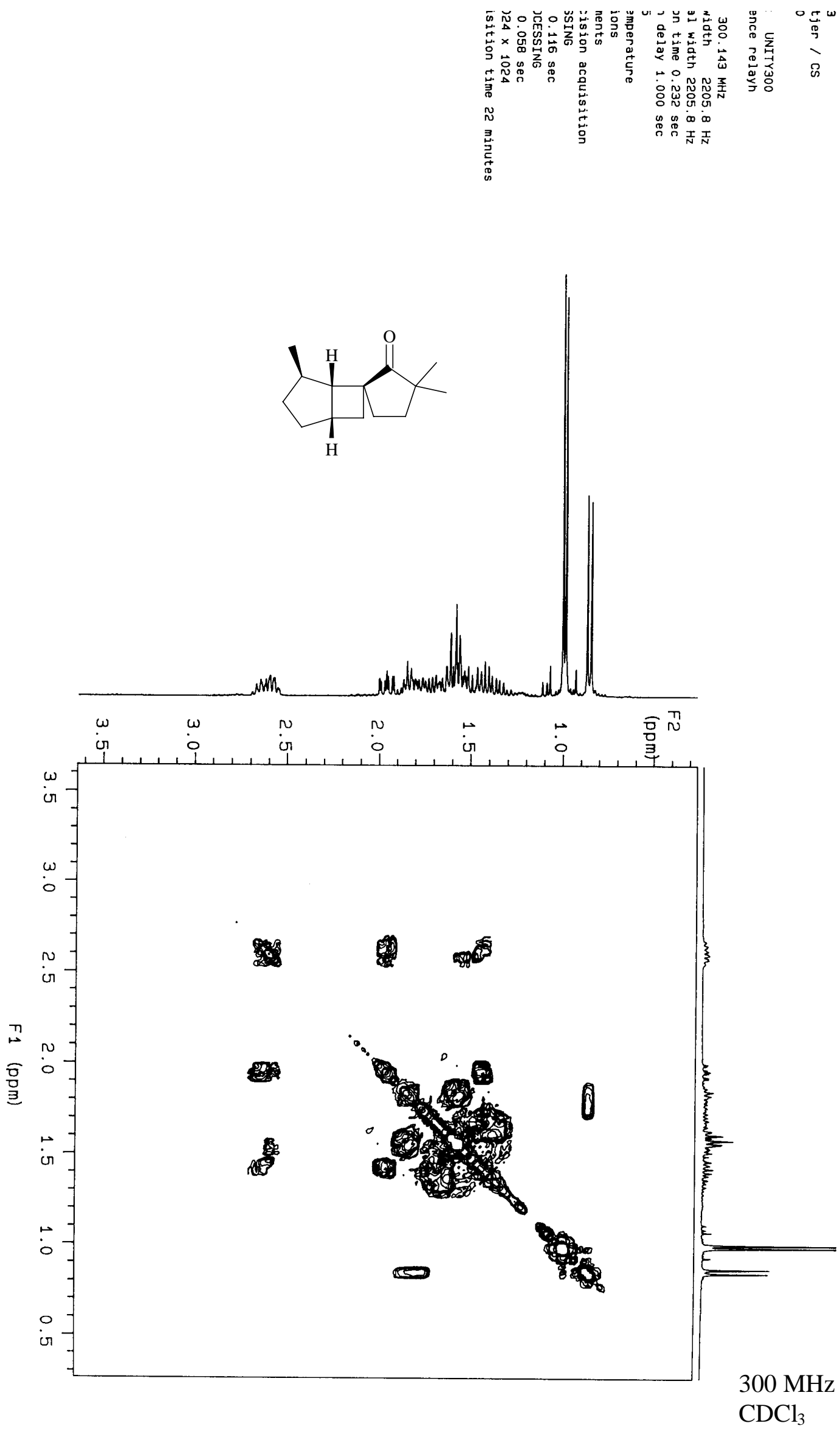

Abb. 122: COSY-Spektrum von (1R*,4S*,5R*,6R*)-4,3',3'-Trimethyl-spiro \{bicyclo[3.2.0]heptan-6, $1^{\prime}$-cyclopentan-2'-on $\}$ (95) 

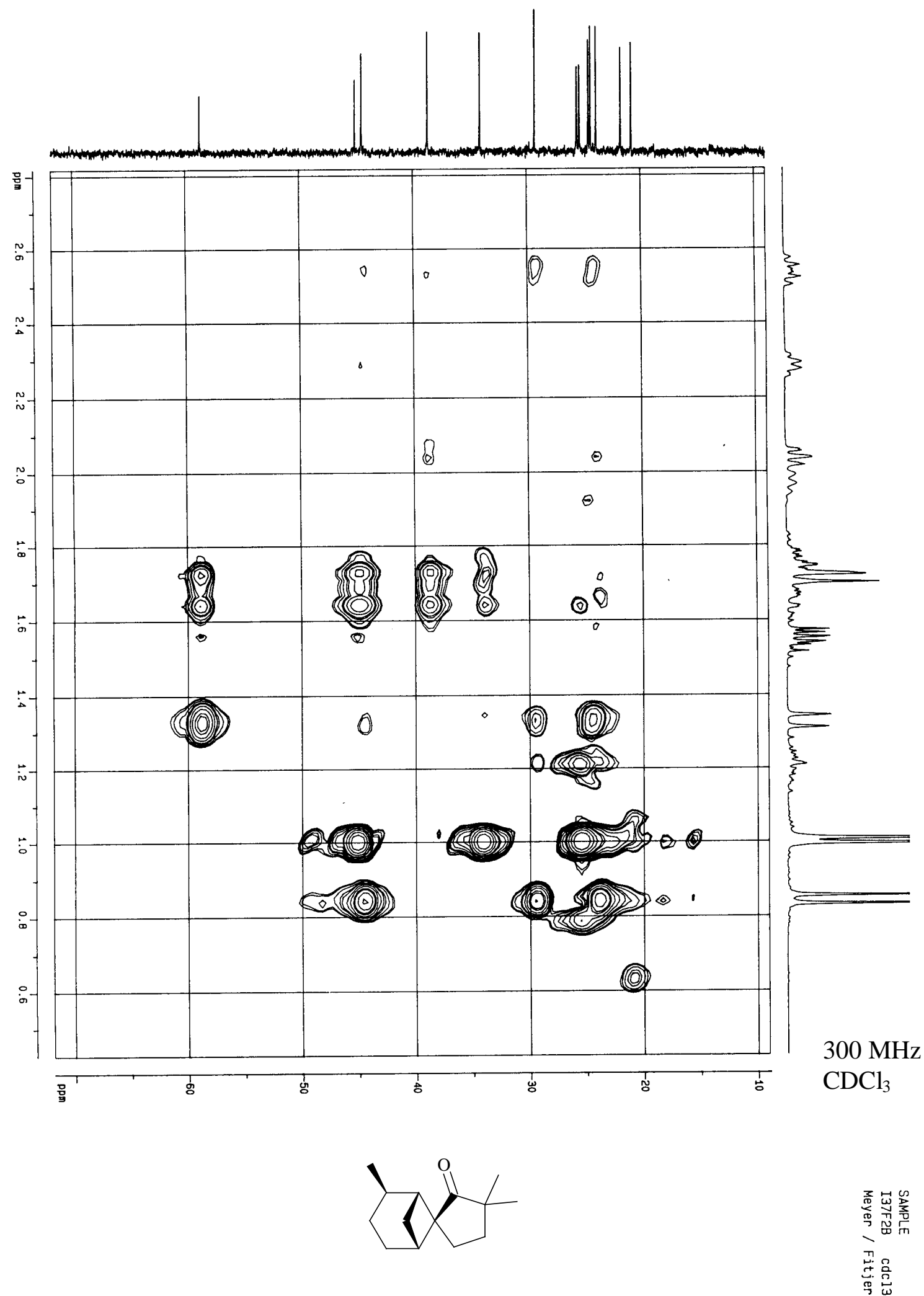

Abb. 123: HMQC-Spektrum von $\left(1 \mathrm{R}^{*}, 2 \mathrm{R}^{*}, 5 \mathrm{R}^{*}, 6 \mathrm{~S}^{*}\right)-2,3^{\prime}, 3^{\prime}$-Trimethyl-spiro $\{$ bicyclo[3.1.1]heptan-6,1'-cyclopentan-2'-on\} (96) 


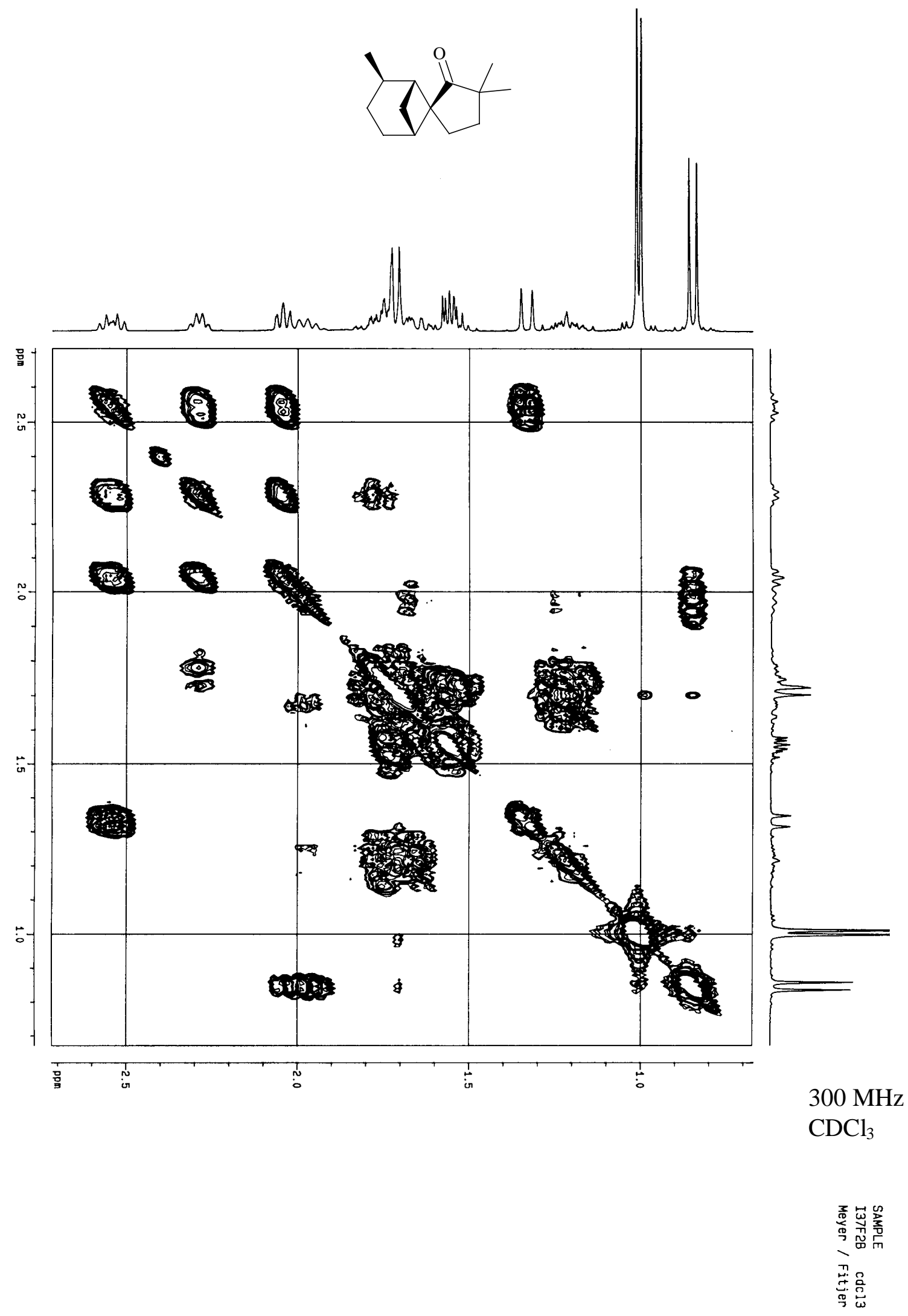

Abb. 124: COSY-Spektrum von $\left(1 \mathrm{R}^{*}, 2 \mathrm{R}^{*}, 5 \mathrm{R}^{*}, 6 \mathrm{~S}^{*}\right)-2,3^{\prime}, 3^{\prime}-$ Trimethyl-spiro $\{$ bicyclo[3.1.1]heptan-6,1'-cyclopentan-2'-on\} (96) 

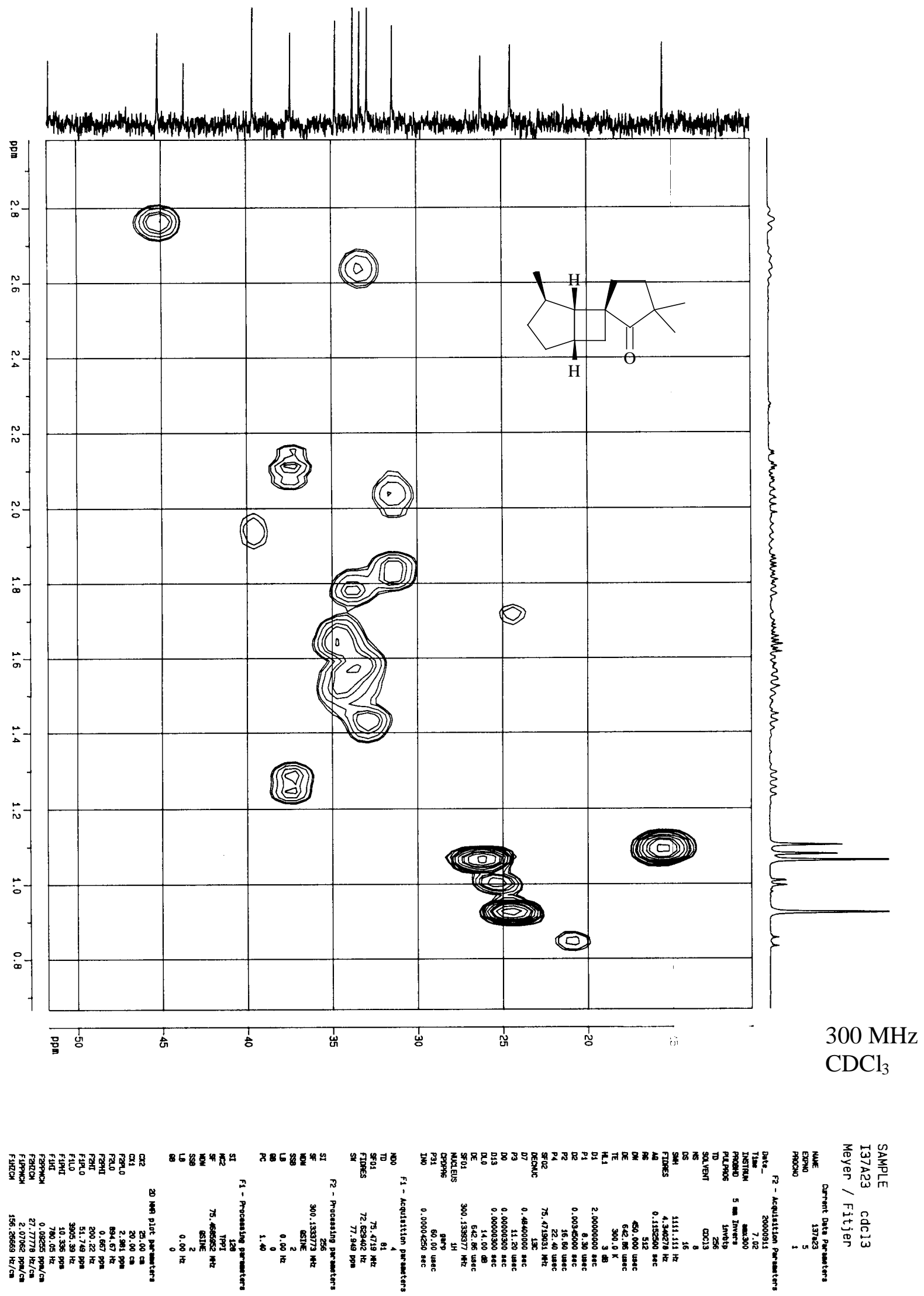

Abb. 125: HMQC-Spektrum von $\left(1 \mathrm{R}^{*}, 4 \mathrm{~S}^{*}, 5 \mathrm{R}^{*}, 6 \mathrm{~S}^{*}\right)-4,3^{\prime}, 3^{\prime}-$ Trimethyl-spiro \{bicyclo[3.2.0]heptan-6,1'-cyclopentan-2on\} (94) 


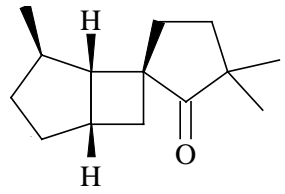

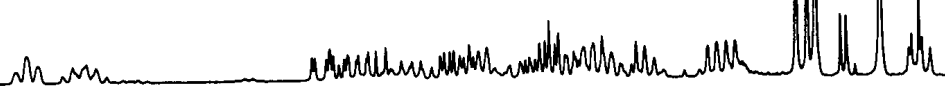
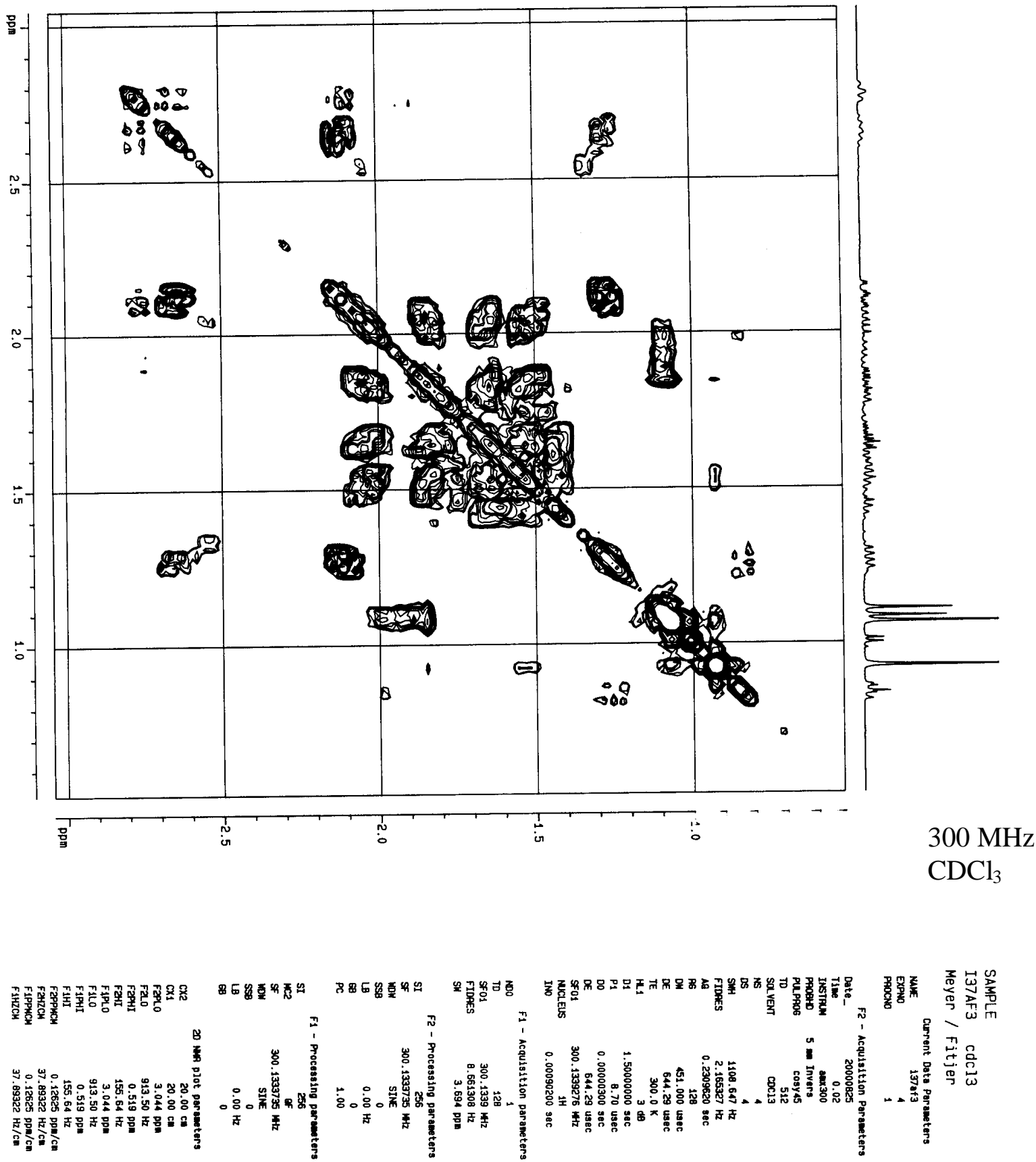

Abb. 126: COSY-Spektrum von $\left(1 \mathrm{R}^{*}, 4 \mathrm{~S}^{*}, 5 \mathrm{R}^{*}, 6 \mathrm{~S}^{*}\right)-4,3^{\prime}, 3^{\prime}$-Trimethyl-spiro $\{$ bicyclo[3.2.0]heptan-6,1'-cyclopentan-2'-on\} (94) 

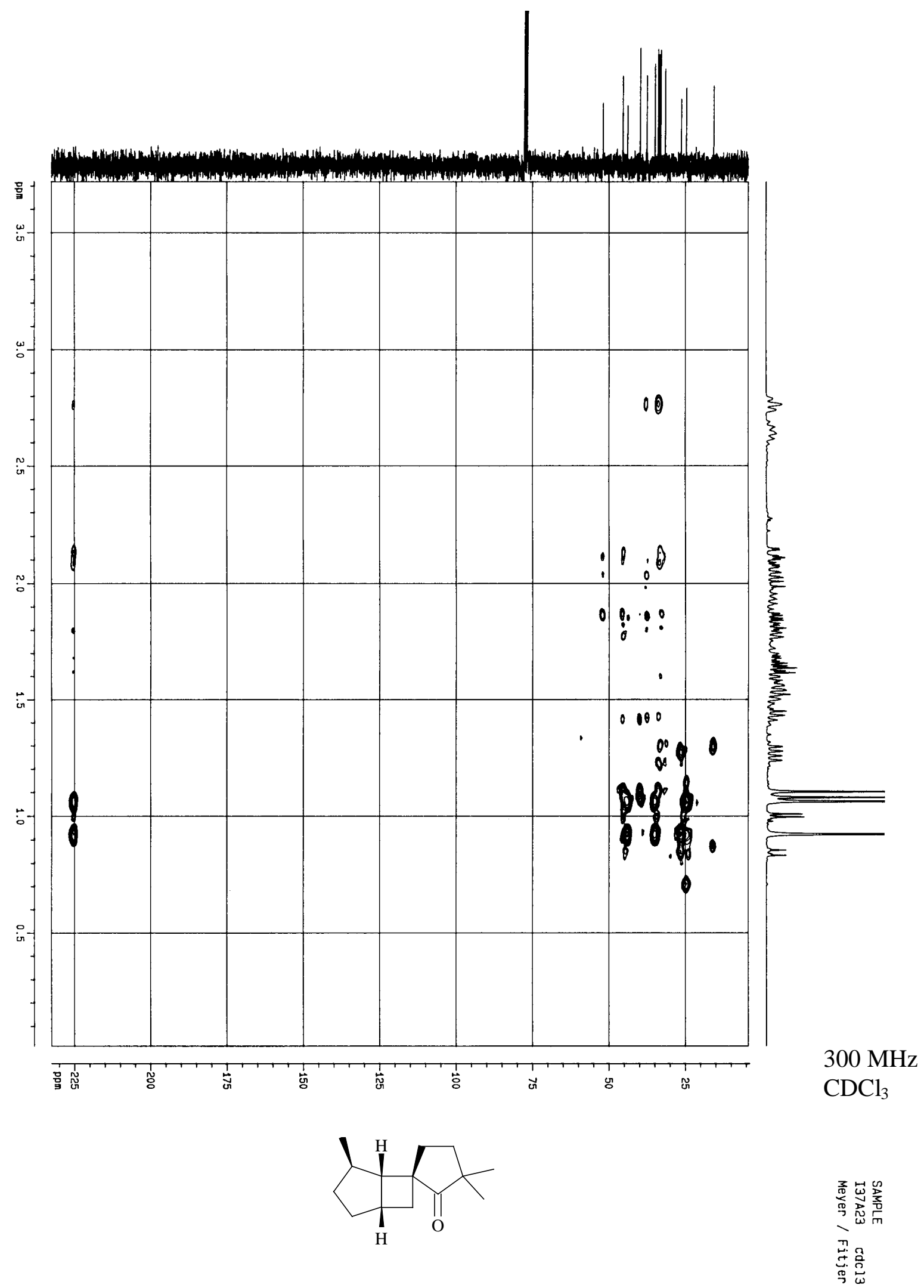

Abb. 127: HMBC-Spektrum von $\left(1 \mathrm{R}^{*}, 4 \mathrm{~S}^{*}, 5 \mathrm{R}^{*}, 6 \mathrm{~S}^{*}\right)-4,3^{\prime}, 3^{\prime}$-Trimethyl-spiro $\{$ bicyclo[3.2.0]heptan-6,1'-cyclopentan-2'-on\} (94) 

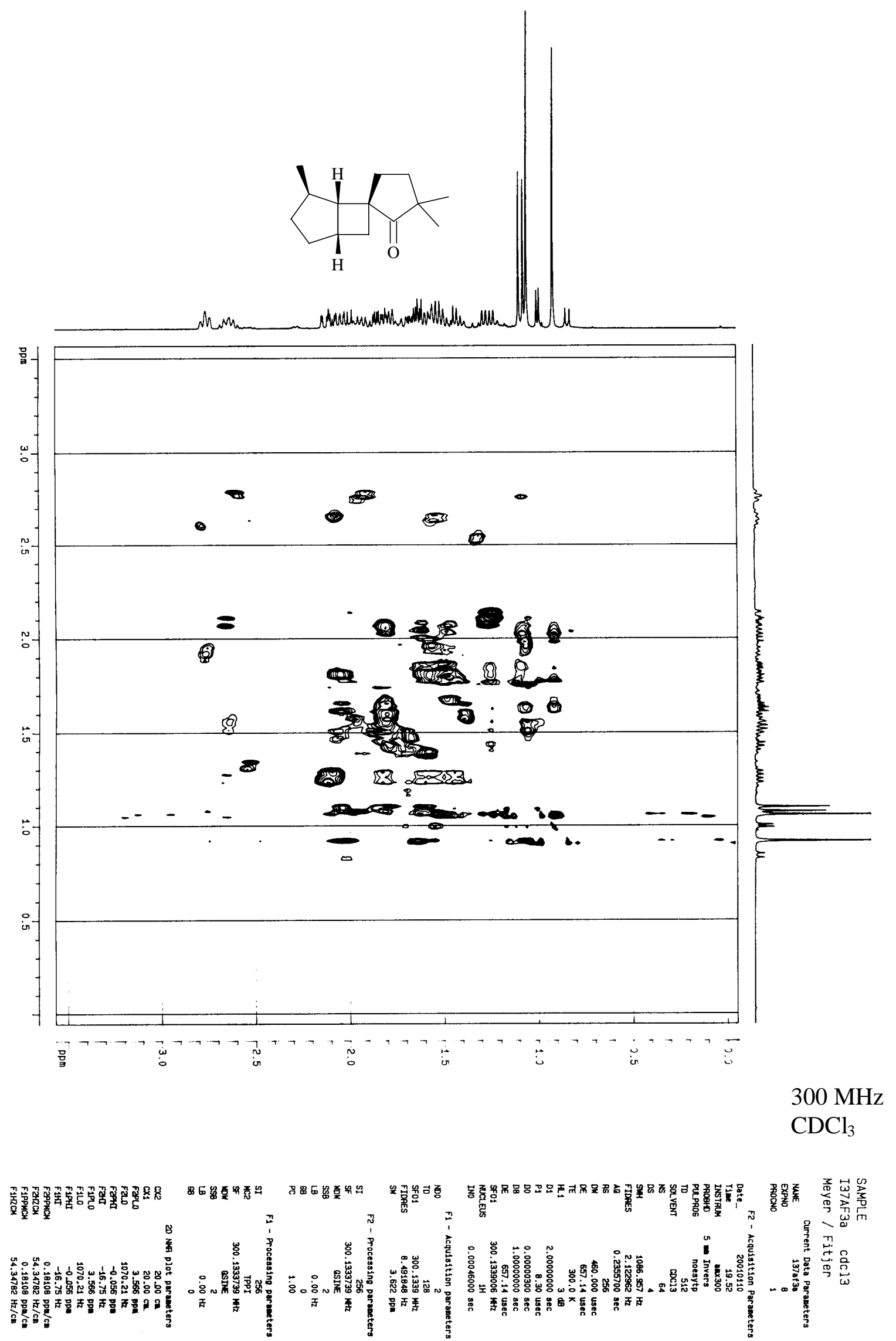

Abb. 128: NOESY-Spektrum von $\left(1 \mathrm{R}^{*}, 4 \mathrm{~S}^{*}, 5 \mathrm{R}^{*}, 6 \mathrm{~S}^{*}\right)-4,3^{\prime}, 3^{\prime}$-Trimethyl-spiro $\{$ bicyclo[3.2.0]heptan-6,1'-cyclopentan-2'-on\} 

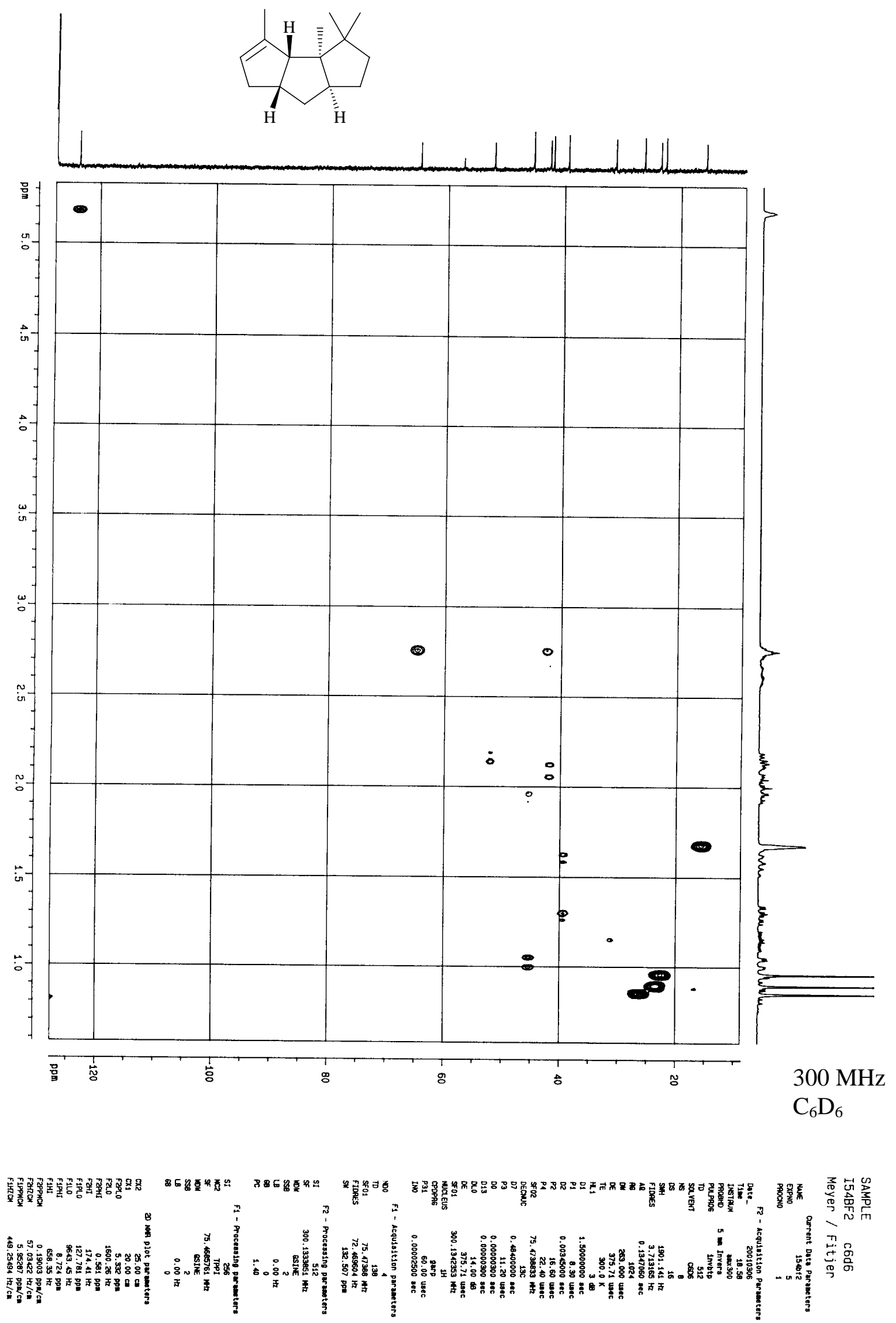

Abb. 129: HMQC-Spektrum von (3aR*,3bR*,6aR*,7aR*)-3,3,3a,4-Tetramethyl-2,3,3a,3b, 6,6a,7,7a-octahydro- $1 H$-cyclopenta[a]pentalen (119) 

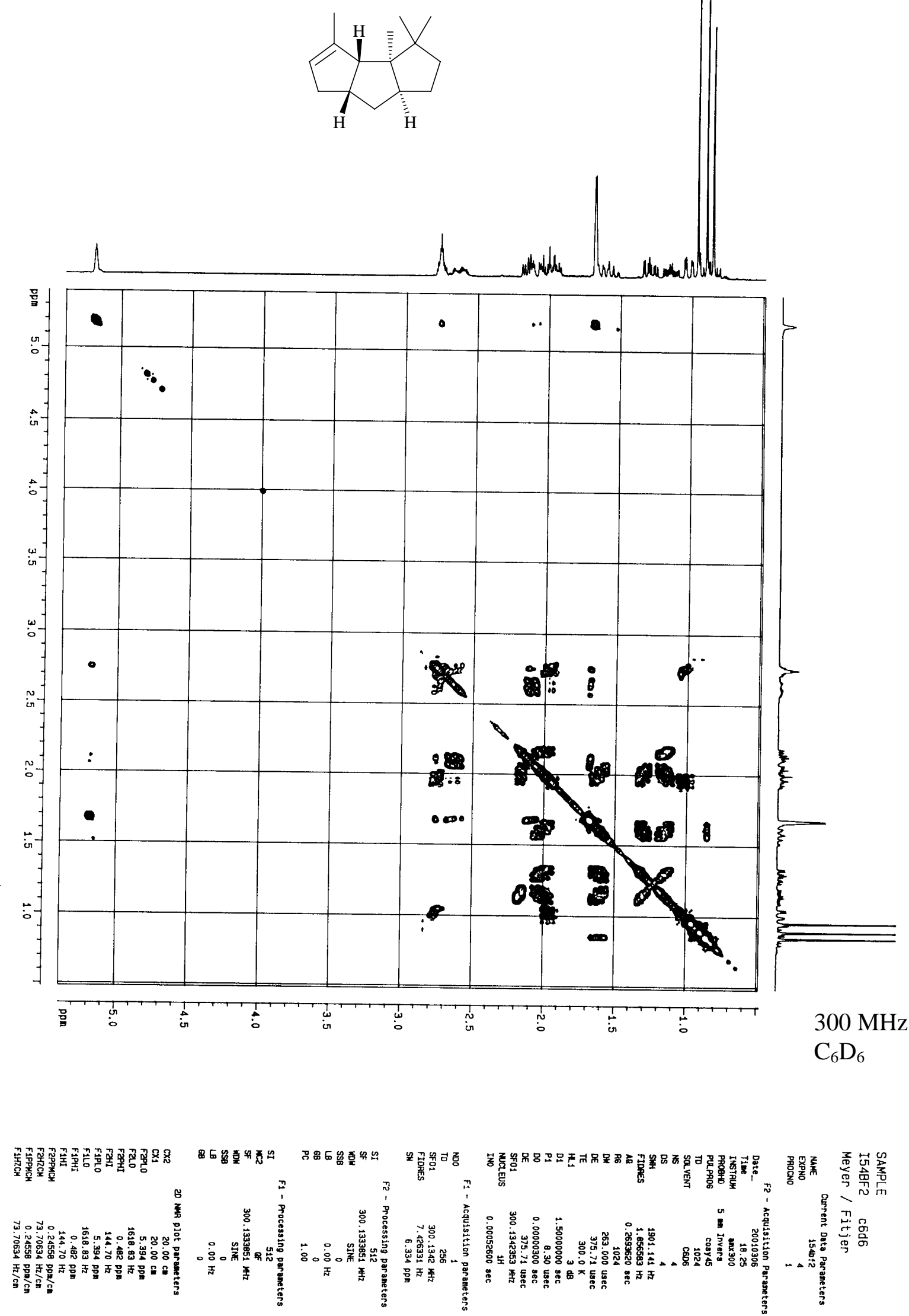

Abb. 130: COSY-Spektrum von (3aR*,3bR*,6aR*,7aR*)-3,3,3a,4-Tetramethyl2,3,3a,3b,6,6a,7,7a-octahydro-1H-cyclopenta[a]pentalen (119) 

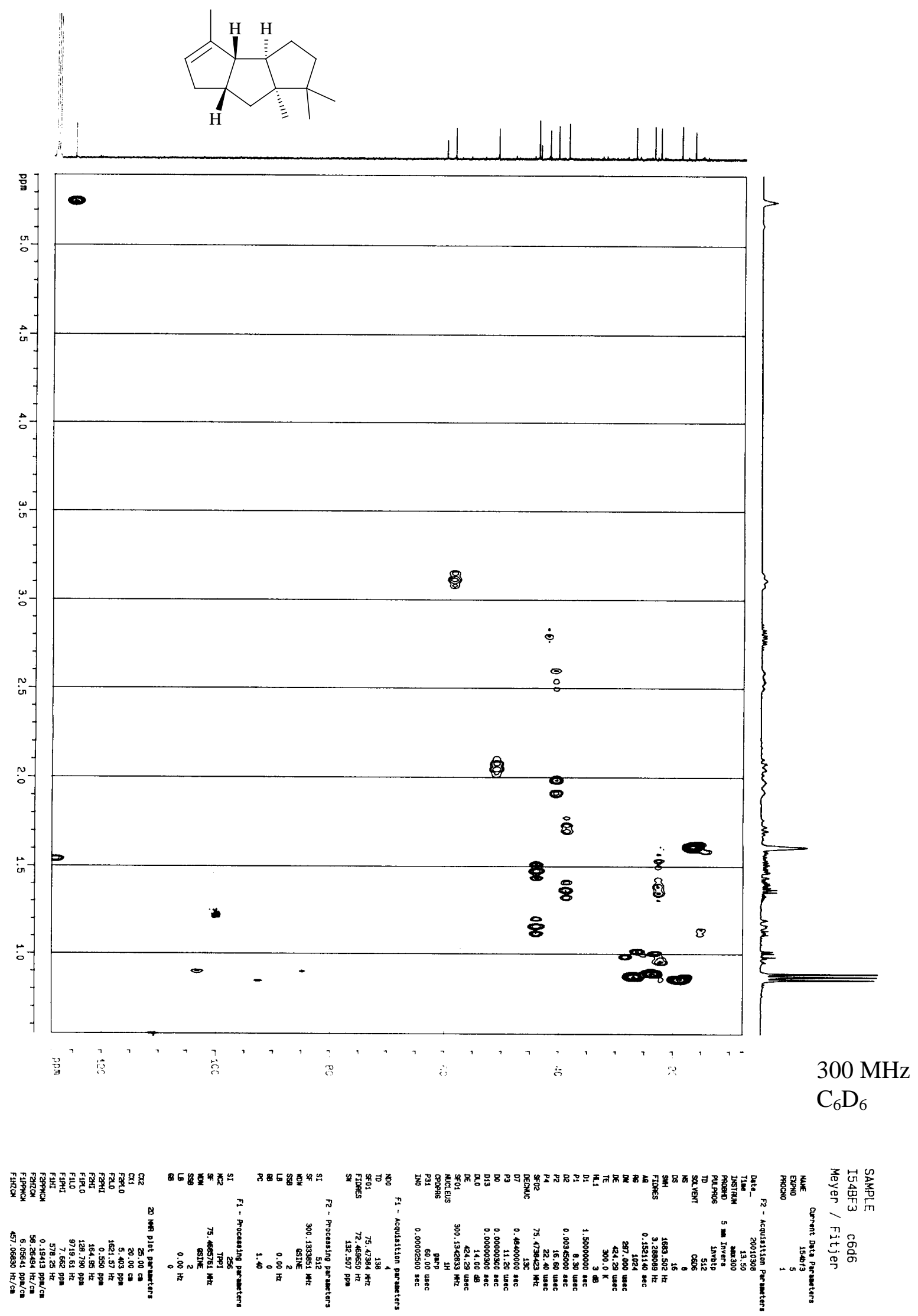

Abb. 131: HMQC-Spektrum von (3aR*,3bR*,6aS*,7aS*)-1,1,4,7a-Tetramethyl-2,3,3a,3b, 6,6a,7,7a-octahydro- $1 H$-cyclopenta[a]pentalen (120) 

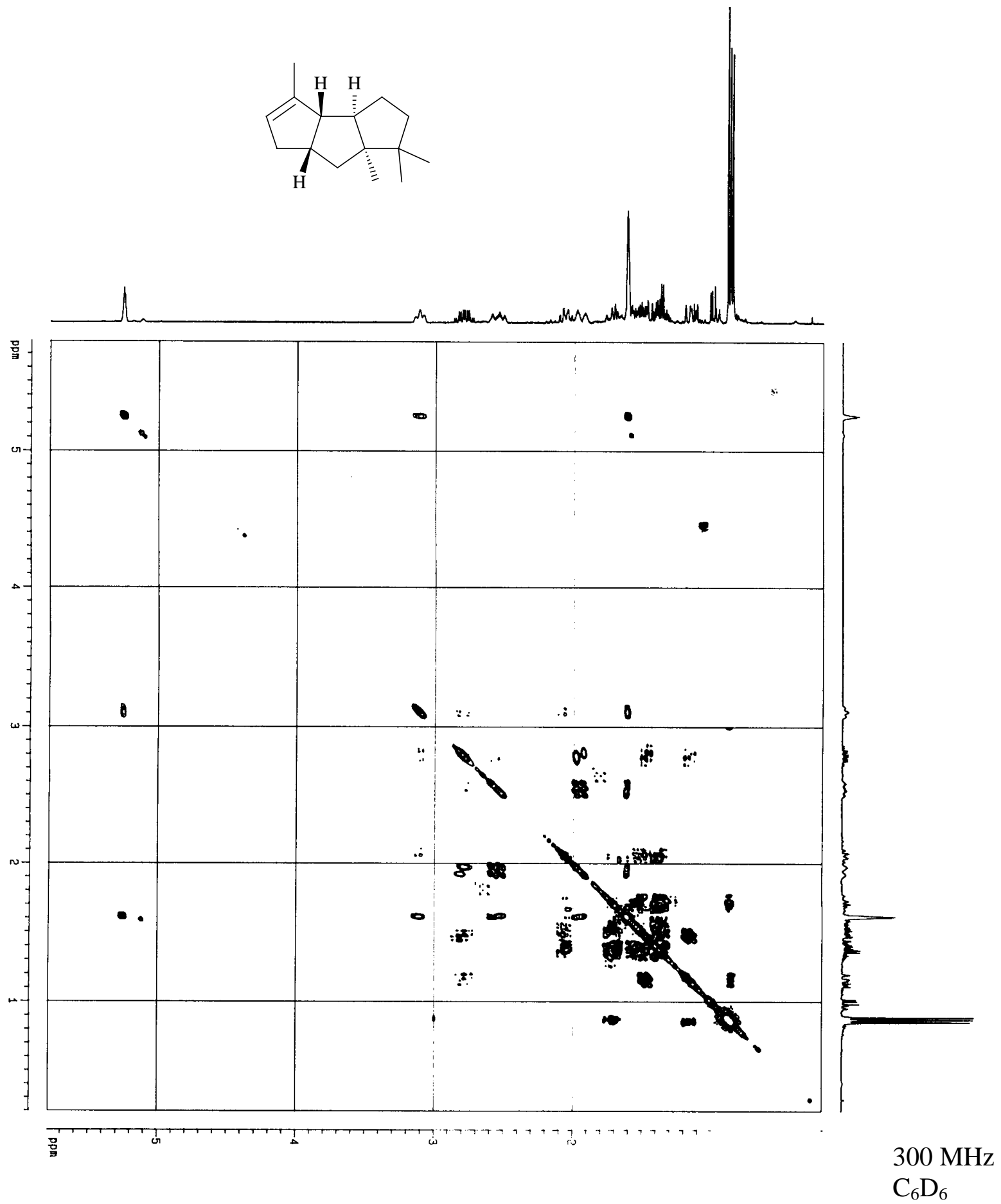

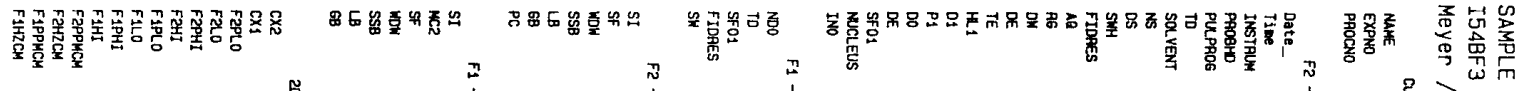

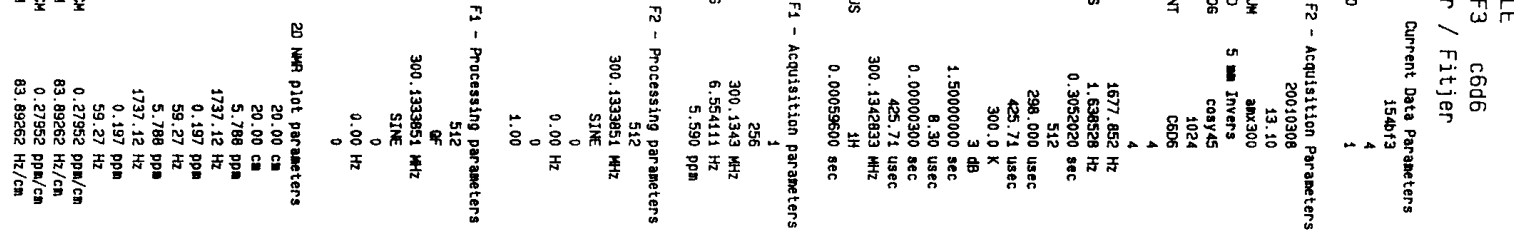

Abb. 132: COSY-Spektrum von $\left(3 \mathrm{aR}^{*}, 3 \mathrm{bR}^{*}, 6 \mathrm{aS} *, 7 \mathrm{aS} *\right)-1,1,4,7 \mathrm{a}-$-Tetramethyl-2,3,3a,3b, 6,6a,7,7a-octahydro- $1 H$-cyclopenta[a]pentalen (120) 


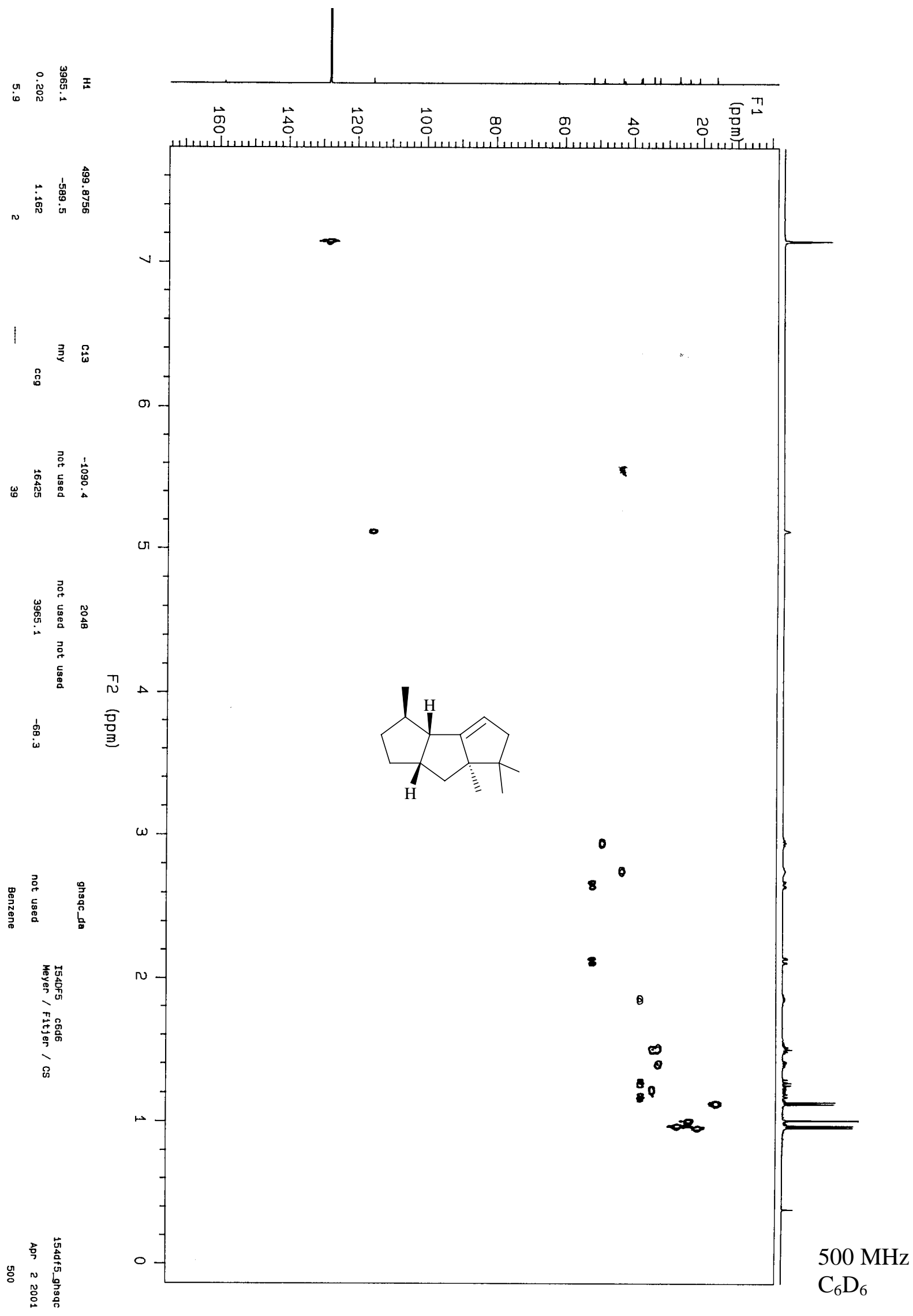

Abb. 133: HMQC-Spektrum von (3R*,3aS*,6aR*,7aR*)-3,6,6,6a-Tetramethyl-2,3,3a,5,6, 6a,7,7a-octahydro- $1 H$-cyclo-penta[a]pentalen (118) 

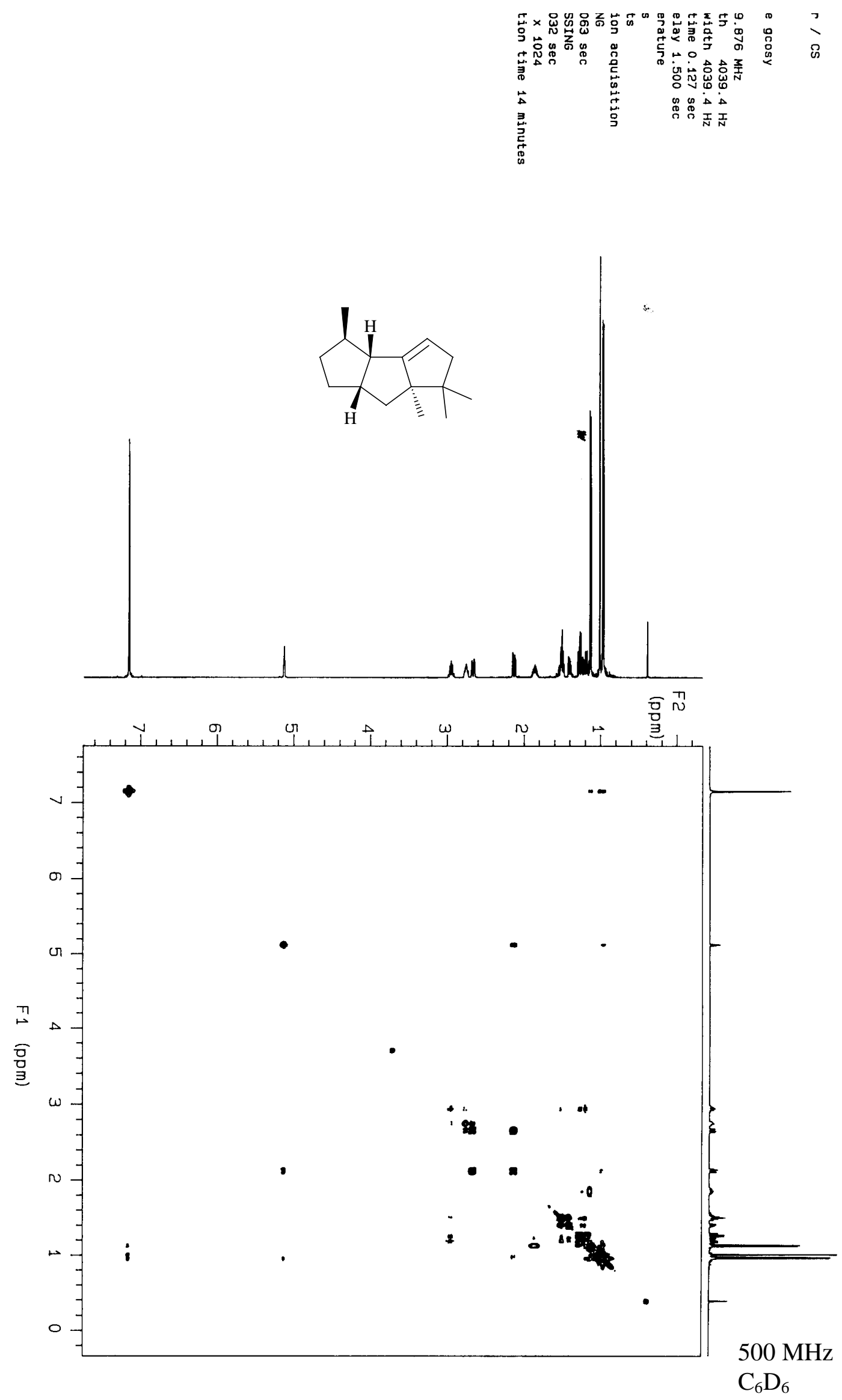

Abb. 134: COSY-Spektrum von $\left(3 \mathrm{R}^{*}, 3 \mathrm{aS} *, 6 \mathrm{aR}^{*}, 7 \mathrm{aR} *\right)-3,6,6,6 \mathrm{a}-$ Tetramethyl-

2,3,3a,5,6,6a,7,7a-octahydro-1H-cyclo-penta[a]pentalen (118) 


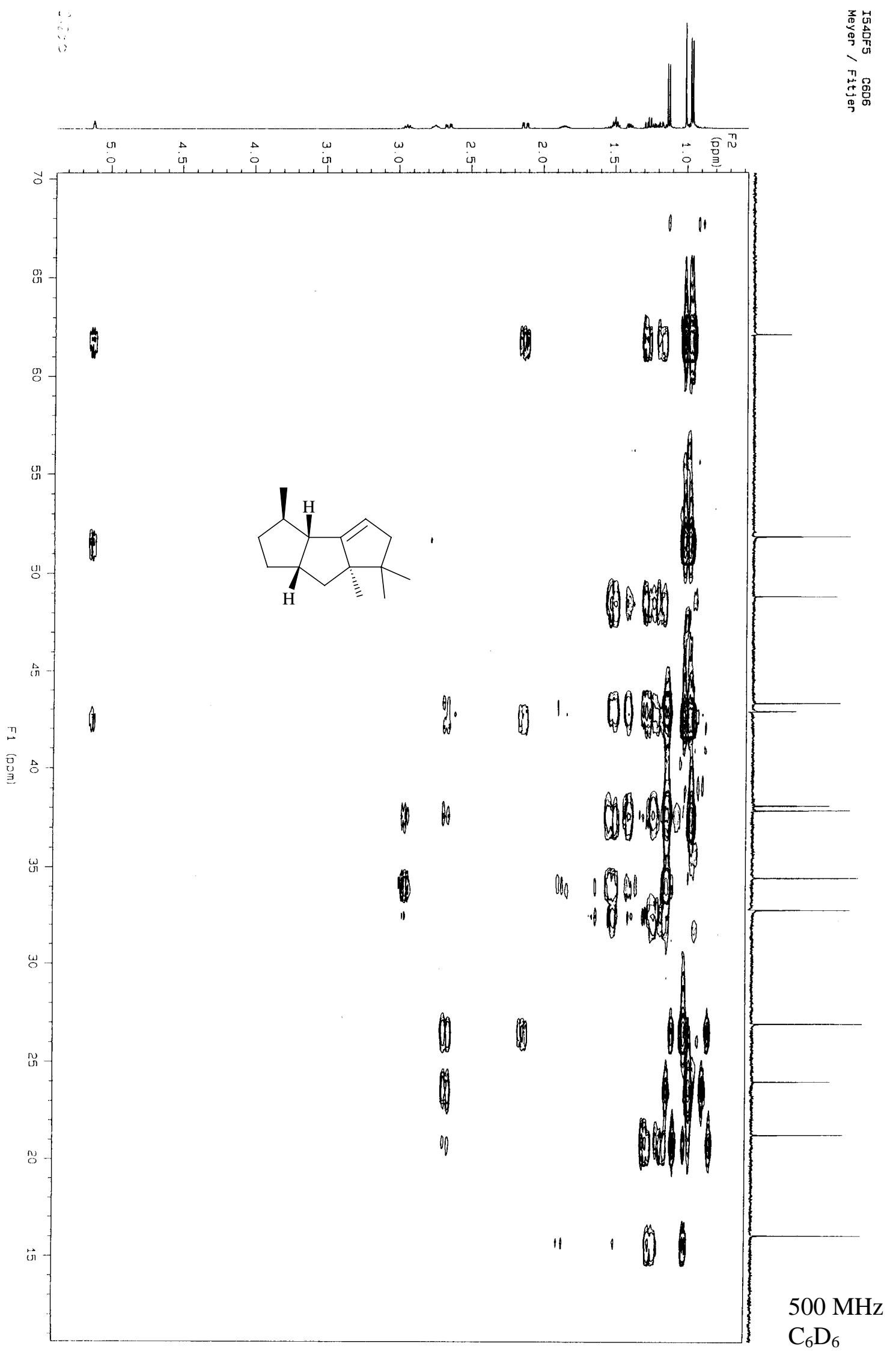

Abb. 135: HMBC-Spektrum von (3R*,3aS*,6aR*,7aR*)-3,6,6,6a-Tetramethyl-2,3,3a,5,6, 6a, 7,7a-octahydro-1H-cyclo-penta[a]pentalen (118) 


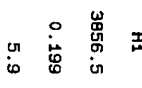

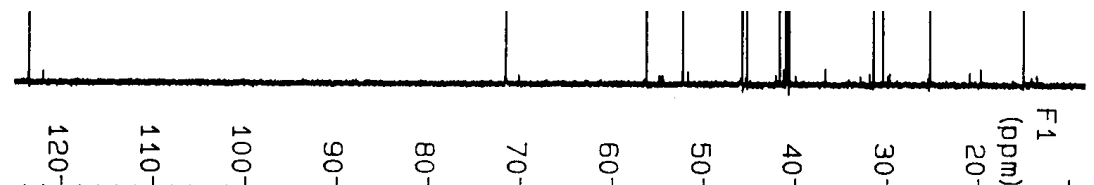

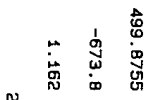

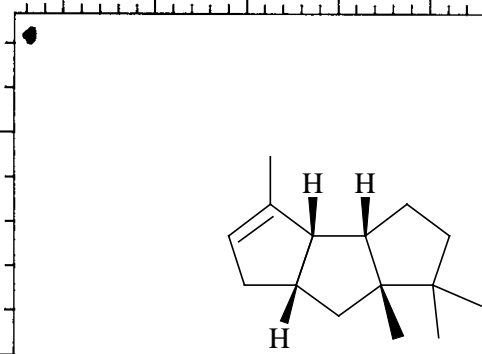

i

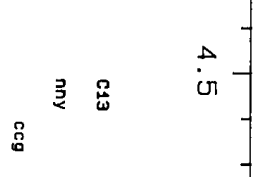
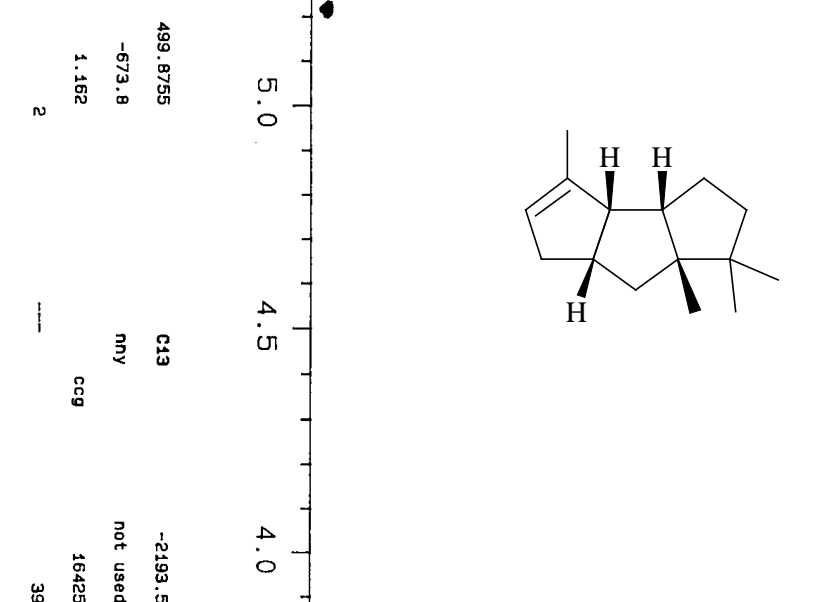

(1)

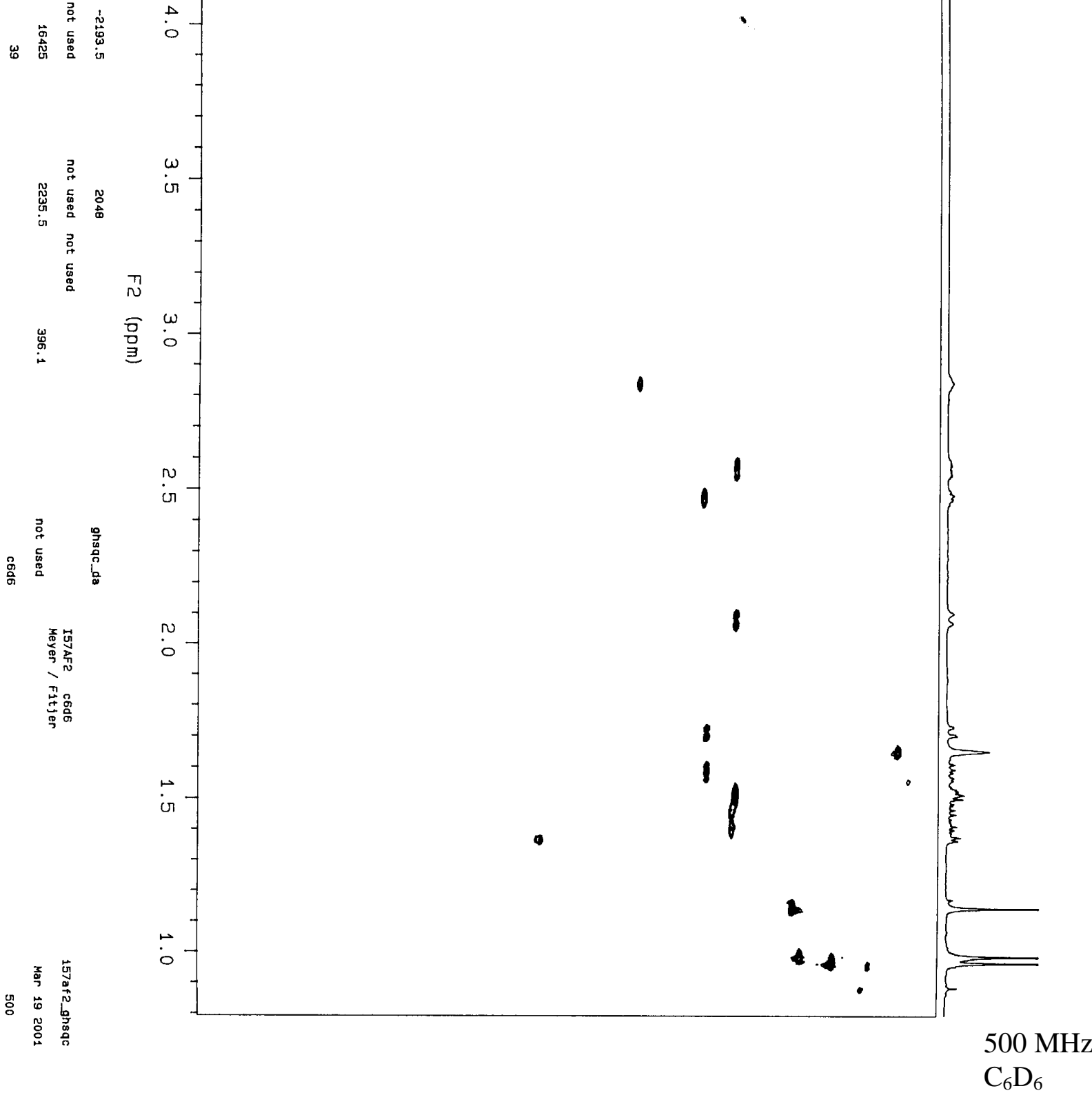

Abb. 136: HMQC-Spektrum von (3aR*,3bS*,6aR*,7aS*)-1,1,4,7a-Tetramethyl-2,3,3a,3b,6, 6a,7,7a-octahydro-1 $\mathrm{H}$-cyclopenta[a]pentalen (110) 


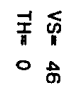

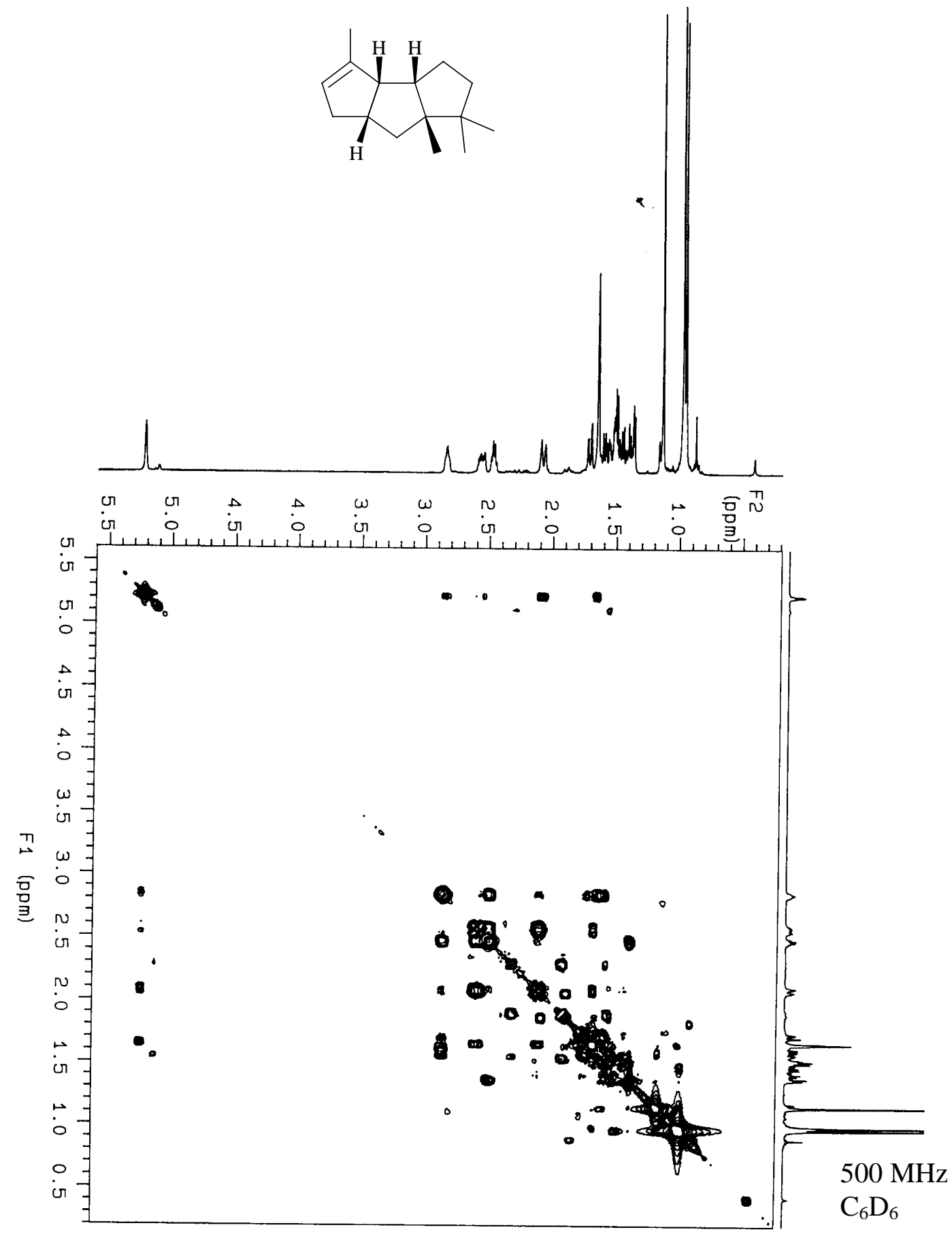

Abb. 137: COSY-Spektrum von ( $\left.3 \mathrm{aR}^{*}, 3 \mathrm{bS}^{*}, 6 \mathrm{aR}^{*}, 7 \mathrm{aS} *\right)-1,1,4,7 \mathrm{a}-$ Tetramethyl-

2,3,3a,3b,6,6a,7,7a-octahydro- $1 H$-cyclopenta[a]pentalen (110) 


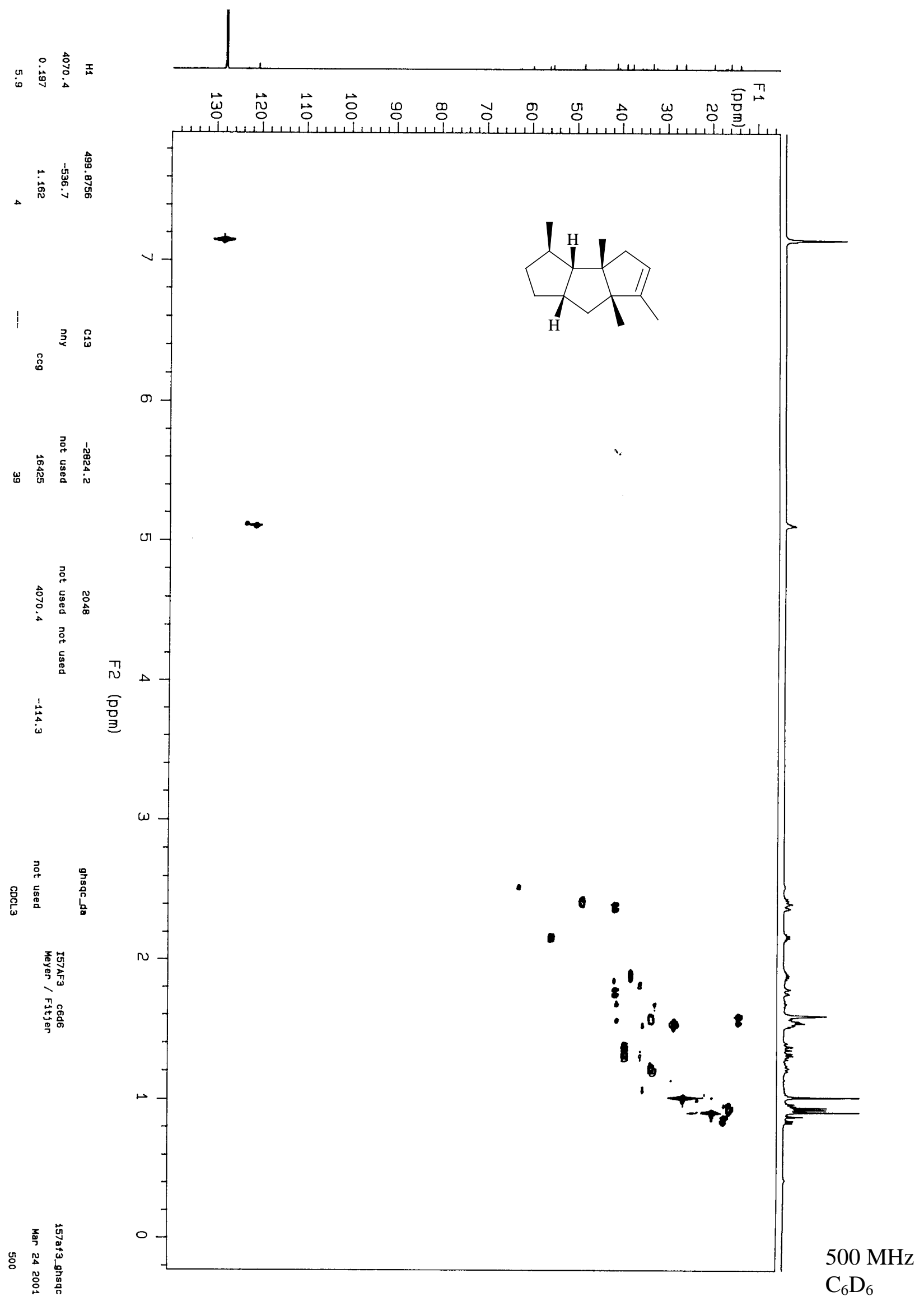

Abb. 138: HMQC-Spektrum von (3R*,3aS*, 3bS*,6aS*,7aS*)-3,3b,6,6a-Tetramethyl-2,3,3a, 3b,4,6a, 7,7a-octahydro-1H-cyclopenta[a]pentalen (111) 


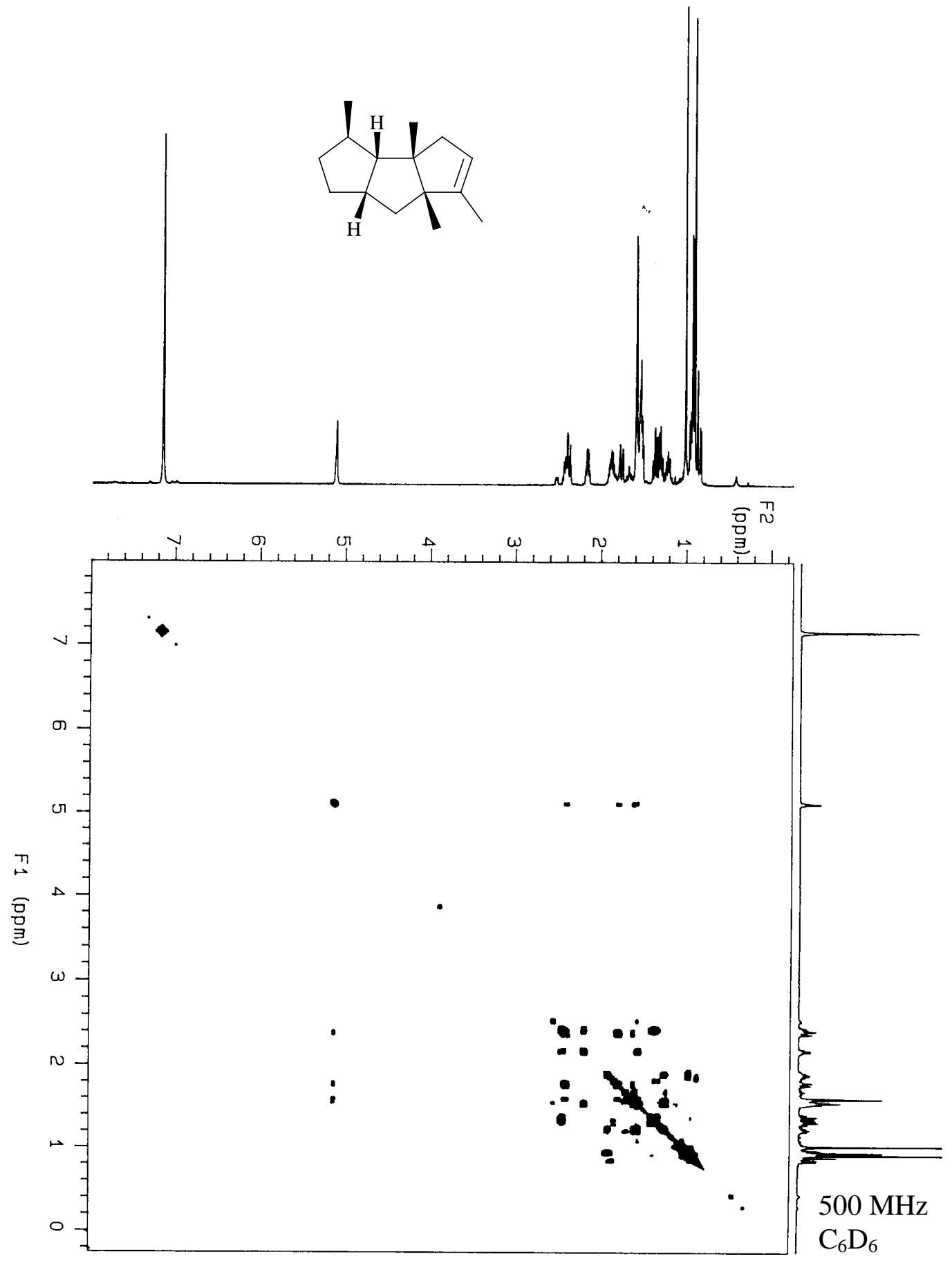

Abb. 139: COSY-Spektrum von (3R*,3aS*, 3bS*,6aS*,7aS*)-3,3b,6,6a-Tetramethyl-2,3,3a, 3b,4,6a,7,7a-octahydro-1H-cyclopenta[a]pentalen (111) 


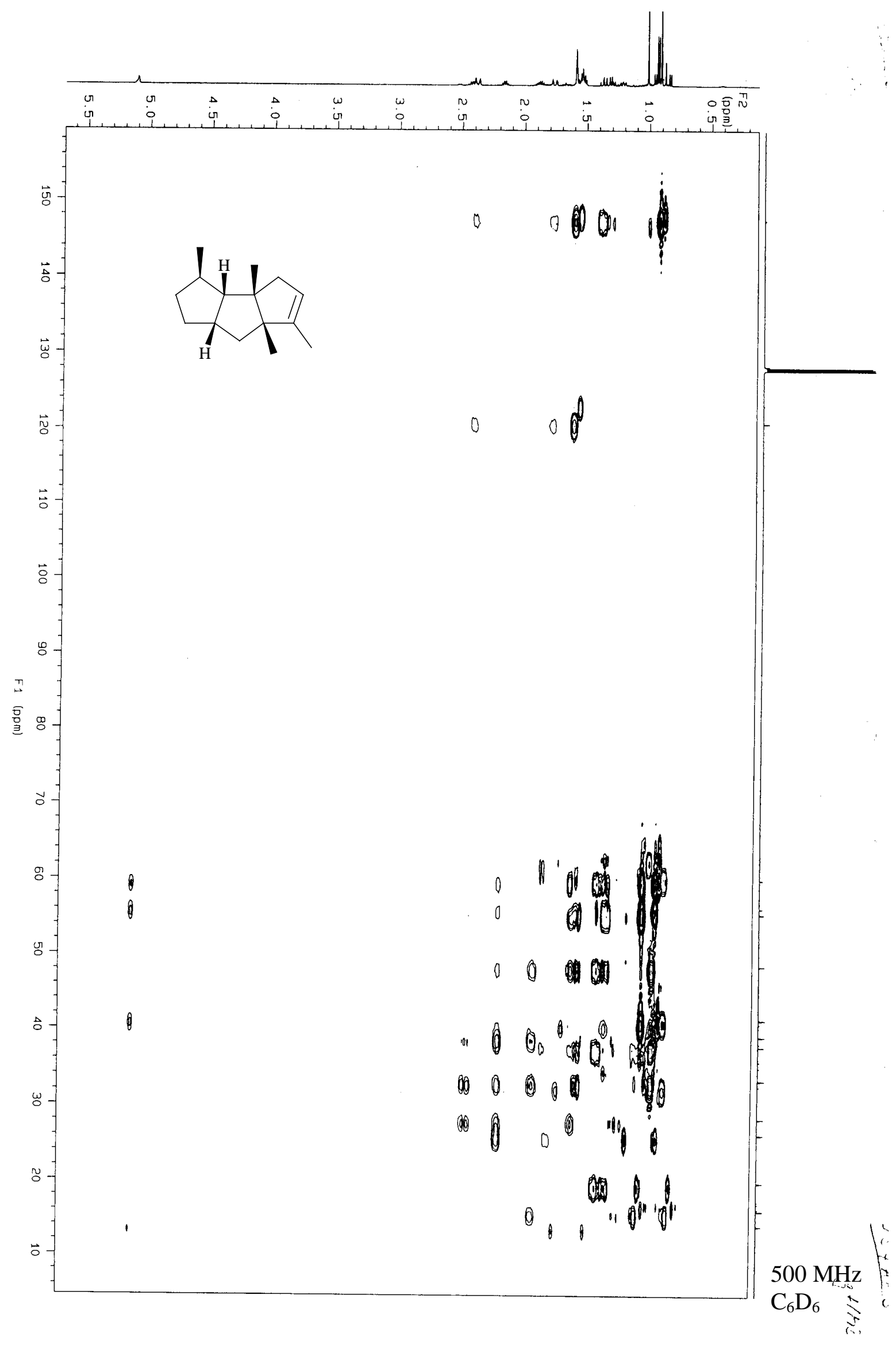

Abb. 140: HMBC-Spektrum von (3R*,3aS*, 3bS*,6aS*,7aS*)-3,3b,6,6a-Tetramethyl-2,3,3a, 3b,4,6a, 7,7a-octahydro-1H-cyclopenta[a]pentalen (111) 


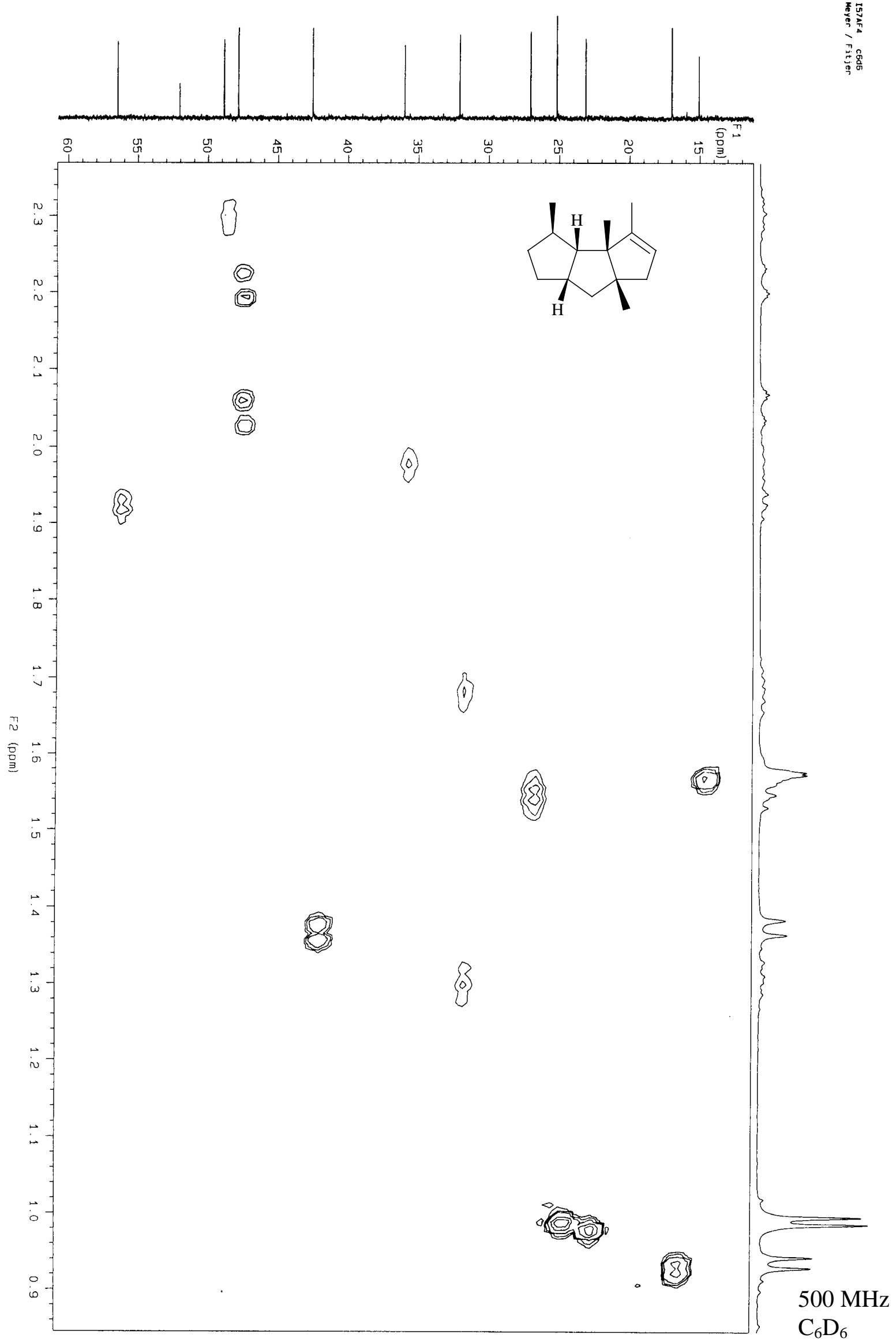

Abb. 141: HMQC-Spektrum von (3R*,3aS*,3bS*,6aS*,7aS*)-3,3b,4,6a-Tetramethyl-2,3,3a, 3b,6,6a,7,7a-octahydro-1H-cyclopenta[a]pentalen (112) 


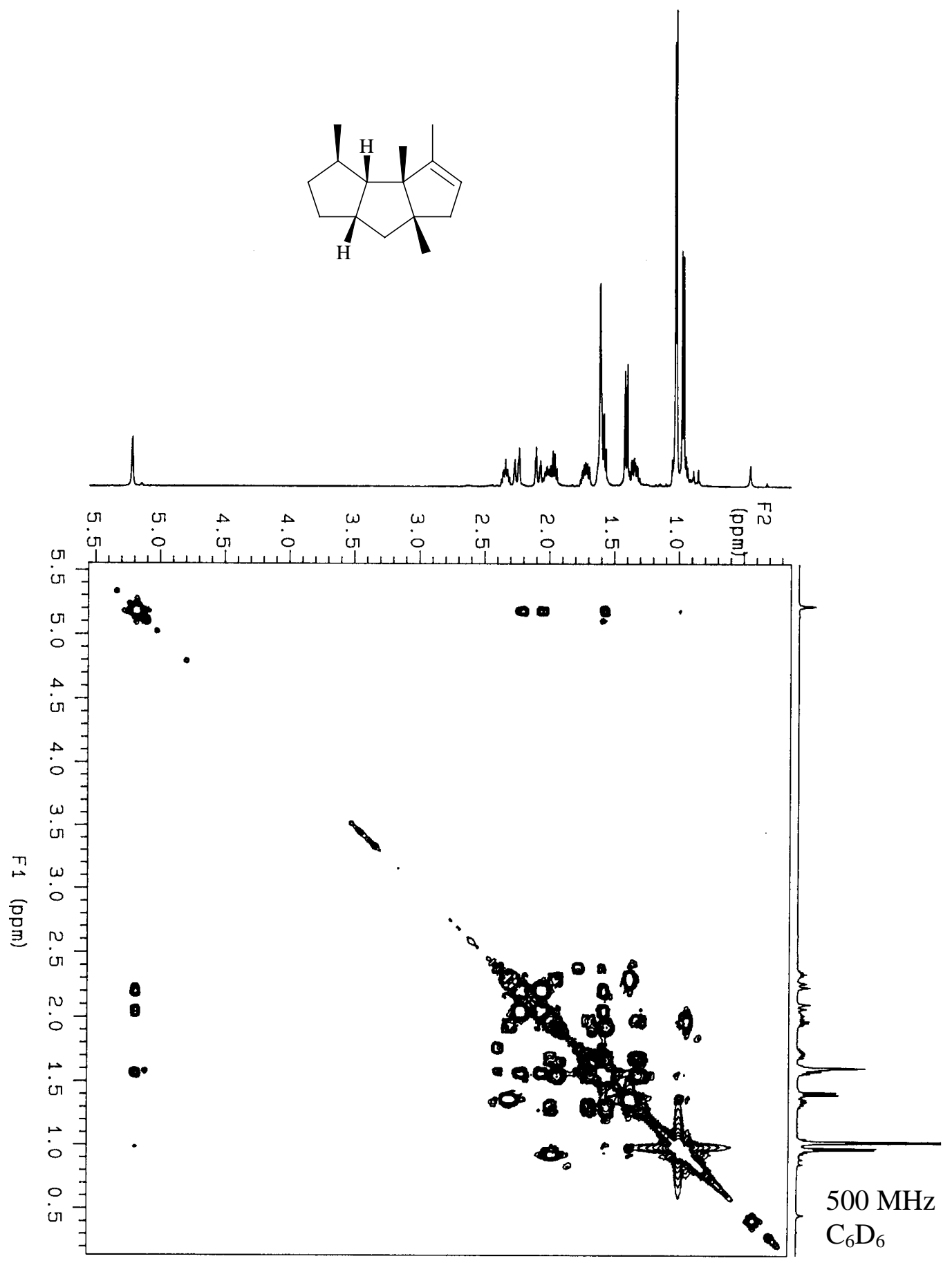

Abb. 142: COSY-Spektrum von (3R*,3aS*,3bS*,6aS*,7aS*)-3,3b,4,6a-Tetramethyl2,3,3a,3b,6,6a,7,7a-octahydro- $1 H$-cyclopenta[a]pentalen (112) 


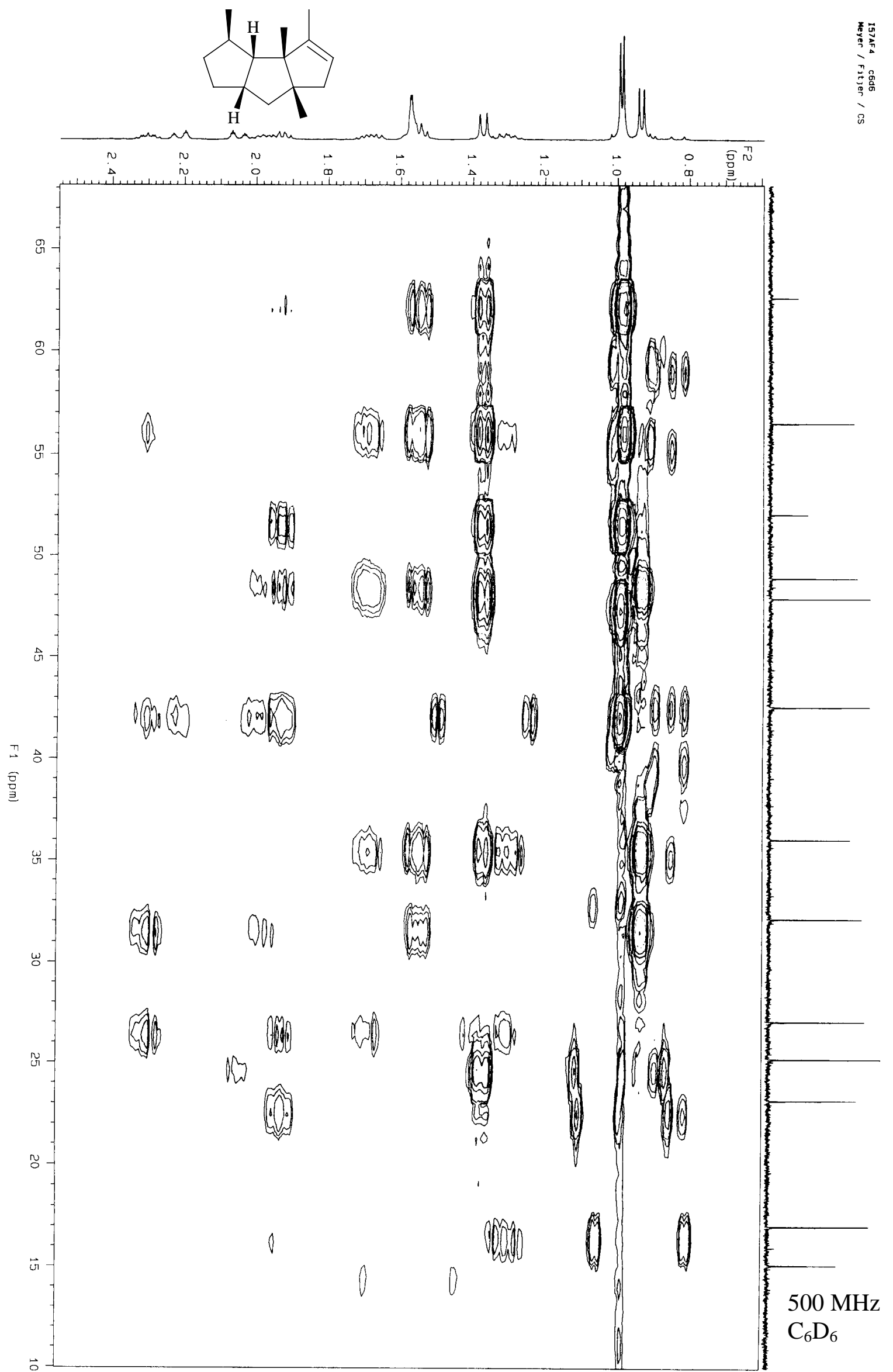

Abb. 143: HMBC-Spektrum von (3R*,3aS*,3bS*,6aS*,7aS*)-3,3b,4,6a-Tetramethyl-2,3,3a, 3b,6,6a,7,7a-octahydro-1 $H$-cyclopenta[a]pentalen (112) 


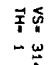
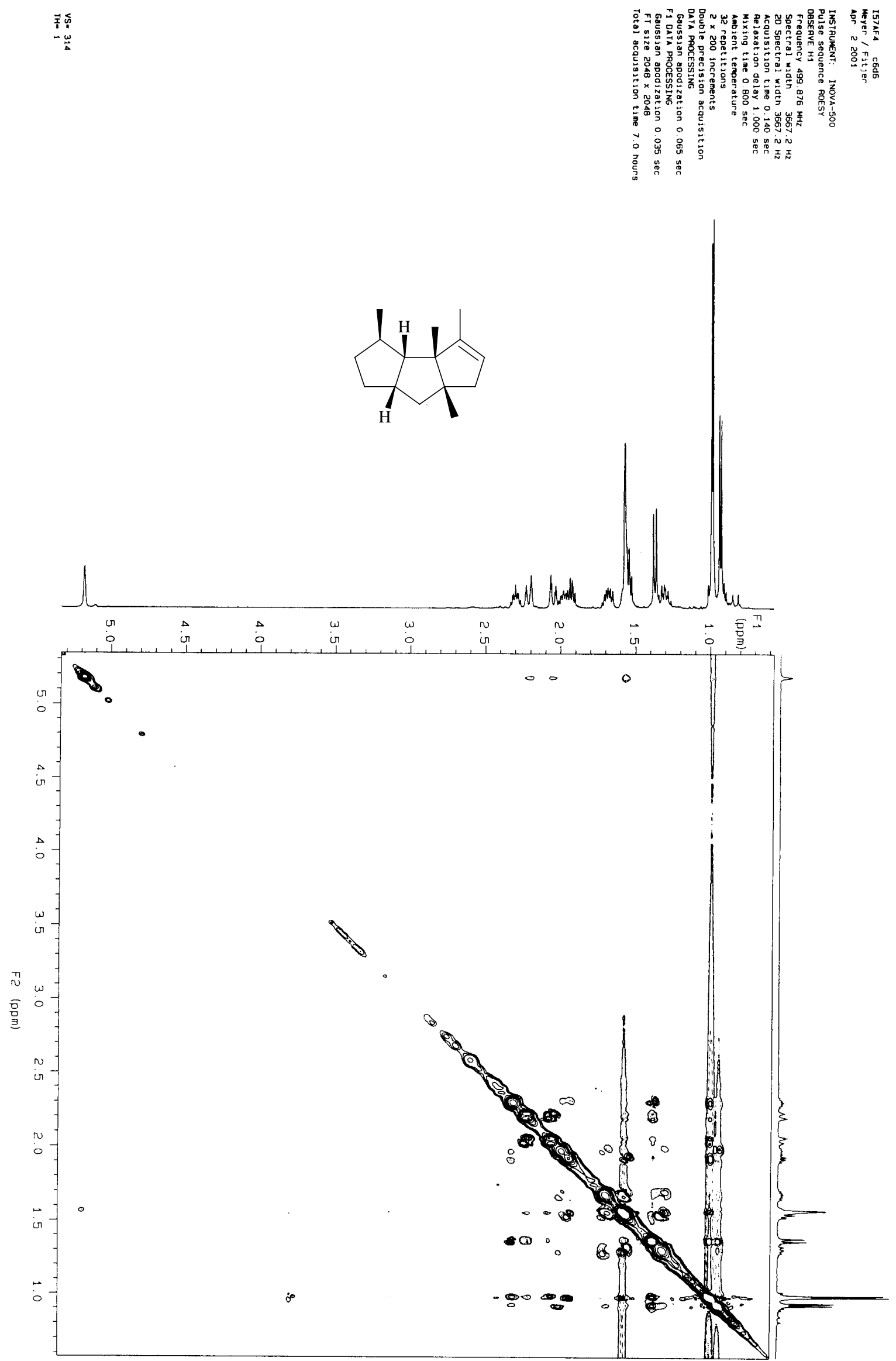

Abb. 144: NOESY-Spektrum von (3R*,3aS*,3bS*,6aS*,7aS*)-3,3b,4,6a-Tetramethyl2,3,3a,3b,6,6a,7,7a-octahydro-1H-cyclopenta[a]pentalen (112) 


\section{Abbildungsverzeichnis}

Abb. 1: $\quad$ IR-Spektrum von 6,6-Dimethyl-spiro[3.4]octan-5-on (51) 96

Abb. 2: $\quad$ IR-Spektrum von 6,6-Dimethyl-spiro[3.4]octan-5-ol (24) 96

Abb. 3: IR-Spektrum von 5,6,6-Trimethyl-spiro[3.4] octan-5-ol (25) 97

Abb. 4: $\quad$ IR-Spektrum von (3aR*,6aR*)-3a,6a-Dimethylhexahydro- 97

pentalen-1-on (30)

Abb. 5: $\quad$ IR-Spektrum von 2,2-Dimethylbicyclobutyliden (26) 98

Abb. 6: $\quad$ IR-Spektrum von 3,3,2',2'-Tetramethyl-bicyclobutyliden-2-on (62) 98

Abb. 7: $\quad$ IR-Spektrum von 3,3,2',2'-Tetramethyl-bicyclobutyliden-2-on (63) 99

Abb. 8: $\quad$ IR-Spektrum von 1,1-Dimethyl-9-oxa-dispiro[3.0.3.1]nonan (64) 99

Abb. 9: $\quad$ IR-Spektrum von 1,1-Dimethyl-spiro[3.4]octan-5-on (27) 100

Abb. 10: IR-Spektrum von 8,8-Dimethyl-spiro[3.4]octan-5-on (67) 100

Abb. 11: $\quad$ IR-Spektrum von 6-Methyl-spiro[3.4]octan-5-on (50) 101

Abb. 12: $\quad$ IR-Spektrum von 5-Methyl-spiro[3.4]octan-5-ol (21) 101

Abb. 13: $\quad$ IR-Spektrum von (5R*,6R*)-5,6-Dimethyl-spiro[3.4] octan-5-ol (23a) 102

Abb. 14: IR-Spektrum von (5S*,6R*)-5,6-Dimethyl-spiro[3.4] octan-5-ol (23b) 102

Abb. 15: IR-Spektrum von (5R*,6R*)-6-Methyl-spiro[3.4] octan-5-ol (22a) 103

Abb. 16: IR-Spektrum von (5S*,6R*)-6-Methyl-spiro[3.4] octan-5-ol (22b) 103

Abb. 17: $\quad$ IR-Spektrum von 1,1-Bis(phenylseleno)-2,2-Dimethylcyclobutan (48) 104

Abb. 18: $\quad$ IR-Spektrum von (1R*,2S*,5R*)-7-Cyclobutyliden-2-methyl- 104

Abb. 19: $\quad$ IR-Spektrum von (1R*,2R*5R*)-7-Cyclobutyliden-2-methyl- 105 bicyclo[3.2.0]heptan (91)

Abb. 20: $\quad$ IR-Spektrum von 2,2-Spiro-cyclobutyl-3,3-spiro[(1R*,4S*,5R*,6S*)- 105 2-methyl-bicyclo[3.2.0]heptan-6,6-yl]-oxiran (92)

Abb. 21: IR-Spektrum von 2,2-Spiro-cyclobutyl-3,3-spiro[(1R*,4S*,5R*,6R*)- 106 2-methyl-bicyclo[3.2.0]heptan-6,6-yl]-oxiran (89)

Abb. 22: $\quad$ IR-Spektrum von $\left(1 \mathrm{R}^{*}, 4 \mathrm{~S}^{*}, 5 \mathrm{R}^{*}, 6 \mathrm{~S}^{*}\right)-4-$ Methyl-spiro\{bicyclo[3.2.0]heptan-6,1'-cyclopentan-2'-on\} (87) und (1R*,2R*,5R*,6S*)2-Methyl-spiro \{bicyclo[3.1.1]heptan-6,1'-cyclopentan-2'-on $\}$ (93) 
Abb. 23: IR-Spektrum von (1R*,4S*,5R*,6R*)-4-Methyl-spiro\{ bicyclo-

[3.2.0]heptan-6, $1^{\prime}$-cyclopentan-2'-on\} (88)

Abb. 24: IR-Spektrum von (1R*,4S*,5R*,6R*)-4,3',3'-Trimethyl-spiro-

\{bicyclo[3.2.0]heptan-6, $1^{\prime}$-cyclopentan-2'-on\} (95)

Abb. 25: IR-Spektrum von $\left(1 \mathrm{R}^{*}, 2 \mathrm{R}^{*}, 5 \mathrm{R}^{*}, 6 \mathrm{~S}^{*}\right)-2,3^{\prime}, 3^{\prime}$-Trimethyl-spiro

\{bicyclo[3.1.1]heptan-6,1'-cyclopentan-2'-on $\}$ (96)

Abb. 26: IR-Spektrum von $\left(1 \mathrm{R}^{*}, 4 \mathrm{~S}^{*}, 5 \mathrm{R}^{*}, 6 \mathrm{~S}^{*}\right)-4,3^{\prime}, 3^{\prime}$-Trimethyl-spiro-

\{bicyclo[3.2.0]heptan-6,1'-cyclopentan-2'-on\} (94)

Abb. 27: IR-Spektrum von $\left(1 \mathrm{R}^{*}, 4 \mathrm{~S}^{*}, 5 \mathrm{R}^{*}, 6 \mathrm{~S}^{*}, 2^{\prime} \mathrm{S}^{*}\right)-4 ; 2^{\prime}, 3^{\prime}, 3^{\prime}-$ Tetramethyl-

spiro $\{$ bicyclo[3.2.0]heptan-6,1'-cyclopentan-2'-ol $\}$ (45)

Abb. 28: IR-Spektrum von $\left(1 \mathrm{R}^{*}, 4 \mathrm{~S}^{*}, 5 \mathrm{R}^{*}, 6 \mathrm{~S}^{*}, 2^{\prime} \mathrm{R}^{*}\right)-4,2^{\prime}, 3^{\prime}, 3^{\prime}-$-Tetramethyl-

109

spiro $\{$ bicyclo[3.2.0]heptan-6,1'-cyclopentan-2'-ol $\}$ (44)

Abb. 29: $\quad$ IR-Spektrum von $\left(1 R^{*}, 4 S^{*}, 5 R^{*}, 6 R^{*}, 2^{\prime} R^{*}\right)-4,2^{\prime}, 3^{\prime}, 3^{\prime}-$

Tetramethyl-spiro $\{$ bicyclo[3.2.0]heptan-6,1'-cyclopentan-2'-ol $\}$ (41)

und $\left(1 \mathrm{R}^{*}, 4 \mathrm{~S}^{*}, 5 \mathrm{R}^{*}, 6 \mathrm{R}^{*}, 2^{\prime} \mathrm{S}^{*}\right)-4,2^{\prime}, 3^{\prime}, 3^{\prime}-$ Tetramethyl-spiro\{ bicyclo-

[3.2.0]heptan-6, $1^{\prime}$-cyclopentan-2'-on\}

$(42)$

Abb. 30: IR-Spektrum von 1-[(1'-Phenylseleno)cyclobutyl]-2,2-dimethyl-

110 cyclobutan-1-ol (76)

Abb. 31: IR-Spektrum von 2,2-Dimethyl-bicyclobutyl-1'-en-1-ol (78)

Abb. 32: IR-Spektrum von 1-[(1'-Phenylseleno)-2',2'-dimethylcyclobutyl]cyclobutan-1-ol (49)

Abb. 33: $\quad{ }^{1}$ H-NMR-Spektrum von 6,6-Dimethyl-spiro[3.4] octan-5-on (51)

Abb. 34: $\quad{ }^{1}$ H-NMR-Spektrum von 6,6-Dimethyl-spiro[3.4] octan-5-ol (24)

Abb. 35: $\quad{ }^{1}$ H-NMR-Spektrum von 5,6,6-Trimethyl-spiro[3.4] octan-5-ol (25)

Abb. 36: $\quad{ }^{1} \mathrm{H}-\mathrm{NMR}-$ Spektrum von (3aR*,6aR*)-3a,6a-Dimethylhexahydropentalen-1-on (30)

Abb. 37: $\quad{ }^{1}$ H-NMR-Spektrum von 6,6a-Dimethyl-1,2,3,3a,4,6a-hexahydropentalen (60)

Abb. 38: $\quad{ }^{1}$ H-NMR-Spektrum von 2,2-Dimethylbicyclobutyliden (26)

Abb. 39: $\quad{ }^{1}$ H-NMR-Spektrum von 3,3,2',2’-Tetramethyl-bicyclobutyliden-2on (62)

Abb. 40: $\quad{ }^{1}$ H-NMR-Spektrum von 3,3,2',2'-Tetramethyl-bicyclobutyliden-2on (63)

Abb. 41: $\quad{ }^{1}$ H-NMR-Spektrum von 1,1-Dimethyl-9-oxa-dispiro[3.0.3.1]nonan (64) 116 
Abb. 42: $\quad{ }^{1}$ H-NMR-Spektrum von 1,1-Dimethyl-spiro[3.4] octan-5-on (27) 116

Abb. 43: $\quad{ }^{1}$ H-NMR-Spektrum von 8,8-Dimethyl-spiro[3.4] octan-5-on (67) 117

Abb. 44: $\quad{ }^{1}$ H-NMR-Spektrum von 6-Methyl-spiro[3.4] octan-5-on (50) 117

Abb. 45: $\quad{ }^{1}$ H-NMR-Spektrum von 5-Methyl-spiro[3.4] octan-5-ol (21) 118

Abb. 46: $\quad{ }^{1}$ H-NMR-Spektrum von (5R*,6R*)-5,6-Dimethyl-spiro[3.4]- 118 octan-5-ol (23a)

Abb. 47: $\quad{ }^{1} \mathrm{H}-\mathrm{NMR}-$ Spektrum von $\left(5 \mathrm{~S}^{*}, 6 \mathrm{R}^{*}\right)$-5,6-Dimethyl-spiro[3.4]octan-5-ol (23b)

Abb. 48: $\quad{ }^{1}$ H-NMR-Spektrum von (5R*,6R*)-6-Methyl-spiro[3.4] octan-5-ol (22a) 119

Abb. 49: $\quad{ }^{1}$ H-NMR-Spektrum von (5S*,6R*)-6-Methyl-spiro[3.4] octan-5-ol (22b) 120

Abb. 50: $\quad{ }^{1}$ H-NMR-Spektrum von 1,1-Bis(phenylseleno)-2,2-Dimethylcyclo- $\quad 120$ butan (48)

Abb. 51: $\quad{ }^{1} \mathrm{H}-\mathrm{NMR}-\mathrm{Spektrum}$ von $\left(1 \mathrm{R}^{*}, 2 \mathrm{~S}^{*}, 5 \mathrm{R}^{*}\right)$-7-Cyclobutyliden-2-methyl- $\quad 121$ bicyclo[3.2.0]heptan (40)

Abb. 52: $\quad{ }^{1} \mathrm{H}-\mathrm{NMR}-$ Spektrum von $(1 \mathrm{R} *, 2 \mathrm{R} * 5 \mathrm{R} *)$-7-Cyclobutyliden-2-methyl- $\quad 121$ bicyclo[3.2.0]heptan (91)

Abb. 53: $\quad{ }^{1}$ H-NMR-Spektrum von 2,2-Spiro-cyclobutyl-3,3-spiro-

[(1R*,4S*,5R*,6S*)-2-methyl-bicyclo[3.2.0]heptan-6,6-yl]-oxiran (92)

Abb. 54: $\quad{ }^{1}$ H-NMR-Spektrum von 2,2-Spiro-cyclobutyl-3,3-spiro-

$\left[\left(1 \mathrm{R} *, 4 \mathrm{~S}^{*}, 5 \mathrm{R} *, 6 \mathrm{R} *\right)\right.$-2-methyl-bicyclo[3.2.0]heptan-6,6-yl]-oxiran (89)

Abb. 55: $\quad{ }^{1} \mathrm{H}-\mathrm{NMR}-$ Spektrum von $\left(1 \mathrm{R}^{*}, 4 \mathrm{~S}^{*}, 5 \mathrm{R}^{*}, 6 \mathrm{~S}^{*}\right)$-4-Methyl-spiro

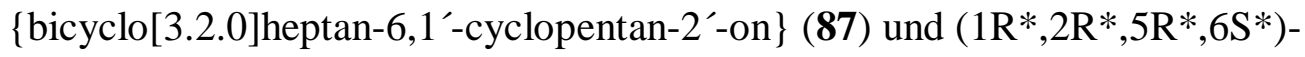

2-Methyl-spiro \{bicyclo[3.1.1]heptan-6,1'-cyclopentan-2'-on\} (93)

Abb. 56: $\quad{ }^{1} \mathrm{H}-\mathrm{NMR}-$ Spektrum von $\left(1 \mathrm{R}^{*}, 4 \mathrm{~S}^{*}, 5 \mathrm{R}^{*}, 6 \mathrm{R}^{*}\right)-4-$ Methyl-spiro-

\{bicyclo[3.2.0]heptan-6,1'-cyclopentan-2'-on $\}$ (88)

Abb. 57: $\quad{ }^{1} \mathrm{H}-\mathrm{NMR}-\mathrm{Spektrum}$ von $\left(1 \mathrm{R}^{*}, 4 \mathrm{~S}^{*}, 5 \mathrm{R}^{*}, 6 \mathrm{R}^{*}\right)-4,3^{\prime}, 3^{\prime}$-Trimethyl-

spiro $\{$ bicyclo[3.2.0]heptan-6,1'-cyclopentan-2'-on $\}$ (95)

Abb. 58: $\quad{ }^{1} \mathrm{H}-\mathrm{NMR}-$ Spektrum von $\left(1 \mathrm{R}^{*}, 2 \mathrm{R}^{*}, 5 \mathrm{R}^{*}, 6 \mathrm{~S}^{*}\right)-2,3^{\prime}, 3^{\prime}-$ Trimethyl-spiro

\{bicyclo[3.1.1]heptan-6,1'-cyclopentan-2'-on\} (96)

Abb. 59: $\quad{ }^{1} \mathrm{H}-\mathrm{NMR}-$ Spektrum von $\left(1 \mathrm{R}^{*}, 4 \mathrm{~S}^{*}, 5 \mathrm{R}^{*}, 6 \mathrm{~S}^{*}\right)-4,3^{\prime}, 3^{\prime}$-Trimethyl-

spiro\{bicyclo[3.2.0] heptan-6,1'-cyclopentan-2'-on $\}$ (94)

Abb. 60: $\quad{ }^{1} \mathrm{H}-\mathrm{NMR}-$ Spektrum von $\left(1 \mathrm{R}^{*}, 4 \mathrm{~S}^{*}, 5 \mathrm{R}^{*}, 6 \mathrm{R}^{*}\right)-2^{\prime}$-Methylen-

4,3',3'-Trimethyl-spiro\{bicyclo[3.2.0]heptan-6,1'-cyclopentan\} (104)

Abb. 61: $\quad{ }^{1} \mathrm{H}-\mathrm{NMR}-$ Spektrum von $\left(1 \mathrm{R}^{*}, 4 \mathrm{~S}^{*}, 5 \mathrm{R}^{*}, 6 \mathrm{~S}^{*}\right)-2^{\prime}$-Methylen- 
4,3',3'-Trimethyl-spiro\{bicyclo[3.2.0]heptan-6,1'-cyclopentan $\}$ (103)

Abb. 62: $\quad{ }^{1} \mathrm{H}-\mathrm{NMR}-\mathrm{Spektrum}$ von $\left(1 \mathrm{R}^{*}, 4 \mathrm{~S}^{*}, 5 \mathrm{R}^{*}, 6 \mathrm{~S}^{*}, 2^{\prime} \mathrm{S}^{*}\right)-4,2^{\prime}, 3^{\prime}, 3^{\prime}-$

Tetramethyl-spiro $\{$ bicyclo[3.2.0]heptan-6,1'-cyclopentan-2'-ol $\}$ (45)

Abb. 63: $\quad{ }^{1} \mathrm{H}-\mathrm{NMR}-$ Spektrum von $\left(1 \mathrm{R}^{*}, 4 \mathrm{~S}^{*}, 5 \mathrm{R}^{*}, 6 \mathrm{~S}^{*}, 2^{\prime} \mathrm{R}^{*}\right)-4,2^{\prime}, 3^{\prime}, 3^{\prime}-$

Tetramethyl-spiro $\{$ bicyclo[3.2.0]heptan-6,1'-cyclopentan-2'-ol $\}$ (44)

Abb. 64: $\quad{ }^{1} \mathrm{H}-\mathrm{NMR}-$ Spektrum von $\left(1 \mathrm{R}^{*}, 4 \mathrm{~S}^{*}, 5 \mathrm{R}^{*}, 6 \mathrm{R}^{*}, \mathrm{2}^{\prime} \mathrm{R}^{*}\right)-4,2^{\prime}, 3^{\prime}, 3^{\prime}-$

Tetramethyl-spiro $\{$ bicyclo[3.2.0]heptan-6,1'-cyclopentan-2'-ol $\}$ (41) und $\left(1 \mathrm{R}^{*}, 4 \mathrm{~S}^{*}, 5 \mathrm{R}^{*}, 6 \mathrm{R}^{*}, 2^{\prime} \mathrm{S}^{*}\right)-4,2^{\prime}, 3^{\prime}, 3^{\prime}$-Tetramethyl-spiro \{ bicyclo[3.2.0]heptan-6,1'-cyclopentan-2'-ol\}

Abb. 65: $\quad{ }^{1}$ H-NMR-Spektrum von 1-[(1'-Phenylseleno)cyclobutyl]-2,2-dimethylcyclobutan-1-ol (76)

Abb. 66: $\quad{ }^{1} \mathrm{H}-\mathrm{NMR}-$ Spektrum von 2,2-Dimethyl-bicyclobutyl-1'-en-1-ol (78) 128

Abb. 67: $\quad{ }^{1} \mathrm{H}-\mathrm{NMR}-$ Spektrum von 1-[(1'-Phenylseleno)-2',2'-dimethylcyclobutyl]cyclobutan-1-ol (49)

Abb. 68: $\quad{ }^{1}$ H-NMR-Spektrum von 116/117

Abb. 69: $\quad{ }^{1} \mathrm{H}-\mathrm{NMR}-$ Spektrum von $\left(3 \mathrm{aR}^{*}, 3 \mathrm{bR}^{*}, 6 \mathrm{aR} *, 7 \mathrm{aR} *\right)-3,3,3 \mathrm{a}, 4-$ Tetramethyl-2,3,3a,3b,6,6a,7,7a-octahydro-1 $H$-cyclopenta[a]pentalen (119)

Abb. 70: $\quad{ }^{1} \mathrm{H}-\mathrm{NMR}-$ Spektrum von $\left(3 \mathrm{aR}^{*}, 3 \mathrm{bR}^{*}, 6 \mathrm{aS} *, 7 \mathrm{aS} *\right)-1,1,4,7 \mathrm{a}-\mathrm{Tetra}-$ 130 methyl-2,3,3a,3b,6,6a,7,7a-octahydro-1H-cyclopenta[a]pentalen (120)

Abb. 71: $\quad{ }^{1} \mathrm{H}-\mathrm{NMR}-$ Spektrum von $\left(3 \mathrm{R}^{*}, 3 \mathrm{aS} *, 6 \mathrm{aR} *, 7 \mathrm{aR} *\right)-3,6,6,6 \mathrm{a}-$ Tetramethyl-2,3,3a,5,6,6a, 7,7a-octahydro-1H-cyclopenta[a]pentalen (118)

Abb. 72: $\quad{ }^{1} \mathrm{H}-\mathrm{NMR}-$ Spektrum von $\left(3 \mathrm{R}^{*}, 3 \mathrm{bS} *, 6 \mathrm{aS} *\right)-$ und $\left(3 \mathrm{R}^{*}, 3 \mathrm{bR}^{*}, 6 \mathrm{aR} *\right)$ 3,6,6,6a-Tetramethyl-2,3,3a,5,6,6a,7,7a-octahydro- $1 \mathrm{H}$-cyclopenta[a]pentalen (108/109)

Abb. 73: $\quad{ }^{1} \mathrm{H}-\mathrm{NMR}-$ Spektrum von $\left(3 \mathrm{aR} *, 3 \mathrm{bS}^{*}, 6 \mathrm{aR} *, 7 \mathrm{aS} *\right)-1,1,4,7 \mathrm{a}-$ Tetra132 methyl-2,3,3a,3b,6,6a,7,7a-octahydro-1H-cyclopenta[a]pentalen (110)

Abb. 74: $\quad{ }^{1} \mathrm{H}-\mathrm{NMR}-$ Spektrum von $\left(3 \mathrm{R}^{*}, 3 \mathrm{aS} *, 3 \mathrm{bS} *, 6 \mathrm{aS} *, 7 \mathrm{aS} *\right)-3,3 \mathrm{~b}, 6,6 \mathrm{a}-$ Tetramethyl-2,3,3a,3b,4,6a,7,7a-octahydro-1H-cyclopenta[a]pentalen (111)

Abb. 75: $\quad{ }^{1} \mathrm{H}-\mathrm{NMR}-$ Spektrum von $\left(3 \mathrm{R}^{*}, 3 \mathrm{aS} *, 3 \mathrm{bS} *, 6 \mathrm{aS} *, 7 \mathrm{aS} *\right)-3,3 \mathrm{~b}, 4,6 \mathrm{a}-$ Tetramethyl-2,3,3a,3b,6,6a,7, 7a-octahydro-1H-cyclopenta[a]pentalen (112)

Abb. 76: $\quad{ }^{13}$ C-NMR-Spektrum von 6,6-Dimethyl-spiro[3.4] octan-5-on (51)

Abb. 77:

${ }^{13}$ C-NMR-Spektrum von 6,6-Dimethyl-spiro[3.4] octan-5-ol (24)

Abb. 78:

${ }^{13}$ C-NMR-Spektrum von 5,6,6-Trimethyl-spiro[3.4] octan-5-ol (25)

Abb. 79:

${ }^{13} \mathrm{C}-\mathrm{NMR}-$ Spektrum von $(3 \mathrm{aR} * ; 6 \mathrm{aR} *)$-3a,6a-Dimethylhexahydro- 
pentalen-1-on (30)

Abb. 80: $\quad{ }^{13}$ C-NMR-Spektrum von 6,6a-Dimethyl-1,2,3,3a,4,6a-hexahydropentalen (60)

Abb. 81: $\quad{ }^{13}$ C-NMR-Spektrum von 2,2-Dimethylbicyclobutyliden (26) 136

Abb. 82: $\quad{ }^{13}$ C-NMR-Spektrum von 3,3,2',2'-Tetramethyl-bicyclobutyliden-2on (62)

Abb. 83: $\quad{ }^{13}$ C-NMR-Spektrum von 3,3,2',2'-Tetramethyl-bicyclobutyliden-2on $(63)$

Abb. 84: $\quad{ }^{13}$ C-NMR-Spektrum von 1,1-Dimethyl-9-oxa-dispiro[3.0.3.1]nonan $(\mathbf{6 4})$

Abb. 85: $\quad{ }^{13}$ C-NMR-Spektrum von 1,1-Dimethyl-spiro[3.4] octan-5-on (27)

Abb. 86: $\quad{ }^{13}$ C-NMR-Spektrum von 8,8-Dimethyl-spiro[3.4]octan-5-on (67)

Abb. 87: $\quad{ }^{13}$ C-NMR-Spektrum von 6-Methyl-spiro[3.4] octan-5-on (50)

Abb. 88: $\quad{ }^{13}$ C-NMR-Spektrum von 5-Methyl-spiro[3.4] octan-5-ol (21)

Abb. 89: ${ }^{13} \mathrm{C}-\mathrm{NMR}-$ Spektrum von $\left(5 \mathrm{R}^{*}, 6 \mathrm{R} *\right)$-5,6-Dimethyl-spiro[3.4]octan-5-ol (23a)

Abb. 90: $\quad{ }^{13}$ C-NMR-Spektrum von (5S*,6R*)-5,6-Dimethyl-spiro[3.4] octan-5-ol (23b)

Abb. 91: $\quad{ }^{13} \mathrm{C}-\mathrm{NMR}-$ Spektrum von (5R*,6R*)-6-Methyl-spiro[3.4]octan-5-ol (22a)

Abb. 92: $\quad{ }^{13}$ C-NMR-Spektrum von (5S*,6R*)-6-Methyl-spiro[3.4]octan-5-ol (22b)

Abb. 93: $\quad{ }^{13}$ C-NMR-Spektrum von 1,1-Bis[phenylseleno]-2,2-Dimethylcyclobutan (48)

Abb. 94: $\quad{ }^{13} \mathrm{C}-\mathrm{NMR}-$ Spektrum von $\left(1 \mathrm{R}^{*}, 2 \mathrm{~S} *, 5 \mathrm{R}^{*}\right)$-7-Cyclobutyliden-2-methylbicyclo[3.2.0]heptan (40)

Abb. 95: $\quad{ }^{13} \mathrm{C}-\mathrm{NMR}-$ Spektrum von $\left(1 \mathrm{R}^{*}, 2 \mathrm{R} * 5 \mathrm{R}^{*}\right)$-7-Cyclobutyliden-2-methylbicyclo[3.2.0]heptan (91)

Abb. 96: $\quad{ }^{13}$ C-NMR-Spektrum von 2,2-Spiro-cyclobutyl-3,3-spiro[(1R*,4S*,5R*,6S*)-2-methyl-bicyclo[3.2.0]heptan-6,6-yl]-oxiran (92)

Abb. 97: $\quad{ }^{13}$ C-NMR-Spektrum von 2,2-Spiro-cyclobutyl-3,3-spiro144 [(1R*,4S*,5R*,6R*)-2-methyl-bicyclo[3.2.0]heptan-6,6-yl]-oxiran (89)

Abb. 98: $\quad{ }^{13} \mathrm{C}-\mathrm{NMR}-$ Spektrum von $\left(1 \mathrm{R}^{*}, 4 \mathrm{~S}^{*}, 5 \mathrm{R}^{*}, 6 \mathrm{~S}^{*}\right)-4-$-Methyl-spiro \{bicyclo[3.2.0]heptan-6,1'-cyclopentan-2'-on\} (87) und 
$\left(1 \mathrm{R}^{*}, 2 \mathrm{R}^{*}, 5 \mathrm{R}^{*}, 6 \mathrm{~S}^{*}\right)$-2-Methyl-spiro\{bicyclo[3.1.1]heptan-6,1'-cyclopentan-2'-on $\}(\mathbf{9 3})$

Abb. 99: $\quad{ }^{13} \mathrm{C}-\mathrm{NMR}-$ Spektrum von $\left(1 \mathrm{R}^{*}, 4 \mathrm{~S}^{*}, 5 \mathrm{R}^{*}, 6 \mathrm{R} *\right)-4-$ Methyl-spiro-

\{bicyclo[3.2.0]heptan-6, $1^{\prime}$-cyclopentan-2'-on\} (88)

Abb. 100: $\quad{ }^{13} \mathrm{C}-\mathrm{NMR}-$ Spektrum von $\left(1 \mathrm{R}^{*}, 4 \mathrm{~S}^{*}, 5 \mathrm{R}^{*}, 6 \mathrm{R}^{*}\right)-4,3^{\prime}, 3^{\prime}$-Trimethyl-

spiro $\left\{\right.$ bicyclo[3.2.0]heptan-6, $1^{\prime}$-cyclopentan-2'-on $\}$ (95)

Abb. 101: $\quad{ }^{13}$ C-NMR-Spektrum von $\left(1 R^{*}, 2 R^{*}, 5 R^{*}, 6 S^{*}\right)-2,3^{\prime}, 3^{\prime}-$ Trimethyl-spiro

\{bicyclo[3.1.1]heptan-6,1'-cyclopentan-2'-on\} (96)

Abb. 102: $\quad{ }^{13} \mathrm{C}$-NMR-Spektrum von $\left(1 \mathrm{R}^{*}, 4 \mathrm{~S}^{*}, 5 \mathrm{R}^{*}, 6 \mathrm{~S}^{*}\right)-4,3^{\prime}, 3^{\prime}$-Trimethyl-

spiro $\left\{\right.$ bicyclo[3.2.0]heptan-6, $1^{\prime}$-cyclopentan-2'-on $\}$ (94)

Abb. 103: $\quad{ }^{13} \mathrm{C}-\mathrm{NMR}-$ Spektrum von $\left(1 \mathrm{R}^{*}, 4 \mathrm{~S}^{*}, 5 \mathrm{R} *, 6 \mathrm{R} *\right)-2^{\prime}-$ Methylen-

4,3',3'-Trimethyl-spiro \{bicyclo[3.2.0] heptan-6,1'-cyclopentan\} (104)

Abb. 104: $\quad{ }^{13} \mathrm{C}-\mathrm{NMR}-$ Spektrum von $\left(1 \mathrm{R}^{*}, 4 \mathrm{~S}^{*}, 5 \mathrm{R}^{*}, 6 \mathrm{~S}^{*}\right)-2^{\prime}-$ Methylen-

4,3',3'-Trimethyl-spiro\{bicyclo[3.2.0]heptan-6,1'-cyclopentan\} (103)

Abb. 105: $\quad{ }^{13} \mathrm{C}-\mathrm{NMR}-$ Spektrum von $\left(1 \mathrm{R}^{*}, 4 \mathrm{~S}^{*}, 5 \mathrm{R}^{*}, 6 \mathrm{~S}^{*}, 2^{\prime} \mathrm{S}^{*}\right)-4,2^{\prime}, 3^{\prime}, 3^{\prime}-$

Tetramethyl-spiro $\left\{\right.$ bicyclo[3.2.0]heptan-6, $1^{\prime}$-cyclopentan-2'-ol $\}$ (45)

Abb. 106: $\quad{ }^{13} \mathrm{C}-\mathrm{NMR}-\mathrm{Spektrum}$ von $\left(1 \mathrm{R}^{*}, 4 \mathrm{~S}^{*}, 5 \mathrm{R}^{*}, 6 \mathrm{~S}^{*}, 2^{\prime} \mathrm{R}^{*}\right)-4,2^{\prime}, 3^{\prime}, 3^{\prime}-$

Tetramethyl-spiro $\{$ bicyclo[3.2.0]heptan-6,1'-cyclopentan-2'-ol $\}$ (44)

Abb. 107: $\quad{ }^{13} \mathrm{C}-\mathrm{NMR}-\mathrm{Spektrum}$ von $\left(1 \mathrm{R}^{*}, 4 \mathrm{~S}^{*}, 5 \mathrm{R}^{*}, 6 \mathrm{R}^{*}, 2^{\prime} \mathrm{R}^{*}\right)-4,2^{\prime}, 3^{\prime}, 3^{\prime}-$

Tetramethyl-spiro $\{$ bicyclo[3.2.0]heptan-6,1'-cyclopentan-2'-ol $\}$ (41) und $\left(1 \mathrm{R}^{*}, 4 \mathrm{~S}^{*}, 5 \mathrm{R}^{*}, 6 \mathrm{R}^{*}, 2^{\prime} \mathrm{S}^{*}\right)-4,2^{\prime}, 3^{\prime}, 3^{\prime}$-Tetramethyl-spiro\{ bicyclo[3.2.0]heptan-6,1'-cyclopentan-2'-ol\}

Abb. 108: $\quad{ }^{13}$ C-NMR-Spektrum von 1-[(1'-Phenylseleno)cyclobutyl]-2,2-dimethylcyclobutan-1-ol (76)

Abb. 109: $\quad{ }^{13}$ C-NMR-Spektrum von 2,2-Dimethyl-bicyclobutyl-1'-en-1-ol (78) 150

Abb. 110: $\quad{ }^{13}$ C-NMR-Spektrum von 1-[(1'-Phenylseleno)-2',2'-dimethylcyclobutyl]cyclobutan-1-ol (49)

Abb. 111: $\quad{ }^{13}$ C-NMR-Spektrum von 116/117

Abb. 112: $\quad{ }^{13} \mathrm{C}-\mathrm{NMR}-$ Spektrum von (3aR*,3bR*,6aR*,7aR*)-3,3,3a,4-Tetramethyl-2,3,3a,3b,6,6a,7,7a-octahydro-1H-cyclopenta[a]pentalen (119)

Abb. 113: $\quad{ }^{13}$ C-NMR-Spektrum von $\left(3 \mathrm{aR}^{*}, 3 \mathrm{bR}^{*}, 6 \mathrm{aS} *, 7 \mathrm{aS} *\right)-1,1,4,7 \mathrm{a}-$ Tetramethyl-2,3,3a,3b,6,6a,7,7a-octahydro-1H-cyclopenta[a]pentalen (120)

Abb. 114: $\quad{ }^{13} \mathrm{C}-\mathrm{NMR}-$ Spektrum von $\left(3 \mathrm{R}^{*}, 3 \mathrm{aS} *, 6 \mathrm{aR} *, 7 \mathrm{aR} *\right)-3,6,6,6 \mathrm{a}-$ Tetramethyl-2,3,3a,5,6,6a,7,7a-octahydro- $1 H$-cyclopenta[a]pentalen (118) 
Abb. 115: $\quad{ }^{13} \mathrm{C}-\mathrm{NMR}-$ Spektrum von $\left(3 \mathrm{R}^{*}, 3 \mathrm{bS} \mathrm{S}^{*}, 6 \mathrm{a} \mathrm{S}^{*}\right)-$ und $\left(3 \mathrm{R}^{*}, 3 \mathrm{bR}^{*}, 6 \mathrm{aR} *\right)-$

3,6,6,6a-Tetramethyl-2,3,3a,5,6,6a,7,7a-octahydro-1H-cyclopenta[a]pentalen (108/109)

Abb. 116: $\quad{ }^{13}$ C-NMR-Spektrum von $\left(3 \mathrm{aR}^{*}, 3 \mathrm{bS} *, 6 \mathrm{aR} *, 7 \mathrm{aS} *\right)-1,1,4,7 \mathrm{a}$-Tetramethyl-2,3,3a,3b,6,6a,7,7a-octahydro-1H-cyclopenta[a]pentalen (110)

Abb. 117: $\quad{ }^{13}$ C-NMR-Spektrum von (3R*,3aS*, 3bS*,6aS*,7aS*)-3,3b,6,6a-Tetra154 methyl-2,3,3a,3b,4,6a,7,7a-octahydro-1H-cyclopenta[a]pentalen (111)

Abb. 118: $\quad{ }^{13}$ C-NMR-Spektrum von $\left(3 \mathrm{R}^{*}, 3 \mathrm{aS} *, 3 \mathrm{bS} *, 6 \mathrm{aS} *, 7 \mathrm{aS} *\right)-3,3 \mathrm{~b}, 4,6 \mathrm{a}-$ Tetra155 methyl-2,3,3a,3b,6,6a,7,7a-octahydro-1H-cyclopenta[a]pentalen (112)

Abb. 119: HMQC-Spektrum von (5R*,6R*)-5,6-Dimethyl-spiro[3.4]octan156 5-ol (23a)

Abb. 120: HMQC-Spektrum von (5S*,6R*)-5,6-Dimethyl-spiro[3.4]octan5-ol (23b)

Abb. 121: HMQC-Spektrum von $\left(1 \mathrm{R}^{*}, 4 \mathrm{~S}^{*}, 5 \mathrm{R}^{*}, 6 \mathrm{R}^{*}\right)-4,3^{\prime}, 3^{\prime}$-Trimethylspiro $\{$ bicyclo[3.2.0]heptan-6,1'-cyclopentan-2'-on $\}$ (95)

Abb. 122: COSY-Spektrum von (1R*,4S*,5R*,6R*)-4,3',3'-Trimethyl159 spiro \{bicyclo[3.2.0] heptan-6, $1^{\prime}$-cyclopentan-2'-on $\}$ (95)

Abb. 123: HMQC-Spektrum von $\left(1 \mathrm{R}^{*}, 2 \mathrm{R}^{*}, 5 \mathrm{R}^{*}, 6 \mathrm{~S}^{*}\right)-2,3^{\prime}, 3^{\prime}$-Trimethyl-spiro 160 \{bicyclo[3.1.1] heptan-6,1'-cyclopentan-2'-on\} (96)

Abb. 124: COSY-Spektrum von $\left(1 \mathrm{R}^{*}, 2 \mathrm{R}^{*}, 5 \mathrm{R}^{*}, 6 \mathrm{~S}^{*}\right)-2,3^{\prime}, 3^{\prime}$-Trimethyl-spiro 161 \{bicyclo[3.1.1] heptan-6,1'-cyclopentan-2'-on \} (96)

Abb. 125: HMQC-Spektrum von $\left(1 \mathrm{R}^{*}, 4 \mathrm{~S}^{*}, 5 \mathrm{R}^{*}, 6 \mathrm{~S}^{*}\right)-4,3^{\prime}, 3^{\prime}$-Trimethyl162 spiro $\{$ bicyclo[3.2.0]heptan-6,1'-cyclopentan-2'-on $\}$ (94)

Abb. 126: COSY-Spektrum von $\left(1 \mathrm{R}^{*}, 4 \mathrm{~S}^{*}, 5 \mathrm{R}^{*}, 6 \mathrm{~S}^{*}\right)-4,3^{\prime}, 3^{\prime}$-Trimethyl163 spiro $\{$ bicyclo[3.2.0]heptan-6,1'-cyclopentan-2'-on $\}$ (94)

Abb. 127: HMBC-Spektrum von $\left(1 \mathrm{R}^{*}, 4 \mathrm{~S}^{*}, 5 \mathrm{R}^{*}, 6 \mathrm{~S}^{*}\right)-4,3^{\prime}, 3^{\prime}$-Trimethyl164 spiro $\left\{\right.$ bicyclo[3.2.0]heptan-6, $1^{\prime}$-cyclopentan-2'-on $\}$ (94)

Abb. 128: NOESY-Spektrum von $\left(1 \mathrm{R}^{*}, 4 \mathrm{~S}^{*}, 5 \mathrm{R}^{*}, 6 \mathrm{~S}^{*}\right)-4,3^{\prime}, 3^{\prime}$-Trimethyl165 spiro $\{$ bicyclo[3.2.0]heptan-6,1'-cyclopentan-2'-on $\}$ (94)

Abb. 129: HMQC-Spektrum von $\left(3 \mathrm{aR} *, 3 \mathrm{bR}^{*}, 6 \mathrm{aR} *, 7 \mathrm{aR} *\right)-3,3,3 \mathrm{a}, 4-$ Tetra166 methyl-2,3,3a,3b,6,6a,7,7a-octahydro-1H-cyclopenta[a]pentalen (119)

Abb. 130: COSY-Spektrum von (3aR*,3bR*,6aR*,7aR*)-3,3,3a,4-Tetra167 methyl-2,3,3a,3b,6,6a,7,7a-octahydro-1H-cyclopenta[a]pentalen (119)

Abb. 131: HMQC-Spektrum von $\left(3 \mathrm{aR}^{*}, 3 \mathrm{bR}^{*}, 6 \mathrm{aS} *, 7 \mathrm{aS} *\right)-1,1,4,7 \mathrm{a}-\mathrm{Tetra}-$ 
methyl-2,3,3a,3b,6,6a,7,7a-octahydro-1H-cyclopenta[a]pentalen (120)

Abb. 132: COSY-Spektrum von (3aR*,3bR*,6aS*,7aS*)-1,1,4,7a-Tetra-

methyl-2,3,3a,3b,6,6a,7,7a-octahydro-1 $H$-cyclopenta[a]pentalen (120)

Abb. 133: HMQC-Spektrum von (3R*,3aS*,6aR*,7aR*)-3,6,6,6a-Tetra170 methyl-2,3,3a,5,6,6a,7,7a-octahydro-1 $H$-cyclopenta[a]pentalen (118)

Abb. 134: COSY-Spektrum von (3R*,3aS*,6aR*,7aR*)-3,6,6,6a-Tetramethyl-2,3,3a,5,6,6a, 7,7a-octahydro- $1 H$-cyclopenta[a]pentalen (118)

Abb. 135: $\quad$ HMBC-Spektrum von (3R*,3aS*,6aR*,7aR*)-3,6,6,6a-Tetramethyl-2,3,3a,5,6,6a, 7,7a-octahydro-1 $H$-cyclopenta[a]pentalen (118)

Abb. 136: HMQC-Spektrum von (3aR*,3bS*,6aR*,7aS*)-1,1,4,7a-Tetramethyl-2,3,3a,3b,6,6a,7,7a-octahydro-1H-cyclopenta[a]pentalen (110)

Abb. 137: COSY-Spektrum von (3aR*,3bS*,6aR*,7aS*)-1,1,4,7a-Tetra174 methyl-2,3,3a,3b,6,6a,7,7a-octahydro-1H-cyclopenta[a]pentalen (110)

Abb. 138: HMQC-Spektrum von $\left(3 \mathrm{R}^{*}, 3 \mathrm{aS} *, 3 \mathrm{bS} *, 6 \mathrm{aS} *, 7 \mathrm{aS} *\right)-3,3 \mathrm{~b}, 6,6 \mathrm{a}-$ Tetra175 methyl-2,3,3a,3b,4,6a,7,7a-octahydro-1H-cyclopenta[a]pentalen (111)

Abb. 139: COSY-Spektrum von (3R*,3aS*, 3bS*,6aS*,7aS*)-3,3b,6,6a-Tetra176 methyl-2,3,3a,3b,4,6a,7,7a-octahydro-1H-cyclopenta[a]pentalen (111)

Abb. 140: HMBC-Spektrum von (3R*,3aS*, 3bS*,6aS*,7aS*)-3,3b,6,6a-Tetra177 methyl-2,3,3a,3b,4,6a,7,7a-octahydro-1 $H$-cyclopenta[a]pentalen (111)

Abb. 141: HMQC-Spektrum von (3R*,3aS*,3bS*,6aS*,7aS*)-3,3b,4,6a,-Tetra178 methyl-2,3,3a,3b,6,6a,7,7a-octahydro- $1 H$-cyclopenta[a]pentalen (112)

Abb. 142: $\quad$ COSY-Spektrum von (3R*,3aS*,3bS*,6aS*,7aS*)-3,3b,4,6a-Tetra179 methyl-2,3,3a,3b,6,6a,7,7a-octahydro-1H-cyclopenta[a]pentalen (112)

Abb. 143: HMBC-Spektrum von $\left(3 \mathrm{R}^{*}, 3 \mathrm{aS} *, 3 \mathrm{bS} *, 6 \mathrm{aS} *, 7 \mathrm{aS} *\right)-3,3 \mathrm{~b}, 4,6 \mathrm{a}-$ Tetra180 methyl-2,3,3a,3b,6,6a,7,7a-octahydro-1H-cyclopenta[a]pentalen (112)

Abb. 144: NOESY-Spektrum von (3R*,3aS*,3bS*,6aS*,7aS*)-3,3b,4,6a-Tetra181 methyl-2,3,3a,3b,6,6a,7,7a-octahydro-1H-cyclopenta[a]pentalen (112) 


\section{Literaturverzeichnis}

1 (a) Whitesell, J. K.; Matthews, R. S.; Helbing, H. J. Org. Chem. 1978, 43, $784-786$.

(b) Paquette, L. A.; Leone-Bay, A. J. J. Am. Chem. Soc. 1983, 105, 7352 - 7358.

2 Wong, H. N. C. In Methods of Organic Chemistry (Houben-Weyl); de Meijere, A., Ed., Georg Thieme Verlag, Stuttgard 1997,; Vol. E 17e, S. 495 ff.

3 Fitjer, L.; Gerke, R.; Anger, T. Synthesis 1994, 893 - 894.

4 El-Hachach, N.; Fischbach, M.; Gerke, R.; Fitjer, L. Tetrahedron 1999, 55, 6119 6128 .

$5 \quad$ Mandelt, K.; Fitjer, L. Synthesis 1998, 1523 - 1526.

6 Mandelt, K. Dissertation, Universität Göttingen, 2000.

7 Anger. T.; Graalmann, O.; Schröder, H.; Gerke, R.; Kaiser, U.; Fitjer, L.; Noltemeyer, M. Tetrahedron 1998, 54, 10713 - 10720.

8 (a) Anderson, W. K.; Veysogen, J. Org. Chem. 1973, 38, 2267 - 2268.

(b) Everett, J. W.; Garratt, P. J. . J. Chem. Soc., Chem. Commun 1972, 642.

$9 \quad$ Fitjer, L.; Kanschik, A.; Majewski, M: Tetrahedron Lett. 1985, 26, 5277 - 5280.

10 Fitjer, L.; Majewski, M.; Kanschik, A. Tetrahedron Lett. 1988, 29, 1263 - 1264.

11 Fitjer, L.; Majewski, M.; Kanschik, A. Tetrahedron Lett. 1988, 29, 5525 - 5528.

12 Fitjer, L.; Kanschik, A.; Majewski, M. NATO Adv. Study Inst. Ser. C 273, 1989, 431 437. 
13 Fitjer, L.; Monzó-Oltra, H.; Noltemeyer, M. Angew. Chem. 1991, 103, 1534 - 1536; Angew. Chem. Int. Ed. Engl. 1991, 30, 1492 - 1494.

14 Fitjer, L.; Monzó-Oltra, H. J. Org. Chem. 1993, 58, 6171- 6173.

15 Takeda, R.; Naoki, H.; Iwashita, T.; Hirose, Y. Tetrahedron Lett. 1981, 52, 53075310

16 Die Synthese eines Stereoisomers ist beschrieben: Solaja, B.; Huguet, J.; Karpf, M.: Dreiding, A. S. Tetrahedron 1987, 53, 4875 - 4886.

17 (a) Hansen, H. P.; Abraham, W. R.; Tetrahedron, 1988, 44, 2175 - 2180.

(b) Metha, G.; Rao Karra, S. J. Chem. Soc., Chem. Commun. 1991, 1367 - 1368.

(c) Clieve, D. L. J.; Magnuson, S. R.; Manning, H. W.; Mayhew, D. L. J. Org. Chem. 1996, 61, 2095 - 2108.

18 Meyers, A. I.; Bienz, S. J. Org. Chem. 1990, 55, 791 - 798 und dort angegebene Literatur.

19 Weiser, Dissertation, Universität Göttingen 1994.

20 Weiser, J.; Holthausen, M. C.; Fitjer, L. J. Comput. Chem. 1997, 18, 1265 - 1281.

21 Quantum Chemistry Programm Exchange (OCPE), University of Indiana, Bloomington, IN 47405.

22 Dowd, P.; Zhang, W. J. Am. Chem. Soc. 1992, 114, 10084 - 10085.

Dowd, P.; Zhang, W.; Geib, S. J. Tetrahedron 1995, 51, 3435 - 3454.

23 Fitjer L.; Quabeck, U. Synth. Commun. 1985, 15, 855 - 864.

24 Fitjer, L.; Wehle, D.; Scheuermann, H. -J. Chem. Ber. 1986, 119, 1162 - 1173. 
26 Fitjer, L.; Quabeck, U. Synthesis, 1987, 299 - 300.

26 Mayer, R.; Wenschuh, G.; Töpelmann, W. Chem. Ber. 1958, 91, 1616.

Wilcox, C. F.; Whitney, Jr. G. C. J. Org. Chem. 1967, 32, 2933.

27 (a) Eliel, E. L.; Pietruiewicz; K. M. Org. Magn. Res. 1980, 13, 193 - 196.

(b) Hesse, M.; Meier, H.; Zeeh, B. Spektroskopische Methoden in der organischen Chemie, Georg Thieme Verlag, Stuttgart 1987.

28 Whitesell, J. K.; Matthews, R. S. J. Org. Chem. 1977, 42, 3878 - 3882.

29 Brown, H. C.; Hammar, W. J. Tetrahedron 1978, 34, 3405 - 3411.

30 Gassmann, P. G.; Valcho, J. J.; Proehl, G. S.; Cooper, C. F. J. Am. Chem. Soc. 1980, $102,6519-6526$.

31 Billington, D. C.; Kerr, W. J.; Pauson, P. L.; Farnocchi, C. J. Organomet. Chem. 1988, $356,213-219$.

32 Thiele, S.; Erker, G. Chem. Ber. 1997, 130, 201 - 207.

33 Whitesell, J. K.; Matthews, R. S.; Solomon, P. A. Tetrahedron Lett. 1976, 47, 1549 1552.

34 Haufe, G.; Wolf,, A.; Schulze, K. Tetrahedron 1986, 42, 4719 - 4728.

35 Mallien, M.; Haupt, E. T. K.; tom Dieck, H. Angew. Chem. 1988, 100, 1091 - 1092.

36 Baldenius, K. -U.; tom Dieck, H. Angew. Chem. 1992, 104, 338 - 340; Angew. Chem. Int. Ed. Engl. 1992, 31, 305 - 307.

37 (a) Labar, D.; Krief, A. J. Chem. Soc., Chem. Commun. 1982, 564 - 566. 
(b) Labar, D.; Laboureur, J. L.; Krief, A. Tetrahedron Lett. 1982, 53, 983 - 986.

(c) Krief, A.; Dumont, W.; Laboureur, J. L. Tetrahedron Lett. 1988, 29, 3265 - 3268.

(d) Krief, A. Tetrahedron Lett. 1984, 25, 2713 - 2716.

38 Krief, A.; Clarembeau, M.; Cravador, A.; Dumont, W.; Hevesi, L.; Luchetti, J.; van Ende, D. Tetrahedron, 1985, 41, 4793 - 4812.

39 Krief, A. Tetrahedron, 1980, 36, 2531 - 2640.

40 Fitjer, L.; Steeneeck, Ch.; Gaini-Rahimi, S.; Schröder, U.; Justus, K.; Puder, P.; Dittmer, M; Haßler, C.; Weiser, J.; Noltemeyer, M.; Teichert, M. J. Am. Chem. Soc. 1998, 120,317 - 328.

41 Dittmer, M. Dissertation, Universität Göttingen, 1990.

42 Fitjer, L.; Kanschik, A.; Majewski, M: Tetrahedron 1994, 50, 10867 - 10878.

43 Olah, G. A.; Pradeep, S. I.; Prakash, G. K. S. Synthesis, 1986, 513 - 531.

44 (a) Forsyth, D. A.; Mahmoud, S.; Giessen, B. C. Org. Magn. Reson. 1983, 19, 36 - 38.

(b) Weigand, E. W.; Schneider, H. -J. Org. Magn. Reson. 1979, 12, 637 - 644.

(c) Holden, C. M.; Whittaker, D. Org. Magn. Reson. 1975, 7, 125127.

(d) Lysenkov, V. I.; Pekhk, T. I.; Lippmaa, E. T.; Zheleznyak, T. L. J. Org. Chem. USSR 1981, 17, 1436 - 1445.

(e) Grover, S. H.; Marr, D. H.; Stothers, J. B.; Tan, C. T. Can. J. Chem. 1975, 53, 13511361.

45 Pretsch, E.; Clerc, T.; Seibl, J. Simon, W. Tabellen zur Stukturaufklärung organischer Verbindungen mit spektroskopischen Methoden, Springer Verlag, Berlin, Heidelberg, New York, 2. Auflage, 1989.

46 Apt-Pulsseqenz: Patt, S. L.; Shoolery, J. J. Magn. Res. 1982, 46, 535 - 539. 


\section{Danksagung}

Für die Messungen der NMR-Spektren danke ich Frau Carola Zolke, Herrn Ulrich Leonhardt und Herrn Reinhard Machinek.

Dank an Dr. Frauendorf und Frau Györgi Udvarnoki für die Aufnahme der Massenspektren.

Für die Bearbeitung der Elementaranalysen bedanke ich mich bei Herrn Hambloch.

Für die Bereitstellung von ausgewählten Chemikalien, die allgegenwärtige Unterstützung und die vielen Tassen Kaffee bedanke ich mich bei Herrn Ralf Gerke.

Bei Frau Uta Kaiser bedanke ich mich für die nette Gesellschaft im Labor.

Ich danke allen Mitgliedern der Abteilung Fitjer für ihr unkompliziertes Verhalten im Labor, ihre stete Hilfsbereitschaft und das angenehme Arbeitsklima. Onno Graalmann und Klaus Mandelt danke ich für die zahlreichen fördernden Diskussionen. Bei Klaus Mandelt möchte ich mich besonders für seine nette Labornachbarschaft bedanken.

Weiterhin gilt mein Dank für die angenehme Ablenkung und Zerstreuung außerhalb des Labors Almut, Andi, Barney, Cörnholt, Dirk, Fred, Jochen, Katie, Melli, Sandra, Saturn, Stefan und Winny.

Ich danke Gero für sein Verständnis und die moralische Unterstützung.

Bei Sandra und Klaus möchte ich mich für das unermüdliche Korrekturlesen bedanken.

Mein besonderer Dank gilt meinen Eltern, die mir dieses Studium überhaupt erst ermöglicht haben und für ihre Unterstützung während des gesamten Studiums. 


\section{Lebenslauf}

Am 10.02.1971 wurde ich, Imelda Meyer, in Emstek als Tochter von Marlene Meyer, geborene Wehry, und Clemens Meyer geboren. Von 1977 - 1981 besuchte ich die Grundschule Emstek, von 1981 - 1983 die Orientierungsstufe in Emstek und von 1983 - 1990 das Gymnasium der Liebfrauenschule in Cloppenburg, wo ich 1990 die Allgemeine Hochschulreife erwarb. Im Wintersemester 1990 nahm ich das Chemiestudium und im Wintersemester 1993 das Biologiestudium an der Georg-August-Universität in Göttingen auf. Im Juni 1995 bestand ich dort die Diplomvorprüfung im Fach Chemie, im Oktober 1995 die Diplomvorprüfung im Fach Biologie. Unter der Leitung von Prof. Dr. L. Fitjer begann ich im Dezember 1997 am Organisch Chemischen Institut in Göttingen mit meiner Diplomarbeit über das Thema „Versuche zur Darstellung acyclisch 1,1-verknüpfter Quinquecyclobutane als potentieller Vorläufer von Tetraspiranen mit einer helicalen Primärstruktur“ und legte im Oktober 1998 die Diplomprüfung im Fach Chemie ab. Seit April 1999 arbeite ich im gleichen Arbeitskreis an meiner Dissertation. 